Abstracts
$\begin{aligned} & \text { www.anatomy.org.tr } \\ & \text { doi:10.2399/ana.11.001s }\end{aligned}$

\title{
Abstracts for the Joint Meeting of Anatomical Societies, 19-22 May 2011, Bursa, Turkey
}

\section{Opening Lecture}

\section{New genoarchitectonic viewpoints on the developing hypothalamus}

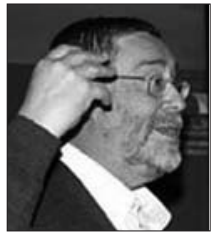

$\underline{\text { Puelles L }}$

Department of Human Anatomy, Faculty of Medicine, University of Murcia, Murcia, Spain

The anatomic concept of the hypothalamus changed considerably since its earliest definition. Tridimensional reconstructions, experiments and many staining methods have expanded considerably the number of anatomical details recognized in this territory, probably one of the most complex in the brain. For a long time the predominant anatomic view has interpreted the hypothalamus as a longitudinal column at the floor of the diencephalon, connected rostrally with the telencephalon and caudally with the midbrain. However, recent advances in our knowledge of early developmental mechanisms seem increasingly contradictory with this columnar viewpoint. Consideration of early ventralization, dorsalization and anteroposterior patterning effects suggests that, rather than being a diencephalic floor element, the hypothalamus is best understood as a transverse region lying ventral to the telencephalon and rostral to the diencephalon; the latter separates it from the midbrain. A number of gene expression patterns observed in the developing forebrain, part of the emergent genoarchitectonic neuroanatomy, have revealed the true topologic position of the hypothalamus, as well as the nature of its fundamental subdivisions. There are interesting parallelisms with genoarchitectonic patterns in the diencephalon and midbrain. In all these cases continuous longitudinal domains can be distinguished, as well as a number of anteroposterior (transverse) neuromeric units of the neural wall. The hypothalamus has been newly recognized to have two anteroposterior neuromeric subdivisions, named terminal and peduncular hypothalamic portions. These ideas give meaning to the tract trajectories through the hypothalamus and serve to localize more precisely the complex chemical structure and circuitry of this brain region. 


\section{Plenary Lectures \\ (PL-1 - PL-9)}

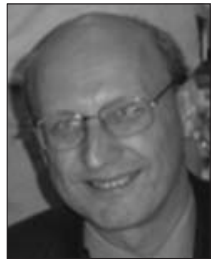

\section{PL-1}

Student directed learning of gross anatomy: how to distinguish between teaching and learning

Moxham BI

Cardiff School of Biosciences, Cardiff University, Cardiff, Wales, United Kingdom

There has been much debate (and controversy) concerning the role of the teacher in biomedical education and training and this has led to many medical courses changing radically to comply with the ideology of "student-directed learning" (SDL). Although there are many possible variants of SDL, most educationalists take this to be synonymous with "problem based learning" (PBL). For this presentation, the variety of SDL approaches will be considered and a critique levelled at PBL as the prime means of acquiring anatomical/medical knowledge and skills. Furthermore, a new approach will be described that, building on the idea of modularisation of courses, sees the development of "shadow modules" run by the students themselves to complement teaching from within standard modular arrangements. The shadow modules will have student shadow module leaders and be provided with a collaboration platform through a universally accessible web portal (including mobiles and tablet PCs). This portal will enable students to recognise, and develop, appropriate learning resources and will encourage student discussion and group learning (as well as gather valuable feedback for module improvement). This arrangement accords with recent technology and pedagogy developments in virtual learning environments (VLEs), including Sakai Open Academic Environment, Blackboard Collaborate (Currently Project Gemini) and Moodle 2.0, and with new websites such as OpenStudy.com where more personalised, social and streamlined teaching and learning experiences are available. Thus, it is proposed that a division of labour is most effective for medical and biomedical courses that involves teachers being responsible for developing syllabuses, teaching methodologies and assessment arrangements while students are empowered to develop learning strategies, learning tools and extramodular resources (e.g. internet resources) that befits their academic and generational needs. Teachers of modules and students on shadow modules should come together to audit arrangements and to devise learning outcomes.

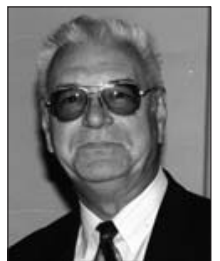

PL-2

Features in teaching human anatomy in Russia

Kolesnikov LL*, Kulikov VV**

Department of Human Anatomy*, Moscow State University of Medicine and Dentistry, Moscow, Russian Federation; Department of Normal Human Anatomy**, Russian State Medical University, Moscow, Russian Federation

The Human Anatomy is taught in 50 Medical Schools of Russian Federation. Total number of students is more than 25 thousands. Each Medical school has a Faculty of Medicine, 37 medical schools have a Faculty of Pediatrics and 30 medical schools have a Faculty of Dentistry (Dental school). Nowadays anatomy at the Faculties of Medicine is taught during first 3 semesters (280 hours totally including 72 hours of lectures plus 140 hours of independent studies.) Histology (180 hours) and topographic anatomy (65 hours) are taught as separate courses. The curriculum of Human Anatomy is determined by the State Educational Standard which is renewed every 5 years. The last Standard corresponds to the "Bologna's consensus". Anatomy occupies traditionally a special place in the higher medical education. It is studied in a systematic way in the following sequence: locomotor apparatus (1-st semester), splanchnology and CNS (2-nd semester), cardiovascular system and PNS (3rd semester). Traditionally Anatomy in Russia is not regarded as a merely descriptive discipline. Its teaching is based on major didactic approaches - historicism, systemic approach, functionality, actuality, sufficiency, necessity and clinical orientation. The anatomical structure is mostly considered to be the component of the ordinal systems in their historical and ontogenic dynamics and functional interrelations. The content of the course should be consistent with the recent concepts relating to the morpho- functional organization of the human body components. It should be sufficient and necessary for further study of basic and clinical disciplines. The ratio of these approaches may vary in different Russian medical schools.

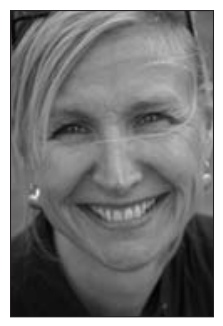

\section{PL-3}

An innovative approach to teach anatomy: the Teatrum Anatomicum experience

Böckers A

Institute of Anatomy and Cell Biology, UIm University Faculty of Medicine, UIm, Germany 
Modern anatomical teaching approaches are embedded in the clinical context today. Teaching methods should alleviate the learning process, increase learning motivation and provide additional skills and attitudes of medical professionalism. Therefore, new information should be based on already learnt knowledge, should stress its clinical relevance to improve learning motivation and should be presented in an empathetic learning environment by role models. The "Theatrum anatomicum" offers an excellent place for this. Premedical students come into contact with clinicians and learn anatomy. The idea of an anatomical theatre is not basically new, however, in 2008 it was reactivated at Ulm University for the first time again in Germany. Faculty and student support made it possible to build an operating theatre which includes a grandstand for 50-60 spectators adjacent to the dissection lab. Clinical colleagues of different disciplines like neurosurgeons instruct students to perform or assist an operation on Thiel fixated cadavers. A high-quality video and audio transmission allows the spectators to pursue the action in the operation field simultaneously. Based on the already gathered knowledge in seminars and the gross anatomy course this clinical experience motivates students to learn and transfer anatomical knowledge into the presented clinical case. Anatomical structures must be recognized and be spared during the surgical procedure, while at the same time the craft challenges of a professional surgical action are experienced. The "Theatrum anatomicum" creates an empathic learning environment between students, who for the first time in their lives enthusiastically operate on a body donor while the surgeon experiencing no time pressure or a risk to harm the patient enjoys teaching. Finally, this setting allows to take over a doctor's social roles of being a team player, teaching instructor and the person responsible.

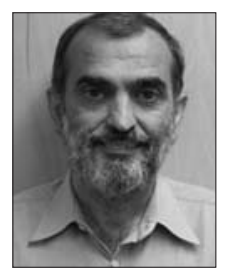

\section{PL-4}

\section{Bridges between neuroanatomy and neuropathology}

$\underline{\text { Salamat MS }}$

Department of Pathology and Laboratory Medicine, University of Wisconsin Medical School, Madison, Wisconsin, USA

The basic structure of the North American system of allopathic medical education was established a century ago and has essentially remained unchanged. This system, which is sought after by students worldwide, was organized around the principal of teaching sciences and included a four-year training program consisting of two years of basic sciences education followed by two years of clinical sciences. Since the early 20th century, both basic and clinical sciences have become more diverse and each area has seen immense advancements. In earlier decades, anatomical sciences, physiology, pathology and pharmacology were the bulk of training in the first two years of medical school, supplemented by smaller courses such as chemistry, bacteriology and clinical medicine. With time, all these subjects expanded, and the new information was incorporated into the medical school curriculum. Fifty years later, by the middle of the second half of the 20th century, newer sciences such as biochemistry, genetics and psychology were added, and toward the end of the century, a cascade of new knowledge on the molecular basis of normal and abnormal structure and function was also being delivered in the medical school classrooms. Despite this increased volume of basic sciences knowledge, which needs to be taught in medical school, the initial two years of basic sciences training has not remained exclusive to basic sciences. Over time, there has been a push to bring more "relevant" clinical sciences into the earlier years of medical training. Perhaps, in early 70s, neuroscience courses that combined neuroanatomy with clinical aspects of the diseases of the nervous system were the beacons of such changes. Also monetary rewards that medical schools allocated for teaching departments, have resulted in greater take-over of basic science training by the clinical faculty, further diluting basic sciences. In the 21 st century, population expansion and increasing costs of health care have become a major concern worldwide. National medical education policy makers press for and provide greater funding for training programs that produce a larger number of generalists than specialty physicians. Medical schools prioritize teaching skills that enable students to work in rural and underprivileged environments such as minority languages and business skills, which further decrease the time available to provide a solid foundation in the basic sciences. No matter how it is regarded, there has been a progressive decline in basic sciences education in medical schools since at least the 70s. One reflection of this is the lack of knowledge of anatomy, and specifically neuroanatomy, exhibited by present-day medical students who reach the clinical years. The teaching of neuroanatomy has been reduced to a level such that, in some medical schools, students have only one lab covering the gross examination of the brain. Even the medical students are becoming unsatisfied with the minimal education they are receiving in these fields. In an era where the education of young physicians is being pulled in different directions, one should not forget that no matter what the societal needs for physicians may be, a quality education should not be sacrificed. Given the ample post-graduate medical training opportunities that are available, it behooves us to protect the quality of basic sciences education in medical schools by providing medical students with both sufficient exposure time to the material and qualified basic science instructors to take charge of basic science training. 


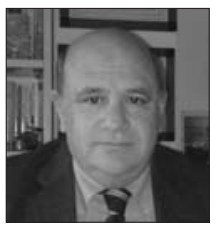

\section{PL-5}

The effect of thyroid hormone and gonadotropins on angiogenesis in the ovary

Macchiarelli G

Department of Health Sciences, Faculty of Medicine, University of L'aquila, Italy

Thyroid hormone may exert a pro-angiogenic effect, as firstly described in studies of T4 induced cardiac hypertrophy. In the ovary several endocrine factors finely regulate follicular and luteal development, ensuring the reproductive success. An important role is exerted by thyroid hormone and gonadotropins, both involved in vivo and in vitro in the regulation of follicle development, ovulation and corpus luteum formation as well as in the pro-angiogenic action necessary to provide an optimal vascular supply during the menstrual cycle and pregnancy. We recently demonstrated, using an infertile model of female hypothyroid rdw rats, that T4 in combination with gonadotropins promotes the development of follicular and luteal microvasculature by up-regulating gene expression of major paracrine angiogenic factors, as VEGF (produced by endothelial cells), bFGF (produced by endothelial cells) and TNF $\alpha$ (produced by smooth muscle cells as pericytes). Numerous pericytes were associated to capillary endothelial cells, mainly in correspondence of sprout origin. The sprout formation was embraced and accompanied by the long pericytic cytoplasmic processes. We suggest that in the ovary the pro-angiogenic effect exerted by thyroid hormones associated to gonadotropins is locally mediated by VEGF, bFGF and TNF $\alpha$ that actively drive this process by increasing capillary sprouting, tube elongation and pericyte recruitment to stabilize the new formed vessels.

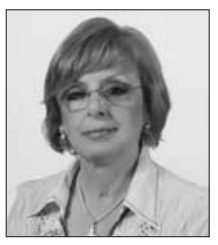

\section{PL-6}

Structural neural tube malformations
leading to functional and behavioral
defects

\section{Emmanouil-Nikoloussi EN}

Laboratory of Histology-Embryology, School of Medicine, Aristotle University of Thessaloniki, Thessaloniki, Greece

Neural tube congenital anomalies are represented as major or minor structural defects. Diverse teratogenetic drugs are causative factors for neural tube birth defects. The aim of this paper is to describe several neural tube defects observed in experimental animal embryos', after teratogenic drugs' administration during diverse timing of gestational life. The congenital anomalies described were registered during our diverse original experimental protocols applied to study teratogenetic pathways of birth defects in experimental animal models. Neural tube anomalies were studied by Histochemical, Immunohistochemical and Electron microscopical methods. Brain defects expressed after teratogenic drug administration to experimental animals, included exencephaly, brain myeloceles, hydrocephalous, anopthalmia or/and micropthalmia. Eye malformations, were always associated with exencephaly and mostly with holoprosencephaly. From all registered brain defects, holoprosencephaly has been the most common brain malformation occurred. Holoprosencephaly of the forebrain, results from a failure to completely septate the left and right hemispheres at the rostral end of the neural tube. As during craniofacial development diverse tissue interactions occurred, which drove cranial vault development, these forebrain defects were accompanied by midline deficiencies of craniofacial structures expressed as orofacial clefts. Neural tube defects affecting spinal cord, were always expressed in combination with vertebrae anomalies and morphologically expressed mostly as spina bifida aperta, or spina bifida occulta. Teratogenic drugs are involved in the control of many developmental processes. Excess and deficiency of them are considered as teratogenic mostly at the Central Nervous System (CNS).The registered structural brain and spinal cord anomalies cannot be considered as incidental findings but must be characterized as an intrinsic part of neural defects occurring during mammalian evolution. This condition, related to drug administration effects' and/or genetic mutations additionally, can lead from structural to functional and behavioral defects of the neonate in experimental animals.

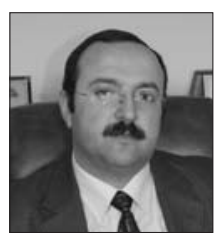

PL-7

Whole rat embryo culture; as a tool for teratological screening

Karabulut AK

Department of Anatomy, Selcuklu Medical Faculty, Selcuk University, Konya, Turkey

Birth defects induced by maternal exposure to exogenous agents during pregnancy are preventable, if the agents themselves can be identified and avoided. The number of candidate chemicals or drugs for registration and autorization is increasing at a fast rate and only few of the existing substances have been tested for teratogenicity to date. Whole rat embryo culture (WEC) as a screening system for safety evaluation procedures was considered as promising alternative test for developmental toxicity and teratogenicity assays. Over the past 30 years, the WEC system has been developed, optimized, and validated as an in vitro teratogenicity assay. According to this method, the rat embryos were cultured in rotated culture bottles during the early organogenesis, typically over a $48 \mathrm{hr}$ period. The culture medium is supplemented with serum and specific percentages of oxygen over the culture period. Test compounds are added into the culture medium. At the end of the culture, embryos are examined and evaluated for abnormalities of developmental morphology and overall embryonic growth. To quantitate embryonic growth and development, six developmental stages of series of structures are defined and scored from 0 to 5 . The total morphological score in combination 
with the other measurable evaluation parameters such as somite number, crown-rump length, yolk sac diameter and protein content reflects the degree of growth and differentiation of the embryo. Furthermore, teratologic doses of the test compounds can be evaluated. The WEC system is considered a well established in vitro model for identifying and characterizing teratogenic properties of test compounds. The successful validation of the procedure by the European Center for the Validation of Alternative Methods (ECVAM) in 1995, makes the WEC a complex in vitro embryotoxicity test with high accuracy and predictability.

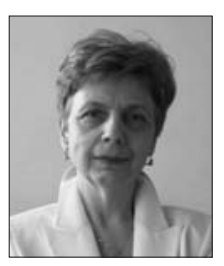

\section{PL-8}

Anatomy - An important tool in cardiologists' decision making

Teofilovski-Parapid G

Institute of Anatomy, School of Medicine, University of Belgrade, Serbia

Although sometimes anatomy might looks like as a subject which importance belongs to the past it is not true. The idea can cross the minds only of those with very specific, hate to say narrow, biomedical education who are nowadays, due to the complexity of economical situation on one side and the demands of science on the other, greatly involved in research and stepping in education of medical students. At the same time, clinicians are at first site doctors and afterward scientists. Shaping their opinion is not that easy, though from time to time astonished by discoveries at micro and molecular levels they can neglect the importance of gross anatomy. Among them, invasive cardiologists are very special. Being exposed to fast decision makings situations they behave very much like surgeons, although doubting the contemporary importance of cardiosurgery, but the best among them appreciate the complexity of knowledge and data that anatomy can provide these days. Our experience is very interesting. In early nineties, we anatomists were the first to call attention of our cardiosurgeons on tunneled coronary arteries and their branches, and we got the information that it has not been frequently seen in our population. The data in literature could support that view; we were the first to call attention of our interventional cardiologists but they have not been excited with that issue as well. However, the autopsy studies that we carried on a relatively modest sample but with revealed incidence of tunneling phenomenon of more than $1 / 3$ of cadavers examined encouraged retrospective analysis of the records, from over 500 consecutive autopsies which at first site surprised us with almost ten times lesser incidence of the phenomenon revealed. Nevertheless, both studies had in common presence of athero- sclerotic lesions proximal to the myocardial bridge (MB), although rarely in the tunneled part of the vessel. Furthermore, the prospective studies revealed relatively frequent presence of multiple MBs in the single heart. Finally, the advances in technology, and coronary computed tomography angiography (CCTA) using multidetector computed tomography (MDCT) scanner enormously improved the visualization of MBs, and all of a sudden increased in vivo diagnosed incidence. Thus, radiologists helped us a lot in convincing interventional cardiologists of the importance of conducting the investigations on tunneling coronary arteries in our populations. The first results with the incidence detected on the coronary angiography much higher than once thought to be, although lesser then on our autopsy studies, have been encouraging. Furthermore, we made them to be aware of multiple presence of MBs in the single heart which is of importance for planning and selecting the therapeutic approaches; of a high risk of coronary perforation during the angioplasty procedure that can be expected due to the extremely thin intima in the tunneled segment of the vessel, and not to mention high risk of restenosis after the stenting, along with extremely severe atherosclerosis lesion proximal to the stent. In months to come, we expect to enrich our knowledge by integrating results from classic coronary angiography with those obtained by MDCT angiography and by IVUS in the same symptomatic patients. In this way, anatomy finally got the place it deserves i.e. in every day interventional cardiologists' decision making situations.

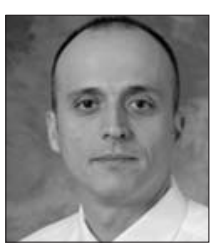

PL-9

Importance of topographical neuroanatomy and skull base anatomy teaching in training neurosurgery residents

Bașkaya MK

Department of Neurological Surgery, School of Medicine and Public Health University of Wisconsin-Madison, Madison, Wisconsin, USA

Despite the advances in technology, the main stone in determining the outcome in neurosurgery is still the individual skill and technique of the neurosurgeon. This is especially pronounced in the lesions involving critical regions or deep-seated locations which are extremely difficult to access. Beyond the surgical technique, the another important determinant in the outcome is the anatomical location of the lesion, the region involved by the lesion and the anatomy of its neighboring structures. In this presentation the importance of the almost forgotten science of topographical gross anatomy, neuroanatomy's contribution in clinical neurosurgery as a basic science and its vital role in resident training will be highlighted with accompanying case presentations. 


\section{Symposium 1}

\section{Neuroanatomy Education}

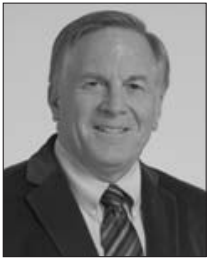

Neuroanatomy/neuroscience education in United States medical schools

Drake R

Cleveland Clinic Lerner College of Medicine, Case Western Reserve University, Cleveland Clinic, Cleveland, Ohio, USA

In our most recent survey of anatomical sciences education in United States medical schools, courses in Neuroanatomy / Neuroscience had the largest drop in total course hours when compared to our previous survey (Drake et al., Anat. Rec. 269:118-122, 2002). Evidence suggests that a major component of this change is related to a sizable decrease in laboratory hours. Additionally, when looking at survey data from 1955 to 2009 it appears that over this time period changes in this discipline may relate to shifts from strictly Neuroanatomy courses to multidisciplinary Neuroscience courses. Other factors impacting not only Neuroanatomy / Neuroscience courses, but also all courses in the anatomical sciences, include changes in presentation styles, the increased use of technology, educational environments becoming more interactive, and a closer connection between the clinical and basic science curriculum. The influence of these factors and how they will shape teaching in the future will be discussed.

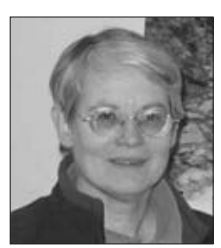

\section{The development of a core syllabus for the teaching of neuroanatomy to medical students}

$\underline{\text { Plaisant } \mathrm{O}^{*},}$ McHanwell S**, Moxham BJ ${ }^{\star * *}$ Laboratoire d'anatomie*, Faculté de Médecine, Université Paris Descartes, Urdia, Paris, France; School of Dental Sciences**, Framlington Place, Newcastle on Tyne, United Kingdom; Cardiff School of Biosciences***, Cardiff University, Cardiff, Wales, United Kingdom

Increasingly, there is a call for clinical relevance in the teaching of biomedical sciences within a medical course. This presupposes that there is a clear understanding of what can be considered "core" material within the medical syllabus. To date, the anatomical sciences have been relatively poorly served by the development of "core" syllabuses. There have been several commendable attempts to define a "core" syllabus in gross anatomy in Europe and the USA, most recently by the Anatomical Society in the UK and Ireland. More specialised core syllabuses are rarer. In association with the TransEuropean Pedagogic Anatomical Research Group (TEPARG), we are engaged in developing a "core" syllabus for neuroanatomy by means of a "Delphi Panel" that consists of anatomists, neuroscientists and clinicians who evaluate syllabus content and accord each element "essential", "important", "acceptable" or "not required" status. This work is ongoing and we present at this conference the concept underlying the investigation and the preliminary findings. The study, while concentrating on the medical curriculum, is also being developed to assess core neuroanatomical knowledge in the dental curriculum.

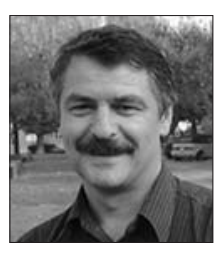

The role of plastination for teaching neuroanatomy

\section{Riederer BM}

Department of Cell Biology and Morphology, Faculty of Biology and Medicine, University of Lausanne, Lausanne, Switzerland

Plastination is also called polymer impregnation of tissue either with silicon S-10 or other polymers such as P-40 and allows a longterm preservation of well dissected body parts or slices. This technique is especially useful for brain tissue and for teaching human neuroanatomy. Here, I will address several points, i) ethical considerations and precautions to foresee in the donation program, ii) some technical aspects and advantages of different plastination techniques, and how to use it in practical teaching, or even outside the dissection room; iii) plastinated specimens are more or less odorless and allow a three-dimensional visualization of complex structures. Yet, there are not only advantages, since soft tissue tends to shrink and becomes more rigid the advantage of solid tissue turns into a disadvantage when it comes to showing the auditory cortex or insula. In addition, plastinated specimens may provide a technical support for the preparation of surgeons when it comes to access frontal cortex areas or brain stem via nasal sinuses. Plastinates of well prepared brain parts or thin tissue sections provide superb teaching tools and are a good complement to wet specimens, in dissection and postgraduate courses and medical imaging and are very much appreciated by staff and students.

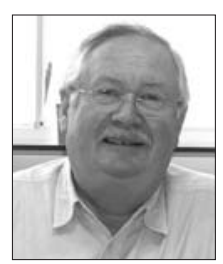

\section{Acquisition and retention of neuroanatomical knowledge by medical and science undergraduate students}

$\underline{\text { Santer RM }}$

\section{School of Biosciences, Cardiff University, Cardiff, United Kingdom}

Introduction and purpose: undergraduates educated in many European schools enter university with a scant knowledge of the nervous system, yet they are required to develop a reason- 
ably detailed knowledge, particularly of CNS neuroanatomy, by the end of their second year of study. This applies equally to medical students and to science students reading for a degree in neuroscience and/or physiology. Neuroanatomy is traditionally perceived by students as being a challenging and difficult discipline. Few would disagree that the structure and connections of the CNS is astonishingly - and indeed wondrously - complex and presents a serious challenge to any student. Methods: Year 1 and year 2 medical and science students have been surveyed to determine the extent of their knowledge of neuroanatomical terminology on entry to university and to assess their progress in learning neuroanatomy over the su, cceeding two years at university. Results and interpretation: The results for students in their first two years are encouraging as they perceive the need to acquire neuroanatomical knowledge ahead of examinations necessary to progress as undergraduates. In conjunction with this survey, medical and science students have been surveyed in order to explore their attitudes towards the importance of an understanding of neuroanatomy in relation to their chosen career pathway, their problems in learning neuroanatomy and their suggestions on how the delivery of the subject could be altered to improve their performance. Retention of neuroanatomical knowledge has been investigated by analyzing examination results of science students at the end of Year 2 and by surveying medical students' ability to answer Year 2 examination questions at the end of Year 4 of study. These results are not encouraging for teachers of neuroanatomy who will need to develop strategies to assist students in learning and retaining a vital basic and/or clinically relevant core of neuroanatomical knowledge.

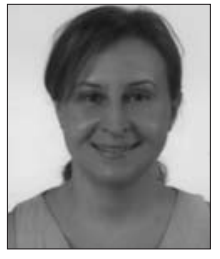

A new approach in neuroanatomy education: teaching based on contemporary neuroscience research rather than old-style medical school neuroanatomy teaching

Ş̧engül G

Department of Anatomy, School of Medicine, Ege University, Bornova, Izmir, Turkey

Neuroanatomy is regarded as one of the cornerstones of basic medical science. With the advance of neuroscience methods and imaging techniques, there is an enormous increase in neuroanatomical knowledge. This makes neuroanatomy education even more challenging. Students often complain about the dryness of the subject and the difficulties they encounter when trying to memorize all the terms, areas and connections. This talk presents a new approach in neuroanatomy education: teaching based on contemporary neuroscience research rather than oldstyle medical school neuroanatomy teaching.Instead of commencing with the anatomy of the human brain, teaching starts with a presentation of the organization of a simple mammalian brain, that of the rat.Apart from the cerebral cortex which is highly developed in humans and a few mammals, the human and rodent brains have all the basic features of a mammalian brain. Also, many important studies on the cause of human brain disease have been based on work on the rat brain. When concepts from molecular biology, pathology, physiology, neurological diseases, neuroscience research or operative procedures are introduced to neuroscience teaching, this will serve to stimulate a curiositydriven approach and motivation, and thus improve quality of teaching.

\section{Symposium 2 Translational Research in Epilepsy}

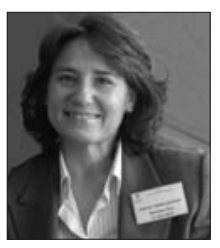

\section{The role of thalamus in epileptic network \\ Çavdar S \\ Departmet of Anatomy, Faculty of Medicine, Marmara University, Istanbul, Turkey}

Thalamus, is an oval shaped gray mass located one on each side of the third ventricle. The internal medullary lamina divided the thalamus into nuclear groups, each thalamic nucleus is assosiated with different functions. Each thalamic nucleus sends impulses to spesific regions of the cortex. Recent studies on different epilepsy types have shown that thalamus plays an important role in the mechanism of epilepsy. We will deal with two examples one absence epilepsy which is classified as generalized seizure and mesiotemporal lobe epilepsy (MTLE) or limbic epilepsy is classified as complex partial seizure. In the absence epilepsy the primary network has been defined as resiprocal circuit involving neocortex (corticothalamic) and thalamic relay nuclei (thalamocortical) with the TRN serving as a key modulator. The TRN is composed of GABAergic neurons. The $75-85 \%$ of the thalamic nuclei are composed of relay and $15-25 \%$ of the neurons are composed of interneurons. Thalamic relay neurons can fire in tonic or burst modes. The tonic mode is excitatory and transfer of linear information occurs in the awake state whereas burst mode is inhibitory and transfer of non-linear information as it is in sleep and epileptic discharges states. TRN plays an important role in which phase the thalamic relay neurons will fire. In MTLE seizure onset was shown to be associated with hippocampus in the temporal lobe. However, there is increasing evidence that other limbic structures such as amygdala, entorhinal cortex, perirhinal cortex, neocortex and thalamus plays an important role in the initiation, propagation and spred of seizures. Recent experiments has supported the concept that the thalamus, particularly the midline thalamus takes part in the limbic seizure circuit. Several limbic sites initially interacts with one another independently. The mid- 
line thalamus has reciprocal connections with all these regions, the midline thalamus constitute part of the initiating circuit, acting as a subcortical synchronizing site. Generalization can occur through spred to the adjacent neocortex.

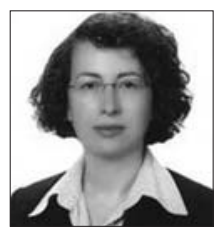

\section{Bridging basic with clinical epilepsy}

\section{Onat F}

Department of Pharmacology and Clinical Pharmacology, Faculty of Medicine, Marmara University, Istanbul, Turkey

Non-convulsive seizures are suggested to be related to a predominance of inhibitory activity, and typically characterized by spike-and-wave discharges in the EEG, whereas convulsive seizures are related to predominance of excitatory activity. The coexistence of idiopathic generalized typical absence epilepsy and partial temporal lobe epilepsy in the same patient is extremely rare. In agreement with this clinical observation experimental studies of temporal lobe epilepsy in models of genetic absence epilepsy have shown a resistance to secondary generalization of focal limbic seizures. In Genetic Absence Epilepsy Rats from Strasbourg (GAERS) or Wistar Albino Glaxo Rats from Rijswijk (WAG/Rij) rats, both well-validated genetic models of absence epilepsy, high level of spike-and-wave discharge activity correlates negatively with kindling rates. This demonstrates that a high level of spike-and-wave discharge activity in absence epilepsy reduces the effectiveness of kindling. We have further evaluated the interplay between absence epilepsy and limbic epilepsy by using intraamygdaloid injection of kainic acid in adult GAERS as another model of temporal lobe epilepsy to compare with intraamygdaloid kindling. In the acute period the first convulsive seizure was significantly delayed in GAERS compared to control animals. Thereafter, the animals in both groups became epileptic although convulsive seizures were delayed for GAERS relative to $W$ istar rats. There is no difference in the survival rates of Wistar and GAERS. Intensive basal spike-and-wave discharge activity in GAERS was completely suppressed immediately after kainic acid injection and gradually reappeared. Our findings indicate an interaction between 2 types of epilepsy and further a mutual cross inhibition of circuits involved in absence epilepsy and temporal lobe seizures.

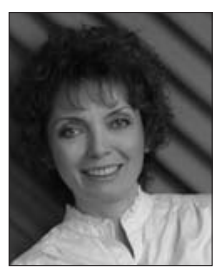

\section{Generalised and focal epilepsies in human}

\section{Özkara C}

Department of Neurology, Cerrahpaşa Medical School, Institute of Cardiology, University of Istanbul, Turkey

An epileptic seizure is a transient occurrence of signs and/or symptoms due to abnormal excessive or synchronous neuronal activity in the brain. Seizure is a symptom with an incidence of approximately $80 / 100,000$ and lifetime prevalence is $9 \%$ per year. Epilepsy is a chronic condition of the brain characterized by an enduring propensity to generate epileptic seizures, and by the neurobiological, cognitive, psychological, and social conse- quences of this condition. Incidence: approximately 45/100,000 per year with point prevalence: $0.5-1 \%$ There are several etiological factors that gives rise to epilepsy which may differ according to the age including pre and post natal trauma, metabolic disorders, vascular malformations, tumor, malformations of cortical development, stroke, degenerative diseases, infections etc. Seizures and epilepsies are classified as partial or generalized. Generalized epileptic seizures are conceptualized as originating at some point within, and rapidly engaging, bilaterally distributed networks. Such bilateral networks can include cortical and subcortical structures, but do not necessarily include the entire cortex. They can be tonic-clonic, absence, myoclonic, clonic, tonic and atonic types and usually have genetic etiology. Focal epileptic seizures are conceptualized as originating within networks limited to one hemisphere and could be genetically determined or related to structural lesions in majority of the cases. There are descriptors of focal seizures according to the degree of impairment during a seizure. Furthermore, epilepsies are classified according to their etiology as genetic, structural/metabolic and unknown. There is a list of epileptic diseases and syndromes which could be identified up to date and arranged by age at onset including some constellations.

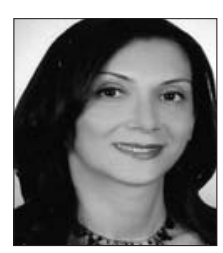

\section{Imaging of epilepsia}

\section{$\underline{\text { Kara SA }}$}

Department of Radiology, School of Medicine, University of Kırıkkale, Kırıkkale, Turkey

Patients with intractable epilepsy represent a challenging clinical population. Recent advences in neuroimaging, with a multimodality imaging approach that combines 18 fluorodeoxyglucose positron emission tomography 18 FDG PET, diffusion tensor imaging (DTI), and magnetic resonance imagin(MRI) continue to improve evaluation, diagnosis and treatment in epilepsy patients and seizure disorders. Potential advantages of imaging patients at $3 \mathrm{~T}$ MR include better image quality with good signal to nose ratio and spatial resolution even for a small field of view. These are also helpful in imaging small children and infants with varying body sizes. These advances are increasing the understanding of the underlying disease process and improving the ability to noninvasively detect epileptogenic foci that in the past went undetected and whose accurate localization is crucial for a good outcome following surgical resection. Magnetic source imaging, like MR/FDG-PET fusion imaging, allows the detection of many subtle abnormalities that may otherwise go undetected with conventional MR imaging. Focal cortical displazi (FCD) is now recognized as one of the most common causes of seizures in patients espacially in children with intractable epilepsy, accounting for nearly $80 \%$ of all surgically treated cases in children under 3 years of age. Regardless of the underlying disease, patients in whom the lesions are visualized at preoperative MR imaging tend to have a beter outcome after surgery for epilepsy than do patients in whom the lesions are not delineated with MR imaging, so imaging of anatomical detail in epilepsy patients is very important for treatment, surgery and outcome. 


\section{Symposium 3}

\section{Steroids in Brain}

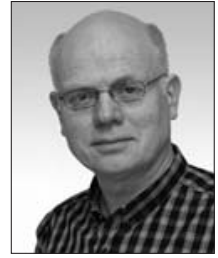

The role of steroid-hormone binding globulins in brain

Caldwell JD*, Jirikowski GF**

Lake Erie College of Osteopathic Medicine*, Erie, PA, USA; Department of Anatomy**, Friedrich Schiller Universität Jena, PF, Jena, Germany

Several steroid-binding globulins, including sex hormone binding globulin (SHBG), corticosteroid binding globulin (CBG), and vitamin-D binding protein (DBP), have been discovered in the brain. All three of these are expressed in the hypothalamus, an area important for control of reproductive endocrinology, stress, and the behaviors that mediate these physiological states. We propose that these proteins, much like intracellular steroid receptors, mediate physiological effects of steroids in the brain. As evidence of this we have demonstrated that infusions of binding globulins into the brain resulted in their being taken up by select brain cells and also in activating specific reproductive behaviors. SHBG infused into the lateral cerebroventricles was taken up by specific cells near the lining of the third ventricle, by cells of the subfornical organ, and the paraventricular nucleus. Infusing SHBG into the reproductively important medial preoptic area and medial basal hypothalamus resulted in significantly elevated reproductive behaviors in female rats. When SHBG was pre-incubated with the androgen steroid dihydrotesterone (DHT), it no longer activated female sexual behavior, suggesting that there is a receptor for SHBG in brain that binds SHBG but not SHBG bound to DHT. Further evidence of a receptor for SHBG in brain was the isolation using affinity purification of 70 and $160 \mathrm{kDa}$ bands that were eluted from SHBG bound on the column by DHT. One postulate is that steroid-binding globulins like SHBG are produced by brain cells to sequester excess steroids away from cells. However, this does not agree with our analysis of SHBG mRNA levels. If SHBG is important in sequestering excess steroids, then one would expect its production to be elevated under high steroid conditions; but high concentrations of estradiol actually significantly reduced SHBG production, as occurs with intracellular estradiol receptors in brain. This suggests that SHBG, like intracellular steroid receptors, is important for neurophysiological actions of steroids. CBG is also found in the limbic system, suggesting that it is involved in the endocrine response to stress as well as modulating behaviors important for stress responsiveness. Like SHBG, CBG may be important for delivery of glucocorticoids (GC) to intracellular receptors. However, in vitro analysis suggests that at least some cells lacking in GC intracellular receptors produce and internalize CBG. We demonstrated that the GC cortisol stimulated rapid release of CBG from glioblastoma cells. Our findings suggest that steroid-binding globulins are made in neurons and released in response to stimulation by steroids, once released they bind extracellular steroids and then the combination is bound by membrane receptors on the same cell or different brain cells. Binding to receptors on the cell membrane then results in changes in intracellular second messengers and/or results in internalization of the steroid-binding globulin with its steroid, this then may move within the cell to specific cell organelles, to other intracellular steroid receptors, or transport steroids into axons.

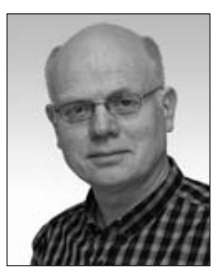

\section{Steroid actions in the brain, a morphologist's point of view}

Jirikowski GF

Department of Anatomy, Friedrich Schiller Universität Jena, PF, Jena, Germany

Gonadal and adrenal steroids as well as vitamin D are among a group of hormones known to act directly on the brain. Being abundant in the systemic circulation and due to their lipophilic nature, most steroids are capable of crossing the blood brain barrier. Thus they seem to be predestined peripheral mediators of central functions including behavioural changes. Sexual and maternal behaviours as well as central stress response have been linked to altered levels of systemic steroid hormones. Numerous neuroanatomical studies assessed the distribution of nuclear steroid receptors in various brain regions because the classical concept of steroid action involves the direct genomic effect of the steroid-steroidreceptor-complex which is known to function as transcription factor. Most of these studies ignored the fact that steroid effects on the central nervous system are in mostly rapid, probably mediated through membrane receptors. Such receptors have been postulated but not unequivocably shown sofar. Hypothalamic neuroendocrine nuclei respond to steroid treatment with increased liberation of neuropeptides although these neurons lack nuclear receptors. Recent studies demonstrated the expression of metabolic enzymes in hypothalamic neurons, indicating intrinsic synthesis of steroid hormones in the brain, now termed "neurosteroids". Aromatisation of testosterone to estradiol in the fetal central nervous system is likely to be responsible for gender specific brain development. In addition to established genomic effects, nongenomic steroid actions, which involve membrane receptors may be important for developmental and functional importance of steroid hormones. 


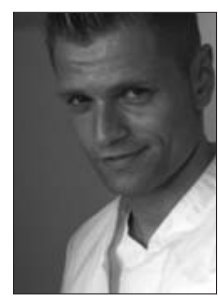

\section{SHBG and oxytocin receptor in the olfactory system of schizophrenic patients}

\section{$\underline{\text { Schäfer HH}}$}

Department of Cardiothoracic Surgery, Cape Heart Centre, University of Cape Town, Cape Town, South Africa

Schizophrenia (S) is a multifactorial disease and associated with an impairment of cognition and olfactory processing. Degeneration of the olfactory bulb (BO) and structures of the limbic system might be responsible for the early onset of the psychosis manifested in hallucinatory symptoms. There is evidence for changing levels of neurohormons in CSF of schizophrenic patients $(\mathrm{SciP})$ resulting in clinical deficits of odor discrimination. Oxytocin (OT) may interact with olfactory processing as it is known to lower the consolidation of memory. It has been shown that in cortical and limbic structures receptors for oxytocin (OTR) are expressed by different pathways triggered by estradiol which might be internalized by Sex Hormone Binding Globuline (SHBG). We analyzed immuncytochemically the expression of OTR and SHBG in SciP-BO and controls. Furthermore, using Western Blots and SELDI TOF mass spectrometry, we confirmed the presence of both proteins. OTR immunoreactivity was observed in a portion of Mitral cells and in Tuffed cells, partial co-localized with SHBG. OTR was much more abundant in SciP-BO than in controls which may occur as a consequence of reduced OT levels in S. Our findings indicate that a portion of neurons in $\mathrm{BO}$ is capable of producing SHBG and OTR. Since both, synthesis of OT and expression of OTR is modulated by estradiol and thus may explain underlying cellular mechanisms. it is likely that an intrinsic expression of SHBG may enhance bioavailability of the steroid in the brain as it has been shown that estradiol treatment improves symptoms in acute state of $\mathrm{S}$.

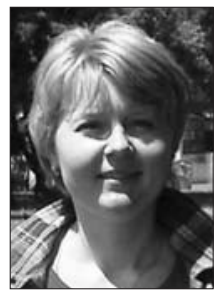

\section{Neuroendocrine stress response: adrenal steroids in the hypothalamo-hypophyseal system}

\section{Sivukhina E}

Institute of Anatomy, Friedrich-Schiller University, Jena, Germany

Adrenal steroids have tremendous importance in the brain. Stress response is controlled by glucocorticoids (GCs). Steroids are capable of crossing the blood brain barrier thus being predestined peripheral mediators of central functions through a feedback loop. The hypothalamo-pituitary adrenal (HPA) axis is the best described circuit in this respect. HPA axis is the key biological machinery, which is primarily activated in response to stress and regulated via feedback mechanisms. While GCs play crucial role in the metabolic, immune and autonomic adaptation to stress, their chronic excess leads to deleterious effects on many systems. We performed an analysis of plasma cortisol in patients suffered from chronic heart failure (CHF) combined with post- mortem morphological examinations of hypothalami, pituitary and adrenal glands. No changes in cortisol concentration were found in patients even with severe form of CHF, which was accompanied by dystrophic changes in adrenal cortex. These findings coincided with a profound increase of CRH and ACTH expressions, suggesting a disbalance in a feed-back signaling in the HPA axis. Chronic alcoholic disease is also known to be related to the disturbances of the HPA axis. We demonstrated the enhancement of CRH-immunoreactivity and increased coproduction of $\mathrm{CRH}$ and VP in parvocellular neurons of alcoholics. This may be due to a decline in GCs production, implied by the hypoplasic impairment of adrenal cortex. It is likely that known central actions of adrenal steroids may not be confined to genomic effects through a nuclear receptor but may include other functional pathways that involve the intrinsic binding globulin. Taking together, our results provide further evidence for the important involvement of adrenal steroids in the regulation of the hypothalamo-hypophyseal system during chronic stress. The disregulation between the central and peripheral components of the HPA axis is a very important question, which will be addressed in our further studies.

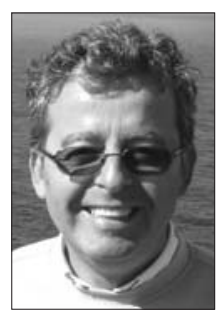

\section{Changes of sex hormone binding globulin (SHBG) expression in the hypothalamo-hypophyseal system of rats during pregnancy, parturition and lactation}

\section{Sendemir E}

Department of Anatomy, School of Medicine, Uludag University, Bursa, Turkey

Magnocellular neurons in the supraoptic and paraventricular nuclei have recently been shown to express both SHBG and the labor inducing and milk ejecting posterior lobe hormone, oxytocin (OT). The known increase in female sexual receptivity by SHBG and OT infusions into the medial pre-optic area suggests the concomitant function and possible co-regulation of these two factors. In recent studies we could show that hypothalamic SHBG and OT expression are malleable to changing steroid conditions. In the present study we examined immunocytochemically the occurrence of SHBG and OT in the late pregnant, parturient and early lactating rats. Consecutive semithin sections of the posterior lobes (PPL) and the median eminence (ME) were immunostained for either SHBG or OT. In animals that were killed at day 21 of gestation, we found scattered SHBG staining in Herring bodies of the posterior lobe, in part co-localized with OT. In parturient rats we observed a dramatic decrease of SHBG staining as well as OT, in the posterior lobe. A similar low staining distribution of SHBG and OT was seen on day two of lactation while in day six, the immunoreactive patterns resembled the staining observed in normal cycling controls rats. The median eminence on the $21 \mathrm{st}$ gestational day is stained darkly with OT while SHBG immunoreacitivity was low. In parturient rats SHBG immunoreactive patterns are similar to OT immunoreactivity. 
From the 2nd day of lactation, the prominent staining of SHBG is maintained until day 6 of lactation, while intensity of OT staining gradually declines. Our findings suggest that hypothalamic SHBG expression is malleable to changing endogenous steroid levels, similar to the SHBG expression in the reproductive organs. In previous electron microscopic studies we found that SHBG and OT are colocalized in secreting vesicles in the hypothalamo-neurohypophysial tract. It is likely that both antigens are coreleased upon specific stimulation. Coexpression of SHBG and OT may be of significance for known non-genomic actions of ovarian steroids for the endocrine control of parturition and lactation.

\section{Symposium 4}

\section{Neuroimaging}

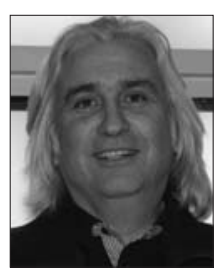

\section{Advanced neuroimaging}

Ișlak C

Department of Radiology, Section of Neuroradiology, Cerrahpaşa Medical Faculty, Istanbul University, Istanbul, Turkey

Several steroid-binding globulins, including In this topic we are going to talk about new techniques of mainly MR imaging and combined modalities. Perfusion MR studies with arterial spin labelling or with external contrast administration is a relatively new technique of imaging which gives idea about global and focal perfusion changes in brain parenchyma. Diffusion-weighted imaging is an MRI method that produces in vivo magnetic resonance images of biological tissues weighted with the local characteristics of water diffusion.Diffusion-tensor magnetic resonance imaging and fiber tractography are new methods that can demonstrate the orientation and integrity of white matter fibers in vivo. These techniques allow detection of abnormalities of the brain tissue in an earlier stage than conventional MR imaging sequences. Diffusion tensor imaging can be used to assess gray matter abnormalities like cortical infarction or malformations of cortical development. Functional Magnetic Resonance Imaging (FMRI) is a type of specialized MRI scan. It measures the hemodynamic response (change in blood flow) related to neural activity in the neuronal tissue. Blood-oxygen-level dependence (BOLD) is the MRI contrast of blood deoxyhemoglobin. Image fusion is a new area of imaging, it is the domain of combining two or more datasets in a combined visualization. It allows to use datasets of different imaging modalities during diagnostic workup and endovascular treatment.

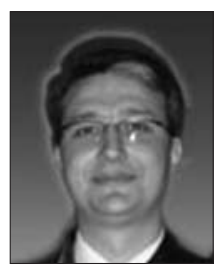

\section{Imaging of sellar and parasellar region} $\underline{\text { Kizılkılıç O }}$

Department of Radiology, Cerrahpaşa Medical Faculty, Istanbul University, Istanbul, Turkey

The imaging modality of choice for the pituitary gland and parasellar region is MRI. Coronal CT with contrast and thin slices $(1.5-2 \mathrm{~mm})$ may be obtained in patients who have con- traindications to MRI. Contiguous slices with no gap are obtained in the coronal and sagittal planes.Adenomas: For evaluation of pituitary microadenomas, a dynamic contrast-enhanced study is essential to detect subtle lesions. The dynamic study is performed by obtaining five T1-weighted, turbo spin-echo,3mm thick slices repetitively 20 seconds, 40 seconds, 60 seconds, 80 seconds, and 100 seconds after the injection of contrast material using an injector. Macroadenomas are usually easy to diagnose on coronal postcontrast images and are seen as enhancing masses. Bony erosion by the tumor mass causes the sellarturcica to enlarge. Other lesions: Pituitary apoplexy, craniophayrngioma, hypothalamic astrocytoma, Rathke cleft cyst, dermoid and epidermoid tumors, meningioma, hemangiopericytoma, schwannoma, germinoma, pituitary abscess, histiocytosis $\mathrm{X}$, suprasellar or intrasellar arterial aneurysm, carotid-cavernous fistula, thrombosis of cavernous sinus clival tumors such as metastases, chordoma, clival lymphoma, plasmocytoma, and suprasellar arachnoid cysts are the other lesions involving this region.Infiltrating lesions of the sellar and parasellar region include metastatic disease, sarcoidosis, lymphoma and leukemia, pseudotumor, lymphocytic hypophysitis, and other granulomatous disease as tuberculosis.

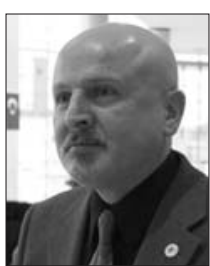

\section{Anatomy of anatomy atlases}

Sinav A

Professor \& Dean, School of Fine Arts, Trakya University, Edirne, Turkey

Throughout history, anatomical information has been conveyed visually from teacher to student. Even in our modern era with advanced imaging technologies, still, no one can imagine an anatomy curriculum without hand illustrated anatomy atlases. The anatomical illustrations in atlases are used as self study materials by students, as slides for lectures etc... Atlases are especially crucial in countries where cadavers are not readily available for dissection. In such cases, anatomical illustrations provide students the sole point of access to internal structure of the human body. Unfortunately, atlas illustrations sometimes misrepresent anatomical information. The explanations for such inaccuracies are numerous. First, some medical illustrators do not possess sufficient understanding of anatomical details. Second, illustrators may pay more attention to the artistic features of their illustra- 
tions than to their anatomic accuracy. Finally, and most importantly, medical illustrators often use existing illustrations as resources rather than drawing on observations from actual dissections. This practice propagates errors through generations. Anatomy education can be considered the foundation of medical sciences. Therefore, visual materials must be prepared with painstaking accuracy. Anatomists need to assume responsibility for collaborating illustrators, in order that the mistakes of history may be rectified.

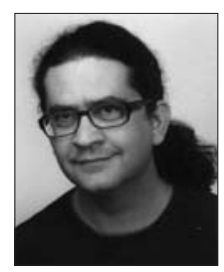

esfMRI of the upper STS: further evidence for the lack of electrically induced polysynaptic propagation of activity in neocortex

$\underline{\text { Sultan F }}$

Department of Cognitive Neurology*, HIH for Clinical Brain Research, Tuebingen \& Max Planck Institute for Biological Cybernetics, Tuebingen, Germany
Combining electrical stimulation with functional magnetic resonance imaging (esfMRI) has proven to be an important tool to study the global effects of electrical stimulation on neural networks in the brain. Here we extend our previous studies to stimulating the cerebral cortex in the upper superior temporal sulcus (STS) in the anaesthetized monkey. Our results show that stimulating area V5/MT and surrounding areas leads to positive blood-oxygen-dependant responses in the majority of cortical areas known from previous studies to receive direct/ monosynaptic connections from the stimulation site. We confirm our previous results from stimulating primary visual cortex that the propagation of electrically induced activity is limited on the cerebral cortex in its transsynaptic propagation to the first synapse likely due to the artificial nature of the electrical stimulus and the dominance of inhibitory responses to such synchronous stimuli.

\section{Symposium 5}

\section{Human and Experimental Encephalopathies}

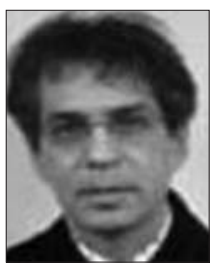

Encephalopathy in septic patients; from pathophysiology to treatment

Sharshar T

Department of Neurology, Hopital Raymond Poincaré, Garches, France

Several steroid-binding globulins, including In this topic we are going to talk about new techniques of mainly MR imaging and combined modalities. Perfusion MR studies with arterial spin labelling or with external contrast administration is a relatively new technique of imaging which gives idea about global and focal perfusion changes in brain parenchyma. Diffusionweighted imaging is an MRI method that produces in vivo magnetic resonance images of biological tissues weighted with the local characteristics of water diffusion.Diffusion-tensor magnetic resonance imaging and fiber tractography are new methods that can demonstrate the orientation and integrity of white matter fibers in vivo. These techniques allow detection of abnormalities of the brain tissue in an earlier stage than conventional MR imaging sequences. Diffusion tensor imaging can be used to assess gray matter abnormalities like cortical infarction or malformations of cortical development. Functional Magnetic Resonance Imaging (FMRI) is a type of specialized MRI scan. It measures the hemodynamic response (change in blood flow) related to neural activity in the neuronal tissue. Blood-oxygen-level dependence (BOLD) is the MRI contrast of blood deoxyhemoglobin. Image fusion is a new area of imaging, it is the domain of combining two or more datasets in a combined visualization. It allows to use datasets of different imaging modalities during diagnostic workup and endovascular treatment.

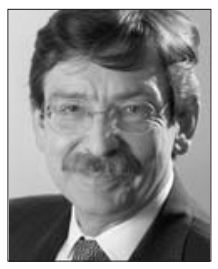

Encephalopathy in the rat caecalligation and puncture model of sepsis

Davies DC

Human Anatomy Unit, Department of Surgery, Imperial College, London, United Kingdom

Sepsis is a major clinical challenge that is associated with encephalopathy and multi-organ dysfunction. Sepsis associated encephalopathy (SAE) develops in as may as $50 \%$ of patients with sepsis, but despite the association of SAE with increased mortality, brain dysfunction remains a poorly understood complication of sepsis. Caecal ligation and puncture (CLP) in rats causes bacteraemia, reduced motor activity and pyrexia followed by hypothermia. It also results in reduced blood glucose, reduced white blood cell and platelet counts and an increased neutrophil: lymphocyte ratio. CLP also causes histopathological changes in the cerebral cortex, liver, lungs and vascular system indicative of multi-organ dysfunction and thus, CLP mimics the cardinal features of human sepsis. CLP results in signif- 
icantly increased in brain water content in rat frontal cerebral cortex and electron microscopy revealsthat this is associated with significant perimicrovessel oedema, collapse of microvessellumina, and decreased microvessel endothelial cell cross-sectional area. Tracer studies demonstrate that the blood-brain barrier in CLP rats is impermeable to large molecules such as Evans Blue, but particles of the ionic tracer lanthanum nitrate appear to pass into the neuropil. CLP also results in significantly reduced synapse density in the frontal cortex and synapse density decreases as perimicrovessel oedema increases. The increased brain water content and reduction in synapse density in the cortex of CLP rats could explain the cognitive deficits that are frequent sequelae of sepsis. To date the treatment of SAE has primarily been focussed on the control of sepsis, but the CLP model now provides a clinically accurate model for the development of novel therapies directed at the protection of the brain in sepsis.

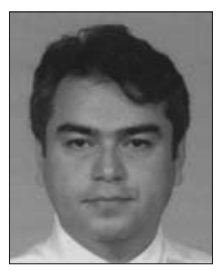

Neuronal injury and regeneration in caecal ligation and puncture model of sepsis

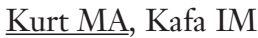

Department of Anatomy, School of Medicine, Uludag University, Bursa, Turkey
Sepsis occurs in $14-37 \%$ of patients admitted to intensive care units and sepsis associated encephalopathy (SAE) is its severe complication. Although recent research has improved our understanding of the pathophysiology of sepsis, underlying molecular and cellular mechanisms in SAE is still poorly understood. We have previously demonstrated the presence of significantly more apoptotic cell death, in five brain regions (Median preoptic nucleus, Subventricular Zone of the lateral ventricles (SVZ) and CA1, CA3 and dentate gyrus (DG) of the hippocampus) in caecal ligation and puncture (CLP) model of sepsis in rats; suggesting that the apoptotic cell death would bare a major role in the pathogenesis of the SAE. Our previous studies have also revealed an increased cellular proliferation in SVZ but not in DG of hippocampus of the CLP rats, which are both considered as the well-established brain sites for the adult neurogenesis. In the light of our previous results and recent research findings of other groups on the subject, we present and discuss the potential effects of sepsis and SAE on the neuronal injury and regeneration in the CLP model which has been widely used and considered as the gold standard for sepsis studies. Furthermore, we seek answers to the questions whether neurogenesis is in fact triggered by an accompanying cell death observed in CLP rats to counterbalance the cellular loss and to contribute to the selfrenewal mechanism of the brain in sepsis. 


\title{
Oral Presentations
}

\author{
(OP-1 - OP-73)
}

\author{
OP-1 \\ Dorsal commissural nucleus: the key position of \\ visceral sensory and somatic sensory information con- \\ vergence \\ Zhang MM, Li YQ \\ Department of Anatomy, Histology \& Embryology, K. K. Leung Brain \\ Research Centre, The Fourth Military Medical University, Xi'an China
}

Referred pain is an important clinical phenomenon often associated with visceral diseases to somatic structures, especially to the body area of homo-segmental innervations. After pelvic visceral noxious stimulation, there were more Fosimmunopositive (Fos-ip) neurons in the dorsal commissural nucleus (DCN), a special area located dorsally to the central canal in the lower lumbar and sacral segments, than that in the spinal dorsal horn of corresponding segments in the rat. Immunohistochemical staining also showed that Substance P receptor (SPR) -containing endosomes migrated to the interior of the neurons in DCN. Through electron microscopy combined with tracing techniques, it was observed that DCN neurons innervated by primary afferents originated from both pelvic nerve (visceral sensation) and sciatic nerve (somatic sensation) and projected to parabrachial nucleus (PBN). And there were dense Substance P (SP)-, enkephalin (ENK)-, GABA-, glycine-immunopositive (Gly-ip) terminals and SPR-ip neurons in DCN. It was also found that SP-ip and ENK-ip terminals to make asymmetrical synapses with SPR-ip PBN projecting DCN neurons, while GABA-ip, Gly-ip and GABA/Gly-ip terminals to make symmetrical synapses with SPR-ip PBN projecting DCN neurons. These results suggested that: (1) DCN might be the convergence point of the referred pain associated with pelvic visceral organs to lower limbs; (2) The projection from DCN to PBN might contribute to the pelvic visceral information transmission; (3) The neurotransmission in DCN might exert precise regulatory influences upon the process of visceral nociceptive information by the relative amount of neuroactive substances, such as SP, ENK, GABA and Gly, released from the presynaptic terminals.

\section{OP-2}

Functional repair of Parkinsons disease by olfactory epithelial stem cells in rat

Safari $M^{*}$, Ghahari L**

Department of Anatomy, Semnan University of Medical Sciences, Semnan, Iran*; Department of Anatomy, Artesh University of Medical Sciences, Tehran, Iran**
Introduction: Parkinson's disease (PD) is a disorder in basal ganglia that is a slow progressive disease and is characterized by the loss of dopaminergic neurons in the pars compacta of the substantia nigra (NG). There is a strong evidence the oxidative stress participates in the etiology of PD. Materials and

Methods: 30 male rats from vistar spring were divided into 3 groups (10 animals in each group) control, sham and injection. stem cells divided from olfactory epithelium of a young mail rat. Cells cultured for a week in DMEM/F12 including 10\% FBS. After a week Immunocytochemistery were done for detection of nestin positive cells. Then cells differentiated with FGF $20 \mu \mathrm{m}$. After 2 weeks all cells detached from the flasks and counted with Trypan blue then 10000 to 20000 cells injected in compact part of substantia nigra. 4 weeks after injection Immunocytochemistery were done again for detection of alive cells and behavioral test were done for all groups. All data analyze by SPSS.

Results: Some cells after 7 days culture were nestin positive. After 2 weeks culture and $20 \mu \mathrm{m}$ FGF some cells express tyrosine hydroxyls precursor of dopamine. 4 week after injection some injected cells were alive and express Brdu. But behavioral test were not significant in all groups.

\section{OP-3}

\section{Neurodegeneration in the frontal cortex and hippocampus of Sprague Dawley rats administered with aqueous seed extract of Datura stramonium}

Adekomi AD*, Tijani AA**

Department of Human Anatomy, Faculty of Basic Medical Sciences, University of Ilorin, Ilorin, Nigeria*; Osun State University, Osun State Nigeria**

This study was carried out in order to investigate some of the effects of aqueous seed extract of Datura stramonium on the frontal cortex and hippocampus of Sprague Dawley rats. A total of sixteen adult male Sprague Dawley rats weighing about 160$190 \mathrm{~g}$ in weight were used for this study. The experimental animals were randomly distributed into two groups designated as A and B. The animals in group B serves as the treatment group and were administered with $300 \mathrm{mg} / \mathrm{kg}$ bwt of the aqueous seed extract of Datura stramonium while the animals in group A which serves as the control were administered with equal volume of phosphate buffered saline (PBS). The mode and duration of administration was orally with the aid of a sterilized 
flexible orogastric tube and for fourteen days respectively. All the experimental animals were sacrificed using cervical dislocation 3 hrs after the last administration. The brains were extracted from the skulls of the animals and were completely fixed in $10 \%$ formol calcium. Eighteen hours after fixation, the frontal cortex and the hippocampus were extracted from the fixed brain and were processed for routine hematoxylin and eosin (H\&E) stains. Microscopic observations made from the permanent photomicrographs revealed alterations in the histology of the frontal cortex and hippocampus. These findings provide evidence that suggests that the oral administration of aqueous seed extract of Datura stramonium causes neurodegeneration on the frontal cortex and hippocampus of Sprague Dawley rats.

\section{OP-4}

\section{Proposals for "More Intelligent Design" of Homo sapiens and other vertebrates}

\section{$\underline{\text { Kurz H }}$}

Tissue Dynamics Lab, Institute of Anatomy and Musculoskeletal Research, Paracelsus Private Medical University, Salzburg, Austria

Medical doctors all over the world are trained to diagnose and eventually provide therapy for the numerous shortcomings and lesions of human psycho-somatic constitution at all levels of organization, from elementary particles to social interactions. Yet, and despite overwhelming evidence for nonsentient and not goal-directed evolution of form and function in all life forms including $\mathrm{H}$. sapiens, a not negligible fraction of doctors and scientists argue in favour of their being "intelligently designed". However, as morphologists and academic teachers, we should be aware of pervasive bad or "unintelligent design", i.e., of structural and functional flaws at the macroscopic, microscopic and molecular levels: 1) Non-redundancy of vital structures (heart, respiratory pacemaker etc.); 2) Lack of wellpossible, useful functions (detection of broader spectra of electromagnetic radiation or acoustic waves, regeneration of limbs, or at least joint cartilage etc.); 3) Grotesque or dangerous contrivances (descensus testis, obligatory aerobic metabolism in neurons and cardiomyocytes etc.); 4) Inefficiency of reproduction (high rates of embryo loss, premature birth, malformations etc.); 5) Low fidelity of molecular mechanisms (DNA replication, transcription etc.). Some of these "faulty designs" - which all can be understood in the light of evolution and embryology - have already been amended or overcome by science, medical practice and widespread acceptance in the population, but a majority are awaiting "More Intelligent Design", i.e., intervention by well-educated, ethically competent and publicly accepted scientist doctors. More frequent demonstration of "unintelligent design" for educational purposes in high school biology, anatomy and medicine is proposed.

\section{OP-5}

\section{Analgesic mechanism of endomorphin in the spinal dorsal horn}

\section{Li YQ}

Department of Human Anatomy, The Fourth Military Medical University, Xi'an, China

Background: Endomorphin (EM) is a powerful endogenous analgesic. In the dorsal root ganglion (DRG) and superficial layers (laminae I and II) of the spinal dorsal horn, many substance $\mathrm{P}(\mathrm{SP}), \mathrm{EM}$ and $\mu$-opioid receptor (MOR) immunpositive neurons and dense terminals are found, respectively. We proposed that EM binding to its autoreceptor MOR on the pre-synaptic buttons might inhibit further release of the noxious neurotransmitters, such as SP.

Aims: To prove whether the hypothesis is true or not, the analgesic effects of EM in the spinal dorsal horn were investigated.

Methods: Behavioral observation, patch clamp recording, immunohistochemical staining methods were used.

Results: (1) EM pre-treatment through intrathecal administration could dose-dependently inhibit the primary mechanical allodynia induced by subcutaneous injection of complete Freund's adjuvant. This effect might be reversed by antagonists of neurokinin 1 receptor, and attenuated by SP or co-administration of SP and EM, respectively. (2) EM remarkably reduced the frequency instead of the amplitude of miniature EPSCs in lamina II. This effect was antagonized by MOR, antagonist beta-FNA. EM could also inhibit the EPSCs induced by stimulating of the attached dorsal root. The average amplitude of the residual EPSCs increased during high concentration of SP being perfused. (3) SP, EM and MOR were co-localized in the DRG neurons. MOR was localized on the pre-synaptic membrane in which SP and EM were found to co-exist in the same large dense-coated granular synaptic vesicles.

Conclusions: SP and EM are co-localized in the pre-synaptic button, of which MOR can be found on the pre-synaptic membrane. EM might inhibit excitatory synaptic transmission and decreases SP release through activating MOR on pre-synaptic terminals to exert analgesic effects.

\section{OP-6}

\section{The enigmatic claustrum}

Reblet $\mathrm{C}^{*}$, De La Cuerda $\mathrm{MF}^{* *}$, Alejo $\mathrm{A}^{* *}$, MendizabalZubiaga JL*, Chara-Ventura JC**, Bueno-Lopez JL*

Department of Neurosciences, School of Medicine and Dentistry, the University of the Basque Country, Spain*; Animal Facility, School of Medicine and Dentistry, the University of the Basque Country, Spain**

Gene-expression and molecular studies in amniotes have suggested that the claustrum (CL) originates from the ventral and 
lateral pallium together with part of the amygdala and piriform cortex. Nonetheless, CL origin, anatomy and function remain poorly understood. Our studies with BrdU and calcium-binding-proteins immunocytochemistry in rabbits showed that the pre and postnatal development of the lateral pallium, dorsal claustrum and insular cortex are coherent with one another, as in turn they are those of the ventral pallium, ventral claustrum and piriform cortex. We additionally studied the axon connections of the claustrum with the limbic cortex and neocortical sensory areas by injecting anterograde and retrograde tracers in the dorsal CL and retrograde tracers in the adult rabbit cortex. Injections in the anterior cingulate cortex and injections in the claustrum produced similar retrograde-labelling of cells in the anterior subventricular zone (SVZ) and white mater of the corona radiata and external capsule; the morphology of these cells was like that of the migrating neuroblasts generated in SVZ of adult rabbits in other studies. This suggests that neurons originated in the adult pallial SVZ project to the limbic cortex or the claustrum during their migration. In our studies, the connectivity of the claustrum with the anterior cingulate area was estimated to be heavier than that to the neocortical sensory areas. Neurons projecting to the anterior limbic cortex occupied all antero-posterior extension of the dorsal claustrum whereas only few among them lay in the anterior ventral claustrum. Claustro-cortical axons to the cingulate cortex were thicker and spread more extensively than axons to sensory areas. All this implies that the claustrum is indeed a limbic domain. This agrees with the outcome of recent studies of others on the expression of the limbic system-associated membrane protein in human claustrum. Supported by Grant GIU07/14 of the UPV/EHU

\section{OP-7}

Innervation on axon-initial-segment of cortical pyramidal neurons is correlated with axon projection and layer position of parent neuron but not with length and thickness of axon initial segment proper

Bueno-Lopez JL, Chara-Ventura JC, Reblet C, MendizabalZubiaga JL

Department of Neurosciences, School of Medicine and Dentistry, the University of the Basque Country (Upv/Ehu), Spain

This is the outcome of correlated LM/EM studies on the axon initial segment (AIS) of pyramidal neurons in the rat cerebral cortex. The radial place (either supra- or infragranular) and axon-projection (either contralateral from area 17 or ipsilateral from area 18) as well as the spiny nature of the cells were observed following retrograde-tracing of BDA injected in lateralmost area 17. Examined features were AIS-length, AISthickness, number and distribution of AIS-synapsing boutons and density of these synaptic boutons per AIS-length unit.
Statistics $\left(\mathrm{P}_{4} 0.05\right)$ were Student's T-test and linear-regression analysis. The number of synaptic boutons consistently decreased during the infragranular-neuron-AIS courses. Throughout supragranular-neuron AISs, such a distribution was uneven not only regarding types of participated projection but also within each type. All features but length and thickness were greater in AISs belonging to neurons furnishing the ipsilateral projection. This finding was significant concerning the total number of synaptic boutons and also its density per AISlength unit. Consequently, the correlation between the number of boutons synapsing the AIS and the type of cortico-cortical projection participated by the parent neuron was positive and highly significant. The total number of synaptic boutons as well as its density per AIS-length unit was significantly greater for supragranular neurons than for infragranular ones. Oppositely, AIS length and thickness were significantly greater in infragranular rather than in supragranular neurons. Conclusively, the correlation between the number of boutons synapsing the AIS and the cortical layer in which the parent neuron lay was positive and highly significant. AIS-length and -thickness were positive and significantly correlated with each other in all subgroups. On the contrary, the correlation between AIS-length and number of synaptic boutons was inverse but not significant. In spiny inverted pyramidal neurons previously studied, none of these last two correlations were significant. Supported by Grant GIU07/14 of the UPV/EHU.

\section{OP-8}

Gene expression in the spinal cord: implications in spinal cord anatomy and research

$\underline{\text { Sengul G }}$

Department of Anatomy, Faculty of Medicine, Ege University, Izmir, Turkey

Humans and mice share approximately 90 percent of their genetic code. Therefore, a spinal cord gene atlas will contribute significantly to spinal cord research, serving as a model to study the mechanisms behind degenerative diseases such as amyotrophic lateral sclerosis (ALS) and multiple sclerosis (MS). A spinal cord gene atlas can also point to new gene-based methods for promoting regeneration for spinal cords that have been injured. This presentation is mainly on the recently completed Allen Spinal Cord Project, an interactive atlas database of gene expression mapped in the mouse across all anatomic segments of the cord at postnatal days 4 (P4) and 56 (P56). The indexed set of images for the entire genome is based on RNA ISH data, and is searchable and sortable by gene, age, expression, and spinal cord segments. For this project, we have prepared reference atlases of the $\mathrm{P} 4$ and P56 C57Bl/6J male mouse on the Nissl sections (Allen Spinal Cord Reference Atlas, Kayalioglu and Watson, 2009), identifying over 80 struc- 
tures in the spinal cord. Scientists from the spinal cord community including us further analyzed the sets of 1250 interesting genes annotated by insitute experts, to identify putative cell types, new markers for cell types, novel boundaries defined by gene expression, differences between $\mathrm{P} 4$ and P56, and regionally-restricted patterns along the dorsoventral and rostrocaudal axes. Additionally, all the genes expressed in the dorsal root ganglion neurons of the entire $\mathrm{P} 4$ data set were analyzed. The cytoarchitectonic localization of genes and their specific expression in the spinal cord contributes to our understanding of their function in the context of the circuitry of the dorsal horn. This resource is freely available at www.brain-map.org and serves neuroscience community as an importantr platform for exploring the spinal cord at the cellular and molecular levels.

\section{OP-9}

An integrating system for assessing students' progress in the anatomical dissection lab

\author{
Brenner E, Moriggl B, Fritsch H \\ Division of Clinical and Functional Anatomy, Innsbruck Medical \\ University, Innsbruck, Austria
}

Learning anatomy in anatomical dissection lab can be seen as a multiple step task based curriculum. Doing so, they gain practical knowhow, manual skills, as well as factual and procedural knowledge. Furthermore, they are trained to teamwork. Assessing these learning objectives must comprise of as many of these goals as possible. We designed a multimodal assessment system comprising four different aspects: 1 . Oral contributions of factual and procedural knowledge are assessed by five independent short structured oral exams (sSOEs). These sSOEs are dedicated to different topics and comprise three questions each. Each question is judged with up to three scores. 2. Interaction with other students is assessed by structured observation. Good interaction with and proper contributions to their group are awarded with one score per fortnight. 3 . The main product of their work, the specimen, is assessed on a twoweeks basis. Students are awarded up to three scores for a perfect dissection. Two scores are awarded when the specimens show minor destruction such as removal or cutting apart of minor structures or when the borders or limit of the region dissected are spared. One score is awarded when there are major destructions such as cutting apart a major nerve or vessel. Completely inadequate dissection doesn't get any score. 4. Finally, the students' documentation of their work including their findings in their specimen is assessed by their courseportfolio. This portfolio should contain proper documentation of the work's progress, actual findings in their specimens (i.e. anatomical variants and variations), reflection on their work and contributions to ethical issues. The portfolio is a team product and is assessed as a whole, so each student is awarded the same amount of up to six scores.

\section{OP-10}

\section{Learning anatomy across medical, dental and allied health professions}

Smith $\mathrm{C}^{*}$, Martínez $\mathrm{C}^{* *}$, Mchanwell $\mathrm{S}^{* * *}$

Centre for Learning Anatomical Sciences, Faculty of Medicine, University of Southampton, Hampshire, United Kingdom*; Departamento de Anatomía y Embriología Humana I, Facultad de Odontología, Universidad Complutense, Madrid, Spain**; Department of Oral Biology, School of Dental Sciences, University of Newcastle, Newcastle, United Kingdom***

This study set out to ascertain if the context of learning anatomy made a difference to students' perceptions. A 31 question Likert scale questionnaire was administered to 224 students (77 dental, 132 medical and 19 speech language). Statistical analysis revealed significant associations between course of study and students' responses. Overall dental students responded to being able to use their anatomy knowledge in the context of surface and radiological anatomy more so than other groups $(\mathrm{p}=<0.000)$. Medical students interestingly found the dissecting environment an intimidating one and subsequently reported finding online anatomy resources helpful ( $\mathrm{p}=0.015$ and 0.003 respectively). Speech therapy students had greater issues, they reported finding the amount to learn daunting $(\mathrm{p}=0.007)$, struggled to remember what they did last semester and were not confident in their knowledge ( $\mathrm{p}=0.032$ and $\mathrm{p}=<0.000$ respectively). The remaining 25 questions were relatively uniformly answered irrespective of course of study. The results were cross tabulated with the students' approaches to learning. Significant associations were found between a surface approach and students having problems using their knowledge, who were not confident in their knowledge, who found the amount to learn daunting, who did not see the point to learning, whose main motivation was to pass exams, who found anatomy memorisation based, and who forgot what they learn previously. All students responded highly to the statement 'I feel that working with cadaveric material is an important part of becoming a doctor/dentist/healthcare professional', this is associated with a deep approach $(\mathrm{p}=<0.000)$. Importantly this study elucidated that whilst local curriculum factors are important in creating an enabling learning environment there are many generic elements that are inherent in anatomy as a discipline and experienced across courses and institutions alike. Research and recommendations made regarding anatomy education can now confidently be applied to the wider anatomy community.

\section{OP-11}

Imorphology: a novel concept for co-teaching anatomy and diagnostic imaging in an integrated curriculum

\section{Streicher I}

Integrative Morphology Group, Medical University of Vienna, Vienna, Austria

Introduction: In integrated curricula anatomy teaching frequently integrates chronologically with other subjects, but fails 
to integrate with regard to contents; especially, if higher numbers of students shall be taught in small groups. This was also the case in the first approach at our university, where 720 students are enroled per year. After evaluation giving unsatisfactory results, we have elaborated the iMorphology-concept for integrated teaching of anatomy and diagnostic imaging.

Didactic Setting: In the same module, students switch between anatomical preparation of an organ system at the dissection hall and according case-based learning of diagnostic imaging of the respective organ system guided by radiologists. In the dissection hall, ceiling mounted iMacs and large-screen interactive whiteboards provide the students with the images from these cases, additional clinical cases, preparation-videos, videos of relevant surgical/clinical interventions, animations illucidating development, and 3D-reconstructions from crosssectional image series. The diagnostic imaging case-presentations are provided as e-learning materials supplemented by internet-discussion-fora, that are supervised by radiologists. Assessment combines immanent evaluation of dissection and respective background knowledge, mutual cooperative teaching of students among each other, and diagnostic imaging-tests using interactive learner response devices.

Results and Conclusions: The "iMorphology" didactic setting provides flexible and accelerated access to comprehensive understanding of complex spatial relations and their clinical implications, and is readily adopted by the students. The diagnostic imaging tests provide teachers with valuable, objective feedback on the achieved clinical diagnostic competence of the students. With this approach, we on the one hand achieve faster and indepth morphological knowledge base in a limited time frame, and on the other hand become able to rapidly accommodate contents and objectives to the students actual level of competence.

\section{OP-12}

Body explorer 3.0: an easily accessed multi user web system for studying cross-sectional anatomy

Lazarov $\mathrm{N}^{*}$, Lazarov $\mathrm{V}^{* *}$, Bulling $\mathrm{A}^{* * *}$, Gratzl $\mathrm{M}^{* * * *}$

Department of Anatomy and Histology, Medical University, Sofia, Bulgaria*; Technical University, Munich, Germany**; Department of Anatomy, Ludwig Maximilian University, Munich, Germany***; Department of Anatomy, Ludwig Maximilian University, Munich, Germany****

Sectional techniques are widely used in the medical field (computer tomography, magnetic resonance imaging and sonography). In order to facilitate successful interpretation of the anatomical structures, in 1997 we published our first interactive program on the cross-sectional anatomy of the Visible Human Male, termed BodyExplorer. Terminologia Anatomica, since 1998 the internationally accepted source for human anatomical terminology, was used in Latin and English. In 2001, the completely revised multilingual (six languages) BodyExplorer 2.0 was launched containing important additional information, including images of the female pelvis. The novel web version of the BodyExplorer 3.0 features more than 2000 high resolution images of a human body and will enable users to easily and comprehensively navigate through the body, to display relevant sections and to achieve interpretive excellence in 12 languages, including Latin, German, English, French, Italian, Spanish, Portuguese, Bulgarian, Russian, Turkish, Japanese and Chinese. Further languages are under way. Our multilingual approach will facilitate successful communication not only within the anatomical community but also in many other medical disciplines worldwide.

\section{OP-13}

\section{Future contributions to the education of anatomy}

Yazar F, Develi S

Department of Anatomy, Faculty of Medicine, Gülhane Military Medical Academy, Ankara, Turkey

Objectives: Medical simulation stands out with new products and developing with each passing day. Make it more interactive and to facilitate the training of Anatomy, simulators and softwares should be used.

Introduction: Anatomy is often difficult to engage and motivate students to digest the vast numbers of structures and names and, once this is achieved, the crammed knowledge is often forgotten or difficult to apply in clinical practice. Thanks to the incredible advances in medicine and computer technology, changes in anatomy education, there is also very versatile. The expanding radiological imaging technology offers powerful educational tools for visualizing and understanding the organization and functionality of the human body. Advancements in technology and personal computing have allowed for the development of novel teaching modalities such as online web-based modules. Using with $3 \mathrm{D}$ models; learn interactively, view structures and systems from any angle and zoom level, hide structures and systems, or make them transparent, to see through muscle and bone and into organs. New anatomy educational apps for the smart phones and tablets have been releasing over at app stores that should provide students additional content customization and learning assistance. Further more nowadays; anatomy education in a computer game even more fun to make the models began to develop.

Conclusion: This study reviewed the ways of future anatomy education diversification and renewal. Technological changes in this area of medical education to recognize and reflect them as possible, will give a better anatomy education. We believe that these educational ways have the potential of significantly enriching anatomy education by helping students better appre- 
ciate spatial relationships, especially in areas of the body with greater anatomical complexity.

\section{OP-14}

\section{Visual history of human anatomy}

Kasirga B, Gulbar S

Department of Anatomy, Medical Faculty, Hacettepe University, Ankara, Turkey

Objective: Through out the years anatomical illustrations contained different images sometimes bizarre, sometimes like a grotesque nightmare. However human body is the product and paradoxically, the urge to discover the truth about that bodies was always the motivation propelling artists and researchers. In this study, it is aimed to bring many drawings together in a historical manner from various ages to make a brief and visual history of anatomical illustrations.

Materials and Methods: In 1543 Andreas Vesalius produced De Humani Corporis Fabrica. It is the first profusely and lavishly illustrated anatomy book published ever. Besides those basic references online catalogue series and medical library sources searched and explored to collect visual data and images.

Results: Earlier than the 15th century, only the haziest ideas about human anatomy prevailed. Then, suddenly, artists began to dig. By dissecting cadavers, bodies they unearthed a wealth of revelatory information about the complex structures hidden under the skin.

Conclusion: Hitherto, the leap is huge for medical illustrations from the beginning to today's digital age; it is clear that knowing the progress and real content of artwork will be contributing to the available bibliography in this area. Key words: Anatomical illustration, medical illustration, medical illustrations history.

\section{OP-15}

DryCon $\mathrm{HPCS}^{\odot}$ - A new method for preservation and mummification of whole bodies in a closed system without formalin

\section{Weber G}

Medis-Kugel Gmbh, R\&D Department, Buseck, Germany

This new anatomical technology, a first worldwide, was designed to eliminate the use of formalin and to create a closed system for the processing of whole bodies for teaching purposes in anatomy. Consisting of modular units that work autonomous and may be pro- grammed individually, the system also allows the modular units to be fitted together to allow preservation of an unlimited number of cadavers. A special advantage is the processing not just of the whole body but also of organs and body parts in a fully closed process chamber, whereby the infiltration solution is pumped into the chamber until the body / organ is fully covered (akin to a closed Tissue Processor in pathology). After this procedure, the body maybe "mummified" for longterm storage in a dry environment on a simple shelf. If the body is to be used for a student dissecting course, it can be readily restored to its original "soft" condition. This system is also designed for the regeneration, resoftening, examination and repair of ancient mummies in a careful manner. This unit consists of a solid, and self-carrying construction with automatic electronic control functions to enable one-man operation. Specially developed to guarantee pollution-free fixation and conservation of whole bodies without the use of formalin in a fully closed system that is pressurized with 3 bars in order to enhance the infiltration process. The infiltration / preservation solution (DIS) is a special, quality controlled solutionbased on a harmless, none-toxic, organic product called "Shellac" (with low concentrations of ethanol). With the approval of leading anatomists, because of the use of Shellac, the method is termed "Lacservation". 10 of the DryCon units are in full routine use at the King Abdul Aziz University, Dpt. of Anatomy (Dir. Prof. A. Al-Hayani) in Jeddha / KSA.

\section{OP-16}

\section{Effects of sodium nitrite-induced hypoxia on the cerebellar Purkinje cells in adult rats}

Zaidi Z

College of Medicine, King Saud University, Riyadh, Saudi Arabia

Objectives: To explore the changes in Purkinje cells in adult rats after inducing chemical hypoxia by sodium nitrite

Methodology: Adult male albino rats, weighing 180-200 gm were used in this study. The animals were fasted for three hours prior to drugs administration. One hour after sodium nitrite (75 $\mathrm{mg} / \mathrm{kg}$ ) subcutaneous injection, rats were decapitated. The brains were removed and placed overnight in fixative containing $10 \%$ formalin. Cerebellum were paraffin- embedded for hematoxylin and eosin staining and cut at $5 \mu \mathrm{m}$ in the coronal plane. Fifty Purkinje cells along the line of cells from two randomly chosen portions of each section were counted at a magnification of $x 20$. Cells in a total of five sections were counted to compute the ratio of damaged cells to intact neurons.

Results: There was no marked difference in the general appearance and thickness of the cerebellar cortices of the control and the sodium nitrite treated rats. The principal findings in the treated group were that almost all the Purkinje cells showed autolytic changes. Their arrangement as a single layer was also deranged in certain areas. Some areas showed complete loss of Purkinje neurons.

Conclusion: Sodium nitrite-induced hypoxia results in severe damage to the Purkinje neurons. 


\section{OP-17}

Systemic and local state of the head vessels under condition of alcoholic intoxication

Moldavskaja A, Kalaev A, Petrov V, Gorbunov A

Russia

Vascular diseases, both of traumatologic and non traumatologic genesis, is one of the main causes of mortality in the industrialized countries. Prophylaxis and treatment of the mentioned pathology is one of the most actual problems of modern medicine. The aim of the research was to study the head vessels state in norm and under conditions of alcoholic intoxication. On the basis of the acquired data to find out and study adaptational processes in the head vessels under conditions of alcoholic intoxication. Microcirculatory route, brain vessels, dura mater vessels, vessels of mucous membrane of the nasal cavity due to connection of their bloodstream have been studied both in norm and under conditions of alcoholic intoxication on biopsy and autopsy material and under experimental on the laboratory animals. Under conditions of chronic alcoholic intoxication in the microcirculatory route of the dura mater (DM) the following adaptation mechanisms have been found out: 1 . The stage of formation of venulo-venular anastomoses between the vessels of the external and internal layers of DM through the zone without vessels; 2 . The stage of formation of the intervascular network around the arteries. We consider it is possible to present the following results: 1. Morphologic reconstruction of the vessels in the DM of the experimental animals under condition of alcoholic intoxication is accompanied by the structural reconstruction of all the chains in the microcirculatory route, reflecting the structural mechanisms of adaptation; 2. Morphological changes under long-term alcohol stress has irreversible generalized character, and the adaptation mechanisms under conditions of pathology both traumatic and non-traumatic genesis, become insufficient for providing vascular compensation and liquidation of the pathologic process. The elements of the evident adaptation process in DM are noted in the whole vascular system of the head.

\section{OP-18}

\section{A simplified model of the neural stem cell niche featuring surrogate elements}

Kahn N, Kaisman T, Fishman D, Brodski C, Silverman Z

Ben-Gurion University, Israel

Stem cells in the body are maintained and regulated in a complex microenvironment known as the 'stem cell niche'. While the identity and behavior of the somatic and supporting elements of the niche in any particular tissue are mostly unknown, the seminiferous tubule in the testis is an exception. Here,
Sertoli cells, with support from identified stromal and endocrine components control a stem cell population that generates the male germ cells. We posited that aspects of stem cell regulation are sufficiently conserved between tissues to allow Sertoli cells to be used to model a non-testis niche. To test this, we established co-cultures of Sertoli cells and a fluorescentlylabeled surrogate neural progenitor population, pheochromocytoma (PC12) cells. PC12 cells are progenitor-like cell line that responds to recognized signals by differentiating into neuron-like cells. The cultures were initially monitored for development of reciprocal cellular influences by stereology and confocal microscopy. Co-culture of PC12 cells with Sertoli but not HEK293 cells induced extensive neurite extension. Similarly, exposure to PC12 cells induced robust morphologic changes in Sertoli cells. The two cell populations were then separated by FACS after 5 days in culture and molecular influences of the interacting cell populations assessed by RT-PCR. Gene expression relevant to neural development and signal transduction was monitored in separate or co-cultured PC12 and Sertoli cells. Our data indicate that the GDNF co-receptor, Ret was up-regulated in the PC12 cells while Wnt-5 and transferrin were down-regulated in Sertoli cells in the co-cultures. Our results demonstrate that stem and niche surrogates establish reciprocal communication in a manner that represents a proof-of-concept for future studies designed to model the neural stem cell niche.

\section{OP-19}

\section{Sildernafil citrate induces reactive astrocytes formation in the hippocampus}

\section{Oyesomi TO* , Ajao MS**}

Department of Anatomy, Faculty of Basic Medical Sciences, University of Ilorin, Kwara State. Nigeria*; School of Anatomical Sciences, University of Witwatersrand, Johannesburg, South Africa**

The normal adult vertebrate nervous system is a relative quiescent tissue in terms of cell proliferation. However astrocytes in many region of the central nervous system (CNS) like the hippocampus retain the capacity to undergo cell division. Understanding the growth of this glial cell is a key to repairing neurons in response to insult. We studied reaction of astrocytes in the hippocampus after a thirty days administration of Sildernafil citrate to twenty-four wistar rats. The rats of both sexes $(\mathrm{n}=24)$, average weight of $202 \mathrm{~g}$ were randomly assigned into three treatment $(\mathrm{n}=18)$ and control $(\mathrm{n}=6)$ groups. The rats in the treatment groups 'A', 'B' and ' $\mathrm{C}$ ' received respectively, $0.25 \mathrm{mg} / \mathrm{kg}, 0.70 \mathrm{mg} / \mathrm{kg}$ and $1.43 \mathrm{mg} / \mathrm{kg}$ body weight of sildenafil citrate base dissolved in distilled water daily for 30 days, through orogastric feeding tube, while that of the control group $\mathrm{D}$, received equal volume of distilled water daily during the period of the experiment. Hippocampal sections of the treated rats stained by Hortegas lithium carbonate hitochemi- 
cal method revealed astrocytes stained black as in control, but hippocampal sections of rats treated with, $0.25 \mathrm{mg} / \mathrm{kg}, 0.70$ $\mathrm{mg} / \mathrm{kg}$ and $1.43 \mathrm{mg} / \mathrm{kg}$ body weight of sildenafil citrate base showed large, numerous and some few impaired astrocytes. We conclude that sildenafil citrate induced dose dependent reactive astrocytes formation in the hippocampus. This may impairs uptake of neurotransmitters and alter neuronal environment thus altering hippocampal function like memory and learning.

\section{OP-20}

The effects of Garcinia kola (bitter kola) on the memory of malnourished mice

Ajayi SA, Nwoha PU

Osun State University, Osogbo, Osun State, Nigeria

The present study was undertaken to assess the neuroprotective effects of aqueous extract of Garcinia kola on neurotoxin administered malnourished mice. The study was carried out using thirty-two adult Malnourished mice which were randomly assigned into four groups $(\mathrm{n}=8)$ : A, B, C, and D. Group A served as control, while the other groups served as the experimental groups. Animals in group A were fed malnourished diet ad libitum and water given liberally. Animals in group B were administered with 3-Nitropropionic acid (3-NP) (neurotoxin) only (20 mg/kg body weight), group $\mathrm{C}$ were given only Garcinia kola extracts, and group D were pre-treated with Garcinia kola extracts for seven days $(200 \mathrm{mg} / \mathrm{kg}$ ) prior to administration of neurotoxin (20 mg/ $\mathrm{kg}$ body weight). After three days of neurotoxins administration in the relevant groups, the brains were excised and fixed in formal calcium for histological processing. The study showed that hippocampal neurons of animals in group B exhibited some cellular degeneration and blood vessel blockage, which were not seen in groups A, C and D. Cresyl violet staining was least intense in group B than in groups A, C and D. Despite the fact that animals in group $\mathrm{D}$ has equal administration of 3-Nitropropionic acid concentration, there were no traces of neural degeneration as it was evidenced in group B. It was concluded that Garcinia kola has protective effects on the neurons of the hippocampus of malnourished mice.

\section{OP-21}

\section{Histological and stereological studies of apoptosis in adrenal cortex following dexametasone administration and evaluation of silymarine effects on this process}

Hamidian $\mathrm{G}^{*}$, Majd $\mathrm{NE}^{* *}$, Varzi $\mathrm{HN}^{* *}$

Department of Histology, Faculty of Veterinary Medicine, University of Tabriz, Iran*; Department of Histology, Faculty of Veterinary Medicine, Shahid Chamran Universit of Ahvaz, Iran**
Adrenal cortex is an essential tissue for life and its function can be affected by many chemical agents and drugs such as dexamethasone. In this study 20 young adult male hamsters were randomly divided into four groups: group 1 as control group received no drug, group 2 received $7 \mathrm{mg} / \mathrm{kg}$ dexamethasone, group 3 received $100 \mathrm{mg} / \mathrm{kg}$ silymarin and group 4 which received $7 \mathrm{mg} / \mathrm{kg}$ dexamethasone and $100 \mathrm{mg} / \mathrm{kg}$ silymarin. All animals were injected IP for seven consecutive days and conducted in accordance with humane care and ethical animal welfare. At the eighth day, animals were euthanized and the adrenal glands were quickly removed, weighted and fixed in buffered formalin. The samples were processed by routine and standard paraffin embedding and serially sectioned. The total volume of adrenal gland, adrenal cortex and cortical zones were estimated by Cavalieri's point-counting principle using Weibel's multipurpose test grid M42. Total number of cortical cell in each zona was estimated by unbiased stereological methods. Detection of apoptotic cell was performed by using TUNEL immunocytochemical technique and apoptotic index was assessed in different adrenocortical zones. The results showed that there are no significant differences in the zona glomerulosa volume and cell number among examined groups. The volume of cortex, zona fasciculata, zona reticularis and cell number of these regions were significantly reduced in dexamethasone treated hamsters compared to controls $(\mathrm{P}<0.05)$, whereas in group 4 this reduction was not observed. TUNEL test revealed that the greatest and the smallest apoptotic index were observed in zona reticularis and zona glomerulosa of all groups, respectively. It is also showed that silymarin can reduce normal and dexamethasone-induced apoptosis in all adrenocortical zones of hamster $(\mathrm{P}<0.01)$. Finally, it can be concluded that silymarin is a suitable protective drug for side effect of glucocorticoid therapy in adrenal gland.

\section{OP-22}

\section{C-kit positive trigeminal neurons and interstitial Cajal like cells}

$\underline{\text { Rusu } M C^{*}}$, Pop $F^{*}$, Hostiuc $\mathrm{S}^{* *}$, Dermengiu $\mathrm{D}^{*}$, Ion $\mathrm{DA}^{*}$, Mirancea $\mathrm{N}^{* * *}$

Arol Davila" University of Medicine and Pharmacy Bucharest, Romania*; Mina Minovici National Institute of Legal Medicine, Bucharest, Romania**; Institute of Biology, Romanian Academy, Bucharest***

Objectives: c-kit positive neurons of sensory ganglia were proven in various species but not in humans as being nociceptive. Our aim was to identify whether or not human primary trigeminal neurons label with c-kit/CD117 and so the few existing experimental data can be extrapolated to humans. We also looked to establish whether or not non-neuronal cells also label with c-kit in the trigeminal ganglion. 
Methods: Human adult trigeminal ganglia resulted from eight donor cadavers were processed for immunohistochemistry on paraffin embedded samples using monoclonal antibodies for CD117/c-kit and for neurofilaments; four additional trigeminal ganglia were used for transmission electron microscopy (TEM). Results: We identified trigeminal neurons that were ckit positive and neurofilaments negative, on successive slides as the trigeminal nociceptive neurons are known as small dark cells that are neurofilaments-poor we concluded that c-kit labeled such neurons. c-kit also labeled mast cells and interstitial cells (ICs) within the trigeminal ganglion. c-kit+ ICs were located within and beneath the neuronal capsule built up by satellite glial cells (SGCs) and sent long, thin and occasionally moniliform processes projected on the neuronal somata. TEM evaluations confirmed the mixed composition of the neuronal envelopes, consisting of SGCs and ICs that had the ultrastructural gold standard for interstitial Cajal-like cells (ICLCs) and/or telocytes. Conclusion: c-kit labeling of nociceptive neurons is confirmed now in humans as it was previously described in rodents. Moreover, the neuronal envelopes appear more complex morphologically and functionally, as they consist not only of SGCs but also of ICLCs/telocytes. Acknowledgements: (1) Research Grant UEFISCSU, 317/2007. (2) This study was also supported by the SOP HRD, financed from the ESF and by the Romanian Government under the contract number POSDRU/89/1.5/S/64109.

\section{OP-23}

\section{The effects of grape seed extract on learning and memory}

Ozdemir MB*, Yonguc $\mathrm{N}^{*}$, Hatip-Ai-Khatip I**, Adiguzel E*

Department of Anatomy, Pamukkale University, Faculty of Medicine, Denizli, Turkey*; Department of Pharmacology, Pamukkale University, Faculty of Medicine, Denizli, Turkey**

It has been shown previously that neonatal hypoxic ischemic encephalopathy results in neuronal damage, learning and memory impairments. The aim of this study is to examine the effects of grape seed extract (GSE) on learning and memory impairments in hypoxic ischemic (modified Levine model) neonatal rats. For this purpose thirty male, twelve-days-old Wistar albino rat pups were divided into four groups. Treatments were given during three days after the hypoxic ischemia operation. Distribution of rats and procedures: group 1 (n:5): sham operated; group 2 (n:6): hypoxic ischemic and received \%0.9 saline ( $1 \mathrm{ml} /$ day; intraperitoneally); group 3 (n:9): received $50 \mathrm{mg} / \mathrm{kg} /$ day GSE (intraperitoneally); group 4 (n:10): hypoxic ischemic and received $50 \mathrm{mg} / \mathrm{kg} /$ day GSE (intraperitoneally). Water maze and eight arm radial maze tests were performed respectively eight weeks after hypoxic ischemic brain injury to test learning and memory. In water maze test, time to reach the platform (Escape Latency) was increased in group 4 according to groups 1 and $3(\mathrm{p}<0.05)$. Path length to reach the platform was increased in group 4 according to group $3(\mathrm{p}<0.05)$. The swimming speed (velocity) of rats was not different between groups $(\mathrm{p}>0.05)$. In eight arm radial maze test correct choices were decreased in group 2 according to group $1(\mathrm{p}<0.05)$. The number of errors in choosing arms and total errors were increased in group 2 according to group 1 ( $\mathrm{p}<0.05)$. Running time of rats was not different between groups $(p>0.05)$. Hypoxic ischemic encephalopathy led to impairments in eight arm radial maze test in rats. Treatment with grape seed extract showed positive effects on the impairments in eight arm radial maze test but these results were not statistically significant $(\mathrm{p}>0.05)$. This study was supported by TUBITAK (SBAG-3994. 108S157) and Pamukkale University Research Grant (2008TPF005).

\section{OP-24}

\section{Smoke extract of Datura stramonium leaf; effects on the heart, liver, lungs, kidney and testes of male Sprague Dawley rats}

Tijani-Adekilakun A*, Adekomi DA**, Muhammed AO***

Department of Anatomy, College of Health Sciences, Osun State University, Osogbo, Nigeria. *; Department of Anatomy, Faculty of Basic Medical Sciences, University of Ilorin, Ilorin, Nigeria. ${ }^{*}$; Department of Histopathology, University of Ilorin Teaching Hospital, Ilorin, Nigeria***

This study evaluated some of the toxic effects of smoke extract of Datura stramonium on the heart, liver, kidney and testes of male Sprague Dawley rats using histological studies. Ten male rats weighing between 100-120 g were used. The animals were randomly assigned into two (2) groups; A and B. Animals in group A were exposed to smoke from a completely burnt 0.74 $\mathrm{g}$ leaf extract of Datura stramonium, wrapped in $0.5 \mathrm{~g}$ of sterilized cotton wool for 5 minutes three times daily ( 7 am, 10 am, and $1 \mathrm{pm}$ ) respectively. The animals in group B were exposed to smoke from completely burnt $0.5 \mathrm{~g}$ of sterilized cotton wool with the same parameters as observed with the treatment groups. The duration of exposure was for five (5) days. Three hours after the last administration, all the animals were sacrificed by cervical dislocation. The heart, liver, lungs, kidney and testes were carefully excised, blotted dry, and fixed in formol saline for histological analysis using Hematoxylin and Eosin (H\&E) stain. Histopathological observations of the heart and kidney showed no significant histological alteration. Several histological deviations were seen in the sections of the liver, 
lungs and testes of the animals in group A (ranging from distortion and degeneration of the hepatocytes, reduction in the population of the germ cells and enlargement of the alveoli). These histological deviations indicate that the smoke extract of Datura stramonium has deleterious effects on the liver, lungs and testes of male Sprague Dawley rats. There was also a statistical significant decrease in the body weight of the animals in group A when compared with group B. From these, we inferred that exposure of male Sprague Dawley rats to the smoke extract of Datura stramonium is associated with structural damage to some vital organs.

\section{OP-25}

\section{Reversal of propoxur induced testicular alteration by Cissus populnea in Wistar rats}

Oyewopo O* Saalu LC**, Osinubi AAA***, Ashiru OA

Department of Anatomy, University of Ilorin, lorin, Nigeria*; Department of Anatomy, Lagos State University, College of Medicine, Ikeja, Lagos**; Department of Anatomy, University of Lagos, Lagos, Nigeria*** MART Medical Center, Ikeja, Lagos, Nigeria****

Background: Propoxur (2-Isopropoxy-phenyl-N-methylcarbamate) is a carbamate insecticide commonly used in private and public places to control insects and pests. Numerous incidents of acute carbamate poisoning and testicular toxicity have resulted from inhalation of sprays or contamination of crops or food. However, cissus populnea is a plant use as aphrodisiac and fertility enhances amongst the males attributed to the declining fertility tread that has been established in this population over the year, coupled with the attendant increasing level of erectile dysfunction. This study, therefore investigated the attenuating effects of cissus populnea on propoxur-induced testicular alteration.

Methods: 50 adults wistar rats were randomly divided into 5 groups of 10 rats each. Group I (control) were given distilled water, group 2 were given cissus populnea for 16 weeks, group 3,4 and 5 were exposed to 1 ppm propoxur every other day for 8 weeks. Thereafter, for another 8 weeks, group 3 was exposed to propoxur and cissus populnea, group 4 was left unexposed to propoxur and group 5 were exposed to propoxur. The rats sacrificed on the 17 weeks.

Results: The histological findings in group 1, 2 and 3 showed normal testicular cytoarchitecture, while group 4 and 5 showed cellular degeneration, tubular hyalination and vacuolation which are evidence of hypertrophy and a markedly extensive fibrosis.

Conclusions: In conclusion, propoxur is testicular cytotoxic, however, cissus populnea is capable of attenuating the toxic effect.

\section{OP-26}

The protective effect of omega-3 against the neurotoxicity of ifosfamide in male rats

Aziz F, Asad H

Salahaddin University, Iraq

Introduction: Although ifosfamide (IF) is a highly effective chemotherapeutic agent for treating a variety of pediatric and adult solid tumors, it has been shown to induce many side effects including neurotoxicity due to its oxidative stress. For this reason, researchers for improving the therapeutic efficacy of IF, used different antioxidants such.

Tools and Methods: The present study is performed to evaluate the ability of omega-3 fatty acids (Omega-3 FAs) to protect ifosfamide (IF) treated rats against the neurotoxicity of this chemotherapeutic drug. Thirty adult Wister male albino rats were used in the present study, in which they were randomly divided into six groups (each contained five rats). These groups included: Group 1: control (given only saline), Group 2: omega-3 (4 gm/kg diet), Group 3: IF (50 mg/kg b.w.), Group 4: IF(80 mg/kg b.w.), Group 5: IF (50 mg/kg b.w.) plus omega3, Group 6: IF (80 mg/kg b.w.) plus omega-3.The IF was administrated intraperitoneally for five consecutive days, while omega-3 was administrated orally within the diet for six consecutive days (started the day before IF administration). For the histological study, paraffin and plastic methods were employed for the preparation of cerebrum and cerebellum slides.Result: Both doses of Ifosfamide have caused degeneration of the pyramidal neurocytes of the 2 nd and 3 rd layers of cerebral cortex and the Purkinje cells of the middle layer of cerebellar cortex. These cells have been approximately histologically protected from the effect of IF when a combination of Omega-3 FAs and IF was administrated

\section{OP-27}

\section{Effects of smokeless tobacco use during pregnancy on liver of off springs of Swiss mice}

Mughal F, Memon Z, Kazi AS

Isra University Hyderabad, Pakistan

Nicotine in smokeless tobacco plays a significant role in fetal weight reduction, fetal death. Smokeless tobacco use is associated with reduction of gestational age. Histological changes have also been reported in the liver, since it is the major organ where nicotine, a component of tobacco is metabolised. 20 pregnant Swiss albino mice were selected and divided into two equal groups; Experimental group treated with tobacco powder mixed in normal diet at libitum) while Control group was treat- 
ed with normal feed at libitum for the same period. After termination of pregnancy 20 offspring's 10 male and 10 female each from experimental groups. Their weight was recoded and sacrificed after birth for histological analysis of their livers. Weight of liver in male offspring experimental group was $0.58 \pm 0.05 \mathrm{gm}$ and in female offspring was $0.50 \pm 0.03 \mathrm{gm}$ and in control group male offspring mice was $0.82 \pm 0.03 \mathrm{gm}$ and the female offspring was $0.78 \pm 0.02 \mathrm{gm}$, the differences in the weight of liver was found highly significant among both groups. Histological changes: Fatty changes were seen in liver parenchyma in which 14 out of 20 cases of livers from the offsprings of experimental mice, out of which 5 cases were accompanied by wide spread swellings and ballooning of hepatocytes indicating hydropic degeneration. However no significant differences were observed in the gestational period, the litter size. The use of smokeless tobacco during pregnancy has adverse effects on the offspring manifested by the cellular changes to liver parenchyma especially the fatty infiltration and hydropic degeneration.

\section{OP-28}

\section{Study of leptin expression changes during human fetal kidneys development}

Rancic $\mathrm{G}^{*}$, Atanossova $\mathrm{P}^{* *}$, Chalakov $\mathrm{G}^{* * *}$, Cvetanović $\mathrm{J}^{*}$, Rancic I*, Lestarevic $\mathrm{S}^{* * * *}$

Institute of Histology and Embryology, Medical University Niš, Serbia* Department of Anatomy, Histology and Embryology, Medical University Plovdiv, Bulgaria**; Department of Cell Biology, Medical University Varna, Bulgaria***; Institute of Histology and Embryology, Medical University Niš, Serbia****; Institute of Histology and Embryology, Medical University Priština, Serbia*****

Introduction: Leptin distribution was found in a number of non-neuronal tissues such as the fat, lung, liver, heart, reproductive organs and when expressed, could mediate a variety of direct peripheral actions. Leptin is expressed in placenta and in certain fetal tissues; however, little is known with regard to the function of this hormone in these tissues. Preliminary findings implicate leptin in pathways other than energy metabolism but most evidence suggests that placental and/or fetal leptin acts as a fetal growth factor. In an attempt to determine the role of leptin in the regulation of the renal development and later renal function, the purpose of the present study was to investigate the expression and distribution of leptin in the human fetal kidneys.

Material and Methods: Four complete human early fetuses (9.-10. weeks old) and 14 kidneys with surrounding adipose tissue of 12.- 40. weeks old fetuses were obtained after pregnancy termination. Samples were fixed and processed. Tissue sec- tions were stained with $\mathrm{HE}$, Masson, Jones methods and immunocytochemical ABC technique with specific antibodies to leptin. Results.Leptin labeling was found to be weekdependent and present perirenally, in mesonephros and in metanephric blastema; mainly associated with ureteric bud, vascular structures near $\mathrm{S}$ form during nephrongenesis and in medulla on papillary epithelium and its larger ducts.

Conclusion: The kidney is not only a site of leptin metabolism, but is also a target organ for leptin action during kidney development. The data indicate that leptin expression during renal development acts as a temporary fetal growth factor, but this role is far from clear.

\section{OP-29}

\section{Novel synthetic retinoids demonstrate different effects on limb and face development}

Lopez-Real RE*, Budge JJR*, Collings $\mathrm{JC}^{* *}$, Barnard $\mathrm{JH}^{* *}$, Marder TB ${ }^{* *}$, Whiting A**, Hunt $\mathrm{PN}^{*}$, Przyborski SA*

Department of Biological and Biomedical Sciences, Durham University, UK*; Department of Chemistry, Durham University, UK**

All-trans retinoic acid (ATRA) is known to affect the expression of developmental genes during embryogenesis. Incorrect levels of ATRA can induce serious teratogenic defects including craniofacial and limb malformations. Therefore, metabolism and availability of ATRA is tightly controlled in vivo. ATRA can isomerise upon exposure to light and this may induce different effects compared to pure ATRA. Therefore, photostable analogues are advantageous for future research. Two photostable, novel retinoids, EC23 and EC19, can induce neural and epithelial differentiation respectively in cultures of human pluripotent stem cells (Christie, Organic and Biomolecular Chemistry, 6; 3497, 2008). The aim of our work was to investigate the activity and mechanism of EC19 and EC23 in vivo. Retinoids were supplied to chick wing buds using in ovo microsurgery. EC23 is more toxic than ATRA whereas EC19 exhibits a similar toxicity to ATRA. EC23 has induced digit duplications and craniofacial abnormalities at concentrations two orders of magnitude lower than ATRA. This suggests that it is a more potent retinoid and mimics the effects of ATRA. EC23 also generates a shortened scapula which has not been seen with other retinoids. Current work is to characterise these novel retinoids further by investigating their mode of action and metabolism. This includes analysis of retinoid receptor expression and the action of EC23 on tissue development ex vivo in comparison to other synthetic retinoids using a chondrogenesis assay. Preliminary data indicates that the potency of EC23 is partly due to its resistance to metabolism unlike EC19 or ATRA. 
OP-30

CpG oligodeoxynucleotides induce antimicrobial peptide cathelicidin expression in primary glial cells

Brandenburg $\mathrm{LO}^{*}$, Tauber $\mathrm{S}^{* *}$, Albrecht $\mathrm{LJ}^{*}$, Jansen $\mathrm{S}^{*}$, Gerger J**, Wruck CJ*, Pufe T*

Institute of Anatomy and Cell Biology, RWTH Aachen University, Germany*; Department of Neurology, RWTH Aachen University, Germany**

Meningitis is a lethal disease characterized by the inflammation of the meninges. Due to the bacterial infection the immune system is stimulated to produce antimicrobial peptides as important part of the innate immunity, but little is known about their expression and function in the brain. The aim of this study is to investigate the involvement of the patternrecognition-receptor toll-like receptor 9 (TLR9) in the expression of cathelin-related antimicrobial peptide (CRAMP) in primary glial cells (astrocytes and microglia). We examined the CRAMP expression after treatment with TLR9 agonist unmethylated cytosine-guanine oligodeoxynucleotide motifs (CpG-DNA) in primary glial cells using real-time RT-PCR in vitro and in vivo by intracerebroventricular infusions of $\mathrm{CpG}$ DNA in mice using immunofluorescence. The pathways which regulate the expression of CRAMP have been identified. Using a mouse meningitis model, we investigated the CRAMP expression in TLR9-knock out and wildtype mice using immunofluorescence. Furthermore we examined for changes in the extracellular signal-regulated kinase (ERK) signal transduction pathway after stimulation with toll-like receptor 9 ligands in glial and transfected HEK293 cells. We show CpGDNA-induced increase of CRAMP expression by real-time RT-PCR and immunofluorescence in glial cells. The expression of CRAMP in glial cells involved different signal transduction pathways. Furthermore, we demonstrated that $\mathrm{CpG}-$ DNA-induced ERK1/2 phosphorylation is depended on TLR9, but also the involvement of scavenger receptor MARCO (macrophage receptor with collagenous structure) in CpG-DNA-induced cell activation and CRAMP expression. Together, these results suggest that cathelicidins produced by glial and other cells play an important part in the innate immune response against pathogens in central nervous system bacterial infections.

\section{OP-31}

Consanguinity, maternal age and parity influence the incidence of congenital malformations in fetus

Kaki SB, Abdul Latif AZ, Hadi SA, Zin T

(Unisza) Faculty of Medicine Kampos Kota Jalan Sultan Mohmad Kuala Terengganu, University Sultan Zainal Abidin, Malaysia
For the present study 1010 infants/ fetuses from King George Hospital Visakhapatnam, India, during the period 2004 and 2006 were taken and studied that 17 subjects were found with congenital anomalies. Personal and maternal details of pregnant women like age, consanguinity and maternal details, history of any affected siblings in the family were recorded. Photographs and X-ray photographs of infants/fetuses were taken. The dead fetuses were embalmed with $10 \%$ formalin and preserved for further investigations. From the above study of 17 congenital anomalous babies, 11 (64.70\%) had consanguineous parents. 15 out of 17 (88.23\%) abnormal babies were born to young mothers, whereas out of 76 elderly (above 35 years of age) mothers, only 2 mothers $(2.63 \%$ ) delivered anomalous babies and out of 934 young mothers only 15 (1.60 $\%)$ gave birth to abnormal babies. Comparative study showed that $9(1.11 \%)$ out of 808 primi gravidas, 7 (3.68\%) out of multigravidae, and $1(8.33 \%)$ out of total grand multigravidae, gave birth to abnormal babies. It shows that there is significant increase in incidence of abnormal babies to beget to elderly women. There are 13 types of anomalies recognised such as-anencephaly-5, Hydrocephaly-1, Cleft lip and palate1,Talipus equinovarus-1, Congenital hydrocoele-1, Turner's syndrome-1, Spinabifida with meningocoele-1, Exompholus-1, Bifid scrotum-1, Recto-urethral fistula-1, Sacrococcygeal tertoma-1, Ectopiavesicae-1 and Meningocoele-1. The total number of infants/fetuses born are 1010 of which 563 were male, 447 are female. Among the 1010 new born 17(1.68\%) were having congenital abnormaliti es. Out of 17 anomalous babies 14 are male and 3 are female. Consanguinity, maternal age, parity etc. influence the incidence of congenital anomalies.

\section{OP-32}

Role of Ste20-like kinases SPAK and OSR1 in regulation of kidney electroneutral cation-Cl cotransporters by vasopressin

Saritas $\mathrm{T}^{*}$, Palige $\mathrm{A}^{*}$, Ellison $\mathrm{D}^{* *}$, Mccormick $\mathrm{J}^{* *}$, Delpire $\mathrm{E}^{* * *}$, Borschewski A*, Uchida $\mathrm{S}^{* * *}$, Bachmann $\mathrm{S}^{*}$, Mutig $\mathrm{K}^{*}$

Department of Anatomy, Charité University Berlin*; Division of Nephrology and Hypertension, Oregon Health \& Science University, Portland, Or**; Anesthesiology, Vanderbilt University School of Medicine, Nashville, Tn***; Nephrology, Tokyo Medical and Dental University, Tokyo, Japan $* * * *$

Aim of the present study was to elucidate the roles of sterile 20/SPS1-related proline/alanine-rich kinase (SPAK) and oxidative-stress response kinase 1 (OSR1) during acute and chronic regulation of the renal $\mathrm{Na}+\mathrm{K}+, \mathrm{Cl}--(\mathrm{NKCC} 2)$ and $\mathrm{Na}+, \mathrm{Cl}-\mathrm{NCC})$ cotransporters by vasopressin. For evaluation of acute effects adult SPAK-knockout (SPAK-/-) and wildtype mice (WT) were treated with the vasopressin $\mathrm{V} 2$ receptor agonist desmopressin (dDAVP, $1 \mu \mathrm{g} / \mathrm{kg}$ bodyweight, $30 \mathrm{~min}$ ) or vehicle. Chronic effects were studied on vasopressin-deficient 
Brattleboro rats (DI) treated with $\operatorname{dDAVP}(2.5 \mathrm{ng} / \mathrm{h})$ or vehicle for 3 days. Abundance and phosphorylation of SPAK, OSR1, NKCC2 and NCC were determined by immunoblotting and confocal microscopy. During steady state SPAK -/- mice showed markedly increased levels of phosphorylated NKCC2 (+320\% vs. WT mice; $\mathrm{p}<0.05$ ) without concomitant changes of total NKCC2 levels. Levels of total and of phosphorylated NCC were reduced compared to WT mice (-72\% for NCC,$58 \%$ for $\mathrm{pS} 71-\mathrm{NCC},-83 \%$ for $\mathrm{pT} 58-\mathrm{NCC}$; $<<0.05)$. Short term treatment with dDAVP induced comparably similar increases of NKCC2 phosphorylation in WT $(+55 \%$; $<<0.05)$ and SPAK-/- mice (+55\%; $<<0.05)$, whereas NCC phosphorylation was significantly enhanced only in WT (+84\% for pS71NCC and +50\% for pT58-NCC; p<0.05) but not in SPAK-/mice. Phosphorylation levels of the full-length and truncated SPAK forms were significantly increased in WT mice upon dDAVP $(+89 \%$ and $+85 \%$ respectively, $\mathrm{p}<0.05)$. In long term, dDAVP increased abundances of the full-length SPAK in the renal medulla $(+31 \%)$ and renal cortex $(+95 \%$; $<<0.05)$ of DI rats, whereas abundance of OSR1 was significantly enhanced only in the renal medulla $(+22 \%$; $<0.05)$. In conclusion, this study provides in-vivo evidence for divergent roles of SPAK and OSR1 in TAL and DCT and confirms the involvement of these kinases in the action of AVP. Work was supported by the German Research Foundation (FOR667).

\section{OP-33}

\section{Microscopic study on the pineal calcification of Bangladeshi cadavers}

\author{
Afroz $\mathrm{H}^{*}$, Nurunnabi ASM ${ }^{* *}$, Ara $\mathrm{S}^{* * *}$ \\ Department of Anatomy, Green Life Medical College, Dhaka, \\ Bangladesh*; Department of Anatomy, Dhaka Medical College, \\ Dhaka, Bangladesh**; Department of Anatomy, Dhaka Medical \\ College, Dhaka, Bangladesh***
}

Context: The human pineal gland is characterized by the presence of the calcified concretions, called 'pineal acervuli' or brain sand. These are basophilic extracellular bodies. The aim of the present study was to describe the microscopic features of the pineal calcifications and find out age related changes.

Materials and Methods: A cross-sectional, descriptive type of study was done in the Department of Anatomy, Dhaka Medical College, Dhaka, from July 2009 to June 2010, based on the collection of 60 human pineal glands from whole human brains of unclaimed dead bodies that were under postmortem examination in the Department of Forensic Medicine. After collection of the pineal gland, all the samples were divided into group A (15-45 years), group B (31-40 years), group C (41-50 years) and group D ( $>50$ years). Slides were produced by following the standard procedure and routine staining was done by using Harris' Haematoxylin and Eosin. The slides were observed for the pineal calcifications under the light microscope.
Results: In group A, $53.33 \%$ were found to be round, $40 \%$ oval and $6.67 \%$ of irregular shape. In group B, there were $45.46 \%$ round, $40.90 \%$ oval and $13.64 \%$ of irregular shape. In group C, there were $75 \%$ of irregular shape, $21.47 \%$ mulberry shaped and $3.53 \%$ oval. In group D, there were $80.65 \%$ mulberry shaped and $19.35 \%$ of irregular shaped. The mean diameter of the pineal calcification found were $351.14 \pm 111.69 \mu \mathrm{m}$ in group A, 600.00 $\pm 232.69 \mu \mathrm{m}$ in group in group B, $909.43 \pm 124.18 \mu \mathrm{m}$ in group $\mathrm{C}$ and $1541.67 \pm 224.54 \mu \mathrm{m}$ in group D. The differences of diameter of the pineal calcifications between group A \& C, A \& D, B \& C, B \& D and C \& $\mathrm{D}$ were statistically significant $(\mathrm{p}<0.05)$.

Conclusion: Age related variations were found in shape and diameter of the pineal calcification in Bangladeshi cadavers.

\section{OP-34}

\section{Pre- and postnatal development of the mandible}

Mihaela $M^{*}$, Vascu $\mathrm{AM}^{*}$, Vascu MB*, Fatu $\mathrm{IC}^{*}$, Fatu $\mathrm{C}^{* *}$

Gr. T. Popa University of Medicine and Pharmacy, iasi, Romania*; Apollonia University, lasi, Romania**

Mandible develops from the anterior half of the first pharyngeal arch. This process starts early, from the 3rd week of development. Its ossification begins in the 30th-40th day of intrauterine development, but some areas are ossified later, about 20 years after birth, as the chin and mental suture. We have studied 24 human fetuses of different ages and gender and we have analyzed the distribution of inferior alveolar nerve, the opening of the gonion and the parameters of the proximal end of mandibular ramus. Anatomical and imagistic investigations were performed on 35 postnatal mandibles of different ages to analyze the same parameters. Our results point out modifications in the relations of mandibular canal with the alveolar margin, modifications of gonion and mandibular condyle, in relation with age and health status of the inferior stomatognathic system. In conclusion, mandible undergoes significant changes during both pre- and postnatal life, mainly in relation with different stages of postnatal evolution (prepuberty, postpuberty, adult or elderly).

\section{OP-35}

The corpse preserved with Thiel's method and with reestablishment of the circulation by pumping seudo blood, as model of continued learning of innovative surgical technologies

del Campo FS, Ferrer MS, Ferrer MLS, Lara MH, Garcia VA, Calpena FT, De La Blanca AS

Histology and Anatomy Department. Miguel Hernández University, Spain 
We propose a technique to use the corpses prepared in such a way that, not only they have the appearance, colour, texture and distention to allow us to perform arthroscopic, endoscopic or laparoscopic techniques, but in addition, its vascular recirculation presents the challenge of surgical bleeding and therefore the need to carry out preventive hemostasis or, if necessary, aspiration and clamping of a bleeding pedicle.

Materials and Methods: We have used corpses prepared with Thiel's technique, modified only by the dissection of the Carotid artery and the Jugular vein, and the cannulation using T-cannulas. We perfuse with Thiel's solution through the carotid until it goes out through the Jugular. Then, the Jugular is clamped and we perfuse more solution. 24 hours later we can use different procedures: a) If abundant liquid is drained from the Jugular, we will restore the perfusion by means of Thiel's solution coloured with water-based painting. It has the property of not spreading its colouring, allowing the wash and aspiration of the cavities . To perfuse we use a peristaltic pump. b) If the intracapillary coagulation does not allow the free circulation of the fluid, the femoral vessels are dissected at level of the Scarpa's Triangle. Then we create an arteriovenous fistula and finally restore the recirculation. c) The third technique consists of establishing the recirculation by means of the reperfusion, entering through the carotid and going out through the femoral artery. This allows us to have enough pressure in the arterial tree (pulsing) and very little at venous level.

Results and Conclusions: Different specialists have very favourably considered this model which, in addition, permits the vascular reconstructive surgery, the microsurgery. Moreover, it permits the investigation of new approaches in non-regulated surgery and the learning by the specialists, reducing the adverse results of the learning curves.

\section{OP-36 \\ Preservation of specimens using new flexible unsaturated polyester resin (UP 89) \\ Esfandiari E, Mehr MS, Rabiei AA, Dashti GR \\ Department of Anatomical Sciences, Medical School, Isfahan University of Medical Sciences, Isfahan, Iran}

Introduction: Preservation of specimens using new flexible unsaturated polyester resin (UP89) were synthesized in the plastination laboratory of Isfahan University was studied. As plastination was first introduced by Von hagens in 1978. In plastination technique specimens have unique position as teaching aids for easy store and handle by students. In this study we applied UP89 technique to examine the new synthesized resin instead of the silicon resin in conventional (S-10) technique.
Materials and Methods: This study was carried out by using suitable raw materials of modified unsaturated polyester resin (UP89). The synthesized resin was found to be transparent and flexible enough to be used in plastination. The specimens such as heart, liver, femur of sheep, fish, lizard and human heart were utilized. Initially they were fixed by $5 \%$ formaldehyde, then dehydrated in cold acetone $\left(-25 \mathrm{C}^{\circ}\right)$ and defatted in warm acetone $\left(+25 \mathrm{C}^{\circ}\right)$. In forced impregnation stage, $1 \%$ peroxide (MEKP) was added to resin, and Forced impregnation was performed at a low temperature $\left(10 \mathrm{C}^{\circ}\right)$ in order to avoid the gelation of resin. The curing of the specimens was carried out in the UV light and heat cabinet. After a period of two months, appropriate curing was obtained and the specimens were found to be dry.

Results: The specimens obtained by UP89 technique were found to show acceptable appearance for teaching anatomy and museum specimens. The specimens were odorless, dry and flexible compared to the specimens prepared by the S-10 technique. Since the cost was observed to be lower than the prepared plastinated specimens by the S-10 technique.

Conclusion: The synthesized resin UP89 provides an excellent opportunity to preserve and study the different tissues of human and animal specimens. The unsaturated polyester resin (UP89) can be an appropriate material in place of silicon resin for S-10 technique.

\section{OP-37}

Evaluation of variations and asymmetry in cerebral sulci Songur $\mathrm{A}^{*}$, Gonul $\mathrm{Y}^{* *}$, Uzun I**, Uygur R**, Alkoc OA***, Caglar $\mathrm{V}^{* *}$, Kucuker $\mathrm{H}^{* * * * *}$

Department of Anatomy, Faculty of Medicine, Afyon Kocatepe University, Afyonkarahisar, Turkey*; Department of Anatomy, Faculty of Medicine, Namik Kemal University, Tekirdag, Turkey**; Council of Forensic Medicine, Istanbul, Turkey***; Department of Anatomy, Faculty of Medicine, Düzce University, Duzce, Turkey***; Department of Forencis Medicine, Faculty of Medicine, Sakarya University, Sakarya, Turkey*****

Functional asymmetry (dominancy) in cerebral structures which emerges during postnatal development and related to neural development is a known feature. It is a matter of curiosity that whether there is a convergence between the morphological asymmetry and the functional asymmetry, and is significant in surgery. The aim of our study was to investigate the variations of several sulci on the lateral aspect of the cerebrum in regard to main sulci and related reference key points and to compare morphometric measurements of the hemispheres. Our study was carried out on forensic autopsy cadavers having no cerebral damage. A total of 100 cerebral hemispheres from 50 cadavers were examined. Length of several sulci on the lateral aspect of the hemispheres and the distances between the sulci and nearby sulci and reference key points were measured. Digital compass and fold- 
ing plastic ruler were used for measurements. Encountered variations were examined and photographed. Variations: Superior frontal sulcus (SFS), inferior frontal sulcus (IFS), superior temporal sulcus (STS), precentral sulcus (preCS) and postcentral sulcus (postCS) were found to be discontinuous in $60 \%, 46 \%$, $41 \%, 84 \%$ ve $70 \%$ of the hemispheres, respectively. Asymmetry: Many differences in morphometric measurements were seen between left and right hemispheres. However, only four of them showed statistically significant results as follows: the distances between SFS posterior end and longitudinal fissure (right $26.52 \pm 6.085 \mathrm{~mm}$, left $22.36 \pm 4.411 \mathrm{~mm}, \mathrm{p}=0.000)$, STS posterior end and lateral sulcus posterior end (right $27.50 \pm 5.898 \mathrm{~mm}$, left $34.28 \pm 5.562 \mathrm{~mm}, \mathrm{p}=0.000$ ), as well as lengths of external occipital fissure (right $27.94 \pm 4.206 \mathrm{~mm}$, left $34.00 \pm 4.562 \mathrm{~mm}$, $\mathrm{p}=0.000$ ), and discontinuous course of STS (right $26 \%$, left $56 \%$,). It is difficult to recognize cerebral sulci during surgery and variations are frequently encountered. Furthermore, there is usually a morphological asymmetry between the right and left hemispheres for any individual. Thus, we think it may be important to consider variations and asymmetry in neurosurgery as well as in anatomy education.

\section{OP-38}

Invasive complimentary medicinal methods and related anatomical structures

$\underline{\text { Kalaycioglu A }}$

Department of Anatomy, Faculty of Medicine, Karadeniz Technical University, Trabzon, Turkey

Practice of complimentary and modern medicine techniques including acupuncture, neural therapy and hirudotherapy may necessitate use of invasive methods. Acupuncture points are especially utilized during these procedures. Determination and preference of these points are reviewed in several studies and studies in this field are ongoing. Investigation of these points from perspective of anatomy highlights relations with peripheral nerves, arteries and fascias. Additionally, establishment of their relationship with the central nervous system structures including the thalamus, limbic system the value of these techniques in modern medicine has been further strengthened.

\section{OP-39}

\section{Nano-bio glass biomimic scaffold fabrication for calvarial bone}

Hossennejad $\mathrm{F}^{* *}$, Hafezi $\mathrm{F}^{* *}$, Amiri $\mathrm{A}^{* *}$, Fooladi AAI***, Nourani MR*

Nano-Bio Technology Research Center, Baqiyatallah University of Medical Sciences, Tehran-Iran*; Department of Chemistry, Islamic Azad University, Central Tehran Branch, Tehran, Iran**; Molecular Biology Research Center, Baqiyatallah University of Medical Sciences Tehran, Iran***
Aim: The repair of large segmental of calvarial bone defects due to trauma, inflammation and tumor surgery remains a major clinical problem. Surgeons have been trying to restore functionality appearance using autografts, allografts, and xenografts without totally acceptable results. As a result, physicians, scientists, and engineers have been trying to expand new techniques to improve bone growth and bone healing. Calvarial bone structure consists of compact bone in outer and inner tables can be seen with the diploe sandwiched in between.

Methods: 6-month old New Zealand rabbits parietal bones were sectioned and morphometrically measured the different parameters such as: whole thickness, compact tables thickness and diploe or cancellous table as well. The porous size of cancellous table also analyzed. Nano-bio Glass/gelatin scaffold fabricated was made through Sol-Gel in different percentage of Nanoparticle and finally the $10 \%$ and $30 \%$ were similar to the compact and cancellous bone respectively. Fabricated three lamellar bone, of two compact lamellae in outer and one porous layer in the middle to mimic the composition and structure of natural bone. The composition and the morphology of the scaffold were characterized by X-ray diffraction, thermal gravimetric analysis and field emission scanning electron microscope, respectively. The cytocompatibility of the scaffold was evaluated by in vitro cell culture carried out using fibroblast.

Result: It was found that Nano-bio Glass/gelatin scaffold structurally, had three layers and interconnected porous structure and its pore size. After 10-day culture, fibroblast cells were attached and proliferated on the surface of the scaffold. Some cells penetrated into the scaffold and spread within the scaffold.

Conclusion: Because of biocompatible and biodegradable charachtristic of Nano-bio Glass/gelatin scaffold and mimic fabrication to natural bone, it could be an excellent candidate as a synthetic calvarial bone graft instead of autograft and so on.

\section{OP-40}

\section{Potential role of nuclear factor erythroid 2-related factor 2 (NRF2) in the amelioration of amyotrophic lateral sclerosis (ALS)}

\author{
Rosen C, Fragoulis A, Pufe T, Wruck CJ \\ Institute of Anatomy and Cell Biology, RWTH Aachen University, \\ Germany
}

Introduction: Mutations in $\mathrm{Cu} / \mathrm{Zn}$ superoxide dismutase (SOD1) are a cause of motoneuron death in about $20 \%$ of cases of familial amyotrophic lateral sclerosis (ALS). Although the molecular mechanism of which these mutations induce motoneuron cell death is to a large extent unknown. There is significant evidence that oxidative stress makes a major contri- 
bution to the selective death of motoneurons in this disease. Nuclear factor erythroid 2-related factor 2 (Nrf2) is known as the major regulator of a battery of genes encoding detoxifying and antioxidative enzymes via binding to the antioxidant response element (ARE). We and others had shown that activation of Nrf2 protects against a large variety of insults, particularly in neuronal cells. The aim of the current in vitro study was to elucidate the protective role of the Nrf2/ARE-System against oxidative stress in the motoneuron-like cell line NSC34, transfected with human SOD1G93A.

Methods: The Nrf2/ARE-System in the motoneuron-like cell line NSC34, transfected with hSOD1G93A, was activated via the stable/transient transfection of shRNA against the Nrf2 inhibitor Keap1. ARE activation was measured in motoneuronlike cell line NSC34, transfected with hSOD1G93A, in a dose response assay utilising a Promega Luminometer. The potential of Keap1-RNAi knockdown NSC34 cells transfected with hSOD1G93A was measured via cell viability assays. The expression of $\mathrm{Nrf} 2$ target genes and the effect of the gene silencing by RNAi were monitored by rael time RT-PCR.

Results: WST and MTT assays show a higher viability of NSC3 4 cells, transfected with shKeap1, compared to untransfected NSC34 cells. Corresponding real time RT-PCR show a higher expression of $\mathrm{Nrf} 2$ target genes compared to untransfected NSC34 cells.

Conclusion: We demonstrate that the activation of the Nrf2/ARE-System in the motoneuron-like cell line NSC34, transfected with hSOD1G93A, leeds to a lower vulnerability against oxidative stress.

\section{OP-41}

\section{TNF- $\alpha$ induced NRF2 activation in monocytes}

Fragoulis A, Döring M, Rosen C, Brandenburg LO, Pufe T, Wruck CJ

Institute of Anatomy and Cell Biology, Medical Faculty, RWTH Aachen University, Aachen, Germany

Background: Oxidative stress has been implicated in a variety of inflammatory diseases including Rheumatoid Arthritis (RA). The transcription factor Nuclear factor-erythroid 2 (NF-E2)related factor $2(\mathrm{Nrf2})$ is known to maintain the cellular defence against oxidative stress via binding to the antioxidant response element (ARE) within the regulatory regions of antioxidative and detoxifying genes. Nrf2 has also been suggested as critical regulator of the innate immune response. Our aim was to investigate if TNF- $\alpha$ plays a role in $\mathrm{Nrf} 2$ activation in monocytes.

Methods: The Nrf2-activity was measured using an AREluciferase mouse treated with antibody-induced arthritis AIA (a
RA inflammation model) in the IVIS imaging-system. For in vitro studies, Nrf2/ARE activation was analyzed in luciferase assays with the human monocytic cell line THP-1 stable transfected with pGL4-ARE. We used various kinase inhibitors and antioxidants to elucidate the involved signal transduction. Gene expression of Nrf2 target genes HO-1 and NQO1, was studied by qRT-PCR. ROS production and accompanying oxidative stress damage in TNF $\alpha$ stimulated monocytes and murine macrophages was investigated using H2DCF-DA and oxyblot technique. Results: IVIS imaging-system data illustrate a time-dependent Nrf2 activation in the course of AIA. Furthermore, we show that TNF- $\alpha$ stimulation of THP-1 cells results in Nrf2 activation and subsequent upregulation of $\mathrm{HO}-$ 1 and NQO1. This Nrf2 activation depends on ERK and p38 kinase activity and NADPH Oxidase activation.

Discussion: These data demonstrate that TNF- $\alpha$ stimulation leads to Nrf2 activation in phagocytotic cells. This may lead to increased protection against oxidative stress in the course of phagocytosis-associated ROS production.

\section{OP-42}

\section{Correlation dependence of rats small intestine Peyer's patches' morphometric parameters}

Kaschenko S, Morozova E

Department of Histology Lugansk State Medical University, Cytology and Embryology Luhansk, Ukraine

Lugansk state medical university, Actuality Mucous membranes are one of the main barriers on the way of antigens penetration, in which an important role is played by the Peyer's patches of small intestine. The objective of this work was to detect a correlation dependence of morphometric parameters of small intestine Peyer's patches of white genesial rats-males. Metods The linear indexes of Peyer's patches of small intestine were studied on 30 animals with mass 50-100 g (I group), 150$200 \mathrm{~g}$ (II group), 250-300 g (III group), 350-400 g (IV group). The amount of patches was counted; their length, width and distance between them were measured in mm. A cross-correlation analysis was conducted with calculation of Bravo-Pirson's correlation coefficient. Results It was determined, that a correlation dependence took place between amount of Peyer's patches and: their length in groups I, III and IV, their width in the III group, and also between distance from ileocecal corner to the first Peyer's patch in the IV group; between the width and the length in the I and the III groups; the width and the distance from ileocecal corner to the first Peyer's patch in the I group of the animals (correlation coefficients $|0.7|-|1|$ ). Conclusions Findings obtained testify to strong cross-correlation dependence between linear indexes in each group of animals. 


\section{OP-43}

Ethanol and nicotine developmental toxicity is mediated by gap junction disruption in cardiomyocytes in culture

\author{
Memon $S^{*}$, Pratten $M^{* *}$ \\ Liaquat University of Medical \& Health Sciences, Pakistan*; The \\ University of Nottingham, UK**
}

Connexin channels (gap junctions), provide communication between cells and in the heart they are also involved in embryonic development. Congenital heart defects are the leading cause of infant mortality and morbidity and excessive consumption of social drugs, particularly alcohol and tobacco, is one of the major contributing factors. Here the potential of disruption to connexin 43 expression as a mechanism for ethanol and nicotine induced teratogenesis was investigated. Nutritional deficiency prenatally is also an important risk factor and the preventative role of folic acid and vitamin $\mathrm{C}$ was also studied. Cardiomyocytes were isolated from 5 day old white leghorn chick embryos and cultured in 8-well chamber slides. They were exposed to $10 \%$ ethanol or $10 \mu \mathrm{M}$ nicotine only, or with $100 \mu \mathrm{M}$ vitamin $\mathrm{C}$ and $1 \mathrm{mM}$ folic acid in addition, on day 2 of incubation. 12-O-Tetradecanoylphorbol-13acetate (50nM TPA) a positive control for phosphokinase C (PKC) activated pathways and dibutyryl cAMP (100 $\mu \mathrm{M}$, for phosphokinase A activated pathways) was added. On day 6 of incubation, after fixing and permeabalizing the cells, Connexion-43 antibody was applied for overnight incubation at $4{ }^{\circ} \mathrm{C}$. Then the secondary antibody was applied for 1 hour at room temperature in the dark. Slides were rinsed in fresh BSA solution, then in water and mounted using Vectashield ${ }^{\mathrm{TM}}$ containing DAPI. The slides were viewed under a confocal microscope. Ethanol and nicotine caused a reduction in expression of Connexin-43 which was not evident when vitamin $\mathrm{C}$ or folic acid are co-administered. A re-distribution of the label from the cell surface to a juxtanuclear location was evident, with addition of ethanol or nicotine. Addition of TPA also showed a similar intracellular distribution, whereas addition of cAMP increased the surface expression of connexion 43. It is therefore likely that both ethanol and nicotine act via PKC and their effects can be negated by vitamin supplementation.

\section{OP-44}

Role of connexin40 in developing ventricular conduction system

Benes J*, Sankova B*, $\underline{\text { Sedmera D** }}$

Institute of Physiology, Academy of Sciences of the Czech Republic*; Institute of Anatomy, First Faculty of Medicine, Charles University in Prague**
Connexin40 (Cx40) is a major gap junction protein that is expressed in His-Purkinje system. During development, its expression is more widespread. In adult mice lacking $\mathrm{Cx} 40$, slowing of conduction in the subendocardial layers and right bundle branch (RBB) block were reported. In our study, we focused on the impact of $\mathrm{Cx} 40$ deficiency during development and maturation of murine His-Purkinje system. We used optical mapping at ED12.5-18.5 Cx40 deficient mice and compared them with wild type and heterozygous embryos. There was no major difference in ventricular activation patterns at ED12.5. In ED14.5 mutant embryos, there was an apparent delay in conduction system maturation manifesting as persistence of primitive activation pattern through the primary ring, and a significant decrease in proportion of functioning left bundle branches. RBB function, evidenced by presence of epicardial breakthrough near the apex of the right ventricle, was not affected by $\mathrm{Cx} 40$ deficiency at these developmental stages, but RBB block progressively appeared at ED16.5 and was significantly more prevalent among mutants compared with controls at ED18.5. We conclude that $\mathrm{Cx} 40$ is not essential for the early functionality of RBB and tissue geometry has a major role in appearance of the right apical breakthrough. Left bundle branch seems to be more sensitive initially, but later on, its more robust architecture compensates for $\mathrm{Cx} 40$ deficiency, highlighting thus asymmetries in form and function of the ventricular conduction system. Supported by MFM VZ 0021620806, AV0Z50450515 and AV0Z50110509, and GACR 304/08/0615.

\section{OP-45}

\section{Biological roles of clusterin in pancreatic beta-cell neogenesis}

\section{Park $\mathrm{IS}^{*}$, Min $\mathrm{BH}^{* *}$}

Department of Anatomy and Center for Advanced Medical Education by BK21 project, College of Medicine, Inha University, Korea*; Department of Pharmacology \& BK21 program for Medical Sciences, College of Medicine, Korea University, Korea**

Based on our previous observations that clusterin induction accompanies pancreas regeneration in the rat, we sought to determine if regeneration might be impaired in mice that lacked clusterin. We studied the impact of absent clusterin on morphogenic and functional features of regenerating pancreas. Clusterin induction was accompanied in the regenerating pancreas by a robust development of new lobules with ductules, acini and endocrine islets in wild type after partial pancreatectomy. In clusterin knock-out mice, however, pancreatectomy resulted in a poor formation of regenerating lobule. In particular, regeneration of beta-cells was also significantly reduced 
and was associated with persistent hyperglycemia. Duct cells obtained from pancreatectomized clusterin knock-out mice exhibited impaired beta-cell formation in vitro; this was restored by administration of exogenous clusterin. We suggest that clusterin plays a critical role to promote both exocrine and endocrine regeneration following pancreas injury, as well as for in vitro beta-cell regeneration.

\section{OP-46 \\ PRP (platelet rich plasma) leads to an increase of VEGFR-2 expression in a microvascular tissue flap a study in the VEGFR-2luc mouse}

Sonmez TT*, Vinogradov $\mathrm{A}^{* *}$, Tohidnezhad $\mathrm{M}^{* *}$, Zor $\mathrm{F}^{* * *}$, Riediger D*, Wruck CJ**, Pufe T**

Department of Oral, Maxillofacial and Plastic Facial Surgery, University Hospital RWTH-Aachen, Aachen, Germany*; Department of Anatomy and Cell Biology, RWTH Aachen University, Wendlingweg 2, 52074 Aachen, Germany**; Department of Plastic and Reconstructive Surgery, Gulhane Military Medical Academy, Ankara, Turkey***

The loss of vascularized soft tissue graft is a major problem in reconstructive surgery. Vascular endothelial growth factor (VEGF) is a potent angiogenic, mitogenic and vascular permeability-enhancing protein, which promotes the survival of microvascular tissue flaps. The platelet rich plasma (PRP) is due to the abundance of growth factors in various clinical areas in application. In the present study, the VEGFR-2 expression after use of PRP during the healing of microvascular skin flap was evaluated in a transgenic mouse model. Additionally, the local VEGF production in the microvascular tissue flaps with ischemia and after PRP application was examined via ELISA and immunohistochemistry. In 4 groups of operations, the effects of ischemia and local PRP application on the healing of the skin flap were studied in the mouse. The pedicle skin flap healing could be successfully visualized with a special Xenogen imaging device in the transgenic VEGFR-2luc mice. The longitudinal in vivo measurements were made in two day intervals until the 14th postoperative day. Finally tissue samples were harvested for immunohistochemical and biochemical investigations. The results of both methods showed that the VEGFR-2 was during the wound healing adjacent to the skin flap in particular between 4th and 12th days present. After application of PRP an increase could be detected. The investigations under ischemia with and without PRP application showed that PRP had a beneficial influence. In summary, we have shown that the local application of PRP in wound healing of the skin flaps has a positive effect concerning vascularization. This study further provides a good perspective concerning the use of new and innovative imaging technology and a practical methodology to support the routine tissue analysis. Due to the longitudinal analysis in one and the same animal the inter-individual variations are eliminated, which often complicate the analysis of conventional animal studies.

\section{OP-47}

Histological and immunohistochemical studies on the postulated protective and therapeutic roles of pentoxifylline on thioacetamide induced hepatic injury in adult male albino rats

Ahmed MS

King Fahad Medical City, Saudi Arabia

Introduction: Thioacetamide induces liver injury, with histological alterations similar to those observed in human cirrhosis, through releasing of tumour necrotising factor-alpha. Pentoxifylline is an anti-tumour necrotising factor-alpha which might decrease the hepatic injury. This study investigated the histological basis for the postulated beneficial role of pentoxifylline on the thioacetamide induced liver injury.

Material and Methods: Fifty adult male albino rats were divided into 5 groups; Group I: rats were intraperitoneally injected once daily with distilled water for 8 days; Group II: rats were intraperitoneally injected with pentoxifylline (200 $\mathrm{mg} / \mathrm{kg}$ body wt) once daily for 8 days; Group III: rats were intraperitoneally injected with thioacetamide $(50 \mathrm{mg} / \mathrm{kg})$ once daily for 8 days; Group IV: rats were concomitantly intraperitoneally injected with pentoxifylline and thioacetamide in doses and duration similar to groups II and III respectively; Group V: rats were injected with thioacetamide for 8 days as in group III and then injected with pentoxifylline from day 9 to day 16 in similar doses as in group II. Blood samples were collected for estimation of liver enzymes. Liver was removed and used for light and transmission electron microscopic studies. Using image analyzer, quantitative measurements and analysis of the optical densities for the Periodic Acid Schiff, Azan and tumour necrotising factor-alpha positive reactions were done. One-way ANOVA followed by Dunnett's test, and Pearson's correlation coefficient (r) were used.

Results: Thioacetamide led to elevated liver enzymes. Light microscopic study revealed degeneration and necrosis of hepatocytes, reduced glycogen, increased collagen and increased expression of tumour necrotising factor-alpha in the liver tissue. Electron microscopic study revealed degeneration of the cellular organelles. These changes were attenuated by using the pentoxifylline in preventive and to some extent in therapeutic groups.

Conclusion: Thioacetamide induced hepatic injury can be prevented and treated by using pentoxifylline. 


\section{OP-48}

NRF2 protects hepatocytes against liver injury and has a role in liver regeneration via stem cell activation

Wruck CI, Streetz KL, Fragoulis A, Rosen C, Kan YW, Kensler TW, Pufe T

Institute of Anatomy and Cell Biology, Medical Faculty, RWTH Aachen University, Aachen, Germany

Background: Increasing evidence suggests that oxidative stress play a key role in the etiology of sclerosing cholangitis. Aim of this study was to elucidate the hepatic defence mechanisms employed against oxidative stress and, in particular, the specific role of nuclear factor erythroid 2-related factor 2 (Nrf2). The DDC-model leads to chronic cholestatic liver injury and therefore resembles human diseases like sclerosing cholangitis and forms of metabolic liver diseases.

Methods: Mice were treated with 3,5-diethoxycarbonyl-1,4dihydrocollidine (DDC) containing diet and analysed over time.

Results: Mice deficient in Nrf2 showed significant more inflammatory cytokine expression and lymphocyte infiltration as wild type mice. Over time significantly more necrosis, apoptosis and cholestasis became evident in Nrf2-knockout mice. This was associated with stronger periportal oval cell activation. In contrast, mice with hepatocyte specific knockout of Keap1, the inhibitor of Nrf2, showed significant less liver damage, less inflammatory cytokine expression and lymphocyte infiltration. Interestingly, Keap1-liver knockout leads to enhanced stem cell proliferation in response to DDC feeding.

Conclusion: We show that Nrf2-dependent signalling protect liver from tissue damage induced by DDC feeding. Beyond, we showed that $\mathrm{Nrf2}$ has a role in liver regeneration via stem cell activation.

\section{OP-49}

\section{Plurihormonal lactotroph/gonadotroph cells in the human foetal pituitary}

Chirculescu ARM* ${ }^{*}$, Chirculescu $M^{* *}$, Coculescu $M^{* * *}$, Morris $\mathrm{JF}^{* * * *}$

Department of Anatomy and Embryology, C. Davila University, Bucharest, Romania and Department of Physiology, Anatomy and Genetics, University of Oxford, Oxford, UK*; Department of Anatomy and Embryology, C. Davila University, Bucharest, Romaina**; Department of Endocrinology, C. Davila University, Bucharest, Romaina***; Department of Physiology, Anatomy and Genetic, University of Oxford, Oxford, UK****

Pituitary cell lineages are defined from about the 6th week of human embryonic development (Puy \& Asa 1996).
Colocalisation for growth hormone (GH) and prolactin (PRL) is well recognized in normal adult human pituitary (somatomammotroph) cells, and for other hormones in pituitary adenomas which not infrequently express FHS (Coculescu et al 2006). We have previously demonstrated colocalisation of FSH or LH with GH in human embryo-fetal pituitaries (Chirculescu et al 2007) and have now studied colocalisation of PRL and gonadotrophins FSH and LH during human ontogenesis. Sections from pituitaries of therapeutically aborted (ethical permission) male human fetuses of 10-20 weeks were used. Colocalisation of FSH or LH (human monoclonal) and PRL polyclonal antibody immunostaining was studied using all combinations of primary and secondary antibodies coupled to FICT, Texas red or rhodamine. Controls used nonimmune serum or omission of one or other stage. PRL immunostaining was detected in about $10-15 \%$ of gonadotrophin-immunoreactive cells (i.e. less than with $\mathrm{GH}$ ), and appeared greatest in the 16-18 week foetus. PRL immunoreactivity in gonadotroph cells appeared as small spots, randomly spread on the clusters/rows of immunopositive cells; complete overlay was never observed. The data from direct and overlaid fluorescence images suggest that immunoreactivity is located in some secretory vesicles and varies among the pituitaries tested. These data show that colocalisation of PRL with FSH/LH can occur in apparently normal human foetal pituitaries, though it is less frequent than for $\mathrm{GH}$ and, as with $\mathrm{GH}$, decreases markedly from 16 to 20 weeks gestation. We hypothesize that the potential for coexpression of a gonadotrophin with GH and PRL is preserved and can be reactivated in adulthood during tumoral transformation, perhaps from progenitor cells.

\section{OP-50}

\section{Cortical branches of the superior cerebellar artery}

Esmer AF, Sen T, Tuccar E, Karahan T

Ankara University Faculty of Medicine Department of Anatomy, Ankara, Turkey

Cortical branches of the superior cerebellar artery (SCA) mainly distribute of the tentorial surface of the cerebellar hemispheres and vermis. Also some areas of the suboccipital and petrosal surfaces of the cerebellum could supply by the branches of the SCA. The study aimed to provide detailed information about cortical branches of the SCA and to shed light on the issues, which have not been adequately discussed. A total of 120 cerebellar hemispheres of 60 adult human cadaveric brains were used. Basilar arteries were cannulated and injected with colored latex. Then, the brains were embalmed in formaldehyde. The cortical branches of the SCA were investigated. Researchers found three different kinds of arteries originated from SCA and distributed at the tentorial surface of the cere- 
bellum: Hemispheric arteries (100\%), vermian arteries (98.34\%) and marginal branches (35\%) of the SCA. Also anastomoses between the cortical branches of the SCA and the cortical branches of the anteroinferior and posteroinferior cerebellar arteries were investigated. These anastomoses especially concentrated at the area of the postclival fissure of the cerebellum. An infratentorial-supracerebellar approach directed through a suboccipital craniotomy provides satisfactory exposure of the cortical branches. In each cerebellar hemisphere, the vascularization pattern of the cortical branches of the SCA is different to a greater or lesser extent. We believe that an awareness of this fact and sufficient anatomy knowledge about the cortical supply of the cerebellum will contribute significantly to surgical interventions.

\section{OP-51}

Entrapment of median nerve after fracture dislocation of elbow: a proposal of a novel approach

Armangil $M^{*}$, Bilgin SS*, Sen $\mathrm{T}^{* *}$, Basarir $\mathrm{K}^{*}$, Esmer $\mathrm{AF}^{* *}$, Demirtas $M^{*}$

Ankara University School of Medicine Department of Orthopedics and Traumatology Ankara, Turkey*; Ankara University School of Medicine Department of Anatomy Ankara, Turkey**

Introduction: Neurovascular injury after elbow fracture dislocation is not so often but when initiated can result in serious morbidity. Isolated median nerve paralysis could be seen especially in children after closed or surgical treatment of these cases. The aim of this study is to evaluate the innervation of the flexor pollicis longus (FPL) due to its proximal innervation and ease of assessment to define the time interval to the exploration of the median nerve and to propose an approach based on a cadaveric study.

Methods: A total number of 20 upper extremities of 10 fixed cadavers were dissected. FPL and its motor branches were evaluated. and also, three patients who had median nerve paralysis after treatment of elbow fracture dislocation at mean time of 15(13-18) months after injury underwent exploration.

Results: In 15 extremities the FPL was found to be innervated by two motor branches, in four three motor branches and in one extremity one motor branch was isolated. The length from the medial epicondyle to the point where motor branches inserted the FPL and the length from olecranon to the ulnar styloid were measured. In the light of our results, by assuming that after axonotmesis type injury the nerve should heal approximately one millimeter a day, the time interval to the exploration of the median nerve by using a formula.

Conclusion: If a nerve lesion does not heal in the anticipated time or the clinical sings of healing is beyond expectations, exploration of the injured nerve should be considered. It can be said that if a median nerve lesion occurs after elbow fracture dislocation, FPL function could be used to assess the nerve function whether it is healing.

\section{OP-52}

Involvement of the ophtalmic nerve in Herpes zoster keratoconjunctivitis

Fatu $C^{*}$, Vascu MG**, Fatu IC**, Vascu AM**, Moscu $M^{* *}$

Apollonia University, lasi, Romania*; "Gr. T. Popa" University of Medicine and Pharmacy lasi, Romania**

Ophtalmic nerve is one of the sensitive branches of trigeminal nerve. It supplies the sensitive territories around the eyeball, including palpebral and bulbar conjunctiva, palpebral, nasal and frontal skin. Herpes Simplex Virus has tropism for the peripheral nervous fibers. In the studied area, taking into account the histological particularities of the territory, it affects the anterior part of cornea, bulbar and palpebral conjunctiva and the lacrimal gland. A number of 60 cases with zoster keratoconjunctivitis were studied. Clinical signs were pain, tear drop, decrease visual acuity and rush at the anterior pole of eyeball. Results: the incidence was higher at 65-75 years old, in females and the lacrimal nerve was affected in most of the cases. In conclusion, our clinical study emphases the affinity of herpes zoster infection for the sensitive trigeminal branches, especially for the ophthalmic nerve, with severe involvement of the eyeball structures.

\section{OP-53}

Variations of the hypothalamic perforating branchs in patients with anterior communicating artery aneurysms: a cadaveric anatomical study

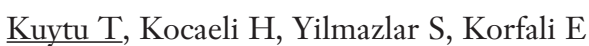

Department of Neurosurgery Uludag University Medical Faculty, Bursa, Turkey*

Objective: Aneurysms of the anterior communicating artery (AComA) are challenging lesions with their complex neurovascular anatomy and variations. The principal difficulty and source of complication during surgery of AComA aneurysms is the dissection of the hypothalamic arteries from the neck of the aneurysm. Previous studies that were performed on cadaveric specimens without an aneurysm on the AComA have reported variations of the perforating branches of the AComA, however, no such study have been reported on cadaveric specimens with AComA aneurysms.

Material and Methods: The anatomy of the AComA was studied in 40 formalin-fixed adult human brain specimens that were obtained after routine autopsy procedures. The specimens were assigned into two groups. The first group $(n=26)$ was 
formed by the specimens without ACoA aneurysms whereas the second group $(n=14)$ was formed by the cases with ACoA aneurysms. The dissections were performed under the microscope with fine instruments.

Results: Hypothalamic perforating arteries (HPA) were identified in $73 \%$ and $57 \%$ in groups 1 and 2 respectively. The diameter of the HPA were $0.34 \pm 0.14 \mathrm{~mm}$ and $0.39 \pm 0.10 \mathrm{~mm}$ in groups 1 and 2, respectively.In the ACoA aneurysms with anterior projection, the hypothalamic perforators did not show any relation with the aneurysm. In the aneurysms with posterior and superior projections, the hypothalamic perforators were displaced inferiorly and were frequently seen at the anteroinferior part of the aneurysm neck. In one case with an inferiorly projecting aneurysm, the HPA were located at the lateral side of the aneurysm neck. In most of the cases in whom one of the A1s was larger at one side, the HPA were found to arise on the larger A1 side.

Conclusion: In patients with superior and posterior projecting AComA aneurysms, if the approach was performed from the dominant A1 side the HPA should be looked for primarily at the anteroinferior part of the aneurysm neck. This knowledge may help reduce the mortality and morbidity associated with HPA injury during AComA aneurysm surgery.

\section{OP-54}

The morphometric evaluation of the tracheobronchial tree by multidetector computed tomography

Ulusoy $M^{*}$, Uysal II*, Kivrak AS ${ }^{* *}$, Karabulut AK ${ }^{*}$, Ozbek S**, Paksoy $\mathrm{Y}^{* *}$

Deparment of Anatomy, Selcuklu Medical Faculty, Selcuk University, Konya, Turkey*; Deparment of Radiology, Selcuklu Medical Faculty, Selcuk University, Konya, Turkey**

Objective: To evaluate the tracheobronchial tree by multidetector computed tomography (MDCT) in several age groups and in both sexes.

Methods: The measurements were made in axial, sagittal and coronal images of 4 slice computed tomography (CT) of 253 patients without any pathology (142 male, 111 female; age range: $0-74$ years). The patients were grouped into four age groups (018, 19-37, 38-56, 57 and older) in both sexes. The length and diameter of trachea, main bronchi and lobar bronchi were measured. and, also the subcarinal angles, interbronchial angles and several angles between the lobar bronchi were measured.

Results: Four type of trachea were detected as round $(33.9 \%)$, oval (45.8\%), square (3.5\%) and horseshoe shaped (16.6\%). Tracheal volume was significantly higher in men $(248.5 \pm 80.8$ $\left.\mathrm{mm}^{3}\right)$ than in women $\left(149.6 \pm 50.2 \mathrm{~mm}^{3}\right)$. Antero-posterior diameter of trachea was significantly higher in 38-56 year-old group than in $19-37$ year-old group $(18.4 \pm 2.3 \mathrm{~mm}$ versus 17.0 $\pm 6.5 \mathrm{~mm}$ ). And there are some significant differences in the measures of lobar bronchi between the age groups and the sex.
Conclusion: There are some significant differences in the measures of tracheobronchial tree. Awareness of these differences help achieving lesions and lower the complications in bronchoscope.

\section{OP-55}

\section{Aberrant right subclavian artery: a MDCT study}

Fazliogullari $Z^{*}$, Kivrak AS**, Dogan NU*, Karabulut AK*, Uysal II*, Ozbek S**

Department of Anatomy, Selcuklu Medical Faculty, Selcuk University, Konya, Turkey*; Department of Radiology Selcuklu Medical Faculty, Selcuk University, Konya, Turkey**

Introduction and Objectives: Aberrant subclavian artery is a rare defect where one of the subclavian arteries arise from an abnormal location on the aortic arch and is asymptomatic. The aberrant right subclavian artery (ARSA) usually arises as the fourth branch of the aorta just distal to the origin of the left subclavian artery and passes posterior to the esophagus or trachea on its way to the right upper extremity. The purpose of our study was to determine the prevalence and course of ARSA, anomalies of the aortic arch branches and origins of the vertebral artery.

Method: 2700 patients (1500 males, 1200 females), their ages ranging from 1 to 95 years, who had undergone multidetector computed tomography (MDCT) examination of the thorax for a variety of medical conditions were evaluated. For all of the patients, the prevalence of ARSA, the variations of the aortic arch and origins of the veretbral artery in those with ARSA were evaluated. The distance from the point of narrowing in the esophagus to the apex of the lung was measured.

Results: We identified 24 patients (\%0.89) who had ARSA. The ARSA followed a retroesophageal course in all cases and resulted in a mean narrowing of $6.31 \mathrm{~mm}$ in the esophageal diameter at a mean distance of $42.6 \mathrm{~mm}$ from the apex of the lung. In 4 of the 24 cases, truncus bicaroticus was determined. The origin of the vertebral artery was observed to be non-variant.

Conclusion: Different courses of this aberrant vessel may cause compression of the esophagus and mediastinal structures. The importance of MDCT in the evaluation of such variations in patients with dysphagia is emphasized.

\section{OP-56}

Study, in fetuses, of thoracic vagus nerves: their position and relation with gastric rotation

Biasutto SN, Ceccon GAF, de la Rosa M, Bortolin PA, Aliendo M, Repetto E

Chair and Institute of Normal Anatomy, Faculty of Medical Sciences, National University of Cordoba, Cordoba, Argentina

Studies on vagus nerves and traditional literature associate their final position with the gastric rotation occurred between the 4th 
and 6th week of gestation. The objective of this study was to observe vagus nerves in the thorax, specially, their position while passing the diaphragm, and determining the relation with the gastric rotation. We dissected vagus nerves from the inferior neck to the abdomen, in 20 fetuses with a crown-rump length of 64.4 to 153.7 (14 and 22 weeks of gestation). Most of them were male and only 2 female. Cardiac and pulmonary branches were cut to continue dissecting the nerves around the esophagus. The diaphragm hiatus was opened and the gastric rotation determined. Vagus nerves entered the thorax laterally to the common carotid arteries and included in the same sheath. At this level it was a big nerve, nearly as wide as the carotid artery. After giving the recurrent nerve, the main branch addressed to the pulmonary pedicle and provided the cardiac and pulmonary branches. Under the tracheal division, vagus nerves remained as a thin branch $(1 / 3$ or $1 / 4)$ in relation to the neck, but its distribution and location is not as simple as usually described. The right nerve showed many variations under the pulmonary pedicle: dividing into anterior and posterior branches, lateral going backward or frontward, anterior or posterior to the esophagus, while descending. The left nerve was laterally moving to the anterior wall of the esophagus (65\%), or anterior, or branching into anterior and posterior. The 2 cases with unrotated stomach had a multiple divided right nerve and an anterior left nerve. Instead this is a preliminary presentation, it does not seem so easy to associate vagus nerves location with the stomach rotation.

\section{OP-57}

Engineering of a dermal matrix scaffold with adult mesenchymal stem cells and platelet rich plasma: an innovative potential tool for tissue repair and regeneration

Zecchi-Orlandini $\mathrm{S}^{*}$, Benvenuti $\mathrm{S}^{* *}$, Mercatelli $\mathrm{R}^{* * *}$, Mirabella $\mathrm{C}^{* * * *}$, Saccardi $\mathrm{R}^{* * * *}$, Formigli L*, Orlandini GE*

Department of Anatomy, Histology, Forensic Medicine - University of Florence*; Department of Clinical Physiopathology - University of Florence**; Institute of Complex Systems - National Council of Research, Florence ${ }^{* * *}$; Immunoematology and Transfusion Medicine, University Hospital of Careggi, Florence****; Department of Hematology, Cord Blood Bank, University Hospital of Careggi, Florence $* * * *$

Background and Methods: The purpose of this study was to investigate the efficacy of Integra ${ }^{\circledR}$, an artificial dermal matrix used as a dermal template for skin regeneration, to form a multifunctional scaffold with human bone marrow-derived mesenchymal stem cells (hMSCs) and platelet-rich plasma (PRP) for tissue engineering and regenerative technology.

Results: First, we showed that PRP, used as a supplement for growth medium represented an optimal substitute for animal serum as well as a source of multiple growth factors, was able to satisfactorily support cell viability, cell proliferation and influence stemness gene expression in hMSCs. Moreover, Integra ${ }^{\circledR}$ appeared to be a suitable substrate for hMSCs colonization, as judged by two-photon microscopy combined with fluorescence lifetime imaging (FLIM) and confocal analysis. The cells were then seeded on Integra ${ }^{\circledR}+$ PRP for 24 and $48 \mathrm{~h}$. Notably, in these conditions, the seeded cells exhibited a greater attitude to colonize the scaffold, showed improved cell adhesion and spreading as compared with those cultured on Integra ${ }^{\circledR}$ alone, and acquired a fibroblast-like phenotype indicating that the bioengineered scaffold provided an appropriate environment for cellular growth and differentiation.

Discussion: In conclusion, these results, even though preliminary, provide clues for the design of new therapeutic strategies for skin regeneration, consisting in the combination of mesenchymal stem cells with engineered biomaterials.

\section{OP-58}

\section{Incidence of congenital malformations in fetal autopsies - Profile of 150 cases}

Kapoor K, Singh B, Sharma A

Government Medical College \& Hospital, Sector 32, Chandigarh, India

Better knowledge of unexpected fetal loss is the basis for better parental counselling and for prevention of recurrences. Fetal autopsy can provide a clue to ascertain cause of death in these cases. There are many variations in the incidence of CMF out of total fetal autopsies in a given time. The variations in the incidence can be attributed to factors like geography, environment, socio-economic conditions, nutritional status, history of medication and genetic disorders. Further, there is a need to classify the types of CMF in a given population. The present study was done to help us to find incidence and types of CMF in North-West Indian Population. Autopsy was done in 150 fetuses as per guidelines provided by fetal autopsy protocol. Consent was taken from the parent/parents to coduct the autopsy according to institutional ethical guidelines. A brief maternal and family history was noted down. FINDINGS: Out of total 150 autopsies, $83(55 \%)$ were induced abortions, 40(27\%) spontaneous abortions and $27(18 \%)$ were intra uterine deaths. In total the incidence of CMF was $103(69 \%)$ of the fetal autopsies. The types of CMF were classifed as CNS defects in 49(32.6\%), GIT disorders in 48(32\%), musculo-skeletal in 31(21\%), genito-urinary in $25(16.6 \%$ ), and genetic disorders in $12(8 \%) .40 \%$ fetuses presented with multiple CMFs. Anencephaly turned out to be most prevalent anomaly (28.5\%). The other common anomalies were rachischisis, congenital polycystic kidney, omphalocoele and malrotation of gut. A few cases showed the occurrence of some uuncommon syndromes including split notochord syndrome, hand-foot-genital syndrome, aplasia cutis congenita; cloacal dysgenesis and holoprosencephaly. Major CMFs manifested very early in the intra-uterine life and could lead to early MTP. Therefore percentage of incidence of CMF 
at birth can be quite misleading while formulating the policies regarding prevention of congenital anomalies.

\section{OP-59 \\ Prophylactic effects of melatonin on cyclophosphamide induced skeletal and neural tube defects in mice embryos}

Mahabady $\mathrm{MK}^{*}$, Varzi $\mathrm{HN}^{* *}$, Bakhtiari $\mathrm{E}^{* * *}$

Department of Anatomy and Embryology, Faculty of Veterinary Medicine, Shahid Chamran University, Ahvaz, Iran. *; Department of Pharmacology and Toxicology, Faculty of Veterinary Medicine, Shahid Chamran University, Ahvaz, Iran.**; Graduated From Faculty of Veterinary Medicine, Shahid Chamran University, Ahvaz, Iran. ***

Cyclophosphamide is used for cancer treatment and as an immunosuppressive is also used for preventing of tissue rejection. There are many reports that show the teratogenic effect of cyclophosphamide can be decreased by application of antioxidant drugs and stimulation of maternal immune system. It is appearing that melatonin has antioxidant effect. Thus, the aim of this study was prevention or decreases of teratogenicity from cyclophosphamide in fetuses of mice by melatonin This study was performed on 15 pregnant mice that were divided into three groups. First group (control group) received normal saline (1 $\mathrm{mg} / \mathrm{kg}$ ) and test groups received cyclophosphamide $(20 \mathrm{mg} / \mathrm{kg})$, and cyclophosphamide $(20 \mathrm{mg} / \mathrm{kg})$ plus melatonin $(10 \mathrm{mg} / \mathrm{kg})$ at 10th day gestation, respectively. All drugs were administrated interaperitonealy. Fetuses were carried out in 20th day of gestation by cervical dislocation of pregnant mice and after determination gross malformations; they were stained by alizarin redalcian blue method and investigated by stereomicroscope for teratogenic effec The results have shown that cleft palate, spina bifida and open eye incidence were $62.79 \%, 62.79 \%$ and $34.88 \%$ in fetuses of mice that received only cyclophosphamide, respectively. But it decreased to $62.5 \%, 45.83 \%$ and $4.16 \%$ in group which received cyclophosphamide plus melatonin, respectively. In addition skeletal anomalies incidence including limbs, sternum and vertebrae were decreased by melatonin. On the basis of results melatonin can significantly decreased teratogenicity induced by cyclophosphamide.

\section{OP-60}

Characterization of haemathrosis derived stem cells after abrasion arthroplasty

Rainer $\mathrm{B}^{*}$, Hartz $\mathrm{C}^{* *}$, Lippross $\mathrm{S}^{* *}$, Pries $\mathrm{F}^{* * *}$, Neuß-Stein $\mathrm{S}^{* * * *}$, Seekamp $\mathrm{A}^{* *}$, Varoga $\mathrm{D}^{* *}$, Pufe $\mathrm{T}^{*}$

Department of Anatomy and Cell Biology, RWTH Aachen University;Aachen, Germany*; Department of Trauma Surgery, University Hospital Schleswig Holstein, Campus Kiel, Germany**; Department of Arthroscopical Surgery and Sports Traumatology, Mare-Hospital, Kiel, Germany***; Department of Pathology; RWTH Aachen University; Aachen; Germany****
There are different approaches to therapy osteoathritic lesions. Arthroscopic treatment of OA includes bone marrow stimulation technique such as abrasion arthroplasty (AAP) and microfracturing (MF). Beside the support of chondrocyte progenitor cells the environment is also important for the commitment to chondrocytes. Therefore insulin-like growth factor-1 (IGF-1) and transforming growth factor beta-1 (TGF$\beta 1)$ are important factors during the regeneration process. Our aim was to characterize the released cells after AAP and its ability to differentiate into the chondrocyte linage.

Material and Methods: Postoperative haemarthrosis was taken 5, 22 or 44 hours after surgery from the drainage bottle. $7.5 \mathrm{mg}$ Dexamethasone (Corticosteroid) was administered into the knee joint to prevent postoperative inflammation. Mononuclear cells were isolated from haemarthrosis by ficoll density gradient centrifugation. The isolated cells were characterized using fluorescence-activated cell-sorting (FACS) analysis for characteristic markers of MSC such as CD 44, 73, 90, 105. After expanding; cells were cultured in a pellet culture. After 3 weeks, histochemistry and immunohistochemistry against CD44, collagen 1, proteoglycan were preformed. The IGF1 and TGF $\beta$ release was analysed in haemathrosis serum by ELISA.

Results: The isolated cells after AAP are positive for the mesenchymal stem cell marker CD105, CD90, CD73, CD 44 and negative for the marker of hematopoetic stem cells CD 34. The staining of the $3 \mathrm{D}$-culture revealed a positive signal for proteoglycan and collagen I. The haemathrosis serum contains IGF1 and TGF $\beta$.

Discussion: We could demonstrate that the released mesenchymal stem cells during abrasion cartilage arthroplasty are able to differentiate into chondrocyte-like cells. In pellet culture they produce a collagen proteoglycan rich matrix. The haemathrosis contains chondrogenic factors IGF1 and TGF $\beta$ which could enhance the commitment of the released MSCs to the chondrogenic lineage. Our study provides the evidence for a therapeutic benefit of opening bone marrow like AAP.

\section{OP-61}

Structural divergence and spatial synexpression of nodal and dynein in the posterior notochord during left-right differentiation of the mammalian embryo

Schröder $\mathrm{S}^{*}$, Tsikolia $\mathrm{N}^{*}$, Feistel $\mathrm{K}^{* *}$, Blum $\mathrm{M}^{* *}$, Hue $\mathrm{I}^{* * *}$, Rath D ${ }^{* * *}$, Schwartz $\mathrm{P}^{*}$, Viebahn $\mathrm{C}^{*}$

Göttingen University, Germany*; Hohenheim University, Germany**; Institut National De La Recherche Agronomique, Jouy-En-Josas, France ${ }^{* *}$; Institute of Farm Animal Genetics, Friedrich-Löffler-Institut, Neustadt-Mariensee, Germany $* * * *$

Molecular left-right (LR) differentiation in vertebrates is irreversibly fixed through left-sided nodal expression in the lateral 
plate mesoderm (LPM) at the 4-somite stage in birds and mammals. A preceding labile phase of symmetry breaking is marked by paramedian nodal expression at the level of the posterior notochord (PNC). This nodal domain is bilaterally symmetrical in mouse and rabbit but restricted to the left side in chick and pig, a specific difference matched by the presence of cilia on the ventral free surface of the PNC (creating leftward "ciliary flow") in rabbit and mouse, on the one hand, and by the absence of cilia in chick and pig, on the other. To answer the question as to which scenario is applicable to mammals in general PNC morphology and the sequence of nodal expression during early LR differentiation was analysed in pig and bovine embryos. In both species, the ventral PNC surface is covered by a sheet of mesenchymal cells which we call "subchordal mesoderm"; the paramedian nodal expression emerges as a leftsided domain at the late pre-somite stage 5 or 6 und is confined to lateral cells of the PNC. These results add the bovine embryo to the "non-ciliated league" of pig and chick, but raise the question as to the topographical relationship between the expression domains of nodal and dynein axonemal heavy chains (DNAHs), ciliary motorproteins known to be associated with physiological ciliary flow and LR differentiation. Initial analyses carried out in the rabbit show that DNAH9 expression expression correlates with the domain of rotating cilia of the PNC at stage 6 and 7 and lies at the same level as the paramedian (symmetrical) nodal expression domain. Spatial synexpression of DNAH9 and nodal within the PNC thus supports an intracellular planar symmetry-breaking function of DNAHs during amniote LR differentiation.

\section{OP-62}

\section{Prenatal pathology does not influence significantly the vertical growth of long bones}

Kaki SB, Abdul Latif AZ, Hadi SA, Zin T

Faculty of Medicine Kampos Kota Jalan Sultan Mohmad Kuala Terengganu University of Sultan Zainal Abidin, Malaysia

Materials and Methods: The present study analyses the relative linear growth and proportionality of the long bones of 50 aborted fetuses and 117 live fetuses based on the radiographic measurements, anthropometric measurements, ultrasonographic values of diaphyseal length and crown heel length (CHL), biparietal diameter (BPD) etc. The data was generated from the radiographs and ultrasonographs of the above 50 dead fetuses and 117 live fetuses. The second data was generated from the autopsy records of the fetuses of soft tissue examination. In many cases it was found that there was no pathology while others showed some or other problems like placental disorders and some fetuses showed acute pathology like hyaline membrane disease. These cases were compared with those in which the pathology is proved in the postmortem examination.
The above subjects were divided in to groups according to the femur length and HCL measurements corresponding to the gestational ages between 16th and 40th weeks.

Results: Observations from our data of study are:- average weekly growth of femur $=1.975 \mathrm{~mm}$, tibia $=1.825 \mathrm{~mm}$; the ratio of Femur Vs Tibia =1.146: 1.0: the ratio of Humorus Vs Ulna = 1.0845: 1.0; the ratio of lower limb Vs upper limb = 1.0852: 1.0

Inference: In this study it was revealed that the linear growth of the long bones is not significantly affected by the pre-natal pathology. It is also revealed that the proportionate relationship between the linear length of long bones and the crown heel length are stable and predictable. This fact is consistently utilised clinically by the radiologists and ultrasonologists as well.

\section{OP-63}

\section{A new paradigm for learning and teaching histology}

Meyer G

Department of Anatomy and Human Biology, University of Western Australia, Nedlands, Western Australia

The teaching of histology is expensive particularly in some universities with limited or aging resources such as microscopes and inadequate histological slide collections. Increasing numbers of students has often required duplications of laboratory classes. Laboratory classes are staff intensive and so teaching hours are increased. Technology can now solve many of these issues and also caters for the self-directed and independent learning needs of students. A visual presentation will simulate how students can now study histology online. I will explain how using this innovative histology resource has reduced our need for students to attend many practical histology laboratory sessions. Students can now study histology successfully in their own time - and have fun! There is no need for academic staff to direct students through their learning objectives - even in practical classes! You will be able to see how students interact with over 6600 high quality images and click on hyperlinked text to identify key features of histological structures. Students use a computerized microscope to examine tissue/organ structures at increasing levels of magnification then view digitized histological sections using an ImageScope. Many quizzes provide instant feedback to students, making histology learning efficient and rewarding, and have significantly improved students' grades/marks in examinations. This learning system promotes student engagement and learning effectiveness. Using this resource has reduced our need for students to attend many practical histology laboratory sessions and so reduced our teaching workloads and reduced the costs for our Department/School providing these practical histology sessions. The learning platform I will demonstrate exploits the Internet's capacity for the rapid, shared dissemination of knowledge and for enhancing student learning. As a histology teacher you will see how you can add you own histology content and customize the resource to suit your own histology curricula at your institution/university. 


\section{OP-64}

Volume and cell number of the carotid body in human: a stereological study

Acar T $T^{*}$, Songur A**, Kaplan $\mathrm{S}^{* * *}$, Uzun I**** , Kucuker $\mathrm{H}^{\star * * *}$

Department of Anatomy, School of Medicine, Sifa University, Izmir, Turkey*; Department of Anatomy School of Medicine Afyon Kocatepe University, Afyonkarahisar, Turkey**; Department of Histology and Embryology, School of Medicine, Ondokuz Mayis University, Samsun, Turkey. ${ }^{* *}$; Department of Forensic Medicine School of Medicine, Sakarya University, Sakarya, Turkey****; Council of Forensic Medicine, Istanbul, Turkey $* * * * *$

The Carotid body (CB) is a very small mass of tissue located on the carotid bifurcation. CB functions in the autonomic control of the respiratory and cardiovascular systems. It detects hypercapnia, hypoxia, and acidosis. In this study, we examined total cell number and volume of $\mathrm{CB}$ and correlations to the other structures, i.e. BMI, common carotid artery (CCA), carotid sinus (CS), lung and heart. Our study was conducted on 25 male forensic autopsy cadavers who were between 3rd to 5 th decades. Demographic information and weights of heart and lungs were noted. Hemotoxilen-eozin staining methods were used for stereological study. Stereologic studies consisted of volume estimation and T1,T2 and total cell counting in CB. Cavalieri method was used for volume estimation and optic fractionator was used for cell counting. CB volume was found to be $4.677 \pm 2.236 \mathrm{~mm}^{3}$. T1, T2 and total cell number was calculated as $2.326 .328 \pm 1.215 .328, \quad 1.413 .927 \pm 768.210$ and 3.740.256 \pm 1.982.678, respectively. Also the cell density, which means cell number per volume unit, was $509.685 \pm 157.835 \mathrm{cell} / \mathrm{mm} 3$ for $\mathrm{T} 1,308.437 \pm 97.483 \mathrm{cell} / \mathrm{mm}^{3}$ for $\mathrm{T} 2$. T1/T2 ratio was $1.657 \pm 0.069$. There was a positive correlation between CB volume and BMI ( $\mathrm{r}=0,472 ; \mathrm{p}=0.017)$. There was a positive correlation between CB volume and CCA thickness ( $r=0.415$; $\mathrm{p}=0.039)$, and between $\mathrm{CB}$ volume and CS thickness ( $\mathrm{r}=0.539$; $\mathrm{p}=0.005)$. $\mathrm{CB}$ volume had positive correlations with $\mathrm{T} 1(\mathrm{r}=0.783 ; \mathrm{p}=0.000)$, $\mathrm{T} 2(\mathrm{r}=0.790 ; \mathrm{p}=0.000)$ and total cell number $(\mathrm{r}=0.786 ; \mathrm{p}=0,000)$. There was a positive correlation between $\mathrm{CB}$ volume and right lung weight $(\mathrm{r}=0,491 ; \mathrm{p}=0.013)$ and total lung weight $(\mathrm{r}=0.444$; $\mathrm{p}=0.026$ ). But no significant correlation between $\mathrm{CB}$ volume and left lung weight was found. We also observed that there was a significant correlation between $\mathrm{CB}$ volume and heart weight $(\mathrm{r}=0.445 ; \mathrm{p}=0.026)$. We think that our results may contribute to the basic and clinical science, may provide a frame of reference for future studies as this is the first study to estimate T1, T2 and total cell numbers

\section{OP-65}

Changes in collagen cross-links: hydroxylysyl pyridinoline, hydroxylysinonorleucin and pentosidine, with advancing age and severe disc degeneration

Al-Abbasi M, Tarlton J

University of Bristol, Bristol, UK
Collagen stability and hence the mechanical integrity of intervertebral discs is dependent on the degree and type of crosslinks between its collagen molecules. Degenerative disease and normal age-related deterioration in discs are both characterized by changes in those cross-links. Little is known about the differences between collagen cross-links (Hydroxylysyl Pyridinoline HL-Pyr, Hydroxylysinonorleucin HLNL and Pentosidine) with advancing age and severe disc degeneration. Twenty one postmortem intervertebral discs levels T9-S1, were obtained from seven individuals with a median age of 81 years. Degree of disc degeneration was graded macroscopically. Each disc was acid hydrolysed and the collagen cross-links analysed by fractionation on an amino acid analyser configured for cross-link analysis using ninhydrin post-column detection and reverse-phase high-performance liquid chromatography for pentosidine cross-links. The findings indicate a decrease in mature cross-link (HL-Pyr) and an increase in both reducible (HLNL) and pentosidine cross-link levels with advancing degree of macroscopic disc degeneration compared with matched normal discs $(\mathrm{P}<0.01)$. That was more obvious in both nucleus pulposus and the posterior annulus. Mature and reducible cross-links declines from the outer annulus through into the nucleus and posterior annulus in both aged and degenerated parts with advancing age $(\mathrm{P}<0.01)$. Pentosidine, in contrast, showed little difference across the disc, but did show the expected age-related increase. Decrease in mature cross-links is a novel finding and may have damaging effects on matrix resilience. On the other hand, increased pentosidine levels could be incriminated in the deterioration of disc integrity and function (weight bearing). These changes in the cross-link profile of the intervertebral disc in aged and degenerative disease are indicative of increased matrix turnover and tissue remodelling and likely to have implications for the progression of these disorders, and may result in a mechanically less stable disc.

\section{OP-66}

\section{Biomechanical and virtual investigation of pelvic open book injuries}

Hammer $\mathrm{N}^{*}$, Steinke $\mathrm{H}^{*}$, Lingslebe $\mathrm{U}^{* *}$, Böhme J**, Slowik $\mathrm{V}^{* * *}$, Bechmann I*

Institute of Anatomy, University of Leipzig, Germany*; Department of Trauma and Reconstructive Surgery,; University of Leipzig, Germany**; Leipzig University of Applied Sciences, Leipzig, Germany***

Summary of Background Data: Little is known about the mechanism of open book fractures (OB) and the resulting instabilities of the pelvic ring. The involvement of the ligaments that stabilize the sacroiliac joint is in the focus of recent discussion. The aim of this study was to investigate the $\mathrm{OB}$ mechanism and the consequences on pelvic ring stability. 
Materials and Methods: 8 human pelves (2 ethanol-fixed, 6 unfixed) were utilized, regarding material deformation. After the tests, the specimens were investigated by anatomical preparation, thin-slice plastination, sacroiliac joint arthroscopy and CT imaging. A Finite Elements (FE) model was created, including the pelvic bones and ligaments. Alterations of pelvic load transfer in the standing position related to OB instability to normal standing were then determined.

Results: In the cadaveric tests, cranial parts of the anterior sacroiliac joint ligament (ASL) were affected frequently after disruption of the pubic symphysis. These findings were confirmed in the FE simulation. Here, the ASL contributed to more than $80 \%$ of the overall ligamentous force transmission. After symphyseal dehiscence, load maxima were found for the cranial parts of the ASL. The altered force distribution, caused by ASL disruption, was redirected to the iliolumbar ligament, the interosseous sacroiliac ligaments, the sacrotuberous and the sacrospinous ligaments.

Conclusions: Close concordances were found for the biomechanical tests and FE simulations. Consecutive instabilities, caused by ASL disruption, were compensated by force distribution to the surrounding ligaments. This supports the thesis that pelvic ligaments are strongly involved in pelvic stabilization. The $\mathrm{OB}$ mechanism can be described distinctively by means of FE analysis.

\section{OP-67}

Positions of the anatomical structures of temporal bone related to pneumatization

Turan B*, Tatlisumak E** , Aslan $\mathrm{A}^{* * *}$

Vocational High School of Health Sciences, Batman University, Batman, Turkey*; Department of Anatomy, School of Medicine, Celal Bayar University, Manisa, Turkey**; Department of Otolaryngology, School of Medicine, Celal Bayar University, Manisa, Turkey***

It is widely accepted that the position of the anatomical structures of temporal bone can change depending on the pneumatization of the bone. The aim of this study was to determine the distances between the anatomical structures important in transtemporal surgical procedures with relations to temporal bone pneumatization. The study was done on the temporal bone CT scans of 60 patients present in the archieve of ENT Department. Measurements were applied on three sections obtained in axial plane (from the levels of basal turn of cochlea, jugular foramen and lateral semicircular canal). The pneumatization of petrous apex and mastoid bones were staged according to the pneumatization levels. Following distances were measured: 1) Sigmoid sinus (SS) to mastoid cortex (MC) 2) SS to external acoustic meatus (MAE) 3) Jugular bulb (JB) to SS 4) Internal carotid artery (ACI) to JB 5) ACI to petrous bone (PB)
6) Facial nerve (NF) to MAE 7) NF to MC 8) Diameter of SS in the horizontal plane 9) Diameter of SS in the vertical plane 10) Diameter of JB 11) Lenght of the anterior wall of internal acoustic meatus (MAI-ant) 12) Lenght of the posterior wall of internal acoustic meatus (MAI-post) 13) Lateral semicircular canal (LSCC) to anterior wall of SS (SS-ant). The measurements of temporal bones with pneumatized and nonpneumatized mastoid processes showed no statistically significant difference except SS to MC. The bones with and without petrous apex pneumatization presented statistically significant differences in the measurements SS to MC, SS to MAE and ACI to PB. There was not a significant relation between the jugular bulb level and mastoid or petrous apex pneumatization. Surgical procedures on the temporal bone have to be applied very close to the important and vulnerable structures. We suggest that these measurements may be important guides for surgeons.

\section{OP-68}

\section{Age-dependent biomechanical properties of the} iliotibial tract for pelvic finite elements analysis

Steinke $\mathrm{H}^{*}$, Hammer $\mathrm{N}^{* *}$, Lingslebe $\mathrm{U}^{* * *}$, Böhme $\mathrm{J}^{* *}$, Hädrich $\mathrm{C}^{* * * *}$, Slowik $\mathrm{V}^{* * *}$, Josten $\mathrm{C}^{* *}$, Bechmann $\mathrm{I}^{*}$

Insitute of Anatomy Leipzig, Germany*; Department for Trauma, Reconstructive and Plastic Surgery, University of Leipzig, Germany**; Leipzig University of Applied Science (HTWK), Germany ${ }^{* * *}$; Institute of Legal Medicine, University of Leipzig, Germany $* * * *$

Background \& Objective: Pelvic Finite Elements Analysis (FEA) lacks in adequate morphological and biomechanical properties of the ligaments. The tissues which are usually subject of materials testing may not reflect the native situation, as regards the mean age of the specimens. The iliotibial tract (IT) serves as a suitable model for FEA due to its parallel fibers and its simple manageability in materials testing. The Young's modulus (YM) of the IT was investigated, regarding correlations of the YM to age, body weight and failure load and gender differences.

Material \& Methods: 33 fresh IT were gained from donators (mean age 31.2 years) and investigated biomechanically. Improved partial plastination was accomplished to prevent material slippage. After preconditioning, the non-linear YM range $\left(\mathrm{YMn} ; 0-4 \mathrm{~N} / \mathrm{mm}^{2}\right.$ ) and the quasi-linear $\mathrm{YM}$ range (YMq; 4-11 N/mm²) were investigated before the failure load was determined.

Results: The YM of the probes averaged $79 \mathrm{~N} / \mathrm{mm}^{2}$ (YMn) and $380 \mathrm{~N} / \mathrm{mm}^{2}(\mathrm{YMq})$, respectively. The YM were significantly smaller than those of old-aged specimens in a previous study (mean age 78.8 years, $\mathrm{YMn}=273 \mathrm{~N} / \mathrm{mm}^{2}, \mathrm{YMq}=597$ $\left.\mathrm{N} / \mathrm{mm}^{2}\right)$. A mean failure load of $36.6 \mathrm{~N} / \mathrm{mm}^{2}\left( \pm 19.8 \mathrm{~N} / \mathrm{mm}^{2}\right)$ was recorded. While the YM and the failure load correlated 
closely ( $\mathrm{r}=0.66$ for $\mathrm{YMn}, \mathrm{r}=0.88$ for $\mathrm{YMq}$ ), no correlations were found for the YM and age or body weight. Gender-related differences could not be determined.

Conclusions: This is the first study to provide age- and failure load-dependent YM of the IT. IT rigidity is significantly smaller in young specimens and is subject to alteration. The phenomenon that failure load and rigidity correlate closely cannot be explained conclusively but gives insight into complexity of ligament biomechanics, which has to be considered in pelvic FEA.

\section{OP-69}

\section{Evidence that palisade endings in extraocular muscles are innervated by motor nuclei}

Zimmermann L, Pastor AM, May PJ, Streicher J, Blumer R

Departamento De Fisiología Y Zoología, Facultad De Biología, Universidad De Sevilla, Sevilla, Spain*

Palisade endings are nerve specializations that are found in the extraocular muscles (EOMs) of almost every mammalian species, including primates. Palisade endings have been postulated to be proprioceptors. However, it was recently demonstrated that palisade endings have a cholinergic nature, which reopened the question of whether palisade endings are motor or sensory structures. In this work, we examined whether the cell bodies of palisade endings lie in EOM motor nuclei by injecting an anterograde tracer, biotinylated dextran amine, into the oculomotor/abducens nucleus of cats and monkeys. Tracer visualization in EOMs was combined with choline acetyltransferase (ChAT) and a-bungarotoxin staining, and was analyzed by conventional light and confocal laser scanning microscopy. We observed tracer-positive nerve fibers in each EOM innervated by nerve fibers from oculomotor/abducens nucleus. The amount of tracer-positive nerve fibers varied between $20-60 \%$ within the muscles. All tracer-positive nerve fibers exhibited ChAT immunoreactivity. As expected, tracer-positive nerve fibers established motor contacts on singly and multiply innervated muscle fibers, which were confirmed by a-bungarotoxin staining. However, at the transition between muscle and distal tendon, we found palisade endings containing tracer. All tracer-positive palisade endings were ChAT-immunoreactive as well. Palisade endings exhibited the classic morphology: axons arising from the muscle extend onto the tendon, then turn back $180^{\circ}$ and terminate in a cuff of terminals around an individual muscle fiber tip. This finding suggests that the cell bodies of palisade endings lie in EOM motor nuclei, which complements prior studies demonstrating a cholinergic, and possibly motor, phenotype for palisade endings.

\section{OP-70}

New anatomoradiological findings about human temporomandibular joint

Mañas CB, Sequeros OG, Cascales MM, Cabrera RS, Reviriego RL

Facultad De Medicina and 2. Facultad De Veterinaria, Universidad De Murcia, Spain

Introduction and Purpose: There are discrepancies in the literature regarding the anatomy of the temporomandibular joint (TMJ), particularly at the level of the capsule and lateral pterygoid muscle, so we decided to compare the techniques of plastinated sections and dissection of the TMJ with MRI images, describing the features o lateral pterygoid muscle and the exact location of its insertions. Tools and method: We studied 20 blocks of humanTMJ, 7 were studied by MRI, with a 0.5 Tesla system (General Electric Healthcare, O'Signa,) with a standard head coil. The images were obtained in the oblique-sagittal, oblique-coronal and axial planes. 4 of the blocks studied by MRI, were processed through the E12 plastination technique and cut in $3 \mathrm{~mm}$ thick, scanned and examined with a magnifying glass to $0.65 \mathrm{X}$. The remaining blocks were dissected. Finally we performed a comparative morphological analysis of the different techniques used. Result and interpretation: We have observed both in plastinated sections as in TMJ dissections that the lateral pterygoid muscle is inserted into the disc and the condyle, through muscle and tendon fibers, giving it the characteristics of multipeniforme muscle. Insertion caudally exceeded the pterygoid fossa and reaches the neck of the condyle near lingula. On insertion we identify different layers that are crossed by the pterygoid venous plexus. It is also possible to see details of the insertions of the joint capsule and its vascular and nerve relations. By comparing these findings with MRI images we found that they were clearly observed at the axial and oblique-sagittal planes. RMI: Scanner Murcia S.L Aids Research Initiation R380/2010. Belonging to the Research Plan of the University of Murcia.

\section{OP-71}

\section{Origin of the sciatic artery}

Soames R, Altalalwah W

University of Dundee, UK

The sciatic artery is said to be an extremely rare anomaly resulting from failure of the primitive sciatic (axial) artery to regress. Based on the embryological development of the vasculature of the lower limb, the proximal part of axial artery becomes either the superior or inferior gluteal artery, while the 
distal part becomes the popliteal artery. The present study in 32 specimens identified several origins of the sciatic artery. The sciatic artery was observed to be a direct (6) or indirect (1, from the internal pundendal artery) branch of the anterior division of the internal iliac artery, a direct (19) or indirect (5, all from the superior gluteal artery) branch of the posterior division. It was also noted that the superior and inferior gluteal arteries were absent in 2 and 8 specimens, respectively. In 2 specimens, 2 sciatic arteries were present, one arising from the anterior and one from the posterior trunk. The sciatic artery can therefore arise from several sources, as well as coexisting with either the superior and/or inferior gluteal arteries: this latter observation does not fit with the established theory of lower limb vascular development.

\section{OP-72}

\section{Limb vein pattern analysis for human forensic identification}

Meadows H

University of Dundee, UK

The superficial veins of our body are so individual to each person that they are effectively a venous biometric barcode. This research project addresses the variability in parts of the human body that have not as yet been investigated and assesses the stability, symmetry and individuality of these patterns in a large sample size. Venous pattern analysis has a significant commercial history with regards to vein patterns of the back of the hand, the palm of the hand and the fingers - but there is no information on the remainder of the body. Veins lend themselves to this type of assessment particularly well, as superficial veins are identifiable in the living using near infra-red light. Consequently reflected or transmitted images of superficial blood vessels are detected via the reaction between IR light and the deoxygenated blood in the subcutaneous vessels. Due to differences in absorbance between veins and other tissues, the reflected near infra-red rays produce an image in which regions of high absorbency (the veins) appear as dark lines in an image. The venous pattern from the captured image is then processed and subjected to mathematical algorithms and modeling profiles for forensic/biometric identification purposes. A compari- son of manual automated and manual tracing methods is presented with inter and intra-observer error rate results.

\section{OP-73}

\section{A comparison of prosections and ultrasound as modalities for teaching cardiac anatomy}

\author{
Griksaitis M, Sawdon M, Finn G
}

Durham University, UK

Traditionally, cardiac anatomy has been taught with prosections of the heart and great vessels. Imaging of the heart is frequently encountered in clinical practice (e.g. FAST scans, echocardiography), and these methods should be utilised in teaching cardiac anatomy. We report how we use a live apical four chamber echocardiography view and compare its effectiveness to a traditional cadaveric prosection approach. 108 first year medical students participated in this study. The year group was divided into 2 equal groups. All students sat an identical MCQ pre-test prior to any teaching, composed of 10 questions covering major cardiac structures (five showing apical four chamber views and five showing pictures of prosections). Students then received facilitated teaching through one modality, either ultrasound or prosection. Both groups then repeated the MCQ (post-test). The preintervention test scores showed no statistical significant difference in base line knowledge between the two study groups $(\mathrm{F}=0.310$ and $\mathrm{p}=0.948$, one way ANOVA). The pre-intervention test showed no statistical significant difference between the scores obtained on the echocardiography questions and the cadaveric questions within each study group ( $\mathrm{p}=0.488$, independent t-test). For the remainder of the calculations we can therefore assume that each study group can be considered as the same. Following the intervention we found no statistical difference in test scores between those taught using ultrasound and those taught using cadaveric prosections ( $\mathrm{p}=0.512$, independent $\mathrm{t}$-test). As we found no significant difference between teaching using cadaveric or ultrasound our results suggest that there is no correct way to teaching cardiac anatomy. Perceived advantages of ultrasound teaching is that it mirrors real life imaging of anatomy and allows students to see dynamic anatomy. Our data adds to the debate that cadaveric anatomy is a superior method for teaching and may encourage institutions to engage students in living anatomy approaches. 


\section{Poster Presentations - A}

$$
\text { (PP-1 - PP-175) }
$$

\section{PP-1}

Time-dependent reactivity of bone after hydroxyapatite (Hap) implants in jaw medicine of experimental animals Pilmane $M^{*}$, Salms G**, Salma I**, Skagers A**

Institute of Anatomy and Anthropology Riga Stradins University, Riga, Letonia*; Institute of Stomatology, Riga Stradins University, Riga, Letonia**

Biocompatibility is main indicator of validity of biomaterials for the implantation in different tissue. However, unclear still are data about the time period when implanted biomaterial shows the best biocompatibility with the tissue. Thus, aim of our work was the detection of tissue changes in jaws of experimental rabbits after HAp implantation in different time period. Tissue with biomaterial was obtained from 4 rabbits jaw 8 months and from 2 rabbits 6 months after the HAp implantation. Control side was sham jaw of other side in the same animal without HAp implantation. Tissues were proceeded for the detection of Osteopretegerin (OPG), BMP2/4, TGF $\beta$, MMP8, MMP9 and defensin2 immunohistochemically. Results demonstrated decrease of BMP2/4-, but not TGF $\beta$-containing structures in bone only in control side of jaw in all investigated times. From rich MMPs expression in 6 months, only expression of MMP9 decreases in bone with 8 months implants. OPG appearance demonstrated increased expression with time in bone. Defensin was mainly expressed by few cells in bone proliferation zone of control side. Intensive expression of MMPs in jaws after implantation suggests about active degeneration processes and probably is common reaction on trauma that decreases with time when only the most stable MMPs like MMP8 still is intensively expressed. Decrease of BMP2/4 with still intensive TGF $\beta$ expression in control bone seems to be a compensatory reaction of tissue on the HAp and/or traumatic injury, while bone growth in experimental side is notably stimulated by rich BMP2/4 expression. Osteoblast activity seems to increase after longer HAp implantation, while antimicrobial defense is seriously affected (decreased) around the implants in any of implantation time.

\section{PP-2}

Urophysis and caudal neurosecvretory system in two species of fishes in river Tigris passing through Mosul city Rahemo Z, Shareef NM

Department of Biology, College of Science, University of Mosul, Mosul, Iraq

Two species of Fishes were studied searching for their urophysis and caudal neurosecretory system namely in Barbus luteus and Varicorhinus trutta. Anatomical examination revealed that two or more enlargement were noticed in the spinal cord of B. luteus while no enlargement was noticed in V. trutta. After histological examination using different stains such as Haematoxyline-eosin, Aldehyde Fucsin and Mallory triple different types of cells revealed in this study in the spinal cord among them Dahlgreen cells. Furthermore a trial was designed to find a possible relation between the ovary and testes activity and the activity of these neurosecretory cells.

\section{PP-3}

\section{Can acetylcysteine have treated effects on} contaminated rat spleen by sulfur mustard?

Kaka G

Applied Neuroscience Research Center of Baqiyatallah Medical Sciences University, Tehran, Iran

Sulfur mustard is a chemical that causes the most damage in the war has had on the Warriors. Including the adverse effects of sulfur mustard is damage to the immune system. One of the drugs effective against thhese lesions is Acetylcysteine. Spleen has responsible part of the body's immune system. Present study is about the effect Acetylcysteine for preventing damage in spleen tissue.

Methods: Twenty one adult male rats were used to perform this study. Animals were randomly divided into three groups consist of solvent (saline), SM with dose 0.25 Percent and treated group with Acetylcysteine. Animals under anesthesia, received normal saline solution and SM received by intratracheal injection. Treated group received drug for fourteen days intraperitoneally. Animals were sacrificed in the end of 14th day of studying with ether. Sections have prepared from rat's spleen. The sections were studied for histomorphology evaluation. To study the photographs of samples produced different microscopic magnification and percentage area of white pulp and germinal center were measured by the hardware and software Motic system. Statistical analysis was performed by ANOVA using Spss software. Data are reported as mean $\pm S E M$ at a significance level of $\mathrm{P}<0.05$.

Results: Our observations showed histomorphometrical average percentage of white pulp area of optical microscope with a magnification 100 in SM group compared to vehicle group was reduced but was not significant. This variable in treated group was greater than SM group and less than vehicle group. Average percentage of germinal center of white pulp in SM group was more than vehicle group but not significant this increasing was not seen in treated group. 


\section{PP-4}

\section{The spirit missing in education and teaching of the anatomy}

Kavak V*, Pilmane $M^{* *}$, Boka $\mathrm{S}^{* *}$

Department of Anatomy, Dicle University, Diyarbakır, Turkey*; Institute of Anatomy and Anthropology, Riga Stradins University, Riga, Letonia**

The spirit that is missing in Anatomy education and teaching is pencil drawings. Pencil drawings provide a better understanding in anatomy lesson. Anatomy is an important tool which is necessary to be used in picture, drawing or sculpture. Moreover, it is a powerful and amazing tool when it is used in the correct way and not permitted to block the creative development. The most common approach on the study of human anatomy is to see it as a tool to use for the understanding of body movements. The forms occurred by bone, muscle and skin movements are completely different from those of the motionless body. The movement is to change the places depending on human's requirements. In human life, there is no even one moment that the movement does not take place in the body. The movement finishes only when the life ends. Therefore, it is a useless effort to study the anatomy by looking at the motionless bone and muscle, as it is also an unnecessary effort for the painter to memorize the bones and muscles in the body. The painters need anatomy to deepen and to increase the power and expression of their art. Before starting the anatomical study, another important point to mention is that the bone, muscle and skin develope and evolve as all living things. If a muscle is continuously worked by repeated movements, it develops in terms of shape and dimension. There would not be any development in an unworked muscle. It is essential to be careful about the proportin during drawings of anatomical figures. We have therefore obeyed these rules. As a conclusion, we have paid attention to proportion and symmetry during the drawings. We have designed them by the pencil drawings to provide the perception of three-dimension of human form profoundessly.

\section{PP-5}

\section{External ear measurements of the students aged} between 18-24

Tuncer I

Department of Anatomy, Meram Faculty of Medicine, Selcuk University, Konya, Turkey

Objectives: It was aimed to present the morphometric properties of the ear, one of the characteristics structures of the face, in this study.

Material and Method: This study was carried out on total 121 Medical faculty students(90 male, 31 Female) aged between 18-
24 and whose parents and themselves were from central Anatolia and had no physical anomalies. The height of the ear, the width of the ear, lobules auricle size, lobules auricle width, tragus-helix distance, tragus-antihelix distance, the distance between the eye and the upper edge of the ear, the distance between the eye and the lower edge of the ear, the distance between the nose septum and the upper edge of the ear, and the distance between the nose septum and the lower edge of the ear were measured using a digital caliper.

Findings: When the obtained data were compared according to the sexes, all values except the height of the right ear were higher in males $(\mathrm{P}<0.00)$. When the values of right and left ears were compared, except for the height of right ear, tragus-helix distance, tragus-antihelix distance, the distance between the eye and the upper edge of the ear, and the distance between the nose septum and the upper edge of the ear, all values were higher in the right ear $(\mathrm{P}<0.00)$.

Results: Different ear growth pattern and maturation size from previously published report have been obtained from different populations. There is still need for further studies comparing populations with different social and ethnic background to interpret common knowledge about the size of the ear. This study gives dimensional information of the auricle, and therefore may reveal important implications for the adequate timing of the surgical treatment of auricular deformity.

\section{PP-6}

\section{Determining the upper border of the leg plasters for preventing the common fibular nerve palsy complication}

Dikici $\mathrm{F}^{*}$, Gayretli $\mathrm{O}^{* *}$, Gurses $\mathrm{IA}^{* *}$, Kale $\mathrm{A}^{* *}$, Erdil $\mathrm{M}^{* * *}$, Ozturk A**, Usta $A^{* *}$

Department of Orthopaedics and Traumatology, Faculty of Medicine, Istanbul University, Istanbul, Turkey*; Department of Anatomy, Faculty of Medicine, Istanbul University, Istanbul, Turkey**; Department of Orthopaedics and Traumatology, Faculty of Medicine, Bezmialem Vakif University, Istanbul, Turkey***

Lower extremity plasters are frequently used in orthopaedic practices. As curable common fibular nerve (CFN) paralysis occurred in 3 patients who had plasters for their lower extremity fractures, we decided to investigate the CFN in adult cadavers. In 22 legs of 11 cadavers, the part of the CFN, where it crossed over the head of the fibula, was examined as this part had the maximum risk of exposure to compression in a lower extremity plaster. The vertical distance between the point where the CFN passed to the anterior part of the fibula and the most proximal palpable point of head of the fibula was measured meanly as $41.7 \mathrm{~mm}$. In order to standardize the vertical distance for people of any fibula length, the distance between 
the most proximal palpable point of head of the fibula and the most proximal palpable point of the lateral malleolus of the fibula was determined as the fibula length and the fibula length was measured meanly as $334.2 \mathrm{~mm}$. Afterwards we divided the mean length of the fibula to the determined vertical distance and this ratio was found approximately as 8 . Consequently we recommend that the upper border of the plasters for leg should end at the upper $1 / 8$ of the fibula length defined.

\section{PP-7}

\section{The proportion between face and hand in terms of artistic anatomy}

$\underline{\text { Yilmaz A }}$

Department of Anatomy, Faculty of Medicine, Trakya University, Edirne, Turkey

\begin{abstract}
Aim:The science that analyzes the sizes and the proportions of the human body in visual arts and statuary is known as "Artistic Anatomy". The artists, who use the human body as a narration symbol, had investigated the human anatomy in terms of artistic anatomy thoroughly. They had accepted their work of arts have some proportions on human body. And they had used these proportions in their works. The aim of study determine correlation between hand length and aesthetic face height.
\end{abstract}

Methods: 96 female volunteers whose 18- 22 years (mean age $18.89 \pm 0.94)$ were included this study. Hand length and aesthetic face height were measured on each subject. Harpenden anthropometer and digital caliper were used in measurements. It was searched the correlation between hand length and aesthetic face height.

Results: The means of hand length and aesthetic face height were $178.89 \pm 10,61 \mathrm{~cm}$ and $180.53 \pm 10.45 \mathrm{~cm}$, respectively. There is a correlation between hand length and aesthetic face height $(\mathrm{p}<0.001, \mathrm{r}=0.684)$

Conclusion: While the artists were studying on human body, they had accepted some proportions as rules. And they had used these proportions in their works. According to this artists, aesthetic face height of the body is equal hand length. There is not this equality as the means values in our study. But there is correlation between them. These results are compared with the literature.

\section{PP-8}

Does naso-oral ratio fit to golden ratio?

Onal T, Yilmaz A

Department of Anatomy, Faculty of Medicine, Trakya University, Edirne, Turkey
The analyze of beauty is an intriguing topic. Many scientists and artists have searched to find a magical mathematical Formula that could explain the ideal beauty through the history. They called that magical Formula as the 'Golden Ratio'. The golden ratio have for long been recognized as one of main forms in architecture, sculpture and music. The propose of this study is to investigate the golden ratio between nose width and mouth width.

Methods: 104 female students educating in Trakya University Medical Faculty who had no orthopaedic and physical defect took place in this study. Harpenden anthropometer and vernier caliper were used in measurements. Body height, body weight, nose width ant mouth width were measured on each subject. The proportion of mouth width to nose width was calculated.

Results: The mean body height $170.77 \pm 7.74 \mathrm{~cm} ; 347,25 \pm 49.73$ $\mathrm{mm}$ and $681.35 \pm 95.95 \mathrm{~mm}$ The proportion of mouth width to nose width was determined $1,988 \pm 0,34$.

Conclusion: We have determined that, the golden ratio which is known as 1,618 does not exist between nose width and mouth width. However, it is supposed that by increasing the number of subjects in this study.

\section{PP-9}

Numerical density of ganglion cells in the parts of sympathetic trunk, in relation with certain content of lipofuscin pigment and ageing

Filipovic $\mathrm{T}^{*}$, Mandić $\mathrm{P}^{*}$, Djukic-Macut $\mathrm{N}^{*}$, Vitošević $\mathrm{Z}^{*}$, Filipovic $M^{* *}$, Teofilofski-Parapid $\mathrm{G}^{* * *}$

Institute of Anatomy, School of Medicine, University in Prishtina*; School of Medicine, University Prishtina**; Institute of Anatomy, School of Medicine, University in Belgrade, Serbia***

Neurons of sympathetic trunk, like the other nerve cells, undergo changes during ageing. The fact that the accumulation of lipofuscin pigment is in relation with ageing first was noted in 1934th by Hamper. The aim of this study is to confirm the presence of pigment in ganglion cells of sympathetic trunk, and when it occurs in greater extent, as well as dynamics of its accumulation (quantification of ganglion cells without pigment, those with partial presence of pigment, and those that were completely filled with pigment) by using morphometric methods (numerical density). The material were ganglions of human sympathetic trunk. For morphometric analysis we used the test system M42. Calibrating the microscope objective was adjusted to incrise $63 \mathrm{X}$, and was determined by surface of test system At, that ten fields in which it was done was $0.23 \mathrm{~mm}^{2}$. To determine the numerical density of ganglion cells we used method for thick cuts by Floderus. Results In all parts of the symphatetic trunk aged 20-39 years, there were approximately constant rate of cells without pigment and those with partial content of lipofuscin. Slightly higher proportion of cells with pigment, in this age 
group, was present in cervical part of trunk (18.5\%), while in other parts of trunk ranges from $13-17 \%$. In the age of 40-69 years there was drastic increase of cells partly filled with pigment as well as cells with full presence of pigment. In the age of 70 or more, the higher share of cells filled with pigment was in the cervical (25\%) and thoracic part (19\%) of sympathetic trunk. Conclusion Numerical density of ganglion cells with pigment increases significantly in the observed parts of sympathetic trunk, during ageing. Significantly increase in density of cells with pigment we noticed in second age group (40-69 years).

\section{PP-10}

Multiple variations along the brachial artery distribution Elvan O, Kara A, Ozturk NC

Department of Anatomy, Faculty of Medicine, Mersin University, Mersin, Turkey

During the left upper limb dissection of a 75-year-old male cadaver variations were encountered in the brachial artery and its branches. A superficial brachioulnar artery arose from the brachial artery in the middle of the arm. The superior and inferior ulnar collateral branches arose from the brachial and superficial brachioulnar arteries, respectively. Then the brachial artery ramified into a radial, common interosseous, recurrent artery and a muscular branch at the level of the neck of the radius. The common interosseous artery first gave off a recurrent artery and then a trunk which ramified into another recurrent artery and a muscular branch and finally terminated giving anterior, posterior and recurrent interosseous arteries. The superficial palmar branch of the radial artery coursed on the dorsum of the hand and by passing through the first web space joined the superficial brachioulnar artery. Additional variations were observed during the courses of the branches of these arteries.

\section{PP-11}

\section{Effects of balneotherapy on the number of blood leukocytes}

Cuce G, Duman S, Aktan TM, Ince B

Department of Histology and Embryology, Faculty of Meram Medicine, University of Selcuk, Konya, Turkey

Introduction and Purpose: We aimed to investigate the effect of hyperthermia as a result of the balneotherapy sessions on the number of blood leukocytes in blood smears.

Tools and Method: Balneotherapy sessions had applied in spa of Governor Saim COTUR (Central Ilica town of Kahramanmaras). Patients entered the pool one hour a day. They waited for 1 hour in water to neck level. Their ages were between 21-65 and they did not have an infectious disease primarily. Blood was collected from 55 persons without distinction of sex. Groups were created according to the number balneotherapy sessions ( 1 hour, 3 hour, 5 hour, 10 hour). Blood smears were stained with May Gronland Giemsa stain and 100 cells were counted at random areas. Monocyte, lymphocyte, eosinophil and neutrophil counts were determined separately. Group numbers were evaluated statistically and blood cells were evaluated morphologically.

Findings: There were significant differences between the groups in terms of the number of monocytes $((\mathrm{P}=0.001)$ and eosinophils $(\mathrm{P}=0.004)$. These significant differences were seen only the group which balneotherapy session applied 1 hour. There was no change in morphology of erythrocytes and leukocytes.

Result and Interpretation: 1 hour hyperthermal stress increases the number of monocytes and eosinophils.

\section{PP-12}

Intra-rater and inter-rater variance of measurement intracranial volume using water filling method

Ozdemir F, Alpay M, Acar M, Sahin B

Department of Anatomy, Faculty of Medicine, Ondokuz Mayis University, Samsun, Turkey

Introduction and purpose: At the age of 16-20, the intracranial volume (ICV) reaches its final size and it is thought that it does not change its size thereafter. At about 20 years of age, the volume of the brain starts to decrease, while it is presumed that the ICV remains constant. By combining measures of brain volume with measures of ICV, investigators can infer how much reduction in volume has occurred. In this study we used water filling method to measurement ICV, and the aim of this study is to find out the intra-rater and inter-rater variance of measurement of ICV.

Tools and Method: Ten skulls were fixed by a plaster before pouring the pressurized water into them. Two balloons were inserted into the skulls via the foramen magnum and filled with pressurized tap water. Finally, the water was poured into a measuring cylinder and the amount of water was recorded as the ICV. Three observers were done the same procedure for each skull 5 times. We analyzed the obtained data of each rater to see intra and inter-rater difference.

Findings: Our results showed that the mean highest ICVs were $1236.5,1284.0,1235.0 \mathrm{~cm}^{3}$ for the first, second and third raters, respectively. The means of the difference between the highest and lowest measures were 13.4, 10.7 and $16.2 \%$ for the first, second and third raters, respectively. The mean difference between the highest and lowest measures of three raters was $23.8 \%$. The most stable rater was the second one. The measures of three performers showed little intra and inter-variance. 
Results and Interpretations: Our results revealed that the measuring ICV five times and recording the highest one may be good for the assessment of more realistic ICV. Even though, there are some differences between the raters the differences are within the tolerable range.

\section{PP-13}

Chondrosarcoma - Histopathologic and clinical aspects

Selaru M, Moise M, Stana LG, Hogea B, Motoc A, Sisu AM, Sava A

University of Medicine and Pharmacy "Victor Babes", Timisoara, Romania

Is the second most common primary malignant bone tumor, occurring more commonly in men that in women, ratio $2 / 1$. It is most frequently in the fourth to sixth decades (average age, 45 years). Chondrosarcoma occurs in three variants, classified according to location: central, peripheral and juxtacortical. Central chondrosarcoma arises in the medullar cavity of pelvic bones, ribs and long bones (femur, humerus), although any site may on occasion be affected (skull bones). Radiological, these tumors are characterized by poor defined borders, a thickened shaft, and perforation of the cortex. Occurs in the fourth to sixth decades, and has a male to female ratio of $2 / 1$. It is most common in the long bones and on the surface of the pelvis. The symptoms are the following: complaining of severe and persistent pain in the left hip joint and presenting limitation of adduction movement, limitation of internal-external rotation movements, and also could not be able to do thigh flexion on the abdomen. Laboratory tests could not show any significance. Other investigations are radiographies and magnetic nuclear resonance. Histopathology indicates the histological feature more precisely and the treatment. Prognosis depends upon stage and histological grade. Metastasis: usually spreads within the medullar cavity; pelvic tumors may invade the adjacent organs. Distant metastases to lung are followed by skin and soft tissue. 5-year survival rates were: $78 \%$ if low grade; $53 \%$ if moderate grade; $22 \%$ if high grade.

\section{PP-14}

\section{A study of sutural morphology of the pterion and} asterion among human adult Indian skulls

\section{Shaik HS}

Department of Anatomy, Jjm Medical College, Davangere, India

A study of sutural morphology of the pterion and asterion among human adult Indian skulls. Hussain Saheb $\mathrm{S}$,
Mavishetter GF, Thomas ST, Prasanna LC, Muralidhar P, Magi. Department of Anatomy, JJM Medical College, Davangere, Karnataka, India. Abstract The pterion and asterion are points of sutural confluence seen in the norma lateralis of the skull. Their patterns of formation exhibit population based variations. The sutural mor-phology of the pterion and asterion is important in surgical approaches to the cranial fossae. 125 human skulls of known gender (83 male, 42 female) were examined on both sides. Four types of pterion were observed - sphenoparietal $69.25 \%$, frontotemporal $17.35 \%$, stellate $9.7 \%$ and epipteric $3.7 \%$. Two types of asterion were also observed - type I in $23.15 \%$ cases and type II $76.85 \%$ cases. These findings should be of use in surgical approaches and inter-ventions via the pterion and asterion.

\section{PP-15}

The morphometric analysis of the thorocal and lumbar vertebraes

Tuncer I

Department of Anatomy, Meram Faculty of Medicine, Selcuk University, Konya, Turkey

Summary: To evaluate the morphometric specialities of the thorocal and lumbar vertebraes in this study, 103 thorocal and 53 lumbar vertebraes were studied in anatomy department of the Selcuk university school of medicine. The mean posterior height of the thorocal and lumbar corpuses respectly as follows $18.96 \pm 2.68 \mathrm{~mm}$ and $25.33 \pm 2.69 \mathrm{~mm}$, anterior height $19.29 \pm$ $2.75 \mathrm{~mm}$ and $25.24 \pm 2.51 \mathrm{~mm}$, the width of pedicules; $27.17 \pm$ $5.50 \mathrm{~mm}$ and $31.84 \pm 4.19 \mathrm{~mm}$, the height of pedicül; $12.67 \pm$ $2.43 \mathrm{~mm}$ and $13.54 \mathrm{~mm} \pm 2.08 \mathrm{~mm}$, the sagittal diameter corpuses; $15.30 \pm 2.54 \mathrm{~mm}$ and $15.08 \pm 2.12 \mathrm{~mm}$ the transvers diameter; $33.31 \pm 5.62 \mathrm{~mm}$ and $48.53 \pm 6.86$ the transvers diameter of the foramen vertebrale $20.38 \pm 3.10 \mathrm{~mm}$ and $23.13 \pm 2.50 \mathrm{~mm}$ the sagittal diameter of the foramen vertebrale $7.83 \pm 4.40 \mathrm{~mm}$ and $11.86 \pm 5.00 \mathrm{~mm}$. The knowledge of mean values and variabilities of thorocal and lumbar vertebraes will decrease the mortality and morbidity in spinal surgery.

\section{PP-16}

Some morphometric features of foramen magnum, orbita and calvaria

Tuncer I

Department of Anatomy, Meram Faculty of Medicine, Selcuk University, Konya, Turkey

Objectives: It was aimed to assess some essential structures formed in the cranium morphometrically and to compare the results according to sexes. 
Material and Method: This study was carried out on total 78 craniums (45 female, 33 male) in the anatomy laboratory of Medical Faculty of Selcuk University. The sagittal and transverse diameter of calvaria (A1A1, A2A2, respectively), transverse diameter of both aditus orbita (right; A3A3, left;A4A4), the distance between the medial edges of aditus orbitas (A6A6) and the sagittal and transverse diameter of foramen magnum (A7A7,A8A8, respectively) were measured in $\mathrm{cm}$ with the help of a caliper. The comparison of male-female was evaluated with student-t test statistically on SPSS program.

Findings: When the parameters were evaluated, it occurred that except for the transverse diameter of calvarias, others were greater in males than females $(\mathrm{P}<0.05)$ Besides, there was moderate and high level correlation between the parameters. Results: The differences established in morphometric data in the particular zones in the cranium of males and females can be helpful in the diagnostic and therapic applications of neuroradiology and neurochirurgia.

\section{PP-17}

3D-morphometry of the subchondral bone plate of human articular processes

Herrera $M^{*}$, Gimeno JS**, Erades D*, Gosalvez ER*, Ferrer $\mathrm{MS}^{* * *}$, Manzanares-Cespedes $\mathrm{MC}^{* * * *}$, Lapresa-Alonso $\mathrm{M}^{\star * * * *}$, Almenar-Garcia $\mathrm{V}^{* * *}$, Del Campo FS***

Department of Optics, Pharmacology and Anatomy, University of Alicante, Spain*; Department of Anatomy and Human Embryology, University of Valencia, Spain**, Department of Histology and Anatomy, Faculty of Medicine, University Miguel Hernandez (Umh), Elx, Spain***; Unitat de Anatomía Humana, Departament de Patologia Y Terapeutica Animal, Bellvitge, Universitat de Barcelona, Spain****; Servicio de Microscopia, C.E.N.I.E.H., Fundación Atapuerca, Burgos, Spain $* * * * *$

The 3D-microarchitecture at the anatomical level of the subchondral bone plate (SCBP) of C3-C5 articular processes (AP) was analysed to define its extension, architectural changes in depth as well as their lateral to medial differences, knowing its respective connections to the costotransverse process and the pedicle. Twenty AP (35-50 y) from a total sample of 400 were obtained from the dissection room of the Faculty of Medicine of the U.M.H (Spain), without macroscopical signs of osteoarthrosis and selected for the CT- 80 and the obtained images weremstudy. The AP were scanned with a Scanco analyzed into regions of interest. The SCBP were firstly divided into lateral and medial parts, and, afterwards the whole SCBP was divided craneocaudally into $\mathrm{m}$ subregions. Standard 3D-morphometry were carried usingmfour one-thousand Scanco software and. 3D reconstructions were made using Mimics (Materialise). The medial part of the SCBP possesses higher BV/TV, Th and ConnD. and lower MV/TV, Tb.Sp and ConnD of marrow cavities with respect to the lateral one $(\mathrm{p}<0.001)$. SMI mean values indicated a stronger plate-like configuration in the medial part than the lat- eral one. Subregional analysis indicated that from upper to lower levels the BV/TV, SMI, and Th decreases progressively while the Tb.Sp and the ConnD of marrow holes increases progressively. There was a clear morphometrical and statistical $(\mathrm{p}<0.001)$ difference between the two more superficial subregions and the deep ones. This was confirmed using the index $\mathrm{Th} / \mathrm{Tb}$.Sp to detect different between the bony and the bone marrow parts of the SCBP $(\mathrm{p}<0.001 ; \mathrm{R}=0.82)$. 3D anatomical reconstructions of the SCBP trabeculae show the external trabeculae of the lateral part ending in the cortical wall approximately 2,500 in depth. By contrast, the trabeculae of their medialmost part end in the medial cortical wall and the pedicle root.

\section{PP-18}

\section{The morphometry of femur's proximal part}

Cikmaz S*, Copuroglu $\mathrm{C}^{* *}$, Yilmaz A*

Department of Anatomy, Faculty of Medicine, Trakya University, Edirne, Turkey*; Department of Orthopaedics and Traumatology, Faculty of Medicine, Trakya University Edirne, Turkey**

Objective: The morphology of femur's proximal part differs among people and societies. Osteometric values of the formations here are significant in terms of choosing the appropriate size of prothesis in the surgery of hip prothesis which is applied in orthopedy.

Material and Method: In our study, we have measured the length of femoral neck axis, femoral head diameter 1-2, femoral neck width 1-2, neck shaft angle, shaft width 1-2 and femur length parameters, of 42 femur in total.

Results: According to this, the measurement values and standard deviations we got from the length of femoral neck axis, femoral head diameter 1-2, femoral neck width 1-2, shaft angle, shaft width 1-2 and femur length parameters, are these in order: $96.98 \pm 7.36 \mathrm{~mm}, 45.34 \pm 3.84 \mathrm{~mm}, 45.40 \pm 3.96 \mathrm{~mm}$, $32.16 \pm 3.17 \mathrm{~mm}, 29.83 \pm 3.80 \mathrm{~mm}, 122.40^{\circ} \pm 5.38,31.71 \pm 2.51$ $\mathrm{mm}, 26.22 \pm 2.02 \mathrm{~mm}, 368.07 \pm 22.64 \mathrm{~mm}$.

Conclusion: We have come to the conclusion that these results we got will be helpful in choosing the appropriate prothesis in the operations of hip prothesis.

\section{PP-19}

\section{Study of effects of high sodium chloride intake on big arterial wall}

\section{Jafarpur S}

Department of Anatomy and Cell Biology, Faculty of Medicine, Mashhad University of Medical Sciences, Mashhad, Iran

Aims: uncontrolled salt intake may increases arterial blood pressure. In many patients, high blood pressure is associated 
with big arterial wall injuries. Either high salt intake causes arterial wall injuries or other factors are responsible. This question induces our investigation.

Methods: 40 male and female, 2 months old, 30-35 grams weight Balb/c mice were divided in 2 experimental and control groups randomly. Sex equality was executed in this manner so there were 10 male and 10 female mice in each group. Experimental group received normal saline as 18 grams $\mathrm{ClNa}$ solved in $1000 \mathrm{cc}$ tap water as drink for 12 weeks. Control group received tap water as drink in this period. Mice of two groups were anesthetized by chloroform and killed after consumption of salt solution by experimental group for a period of 12 weeks. Aorta and carotid arteries were excised from their bodies and placed in 10\% formalin solution for fixation. Other steps of tissue preparation done and sections were obtained from the parafinized blocks finally and stained by Hematoxilin-eosin. Stained sections were observed microscopically and findings were recorded. Elective specimens were photographed. Findings were analyzed statistically at the next step.

Results: Atheromas were observed in big arterial intima in experimental mice. This phenomenon was not seen in control group. Cholesterol deposition was another abnormal change in arterial wall of mice of experimental group that there was not in control group. Other abnormal change in arterial wall of experimental group was many macrophage infiltration into intima that was not in control group.

Conclusion: Atheromas, cholesterol deposition and macrophage infiltration in arterial wall of experimental mice in comparison to control group had significant difference in statistical analysis $(\mathrm{p}<0.001)$. Abnormal changes in experimental mice mentioned above, show atherosclerosis in these laboratory models. So, probably, high salt intake induces atherosclerosis.

\section{PP-20}

\section{Absence of right common iliac vein}

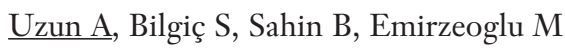

Department of Anatomy, Faculty of Medicine, Ondokuz Mayıs University, Samsun, Turkey

Introduction and Purpose: We observed absence of the right common iliac vein, during educational dissections on a female cadaver at the age of 52 .

Tools and Method: Absence of the right common iliac vein was found by dissection in 52 -year-old Turkish female cadaver who died of heart and respiration failure.

Results and Interpretations: Either common iliac vein may be doubled in part or throughout its length. One of the veins may be absent, in which case the external and internal veins join the opposite common iliac to form the vena cava. The right and left internal iliac veins occasionally unite and open as a common trunk at the confluence of the right and left external iliac veins to form the vena cava. The middle sacral trunk may divide, and one branch may open into the right common iliac vein and the other into the left. The common iliac veins occasionally receive an accessory renal vein or even the entire renal drainage. A connection between the portal and right common iliac has been reported. The internal iliac vein may form an annulus around the internal iliac artery. The internal iliac vein may receive an aberrant vessel representing a confluence of the internal pudendal, obturator, gluteal, and sciatic veins. Anomaly of the common iliac veins occurs infrequently but if unidentified can lead to significant morbidity during surgical exploration.

\section{PP-21}

\section{Morphometry of age related wall remodeling of human left anterior descedent coronary arteries}

$\underline{\text { Rancic } \mathrm{G}^{*}}$, Atanassova $\mathrm{P}^{* *}$, Chaldakov $\mathrm{G}^{* * *}$, Jancic $\mathrm{S}^{* * * *}$, Rancic $M^{*}$

Institute of Histology and Embryology, Medical University Niš, Serbia*; Department of Anatomy, Histology and Embryology, Medical University Plovdiv, Bulgaria**; Department of Cell Biology, Medical University Varna, Bulgaria***; Institute of Pathology, Medical University Kragujevac, Serbia****

Present knowledge of age related human coronary arteries wall remodeling is relatively deficient, especially in fetal life and on left anterior descendent (LAD) artery. Morphometric data concerning these changes could help to understand the arteries wall structure role in atherosclerosis and cardiovascular diseases, and periarterial adipose tissue, referred to as tunica adiposa, significance and its endocrine influence. The aim was to relate coronary artery remodeling progress during the aging through LAD wall structure analysis, and to estimate width numerical parameters of four wall layers, using morphometry to observe possible age related differences between the groups. Study was conducted on fetal LAD proximal segments (10) obtained by autopsy, gestation age $30-40$ weeks and on postmortem LAD samples with uncardiac death (50), aged 45-80 years. Samples were fixed and processed. Tissue sections were stained with HE, Masson and Spicer methods and analyzed through microscopic camera images and ImageJ computer software. Wall structure constituents: intima, media, adventitia and adiposa thickness were morphometricaly analyzed. Intima and adiposa thickness increases progressively with age, as opposed to adventitia thickness which progressively decreases from fetal to old adult group. Media thickness significantly decreases in older adult group comparing to fetal and young adult group. This remodeling process occurs systematically, accompanied by atherosclerosis in vessels, is distributed circumferentially, and precedes the localised lesions development 
in intima, and later, during the aging and atherosclerosis interference, in media and adventitia. Adventia changes are significantly connected with adiposa which increases with age. Age related wall remodeling is present in LAD human coronary artery, characterised by intima and adiposa thickness increase, and media and adventitia thickness reduction. Intima, media, adventitia and adiposa play a role in the remodeling process. Adiposa facilitates the atherogenesis. All four wall layers have anatomical and functional role.

\section{PP-22}

\section{Microvascularisation of carotid body}

José Pedro L*, Luís L*, Pedro R* , Joao O**

Departement of Anatomy, Faculty of Medical Sciences, New University of Lisbon, Portugal*; Director of Department of Anatomy, Faculty of Medical Sciences, New University of Lisbon, Portugal**

Introduction: Our understanding of the microvascularisation of carotid body is still uncomplete. The arterial afferences of this structure have been established by some reports as emerging from either the internal carotid artery or the external carotid body, and in fewer cases from the bifurcation of de common carotid artery.

Material and Method: 24 mice were injected with Mercox® by left ventricular catheterization. By open microsurgery, the anatomical structure composed by the common carotid artery and its terminal branches - internal carotid artery and external carotid artery - was bilaterally extracted. These specimens were prepared and observed by scanning electron microscope (SEM).

Results: We confirmed the existence of arterial afferences from internal and external carotid arteries. There haven't been found afferences from the bifurcation of the common carotid artery. We observed vasa vasorum emerging from internal carotid artery and directly vascularizing the carotid body.

Conclusion: We found evidence that suggests the existence of another carotid body vascularization system apart from the others previously known. It is hypothized that this two systems can co-exist in the same being. This double-vascularization may be related with the physiological importance of this paraganglion.

\section{PP-23}

Effect of aging on elastin distribution pattern in male and female basilar artery media

Valanciute A, Gudiene D, Pangonyte D, Balnyte I

Lithuanian University of Health Sciences, Kaunas, Lithuania

Vascular aging is associated with structural changes in elastin distrubution pattern in the arterial wall. Degradation of elastin during aging influences arterial elasticity and is related to reduced arterial compliance. Purpose of this study is to evaluate morphometrically age related changes of elastin in media of human basilar artery of both genders. Basilar arteries were obtained from 76 human cadavers of both genders (age from 20 to 86 years). Morphometrically were investigated middle segments of basilar arteries. Area of elastic fibers network, perimeter of bundles of elastic fibers and number of bundles were evaluated in media of basilar arteries in 3 different age groups of both sexes. Quantitative assessment of the area of elastin network in media of basilar artery in age groups $<40$ years(young); 40-59 years (middle age) and $\geq 60$ years (old) showed significant decrease of elastin network area with increasing age among all investigated groups. Results showed also significant decrease of perimeter of elastic fibers bundles with increasing of the age. Perimeter of elastic fibers in male young age group was $47.3 \%$ higher than in middle age group and $66.5 \%$ higher than in old age group. Perimeter of elastic fibers in different female age groups showed less expressed differences, but it also significantly decreased with increasing of the age. We obtained strong correlation between area of elastic fibers network and perimeter of fibers in both (male and female) age groups. Correlation between area of elastic fibers and number of fibers in $1 \mathrm{~mm}^{2}$ was strong and significant in both male and female age groups. Such strong correlation between area of fibers, number and perimeter of fibers shows changes in the structure of the fibers: they become smaller, thinner and number of fibers decreases.

\section{PP-24}

\section{The study of the coronary artery in women and men at the age of 20-50 in Kerman city}

\section{Rayegan P, Saba M, Hasan S, Vaghefi E}

Kerman Medical School, Kerman, Iran

Introduction: Heart, as a blood circulation system pump, needs feeding vessels for its muscular wall thickness. These vessels include coronary arteries and its branches. By using the angiographic technique, it is possible to study the situation of the coronary vessels and its branches in people, and to use these results in the clinics.

Materials and Methods: Optimus Biplane angiographic equipment,, M -200 and its peripheral tools were used in this study. Special cutter is inserted in ascending aorta (beginning of right and left coronary steum ) through one of the large arteries (Femoral or Brachial arteries)and during injection of the contrast agent by instrument, the pictures were recorded on the film. Then by using the film we can study the situation of the vessels. 
Results and discussion: Results from the study revealed that in most cases there is a meaningful difference in the number of the branches in the coronary arteries between two sexes. (pv0.05). Acceptable justification for this difference may arisen from the higher weight and volume of the men body in comparison with the females which also results larger heart in males. So the number of feeding arteries in men is more than women for larger heart and increase of its surface and volume.

\section{PP-25}

The morphological base of the cerebral blood circulation in the experimental carotid-jugular fistula

\section{Musienko V}

Lugansk State Medical University, Ukraine

Objectives: Cerebral circulatory disorders at extracranial arteriovenous fistula are the main pathogenetic factor of the brain damage; therefore their morphological base requires detailed study.

Methods: Carotid-jugular fistula was modeled on 40 dogs. The experiments conformed to internationally accepted ethical standards in the care and use of animals. Methods of the intravascular ink-gelatin injection and plastic injection with corrosion were used.

Results: Complex character of the cerebral circulatory disorders has been caused by relative hypovolemia in result of arteriovenous blood shunting, the decrease of the blood inflow to the brain by affected artery and impairment of venous outflow from the skull cavity by the veins engaged in drainage of the shunting blood. Morphological changes of the brain venous system within acute period were characteristic for venous plethora. Reconstruction of the arterial system was directed on improvement of the blood supply of the brain hemisphere on the side of defeat. Transformation of the vascular system was accompanied by formation of the extra- and intracranial arterial and venous collateral ways and the vascular system of the arteriovenous fistula. Development of the last one was expansive that has caused progressing character of the systemic and cerebral circulatory disorders. Involving intact arteries, including intracranial ones, in supply of the arteriovenous fistula led to "robbery" of the cerebral arterial circulation. Increased disproportion of the arterial inflow ways to the brain and the ways of collateral venous outflow formed predispositions for development of the relative arterial stenosis syndrome. Cerebral microcirculatory bed transformation formed the ways for intracerebral arteriovenous blood shunting and pathological acceleration ("centralizations") of the cerebral blood

Conclusion: Morphological transformation of the vascular system at extracranial carotid-jugular arteriovenous fistula forms the basis for hypoxic damage of the brain by the cardiovascular insufficiency, the "robbery" and relative arterial stenosis syndromes, the "centralizations" of the cerebral blood flow.
PP-26

Extensive papillary muscle calcification and ossification in a diabetic patient

Jianu $\mathrm{AM}^{*}$, Rusu MC $\mathrm{MC}^{* *}$, Pop $\mathrm{F}^{* * *}$, Gutu $\mathrm{DE}^{* * *}$, Hostiuc $\mathrm{S}^{* * * * *}$, Curca GC $\mathrm{GC}^{* * * *}$

Department of Anatomy and Embryology, "Victor Babes" University of Medicine and Pharmacy, Timisoara, Romania*, Discipline of Anatomy and Embryology, Faculty of Dental Medicine, "Carol Davila" University of Medicine and Pharmacy, Bucharest, Romania**; Department of Pathology, Faculty of Medicine, "Carol Davila" University of Medicine and Pharmacy, Bucharest, Romania***; Department of Pathology, National Institute of Diabetes, Nutrition and Metabolic Diseases, "Prof. Dr. N. C. Paulescu", Bucharest, Romania****; National Institute of Legal Medicine "Mina Minovici" Bucharest, Romania*****

Cardiovascular calcification has been reported in arterial wall, heart valves, pericardium, myocardium and papillary muscles. Papillary muscle calcifications are usually found in the elderly and are located apically (most frequent), in an arrowhead shape, or extensive (extremely rare cases). Myocardial ossifications are rarely described in human hearts and only exceptionally in papillary muscles. Type II Diabetes Mellitus is often associated with cardiovascular calcification, determined, amongst other causes by increased atherogenesis, high calcium affinity for advanced glycation end-products, diabetic nephropathy with subsequent chronic renal failure, local hypoxia, etc We present here a case, histopathologically documented, of papillary muscle calcification associated with metaplastic ossification in a patient with congestive heart failure, arterial hypertension and complicated type II Diabetes Mellitus (diabetic nephropathy, arteriopathy) and discuss potential causes and mechanisms.

\section{PP-27}

\section{Electrical conduction system apoptosis in type II diabetes mellitus}

Jianu $\mathrm{AM}^{*}$, Rusu $\mathrm{MC}^{* *}$, Pop $\mathrm{F}^{* * *}$, Hostiuc $\mathrm{S}^{* * * * *}$, Curca $\mathrm{GC}^{* * * *}$, Gutu DE****

Department of Anatomy and Embryology, "Victor Babes" University of Medicine and Pharmacy, Timisoara, Romania*, Discipline of Anatomy and Embryology, Faculty of Dental Medicine, "Carol Davila" University of Medicine and Pharmacy, Bucharest, Romania**; Department of Pathology, Faculty of Medicine, "Carol Davila" University of Medicine and Pharmacy, Bucharest, Romania***; Department of Pathology, National Institute of Diabetes, Nutrition and Metabolic Diseases, "Prof. Dr. N. C. Paulescu",Bucharest, Romania****; National Institute of Legal Medicine "Mina Minovici" Bucharest, Romania*****

Even though apoptosis is known to be associated with various cardiovascular pathologies, its presence in cardiac nodal tissue in adults was only scarcely researched. Cardiomyocyte apoptosis was associated with diabetic cardiovascular pathology. Our main 
objective was to test whether programmed cell death is present in nodal tissue in type II diabetes mellitus and, if present to characterize it. The study was designed as a qualitative one. We used autopsy samples of hearts from 10 patients (56 to 73 years old, 6:4 male to female ratio), positive for type 2 diabetes mellitus. Samples from sinoatrial and atrioventricular nodes were stained with hematoxylin-eosin. For immunohistochemistry, we used primary antibodies for caspases 3 and 9, cathepsin B, and TRADD. Nodal tissue in all samples was characterized by diffuse interstitial fibrosis and chronic ischemic lesions; nuclear damage and foci of irreversible ischemic necrosis intermingled with isles of relatively morphologically normal myocytes. Sinoatrial and atrioventricular nodes were caspase- 3 and -9 positive, and also cathepsin-B-positive, suggesting an overlap between apoptotic and necrotic mechanisms. Central area of the sinus node seemed to have the most severe lesions. As a conclusion nodal apoptosis is present in nodal tissue in type II diabetes mellitus, it involves the intrinsic pathway and associated concomitant and/or post-apoptotic necrosis.

\section{PP-28}

\section{Anteroseptal accessory pathways}

Lazarova D, Zivadinovic J, Zafirova B,Trpkovska B

Institute of Anatomy, Medical Faculty, Skopje, Republic of Macedonia

The most common accessory pathways in the anteroseptal place are Kent Bundles. They occupy an area along the right anterior AV junction and then extend anteriorly and laterally to the point where the right coronary artery enters the coronary sulcus. Therefore, it's imperative to understand the anatomy of this area in order to successfully and safely ablate pathways in this location. The anatomical characteristics of the area were reviewed by the dissection of 15 human hearts. The anterior septal area was approached from the superior aspect of the heart. The position of Kent Bundles was determined from a study of serial sections. A variety of stains were employed but the most useful proved to be hematoxillin-eosin and Masson trichrome. One important anatomical finding was the relationship of the atrial septum, the right fibrous trigone and the underlying membranous ventricular septum to the AV node and the His bundle.This study indicates that the Kent Bundles and the His bundle could be within $2 \mathrm{~mm}$ of each other. The AV node and the His bundle are actually in the posterior septal area with the compact AV node enclosed in the posterior aspect of the atrial septum. Based on this information one possible Kent Bundles connection in the septal area could be from the anterior edge of the atrial septum to the muscular ventricular septum, skirting the anterior edge of the right fibrous trigone. The other course from the right atrium to the right ventricle could be a point anterior to the right fibrous trigone to the arbitrary line separating the anterior septal area from the right free wall. The tendon of Todaro was found in 8 hearts.
No other pathways, except Kent Bundles, have been found in otherwise normal human hearts in the anterior septal area.

\section{PP-29}

Human anatomic variation: ascending vermian artery, branch of the vertebral artery

\section{$\underline{\text { Rusu MC }}$}

"Carol Davila" University of Medicine and Pharmacy, Bucharest, Romania

A previously undescribed anatomical variant is reported here, that of vermian arteries (vAs) branched from the vertebral arteries (VAs) and replacing the medial branches of the posterior inferior cerebellar arteries (PICAs) that, in turn, were leaving the basilar artery. Both vAs left the VAs in the foramen magnum. The left vA initially looped in front of the spinal root of the accessory nerve (Sp11) and then looped inferiorly on the dorsal side of the spinal cord, in the vertebral canal and continued ascending on the dorsal surface of the medulla oblongata. The right vA looped dorsally to the Sp11 above the foramen magnum and also ascended dorsally to the medulla. Both vAs were distributed to the vermis. On both sides the PICAs looped above the glossopharyngeal nerves and the jugular foramina and continued as only lateral terminal branches of a normal PICA that descended in countercurrent on the outer side of the variant vAs. Such anatomical variation is relevant for surgery and microsurgery on both sides of the foramen magnum and in the posterior cerebral fossa. Acknowledgements: (1) This study was supported by the SOP HRD, financed from the ESF and by the Romanian.

\section{PP-30}

Human portal interstitial Cajal cells, extrahepatic and intrahepatic

$\underline{\text { Rusu } M C^{*}}$, Pop F* $F^{*}$ Hostiuc $S^{* *}$, Curca GC* ${ }^{*}$ Sztika D ${ }^{* * *}$, Jianu $\mathrm{AM}^{* * *}$

Carol Davila University of Medicine and Pharmacy Bucharest, Romania*; Mina Minovici National Institute of Legal Medicine, Bucharest, Romania**; Victor Babeş University of Medicine and Pharmacy, Timişoara, Romania***

Rationale of the Study: Portal interstitial cells of Cajal (PICCs), acting as vascular pacemakers, were previously identified only in animal species but are not yet confirmed in humans. Moreover, there are not available evidences on the presence of these cells within the liver.

Objective of the Study: to evaluate whether or not PICCs exist also in humans and, if present, whether or not such cells follow within the liver the scaffold of the portal vein and its branches. 
Methods and Results: From five adult human cadavers were obtained in accordance with the ethical rules extrahepatic portal veins (PV) and liver samples. These were $\mathrm{HE}$ stained and then immunohistochemistry on formalin-fixed paraffinembedded specimens was performed for CD117/c-kit, considered as a marker of the Cajal's cells, and for CD34. PICCs were identified within the PV adventitia, closely located to the muscular bundles. Intrahepatic CD117/c-kit positive PICCs were also identified in the portal spaces and septa and on both sides of the limiting membranes. Additional immune labeling for S100 protein, desmin, alpha smooth muscle actin, and CD34 was also performed and the results are discussed.

Conclusions: Human extrahepatic PICCs evidences strongly reinforce the animal studies and support the possible intrinsic pacemaker influence on the human PV function. However the intrahepatic PICCs which were evidenced here for the first time ever need further experimental studies in order to evaluate their functional role. A promising further direction of study is the PICCs role in the idiopathic portal hypertension.

\section{PP-31}

\section{Embryonal persistent arteries}

Albay S, Kastamoni Y, Koyuncu E

Faculty of Medicine, Suleyman Demirel University, Isparta, Turkey

Some arteries that appear in embryonal period (median artery, trigeminal artery etc.) get lost in intrauterine period or form other arteries. However, if a problem exists during regression, these arteries persist in adult period and are named as persistent arteries. Persistent median artery in upper extremity, persistent sciatic artery in lower extremity, persistent trigeminal artery, persistent hypoglossal artery, persistent otic artery and persistent proatlantal intersegmental artery in carotid-vertebrobasiler anastomosis, persistent hyaloid artery in eye and persistent primitive olfactory artery over bulbus olfactorius are the most seen. Some symptoms and clinic problems may be caused by persistent arteries although they are rare. For example; persistent median artery may cause carpal tunnel syndrome or persistent hyaloid artery may cause strabismus and nystagmus. Knowledge of persistent arteries is very important for diagnosis and treatment. Therefore; characteristic features, diagnostic procedures, differential diagnosis and clinical significances of these arteries must be known well.

\section{PP-32}

A biomolecular study on OA cartilage derived chondrocytes encapsulated in poly(ethylene glycol) diacrylate (PEGDA)

Musumeci G, Carnazza ML, Martinez G, Loreto C Department of Bio-medical Sciences, University of Catania, Italy
Introduction: The aim of this study was to investigate and compare the apoptosis through the expression of caspase- 3 in tissue explants, in cells cultured in monolayer, and in cells encapsulated in a hydrogel (PEGDA) scaffold. Osteoarthritis $(\mathrm{OA})$ is characterized by cartilage attrition, subchondral bone remodeling, osteophyte formation and synovial inflammation. Perturbed homeostasis caused by inflammation, oxidative stress, mitochondrial dysfunction and proapoptotic/antiapoptotic dysregulation is known to impair chondrocyte survival in joint microenvironments and contribute to OA pathogenesis. However, the molecular mechanisms underlying the programmed cell death (apoptosis) of chondral cells are not yet well defined. The present study was conducted to evaluate apoptosis of chondrocytes from knee articular cartilage of patients with $\mathrm{OA}$.

Methods: Chondrocytes were also studied following cell isolation and encapsulation in poly(ethylene glycol) diacrylate (PEGDA) hydrogels. Specifically, articular cartilage specimens were assessed by histology (Hematoxlyn and Eosin) and histochemistry (Safranin-O and Alcian Blue). The effector of apoptosis caspase-3 was studied through immunohistochemistry, immunocytochemistry and immunofluorescence. DNA strand breaks were evaluated in freshly isolated chondrocytes from human OA cartilage using the TUNEL assay, and changes in nuclear morphology of apoptotic cells were detected by staining with Hoechst 33258.

Results: The results showed an increased expression of caspase-3 in tissue explants, in pre-confluent cells and after four passages in culture, and a decreased expression of caspase- 3 comparable to control cartilage in cells encapsulated in hydrogels (PEGDA) after 5 weeks in culture. The freshly isolated chondrocytes were TUNEL positive. The chondrocytes after 5 weeks of culture in hydrogels (PEGDA) showed the formation of new hyaline cartilage with increased cell growth, cellular aggregations and extracellular matrix (ECM) production.

Conclusion: This is of particular relevance to the use of OA cells and tissue engineering in the therapeutic approach to patients.

\section{PP-33}

Interaction effects of interleukin-4 and interleukin$1 \alpha$, on bovine nasal cartilage explants

Orazizadeh $M^{*}$, Yadegari $M^{*}$, Hashemitabar $M^{*}$, Khodadadi $A^{* *}$

Cell and Molecular Research Center (Cmrc), Anatomical Sciences Department, Medical School, Ahvaz Jundishapoor University of Medical Sciences (Ajums), Ahvaz, Iran*; Department of Immunology, Medical School, Ahvaz, Iran**

Introduction: The interactions effects of interleukin-4 (IL-4) and interleukin-1 $\alpha$ (IL-1 $\alpha$ ), on tissue characteristics and matrix 
metalloproteinase-1(MMP-1), as an important degenerating factor in bovine nasal cartilage (BNC) explants were investigated.

Materials and Methods: Fresh BNC samples were shortly prepared from slaughterhouse under sterile conditions. Explants of BNC were treated with the both IL-4 $(50 \mathrm{ng} / \mathrm{ml})$ and IL-l $\alpha(10 \mathrm{ng} / \mathrm{ml})$ at the same time for 28 days. The morphological characteristics of explants were assessed by using invert microscope and histology techniques. The membrane integrity and viability of chondrocytes were evaluated by using lactate dehydrogenase (LDH). MMP-1 expression was assessed within the different days by using western blotting.

Results: In the presence of IL-l $\alpha$ alone, an increase in cell: matrix ration was observed on the day 21 . Chondrocytes presumably showed a foamy and vacuolated cytoplasm. Pyknotic and black point nuclei in most chondrocytes were observed. In the presence of both IL-4 and IL-l $\alpha$, morphological changes in chondrocytes and ECM were negligible and LDH level was not significantly increased. The MMP-1 expression in the presence of IL-l $\alpha$ alone was substantially increased during 2128 days of culture. In the presence of both IL-4 and IL- $1 \alpha$, chondrocytes preserve their ordinary phenotype and the edges of BNC explants remained intact. In addition, a significant reduction of MMP-1 expression was observed.

Conclusion: IL-4 could be candidate as a protective factor against the degradation effects of IL- $1 \alpha$ on cartilage. The cytokines interactions may suggest an applied therapeutic method that more researches in this area are needed.

\section{PP-34}

\section{Thyroid cartilage revisited: new insights into gender-specific mineralization and ossification of human laryngeal cartilages}

Claassen $\mathrm{H}^{*}$, Schicht $\mathrm{M}^{*}$, Sel $\mathrm{S}^{* *}$, Paulsen $\mathrm{F}^{* * *}$

Institute of Anatomy and Cell Biology, Martin Luther University HalleWittenberg, Germany*; Department of Ophthalmology, Martin Luther University Halle-Wittenberg, Germany**; Institute of Anatomy, Friedrich Alexander University Erlangen-Nürnberg, Germany***

Human laryngeal cartilages, and especially thyroid cartilage, mineralize and ossify at a snail's pace. The ossification of thyroid cartilage presents sex differences, and, in advanced age, the shape of thyroid "bone" is gender-specific like the shape of male and female pelvis. The mechanism of sexual different ossification of thyroid cartilage is only partly understood. In all life decades, female thyroid cartilages contained more chondrocytes with a territorial rim of chondroitin-4- and 6-sulphates, probably preventing cartilage mineralization, compared with agematched male specimens. Male thyroid cartilages often contain more chondrocytes with a positive enzymohistochemical reac- tion for alkaline phosphatase, as key enzyme of mineralization, than samples from females. Androgen receptor was detected immunohistochemically in chondrocytes of both sexes. However, estrogen receptor alpha and progesterone receptor were not detected. By scanning radiographs of 80 thyroid cartilages from both genders, we found a significant difference in ossification between men and women in the age group 41 to 60 years $(p<0.002)$. Just in the same age group, thyroid cartilages from men revealed a significantly higher percentage of apoptotic chondrocytes than did thyroid cartilages from women $(\mathrm{p}<0.004)$. Taken together, ossification of thyroid cartilage is coupled with apoptosis of chondrocytes similar to endochondral ossification in growth plates. Looking on the expression of sex hormone receptors, only androgen receptors were found, but surprisingly in both genders. At this time, we like to hypothesize that expression of alkaline phosphatase and apoptosis may be influenced by testosterone. However the hormonal influence on both events remains to be elucidated further.

\section{PP-35}

\section{Comparison of cerebellar volume between the people who are bilateral congenital blind with healthy ones}

\section{Kosif R, Gürel K}

Department of Anatomy, Abant Izzet Baysal University, Bolu, Turkey

It is known that when eye and hand need to be worked in combination, cerebellum is active and it provides coordination between eye and hand. Cerebellar atrophy in cortex appears with dismetry and saccadic eye movement. If there is no stimulant related to vision, how cerebellum is adopted under this circumstance? In order to explore this, 27 male and 16 female volunteered people who are bilateral congenital blind were compared with 35 male and 33 female healthy volunteer in this study. MR images of cross-sectional sequential cerebellum of volunteers with $1.5 \mathrm{~mm}$ thickness were realized in coronal plane. The surface area of apparent cerebellum seen in crosssections was calculated by using Onis (Ver. 2.1) programme. Surface area data obtained by systematic randomized sampling were converted to volume by Cavalieri method. Cerebellar volume of bilateral congenital blind male was $128.15 \pm 11.11 \mathrm{~cm}^{3}$, and cerebellum volume of bilateral congenital blind female was $118.60 \pm 10.73 \mathrm{~cm}^{3}$. Cerebellum volume for healthy men and women were $132.89 \pm 12.51 \mathrm{~cm}^{3}$ and $125.97 \pm 10.78 \mathrm{~cm}^{3}$, respectively. It was revealed that cerebellar volume for bilateral congenital blind men was smaller than that of healthy men, but this difference was not significant. On the other hand cerebellar volume of bilateral congenital 0.05 ). No blind women was significantly smaller than that of healthy women ( $p$ asymmetry was detected between right and left side of cerebellum in both bilateral congenital blind and healthy subjects. 


\section{PP-36}

Increased arginases in the spinal cords with clip compression injury and experimental autoimmune encepahlomyelitis

$\underline{\text { Shin } \mathrm{T}^{*}}$, Kim H*, Ahn $\mathrm{M}^{*}$, Moon $\mathrm{C}^{* *}$

Jeju National University, South Korea*; Chonnam National University, South Korea**

Excess production of nitric oxide (NO) has been thought to contribute central nervous system (CNS) injury, while increased inducible nitric oxide synthase (iNOS) is associated with increased arginases in the course of CNS injury. Arginases has been known to be an alternative enzyme to use L-arginine, resulting in the generation of L-citulline and urea. The aim of this study was to address the changes of arginase 1 in the damaged central nervous system tissues including spinal cord injury and experimental autoimmune encephalomyelitis (EAE). Western blot showed that arginase-1 was significantly upregulated in the injured spinal cords and EAE affected spinal cords. Immunohistochemical study showed that arginase-1 was immunodetected in both macrophages and reactive astrocytes in the injured spinal cords and EAE affected lesions. Because arginase-1 is used as a marker for alternatively activated macrophages (AA macrophages), arginase-1 positive macrophages have been supposed as AA macrophages, which may modulate CNS inflammation. Collectively the present finding suggests that arginase-1 is upregulated in the injured CNS tissues, which may lead to CNS remodelling. A precise mechanism of arginase-1 involvement needs further study.

\section{PP-37}

Evaluation of the relation between skull and phantom brain model using computed tomography sections: a stereological study

Sahin $\mathrm{B}^{*}$, Incesu $\mathrm{L}^{* *}$, Uzun $\mathrm{A}^{*}$

Department of Anatomy, Ondokuz Mayis University, Faculty of Medicine, Samsun, Turkey*; Department of Radiology, Ondokuz Mayis University, Faculty of Medicine, Samsun, Turkey**

Introduction and Purpose: By combining measures of brain volume with measures of $\mathrm{ICV}$, investigators can infer how much reduction in volume has occurred since brain volume was at its peak. Computed tomography (CT) imaging is a powerful modality for central nervous system imaging and for subsequent routine ICV measurements and depiction of bony structures. We investigated the effect of section thickness on the estimated volume fraction (VF) of brain in CT scanning using phantom brain modeling.

Tools and Method: Five dry skulls and fresh cattle brains were taken. They were placed within thin plastic bags filled with water and placed into the skulls to produce phantom mod- els. The models were scanned with a CT machine. The section series with thicknesses of $1,4,7$ and $10 \mathrm{~mm}$ were obtained using Osirix software. The cut surface areas of the skulls and brains were obtained. The ICV and brain volume were estimated using the Cavalieri principle of stereological methods. The actual brain volumes were obtained by Archimedean principle. The actual ICV of 5 skulls was measured using the waterfilling method. Findings: The mean actual ICV $( \pm \mathrm{SD})$ of skulls and brains were $1306.02 \pm 134.21 \mathrm{~cm}^{3} 416.98 \pm 17.28 \mathrm{~cm}^{3}$, respectively. The mean actual VF of the brain to ICV was $32.31 \%$. The mean VFs obtained from the CT images were 33.54, $34.46,35.24$ and $36.21 \%$ for $1,4,7,10 \mathrm{~mm}$ section thicknesses, respectively.

Results and Interpretations: Our results revealed that the VF of the brain was changing depending on section thickness. Thicker sections resulted in higher VF. While the ICV was decreasing, the brain volume was increasing depending on the section thickness. The usage of constant section thickness may standardize the deviations from the actuals depending on the section thickness. Resources:

\section{PP-38}

\section{A comparison of numbers of interneurons in two thalamic nuclei of normal and epileptic rats}

Cavdar $\mathrm{S}^{*}$, Hacioglu $\mathrm{H}^{*}$, Yildiz $\mathrm{SD}^{*}$, Akakin $\mathrm{D}^{* *}$, Onat $\mathrm{F}^{* * *}$

Department of Anatomy, Marmara University, Faculty of Medicine, istanbul, Turkey*; Department of Histology, Marmara University, Faculty of Medicine, istanbul, Turkey**

Department of Pharmacology, Marmara University, Faculty of Medicine, istanbul, Turkey***

Introduction and Purpose: The major inhibitory source of thalamic nuclei are local interneurons and neurons of the thalamic reticular nucleus. Absence epilepsy models have shown that the production of seizures is related to an excess of GABAergic neurotransmission in the thalamus. The present study used light microscopical GABA immunocytochemistry to confirm previous data reported for the rat regarding the presence of GABAergic interneurons in the lateral geniculate (LGN) and ventrobasal (VB) thalamic nuclei and to compare these results with data from genetic absence epilepsy rats from Strasbourg (GAERS).

Tools and methods: Light microscopical GABA immunocytochemistry were used to define GABAergic interneurons.

Results: The results are: 1) The interneurons form a minority of the neurons in LGN and VB in both Wistar and GAERS animals. Interneurons represent $19.0 \%$ of the neurons in the LGN of Wistar rats, $22.6 \%$ in the LGN of GAERS, $0.3 \%$ in the VB of Wistar rats and $4.2 \%$ in the VB of GAERS. 2) the packing density of the interneurons was significantly higher in the LGN 
than in VB in both Wistar rats and GAERS. 3) There was no significant difference between Wistar rats and the GAERS regarding the proportion of interneurons in the LGN whereas the proportion of interneurons in VB was significantly higher for GAERS than for the Wistar rats. 4) The comparison of the mean areas of both relay cell profiles and interneuronal profiles showed no significant diference between Wistar rats and GAERS.

Interpretation: These finding suggest that the relatively greater number of GABAergic interneurons in the VB thalamic nucleus of GAERS may be related to the production of absence seizures or it may represent a compensatory response of the thalamocortical circuitry to the absence seizures.

\section{PP-39}

Study of emotional and exploratory behaviors in the first generation of morphine addicted male and female mice

Mahmoudian A

Mashhad University of Medical Sciences, Iran

Introduction: Morphine addiction changes parental endocrine secretions in pregnancy, that affects behavior. If the next generation is susceptible was our question.

Materials and Methods: Emotional and exploratory behaviors of first generation of morphine addicted male and female mice were studied by Elevated Plus Maze (EPM) and Open Field (OF) procedures.

Results: EMP test showed that male offspring of addicted parents are less enthusiastic than control to enter to open arm and also showed that the female offspring of addicted parents spend more time in close arm to decrease the stress and increase the security ratio. Female children of none-addicted parents and female children of addicted mothers are different in two important factors; the number of standing and washing time; it means that some children had been delivered from some kinds of addicted mothers that in OF condition spent more numbers for standing but this group of children spent less time in washing also the children of addicted fathers spent more time in washing in compare with the two other groups.

Conclusion: Many researches had been done and still doing about the effects of addicted parents on physiological and psychological system of their children both in animals and human. But the main goal of this research is the examination about the effects of morphine addicted male parents on their first generation children. What became clear in OF test result is that the wide behavioral changes happen in male and female children of addicted parents in compare with the other groups. But the most important thing is the effects of addicted parents on female children; for analyzing we refer to the OF test result again that female children of addicted fathers showed more seeking reactions.
PP-40

The effect of the Valerian consumption during pregnancy on cortical volume and the levels of zinc and copper in the brain tissue of mouse fetus

\section{Mahmoudian A}

Mashhad University of Medical Sciences, Iran

Valerian (Valeriana Officinallis) is a perennial plant used as a hypnotic, sedative, muscle relaxant, anxiolytic, anticonvulsant and antidepressant. It is traditionally contraindicated in pregnancy, however, there are no studies to warrant this warning. The aim of the present study was to determine the effect of valerian consumption in pregnancy on cortical volume and the levels of zinc and copper, two electrolytes that affect brain development and function, in the brain tissue of mouse fetus.

Materials and Methods: Pregnant female mice were dosed with either saline or a $1.2 \mathrm{~g} / \mathrm{kg}$ valerian extract in $40 \%$ ethanol (ip) daily on gestation days (GD) 7-17. On GD 20, mice were sacrificed and fetuses collected. Fetal brains were dissected, weighed and processed for histological analysis. The volume of cerebral cortex was estimated by Cavalieri principle. The levels of zinc and copper in the brain tissue were measured by atomic absorption spectroscopy.

Results: Our results indicated that valerian consumption during pregnancy caused no significant reduction in brain weight, cerebral cortex volume and copper level in fetal brain. However, it significantly decreased the level of zinc in the brain.

Conclusions: Using valerian during mid-gestation did not have an adverse effect on cerebral cortex, however it caused a significant decrease in zinc level in the fetal brain. Therefore, the utilization of valerian should be limited in pregnancy.

\section{PP-41}

Neuroanatomy aspect of sustation attention in Bosnian war veterans diagnosis with PTSD

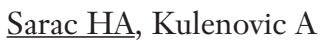

Department for Anatomy, Faculty of Medicine, Bosnia and Herzegovina, University of Sarajevo, Sarajevo, Bosnia and Herzegovina

Objective: Aim of our research is to exam sustained attention on Bosnian War Veterans with PTSD diagnosis.

Material and Methods: As a material for construction of this stady we tested 79 war veteranes of Bosnian Army, wich participate in the former war in BIH from 1992 to 1995 . From 79 tested war veterans on 45 PTSD has developed, wile 34 stayed without PTSD, and thay are belong to control group. All 79 war veterans are ages from $30-50$ years; and with the same education level (elementary and secondary school). We tested all of them with sustained attentions to response task - SART. This task includes frequent button pressing on every targeted 
stimulus and keeping or non-pressing button when targeted stimulus shows. This computerized tests last 4.3 minutes were in this time 225 singular numbers (1-9) shows by accidental order in a center of the computer screen. As a result we got 3 measures: number of faulty positive ones - press / answers on number 3 (maximal error number is 25 ), number of faulty negative - cases when participants did not press the button but the number which is not 3 appear on the screen (maximal error number is 200) and average reaction time on all answers.

Results: On the tests for the sustained attention to response task SART, patients with PTSD made more errors one and other types (positive and negative errors). Statisticaly significant difference between PTSD group and control group does not exist only at the reaction time measurement at SART test.

Conclusion: Patients with PTSD made more errors what is linked to difficulties of maintaining of vigility over the time. Persons with PTSD has shorter reaction time what is related with the anxiety symptoms on persons with PTSD. Over anxiety can lead to this that persons with PTSD faster react in such tests but with more errors.

\section{PP-42}

Evaluation of the ventricle volumes and the projection area of corpus callosum with stereological method in children with cerebral palsy

Dilber $\mathrm{C}^{* *}$, Korkmaz U**, Ozbag D*, Tugtag B*, Geckil E*

Departments of Anatomy, Faculty of Medicine, Kahramanmaraş Sütçü Imam University, Kahramanmaraş, Turkey*; Departments of Pediatric Neurology, Faculty of Medicine, Kahramanmaraş Sütçü Imam University, Kahramanmaraş, Turkey**

Cerebral palsy is a non-progressive motor and posture disorder caused by an injury to the brain due to various etiological factors during prenatal and perinatal periods. The objective of this study was to investigate the difference in the ventricular volumes and the projection area of corpus callosum in children with cerebral palsy with stereological method and planimetry as well as finding an association with the clinical picture. Cranial MR images of $100 \mathrm{CP}$ patients (60 boys, 40 girls), who applied to the Pediatric Neurology clinic between 2008-2010 and diagnosed as CP, were examined and the ventricular volumes and projection area of corpus callosum were measured with stereological method. A total of 116 subjects ( 67 boys, 49 girls) who had normal cranial MRIs were evaluated as the control group. The mean PAL value of corpus callosum in all CP patients was found statistically significantly lower than the control group $(\mathrm{p}<0.05)$. When girl and boy CP patients were evaluated separately, PAL value of corpus callosum was also seen to be lower significantly $(\mathrm{p}<0.05)$. However, that decrease was statistically insignificant in some age groups $(\mathrm{p}>0.05)$. Thinning in the splenium of corpus callosum with a rate of $70 \%$ in $\mathrm{CP}$ patient group was observed in our study.
PP-43

Blood-brain-barrier disruption following subarachnoid hemorrhage may be facilitated through puma induction of endothelial cell apoptosis from the endoplasmic reticulum

\author{
Zhou C, Han J, Yan Y, Zhang W \\ Peking University Health Science Center, China
}

The blood-brain-barrier (BBB) plays a vital role as both a physiologic and physical barrier in regulating the movement of water from the vasculature to the brain. During a subarachnoid hemorrhage (SAH), the BBB is disrupted by a variety of mediators, one of which can result in endothelial cell death. As a result, in the present study, we investigated the role of PUMA (p53 upregulated modulator of apoptosis) following SAH injury in rats. Specifically evaluating whether through the endoplasmic reticulum (ER), PUMA could orchestrate the induction of endothelial cell apoptosis and cause a disruption in the BBB integrity. One hundred twelve male Sprague-Dawley rats were randomly divided into 4 groups: sham, SAH, SAH+control siRNA, SAH+PUMA siRNA. Outcomes measured include mortality rate, brain edema, $\mathrm{BBB}$ disruption, and neurobehavioral testing. We also used western blotting techniques to measure the expression of key pro-apoptotic proteins such as BAX, BAK, and DRP1. PUMA siRNA treatment significantly reduced the mortality rate, cerebral edema, neurobehavioral deficits, and BBB disruption as measured by Evan's Blue assay following SAH injury. The T2WI images showed there was an increase in vasogenic edema in the brain following SAH, which could be alleviated by PUMA siRNA. Immunohistochemical staining and western blot analysis demonstrated an increased expression of PUMA, BAX, BAK, GRP78 and DRP1 in the microvascular endothelial cells of the hippocampus, which was accompanied with endothelium apoptosis. This study showed that PUMA induced endothelial cell apoptosis may in fact play a significant role in BBB disruption following SAH and its mediation may be through the endoplasmic reticulum. By blocking the activity of PUMA using siRNA, we were able to prevent the accumulation of cerebral edema that occurs following BBB disruption. This translated into a preservation of functional integrity and an improvement in mortality.

\section{PP-44}

Neural tube defects: morphologic and genetic check on fetuses with ultrasonographic and/or genetic prenatal diagnosis of malformation

Pirino A, Bandiera P, Fenu G, Fogu G, Moro MA, Sotgiu MA, Montella A

Department of Biomedical Sciences, University of Sassari, Sassari, Italy 
Introduction: The neural tube defects (NTD) are common congenital anomalies, with both genetic and environmental factors implicated in their complex etiology. About 1-2/1000 pregnancies are affected by NTD, both in the USA and in Italy (ISTAT 2007 data). The main aim of the present study is to evaluate the eventual connection between chromosomic alterations and NTD.

Material and Methods: The study has been conducted in Sassari (Sardinia, Italy). 140 human fetuses, from spontaneous or therapeutic abortions, that reached our laboratory from 2001 to 2009 , were evaluated in order to select the ones presenting neural tube defects. 28 fetuses between all the 140 proved to be affected by NTD. A first morphologic study has been conducted on each affected fetus. Then, the possible connection between NTD and cytogenetic anomalies has been also investigated. 3 fetuses showing such connection were investigated by FISH analysis, looking for genotype/phenotype relationship. Two of these foetus showed cariotype $46, \mathrm{XY}$, add4(q35) and one showed a chromosome marker. So, we further investigated these foetus by FISH (Fluorescence hibridation in situ) analysis to find genotype/phenotype correlations.

Results: It was very hard to correlate our results with other previous studies because of the multifactorial nature of these diseases. During this study, we also created a database containing all data related to the foetus and their parents. In this way, this study could represent the departure point to create a data bank in order to monitor DTN morphological and genetics anomalies in population and to put in effect a prevention plan creating a team of different professional figures.

\section{PP-45}

Expression of nestin and glial fibrillary acidic protein in injured spinal cord of adult rats at different time

Heshmati M, Amini H

Shahed University, Tehran, Iran

Introduction: To explore the expression of nestin and glial fibrillary acidic protein at different time and sites after spinal cord injury in adult rats.

Material and Methods: 21 adult Sprague - Dawley rats,aging 8 weeks and weighing from 180 to $220 \mathrm{~g}$. were randomly divided into 3 experimental groups $(18, n=3)$ and 1 control group $(n=3)$. To achieve this aim we used aneurysm clips. After the laminectomy of T9 to T11 vertebrae which is superior to lumbar plexus .we used aneurysm clip to compress the spinal cord. Then expression nestin and GFAP at different time (1-2-4 weeks) were recognized by immunohistochemistry method.In control group,the same site of the rat spinal cord was exposed with out aneurysmclip compression.
Result: one week after injury the number of motoneuron decreased obviously, in control group,the expression of nestin was hardly seen except ependymal cells of central canal,and the low expression of GFAP was seen.in experimental groups, the nestin and GFAP expression increased obviously in the injured sites and reached the peak value after 1 week and followed by gradual decrease.

Conclusion: The above results suggest that spinal cord injury can induce the expression of nestin and GFAP.

\section{PP-46}

The study of TRK receptors after spinal cord injury in adult rats

Heshmati M, Amini H

Shahed University, Tehran, Iran

Introduction: In a number of stress conditions, the biological effects of tumor necrosis factor- alpha,such as the induction of neuronal apoptosis, are presumably attenuated by TNF receptors and TRK receptors. In this study we try to make spinal cord injury in Sprague - Dawley adult male rats and then study the expression of these receptors. Beside neuroprotective effect of deprenyl.

Material and Methods: To achieve this aim we used aneurysm clips. After the laminectomy of T9 to T11 vertebrae which is superior to lumbar plexus. we used aneurysm clip to compress the spinal cord. Then it was done due to classification of rats: Group A or treatment with deprenyl divided to two sub groups:1- laminectomy and 2- laminectomy with aneurysmclip. Group B or control group was given normal saline instead of deprenyl, then divided as the same as treatment group. Expression of mRNA of TrK-A, TRk-B and p75 receptors were studied by RT-PCR. which sampling was taken in 2-4-824-72 hours in 2 groups.

Result: Electrophoresis results showed that in treatment group expression of TRk-A gene was not changed. After 8, 24 and 72 hours the expression $\mathrm{p} 75$ gene increased $(\mathrm{p}<0.05)$. Expression of Trk-B was decreased $(\mathrm{p}<0.05)$.

Conclusion: The expression of TrK-A was seen in all samples but, there was no significant difference between two groups.

\section{PP-47}

The study of gliosis reaction induce of pressure in spinal cord injury in adult rats

Heshmati M, Amini H

Shahed University, Iran

Introduction \& Aim: The most considerable mechanism of spinal cord injuries is fracture of vertebra. One of the methods 
of studying this field is establishment of spinal cord injuries in laboratory animals. In this study we try to make spinal cord injury in Sprague - Dawley adult male rats and then study the gliosis reaction in injuries.

Material and Methods: To achieve this aim we used aneurysm clips. After the laminectomy of T9 to T11 vertebrae which is superior to lumbar plexus we used aneurysm clip to compress the spinal cord. Then classification of rats: group A- laminectomy .group B - laminectomy and aneurysmclip. Study of morphometry and immunohistochemistry which sampling was done in 1,2 and 4 weeks after laminectomy. Immunohistochemistry study was carried out by antibody against astrocytes markers which is GFAP protein, microglial cells by OX-42 antibody, oligodendrocytes by CNPase antibody.

Result: The morphometry study in group B indicates motoneuron decreased and glial cell increased with hemorrhage within the holes in grey matter. By $\mathrm{T}$ test revealed a significant decrease in motoneuron by the time $(\mathrm{p}<0.05)$. The immunohistochemistry study indicates that, the number of glial cells increased and this rises by the time. The number of astrocytes increases and it is significant. Oligodendrocytes raised but this rise was not significant.

Conclusion: Spinal cord compression induses gliosis and it rises by the time.

\section{PP-48}

\section{An anatomical comparative study: The rib as dental implant carrying bone graft compared to the scapula}

Sonmez TT ${ }^{*}$, Ghassemi A*, Zaker-Shahrak A*, Altuntas $\mathrm{SH}^{* * * *}$, Gerressen $M^{*}$, Hilgers RD*** Prescher $\mathrm{A}^{* *}$ Riediger D*

Department of Oral, Maxillofacial and Plastic Facial Surgery, University Hospital RWTH-Aachen, Aachen, Germany*; Institute of Molecular and Cellular Anatomy, University Hospital RWTH-Aachen, Aachen, Germany**; Institute of Medical Statistics, University Hospital RWTHAachen, Aachen, Germany***; Plastic and Reconstructive Surgery, University Hospital RWTH-Aachen, Aachen, Germany****

Complex long bony defects of the maxillofacial region can be reconstructed regularly with microvascular anastomosed bone grafts. This study was designed to evaluate the clinically usable implantable dimensions of the lateral scapular border and the rib for bone flaps including osseointegrated dental implants in a large investigation collective. Furthermore, the possible age, gender and the side choice dependency of the results were investigated. The macerated scapulae and ribs from both sites of 65 central European adult cadavers were used in this study. We applied a clinically adapted measuring program of the osteometry and morphometry to quantify the vertical bone height and transversal bone width. The average data of the two-time measurements on measurement points were evaluat- ed statistically with regard to age, sex and side. The clinically relevant morphological characteristics were documented separately. For safe implantability of each bone graft, a vertical bone height $\geq 10 \mathrm{~mm}$ and a transverse bone width $\geq 5.5 \mathrm{~mm}$ were defined as the minimum requirement. The mean height values of the both donor sites were clearly above the mean width values. The morphology of rib bone in the distal region and the scapular lateral border showed differences in longitudinal sections and cross sections. In all measured bone dimensions, a statistically significant $(\mathrm{p}<0.05)$ gender difference in favor of males was determined. On the other hand we found no statistically significant side differences for the scapula and the rib. Further the rib-width and the scapula-height decreases linearly over age. This study permits the conclusion that the distal segment of the rib bone as well as already established scapula bone fulfils the minimal requirements for volume reconstruction as well as implantability.

\section{PP-49}

\section{Applied anatomical studies on the spinal cord of the goat}

$\underline{\text { Karkora A }}$

Faculty of Veterinary Medicine, Alexandria University, Alexandria, Egypt

The goat has received a little attention in the veterinary anatomy, the aim of this work is to study the morphological features of the spinal cord which can provide an adequate information on some relations between the spinal cord segments and vertebrae, the white and gray mater, this work was carried out on twenty adult clinically healthy goats of both sexes and different ages (1.5-3 years), they were anaesthetized, bled and then injected with $10 \%$ formalin mixed with $4 \%$ glycerin and $1 \%$ phenol solution through the common carotid artery for fixation for ten to days before its manual dissection, the spinal cord was exposed dorsally, ventrally and laterally. The dorsal aspect of the spinal cord was exposed to laminectomy (removal of the lamina of the vertebral arches), while the lateral aspect was exposed to paramedian section of vertebrae by using an electric saw and bone cutter. The spinal cord of the goat started at the middle third of the occipital condyle and terminated at the middle of the first sacral vertebra with an average length of $73.3 \mathrm{~cm}$. The measure of length at both dorsal and ventral root attachment; length of both dorsal and ventral inter-root; length of the segment as well as transverse and dorsoventral diameters for each segment were done. The topographical relationship between the spinal segments and each of the followings (corresponding vertebra, transverse process and the spinous process) were determined. cross section anatomy for each spinal segment were done to study the percentage ratio between the gray and white matters and their percentages. The site of epidural anesthesia is also determined. 
PP-50

Dissecting room accidents in a Venezuelan medical

$\underline{\text { Romero RR }}$

Universidad Central de Venezuela, Caracas, Venezuela

Introduction: The teaching of anatomy has changed substantially over the past two decades. Nevertheless, dissection of human cadavers is still a fundamental method for the teaching of this discipline. Dissecting is coupled with the risk of injury.

Materials and Methods: A prospective cohort analysis of dissecting room accidents in first year students of School of Medicine J.M. Vargas, Universidad Central de Venezuela, were done for the periods 2008-2009. Dissecting room accidents were recorded on anonymous and voluntarily filled standardized forms that contained the following data: type and site of injury, treatment, outcome, previous knowledge of the dissecting technique. The number of enrolled students in each course and the total hours spent in the dissecting room during each course were obtained from the department's data base.

Results: Seven injuries were recorded between 2008-2009 in a group of 431 first year anatomy students. Five were accidental self-inflicted with a scalpel blade. Four out of the five blade injuries were on the left index finger. Two students were injured through formalin fluids or tissue splash in the eyes.

Discussion: The findings from this study suggest that dissecting room accidents occur rarely. Most of them are cuts in fingers from scalpel blades and formalin fluids or tissue splash on the face and eyes. Although none of the physical injuries documented in this study have had any complications so far, this should not deter us from considering preventive strategies. There is no room for complacency. We need consensus in use of protective eyewear. It is important that all dissecting room accidents be recorded in order to both provide immediate care for the student and to better understand how to prevent further injuries.

\section{PP-51}

\section{An alternation from hypogastric area to Ou MC area} Ou MC

Obstetrics \& Gynecology Department of Taipei City Hospital, Taipei Medical University, Yang-Ming University, Taipei, Taiwan

Objectives: Ou MC area (CEM, 2010, UK) is a central trapezoid hypogastric area which is configured by abdominal palpation with Ou MC manipulation (APOM, JEM, 2010). This study compared the results between APOM for Ou MC area and traditional abdominal palpation (AP) to evaluate acute abdomen in women.

Methods: From January 2006 through December 2009, 113 women with acute abdomen attending an emergency depart- ment received APOM and AP. Of the 113 women, 91 had pelvic organ disease while 21 had non-pelvic organ disease and 1 had pelvic and non-pelvic organ disease concurrently.

Results: Excluding the case with concurrent pelvic and nonpelvic organ disease, the sensitivity of APOM for the diagnosis of pelvic organ disease was significantly greater than that of AP $(\mathrm{P}=.003)$. APOM also showed greater specificity in terms of excluding pelvic organ disease than did AP ( $\mathrm{P}=.003)$. Overall, $37.2 \%$ of patients with muscle guarding had repeated APOM or APOMs that eliminated the tenderness beside the diseased organ area (Ou MC decrescendo phenomenon) and made the diseased organ area more distinct to identify,

Conclusions: APOM is more sensitive and specific for the diagnosis of pelvic organ disease than AP. It indicates that the Ou MC area defined by APOM is closely related to the function of pelvic organ. Ou MC area involves less abdominal area outside the pelvic area than hypogastric area does (AJEM), which may account for the high sensitivity and specificity of APOM in the diagnosis of pelvic organ disease. Ou MC area is a superficial abdominal area that warrants further study.

PP-52

\section{Mutual position of the distal fibular physis and the tibiotalar joint space - Radiological typology and clinical significance}

Nanka O* ${ }^{*}$ Havranek $\mathrm{P}^{* *}$, Pesl T**

Institute of Anatomy, 1st Faculty of Medicine, Charles University, Prague, Czech Republic*; Department of Paediatric and Trauma Surgery, 3rd Faculty of Medicine, Charles University, Thomayer Teaching Hospital Prague, Czech Republic**

Aim: The mutual position of the distal fibular physis compared to the tibiotalar joint space in the immature skeleton was investigated in X-ray studies. The clinical relevance of the recorded mutual position was evaluated for pediatric skeletal traumatology.

Materials and Methods: 140 radiographs of immature ankle joints without skeletal injury were reviewed and the mutual position of the distal fibular physis and tibiotalar joint space was tested. We then reviewed a cohort of 30 children with skeletal injuries of both the distal tibial epiphysis and the distal fibula. The type of distal fibular injury was evaluated according to the mutual position of the distal fibular physis and the tibiotalar joint space.

Results: We found that in about one-half of cases the distal fibular physis is located distally to the plane of the tibiotalar joint, which has not been considered in the literature. Thus, we defined three radiological types of immature ankle joint according to the vertical position of the distal fibular physis in relation to the tibiotalar joint space: type 1 - distal fibular 
physis is above the joint space; type 2 - distal fibular physis is on the same level as the joint space; type 3 - distal fibular physis is below the joint space. In the second cohort, we found that type 2 predisposes to physeal fibular injury and type 3 predisposes to metaphyseal fibular injury. All data obtained were statistically evaluated.

Conclusions: There are three radiological types of immature ankle joint. Type 1 is only an evolutionary type without clinical significance, type 2 predisposes to physeal and type 3 to metaphyseal fibular injury in combination with distal tibial physeal injury.

\section{PP-53}

\section{Topography of the phrenic nerve in videothoracoscopic} interventions

Bogojavlenskaya T* ${ }^{*}$ Nikolaev A*, Kuzmichev $V^{* *}$, Dydykin $\mathrm{S}^{*}$, Laptina $\mathrm{V}^{*}$

First Moscow State Medical University I.M.Sechenov, Moscow, Russia*; Moscow Regional Research Clinical Institute M.F.Vladimirsky, Moscow, Russia**

The purpose of our research was the study of features of topography of the phrenic nerve in videothoracoscopic operations. Materials and research method. Research was conducted on 20 unstable corpses of adult people using the standard videothoracoscopic equipments. Internal reference point for identification of $\mathrm{n}$. phrenicus dexter in the upper mediastinum is the triangle between a. subclavia, a. et v. thoracicae internae. In 17 cases $(85 \%)$ n. phrenicus dexter crossed a. subclavia medially to the place where a. thoracica interna arised from it, in 3 cases it occurred laterally to that place. Because of the location of the n. phrenicus dexter on the surface of large vessels the surgical nerve intervention will be most safe in the area between the root of the lung and a pericardium or in the site where nerve runs to the diaphragm. Reference points for the n. phrenicus sinister in the upper mediastinum are a. subclavia sinistra and a. carotica communis sinistra. The nerve lies anteriorly and parallel to these vessels. In our study the nerve was following the bisector of the angle between a. subclavia and a. thoracica interna. The n. phrenicus sinister lies on the lateral wall of the v. brachiocephalica, then runs downwards parallel and anteriorly to the aa. subclavia et carotica. Therefore for the safe nerve intervention we recommend the area before the nerve runs to the $\mathrm{v}$. brachiocephalica and the second area where the nerve runs from the root of the lung to the pericardium Results: As a result of our research, we consider the thoracoscopic access from axillary area as the most adequate for detection of the $n$. phrenicus on all its extent, to level of a dome of the diaphragm. This access minimally damages structures of a thorax and has the best cosmetic properties.
PP-54

\section{Endoscope assisted supracerebellar transtentorial approach to the temporomedial structure: an anatomic study}

Boyaci $\mathrm{S}^{*}$, Seker A**, Atik F**, Ceylan $\mathrm{D}^{* *}$, Cosar $\mathrm{M}^{* *}$, Bayri $\mathrm{Y}^{* *}$, Aksoy $\mathrm{K}^{*}$, Kilic T*

University of Acibadem, Department of Neurosurgery, istanbul, Turkey*; Marmara University Institute of Neurological Sciences, Istanbul, Turkey**

Objectives: Approaching to posterior temporomedial region pathologies such as tumors, vascular lesions and epilepsy surgery is challenging and many approaches to this region such as occipital interhemispheric, subtemporal, or temporal transventricular approaches are favored. Morbidity associated with this approaches are mainly visual field deficits and aphasia. Our aim is to study the endoscopic and microscopic anatomy of the supracerebellar transtentorial (EASCTT) transparahippocampal approach to temporomedial structures and to avoid the morbidities of approaches to this region.

Materials and Methods: Five formalin fixed cadaveric head were used in this study. The vessels were injected with colored silicon. The specimens were stepwise dissected under surgical microscope and endoscope, each step was documented. The EASCTT approach to the temporomedial structure was performed.

Results: The EASCTT approach facilitates simple and minimally invasive access to temporomedial structures without requiring retraction of the temporal or occipital lobe. The craniotomy should be placed paramedian and transverse sinus should be retracted superiorly. At all specimen's both sides, at least one bridging vein from cerebellum to the tentorial sinuses were detected. All of the specimens both sides had temporal sinuses (6 of them were large, 4 of them were small size) were detected. After dividing the tentorium parahippocampal gyrus were reached. Dissection at the level of P2-P3 junction lead to temporal horn of the lateral ventricule. The mean distance from midpoint of the transverse sinus to the P2-P3 junction is $69.4 \mathrm{~mm}$

Conclusion: The EASCTT transparahippocampal approach provides wide access to the parahippocampal gyrus, hippocampus, amygdala, and uncus allowing the surgeon to remove the structures under direct visualization throughout the operation in the cadaveric studies. It provides ease of access to the P2-P3 junction of the PCA.

\section{PP-55}

\section{Anatomical variations of the carotid bifurcation}

Sañudo JR, Navas I, Herencias A, Perez A, Cobiella R, Maranillo E, Rodriguez-Niedenführ M, Pascual-Font A, Vazquez T

Department of Human Anatomy and Embryology I-Faculty of Medicine-Complutense University of Madrid, Spain

Introduction: Although all surgical procedures involving the carotid arteries require meticulous dissection in an anatomically complex area, few reports on the anatomy of the common 
carotid's bifurcation have been published. Furthermore, knowledge of the anatomical variants of the carotid bifurcation is essential for accurate interpretation during radiological procedures The aim of this study is to obtain morphological parameters that improve the identification of the arteries during clinical procedures in the neck. For this purpose, the carotid bifurcation (cb) will be located with respect to the hyoid bone as well as measurements of the diameters of the common carotid artery (cca), internal carotid artery (ica) and external carotid artery (eca) will be taken.

Material and Methods: A total sample of 206 heminecks from human embalmed cadavers were dissected. Diameters of the three arteries and the distance from $\mathrm{cb}$ to the hyoid bone were measured. Statistical comparisons were made using the chisquare test $(\mathrm{p}<0.05)$ and Pearson's correlation test.

Results: The cca's mean external diameter was $0.97 \mathrm{~cm}$, being statistically significant larger in male than in females $(p=.001)$; the ica's mean external diameter was $0.74 \mathrm{~cm}$, and the eca's was $0.49 \mathrm{~cm}$. There was no correlation between cca, ica and eca's external diameters. The carotid bifurcation was located in most cases (44\%) caudally to the hyoid bone, in $35 \%$ at the same level and in $20 \%$ cranially. The bifurcation was located more cranially on the right side $(\mathrm{p}=.046)$.

Conclusion: An accurate knowledge of the morphological details of the carotid arteries could improve surgical and radiological procedures.

\section{PP-56}

Improvement of audiovisual resources to optimize the lab practices of anatomy and surgery at the degree in podiatry

José Iglesias Sánchez M, Martinez A, Martinez R, Pérez-Pico AM, Hidalgo S, Carlos Cuevas J, Escamilla E, Ledesma C, Palomo P, Mayordomo R

University of Extremadura, Meruda, Spain

This communication shows the results of the development of a teaching innovation project, funded by the Vicerreptorado of Quality and Continuing Education, at University of Extremadura (UEX) during curse 2009-2010. The main objective was to obtain educational audiovisual materials in the areas of anatomy and clinical surgery to improve teaching in these subjects, because of the difficult access for students to the operating room. We organized the installation of a video system at the operating room located in the College of Podiatric Clinic of UEX, and a big screen associated display at the contiguous room were a larger number of students could view it at the time of implementation. Also, using the same video system we did videos of anatomy that were subsequently applied to practical classes. Once installed, the system has been used in practical classes in anatomy and in a course of surgery, with a remarkable success because students can see the activity at the time of, without contributing to increased the stress on the patient.
PP-57

\section{The developmental changes of calbindin expressing} neurons in pedunculopontine nucleus

\section{$\underline{\text { Sarioz O, }}$ Sehirli US}

Department of Anatomy, Marmara University School of Medicine, Istanbul, Turkey

The pedunculopontine nucleus $(\mathrm{PPN})$ is located in mesopontine tegmentum. Having connections with the spinal cord, brain stem, thalamus and basal ganglia, the PPN has a role in sleep and wakefulness, arousal and locomotion. It becomes an alternative site of deep brain stimulation in relieving symptoms of Parkinson's disease. The PPN contains morphologically, neurochemically and electrophysiologically diverse neurons including cholinergic, GABA, glutamate and dopamine containing cells. There are also various markers like substance $\mathrm{P}$, calmodulin, calbindin, calretinin,etc. expressed by neurons in PPN. Recent studies showed that non-cholinergic neurons comprise more than $80 \%$ of all PPN neurons in the rat, and include populations of GABAergic and glutamatergic neurons, both of which account for twice the number of cholinergic neurons. The developmental neuronal changes in PPN have not been well known. This study aims to show the developmental changes of one of the calcium binding protein, calbindin in PPN. In this preliminary study, 7, 14 and 21 days of age rats were used. Choline acetyltransferase and calbindin immunohistochemical stainings were applied to the $40 \mu \mathrm{m}$ frozen brain sections. The differences in number, size, location and the types of calbindin expressing neurons in PPN were examined. We did not observe any double stained neurons. Therefore, all the neurons examined were noncholinergic ones. The calbindin positive cells were located mostly in the rostral part of the PPN in the 7 and 14 day rats whereas a diffuse distribution of the cells was observed in the 21 day rat. The mean number of neurons was $30 \pm 2$ in 7 day rat, $12 \pm 2$ in 14 day rat, and $214 \pm 8$ in 21 day rat. The diameters of the neurons were $12.62 \pm 1.5 \mu \mathrm{m}$ in 7 day rat, $12.8 \pm 2.7 \mu \mathrm{m}$ in 14 day, $11.35 \pm 3.6 \mu \mathrm{m}$ in 21 day rat. It was observed that there is a major increase of the number of the cells in between 14 and 21 days.

\section{PP-58}

Unbiased stereological study of regular aerobic exercise training effect of hypertensive rats' kidney

\section{Hamidian G}

Department of Histology, Faculty of Veterinary Medicine, University of Tabriz, Iran

Numerous hypertension treatment trials have been reported during the past decade but there is a clear need for improved management of hypertension via therapeutic lifestyle modification and exercise training. In this study, 36 matured male Wistar rats were randomly allocated to 4 groups (1. control, 2. exercise, 
3. hypertensive and 4. hypertensive + exercise groups). Hypertension was induced by L-NAME. Animals were training for eight weeks. All groups were anesthetized and their left kidney was removed and fixed in formalin buffered $10 \%$. The samples were made on by routine paraffin embedding and stained by H\&E, Mallory's trichrome and PAS. Then Stereological methods were used to obtain quantitative information about estimation of glomerulus and urinary space volume, and diameter and epithelium thickness of urinary tubes by physical dissector/fractionator technique. Finally, data analysis was performed by one way ANOVA and Tukey post hoc. The stereological results showed that mean renal corpuscle and urinary space volume increased significantly and also glomerulus volume and epithelium thickness of urinary tube decreased significantly in group 3 in comparison with other groups $(\mathrm{P}<0.05)$ but there is no significant different among group 1,2 and $4(\mathrm{P}<0.05)$. No significant different were observed in diameter of proximal and distal tubules and collecting ducts of all groups. Histopathological results indicated that the percentage of sclerosed glomeruli, morphological damage and destruction of urinary tubules epithelium decreased in group 4 in comparison with group 3. Finally, it can be concluded that regular aerobic exercise can partially protect renal structure in hypertensive patients.

\section{PP-59}

\section{Immune system as the main conductor of morphogenesis}

\author{
Voloshyn M, Grygorieva O \\ Zaporozhye State Medical University, Zaporozhye, Ukraine
}

The integrity of organism is ensured by nervous, endocrine and immune systems. In the phylogenesis immune system appears at the specific stage of the development of multicellular organisms, while in the ontogenesis at the stage of the formation of trunk in embryo. Initially, obtained results served as the basis of the formulation of hypothesis about the morphogenetic role of lymphocytes, and then the concepts: "Immune system is the factor of morphogenesis". A quantity of lymphocytes and antigen presenting cells in the tissue is strictly determined. Lymphocytes control the number of population of cells in the organ. For each organ there are specific cellular constants of the relationships: lymphocytes/antigen presenting cells; lymphocyte/epithelial cells; lymphocyte/fibroblasts; the lymphocyte/ parenchyma cells. Lymphocytes influence on the speed of the proliferation of the cells of parenchyma, epithelium and contents of extracellular matrix. Lymphocytes stimulate apoptosis of cells with the changed antigenic determinants, that appear in the process of their aging or forcing on them any factors, without the appearance of signs of inflammation. An increase of the content of immunological immature lymphocytes in the fetal organs, is the basic reason for deviation at the rates, within the periods of the completion of molding of the morphofunctional units of the organs of newborns, i.e., changes in organogenesis appear. An increasing number of the immunological immature lymphocytes in the internal organs and the skin of the adult organisms on the background of chronic stress is the basis for the development of pathology. The restoration of the normal relationships between the lymphocytes and other cells of organs depends on the duration of the life cycle of immunological immature lymphocytes and cells with the changed phenotype. The creation of the immune passport of tissues and organs is the promising direction of morphology.

\section{PP-60}

\section{Preparation of applied atlas of computed tomography images of the Iranian Jabeer abdominal region}

\author{
Zamani $M^{*}$, Sajjadian $M^{* *}$, Sohrabzade B*, Nazem MN*
}

Faculty of Veterinarian Medicine, Tehran University, Tehran, Iran*; Faculty of Veterinarian Medicine, Kerman University, Kerman, Iran**

C.T.scan is a technique which can be used as a safe method for taking information of different body regions, specially in animals with the danger of extinction. Nowadays C.T.Scaning is being used extensively as a paracilinical technique in diagnosis and determination of the best surgical approach due to its ability in image analysis and high tissue resolution. In order to reach the best interpretation of the lesions, basic information about normal images of that regions is needed. In this regard we arranged a study prepared an applied atlas of normal C.T images of the abdominal region of Iranian Jabeer, which is in danger of extinction. Three mature male Jabeer with average age of 3 years old were used in this study. The animal after deep sedation were restricted in customized restraining frames and were transferred to the C.T. hall image were then taken from the abdominal region perpendicular to the vertebral axis in $3 \mathrm{~mm}$ intervals. In order to diagnosis of different organs mean number of window level and width were used in taken images. Tomograms were studied with refer to the anatomical texts and compare with dissected goat to determine the relevant structure. Finally the position of each structure was represented in a table according to the body of each abdominal vertebra which could be seen in each image. The results of this study can be used by radiologists and surgeons as a reference in the interpretation of Jabeer abdominal disorders and identification of the best surgical approach respectively.

\section{PP-61}

\section{Bilateral coronary fistulas between coronary arteries is quite rare}

Ciftci $\mathrm{H}^{*}$, Kavak $\mathrm{V}^{* *}$, Efem MS ${ }^{* * *}$, Taçoy $\mathrm{G}^{* * * *}$

Veni Vidi Hospital Department of Cardiology* Department of Anatomy, Dicle University Medical School Diyarbakir, Turkey**; Department of Cardiology, Tekirdag State Hospital Tekirdag, Turkey***; Department of Cardiology, Gazi University Medical School, Ankara, Turkey**** 
Coronary artery fistulas which often draining to venous systme are rare congenital heart disease. Bilateral coronary fistulas between coronary arteries are quite rare. 60-year-old male patient referred because of atypical chest pain for 3 years to our center for coronary angiographic evaluation. His treadmill exercise ECG demonstrated nonspecific ST-T wave alteration in inferior leads. The patient had only a history of smoking as cardiovascular risk factors. On physical examination, there was no pathologic finding. There was no evidence of ischemia on his rest electrocardiogram. Transthoracic echocardiography showed normal heart valves and normal left ventricular systolic and diastolic function. There was no additional pathological findings. The patient's coronary angiogram in right anterior oblique (RAO) view demonstrated ectasia of the left main coronary artery and left anterior descending (LAD) artery and increased tortuosity in the body section of LAD artery. Lateral image showed that after septal branches LAD artery was communicated to right ventricle. Right anterior oblique view demonstrated coronary fistula which had a origin from conus branch of right coronary artery (RCA). With left ventriculography, and aortography, additional congenital anomalies were excluded. For further evaluation of giant coronary artery fistulas which may cause ischemia, Thorax CT and myocardial perfusion scintigraphy were planned and the patient was informed by the need to close fistulas as percutaneous coronary intervention. The patient denied further evaluation and treatment and was discharged with beta-blocker and aspirin therapy. Coronary artery fistulas are uncommon and have an incidence of 0.2 to $0.4 \%$ of all congenital heart diseases. As in our case, the ratio of bilateral fistula is extremely rare $(0.002-0.0013 \%$ incidence in coronary angiographic evaluation). Patients with coronary artery fistulas are often asymptomatic, although they may cause chest pain, dyspnea, and heart failure. In such cases, when the fistula generates ischemia, hemodynamic evaluation and surgical or percutaneous closure of coronary fistulas is required. Coronary artery fistulas in our patient were found arising from bilateral coronary arteries. Although further examination was scheduled, the patient does not accept the actions of this situation to be clarified. Due to important results of coronary fistulas, ischemia, hemodynamic overload should be evaluated carefully.

\section{PP-62}

\section{Topographic and anatomic features of the content of intervertebral channels in the lumbosacral spine region depending on constitution of the person}

Nikolaev A, Telpuhov V, Dydykin S, Nelipa M

First Moscow State Medical University I. M.Sechenov, Moscow, Russia

To prove the participation of soft tissues of intervertebral channels in the lumbosacral region of the spine in development of an ischemia of the inferior part of the spinal cord in anatomic study of the content of the channels in different constitutional types. We studied content of the 620 lateral intervertebral channels in the lumbar spine region. The material was distributed into groups according to the constitution type. In samples from people with dolichomorphic type of constitution sizes of the channel are the smallest (mainly the width of the foramina), and in samples from people with brachymorphic type of constitution these sizes were the greatest. In $54.84 \%$ there were found soft tissue elements in the lateral intervertebral channels in the lumbosacral region - intraforaminal ligaments. $50 \%$ of samples were taken from people with mesomorphic constitutional type, 26.47 $\%$ and $23.53 \%$ from brachymorphic and dolichomorphic types respectively. Histologically these elements had a ligament-like structure. The ligament apparatus reduces from 44.97 to 79.84 $\%$ free space around the vessels running through the intervertebral channel what can result in compression of the intervertebral channels content both after trauma, and in degenerative and dystrophic diseases of the spine. During a compression, arteries and the veins lying on various surfaces of spinal nerves are damaged first, which may result in ischemic lesions of the spinal cord. The worst conditions for the intervertebral channels content were seen in people with dolichomorphic type of constitution who had plural intraforaminal ligaments. Basing on the results of this research, such combination can be found in $25.5 \%$ of people with dolichomorphic type of constitution.

\section{PP-63}

\section{A rare case of an anomalous origin of left main coronary artery from right aortic sinus}

\section{Cilingiroglu $\mathrm{S}^{*}$, Ozbakir $\mathrm{C}^{* *}$, Bozkurt $\mathrm{MC}^{*}$}

Department of Anatomy, Kirikkale University, School of Medicine, Kirikkale, Turkey*; Akay Hospital, Department of Cardiology, Ankara, Turkey**

Objective: Congenital coronary artery anomalies are seen in $0.2-1.2 \%$ of the population. Left coronary artery anomaly originating from right aortic sinus, a rare congenital coronary artery anomaly, is asymptomatic. Detecting the presence of such origin anomaly is crucial as it causes myocardial ischemia without any symptoms or sudden death in many patients, particularly young people. Here is presented a case in which a left coronary artery originating from right aortic sinus, a rare anomaly, was detected as a result of coronary angiographic examination.

Material and Method: The left coronary artery exit anomaly detected in our research was assessed according to the classification of coronary artery anomalies developed by Dr. Angelini. Coronary angiography of the 58-year-old male patient was performed by Judkins method, and the views obtained were analyzed using a digital cardiac imaging system.

Results: On the coronary angiography of the case, on whose myocardial perfusion scintigraphy ischemia was determined, it was detected that left coronary artery originated from right aortic sinus.

Conclusion: Detecting coronary artery anomalies in such quite rare cases with left coronary artery anomaly originating 
from right aortic sinus plays a crucial role in the success of interventional procedures.

\section{PP-64}

\section{Association of an anomalous origin of left main coronary artery from right aortic sinus and multiple coronary atherosclerosis: a case report}

Cilingiroglu $\mathrm{S}^{*}$, Ozbakir $\mathrm{C}^{* *}$, Kirici $\mathrm{Y}^{* * *}$

Department of Anatomy, Kirikkale University, School of Medicine, Kirikkale, Turkey*; Department of Cardiology, Akay Hospital, Ankara, Turkey*; Department of Anatomy, Gulhane Military Medical Academy, Ankara, Turkey***

Objective: Congenital coronary artery anomalies are seen in $0.6-1 \%$ of all angiographies and $0.3 \%$ of autopsies. Recognition of coronary artery anomalies has a great importance in coronary angioplasty and cardiac surgery. Here, a case is presented, in which left coronary artery anomaly originating from right aortic sinus, a rare coronary artery origin anomaly and therosclerotic lesions in coronary arteries are seen together.

Material and Method: The coronary artery anomaly detected in this case was assessed according to the classification suggested by Dr. Angelini and used by Congenital Cardiac Surgery Committee. Coronary angiography of a 58-year-old female patient was performed with Judkins method, and the images were analyzed in various projections.

Results: As a result of coronary angiographic examination, it was found that the left coronary artery originated from the right aortic sinus. Forty percent stenosis was detected in the left coronary artery, $70 \%$ in the left anterior descending coronary artery, $80 \%$ in the circumflex artery, and $90 \%$ in the right coronary artery due to atherosclerotic lesions.

Conclusion: In such rare cases with co-existing left coronary artery originating from right aortic sinus and multiple coronary atherosclerosis, identification of left coronary artery origin anomaly, that is different from anatomical structure of coronary arteries, as a result of examinations performed by coronary angiography and other imaging methods, helps to prevent complications which could rise during percutaneous and surgical interventions and plan the treatment.

\section{PP-65}

A case report in which myocardial bridge and coronary artery anomaly are seen together

Cilingiroglu $\mathrm{S}^{*}$, Ozbakir $\mathrm{C}^{* *}$, Dagli $\mathrm{Z}^{* * *}$

Department of Anatomy, Kirikkale University, School of Medicine, Kirikkale, Turkey*; Department of Cardiology, Akay Hospital, Ankara, Turkey**; Department of Family Medicine, Ankara University, School of Medicine, Ankara, Turkey***
Objective: Myocardial bridges are detected in $0.5-16 \%$ of coronary angiographies performed in general population. A myocardial bridge is a congenital anomaly, where one segment of the superficial epicardial coronary artery is located in the myocardium. Myocardial bridge, which could cause myocardial ischemia, myocardial infarction, exercise-related tachycardia, and sudden death, is asymptomatic. Here is presented a case in which myocardial bridge and anomalous circumflex artery origin, one of the coronary artery origin anomalies, were seen together.

Material and Method: The coronary artery anomaly detected in the present case was assessed according to the classification reported by Dr. Angelini and used by Congenital Cardiac Surgery Committee. Coronary angiography of the 42 -year-old patient was performed with Judkins method, and various cardiac images were examined.

Results: Coronary angiographic assessment showed an isolated myocardial bridge causing $50 \%$ stenosis in the trunk of left descending coronary artery, which had no atherosclerotic lesion. Also, no atherosclerotic lesions were found in other coronary arteries of the case that had an anomalous circumflex artery originating from right coronary artery.

Conclusion: Myocardial bridge, which is also defined as intramural coronary artery or mural coronary artery anatomically, leads to hemodynamic disturbance in the coronary artery through systolic compression. Although rarely seen by cardiologists and cardiac surgeons, it is evident that knowing myocardial bridge is beneficial for successful and appropriate administration of diagnostic and therapeutic interventions.

\section{PP-66}

\section{The iliolumbar artery-anatomic considerations and details on the common iliac artery trifurcation}

Motoc $\mathrm{A}^{*}$, Rusu $\mathrm{MC}^{* *}$, Cergan $\mathrm{R}^{* * *}$, Dermengiu $\mathrm{D}^{* * * *}$, Curca GC ${ }^{* * *}$, Folescu R*, Jianu AM*

Department of Anatomy and Embryology, "Victor Babes" University of Medicine and Pharmacy, Timisoara, Romania* Discipline of Anatomy and Embryology, Faculty of Dental Medicine, "Carol Davila" University of Medicine and Pharmacy, Bucharest, Romania**; Discipline of Anatomy, Faculty of Medicine, "Carol Davila" University of Medicine and Pharmacy, Bucharest, Romania*** National Institute of Legal Medicine "Mina Minovici", Bucharest, Romania****

The iliolumbar artery (ILA) of Haller is the largest nutrient pedicle of the ilium and its detailed knowledge is important for various surgical procedures that approach the lumbosacral junction, the L4/L5 disk space, the sacroiliac joint, the iliac and psoas muscles, or the lumbar spine. Also the ILA is relevant for various techniques of embolization. We aimed to evaluate the anatomic and topographic features of the ILA, by dissection on 30 human adult pelvic halves and on 50 angiograms. ILA was a 
constant presence and it emerged at Level A (from the common iliac artery (CIA), 8.75\%), Level B (from the CIA bifurcation, $2.5 \%$ ), Level $\mathrm{C}$ (from the internal iliac artery (IIA), $52.5 \%$ ), Level D (from the IIA bifurcation, 3.75\%), and Level $\mathrm{E}$ (from the posterior trunk of the IIA, 32.5\%). Level B of origin of the ILA corresponds to a trifurcated CIA (morphology previously unreported), while Level D corresponds to a trifurcated IIA. A higher origin of the ILA corresponds to a more transversal course of it. A descending lumbar branch that leaves the iliac arterial system independently to enter the psoas major muscle, as seen in $48 \%$ of cases, may be misdiagnosed as ILA. Surgical interventions in the lumbar, sacral, and pelvic regions must take into account the variable origins of the ILA from the iliac system that can modify the expected topographical relations and may lead to undesired hemorrhagic accidents.

\section{PP-67}

\section{Somatochart, cardiovacular risk factors, quality of life and physical activity}

Sánchez Ferrer $M^{* *}$, Sánchez Ferrer $M L^{* *} 2$, Sánchez Del Campo $\mathrm{F}^{* *}$, Lara $\mathrm{MH}^{*}$, Garcia $\mathrm{VA}^{* *}$, Hernando Sáez J**, Terol Calpena $\mathrm{F}^{* *}$

Anatomy and Histology Department, Alicante University, Alicante, Spain*; Miguel Hernández University, Alicante, Spain**

Introduction: Our aim is to find out if there is a relation between Human Anatomy and cardiovascular risk factors, quality of life and physical activity. We have made an anthropometric study using the Heath-Carter method. After that we have built somatocharts to view the results.

Material and Method: We have studied 30 to 65 -year-old people, men and women, registered in a Health Centre. We arranged an appointment with each patient. Then, we did the patient's clinical history and took basic measurements following the ISAK's (International Society for Advancements in Kinanthropometry) protocol, restricted profile. Weight, height, girths (waist, hip, relaxed and tensed arm, calf and antero-posterior chest depth), breadths (humerus, femur, distal styloids) and skinfolds (biceps, triceps, subscapular, supraspinale, iliac crest, abdominal, front thight and medial calf). Furthermore, we made a double blood pressure measurement and each patient filled out two surveys about quality of life (Euroqol-5D) and physical activity (LTPA, Minnesota Time Physical Questionnaire - abbreviated version). Results and conclusions: In general, we observed that the higher the value for cardiovascular risk factors, the higher movement in the somatochart to the up-left corner (less $\mathrm{X}$ and higher $\mathrm{Y}$ ). This happened for high levels of blood pressure, dislipemia, diabetes mellitus, alcoholic habit, SCORE scale and REGICOR scale (scales to measure cardiovascular risk). Patients with poor life quality and less physical activity are also reflected in this area.

Conclusion: Healthy patients are in the central area of the somatochart. Therefore, we must change our population's diet and hygienic habits in order to modify their anthropometry and get them in the Realeaux triangle.

\section{PP-68}

\section{The morphometry and variations of confluence of sinuses and related structures: anatomic study}

Cosar $\mathrm{M}^{*}$, Seker $\mathrm{A}^{* *}$, Ceylan $\mathrm{D}^{* * *}$, Tatarli $\mathrm{N}^{* * * *}$, Sahin $\mathrm{F}^{* * * * *}$, Tokmak $\mathrm{M}^{*}$, Songur $\mathrm{A}^{* * * * * *}$, Kilic $\mathrm{T}^{* *}$, Ozen $\mathrm{OA}^{* * * * * * *}$

Department of Neurosurgery, Canakkale 18 March University, Faculty of Medicine, Turkey*; Department of Neurosurgery Marmara University, Faculty of Medicine, Turkey**; Department of Neurosurgery Sakarya University, Faculty of Medicine Turkey***, Department of Neurosurgery, SB Dr Lütfü Kırdar Kartal Education Hospital, Turkey****, Ab Adli Tip Kurumu Baskanligi, Morg ihtisas Dairesi, Turkey*****, Department of Neurosurgery, Medipol University, Faculty of Medicine, Turkey $* * * * *$, Department of Anatomy, Afyon Kocatepe University, Faculty of Medicine, Turkey*******, Department of Anatomy, Namik Kemal University, Faculty of Medicine, Turkey $* * * * * * * *$

The anatomic studies of the dural sinuses are very important dealing pathologies around confluence of sinuses and the variations of this region are rarely studied in the literature. In this study, we aimed to investigate the morphometric and morphological structures of the confluence of sinuses and related structures with silicon injection technique.

Material and Methods: We studied 30 cadaver in this study. Twelve of them were fixed with formaldehyde that were use for documentation and measurements and eighteen of them were fresh cadavers that were used for measurements. Formalin fixed cadavers were washed with tap water and injected with colored silicone via vena jugularis interna (VJI), internal carotid artery (ICA) and vertebral artery (VA's). The confluens of sinuses (CS) and related structures were dissected under surgical microscope, the normal anatomy were investigated and variations were documented. Diameter of the sinus sagittalis superior (SSS), CS, occipital sinuses (OS), sinus rectus (SR), bilateral transvers sinuses (TS) and the angle between SSS-SR were measured.

Results: In this study, the mean diameters were SSS: $11.7 \mathrm{~mm}$, CS:22.3 mm, OS: $5.25 \mathrm{~mm}$, SR: $7.5 \mathrm{~mm}$, TS: (right: 9.7 and left: $9.1 \mathrm{~mm}$ ) and angle between SR-SSS was 58 degree. There were no difference for the bilateral venous structures which drain to SSS and TS. There were some variations that draine venous blood from SSS to TS or some cortical veins that directly drain to CS. The right TS were located superior in 7 cases compared with the left TS and this process related with the type of CS. A local vertically located septum in the SSS was detected in 2 cases. $40 \%$ of cases (12/30) SSS drain to right transverse sinus and this 
is because of a septum (10/30) that is located in the CS directing the blood that comes from SSS to right TS. Additionally, we encountered OS in the $80 \%$ of the cases.

Conclusion: We have reported the angle between the SSS-SR and effect of CS septum on the CS types and TS dominance. We also reported the superior location of TS and the effect of CS septum on it.

\section{PP-69}

\section{D anatomical study of the nose, paranasal sinuses and skull base}

Monjas-Cánovas I*, Abarca-Olivas J**, Sanchez-Del Campo F***, Gras-Albert J*, Lloret García J**, Moreno-López**, Sánchez Ferrer $M^{\star * *}$, Sánchez Ferrer $M L^{* * *}$

Department of Otolaryngology, Hospital General Alicante, Spain*; Department of Neurosurgery, Hospital General Alicante, Spain**; Histology and Anatomy Department,Miguel Hernández University Alicante, Spain $* \star *$

Introduction: Training in dissection of the paranasal sinuses and the skull base is essential for anatomical understanding and correct surgical techniques. The $3 \mathrm{D}$ visualization of the endoscopic skull base anatomy increases the spatial orientation and allows the depth perception.

Methods: We performed endoscopic dissection in cadaveric specimens fixed by the Thiel technique and prepared with intravascular injection of colored material. Endonasal approaches to the midline cranial base were performed with 2D endoscopes. Then we applied the 3D technique to illustrate the 3D pictures.

Results: The most important anatomical structures and landmarks are illustrated in three dimensional images.

Conclusion:The skull base consists of complex three-dimensional (3D) bony and neurovascular structures. Experience with cadaver dissection is essential to understand the complicated anatomy and develop surgical skills. 3D anatomy represents a novel and detailed way of understanding the skull base anatomy.

\section{PP-70}

\section{Agenesia of the pericardium: A case report}

Piera $V^{*}$, Barberia $E^{*}$, Barberia $E^{* *}$, Villa $D^{*}$, Sempere $T^{*}$, Torrente $M^{*}$, Jove $M^{*}$, Cobos $\mathrm{P}^{*}$

Human Anatomy and Embriology Unit Medicine and Health Sciences Faculty University Rovira Virgili, Tarragona, Spain*; Institute of Legal Medicine of Catalonia, Spain**

Introduction: Congenital absence of the pericardium is a rare condition. The incidence of pericardial agenesia (PA) is estimated to be between $0.01 \%$ and $0.02 \%$ in autopsy series and
$0.044 \%$ in surgical series. Contemporary imaging techniques have revealed an increase in incidental pericardial defects. This suggests that most cases follow an asymptomatic course. The aim of this study is to present a case of PA that was incidentally found during a forensic autopsy.

Case Description: A 53-year-old male with medical history of hypertension and polytraumatism (thoracic, abdominal and pelvic) 10 months before, had an accidental trauma at work. The trauma affected the left inferior extremity with open dislocation of the knee, complete tear of the femoral artery and haemorrhagic shock with subsequent death. The autopsy found a laterally displaced heart apex and a tongue shaped pericardium which covered partially the anterior side of heart and its base. No other congenital abnormalities were found; only two lipomatous-like tumours were discovered limited to the epicardium in the diaphragmatic side of the heart.

Discussion: The suspected etiology of pericardial defect is the premature atrophy of the left common cardinal vein that leads to a reduced blood supply to the left pleuropericardial membrane resulting in pericardial agenesia. As in the case described, congenital pericardial defects are non-symptomatic in $70 \%$ of cases, are predominantly found in males (4:1) and are discovered incidentally (by autopsy, thoracotomy or routine pre-surgical radiological studies). In our case, no other congenital abnormalities were found; however, associations with other malformations have been described in $30 \%$ of cases in the literature, these malformations being pentalogy of Cantrell, atrial septal defect, bicuspid aortic valve, bronchogenic cysts and pulmonary sequestration.

\section{PP-71}

\section{Guyon's canal and course of the ulnar nerve at the wrist a foetal study}

Kirici Y, Yildiz S, Kocabiyik N

Department of Anatomy, Faculty of Medicine, Gulhane Military Medical Academy, Ankara, Turkey

The ulnar nerve is often compressed in cubital tunnel and Guyon's canal.The ulnar nerve can compress elsewhere at the wrist. To put this situation, the ulnar nerve was investigated of the course at the wrist. The twenty-four fetuses were selected for this study and were dissected under a stereomicroscope. Firstly, in hypothenar region, the accessory muscles of ulnar nerve compression were examined. Then, the exit level of the ulnar nerve's deep branch researched. The ulnar nerve's compress places were determined followed by ulnar nerve. In additional we reviewed the palmar carpal ligament described the thickened portion of antebrachial fascia on anterior of the wrist. The accessory muscles in hypothenar region were seen three of the fetuses. The ulnar nerve's deep branch occurs between flexor digiti minimi and abductor digiti minimi muscles, and it enters under the opponens digiti minimi muscle. The ulnar nerve's deep branch 
usually leaves after palmar carpal ligament, and it was seen did not enter Guyon's canal. Guyon's canal was seen only a case. The ulnar nerve and the lateral side associated the ulnar artery pass below carpal palmar ligament at the wrist. The ligament forming Guyon's canal was not seen in most fetuses. In most fetuses, Guyon's canal does not exist. We thought that, the ulnar nerve often pass under the palmar carpal ligament. Moreover, the deep branch of ulnar nerve is often divided to distal of the ligament. Therefore, this compression may occur at the level of this ligament was affected ulnar nerve's deep branch.

\section{PP-72}

Impact of cadaver dissection images

Alfonso Arráez-Aybar L, Casado-Morales I, Castaño-Collado G Faculty of Medicine, Complutense University of Madrid, Spain

Introduction: The skills involved in the perception, the use, the comprehension and the regulation of emotions, which depend fundamentally on the knowledge of the emotional processes and on the skills of the processing of the emotional information is defined as Emotional Intelligence (EI). Dissection is a threatening situation which provokes emotional answers in the students of Anatomy. This emotional answers decrease with the exhibition to the situation. Several researches show EI such as an individual relevant variable in the development of strategies of emotional control. The aim of this study is to analyze influence of the EI as modulating variable of the decrease of the emotional reaction.

Material and Methods: There were applied two scales: TMMS-24 was used to measure the dimensions of the emotional intelligence (perception, comprehension and emotional regulation) and the SAM scale to measure the valence and the level of activation of the students before a set of forty images of dissection of human corpse. The scale SAM was applied to the students in two moments: before realizing the first experience of dissection and at the end of the academic semester.

Results: The results show that the emotional intelligence is a psychological dimension that modulates the change in the perception of threat and in the level of activation that anatomy's students prove to human dissection's images.

\section{PP-73 \\ Utility of special projections of magnetic resonance study of the human temporomandibular joint}

Mañas CB, González Sequeros O, Cascales MM, Sarriá Cabrera R, Latorre Reviriego R

Facultad de Medicina and Facultad de Veterinaria, Universidad de Murcia, Spain
Introduction and Purpose: The temporomandibular joint (TMJ) has been studied by different imaging techniques including magnetic resonance imaging (MRI). Our goal is to assess the suitability of the different projections of MRI in the analysis of the lateral pterygoid muscle insertions, one of the anatomical structures involved in temporomandibular disorders.

Tools and Method: Using a 0.5 Tesla system (General and General Electric Healthcare, O'Signa) seven blocks of human TMJ were studied by MRI. We used a standard head coil for sections in the sagittal, coronal and axial planes in relation to the head. Studies were also performed at the oblique-sagittal and coronal- oblique planes, these being, respectively, perpendicular and parallel to the axis of the condyle. The sequence used was: repetition time $420 \mathrm{~ms}$, echo time $17 \mathrm{~ms}$, number of excitations 3-4, thickness sections $3 \mathrm{~mm}$, distance between sections $0.3 \mathrm{~mm}$, field of view $18-20$, matrix $288 \times 192$. Were processed and studied by Kodak DirectView Diagnostic Workstation software. The images obtained were compared with plastinated sections (E12).

Result and Interpretation: We emphasize how in axial images it is possible to observe the layered morphology of the lateral pterygoid muscle and the insertions in the disc and neck. In oblique-coronal images the disc morphology and the uppermost fibers of the lateral pterygoid muscle inserting into the anterior portion of the disc are clearly appreciated. The oblique-sagittal projection provided more information about size, morphology and position of the disc, relation between muscle and disc and stratification of lateral pterygoid muscle insertions. The vascular structures located between the insertions both superior and inferior fascicles are also observed.

\section{PP-74}

\section{Importance of the craniofacial asymmetry in surgery}

Kizilkanat E*, Boyan $\mathrm{N}^{*}$, Ozsahin $\mathrm{E}^{* *}$, Tekdemir I***, Soames $\mathrm{R}^{* * * *}$, Oguz $\mathrm{O}^{*}$

Department of Anatomy, Cukurova University, Faculty of Medicine, Adana, Turkey*; Department of Anatomy, Baskent University, Adana Research and Teaching Hospital Adana, Turkey**; Department of Anatomy, Ankara University, Faculty of Medicine, Ankara, Turkey***; Centre for Anatomy and Human Identification College of Life Sciences University of Dundee Dow Street, Dundee, UK****

Introduction and Purpose: Asymmetry is a common feature in human craniofacial bones being a feature in all individuals. Such right and left side differences in the skull may lead to misleading clinical and radiological evaluation in cases of potential trauma: in addition, a knowledge of asymmetry is important for some surgical procedures and approaches. The purpose of this study was to determine some aspects of craniofacial asymmetry in human skulls. 
Materials and Methods: Measurements, using slide caliper, were taken from the right and left sides of 45 dry Anatolian skulls of unknown age and sex.

Results: The following mean distances and standard deviations, for the right and left sides respectively, were observed between: the infraorbital foramen and the anterior nasal spine (IOFANS) $34.87 \pm 2.65 \mathrm{~mm}, 33.96 \pm 2.59 \mathrm{~mm}$; the greater palatine foramen and the posterior nasal spine (GPF-PNS) 17.72 \pm 1.69 $\mathrm{mm}, 17.37 \pm 1.75 \mathrm{~mm}$; the foramen spinosum and the basion (FS-B) $36.22 \pm 2.80 \mathrm{~mm}, 36.29 \pm 2.76 \mathrm{~mm}$; the foramen spinosum and the zygomatic arch at the zygomatic-temporal suture (FSZTS) $37.60 \pm 3.58 \mathrm{~mm}, 37.88 \pm 3.12 \mathrm{~mm}$. Using these linear measurements an asymmetry index ((right side - left side)/right side)x100 was calculated. The mean (and standard deviation) of the asymmetry indices were: IOF-ANS, 4.69 (3.67); GPF-PNS, 4.51 (4.63); FS-B, 3.25 (3.34); FS-ZTS, 3.71 (3.49).

Conclusion: An awareness of craniofacial asymmetry is important when planning surgical intervention in all patients, in particular in those with recognisable craniofacial asymmetry.

\section{PP-75}

\section{Surgical significance and morphometry of the subtemporal trapezoid}

Boyan $\mathrm{N}^{*}$, Ozsahin $\mathrm{E}^{* *}$, Kizilkanat E* Tekdemir I**, Soames $\mathrm{R}^{* * * *}$, Oguz O*

Department of Anatomy, Cukurova University, Faculty of Medicine, Adana, Turkey*; Department of Anatomy, Baskent University, Adana Research and Teaching Hospital Adana, Turkey**; Department of Anatomy, Ankara University, Faculty of Medicine, Ankara, Turkey*** Centre for Anatomy and Human Identification College of Life Sciences University of Dundee Dow Street, Dundee, UK****

Introduction and Purpose: Important openings in the skull base, such as the foramen ovale, foramen spinosum and jugular foramen, are contained within the confines of the subtemporal trapezoid, which is described by imaginary lines between the articular eminence, medial pterygoid hamulus, occipital condyle, mastoid process and back to the articular eminence. The aim of this study was to determine the distances between the specific bony landmarks within the subtemporal trapezoid.

Materials and Methods: Measurements, using sliding caliper, between pairs of bony landmarks/structures were taken from the right and left sides of 54 dry Anatolian skulls of unknown age and sex.

Results: There was no significant $(\mathrm{P}>0.01)$ difference between right and left sides for any of the measurements taken, consequently data from the two sides was combined. The following mean distances and the associated standard deviations were calculated: articular eminence to the mastoid tip $39.8 \pm 3.59 \mathrm{~mm}$; mastoid tip to the occipital condyle $44.4 \pm 3.26 \mathrm{~mm}$; occipital condyle to the medial pterygoid plate $36.6 \pm 3.72 \mathrm{~mm}$; medial pterygoid plate to the articular eminence $50.7 \pm 4.37 \mathrm{~mm}$; articular eminence to the occipital condyle $55.2 \pm 3.76 \mathrm{~mm}$. The mean distance, and associated standard deviation from the articular eminence to the foramen ovale was $33.2 \pm 3.28 \mathrm{~mm}$; to the foramen spinosum $28.9 \pm 3.07 \mathrm{~mm}$; to the foramen lacerum $44.8 \pm 3.41 \mathrm{~mm}$; and to the jugular foramen $38.6 \pm 3.15 \mathrm{~mm}$. Similarly, from the mastoid tip the mean distance and standard deviation to the foramen ovale was $46.4 \pm 3.31 \mathrm{~mm}$; to the foramen spinosum $39.6 \pm 3.36 \mathrm{~mm}$; to the foramen lacerum $51.2 \pm 3.62 \mathrm{~mm}$; and to the jugular foramen $27.8 \pm 3.15 \mathrm{~mm}$.

Conclusion: These measurements will be useful to neurosurgeons in subtemporal surgical approaches for tumours involving the base of the skull.

\section{PP-76}

\section{Surgical anatomy of the anterior skull base}

Boyan $\mathrm{N}^{*}$, Ozsahin $\mathrm{E}^{* *}$, Kizilkanat E*, Tekdemir I***, Soames $\mathrm{R}^{* * * *}$, Oguz O*

Department of Anatomy, Cukurova University, Faculty of Medicine, Adana, Turkey*; Department of Anatomy, Baskent University, Adana Research and Teaching Hospital Adana, Turkey**; Department of Anatomy, Ankara University, Faculty of Medicine, Ankara, Turkey***; Centre for Anatomy and Human Identification College of Life Sciences University of Dundee Dow Street, Dundee, UK****

Introduction and Purpose: The aim of this study was to define the morphometric relationships between structures surrounding anterior cranial base, such as the cribriform plate and crista galli. An understanding of the anatomy of this region is important for skull base surgery.

Materials and Methods: Measurements, using sliding caliper, between bony landmarks/structures were taken from 46 Anatolian dry skulls of unknown age and sex.

Results: The mean distance, and associated standard deviation, from the nasion to the cribriform plate was $11.0 \pm 1.76 \mathrm{~mm}$ and to the crista galli was $15.3 \pm 2.10 \mathrm{~mm}$. The mean depth of: the anterior cribriform fossa was $7.5 \pm 1.64 \mathrm{~mm}$; the middle cribriform fossa was $5.7 \pm 1.26 \mathrm{~mm}$; and the posterior cribriform fossa was $2.9 \pm 1.04 \mathrm{~mm}$. The mean width and length of the cribriform plate were $9.8 \pm 1.89 \mathrm{~mm}$ and $21.2 \pm 2.25 \mathrm{~mm}$, respectively. The mean height, anteroposterior length and thickness of the crista galli were $13.3 \pm 2.54 \mathrm{~mm}, 12.6 \pm 1.99 \mathrm{~mm}$ and $3.7 \pm 1.42$ $\mathrm{mm}$, respectively; while the mean distance from the tip of the crista galli to the tuberculum sellae was $39.1 \pm 4.21 \mathrm{~mm}$ and to the dorsum sellae was $54.0 \pm 2.83 \mathrm{~mm}$.

Conclusion: Knowledge of the morphometry of the anterior skull base is guide the surgery. 
PP-77

\section{Curriculum of human anatomy in Lithuanian university of health sciences}

\section{Dabuzinskiene A}

Institute of Anatomy, Medical Academy, Lithuanian University of Health Sciences, Lithuania

Introduction and Purpose: The curriculum of human anatomy in faculty of medicine of Lithuanian University of Health Sciences was depended on social requirements in different time periods. There were traditional multiple subject studies system in first three semesters then in 1993 was established single subject- cycle system. Since 2007 problem-based learning is used in faculty of medicine. Given that we decided to make the comparison between curricula and to define their main features.

Methods: We performed analysis of curricula and evaluated efficiency of anatomy studies in problem based learning system. Furthermore we compared results with previous existing cycle system.

Results: In faculty of Medicine human anatomy is carried in undergraduate regular university studies. There are two basic anatomy cycles i.e. 10 weeks in the first year. Final exam consists of theoretical and practical parts. The assessment score consists of in half of cumulative and exam scores. In first year students must complete curriculum of anatomy 160 hrs, lectures: 38 hrs, practical works: $122 \mathrm{hrs}$. In second, third, and fourth years anatomy studies are included in 10 of 12 preclinical problem based modules. Totally there are 131 hrs, 19 hrs lectures, 61 hrs practical works, 13 hrs seminars, and 38 hrs tutorials. In curriculum of fifth year $16 \mathrm{hrs}$ are for clinically applied anatomy: $11 \mathrm{hrs}$ practical works and 5 hrs seminars. In written form of each module's final exam are included 5 questions, commonly one of them is anatomy. In single subject education system there were $280 \mathrm{hrs}$ for anatomy in first three semesters.

Conclusions: Irrespective of changes during decades, importance of anatomy studies is saved in curricula. Moreover the problem based learning enables continuity and vitality of human anatomy studies in faculty of medicine.

\section{PP-78}

An immunohistochemical study of endometrial and endocervical carcinomas using anti-cycind 1 coexpression with anti- ER/PR

Adriana R, Corina Daniela F

Universitatea de Vest Vasile Goldis, Arad, Romania

Purpose: Immunohistochemistry is an important part of the differential diagnosis between endometrial versus endocervical adenocarcinoma, in identifying precursor lesions.
Material and Methods: The hereby study covers a number of 37 patients presenting endometrioid adenocarcinoma of the uterus and 29 patients previously diagnosed with endocervical adenocarcinoma. A bioptic diagnosis is imposed from the prospective of the treatment plan, which can differ by location: cervical or uterine corpus. The solution for the bipotic diagnosis is the use of monoclonal antibodies following well established algorithms. Within our study we analyzed the histological characters and immunohistochemical profiles of the endometrioid type of tumor. The immunohistochemical marking was made using the following monoclonal antibodies: anti-cyclinD1, antiER/PR The solution for the bipotic diagnosis is the use of monoclonal antibodies following well established algorithms. Results and discutions Anti-CyclinD1 coexpression has some importance in the differential diagnosis between uterine endometrioid carcinoma and endocervical adenocarcinoma, especially when the diagnosis is made on bioptic peaces. In the case of the endometrium there is a high expression in most of the analyzed cases. The use of the anti hormonal receptor markers (anti ER/PR) has been intended in the hereby study in order to avoid compromising a diagnosis that can be possible when using a single marker.

Conclusions: Isolated analyses of the markers can lead to diagnostic errors. The precise differential diagnosis can be reached by using.

\section{PP-79}

The IHC expression of morphological changes using antibody anti-CD 34 on placental level in prematurity and developmental abnormalities

Corina Daniela F, Adriana R, Mircea Florin S, Casiana S

Universitatea de Vest Vasile Goldis, Arad, Romania

Purpose: I the hereby study it was made use of the monoclonal antibody CD34 in order to evaluate the structural placental vascular alterations of the placentas resulted from births with newborns that presented multiple malformations and prematurity.

Methods: The immunohistochemical (IHC) expression of CD34 marker was studied on 115 placentas results from newborns with malformations most of them incompatible with life. Another 25 placentas were provided from normal newborns delivered on term and served as a control group.

Results: IHC reactions for CD34 indicate several abnormal diameters of blood vessels, associated with perivascular oedema, villous system conglomerates and many syncytial buds. The CD34 marker had a positive expression in vascular endothelium, but not in the trophoblastic epithelium on the pathological placental level.

Conclusions: Our results emphasize that in the placental fragments coming from newborns that have severe life-incompatible malformations there are multiple pathological vascular alterations. All these changes from normal will lead to several 
hypoxic - type repercussions on the product of conception whose development will be eventually impaired. On the other hand, in the normal placentas we did not find any important vascular modifications.

\section{PP-80}

\section{A three - dimensional model of the knee applied to arthroscopic surgery}

Peces Peña D, Ruiz Mezcua J R, A Sanz Fernando L, Herranz EC, De La Cuadra Blanco C, Mérida Velasco J R

Universidad Complutense de Madrid, Spain

Introduction and Aims: Initiation into arthroscopic procedure of the knee is complex because it demands the exact bi-manual triangulation as well as the transformation of a tri dimensional structure into the two dimensions of a television monitor. Our starting point was the four basic positions of the arthroscopic exploration of the knee from the anterolateral portal. To make the initiation into arthroscopic manipulation more accessible, correlation is carried out on an auxiliary model thus permitting the study of the different structures of the knee from all angles.

Materials and Method: The Department of Human Anatomy and Embryology II of the UCM and the computer technology company ABADIA have used serial sections of RM of 1,5 teslas of living human beings on axial, coronal and sagittal planes. The programme of $3 \mathrm{D}$ reconstruction employed was AMIRAC and a graphics tablet thus enabling us to represent and assign a different colour to each anatomical structure.

Results: The three- dimensional model facilitates the identification of the osseous, muscular, cartilaginous, vascular and nervous structures of the knee articulation on the different sections in the RM images.

Comments and Conclusion: Minimally invasive surgical techniques and, particularly, arthroscopic surgery on the knee require an exact knowledge of this anatomical region in which there is involvement of important neurovascular structures which might otherwise suffer lesions. Pre-operatory planning with this type of model allows for the minimization of surgical risks for both surgeons in formation and other more qualified practitioners in the development of new surgical techniques.

\section{PP-81}

\section{Morphological characteristics of the neonatal adipose tissue}

Atanassova $\mathrm{P}^{*}$, Rancic $\mathrm{G}^{* *}$

Department of Anatomy, Histology and Embryology, Medical University Plovdiv, Bulgaria*; Institute of Histology and Embryology, Medical Faculty, University of Niš, Serbia**
The morphology of neonatal adipose tissue is poorly studied although its knowing would elucidate the intimate mechanisms of one of the clinical problems of modern civilization - obesity. The purpose of the present work was to study the morphological characteristics of the adipose tissue of newborns using mice as an animal model. The experiments were performed keeping the protocols approved by the Institutional Animal Ethical Committee of Medical University, Plovdiv, on 10 newborn mice. The animals were killed and fragments of subcutaneous tissue from the gluteal region were prepared for light microscopical observation (hematoxylin and eosin staining and immunohistochemical reaction for leptin and cyclin D1). The results revealed that neonatal subcutaneous adipose tissue consisted of packets of adipose cells which were surrounded by connective tissue. Most of the adipocytes were unilocular with one lipid drop occupying the whole cell and pushing the cytoplasm and the nucleus to the cell periphery. Among them some multilocular fat cells with several lipid droplets of different size and almost centrally located nucleus were seen. Our immunohistochemical investigations demonstrated that all the adipose cells (unilocular and multilocular) demonstrated positive leptin expression. Having in mind that leptin is the key marker of white adipose cells, these findings make us accept the multilocular adipose cells as preadipocytes present in the neonatal adipose tissue. At the same time the multilocular adipocytes expressed positive immunohistochemical reaction for cyclin D1 which suggested that they possessed proliferative activities. Our data suggest that there are preadipocytes in the adipose tissue of the newborns that have not yet finished their differentiation and proliferation. Their presence could explain their morphofunctional relation with obesity, i.e. under some external and/or internal stimuli they could finish their development and become mature unilocular adipocytes thus increasing fat mass.

\section{PP-82}

\section{Histology and ultrastructure of feather follicles in a flying birds, Columba livia}

\section{Gharzi A}

Lorestan University, Lorestan, Iran

In present research gross histology and detailed structure of feather follicle from pigeon, Columba livia were examined by means of light and electron microscopy. Growing feather follicles displayed histological features quite similar to those of hair follicles. A large body of dermal cells was observed in the middle of the follicle. This dermal structure was comprised of two regions without a clear border in the histological sections. The lower region that is called papilla is composed of fibroblastic cells within a glycosaminoglycan-free extracellular matrix. The 
papilla is surrounded by a thick and multi-layered epidermal compartment, whose cells were small, densely packed and displayed a strong basophilic reaction. This epidermal component, termed the collar, is the region that gives rise to the feather by cell proliferation. The upper three-quarters of the dermal body named the pulp which indeed is made of components secreted by the papilla cells. Large blood vessels enter the follicle through the papilla basal stalk and continued centrally through the papilla and the pulp. Ultrastructurally, the dermal cells of the papilla displayed various morphologies, but were mainly elongated with a few cytoplasmic projections. The papilla cells were separated from outer epithelial cells by a thick basement membrane rich in fibrous elements. The epidermal cells were found to be small with round nuclei and were closely associated with the basement membrane. Amongst the epidermal cells several dendritic cells were also observed which are possibly dormant melanocytes and would serve as producers of pigment for the next feather generation

\section{PP-83}

\section{Morphofunctional characterization of congenital adhesions}

Pilmane $M^{*}$, Valdovska $A^{*}$, Volrats $\mathrm{O}^{* *}$, Jemeljanovs $\mathrm{A}^{* * *}$, Kavak $V^{* * * *}$

Institute of Anatomy and Anthropology, Riga Stradins University, Riga, Latvia*; Clinic of Children Surgery, Riga Stradins University, Riga, Latvia**; Research Instituteo f Biotechnology and Veterinary Medicine "Sigra", Sigulda, Latvia***; Department of Anatomy, Dicle University, Diyarbakir, Turkey****

Adhesions follow to different congenital abdominal anomalies like atresia, malrotations, peritonitis, ileus. Among possible morphopathogenetical changes like innervation disorders, mesothelial and local blood supply disregulation also disregulation of growth factors is mentioned to play a possible role into the remodelation of adhesions. Thus, the aim of work was the detection of factors stimulating the fibrosis in the congenital adhesions. Materials were obtained from 16 children aged from 1 day until the 2 years who underwent adhesiolesion due to the disease on abdominal cavity during embryogenesis. Tissue were stained for IGF, IGF1R, TGFbeta, HGF, CAB, Hox3b and $\mathrm{SHH}$ immunohistochemically. Results showed patchy fibrosis, neoangiogenesis and inflammatory cells infiltration (mainly macrophages) into the adhesions. Modified in shape fibroblasts variously expressed all above factors, but constantly in moderate number of cells - the CAB and TGFbeta. Mesothelium mainly expressed TGFbeta and IGF1R, while their factors expression here was indistinct or absent. Moderate number of endothelium showed TGFbeta, CAB, Hox and HGF positivity, but numerous cells were Shh immunoreactive. Conclusions Fibroblasts and endothelial cells of congenital adhesions demonstrate the main plasticity and abundance of different factors and genes expression, while mesothelium is limited in the expression of growth factors and genes. Only TGFbeta is the commonly expressed growth factor of all cells in congenital adhesions, but other factors expression seems to depend on their functional local role in adhesions.

\section{PP-84}

\section{Comparative microanatomical study of the tongue of rat, bat and pangoloin}

Abayomi T

Osun State Universtiy, Osogbo, Nigeria

The tongue, which plays a very important role in food intake in mammals, exhibit significant morphological differences that, enables them to adapt to their varying feeding patterns. In this comparative study, the microanatomical features of the tongue of the rat, bat and pangolin were examined. The rat and bat were sacrificed by cervical dislocation while the pangolin was sacrificed by anesthetic administration of Ketamine (2-5 $\mathrm{mg} / \mathrm{kg}$ ) intramuscularly. The tongues were excised and fixed in $10 \%$ formal saline. They were processed for paraffin embedding and serial sections with 6um thickness were stained with haematoxylin-eosin, verhoeff-van Gieson and Masson trichrome and studied under light microscope. The results revealed a correlation between structural features and feeding patterns i.e. omnivorous, frugivorous and insectivorous for the rat, bat and pangolin respectively. Though some structural similarities were observed, never the less, the variations in the epithelial lining, papillary morphology, intrinsic musculature, vasculature and innervation, lingual glands and taste buds are adaptations which enable the tongue of these mammals cope with their varying dietary functions.

\section{PP-85}

Immunohistochemical and molecular expression of CB1 receptors in human colonic segment. Preliminary results

Uzzo ML*, Peri V**, Maria Mauro A*, Gerbino A*, Leone A*, Spatola GF*

Bionec - Histology Section, Medical School University of Palermo, Palermo, Italy*; Gastroenterology Unit, Villa Sofia Hospital - Palermo, Palermo, Italy*

Recent studies document that CB1 cannabinoid receptors, considered since its first identification as "brain specific", could be expressed by peripheral tissues targets (adipose organs, enteric nervous system, striated muscle, epatocytes). This receptors were found in the GI tract of different species, including mice, 
rats, guinea pigs, pigs and humans. Endocannabinoids play a role in the pathophysiology of the gastrointestinal tract. They play a role in emesis, acid-related disorders, motility-related disorders, irritable bowel syndrome and diarrhea, and in inflammatory bowel disease. Endocannabinoid system might be involved in cancer differentiation, growth and cell migration. Distribution of receptors in different colonic segment is not known. We studied CB1 receptors distribution in 10 patients colonic segments; the present report shows preliminary results. Fragments of sigma, transverse and ascending human colon were obtained during endoscopic screening procedure. The specimens, for immunohistochemistry (IHC), were fixed in buffered formalin and embedded in paraffin; obtained sections were processed with anti CB1 (Biosource Europe S.A.) by EnVision+System HRP (AEC) and have been studied with photomicroscope Leica DMLB. The specimens for the Reverse Transcription Polymerase Chain Reaction (RT-PCR) were frozen in liquid nitrogen and stored at $-80^{\circ}$ until use. Total RNA extraction was performed using the "illustra RNA spin Mini Kit". RT reaction was performed using the "Enhanced avian HS RT PCR kit" The PCR was performed using the "PCR enzyme Selection Kit-High specificity". This preliminary results show a presence of CB1 receptors expressed with regional differences in the colonic segments examined. These IHC results are supported by RT-PCR effected on the same segments.

\section{PP-86}

BMP-2 and BMP-4 protein expression during the formation of human brain

$\underline{\text { Namm A*}^{*}}$, Arend A**, Aunapuu $M^{* *}$

Department of Veterinary Medicine, Estonian University of Life Sciences, Kreutzwaldi, Tartu, Estonia*; Department of Anatomy, University of Tartu, Ravila, Tartu, Estonia**

Development, regional specification and morphogenesis of the human brain seem to be controlled by Bone Morphogenetic Proteins (BMP-s). In human embryonic development BMP-2 and BMP-4 are critical signaling molecules required for the early differentiation of the embryo and the establishment of the rostral-caudal parts of neural tube. In our studies we investigated BMP-2 and BMP-4 expression in the human embryos and we determined spatial and temporal expression of BMP-s during the early stages of neural tube development. Sixteen human embryos of Carnegie stages (CS) 14-20 were obtained by medical abortions. The embryos were fixed in $4 \%$ formaldehyde, embedded in paraffin and were serially cut in transversal direction. For immunohistochemistry sections were deparaffinized and rehydrated. Peroxidase activity was removed by
$0.6 \%$ hydroperoxides in methanol. Then sections were washed in tap water and in PBS ( $\mathrm{pH}=7.4)$, treated with normal $1.5 \%$ goat serum and incubated with the primary (BMP-2 diluted 1:250, BMP-4 diluted 1:100) and secondary antibody. Peroxidatic activity was detected with DAB. Sections were counterstained with staining method of van Gieson. The BMP labeling was expressed by a subjective scale ranging from 0 to 3. The results showed immunostaining of BMP-2 and BMP-4 in all regions of developing brain, but there seem to be differences in the expression of BMP-s at different developmental stages. Considerable BMP-2 and BMP-4 expression appeared in embryos of CS 14 to 18 , but expression was low level in embryos of CS 20. Considerable BMP immunostaining appeared in the primary brain vesicles, which are the earliest subdivision of the embryonic neural tube, including the prosencephalon, mesencephalon and rhombencephalon. Our investigations confirmed the idea that BMP-2 and BMP-4 are important mediators in the human embryos and may initiate multiple pathways controlling the specification, proliferation and differentiation of neurons in the developing neural tube.

\section{PP-87}

\section{Effects of aspirin on microstructure of placenta of mouse}

Jafarpur M, Jafarpur S

Department of Anatomy and Cell Biology; Faculty of Medicine, Mashhad University of Medical Sciences, Mashhad, Iran

Introduction: Aspirin is used as painkiller and some other therapeutic purposes. This question whether Aspirin has negative effects upon placenta and fetal development, was the cause of this research.

Method: We divide 40 heads BALBC race female rats into 2 experimental and control groups, altogether four groups with 10 cases in each. After fertilization of the mice, Aspirin was given to experimental groups and physiological serum to control groups. Then rats were killed under anesthesia and placentas were drowning out. Placentas were fixed and observed after stain.

Results: In experimental group 1 was 2 cases and in experimental group 2 were 7 cases of fetal abortion. There was no abortion in control groups. There were distinct light microscopic changes in experimental groups but in control groups there was no microscopic change.

Conclusion: Bad effects of Aspirin such as bleeding effect on gastrointestinal system has been reported in others reports. In this study, placental bleeding too and abortion after that has been observed. So, it can be proposed that using Aspirin will cause disorders in septum of blood vessels that is what we observed in light microscopic inspection. 


\section{PP-88}

Apoptosis inhibition effects, using window labelling approach, during early stages of vertebrate retina

Mayordomo R*, Chavarria T**, Sánchez MJI*, Pico AM*

Department of Cellular and Developmental Biology, Centro de Investigaciones Biológicas, CSIC, Madrid, Spain*; Department of Cellular and Molecular Physiopathology, Centro de Investigaciones Biológicas, CSIC, Madrid, Spain**

Programmed cell death is the result of an interaction between induced signals of survival and death, which finally determinates the destiny of cells, tissues and organs. Many different types of cells could be affected by programmed cell death. Neural stem cells, proliferating progenitors, and recently born neurons and glia are also affected by cell death. Using window labelling approach and double labelling we have characterize different population undergoing apoptosis during chick embryo development. We have studied cell death affecting proliferative precursors and new born neuroblasts in an interval time using this technique that has been used previously to characterize the final destiny of proliferative cells through DNA precursor's incorporation along $\mathrm{S}$ phase. The window labelling was opened with an in ovo injection of $\operatorname{BrdU}$ and closed with another injection of timidine. We show quantifications of TUNEL positive cells, BrdU-TUNEL and TUJ-1TUNEL positive cells in whole mount retinas at stage $\mathrm{HH} 21$ (E3.5), after 3 or 6 hours of incubation within the window closing. In order to determinate if cell death was affecting a population of recently new born neuroblasts, we decided to treat eye embryos with a cell death inhibitor and quantify the number of differentiated (TUJ-1) cells in whole mount retinas, at stage $\mathrm{HH} 21$. Boc-fmk was used as a general caspase inhibitor after the window labelling treatment. Our results show a significant increase in the number of differentiated TUJ-1 positive cells. The inhibition of cell death suggests an implication in the regulation of proliferative cells and also new born neuroblasts.

\section{PP-89}

\section{Evaluation of two-dimensional ultrasonographic} parameters in fetuses of 16-36 weeks of pregnancies

Ozbag D*, Yamac E*, KaragoL A**, Gumusalan $\mathrm{Y}^{*}$, Arikan DC ${ }^{* * *}$, Kiran $\mathrm{H}^{* * *}$

Departments of Anatomy, Faculty of Medicine, Kahramanmaraş Sütçü Imam University, Kahramanmaraş, Turkey*; Sultangazi Medical Center, Istanbul, Turkey**; Departments of Obstetrics and Gynecology, Faculty of Medicine, Kahramanmaraş Sütçü Imam University, Kahramanmaraş, Turkey***

The aim of the present study was to supply the need of ultrasonographic examination of intrauterine fetuses in the Turkish population and to overcome the difficulties in accessing the real means due to the calibration of the present ultrasounds according to the averages of the countries of their origin. The study was conducted on the sonograms of 50 pregnant women 19-38 years old with singletone pregnancies at 16-36 weeks of gestation with no systemic disease. Besides the maternal weight and height, ultrasonographic examination of the fetal parameters such as; biparietal diameter, abdominal circumference, and lengths of femur, tibia, fibula, humerus, radius and ulna were recorded. A total of 50 pregnant women with gravida range 2-4 and parity range $0-3$ were included in the study. There was a statistically significant difference between maternal age, gestational age, parity and all measured parameters $(\mathrm{p}<0.05)$. The relation between fetal BPD and FL,TL,FBL,HL,RL,UL was significant $(\mathrm{p}<0.01)$. While similar statistical significance was determined among all these parameters, there was also a statistical similarity between maternal height and weight $(\mathrm{p}<0.05)$. Besides the fetal development normograms show differences among populations, we think that the ultrasonographic equipments used to measure these parameters must be programmed so as to reflect the population characteristics. Almost at that time we can gain access the real values reflecting the features of the individuals of that population.

\section{PP-90}

Role of vitamin $\mathrm{B} 6$ and folic acid in reducing of craniofacial deformity of carbamazepine in Balb/c mic fetuses with alizarin red-alcian blue staining

$\underline{\text { Afshar } M^{*}}$, Moalem SA**

Birjand University of Medical Sciences, Mashhad, Iran*; Mashhad University of Medical Sciences, Mashhad, Iran**

Background: Carbamazepine (CBZ) is a common antiepileptic drug that it's consumption during pregnancy may associate with some malformations. The aim of this study was to evaluate the protective effect of vitamin B6 and folic acid on prevention of craniofacial anomalies due to CBZ in Balb/c mice.

Methods: In this experimental study $80 \mathrm{Balb} / \mathrm{c}$ timed-pregnant mice were divided into 6 experimental and 2 control groups. Two experimental groups received daily intraperitoneal injections of $30 \mathrm{mg} / \mathrm{kg}$ (group I) or $60 \mathrm{mg} / \mathrm{kg}$ (groupII) of CBZ. Two other experimental groups (group III and IV) received similar doses of CBZ with $3 \mathrm{mg} / \mathrm{kg} /$ day folic acid supplement by gavage route. Experimental groups $\mathrm{V}$ and $\mathrm{VI}$ received similar doses of CBZ with $10 \mathrm{mg} / \mathrm{kg} /$ day vitamin B6 supplement by gavage route. Two control groups I and II received normal saline and Tween 20, respectively All injections were done during GD6GD15 and gavage of vitamins done from 10 days before pregnancy to 15 days after GD0. Dams underwent Cesarean section on GD18 and external examination was done. Fetuses were stained with double staining. Data were analyzed by ANOVA, Tukey and $\chi 2$ tests using 15 version of SPSS software. 
Results: Growth indexes of the fetuses in both experimental groups that received CBZ were significantly reduced compared with control groups. Folic acid didn't have any positive effects on growth indexes but Vit B6 had positive effect on growth indexes. Orofacial deformities such as mandibular hypoplasia and calvaria deformity were detected in both experimental CBZ groups. Significant reduction was seen in the incidence of craniofacial deformities in fetuses of group consumed vitamin B6 or folic acid as a supplement.

Discussion: This study showed that administration of vitamin B6 during pregnancy was more effective in reducing craniofacial deformities than folic acid consumption

\section{PP-91}

\section{Acheiropodia: a new case from Turkey}

$\underline{\text { Akyer SP* }}$, Ulker K**, Baylan $\mathrm{H}^{*}$, Yonguc GN*

Anatomy Department, Pamukkale Medical Faculty, Denizli, Turkey*; Department of Gynecology and Obstetrics, Medical Faculty, Kafkas University, Kars, Turkey**

Object: Acheiropodia which is also called Horn Kolb Syndrome is a developmental disorder that presents with symmetrically and bilateral congenital lacks of the upper and lower extremities. The estimated incidence of acheiropodia is approximately 1/250,000 births in Brazil. The extreme rarity of the anomaly makes it of particular interest in the genetic literature and the description of new cases is therefore highly desirable.

Materials and Methods: This female fetus was aborted at the 23th week of the gestation because of the intrauterine exitus.

Results: External appearance is inspected and we found that distal parts of upper and lower extremities are undeveloped. Extremities proximal to the undeveloped parts are appropriate according to the expected term findings but distally to the joints no forearm, crus, wrist and digits are inspected. Diaphysis of femur and distal epiphysis are well-improved. A shapeless and undefined bone is seen to make a joint with femur. Distally to this undefined bone there seen some other smaller structures which we expect to be foot tarsal bones. There were no metatarsus and digits. Humerus is normal and had joint with some small bone structures distally. They are like carpal bones but no radius, ulna, metacarpals and digits are situated there. The other organ systems of fetus have normal findings and are developed properly.

\section{PP-92}

The artery of Percheron revisited: a cadaveric anatomical study

Kocaeli H, Kuytu T, Yilmazlar S, Korfali E

Department of Neurosurgery, Medical Faculty, Uludağ University, Bursa, Turkey
Introduction: The artery of Percheron is a single thalamoperforating arterial trunk that provides bilateral supply to the paramedian thalami and the rostral midbrain. As this rare anatomical variant artery may be involved in endovascular procedures or encountered surgically during basilar terminus aneurysms, the present study seemed warranted.

Materials and Methods: Thirty-four adult (20 male and 14 female) formalin fixed and fresh cadaveric brains underwent a detailed dissection of the 68 posterior cerebral arteries. Observations were made of the presence and variations of the thalamoperforating arteries as well as the presence of the rare variant artery of Percheron. Also 3 cadaveric brains with basilar artery aneurysms were dissected for determining the realationship of the perforating arteries with the aneurysm.

Results: Thalamoperforating arteries arose from the superior, posterior or posterosuperior surfaces of the P1 segment at the mean $1.93 \mathrm{~mm}$ (range, 0.41-4.71 $\mathrm{mm}$ ) distance from the basilar apex and entered the brain through the posterior perforated substance. The average number was 4.25 (range 1-9) and mean diameter was $0.73 \mathrm{~mm}$ (range 0.46-1.16 $\mathrm{mm}$ ). Thalamoperforating arteries were classified into four different types according to their origin at the P1 segment: type I (bilateral multiple, $\mathrm{n}=19$ ), $55.8 \%$; type II (unilateral multiple, unilateral single, $n=4$ ), 11.7\%; type III (bilateral single, $n=7$ ), 20.5\%; type IV (one side single, the other side with no branches"the artery of Percheron", n=4), $11.7 \%$. Of these 4 cases 2 was Percheron type IIb and one was a type III. In one of the type I specimens anastomosis between perforating arteries was seen.

Conclusions: Since preoperative detailed angiographical demonstration of thalamoperforating arteries and their relation to aneurysm neck is very difficult our data may be useful to neurosurgeons and endovascular specialists who treat basilar terminus aneurysms and to clinicians who interpret imaging of this artery.

\section{PP-93}

\section{Cleft palate and alteration of TGF- $\beta 3$ expression and the palatal fusion mechanisms in mice following a folic acid deficiency}

Maldonado $\mathrm{E}^{*}$, Murillo $\mathrm{J}^{*}$, Barrio $\mathrm{C}^{*}$, Río AD*, Miguelsanz $\mathrm{JP}^{*}$, Gordillo $\mathrm{YL}^{*}$, Partearroyo $\mathrm{T}^{*}$, Paradas $\mathrm{I}^{*}$, Maestro $\mathrm{C}^{*}$, Sanz $\mathrm{EM}^{*}$, Moreiras $\mathrm{GV}^{* *}$, Alvarez $\mathrm{CM}^{*}$

University Complutense Madrid, Madrid, Spain*; Universidad Ceu-San Pablo, Madrid, Spain**

Folic acid is essential for numerous bodily functions. Its reduction during pregnancy has been associated with an increased risk of congenital malformations. The relationship between a folic acid deficiency and the appearance of cleft palate is controversial, and little information exists on any possible effect of folic acid on 
palate development. We investigated the effect of an induced folic acid deficiency for two to eight weeks in female mice on the development of cleft palate and the mechanisms leading to palatal fusion in their progeny: cell proliferation, cell death, palatal shelf adhesion and fusion. We showed that a folic acid deficiency lasting eight weeks in the mothers caused a significant appearance of complete cleft palate in the fetuses, although only a two-week folic acid deficiency was needed to alter all the mechanisms analyzed. Since TGF- $\beta 3$ is crucial for palatal fusion and most of the mechanisms impaired by the folic acid deficiency were also observed in the TGF- $\beta 3$ null mutant palate, we investigated the presence of TGF- $\beta 3 \mathrm{mRNA}$, its protein and phophoSMAD2 in folic acid deficient mouse palates. Our results evidenced a large reduction of Tgf- $\beta 3$ expression in eight-week folic acid deficient mouse palates, with a less reduced expression in the two-week group. Addition of TGF- $\beta 3$ to two-week folic acid deficient palatal shelf cultures normalized all the altered mechanisms. Therefore, in mice, an insufficient folate status may be a risk factor for the development of cleft palate, and exogenous TGF- $\beta 3$ compensates this effect in vitro.

\section{PP-94}

\section{Study of the effect of oxidative stress on ovarian morphology and fertility of ovume in invitro in mice}

Roshangar L*, Rad JS**, Sabetkam S**, Rezazadeh A*, Hemmati $\mathrm{A}^{* *}$, Abedellahi $\mathrm{A}^{* *}$

Neuroscience Research Center, Tabriz, Iran*; Faculty of Medicine, Tabriz University of Medical Science, Tabriz, Iran**

Intoduction: Antioxidents and reactive oxygen species (ROS) are in balance in the body. Whenever the balance between these molecules is disrupted towards an overbundance of ROS, oxidative stress (OS) occurs. Mostly, OS is the result of increased ROS generation, rather than a depletion of antioxidants. There are evidence that gluthatione as an intracellular antioxidant plays an important role against ROS in female reproductive system. Regarding the effect of BSO as a selective inhibitor of gluthatione peroxidase the aim of the present study is to investigate BSO induced OS on fertility of ovume.

Method: For this purpose, 30 adult female and 5adult male $\mathrm{bulb} / \mathrm{c}$ mice were kept in standard condition and divided into 3 groups of experimental, sham and control. The experimental group received $2 \mathrm{mmol} / \mathrm{kg}$ BSO daily for 2 weeks as IP injection. The sham group was received the solvent of BSO and the control group didnot received any. After the injection periode the mice in all 3 groups received 7.5 unit hMG and 48 hours later 7.5 unit hCG as IP injection. 36 hours later the mice were killed by cervical dislocation and oocytes were collected by uterine tube flashing and sperms from male mice from the cauda epididymis by incubating the pieces of epididymis in $37^{\circ}$ oven. The sperm were added to the medium drops containing oocytes 50 in each group and embryo formation was consid- ered as fertility. All of the samples proceed for light microscopic study evaluation. The number of embryos in each group was counted and compared with each other by using ANOVA.

Results: The results showed that the percent of embryo formation in experimental group comparing to control and sham groups were significantly reduced $(\mathrm{P}<0 / 01)$, also morphometrical study of ovarian sections showed a remarkable changes on follicular structure and their quality.

Conclusion: The result indicates that BSO-induced OS could affect the fertility of oocytes and reduces embryo formation.

\section{PP-95}

\section{Early development of the ciliary body and iris}

Peña MDP, Cuadra CDL, Velasco JRM, Mezcua JRR

Universidad Complutense De Madrid, Spain

Introduction and Aims: The aim of this work is to study the early development of the ciliary body and the iris and establish the chronology of the stages involved.

Materials and Method: The study was performed using light microscopy on serial sections of 20 human specimens from the collection of the Embriology Institute of the Complutense University of Madrid.

Results and Conclusions: The ciliary body and the iris are formed from two of the three tissues that develop the human eye: the neuroectoderm and mesenchyme (neural crest). The two epithelial layers covering the ciliary body and the iris as well as the iris muscle derive from the neuroectoderm. The connective tissue, blood vessels and ciliary muscle derive from mesenchyme. The development begins in the 11-12th week, the anterior rim of the optic cup contact with the lens previously formed. We have found that the necessary vascular structures for the formation of the conjunctive tissue of the ciliary body and iris such as the annular vessel and the pupillary membrane are present in embryos of 5-8 weeks.

\section{PP-96}

\section{Development of the palatine aponeurosis during the 6th-16th weeks}

Peña MDP, Krielinger JAP, Mezcua JRR, Blanco CDLC, Velasco JRM

Universidad Complutense De Madrid, Spain

Introduction and Aims: The palatine aponeurosis is a fibrous formation on the soft palate and fixed onto the posterior shelf of the osseous palate; its purpose is to anchor the different muscles of the soft palate. The palatine aponeurosis is considered to be the continuation of the veli palatini tensor muscle. However, we have studied its development to determine 
whether it is formed simultaneously with the muscle or is, in fact an independent formation.

Materials and Method: The study was made with a light microscope on serial sections of 60 human specimens from the 6th to the 16th weeks of development (13.6-137 mm greatest length)

Results: The TVP becomes visible in embryos at Carnegie stage 18 (6th week) from a common blastema with the medial pterygoid and tensor tympani muscles. In embryos at Carnegie stage 20 ( 7 th week), the TVP is differentiated and relates to the anlage of the pterygoid hamulus. At Carnegie stage 23 (8th week), when the palatal shelves become horizontal, the presence of the anlage of the palatine aponeurosis is distinguished and is reached by the TVP. At the 9th week, the TVP is continuous with the palatine aponeurosis. Commentary and

Conclusion: We found that the TVP and the palatine aponeurosis developed independently. The TVP was differentiated by Carngie stage 20 ( 7 th week) while the anlage of the palatine aponeurosis appeared at Carnegie stage 23 (8th week, coinciding with the horizontalisation of the palatal shelves. Ross (1971) while studying fetuses with a cleft palate, found that the TVP was normal and the palatine aponeurosis was not present; an observation that would confirm our own findings.

\section{PP-97}

\section{Development of tracheal hyaline cartilage in second and third trimesters of human fetuses: a preliminary report}

$\underline{\text { Cuce } \mathrm{G}^{*}}$, Cicekcibasi $\mathrm{AE}^{* *}$, Yilmaz $\mathrm{MT}^{* *}$, Cuce $\mathrm{H}^{*}$, Aktan TM* Buyukmumcu $M^{* *}$

Department of Histology and Embryology, Meram Medical Faculty, Selçuk Universtiy, Konya, Turkey*; Department of Anatomy, Meram Medical Faculty, Selçuk University, Konya, Turkey**

Introduction and Purpose: Cartilage tissue is a supporter tissue in organism. There were a lots of study about the development of trachea although we could no find a study about the histologic developmental process of hyaline cartilage tissue according to trimesters in human fetuses.

Tools and Method: This study was carried out on spontaneously aborted 12 fetuses ( 6 second trimester and 6 third trimester) between 18 and 32 weeks of gestation without detectable congenital malformations. The tracheal parts were taken from the end of the larynx. The paraffin sections were prepared after routine histologic process and haematoxylin-eosin and Masson's trichrome staining was performed. The tracheal hyaline cartilages were evaluated under Olympus light microscope.

Findings: In second trimesters, the histological appearance of cartilage tissue were not homogeneous and they were in the form of parts. The only one of them (22 of week) was homogen. But, in third trimesters, a half of them were homogen and the others had cartilage parts.

Result and Interpretation: The development of cartilage tissue continued with increasing gestational age and hyaline cartilage parts were combining with each other to create trachea.

\section{PP-98}

\section{Morphological development of vertebral column at embryonic and early fetal periods}

Gómez SG* ${ }^{*}$ Ordoñez FJ**, Rodríguez MR***, Rodríguez IR**, Gómez $\mathrm{NG}^{* * * *}$

Dpto Anatomía Y Embriología Humana li, Facultad De Medicina, Ucm, Madrid, España*; Dpto Anatomía Y Embriología Humana, Facultad De Medicina, Uca, Cádiz, España**; Dpto Patología Médica, Facultad De Medicina, Uca, Cádiz, España***; Dpto Histología, Anatomia Patológica, Biología, Medicina Preventiva Y Medicina Legal Facultad De Medicina, Uca, Cádiz, España****

Introduction: At the end of embryonic period as well as at the earliest fetal period, apoptotic events are found in cartilaginous vertebral column, firstly in the lamina of vertebral arch and later in the vertebral body. These areas are widely, but wrongly, considered as the origin of vertebral column ossification.

Materials and Methods: We studied 21 human embryos from Carnegie Stages 10-23: 9 between Carnegie Stages 10-12; 5 between Carnegie Stages 13-18 and 7 between Carnegie Stages 19-23. Further we studied several fetuses $(n=13)$ ranging from 35-250 mm-crown-rump length. Sections were stained with hematoxylin-eosin and assessed by light microscopy.

Results: The onset of ossification of the vertebral column started at the end of 12 th-week or at the beginning 13 th-week. At that stages, vascularization of apoptotic areas may promote endochondral and perichondral ossification. In thoracic, lumbar and sacral regions ossification took place firstly in vertebral body. On the contrary, in cervical region, ossification started at vertebral arch. In this line ossification of vertebral arch started at cervical area and it advanced in a craniocaudal direction.

\section{PP-99}

Lower jaw primordia in human embryos: a new look at an old discussion

Gómez SG*, Gómez NG**, Ordoñez FJ***, Rodríguez IR*, Rodríguez $M R^{* * * *}$

Dpto Anatomía Y Embriología Humana li, Facultad De Medicina, Ucm, Madrid, España*; Dpto Histología, Anatomia Patológica, Biología, Medicina Preventiva Y Medicina Legal Facultad De Medicina, Uca, Cádiz, España**; Dpto Anatomía Y Embriología Humana, Facultad De Medicina, Uca, Cádiz, España**; Dpto Patología Médica, Facultad De Medicina, Uca, Cádiz, España**** 
Introduction: Although the question of the embryonic origin of facial structures has received considerable attention, the mechanisms that control differential growth of the facial processes and patterning of skeletal tissues within these structures have been difficult to study and still are not well-understood. It may be explained, at least in part, taking into account each lower jaw primordia appeared before Meckel's cartilage ventromedial development in 19 Carnegie Stage. These mandibular primordia appeared to be made of chondroitin sulphate since it was azo-carmine positive and it may be derived from an apoptotic event.

Materials and Methods: We studied human embryos at 19 Carnegie stage. Sections were stained with azocarmine and hematoxylin-eosin and assessed by light microscopy.

Results: We have found an extracellular matrix mass that was identical to that presented at upper jaw. On the contrary fibrous connective tissue formerly accepted as lower jaw primordia was just the posterior margin of that extracellular matrix mass that appeared previously. Mandibular body derived from extracellular matrix mass whereas mandibular ramus derived from fibrous connective tissue. It seemed Meckel's cartilage had no influence in lower jaw development. Further studies on this topic are required.

\section{PP-100}

Involvement of pathological apoptotic teratogenetic mechanisms in brain development after valproic acid administration in Balb/c mice

\section{Nikoloussi ENE, Manthou ME, Likartsis C, Goula CO \\ School of Medicine Aristotle, University of Thessaloniki, Greece}

Introduction: Valproic acid, a pharmaceutical agent widely used for tonic/clonic seizures treatment, has been associated with teratogenesis of the developing embryo when administered during pregnancy. Therefore, a dilemma arises whether valproic acid could safely be administered during pregnancy. Recent studies suggest that the drug is involved in a mechanism triggering pathological apoptosis, fact which plays a major role in teratogenicity.

Aim of the Study: Apoptosis a well regulated process in embryonic/foetal development is normally performed without any tissue damage. The aim of this study was to determine whether Valproic acid triggers pathological apoptotic pathways in embryonic brain development in Balb/C mice.

Materials and Methods: Valproic acid (500 $\mathrm{mg} / \mathrm{kg}$ ) was administered in Balb/C mice, during 7th to 10th gestational day, period considered as a critical one for Balb/C mice embryonic evolution, in regard to organogenesis. Pregnant mice were sacrificed on gestational day 18th. Embryos were collected and registered for congenital anomalies under a stereomicroscope.
Histochemistry (Heidenhein's Azan) and immunohistochemistry (Tunel antibody (Promega\}) were performed on selected brain tissue slides and examined under light microscope.

Results: Registered congenital anomalies included encephaloceles, brain myeloceles, hydrocephalous, exophthalmos and teratomata. In tissue slides stained histochemically by Heindenhein's Azan, condensed nuclei were easily localized and observed into foetal brain tissue, indicating the pathological apoptotic procedure occurred. Additionally, brain tissue slides stained immunohistochemically using Tunel method revealed increased apoptotic activity.

Conclusions: The remarkably intense staining labeled both by histochemistry and immunohistochemistry indicate that valproic acid administration can cause massive cellular apoptosis during brain organogenesis. Most of brain cells prematurely reach a state where apoptosis is well advanced and massive cell death is going to follow. This condition causes a huge imbalance between cell proliferation and cell death, the final result of which is an abnormal formation of central nervous system structures leading to defective central nervous system function.

\section{PP-101}

\section{Osteogenic origin of human mandible and mandibular angle development}

Gómez NG ${ }^{*}$, Gómez SG**, Ordoñez FJ ${ }^{* * *}$, Rodríguez $\mathrm{IR}^{* * *}$, Rodríguez MR****

Dpto Histología, Anatomia Patológica, Biología, Medicina Preventiva Y Medicina Legal Facultad De Medicina, Uca, Cádiz, España*; Dpto Anatomía Y Embriología Humana li, Facultad De Medicina, Ucm, Madrid, España**; Dpto Anatomía Y Embriología Humana, Facultad De Medicina, Uca, Cádiz, España***; Dpto Patología Médica, Facultad De Medicina, Uca, Cádiz, España****

Introduction: Both body and ramus mandibularis derived from mandible primordia by mean of a direct ossification process. The lower jaw's proportions change during fetal period. The biggest overall growth of the jaw can be observed in 6th and 10th month of the fetus development. The values for the mandibular angle also change during that period. This finding is of particular interest since it may play an important role in orthodontic and prosthetic treatments.

Materials and Methods: We studied 8 human embryos from Carnegie Stages 19-23 and 12 fetuses from weeks 9-24. Sections were stained with azocarmine and hematoxylin-eosin and assessed by light microscopy.

Results: We have found several chondrogenic nuclei for muscle process, another one next to mandible angle, and the last one in mylohyod line. In addition the portion of Meckel's cartilage which lies in symphysis region is surrounded and invaded by the membrane bone. Consequently there was an endochondral ossification specially for symphysis region. Mean val- 
ues of mandibular angles in 4rd, 5th and 6th months were $137.00^{\circ}, 136.58^{\circ}, 136.05^{\circ}$. The depth of the mandibular body increases owing to increased growth of the alveolar process. The body also increased in length throughout the posterior border of the ramus. Respectly mandibular angle we concluded what this decreased during fetal period. Further studies on this topic are required since.

\section{PP-102}

Influence of combined treatment with selenium and zinc on lead induced pathophysiology in rats

\section{$\underline{\text { Falana B }}$}

Osun State University, Osogbo, Nigeria

Lead poisoning has actually become a serious health problem especially among indigenous populations with high level of mining activities, for example in Nigeria, lead poisoning claimed 153 lives in 2010, among which $80 \%$ of the victims are children below the age of 5 years. This study seeks to assess the effect of combined treatment with selenium and zinc in reversing lead induced testicular oxidative stress in rats 60 adult male sprague dawley rats weighing $200 \mathrm{~g}$ averagely shall be randomly divided into six groups, group A-F. group A shall be admistered water adlibitum for 60 days, group B will receive $20 \mathrm{mg} / \mathrm{kg}$ bw, IP injection lead acetate, group C will receive $20 \mathrm{mg} / \mathrm{kg}$ bw, IP injection sodium selenite and lead acetate, group D will receive IP injection $20 \mathrm{mg} / \mathrm{kg}$ bw zinc oxide and lad acetate, group E will receive $20 \mathrm{mg} / \mathrm{kg}$ bw of rat IP injection sodium selenite only, and group F will receive $20 \mathrm{mg}$ IP injection of zinc oxide only, follwed by sacrificing with cervical dislocation method and testis quickly fixed in formol saline for histology or $2.5 \%$ glutaraldehyde for ultrastructural studies.Blood samples will be obtained by cardiac puncture and stored in heparinised bottles for full blood count and sample bottles for bio assays, measuring the level of gluthathione peroxidase activity.sperm morphology and motility will be observed. Blinding procedures wil be utilised in the study to remove or minimize bias. this present study is on going and conclusions will be based on observation. Selenium and zinc may be beneficial to the treatment of lead induced testicular toxicity and probably in treating infertility due to heavy metal contamination by its anti -apoptotic functions as a trace element.

\section{PP-103}

Morphometric study of the male reproductive system of rats under the influence of toluene

Koveshnikov V, Luzin V, Voloshina I, Voloshin V

Lugansk State Medical University, Lugansk, Ukraine
Introduction: There is a fairly large number of products made of polymer and synthetic materials and plastics manufactured on the basis of epoxy resins. It is necessary to emphasize that the professional information on their effects on the organism of men is practically not available. The use of polymeric materials (synthetic wall paper, different types of coating, etc.) envisages the presence of quality ventilation in premises and dwellings. The polymer materials have an important feature: they discharge toxic chemical substances into the environment (phenol, acrylate, phthalates, toluene etc.).

Purpose: The aim of this work is the study of the peculiarities of the morphogenesis of the testis, epididymis, ductus deferens, seminal vesicles and prostate after the chronic influence of toluene.

Tools and Methods: The work was done on male rats of puberty age. The rats of the experimental group experienced the influence of toluene within two months. After the term was over, the animals were taken out of the experiment by way of decapitation under ether narcosis. We studied the sizes of the testes, epididymis, seminal vesicles, prostate and ductus deferens, its absolute and relative masses and histological structure.

Result and Interpretation: Using methods of morphological studies pro-vided data on the organization of the convoluted tubules and restructuring in the testis and epididymis, first described morphological relationship of the parenchyma of the prostate were studied macro- and microscopic features of the structure of the seminal vesicles and ductus deferens. For the first time studied the dynamics of morphological changes that occur in vessels and parenchyma of the testis, epididymis, prostate and seminal vesicles after inhalation of toluene at different times. New data on the microscopic structure of the internal organs of the reproductive system of male rats.

\section{PP-104}

The changes of the thyroid gland caused by formaldehyde and protective effects of melatonin and vitamin $\mathrm{C}$

Diramali $\mathrm{M}^{*}$, Arifoglu $\mathrm{Y}^{*}$, Yilmaz $\mathrm{F}^{* *}$, Kukner $\mathrm{A}^{* * *}$, Gepdiremen $\mathrm{A}^{* * * *}$, Serin $\mathrm{E}^{* * * * *}$

Department of Anatomy, Faculty of Medicine, Abant izzet Baysal University, Turkey*; Department of Pathology, Faculty of Medicine, Abant lzzet Baysal University, Turkey**; Department of Histology, Faculty of Medicine, Abant izzet Baysal University, Turkey***; Department of Pharmacology, Faculty of Medicine, Abant lzzet Baysal University, Turkey****; Department of Biochemistry, Faculty of Medicine, Abant izzet Baysal University, Turkey*****

Introduction and Purpose: Formaldehyde is an aldehyde which is used in Gross anatomy laboratories in embalming fluids. Additionally, formaldehyde is used in the cosmetics, dentistry, histology and pathology laboratories. In this study, we determined the effects of formaldehyde on the thyroid gland and compared it with protective effects of melatonin and vitamin C. 
Tools and Method: In this study, 75 albino rats were employed randomly in 8 groups. As control group 5 rats were used as the rest divided into 7 groups. Rats in group II were used as sham. Rats in group III and IV were injected intraperitoneal formaldehyde at doses of 10 and $15 \mathrm{mg} / \mathrm{kg}$ everyday excluding weekends. Rats in group V and VI daily received $100 \mathrm{mg} / \mathrm{kg}$ vitamin C in addition to formaldehyde injection. Rats in group VII and VIII daily received $25 \mathrm{mg} / \mathrm{kg}$ melatonin in addition to formaldehyde. After four weeks of experiment, rats were sacrificed by taking intracardiac blood to determine plasma levels of TSH, fT3 and fT4.Thyroid gland was dissected out and one lobe was stained with Hematoxylin-Eosin, Periodic Acid Schiff, Masson's Trichrome and Alcian Blue while other lobe was used for WST-1 analysis. Findings: Histology showed increase in interfollicular distance and follicular degeneration in only formaldehyde given groups, additionally there was fibrosis in Group IV. These degenerative changes decreased in rats which received antioxidants. Mast cell infiltration was higher in only formaldehyde given groups than control and sham groups. TSH levels were higher in melatonin given groups. There were no differences in WST-1 analysis.

Result and Interpretation: This study showed us that degenerative changes caused by formaldehyde in thyroid gland can be reduced with melatonin or vitamin $\mathrm{C}$ treatment.

\section{PP-105}

\section{Study of endocrine cells in Brunneroma}

Bulatović $V^{*}$, Zindović $M^{*}$, Jančić $N^{* *}$, Rančić $I^{* *}$, Jovanović $\mathrm{D}^{*}$, Rančić $\mathrm{G}^{* *}$, Jančić $\mathrm{S}^{*}$

Institute of Pathology, Faculty of Medicine, University of Kragujevac, Serbia*; Institute of Histology and Embriology; Faculty of Medicine, University of Nis, Serbia**

Background: Brunneroma is extremely rare hamartoma of the duodenum formed by mature Brunner's glands. These hamartomas, have a low propensity for malignant transformation but can be confused with lesions of more oncological importance such as dysplastic duodenal adenomas or duodenal adenocarcinomas. Aim. The objective of this study was to make micromorphological, histochemical and immunocytochemical characteristics of endocrine cells in Brunneroma.

Material and Methods: Operative duodenal material colected of 18 patients with dignostified Brunneroma, was routinely processed and embedded in paraffin. Section $(5 \mu \mathrm{m})$, were stained with HE method, histochemical AB-PAS (ph 2.5) and immunocytochemical $\mathrm{ABC}$ technique with specific antibodies to chromogranin A, serotonin, gastrin and somatostatin.

Results: The tumors were covered by usual intestinal mucosa and composed of lobules of proliferated Brunner's glands with ducts separated by irregular bands of fibromuscular stroma. These ducts were lined either by cylindrical eosinophilic cells or by clear mucinous cells. In tumor's mucus glands a hypersecretion of the neutral mucins were found. Chromogranin Apositive cells were found in eleven cases. Hyperplasia, hypertrophy and hypergranulation of serotonin-endocrine cells were identified in all cases. Perimembranous gastrin deposits were found in individual cells or cell groups in nine cases. Somatostatin secreting cells were found in all tumors. These cells were large with uni- and bipolar cytoplasmatic processes, often single and/or in small groups. The endocrine cells have uniform nuclei and significant dysplasia have not been seen.

Conclusion: The unexpected increase in endocrine cells presence and their distribution suggests that etiologic factors for the Brunner's gland proliferation also act on the endocrine components or that the stimuli that trigger the precursors might undergo dual proliferation. The pathogenesis of Brunneroma remains still unclear.

\section{PP-106}

Influence of salinity challenge on natriuretic peptides in a euryhaline teleost

$\underline{\text { Akgul G}^{*}}$, Link K ${ }^{* *}$, Baroiller JF ${ }^{* * *}$, Reinecke $M^{* * * *}$, Eppler $E^{*}$

Neuro-Endocrine Immune Interactions Research Group, Institute of Anatomy, University of Zurich, Ch-8057 Zurich, Switzerland*; Center For Evolutionary Medicine, Institute of Anatomy, University of Zurich, Ch-8057 Zurich, Switzerland**; Upr20, Aquaculture Unit, Cirad, Campus International De Baillarguet, Montpellier, France ${ }^{* *}$; Division of Neuroendocrinology, Institute of Anatomy, University of Zürich, 8057 Zürich, Switzerland****

Natriuretic peptides (NPs) are a group of hormones found in vertebrates that are primarily released from the heart. Before the discovery of NPs, this organ's function was believed to be solely for the delivery of blood to all parts in the body. After their discovery, it became evident that these peptides also regulate blood pressure, fluid volume and electrolyte balance. Therefore, the heart has at least two major biological functions: in the blood circulatory system and in the endocrine system. Four major members of this peptide family (atrial NP (ANP), brain/B-type NP (BNP), C-type NP (CNP), and ventricular $\mathrm{NP}(\mathrm{VNP})$ ) have been identified thus far in different vertebrate species. CNP is a paracrine factor mainly expressed in the brain, whereas ANP, BNP and VNP are circulating hormones localized mainly to the heart (Cardiac NPs). The current project aims at investigating the osmoregulatory functions of cardiac and encephalic NPs in fish. The focus is thereby laid on ANP and BNP because of indications of a similar role in higher vertebrate species. In order to understand the functional significance of these peptides within piscine osmoregulation, Tilapia were exposed to different salinity levels. Cardiac ANP, BNP and brain BNP gene expression levels are measured by the quantitive real-time PCR method. We assume that increased salinity will have a positive impact on fish osmoregulation indicated by increased ANP and BNP gene expression levels. 


\section{PP-107}

\section{Liver regeneration in fetal rats}

Elchaninov A, Bolshakova G

The Research Institute of Human Morphology, Russian Academy of Medical Science, Moscow, Russia

Introduction and Purpose: The mammalian liver in postnatal period has high regeneration properties; study of fetal liver regeneration is missed in the literature. The purpose of this study was to investigate liver regeneration in fetal rats.

Tools and Method: An excision of $20 \%$ of the liver in outbred albino rat fetuses on day 17 of prenatal development was performed. The animals were sacrificed by ether narcosis at $6 \mathrm{~h}, 1$, 2, 7, 10 days after the intervention. Liver was removed and weighed. All liver specimens were fixed in Carnoy's fluid, than dehydrated and embedded in paraffin wax to process sections at a thickness of $5 \mu \mathrm{m}$. The sections were stained by hematoxylin and eosin. Proliferation was evaluated by the mitotic index (MI) in hepatocytes surrounding the injury, in the area distant from it and in intact liver in the control. Hepatocyte and its nuclear size were measured on liver imprint smears fixed in methanol and stained by hematoxylin and eosin.

Result and Interpretation: Two days after partial hepatectomy the difference between the liver mass in experimental and control group was not significant ( $\mathrm{p}>0.05$ ). Thus, rat fetal liver after $20 \%$ partial hepatectomy requires 2 days to regain its weight. The MI in the area distant from injury and in the perinecrotic zone was higher than in control $(\mathrm{p}<0.05)$ only on day 1 after the injury (day 18 of prenatal development). MI of hepatocytes in the area adjacent to the injury decreased in comparison with the zone distant from it $(\mathrm{p}<0.05)$. The removed parenchyma seems not to grow back from the wound surface. The differences between hepatocyte and its nuclear size in experimental and control groups were not significant $(\mathrm{p}<0.05)$. Thus, rat fetal liver regeneration after partial hepatectomy is achieved by only the hyperplasia of hepatocytes, without increase in cell size.

\section{PP-108}

The morphometric development of the fetal knee during the fetal period

\section{Tuncer I}

Department of Anatomy, Meram Faculty of Medicine, Selcuk University, Konya, Turkey

\begin{abstract}
Aim: The aim of the present study was to investigate morphometric growth patterns of the distal part of the femur, the proximal part of the tibia, the patella, and anterior and posterior cruciate ligaments in human fetal knee.
\end{abstract}

Methods: This study was performed in the Anatomy Department of Meram Faculty of Medicine in 2009. The fetuses were obtained from the Gynaecology Department of the
Meram Faculty of Medicine of Selcuk University, and Dr. Faruk Sükan Maternity Hospital (Konya, Turkey). Fifty spontaneously aborted fetuses (24 male right, 24 male left, 26 female right and 26 female left) without detectable malformations were evaluated with ages ranging between 9 and 37 weeks of gestation. The dimensions of the distal part of the femur,the proximal part of the tibia,the patella,the anterior and posterior cruciate ligaments were measured. A characterization of the aspect ratio (the medial-lateral to anterior-posterior dimensions) was made for the proximal aspect of the tibia and the distal part of the femur. Statistical analysis for comparisons was done by Student's T test.

Results: In all measurements, no gender and sides differences were identified $(\mathrm{P}>0.05)$ and no difference was seen in the femur aspect ratio, tibia aspect ratio between males and females ( $\mathrm{P}>0.05)$ Conversely a significant association was obtained trimesters and all parameters $(\mathrm{P}<0.005)$.

Conclusion: Detailed information of knee joint and its components related to the fetal period will reveal the extent of biological variation of knee joint of its components to be used in future studies. We hope that data acquired in this study will facilitate other studies on knee anomalies,pathologies and variations as well as diagnoses and treatments of such conditions conducted in obtetrics, perinatology, forensic medicine and fetal pathology departments.

\section{PP-109}

\section{The growth of the face of Turkish fetuses during the fetal period}

\section{Tuncer I}

Department of Anatomy, Meram Faculty of Medicine, Selcuk University, Konya, Turkey

It was aimed to obtain normal morphometric values of the face growth and its morphology during the 1st, 2nd and 3rd trimester of human fetuses in this study. 97 (49 male, 48 female) human fetuses with no external anomaly or pathology and aged between 7 and 37 weeks were included in this study. Special face height (en_gn), face head height II (tr_n), nose length (n_sn), lower face height (sn_gn), forehead height I (tr_g), special upper face height (g_sn), ear length (sa_sba), interchantal distance (en_en), left eye fissure length (ex_en), mouth width (ch_ch) and nose width (al_al) of the fetuses were measured. There was significant correlation between all parameters obtained during the postmenstrual week and fetal period $(\mathrm{p}<0.001)$. All measurements except en_gn, sa_sba and ex - en were greater in male fetuses than female fetuses. It is expected that the normal values of face growth, and their proportion to each other and possible changes that may occur at different gestational ages can contribute to the studies of the branches such as growth anatomy, fetopathology, forensic Medicine, medical imaging and obstetry. 


\section{PP-110}

\section{The development of the foot of the Turkish fetuses during the foetal period}

Tuncer I

Department of Anatomy, Meram Faculty of Medicine, Selcuk University, Konya, Turkey

It was aimed to obtain normal morphometric values of the development of foot and its morphology. This study was performed the Anatomy Department of Meram Medical Faculty, at Selcuk University between 2006-2007. 107 (male: 50, female: 57) human fetuses who had no external anomaly or pathology and whose ages were 8-37 weeks were included in the study. Fetuses were divided into three groups as 1 st trimester (8-12 weeks), 2nd trimester (13-25 weeks) and 3rd trimester (26-37 weeks). The length of foot, bimalleolar width, bimalleolar circumference, tarsal height,tarsal and metatarsophalangeal circumference and metatarsophalangeal width were measured. There was significant correlation between all the parameters obtained during the fetal period $(\mathrm{P}<0.001)$. The statistically significant correlation between the measurements of foot length suggests that it is a proper determiner of age. We think that these measurements can be helpful in determining gestational ages in fields such as anatomy, pathologic anatomy (fetopathology), obstetric screening and pediatrics.

\section{PP-111}

The development of upper and lower extremities of turkish fetuses during the fetal period

Tuncer I

Department of Anatomy, Meram Faculty of Medicine, Selcuk University, Konya, Turkey

The aim of this study was to investigate the morphologic structure of the upper and lower extremities of Turkish fetuses during the fetal period and to measure the morphometric values. In this study, 107 human fetuses (50 male, 57 female) that had no external anomaly and aged between 7-37 gestational weeks were used. This study was performed the Anatomy Department of Meram Medical Faculty, at Selcuk University between 2006-2007. Shoulder width (SW), arm circumference (AC), forearm circumference (FC) and hand length (HL) were measured in the upper extremity. Iliac crest width (ICW), thigh circumference (TC), Crus circumference (CC) and foot length were measured in the lower extremity. The measurements were obtained through tape measure and caliper compass and the results were evaluated according to age and sex. There was significant correlation between all parameters taken during the fetal period and gestational age $(\mathrm{p}<0.001)$. All the measurements of the males were greater than those of females. The measurements of the upper and lower extremities during fetal period are reliable parameters for use in the assessments of gestational age. Fetal limb size is strongly correlated with crown-rump length. Despite a significant biological variability of the measurements, availability of reference ranges could be of help in the early diagnosis of fetal skeletal dysplasia.

\section{PP-112}

The morphometric development sphincter of oddi in the human fetus during the fetal period: microscopic study

Desdicioglu K${ }^{*}$, Bozkurt $\mathrm{KK}^{* *}$, Uguz $\mathrm{C}^{*}$, Evcil EH**, Malas $\mathrm{MA}^{*}$

Department of Anatomy, Medical Faculty, Süleyman Demirel University, Isparta, Turkey*; Department of Pathology Medical Faculty, Süleyman Demirel University, Isparta, Turkey**; Vocational School of Health, Mehmet Akif Ersoy University, Burdur, Turkey***

Aim: In this study, it is aimed to study the morphometric development of oddi sfinkter in the human fetus during the fetal period.

Material and Method: 87 human fetuses (57 males, 30 females) whose ages change between 14-40 pregnancy weeks and who do not have any external patology and anomaly have been included. The fetuses are divided into groups in accordance with their gestational week and trimester. After the overall external measurements of the fetus have been done, the abdomen wall dissection has been done. Following the localization of duodenum, pancreas and sphincter of Oddi, histologic tissue samples from the residential area sphincter of Oddi in the duodenum internal wall have been taken for the microscopic research. In the light microscope, ductus choledochus composing the sphincter of Oddi in each fetus and the total external diameter, lumen diameter of ductus pancreaticus, the total wall thickness of the channels and the distance parameters of the channels have been measured.

Findings: The mean and standard deviation of the measured parameters in accordance with their gestational weeks and trimester. There is a meaningful correlation between the other parameters and the gestational age, except the wall thickness of the ductus choledochus $(\mathrm{p}<0.001)$. There is not any difference between genuses in terms of parameters ( $>00.05)$.

Result: We think that the data of our studies will contribute to the extrapolation of the development the sphincter of Oddi in the intrauterine cases during the fetal period. 


\section{PP-113}

\section{Chronological study of the hoof development in sheep}

Rezaian M, Nazem MN

Department of Anatomy, Division of Histology, Faculty of Veterinary Medicine, University o Tehran, Tehran, Iran

In order to study of the hoof development, 16 Healthy sheep embryos with different ages varied from 36 to 140 days were collected. The ages were estimated by measuring the crown- rump length. Each limb as well as each hoof was cut in half sagitally and fixed in $10 \%$ buffered formalin. Routine histologic processes were used and $6 \mu \mathrm{m}$ thick paraffin sections were stained with hematoxylin-eosin and studied under light microscope. Limbs had been formed as small appendages at 36th - 40th days of embryonic ages and consisted of masses of mesenchymal cells covered with thin and uniform epidermis. The preliminary changes have been occurred on the epidermis of the limbs at 46th days of embryonic ages, so that, it has been thicker with more cell layers at the hoof, especially at the wall position. The epidermis of the hooves has continued to increase cell layers at 50 th -55 th days of embryonic ages and the Laminae (tunica interna) of the wall has been formed at 60th day of embryonic age. Papillation of the epidermis of the toe has been started at 68th day of embryonic age. The Stratum spinosum of the epidermises (tunica media) of the wall and the toe had been highly thickened, with swollen cells having small or picnotic nuclei at 75 th day of embryonic age. The Stratum Germinativum at prioplic region has been thickened and the spinosal cells of the coronary region begun to cornfield in tubular arrangement at 80th days of embryonic age. Keratinization in the epidermal cells of the coronary region was further progressed and the germinal epithelium of the sole has been papillated at 86th - 95th days of embryonic ages. Formation of the tunica media of the wall toward the ground surface was further progressed at 110th day of embryonic age. The epidermis of the prioplic region has been papillated and produced more swollen cells, projected over the coronet and started to produce cornfied layer toward the ground surface (stratum tectorium). The tunica media of the wall were then made by the stratum spinosum of the corium and upper $2 / 3$ of the wall. It made up of hard keratin. The tubular layer of the white line was made by the lower $1 / 3$ epidermis of the wall. The papillary layers of other parts of the hoof were made by the papillary epidermis of the same parts. The last layer has been made before birth was the tunica externa. This layer was made by the papillary epidermis of the priople skin.

\section{PP-114}

Histological study of the synovial membrane covering the intercarpal-interosseal ligaments of carpal joints in sheep

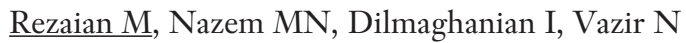

Division of Histology, Department of Anatomy, Faculty of Veterinary Medicine, University of Tehran, Tehran- Iran,
Intercarpal - interoseal ligaments are those intra articular carpal ligaments which connect the carpal bones of each row and the two adjacent rows to each other. These ligaments are covered by synovial membrane at their free surfaces. The present study was undertaken to clarify the histologic appearance of these synovial membranes. For these purpose 19 healthy sheep embryos with different ages were examined. Carpal joints were fixed in $10 \%$ buffered formalin. Routine histologic process was used and $6 \mu \mathrm{m}$ thickness sections were stained with Hematoxylin- eosin for detecting the general characteristics, PAS for carbohydrates, Orcein for elastic fibers, Masson trichrome for collagen fibers, and Verholf for connective tissue fibers and studied under light microscope. Three types of synovial membranes (Areolar, adipose, fibrous) were present at different sites of the membrane. No basement membrane was seen under synovocytes. All fibers of the ligaments were made up of the collagen and no elastic fibers were observed.

\section{PP-115}

\section{Histological study of the guttural pouches in Caspian miniature horses}

Nazem MN, Rezaian M

Department of Basic Sciences, Division of Histology, Faculty of Veterinary Medicine, Tehran University, Tehran, Iran

The guttural pouches (diverticulum's of auditory tubes) of five healthy adult Caspian Miniature Horses were studied histologically. Each pouch was dissected separately and samples were taken from the lateral wall and medial wall of them and fixed with $10 \%$ buffered formalin. Routine histological laboratory methods were used and 6um paraffin sections were stained with hematoxylin- eosin staining method and studied under light microscope. The sacs or pouches were covered by a thin layer of mucous membrane which was loosely attached to the surrounding structures. They lined with pseudo stratified ciliated columnar epithelium with large number of goblet cells. The lamina propria was loose connective tissue and contained small amounts of sero-mucous glands. Serous acini were more abundant. There were no muscularis mucosa and muscularis layer. Submucosa contained dense collagenous connective tissue.

\section{PP-116}

Localization of choline acetyltransferase of the peripheral type in the primary sensory neurons of the guinea pig trigeminal ganglion; comparison with CGRP and SP-containing cells

\section{Elnasharty $M$}

Department of Histology and Cytology, Faculty of Veterinary Medicine, Damanhour University, Egypt 
It has been demonstrated that a splice variant form of choline acetyltransferase (pChAT) is expressed in peripheral organs, including sensory ones, preferentially than the common type (cChAT). In order to identify the possible functional significance of pChAT in sensory functions, we characterized immunohistochemically pChAT-immunoreactive (IR) trigeminal ganglionic neurons in the guinea pig. We observed that the pChATimmunoreactivity occurred in an almost uniform pattern and in a considerable number of all trigeminal neurons throughout the trigeminal ganglion (TG). The vast majority of pChAT-IR neurons were of small to medium-sized cell bodies, although largesized neurons also observed. Most pChAT reactivity was mainly in the cytoplasm with few number of pChAT-IR neurons had nuclear staining. Double immunofluorescent study showed that a great proportion of substance $\mathrm{P}$ (SP)- and calcitonin generelated peptide (CGRP)-immunoreactive trigeminal cells showed pChAT-immunoreactivity, although those with SP was outnumbered those with CGRP. The intracellular expression of pChAT (which differs from that of cChAT) probably reflecting a difference in physiological roles between pChAT and cChAT in ACh production in distinct intracellular compartments. The present data suggest also that pChAT plays roles not only in nociception, but also in other sensory functions such as mechanoreception mediating tactile sensation.

\section{PP-117}

Histological and morphological study of the cerebrum of two reptilian species; wall gecko [Tarentola mauritanica] and skink [Euprepris maculilabris]

\author{
Adekilekun AT*, Adekomi DA** \\ Department of Anatomy, College of Health Sciences, Osun State \\ University, Osogbo, Nigeria*; Department of Anatomy, Faculty of \\ Basic Medical Sciences, University of Ilorin, Ilorin, Nigeria**
}

This study was undertaken in order to compare the cytological and morphological features of the cerebrum of the Moorish Wall gecko (Euprepris maculilabris), and Skink [Euprepris maculilabris]. Eight Wall geckos and six Skinks were used for this study. All reptiles were placed in quarantine for thirty days and were observed for any abnormalities. They were all fed with termites and ants and clean drinking water. The animals were all sacrificed by cervical dislocation and the brain tissues were carefully excised from the brain vault of the animals and were quickly fixed in $10 \%$ formolcalcium for histological processing. Following the histological processes, the cerebral tissues were sectioned and stained with Hematoxylin and Eosin (H\&E) stain. Using the statistical Student's t-test, it was observed that there were significant differences in population, size and distributions of glia cells, pyramidal cells, neuroglia, and oligodendrocytes of the cerebral cortices of the two reptilian species. Also observed in this study was a statistical difference in the body weight, brain weight and relative brain weight of the two reptiles. These differences and observations in the cytological and morphological outline may be as a result of the habitat and biological differences in the two reptiles.

\section{PP-118}

\section{Morphology of non sensory epithelium in developing rabbit vomeronasal organ}

Elgayar SAM, Eltony SA, Othman MA

Assiut University, Assiut, Egypt

Introduction and Purpose: The vomeronasal organ (VNO), through detecting pheromones, has an important role in many social and sexual behaviors in mammals. It also mediates defensive behaviours through detection of protein pheromone homologs. This work provides detailed morphological description of the developing "non sensory" epithelium (NSE) of the female rabbit VNO.

Tools and Method: Histological, immunohistochemical and morphometric techniques were used to study the NSE of the VNO in developing female rabbit. The following postnatal ages (five animals each) were used: new born), one week, two weeks and one month. Result and interpretations: The rabbit NSE of VNO consisted of pseudostratified columnar, partially ciliated epithelium. In addition to basal cells, it contained ciliated and three types of non ciliated columnar cells: dark, light and pale. Mitotic and apoptotic figures were observed during the first week. At birth, the dark cells which were the commonest type showed primary cilia extending from their surfaces. The light cells possessed large nucleus and numerous lysosomes. The pale cells had electron lucent cytoplasm. Their apexes projected above the surface and had constrictions like the olfactory knobs. These pale cells may extend processes around the cells invading the epithelium which included neutrophils, lymphocytes and macrophages. Presence of subsurface mitochondria and clear vesicles in their cytoplasm suggested their involvement in ionic transport. Nucleolus-like bodies were observed in the cytoplasm of dark and basal cells up to two weeks postnatal. The scanning electron microscope revealed ciliated cells to be arranged singly, in clumps or in a dense populations of cells. The structural features of growth of the rabbit VNO-NSE reflected a peculiar structure which would consequently reflect a peculiar function.

\section{PP-119}

Correlation of birth and placental weights amongst hausa speaking parturients: a study conducted at Murtala Muhammed Specialist Hospital Kano, Kano State, Nigeria

Saleh $\mathrm{MS}^{*}$, Yahaya $\mathrm{AI}^{* *}$

Department of Anatomy, Faculty of Medicine, Bayero University Kano, Kano State, Nigeria*; Department of Anthropology, Universtiy College London, London, UK**

Background: Birth weight has been reported to correlate with placental weight in many literatures but unfortunately this cor- 
relation was not reported amongst the Hausa ethnic group in Nigeria who constitutes the majority of the Nation's populace. We believe that the study is a milestone to the pool of the available data not only in the country but to the whole world.

Materials and Methods: The study is a cross sectional, prospective, and descriptive type. 500 healthy term placentae of the Hausa ethnic group parturients were examined and each was cleansed of blood clots and trimmed of umbilical cord near its point of attachment with the placenta. The placental weight was determined using the triple beam balance. The new born baby weight was determined using Birth weight weighing Balance. The data so recorded was analyzed using Minitab software and Pearson' Correlation Coefficient was used to determine the relationship between the weights.

Results: The result indicated that the birth weight correlates positively (P-Value < 0.05) with the placental weight amongst the Hausa ethnic group. It means that as the birth weight increases, the placental weight also increases.

Conclusion: This study shows that the birth weight as well as the placental weight could be used as indicators for poor or good nutrition of the mother.

\section{PP-120}

Expression of gelatinases and cycloxygenases in one case of myoepithelioma

Lipari L, Mauro A, Leone A, Gerbino A, Gallina S, Buscemi M

Bionec - Dipartimento Di Biomedicina Sperimentale E Neuroscienze Cliniche, 1sezione Di Istologia Ed Embriologia, Sezione Di Otorinolaringoiatria, Facoltà Di Medicina E Chirurgia, Università Degli Studi Di Palermo, Italy

Introduction: Myoepithelioma of the salivary gland is a benign tumor made almost of myoepithelial cells only that are positive to some specific antibodies (e.g. keratin, vimentin, S100 protein) [1]. These myoepithelial cells are transformed and not differentiated, therefore, this tumor is considered as the terminal form of the histopathologic spectrum of mixed tumor, but, owing to its monomorphic appearance, is considered an aside form [2].

Purpose: The aim of this study was to investigate the possible expression of gelatinases (MMP-2, MMP-9) and cyclooxygenases (COX-1, COX-2) in myoepithelioma.

Materials and Methods: We investigated one case of myoepithelioma according to the definition of the World Health Organitation 2005 (WHO), through ImmunoHistoChemistry (IHC) and Reserve Transcription Polymerase Chain Reaction (RT-PCR). Results:In myoepithelioma, both IHC and RTPCR, showed the expression of MMPs; in particular, MMP-2 was observed both in ductal and acinar cells while it was not detected the expression of MMP-9 in acinar cells. The IHC and RT-PCR data showed a strong expression of COX-1 in ductal and acinar cells, while, COX-2 was observed only in ductal cells.

Discussion: Unfortunately, in literature there are not data about the expression of these enzymes in myoepithelioma to compare with our results. This study is in progress and we are investigating a higher number of cases

\section{PP-121}

Determination of age in a population of the lizard, ophisops elegans, by mean of skeletochronology

Yari A

Lorestan University, Lorestan, Iran

Age estimation and some growth parameters were studied in a population of Ophisops elegans from Harsin region, Kermanshah province, Iran, by skeletochronology. Age was determined for individual lizards by counting the number of lines of arrested growth (LAG) in cross-sections taken from femoral bones. The maximum life span of females and males was 5 and 4 years, respectively. The average longevity of males was 2.15 years but this value for females was 2.2 years. The mean snout-vent length (SVL) of specimens collected from this region was $48.18 \mathrm{~mm}$ which showed a diameter of $464 \mu \mathrm{m}$ for their femoral bone and $350 \mu \mathrm{m}$ for its lumen. Based on the information provided here and by other workers we concluded that the LAG observed in this specific taxon is result of hibernation and other environmental factors (such as feeding, pray availability) show no influence on producing the LAG.

\section{PP-122}

\section{Effect of green tea on age-related histological changes in thin skin of female albino rat}

Eltony S, Maksoud SA, Ali S

Histology Department, Faculty of Medicine, Assiut University, Assiut, Egypt

Introduction and Purpose: Skin aging is multifactorial phenomena resulted from both environmental and genetically determined factors and accompanied with a decline in skin functions. The improvement of skin quality has gained particular attention especially the number of aged persons is expected to rise in the next decades. Green tea (GT) is a rich source of antioxidant polyphenols which have a health promoting potential. This study aimed to detect the possible effect of GT 
in preventing the skin histological changes associated with aging.

Tools and Method: 30 female albino rats were used and equally divided into 3 groups: Group I (control adult): 6 months old. Group II (control aged): 18 months old. Group III (GT treated): 12 months old, received GT orally at a dose of $120 \mathrm{mg} / 250 \mathrm{gm}$ body weight/ day for 6 month (i.e. till the age of 18 months). Specimens from the back thin skin were processed for light and electron microscopy.

Results and Interpretations: Aged back skin exhibited reduction in the epidermal thickness and flattening of the dermoepidermal junction (DEJ). Keratinocytes proliferation was decreased as detected by the proliferating cell nuclear antigen (PCNA) immunostaining. Ultrastructurally, some keratinocytes exhibited dark nuclei and vacuolated cytoplasm. While, others revealed less electron dense nuclei and cytoplasm. Disorganized collagen fibers, reduced amount of elastic fibers and few mast cells were observed in the dermis. In GT treated group, the epidermal thickness increased and folded DEJ was observed. The number of PCNA positive keratinocytes was similar to that of the aged group. Ultrastructurally, keratinocytes revealed normal electron dense nuclei and cytoplasm. In the dermis, collagen fibers were more organized and elastic fibers increased in amount. The number of mast cells was mildly increased. GT might have a role in preventing the structural changes of the skin occurred with aging.

\section{PP-123}

\section{Effects of fish n-3 fatty acids on testicular injury induced by ethanol in rats}

Uygur R*, Yagmurca $\mathrm{M}^{* *}$, Alkoc OA***, Genc A***, Songur $\mathrm{A}^{* * * * *}$, Ucok $\mathrm{K}^{* * * *}$, Ozen OA*

Namik Kemal University, School of Medicine, Department of Anatomy, Tekirdag, Turkey*; Fatih University, School of Medicine, Department of Histology\&Embryology, Ankara, Turkey**; Duzce University, School of Medicine, Department of Anatomy, Duzce, Turkey***; Afyon Kocatepe University, School of Medicine, Department of Physiology, Afyonkarahisar, Turkey****; Afyon Kocatepe University, School of Medicine, Department of Anatomy, Afyonkarahisar, Turkey*****

Ethanol exposure is known to suppress male reproductive activity in laboratory animals and humans. The aim of this study was to investigate the effects of fish n-3 fatty acids on the changes in testes induced by ethanol. It has been planned to use 27 adult male rats by assigning them into three groups, namely 1) Control group (SF $3 \mathrm{~g} / \mathrm{kg} /$ day, i.g.), 2) Ethanol (40\% 3 g/kg/day, i.g.) group, and 3) Ethanol + Fish n-3 fatty acid (400 $\mathrm{mg} / \mathrm{kg} /$ day, i.g.) group. At the end of 8 -week, all of the rats were sacrificed. Each left testes were used for biochemical stud- ies, while the right ones were used for histopathological analyses. Testicular tissue were stained with hematoxylin and eosin. Germ cell apoptosis was determined by TUNEL technique. SOD, CAT, and GSH-Px enzyme activities as well as MDA and NO levels in the testis were measured. It has been observed that the ethanol-administered rats have retarded in terms of body weight gain, besides developing degenerative changes in histopathologic analyses as well as showing decrease in seminiferous tubule diameters. TUNEL assay also showed an increase in apoptotic cell number. The activities of SOD, CAT, and GSH-Px were decreased as well as the levels MDA and NO were increased in testis tissue of ethanol group compared with the control groups. Morphologic and morphometric changes and damages caused by ethanol have been recovered partly or completely by giving fish n-3 fatty acids. The activities of SOD, CAT, and GSH-Px as well as the levels MDA and NO were increased in testis tissue of ethanol + fish oil fatty acid group compared with the ethanol groups. In conclusion, this study showed that ethanol causes damage in rat testes morphologically and biochemically, and this damage can be recovered by using fish n-3 fatty acids.

\section{PP-124}

\section{Histological study of gut associated lymphoid tissue in Barbus sharpeyi}

Hamidian $\mathrm{G}^{*}$, Nikbakht $\mathrm{M}^{* *}$, Majd NE***

Department of Histology, Faculty of Veterinary Medicine, University of Tabriz, Iran*; Department of Animal Science, Faculty of Agriculture, University of Yasouj, Iran**; Department of Histology, Faculty of Veterinary Medicine, Shahid Chamran University of Ahvaz, Iran***

The GALT as the primary lymphoid tissue is a part of mucosal lymphoid system that there are considerable differences in its structure and distribution among various fishes and also in different parts of the gut. This study made on the 40 normal, mature and same size male and female fish in summer and winter seasons. After biometrical study, the samples were taken from different parts of intestinal bulb and main intestine. Then tissues were fixed in Bouin's solution and were processed by routine paraffin embedding. Tissue sections were stained by H\&E, PAS and TUNEL techniques. The results showed that the lymphoid tissue of intestinal bulb and main intestine of Barbus sharpeyi is present in two different types; the lymphoid cells were scattered in the mucosal epithelium and the lymphoid cells were present as an aggregated cord-like structure in the lamina propria or as diffuse type in the submucosa. The majority of lymphoid cells were lymphocyte, lymphoblast and a few macrophages and plasma cells. Another finding of this study was the lack of lymphoid follicles and Peyer's patches in 
different parts of the Barbus sharpeyi gut. Results showed that there are a significant difference in the distribution of intraepithelial lymphocyte in different parts of intestinal bulb and main intestine in each seasons and sexes $(\mathrm{P}<0.05)$. The most intraepithelial cells were located in anterior part of intestinal bulb and in posterior part of main intestine in both seasons and sexes. Density of lymphoid tissue of basal epithelium in cold season compared to same area was more than ones in warm season. Although, histological and micrometrical study showed that there are a significant difference in distribution and density of intraepithelial lymphocyte among various regions of gut, but TUNEL technique showed that seasonal difference in the apoptotic lymphocytes numbers were not significant $(\mathrm{P}>0.05)$.

\section{PP-125}

\section{Absence of hyaline cartilage in the tongue of "Caspian miniature horse"}

Nazem MN, Rezaian M

Faculty of Veterinarian Medicine, Tehran University, Iran

Histology of the tongue, including apex, root and body, in four adult Caspian miniature horses was examined. Serial sections with 6 micro meter thickness were stained with haematoxylin eosin and Masson trichrome and studies under light microscope. The tongue was covered by stratified squamous epithelium. It was thick and keratinized bearing numerous lingual papillae on the dorsum, mostly filiform with a very fine keratinized thread projecting above the surface and bending backward. The fungiform papillae were sparsely scattered among the filliform papillae and covered with keratinized squamous epithelium. Few taste buds were detected on it. The two very large vallate papillae were detected on the dorsum, just rostral to the root, which were covered with stratified squamous epithelium with relatively high amount of taste buds in the epithelium of the surrounding grooves. The foliate papillae were present near the palatoglossal arch nand had a few taste buds. The epithelium covering the ventral surface of the tongue was thin and keratinized. The lingual muscle core consisted of transverse, longitudinal and perpendicular bundles of skeletal muscle fibers. Clusters of minor salivary glands were present between the muscle fibres and lamina propria. Most of the lingual glands were mucous and most of the gustatory ones were serous type. The mid - dorsal special structure of the tongue (dorsal lingual cartilage) contained sparse skeletal muscle fibres and was rich in white adipose tissue. Hyaline cartilage, routinely observed in this structure in the horses, was not detected in Caspian miniature horse.
PP-126

\section{Changes of thymus structure of immature white rats after imunofan administration}

Kashchenko S, Zakharov A

Luhansk State Medical University, Ukraine

Last decades one of the major problems in pediatrics is growth of quantity of the diseases steady to adequate ethiotropic therapy. Among the reasons of pathology at children the frequent combination of various illnesses with clinical displays of immune insufficiency syndrome is present. Therefore, last years the group of immunomodulating preparations has been developed and successfully introduced in practice. One of them is imunofan, created with nanotechnology using. However, in the literature there are no data about changes of the thymus structure after application of the given Medicine, as was object of our research. Experiment was carried out on 12 immature white rats-males. Immunoactive state was modelled by imunofan introduction under the scheme $(0.7 \mu \mathrm{kg} / \mathrm{kg})$. As the control rats, whom entered $0.9 \%$ solution $\mathrm{NaCl}$, served. Animals were taken out from experiment in 15 days by decapitation method under ether anesthesia. Thymus was extracted, weighed, exposed to the standard histological method and studied by automatic morphometric complex. Significant positive differences of absolute and relative weights of thymus from data of control group of animals on level $12.40 \%$ and $17.28 \%$ accordingly were found out. The width and the area of cortex significantly exceeded control indexes on $7.15 \%$ and $9.30 \%$. The cortex-medulla index of thymic lobules of animals of control group was less on $40.87 \%$ of an indicator of experimental group rats. Thus, dynamics of studied morphometric parameters changes specified on the decrease of natural involutive processes in thymus after imunofan administration.

\section{PP-127}

\section{Thymus of newborn rats after prenatal alcoholisation}

Pugach $\mathrm{P}^{*}$, Kruglov $\mathrm{S}^{* *}$, Karelina $\mathrm{N}^{* *}$, Lukina $\mathrm{N}^{* *}$

I.I. Mechnikov St.-Petersburg State Medical Academy, St.-Petersburg, Russia*; St.-Petersburg State Pediatric Medical Academy, St.Petersburg, Russia**

Ethanol causes considerable infringements of structure and function of all systems, both an organism of mother, and posterity. The purpose - to study features of the newborn rats thymus structure that were developed in the conditions of ethanol influence on females before pregnancy and during its extent.

Material and Methods: Thymuses of 115 newborn rats that were burned by 30 white rats were studied by morph metric and 
histological methods. Females were divided into 4 groups. Rats of I group received drink water before pregnancy and - 15\% ethanol during pregnancy. Females of II, III, and IV groups received $15 \%$ ethanol during 1, 2 and 3 months before pregnancy and on its extent. As control the newborn rats who were born from intact females were served.

Results: At newborn rats of I group decreasing of absolute and relative weight of thymus in 2.4 and 15 times accordingly were found. The quantity of lymphocytes was decreased in parenchyma; erythrocytes located out of vessels were observed. At newborn rats of II group absolute weight of thymus decreased in 2.5, and relative in 52.5 times. In parenchyma the number of lymphocytes sharply decreased, the border between cortex and medulla of thymus was erased. In a significant amount were defined extra vascular erythrocytes. Absolute weight of thymus of newborn rats of III group has been lowered in 1.4 times, relative in 13 times. The number of lymphocytes decreased sharply. The expanded vessels were found, but extra vascular erythrocytes were absent. At newborn rats of IV group absolute and relative weights of thymus essentially didn't change. Decreasing in quantity of lymphocytes was observed only in sub capsular zone.

Conclusions: Prenatal influence of $15 \%$ ethanol causes changes in thymus. Expressiveness of changes depend on duration of pregravidational ethanol influences on females.

\section{PP-128}

\section{Peculiarities of the structure of spleen under the influence of toluene}

Koveshnikov V, Luzin V, Voloshin V, Voloshina I

Lugansk State Medical University, Ukraine

Introduction: At present there is a fairly large number of products made of polymer and synthetic materials and plastics manufactured on the basis of ep-oxy resins which are used in dwellings. The polymer materials discharge toxic chemical substances into the environment (formaldehyde, phenol, toluene, etc.). Purpose. The aim of this work is the study of the peculiarities of the morphogenesis of the spleen after the influence of toluene.

Tools and Methods: The work was done on male rats of puberty age. The rats of the experimental group experienced the influence of toluene within two months. After the term was over, the animals were taken out of the experiment by way of decapitation under ether narcosis. We studied the sizes of the spleen, its absolute and relative masses and histological composition.

Result and Interpretation: The results of the research show the changes of linear sizes of the spleen of the experimental animals as compared to the control ones. At the same time we note the decrease of the absolute and increase of the relative mass of the spleen in the animals which underwent the influence of the toluene. We noted the difference between the control and experimental indices as to the area of the white and the red pulp at the histological preparations. We established the correlation ties between such morphometric indices of the spleen as diameter of lymphatic knots of the white pulp, the wideness of the marginal zone. The received data testify about the morphological changes of the spleen of the animals which were in the conditions of the influence of toluene on the organism. It stipulates a great interest to the further study of the anatomy of the spleen as a secondary lymphoid organ, under the influence of toluene on the organism.

\section{PP-129}

The morphological status of the cerebral cortex, liver, gut mucosa and spleen in cases of SIDS victims

\section{Knipse G, Gramatniece A, Markovs J \\ Department of Anatomy and Histology, University of Latvia}

Sudden infant death syndrome (SIDS) remains a complicated diagnosis with lack of a biologically plausible aetiology. SIDS often is linked to infectionwith a common bacteria. Therefore the toxic infection theory is plausible. The aim of this study was to verify the occurence of morphological changes in neurons, astrocytes and in immunocompetent cells in SIDS victims. Samples from 8 SIDS victims 2-5 months of age and 7 aged mached control infants who died acutely from accidents were compared. Using immunohistochemical methods we examined the distribution of CD68, iNOS positive macrophages and CD20 B lymphocytes in the liver, gut mucosa and spleen of SIDS cases and controls. Neocortical areas (gyrus precentralis and postcentralis) was also investigated for the presence of degenerated neurons and astrocytes using glial fibrillary acidic protein immunohistochemistry. SIDS victims produced more CD20 positive B cells in the germinal centers of the spleen. Thus, there was an increase in the mean diameter of the germinal centers of the spleen in SIDS victims in comparision with controls $(105.2+/-3.0 \mu \mathrm{m}$ v.s. $101.7+/-2.7 \mu \mathrm{m})$. The number of iNOS expressing intestinal macrophages increased in SIDS cases $(23.6+/-3.0$ cells / mm2 v.s. $31.3+/-3.7$ cells / $\mathrm{mm} 2$ ), as well as the cross-sectional surface area (CSSA) of Kupffer cells of the liver parenchyma (4.5\% v.s. $2.7 \%$ ) and the number of CD20 positive B lymphocytes in the portal tracts of the liver ( $12.21+/-1.9$ cells / mm2 v.s. $8.3+/-2.7$ cells / mm2. Morphological changes in neurons and astrocytes of neocortex of SIDS victims were not observed. We hypothesize, that SIDS might have an immunological basis (e.g. a genetically determined inability of the immune system to eliminate an infectious agent). As a result, chronic immune response leads to NO overload, dilatation of the blood vesels and toxic shock. 


\section{PP-130}

The arterial supply of the peroneus brevis muscle, its importance in planning for the muscle flaps

Alkushi A, Elbarrany W

Collage of Medicine Umm Alqura Unversity, Macca, Saudi Arabia

Introduction: The arterial supply of the peroneus brevis muscle, its importance in planning for the muscle flaps Wagih G. Elbarrany and Abdullah Alkheshy Anatomy Department, Faculty of Medicine, Umm Al-Qura University, Makkah, Kingdom of Saudi Arabia Objectives: To study the blood supply to the peroneus brevis muscle to evaluate the anatomical basis of its flaps.

Materials and Methods: Both legs of 10 preserved cadavers were used in the current study. The peroneus brevis was dissected to identify its length, width and ratio of fleshy part to the tendinous one. The sources, lengths and diameters of its arterial pedicles and their distances of the tip of the lateral malleolus were also studied.

Results: The muscle got arterial pedicles from the peroneal artery in all the studied cadavers. The peroneal artery in $70 \%$ of the studies cases continued to anastomose with septal perforating branch of the posterior tibial artery. It got also pedicles from the perforating branches of the anterior tibial artery, the artery accompanying the superficial peroneal artery. The lower part also got vascular branches from the periosteal arteries of the fibula. The number of the pedicles were at an average of 5 pedicles (ranged 4-6 pedicles). The average distance of the distal pedicle from the tip of the lateral malleolus was $4 \mathrm{~cm}$ (ranged, 3-6 cm). The average muscle length was $30 \mathrm{~cm}$ (ranged from 25 to $33 \mathrm{~cm}$ ), the average length of its tendon was at an average of $8 \mathrm{~cm}$ (ranged from 6.8 to $9.3 \mathrm{~cm}$ ) and the average length of the leg was $40 \mathrm{~cm}$ (ranged from 36 to $44 \mathrm{~cm}$ ). The maximum width (the middle third) was at an average of $3 \mathrm{~cm}$ (ranged from 2.6 to $3.7 \mathrm{~cm}$ ).

Conclusion: The peroneus brevis muscle can be used in $70 \%$ of cases as a distally pedicled or proximally pedicled flap and could be used as a free vascularized muscle flap.

\section{PP-131}

Gantzer muscle in fetuses and adult cadavers and its relation to anterior interosseous nerve

Kara A*, Elvan O*, Yildiz $\mathrm{S}^{* *}$, Ozturk $\mathrm{H}^{*}$

Department of Anatomy, Faculty of Medicine, University of Mersin, Mersin, Turkey*; Department of Anatomy, Faculty of Medicine, Gülhane Military Medical Academy, Ankara, Turkey**

The present study was conducted to examine the accessory heads of the flexor pollicis longus (FPLah) and flexor digito- rum profundus muscles (FDPah) in human fetuses and adult cadavers which are also known as Gantzer muscle. Ninety forearms of fetuses and 52 forearms of adult cadavers were dissected in order to evaluate the incidence, morphology and innervation of the Gantzer muscles. The FPLah was observed in $33 \%$ of the fetal forearms and $39 \%$ of adult cadaver forearms and FDPah was observed in 14\% of the fetal forearms and 33\% of adult cadaver forearms. The FPLah was found in 11 fetal and 8 adult cadavers bilaterally, and in 8 fetal and 4 adult cadavers unilaterally. The FDPah was found in 3 fetal and 5 adult cadavers bilaterally, and in 6 fetal and 7 adult cadavers unilaterally. The FDPah was also found in 2 amputated upper limbs. Coexistence of both types was found in 4 fetal and 3 adult cadavers unilaterally. All of the FPLahs were supplied by the anterior interosseous nerve (AIN) both in adults and in fetuses. The FDPah supplied by the MN were determined in 3 sides of adults and in 1 side of fetuses. Both the FPLah and FDPah mostly originated either from the under surface of the flexor digitorum superficialis (FDS) alone or from the medial epicondyle via FDS. Anterior interosseous nerve syndrome (AINS) is an upper extremity neuropathy caused by the compression of the nerve in the forearm by the FPLah as one of the causes. Therefore, relation of FPLah to the anterior interosseous nerve was evaluated and classified.

\section{PP-132}

\section{Morphometry of palmaris longus in human fetuses}

Kocabiyik N, Yildiz S, Develi S, Ozan H

Department of Anatomy, Gülhane Military Medical Academy, Ankara, Turkey

Introduction and Purpose: The palmaris longus arises from the common origin. It is absent in $10-15 \%$ of arms. Its long, flat tendon broadens as it passes in front of the flexor retinaculum, to which it is partly adherent. In the palm it splits to form the longitudinally directed fibres of the palmar aponeurosis. The tendon lies in the front of the median nerve just above the wrist.

Tools and Method: Present study is carried out on 24 human fetuses aged 17-40 weeks of gestation with no external pathology or anomaly. We measured the length and width of the tendinous portion and muscular belly of the palmaris longus in human fetuses.

Findings: The palmaris longus muscles were absent in both arms in 7 fetus and in a unilateral arm in 5 fetus. Reversed palmaris longus muscle was found in 2 arms. Most fetuses had a typical palmaris longus muscle and tendon shape. The mean length and width of the tendons were 18.42 and $0.66 \mathrm{~mm}$, respectively, in second trimester and, 25.9 and $1.71 \mathrm{~mm}$ in third trimester, respectively. The mean length and width of the muscular belly were 13.33 and $2.03 \mathrm{~mm}$, respectively, in second trimester and, 30.42 and $3.77 \mathrm{~mm}$ in third trimester, respectively. 
Result and Interpretation: The results of our study show a relatively high incidence of tendon absence and pointed to more pronounced loss of the muscle on the left side. This detailed fetal study in relationship to the palmaris longus muscle would be useful for educational anatomy dissections and surgical interventions for relevant clinical procedures.

\section{PP-133}

\section{Anatomic and topographic relation of the sciatic nerve to piriformis muscle}

Selaru M, Sisu A, Moise M, Stana LG, Jianu A, Motoc A, Valceanu A

University of Medicine and Pharmacy "Victor Babes", Timisoara, Romania

Sciatic nerve goes under the piriformis muscle at its inferior border. It travels through the greater sciatic foramen, and lies on the thigh, providing motor innervation to the flexors of the knee and all of the muscles of the leg and the foot. Bifurcation into two major divisions (common peroneal and tibial) occurs anywhere between the sacral plexus and the lower part of the thigh. The separation of the nerve into its terminal branches has been reported to occur below the popliteal space. The aim of our study was to reveal the variations in sciatic nerve to piriformis muscle relationship. Our study was made on 30 lower limbs bilaterally (60 cases), with a ratio male/female 2/1 (20/10). In majority of cases, 54, (90\%) the relationship with the sciatic nerve is that it passes from the pelvis beneath piriformis muscle. Piriformis muscle divided into two parts with the peroneal division of the sciatic nerve passing between the two parts of piriformis (a lower majority, 15\%, 9 cases). The peroneal division of the sciatic nerve passes over piriformis muscle and the tibial division passes beneath the undivided muscle (few cases, 5\%, 3 cases).Yeoman (1928) referred to the piriformis muscle as a cause of sciatic pain and to sacroiliac joint lesions as a cause of inflammatory reaction of the piriformis muscle and fascia. The piriformis muscle syndrome complex has been described by Retzlaff and includes low back pain (persistant and severe) from the sacrum over the gluteal region, the posterior portion of the upper leg to the popliteal space. Piriformis syndrome is not life-threatening, but it can have significant associated morbidity.

\section{PP-134}

\section{Complex variation of median nerve: a case report}

Ozen OA*, Songur A**, Gonul Y*, Uygur R*

Department of Anatomy, School of Medicine, Namik Kemal University, Tekirdağ, Turkey*; Department of Anatomy, School of Medicine, Afyon Kocatepe University, Afyonkarahisar, Turkey**
Variations of the vessels and nerves in the arm are common. We saw complex variation of median nerve in the right arm of an adult male cadaver. During routine education dissections of axillary region, a complex variation was observed in the right arm of an adult male cadaver. Lateral cord continued as lateral root of median nerve without giving off musculocutaneous nerve and it formed median nerve joining with medial root of median nerve which arises from medial cord. There was a communicating accessory branch $2.5 \mathrm{~cm}$ long running obliquely between the cords which form the median nerve. Coracobrachialis muscle was innervated by a thin branch arising from lateral cord. We observed that the median nerve divided into two branches at a point $9 \mathrm{~cm}$ distal to its formation. First branch supplied motor innervation to biceps brachii, the second branch gave motor branch to brachialis muscle $7 \mathrm{~cm}$ distant from the division and continued as lateral antebrachial cutaneous nerve. Main trunk passed through the cubital fossa and ran along the forearm following the normal course of median nerve. Peripheral nerve injuries due to traffic accidents, occupational accidents and firearm injuries are increasingly encountered. A good knowledge of classic and variational anatomy of upper extremity peripheral nerve patterns is essential for surgical procedures. Variations of median nerve, which are frequently observed, can increase possibility of sequelae and potential complications in consequence of erroneous clinical practice during surgical procedures and peripheral nerve blocks. Good knowledge about the variations of median nerve and musculocutaneus nerve provides good results concerning the plastic and reconstructive surgical operations on the arm. We think it is important that clinicians performing surgical procedures or nerve blocks in this region should consider this variation in order to avoid failures and complications.

\section{PP-135}

NGFR (nerve growth factor receptor) expression on peripheral nervous system in mice. An immunohistochemical investigation

Erdogan $E^{*}$, Hasirci $Z^{* *}$, Ozturk $G^{* * *}$, Kara $M^{* * * *}$, Dursunoglu D*

Department of Histology and Embryology, Selçuklu Medical Faculty, Selçuk University, Konya, Turkey*; Kızılay Konya Hast, IVF Unit, Konya, Turkey**; Department of Physiology, Medical Faculty, Medipol University, Istanbul, Turkey***; Department of Histology and Embryology, Medical Faculty, Yüzüncü Yıl University, Van, Turkey****

Neurotrophins are trophic factors which affect vital functions as development, differentiation, viability and metabolism of nervous system cells. These soluble and polypeptide form factors secreted from neuron and other target tissues as muscle and connective tissues and transported retrograde from axon to the soma. Their effects were acted via two types of receptor (high affinity tyrosine kinase A, B, C and a low affinity receptor p75). This study was aimed to determine the expression of these receptors. For this 
purpose, L4 and L5 spinal ganglions were taken bilaterally from mice. After fixation (paraformaldehyde 4\%) and sucrose (30\%) processing, 5-30 $\mu \mathrm{m}$ thickness sections were taken by frozen section and then labelled anti Trk A, B, C and P75 primary and fluorescent conjugated secondary antibodies. Tissue samples were examined with confocal and fluorescent microscopes and imagines were captured digitally. In the confocal microscopic examination of sections: the strongest immunoreactivity were in the Trk A and then Trk B while the weakest were of Trk C and p75 respectively. Between the groups of all receptors, showing the highest response were adult male and then adult female groups while showing the least response was the young female group. As a result, the undeniable importance of neurotrophins in neural physiology of the receptor of primary sensory neurons in dorsal root ganglion, expression of these was shown immunohistochemically in different proportions and manner.

\section{PP-136}

Analyses of wildtype and ciliary neurotrophic factor(CNTF)-deficient olfactory ensheathing cell/neuron cocultures indicate indirect effects of CNTF on neurite growth

Boemmel H, Asan E

Institute of Anatomy and Cell Biology, University of Würzburg, Germany

Olfactory ensheathing cells (OEC) provide a microenvironment within the olfactory nerve permitting and promoting targeted outgrowth of olfactory sensory neuron (OSN) axons. Production of neurotrophic factors such as CNTF in OEC has been suggested to modulate axon-growth promoting properties of these unusual glial cells. To investigate functions of CNTF in this context, we designed coculture systems using OEC and neurons (olfactory sensory neurons, OSN and cortical neurons, $\mathrm{CN}$ ) from rat, wildtype (wt) and CNTF-deficient mice. In previous studies, we found that wt and CNTF-deficient OSN exhibited significantly longer mean neurite lengths if cultured on CNTF-deficient compared to wt OEC. Moreover, addition of CNTF-blocking antibodies to rat OEC/OSN and OEC/CN cocultures led to increased neurite lengths. BrdU labelling and TUNEL analyses indicated neither enhanced proliferation of neuronal precursors likely present in OSN cultures nor altered survival rates of OSN in cocultures with wt compared to CNTF-deficient OEC. In monocultures of rat OSN, neurite growth differed significantly depending on the growth substrate (merosin or laminin), but was not influenced by addition of recombinant CNTF to the medium at a concentration suited to induce reduced neurite length in CNTF-deficient mouse OSN/OEC cocultures. Preliminary experiments showed significantly increased neurite length of both wt and CNTF-deficient OSN on extracellular matrix (ECM) from CNTF-deficient versus wt OEC. Our data indicate that CNTF from OEC might indirectly influence neurite outgrowth, possibly via differential- ly modulating production of ECM-associated components by OEC. Currently, microarray studies are underway to assess differences in expression levels particularly of ECM-associated molecules in wt and CNTF-deficient OEC.

\section{PP-137}

\section{Ultrastructural analysis of different injury models of sciatic nerve}

Demirel BM*, Sati L**, Hizay A*, Tanriover G**, Ozsoy U*, Ozturk $\mathrm{S}^{* *}$, Sarikcioglu L*, Demir $\mathrm{N}^{* *}$, Oguz $\mathrm{N}^{*}$

Department of Anatomy, Faculty of Medicine, Akdeniz University, Antalya, Turkey*; Department of Histology and Embryology, Faculty of Medicine, Akdeniz University, Antalya, Turkey**

Objective: The aim of the study was to produce different injury models on sciatic nerve and to examine the effects of different injury models from ultrastructural point of view.

Materials and Methods: A total number of 110 female rats were used. In addition to control and sham groups, the rest of animals were divided into 3 different experimental groups: crush, total cut and hemisection. Experimental groups were examined on postoperative 48th hour, 14th day and 1st month. The sciatic nerve was crushed 30 seconds by a clamp. In total cut groups, transvers sectioning and then coaptation was performed. In hemisection group, the sciatic nerve was cut transversely along the half of the width. After the postoperative period sciatic nerve samples were harvested and were processed for ultrastructural analysis.

Results: According to our results, various ultrastructural differences were observed on sciatic nerve according on the nerve injury model. Less number of remylinazed nerve fibers was found in the total cut groups; on the contrary we observed more and thick remylinazed fibers in the crush group. In the hemisection group, fascicular degeneration, located in different areas of the total area of the nerve, was observed.

Conclusion: Our study is of importance to reveal the effects of different injury models on sciatic nerve. Therefore, our data may yield a very important feature of peripheral nerve injury models and shed light to further studies in this field. Observing fascicular degeneration located in various areas in the hemisection groups reveals that internal topography of sciatic nerve is an important factor during regeneration.

\section{PP-138}

Morphological analysis of neovascularisation of sciatic nerve after applying different sciatic nerve injury models

Demirel BM*, Sati L**, Hizay A*, Tanriover G**, Ozsoy U*, Ozturk $\mathrm{S}^{* *}$, Sarikcioglu L*, Demir N** Oguz N*

Department of Anatomy, Faculty of Medicine, Akdeniz University, Antalya, Turkey*; Department of Histology and Embryology, Faculty of Medicine, Akdeniz University, Antalya, Turkey** 
Objective: The aim of the study was to produce different injury models on sciatic nerve and to examine the effects of different injury models on neovascularization of the sciatic nerve.

Materials and Methods: A total number of 110 female rats were used. In addition to control and sham groups, the rest of animals were divided into 3 different experimental groups: crush, total cut and hemisection. Experimental groups were examined on postoperative 48th hour, 14th day and 1st month. The sciatic nerve was crushed 30 seconds by a clamp. In total cut groups, transvers sectioning and then coaptation was performed. In hemisection group, the sciatic nerve was cut transversly along the half of the width. All injury zones were photographed before and after the operation.

Results: According to our results, various morphological differences were observed on sciatic nerve neovascularisation according on the nerve injury model. Neovascularization was observed both at site of the injury and at proximal side of the injury zone. Neovascularization was observed more organized in the hemisection groups; on the contrary neovascularization was poorly organized in the other groups.

Conclusion:We think that organized neovascularization of the sciatic nerve as observed in the hemisection groups may play an important role during regeneration.

\section{PP-139}

The suprascapular nerve: macroscopic and microscopic study and implications in the entrapment syndrome

Porzionato $A^{*}$, Stecco $C^{*}$, Todros L**, Gagliano G**, Macchi $\mathrm{V}^{*}$, Parenti $\mathrm{A}^{*}$, Aldegheri $\mathrm{R}^{* *}$, De Caro $\mathrm{R}^{*}$

Department of Human Anatomy and Physiology, University of Padua, Padua, Italy*; Department of Medical and Surgical Specialities, University of Padua, Padua, Italy**

The suprascapular nerve entrapment syndrome is an underestimated cause of shoulder pain. Ten fresh cadavers were dissected to analyze the course of the suprascapular nerve. From each cadaver, selective samples of the nerve together with the tissue around were taken for an histological study. The anatomic study demonstrates the presence of 3 different ramification patterns of the suprascapular nerve in the infraspinatus muscle. Relevant data were also the observations of the different shape of the suprascapular notch, the variable thickness of the superior transverse ligament, the course of the suprascapular artery with respect to the nerve and the transverse ligament and finally the relationships between the suprascapular nerve, the supraspinatus ditch and the homonymous muscle. The histological study demonstrates the presence of fibro-adipose tissue surrounding the nerve, forming a "connective small cushion". This structure provides protection and allows a free sliding of the nerve inside the muscle. In cases of thinner fibro-adi- pose tissue, the nerve could be exposed to mechanical stress under contraction. These remarks allow to suppose that the suprascapular nerve entrapment syndrome can arise not only at the suprascapular or at the spinoglenoid notch, but also inside the muscle during contraction.

\section{PP-140}

\section{Anatomical and radiological study of the superior gluteal nerve and artery: implications for hip surgery}

Stecco $C^{*}$, Porzionato $\mathrm{S}^{*}$, Macchi $\mathrm{V}^{*}$, Baggio L* ${ }^{* *}$ Gagliano $\mathrm{G}^{*}$, Parenti A*, Aldegheri R**, De Caro R*

Department of Human Anatomy and Physiology, University of Padua, Padua, Italy*; Department of Medical and Surgical Specialities, University of Padua, Padua, Italy**

The purpose of this study is to identify, in the hip prosthesis with Hardinge's direct lateral approach, the site of incision with the lowest risk of injury of the superior gluteal nerve (SGN). Four fresh cadavers were dissected and nerve branches of the SGN and superior gluteal artery (SGA) were isolated. The distance between the SGA and the trochanter major (TM) was also evaluated in vivo in 29 patients by CT-Angiography. In cadavers we observed 3 spray pattern and 1 trunk pattern of the SGN. In all cases the nerve runs inferiorly to the artery, with a mean distance of $0.5 \mathrm{~cm}$. At the CT-angiography the average distance between the main branch of SGA and the trochanter major was $5.2 \mathrm{~cm}$, indicating a mean distance of 4.7 $\mathrm{cm}$ from the SGN to the TM. Terminal branches of SGA are found until $2.7 \mathrm{~cm}$ from trochanter major. This study have analyzed for the first time the relationships between superior gluteal neurovascular bundle and the trochanter major in vivo (considering also the muscular tone), showing that during the direct lateral access exists a safe area of $4.7 \mathrm{~cm}$ over the trochanter major.

\section{PP-141}

\section{Radiographic examination of the greater sciatic notch in determining the sex among the Iranian people}

Showra A, Ali H, Zohreh A

Department of Anatomical Science, Hamedan Medical University, Iran

It is well known that there are metric and nonmetric variations in the expression of sexual dimorphism between racial phenotypes and populations. The creation of skeletal anthropometric measurements of different populations is useful for both comparisons with similar studies and for improving the identifica- 
tion of human skeletal remains. The distinctive morphology of the human hip bone (os coxae) and its clear sexual dimorphism make it of interest from anatomical, anthropological and forensic points of view. The features of the greater sciatic notch of the coxae are characteristic and are commonly used to determine sex in unknown individuals. In this study, several measurements of the greater sciatic notch including width $(\mathrm{AB})$, depth (OC) and width of the posterior segment (OB) were taken and indices I and II were calculated in 64 adult male coxae radiography. Results indicated that there are significant differences in $\mathrm{AB}$ ( $\mathrm{p}$-value $=0.000<0.05$ ) between the both sexes of left and right hip bone. There aren't significant differences in OC ( $p$-value> 0.05). There are significant differences in $\mathrm{OB}(\mathrm{p}$-value $=0.000<0.05)$ between the both sexes of left and right hip bone. There aren't significant differences index I and II using t-test. Some of these results can be used to aid in the identification of human skeletal remains in Iranian people.

\section{PP-142}

\section{The relationship between parental and neonatal anthropometric parameters}

\author{
Ghasemi M, Mahmoodabadi N, Kermani T \\ Birjand University of Medical Science, Medica Schooll, Birjand, Iran
}

Purpose: The present paper deals with the relationship of parental and neonatal anthropometric measurements, due to the fact that it is a basic index for development and growth in next years.

Material and Methods: This is a cross -sectional descriptive study. Measurements were made on 150 neonates with their parents from September 2009 to March 2010. Tools include caliper tape-measure, scale. Measurements included length and height of face and head, auricular height, head circumference height, weight presentational weight, biparietal diameter, humeral circumference, chest circumference, femoral circumference, foreleg circumferences, abdominal circumference and shoulder width. Data were analyzed by SPSS software.

Results: Significant relationships were found between neonatal and maternal foreleg Circumference $(\mathrm{p}=0.002 \mathrm{r}=0.3)$ as well as between neonatal and maternal current weight $(\mathrm{p}=0.002$ $\mathrm{r}=0.293$ ).

Conclusions: According to this study, a significant relationship between maternal and neonatal weight was found. Other researches in this field have confirmed that this parameter can be a good predictor for neonatal birth weight. There was also a significant correlation between maternal and neonatal foreleg circumference. Hence, it can be a useful index for anthropometry. No significant relationship was found between paternal and neonatal anthropometric measurements. Also, other studies have confirmed there is not such a relationship.

\section{PP-143}

\section{Anthropometry of the external ear in $\mathbf{4 0 0}$ Iranian people}

Rayegan P, Hasan S, Vaghefi E, Kohestani HR, Zargarpoor R, Raygan R

Kerman Medical School, Kerman, Iran

Human beings can be seen in different shapes. The external particulars especially different aspects of the face are important in recognition of the people and are varied depending on the face, race, kind, ecological particulars and acquired changes from one person to another. In close community, these particulars are close to each other and even caused distinction from the other community. Face has the main role in recognition of people. Considering the shape of the auricle from the point of the beauty and recognition some of the congenital diseases related to the external ear is important. To measure different dimensions of auricle, we use instruments such as caliper cephalometer, Mourison's compasses, and the ruler. About 400 men and women (200 men, 200 women) at the age of 20-30 were selected for anthropometry in Kreman. Choice criterion were healty people (lack of former troma e.g. in wrestlers) and from different communities (students from Kerman medical university and Bahonar university, employees of university's hospital, references to hospitals, especially visitors, patients that were hospitalized in different wards except ear, nose, throat wards) that selected in the form of racemose. We try to choose samples from original Kermanian people( at least two former generations that live in Kerman). The average length \& width of the ear, shape of the louble, length \& width of the conchae, angle of the cephaloauricular, ear-skull \& tragus-alar distances and the length of the nose in men and women were $59.86 \mathrm{~mm}, 30.71 \mathrm{~mm}$, round, $24.32 \mathrm{~mm}, 17.83 \mathrm{~mm}, 57.63 \mathrm{~mm}, 8.71 \mathrm{~mm}, 101.72 \mathrm{~mm}$, $50.31 \mathrm{~mm}$, and $60.12 \mathrm{~mm}, 31.36 \mathrm{~mm}$, round, $25.32 \mathrm{~mm}, 17.73$ $\mathrm{mm}, 55.68,8.40 \mathrm{~mm}, 101.36 \mathrm{~mm}$, and $40.76 \mathrm{~mm}$, respectively. Comparison between the right and the left auricle revealed that the average of length \& width of the ear, the shape of the lobule, the length \& the width of the conchae, the angle of the cephaloauricular, the ear-skull \& the tragus-alar distances in the right and the left ear were $59.86 \mathrm{~mm}, 30.63 \mathrm{~mm}$, round, $24.46 \mathrm{~mm}, 17.72$ $\mathrm{mm}, 54.83,8.30 \mathrm{~mm}, 101.56 \mathrm{~mm}$, and $60.13 \mathrm{~mm}, 3.43 \mathrm{~mm}$, round, $25.19 \mathrm{~mm}, 17.84 \mathrm{~mm}, 58.48,8.81 \mathrm{~mm}$, and $101.52 \mathrm{~mm}$, respectively. Also the amount of the lop-ear deformity (auricle) in men and women, were $10.5 \%$ and $7.8 \%$, respectively.

\section{PP-144}

\section{The relationship between anthropometric measurements and antero-posterior balance in young adults}

Zagyapan R, Iyem C, Kurkcuoglu A, Pelin C

Department of Anatomy, Faculty of Medicine, Baskent University, Ankara, Turkey

Evaluation of balance control and postural stability is of importance for physiatrist and physiotherapists not only for more 
reliable diagnoses but for a better treatment planning as well. Postural stability is thought to be affected from body characteristics apart from age and gender. However studies on the relationship between anthropometric dimensions and body equilibrium are highly limited though postural disorders are common in our country. In the present study the relationship between anthropometric variables and body equilibrium had been evaluated in young Turkish adults. Subjects and methods The study was carried out on 116 female and 124 male individuals. The standing posture of the subjects was evaluated by lateral alignment tests and muscular shortenings were evaluated for pectoral, hamstring group, iliopsoas, gastrocnemius muscles and lumbar extensor muscles. Besides five anthropometric measurements as stature, body weight, waist and hip circumferences and supraspinal skin-fold thickness were taken. Body mass index and waist / hip ratio were also calculated. During statistical analysis as a method of testing for the two groups t-test, one way analysis of variance for three or more groups, and Bonferroni method for multiple comparisons were used. Chi-square analysis was used for discrete measurements. For all statistical tests $\alpha=0.05$ and the first type of error as \%5 was kept constant. Results Lateral alignment tests indicated that 107 of 240 subjects had an anterior balance while 89 had posterior, and 44 had neutral balance. When the individuals in different type of anterior-posterior balance groups were compared with each other from the view point of anthropometric measurements waist and hip circumferences were significantly higher in anterior balance group. Besides the absolute circumference values, waist / hip ratio was also higher in anterior balance group. Shortening in hip flexors and hamstring group muscles was significantly prominent in the subjects with anterior balance $(\mathrm{p}<0.05)$. No statistically significant difference was found between the balance groups from the view point of the other anthropometric variables. Conclusion The results of the present study indicate that anterior balance is significantly related with circumference values. On the other hand though shortening in hip flexors and hamstring group have a significant affect on anterior balance shortening in lumbar extensors were not related with posterior balance.

\section{PP-145}

\section{Limb vein pattern analysis for human forensic identification}

\author{
Meadows H, Bellini A \\ Universtiy of Dundee, Dundee, UK
}

The venous vascular pattern is influenced by both genetic and environmental factors. The influence of individual factors results in a pattern that is unique to the individual and can therefore be utilized for personal identification. Vein Mapping and Pattern Recognition is currently employed as a biometric authenticator in a number of high security areas to heighten the degree of sensitivity surrounding confirmation of personal identity. Veins can be identified in the living using near infrared light, whereby reflected or transmitted images of blood vessels can be detected via the reaction between light and the deoxygenated blood in the subcutaneous vessels. Infrared images are analyzed to develop an automatic way to identify, represent the superficial vasculature of the back hand and investigate its discriminative power for human forensic identification. A prototype of a system that extracts the superficial venous pattern of the dorsum of the hand from infrared images For forensic identification purposes is described.

\section{PP-146}

\section{D CT anatomy of the pneumatizations around the pterygopalatine fossa}

\author{
Rusu MC, Sandulescu M, Jianu AM
}

Carol Davila University of Medicine and Pharmacy Bucharest, Romania

Even though the pterygopalatine fossa $(\mathrm{PPF})$ is a region of surgical interest, yet there are no details of the variable pneumatization pattern around it and the usual descriptions of its limits are usually bone-related. The objective of the study was to evaluate the variation of pneumatization neighboring the PPF and to pattern it, using not dry skulls but computer tomograms and $3 \mathrm{D}$ reconstructions. One hundred patients cone beam CTs were so retrospectively evaluated to determine the pneumatization pattern of the PPF walls. The fossa was divided in two floors, upper, orbital, and lower. Variability of the upper floor was determined by: (1) the variable topography of the posterior ethmoid cells; (2) the variable pneumatization of the sphenoidal sinus. The pterygoid recess of the sphenoidal sinus was the variable feature of the lower floor of the PPF. As it resulted, although the osseous anatomy of the PPF is relatively constant, the pneumatization pattern around the fossa is individually variable, mostly related to the variation of the sphenoidal sinus and ethmoid cells. These variables must be taken into account when the PPF is surgically accessed.

\section{PP-147}

\section{Estimation of the volume of the lung using stereological methods on computed tomography scans: a gold standard study}

Edizer $M^{*}$, Sahin $B^{* *}$, Taslak Sengul $A^{* * *}$, Celenk $C^{* * * *}$, Basoglu $\mathrm{A}^{* * *}$

Department of Anatomy, Medical School, Dokuz Eylül Universtiy, Izmir, Turkey*; Department of Anatomy, Medical School, Ondokuz Mayıs University, Samsun, Turkey**; Department of Thoracic Surgery, Medical School, Ondokuz Mayıs University, Samsun, Turkey*** Department of Radiology, Medical School, Ondokuz Mayıs University, Samsun, Turkey****

Introduction and Purpose: The lung volume is a determining factor for the monitoring and surgical procedures of the 
patients. However, we have not found a study evaluating the accuracy of the lung volume estimations using computed tomography images. In the present study we aimed to evaluate to compare the estimated volumes with the actual volumes of cadaveric lungs.

Tools and Method: Twenty lungs were obtained from the cadavers. The lungs were scanned with a CT machine using high resolution computed tomography protocol. The section series with thicknesses of $1 \mathrm{~mm}$ were obtained. The cut surface areas of the lungs were obtained using ImageJ software. The lungs volumes were estimated using the Cavalieri principle of stereological methods. The actual lungs volumes were obtained by Archimedean principle.

Findings: The mean actual lung volume was $1366.55 \mathrm{~cm}^{3}$. The mean estimated lung volume from the CT images was 1380.25 $\mathrm{cm}^{3}$ respectively. The estimated volumes were over-estimated from the actual volumes and the deviation was $0.83 \%$. There is no significant difference between the measured values with actual values.

Results and Interpretations: Our results showed that the estimated lung volume was close to actual volumes. The combination of stereological methods with CT scanning can be used safely to obtain quantitative data for the lung.

\section{PP-148}

Comparison of the calcaneal pitch angle method and modified projection area per length squared method for medial longitudinal arch evaluation

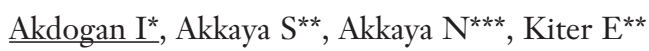

Department of Anatomy, Faculty of Medicine, Pamukkale University, Denizli, Turkey*, Department of Orthopedics and Traumatology, Faculty of Medicine, Pamukkale University, Denizli, Turkey**, Department of Physical Medicine and Rehabilitation, Faculty of Medicine, Pamukkale University, Denizli, Turkey***

Introduction and Purpose: The medial longitudinal arch (MLA) has been used as a main reference to diagnose flatfoot or to assess the results of the treatments. There are many methods currently being used to classify MLA structures. The calcaneal pitch angle (CPA) method is one of these methods. The purpose of this study is the comparison of the CPA values measured on the direct lateral radiographs of feet and modified Projection area per length squared (PAL) calculated as a new method.

Tools and Method: 50 subjects were evaluated (27 female and 23 male, mean age: 40.12, min: 4, max: 78). Direct lateral radiographs were placed on a transparent sheet. Straight line was drawn between the most plantar process of the calcaneus and the head of the first metatarsal bone for the calculation of PAL of MLA. Two arc (semilunar) lines were drawn upon this straight line. One of the arc lines was tangential to inferior margin of calcaneus, and the other crossing the sustantaculum tali. Both areas (PAL1 and PAL2) were estimated by using point counting technique. The CPAs were measured on the same radiographs for each subject. The correlation between PAL1, PAL2 and CPA by using Pearson correlation test-two tailed. Findings: The mean \pm SD of right PAL1, PAL2, and CPA were $8.28 \pm 1.41,11.26 \pm 11.73$, and $22.98 \pm 4.01$, respectively. The mean \pm SD of left PAL1, PAL2, and CPA were $8.37 \pm 1.41,11.21 \pm 1.75$, and $21.52 \pm 4.32$, respectively. The significant correlation was detected between right PAL1, right PAL2 and right CPA ( $r=0.644, r=0.595 ; \mathrm{p}<0.01)$. The significant correlation was detected between left PAL1, left PAL2 and left CPA ( $r=0.703, \mathrm{r}=0.769 ; \mathrm{p}<0.01)$.

Results and Interpretation: A significant correlation was detected between modified PAL method as a new technique and a classical CPA method for MLA evaluation. The PAL method can be suggested as a practical method for evaluation of MLA.

\section{PP-149}

The correlation among neck pain, body mass index and the estimates of projection area per length squared approach on cervical radiographs

Akdogan $I^{*}$, Sarsan $\mathrm{A}^{* *}$

Department of Anatomy, Faculty of Medicine, Pamukkale University, Denizli, Turkey*; Department of Physical Medicine and Rehabilitation, Faculty of Medicine, Pamukkale University, Denizli, Turkey**

Introduction and Purpose: This study is planned to investigate the correlation among neck pain, body mass index (BMI) and the estimates of Projection area per length squared (PAL) method (a practical method for evaluating the degree of cervical lordosis).

Tools and Method: This study was performed on 26 patients with neck pain (mean age: $52.07 \pm 8.51$, min: 41 max: 73 ). Body weights and heights of patients were measured to calculate BMI. BMI was calculated with the formula (BMI: Body weight (kg)/Height $\left.(\mathrm{m})^{2}\right)$. Cervical lordosis was determined with the PAL that was estimated on direct lateral radiographs. The length between the superior and inferior angles of the second and seventh cervical vertebral bodies, and the area behind the cervical vertebral bodies, was estimated using the point counting technique. Degree of pain during motion, rest and night were evaluated by Visual Analogue Scale (VAS). Thus, the patients' cervical PAL values were estimated as a percentage, VAS values were estimated and BMI values were calculated.

Findings: We determined that cervical PAL was increased with the decrease in VAS value at rest (Pearson correlation test, $\mathrm{r}=-0.298, \mathrm{p}=0.029)$. In addition, we determined that increase 
of BMI was increased VAS value during motion (Pearson correlation test, $\mathrm{r}=0.284, \mathrm{p}=0.032)$.

Result and Interpretation: There was a correlation between the increase of cervical lordosis described with PAL and the decrease of neck pain at rest. In addition, the increase of BMI causes the increase of neck pain during motion. PAL cervical approach could provide reproducible data for evaluating the degree of cervical lordosis and neck pain at rest.

\section{PP-150}

\section{Kidney dimensions in Tc-99m dmsa scan compared to somatometric parameters in normal children}

\section{Tuncer $\mathrm{I}^{*}$, Tastekin $\mathrm{G}^{* *}$}

Department of Anatomy, Meram Faculty of Medicine, Selçuk University, Konya, Turkey*; Department of Nuclear Medicine, Meram Faculty of Medicine, Selçuk University, Konya, Turkey**

Objectives: It was aimed to study the morphometric features of the kidney considering the clinical significance of it.

Material and Method: Out of 230 subjects, 83 were boys and 147 girls. The children aged between 7-11 were included in the study. The height and weight of the children, and the width, height and thickness of both kidneys were measured in the Nuclear Medicine Department of Selcuk University. The sizes of the kidneys were researched statistically proportional to the weight and height of the subjects. The results were assessed with Pierson analyzes and t-test.

Findings: The average length of the right ear was $69.21 \pm 13.8$ $\mathrm{mm}$, width $32,66 \pm 6 \mathrm{~mm}$, thickness $18,59 \pm 3,5 \mathrm{~mm}$, the mean length of the left ear was $70.97 \pm 14.2 \mathrm{~mm}$ width $33.51 \pm 6 \mathrm{~mm}$, thickness $18.97 \pm 3.6 \mathrm{~mm}$, height $109.40 \pm 31.1 \mathrm{~cm}$, and weight was $23.32 \pm 15.7 \mathrm{~kg}$. When the parameters were compared wit each other, the mean width, size and thickness of the right ear were lower than the mean width, size and thickness of the left ear, and the sizes of the kidneys were higher in females.

Results: When the clinical significance of the kidney and the problems encountered in surgical practices are taken into account, we are of the opinion that knowing the morphometric properties of the kidney can contribute to clinic considerably.

\section{PP-151}

The determination of the changes in the size of kidneys related with gender, age and lateralization with ultrasound

Tuncer I

Department of Anatomy, Meram Faculty of Medicine, Selcuk University, Konya, Turkey
Objective: To evaluate the morphometric development of the kidneys according to age and gender and to analyze the obtained data statistically.

Material Method: This study was conducted on 100 volunteers (32 Male, 68 female) whose ages were between 4 months to 73 year-old at the radiology department of the Selcuk University, Faculty of Medicine Two hundered kidneys were evaluated with ultrasound. Their lenght, width and thickness were measured and renal volume and shape indexes were calculated. The data were evaluated statistically with paired sample T-test and T-Tukey HDS test in SPSS program (for Windows 10.0).

Results: The length and thickness of the left kidney in females was found greater than the right kidney and males. All parameters had the greatest value at the ages of 20-40 and 40-60. They were lower at the ages of 20 and below and 60 and over. All the results were compared with the results in the literature and discussed.

Conclusions: It was concluded that the results of this study can be additional resource about the development of kidney and can contribute to urology used for nephrectomy and renal transplantation.

\section{PP-152}

\section{Analysis of MR imaging of the conus medullaris position in an adult population}

Karabulut $\mathrm{O}^{*}$, Akay $\mathrm{H}^{* *}$, Karabulut $\mathrm{Z}^{* * *}$, Saka $\mathrm{G}^{* * * *}$, Acikgoz A**

Department of Anatomy, Faculty of Medicine, Dicle University, Diyarbakır, Turkey*; Department of Radiology, Ozel Veni Vidi Hospital, Diyarbakır, Turkey**; Department of Physical Medicine, Ozel Veni Vidi Hospital, Diyarbakır, Turkey***; Department of Public Health, Faculty of Medicine, Dicle University, Diyarbakır, Turkey****

The objective of the study that involved 921 patients with the mean age of 42.86 was to evaluate the variation in position of the conus medullaris in male and female patients without spinal deformity, to correlate the MRI findings of changing termination level by sequential study of magnetic resonance images of the lumbar spine. Study group was comprised of 607 female patients with the mean age of $43.72 \pm 15.01$ and 314 male patients with the mean age of $40.57 \pm 15.383$.The termination level of the conus was recorded in relation to the upper, middle or lower third of the vertebrae and the intervertebral disc space. The distribution of conus localization was determined in a range between upper third of T12 vertebrae and L2-L3 intervertebral disc. When we analysed the two groups according to age decades, there was a significant difference with increasing age in women but not in men. Also the conus positions were compared in two genders. There was a significant difference in the mean conus position related to gender. 


\section{PP-153}

\section{Absence of the internal carotid artery: case report}

Acar $M^{*}, \underline{\text { Salbacak A*}}$, Sakarya $\mathrm{ME}^{* *}$

Department of Anatomy, Meram Medical Faculty, Selçuk University, Konya, Turkey*; Department of Radiology, Meram Medical Faculty, Selçuk University, Konya, Turkey**

The common carotid artery (CCA) usually divides into the internal carotid artery (ICA) and the external carotid artery (ECA) at the level of the 3-4th cervical vertebrae. Absence of the ICA is a rare congenital anomaly. We detected a variation in the carotid arterial system in multidetector computed tomography angiography (MDCTA) of a 42-years-old man in radiology department of Meram Medical Faculty of Selcuk University. There was no bifurcation in ACC at the right. The flow of blood provided by the circle of Willis, from left to right hemisphere of the brain. Recognition of this anomaly becomes important in thromboembolic disease. Consideration of this anomaly will be of utmost importance when planning carotid endarterectomy, as both cerebral hemispheres may be dependent upon the atheromatous carotid.

\section{PP-154}

The morphometric analysis of external carotid artery and its branches with multidetector computed tomography angiography (MDCTA)

\author{
Acar $M^{*}$, Salbacak A*, Sakarya $\mathrm{ME}^{* *}$ \\ Department of Anatomy, Meram Medical Faculty, Selçuk University, \\ Konya, Turkey*; Department of Radiology, Meram Medical Faculty, \\ Selçuk University, Konya, Turkey**
}

Introduction and Purpose: A knowledge of variations in the origin and course of the common carotid artery (CCA) and the external carotid artery (ECA) and its branches is of great importance in surgery and radiologic examinations. The aims of the present study were to describe and quantify variations of the ECA and its branches, and to measure the bifucatio carotidis distance from clinical relevant landmarks. Tools and Method: All measurements were performed on 100 (50 male, 50 female ) patients which were examined multidetector computed tomography angiography (MDCTA) images of the carotid system. All MDCTA examinations were performed with a 64-channel MDCTA scanner (Somatom Sensation 64, Siemens, Germany). The images were retrieved to the Leonardoworkstation (Siemens Medical Solutions). Findings: The bifurcation level of the CCA was determined to be $7 \%$ at the C2-C3 level, $19.5 \%$ at the $\mathrm{C} 3$ level, $34.5 \%$ at the $\mathrm{C} 3-\mathrm{C} 4$ level, $22 \%$ at the $\mathrm{C} 4$ level, $8.5 \%$ at the $\mathrm{C} 4-\mathrm{C} 5$ level, $8.5 \%$ at the C5 level. The variational structure of the ECA and its branches was observed in $31 \%$ of all cases. Result and Interpretation: The patterns of variability in the branches of the carotid artery are extreme important for radiographic evaluations and surgical interventions.

\section{PP-155}

\section{Anatomy and variations of human carotid body and carotid sinus}

Songur $\mathrm{A}^{*}$, Acar T**, Kaplan $\mathrm{S}^{* * *}$, Uzun I**** , Kucuker $\mathrm{H}^{\star * * *}$ Department of Anatomy, School of Medicine, Afyon Kocatepe University, Afyonkarahisar, Turkey*; Department of Anatomy, School of Medicine, Sifa University, Izmir, Turkey**; Department of Histology and Embryology, School of Medicine, Ondokuz Mayıs University, Samsun, Turkey*** Department of Forensic Medicine, School of Medicine, Sakarya University, Sakarya, Turkey****; Council of Forensic Medicine, Istanbul, Turkey ${ }^{\star \star * \star *}$

Carotid Body (CB) is one of the most important paraganglia which functions as a chemoreceptor organ modulating blood carbondioxide and oxygen pressures and blood $\mathrm{pH}$ levels. Carotid sinus (CS) contains baroreceptors which detect the changes in blood pressure and then relay the information to central nervous system. In this study, we aimed to examine morphology and morphometry of CB and CS, and investigate correlations with other structures such as common carotid artery(CCA) and external carotid artery (ECA). Our study was performed on 25 forensic autopsy cadavers who were between 3 rd and 5th decades and male. CB area was dissected, and the possible types of variation were noted. In the anatomic study, $80 \%$ of them were ovoid, $8 \%$ of them had two lobes connected at one end forming a"V" shape, $8 \%$ of them had two lobes with separate stalks and $4 \%$ of them were leaf-shaped. Body and stalk length of CB were found to be $3.376 \pm 0.644 \mathrm{~mm}$ and $2.216 \pm 1.340 \mathrm{~mm}$, respectively. 21 CBs started superior to the CB level, two GCs started immediately below the superior end of CB. Two GCs started at the level of CB and had no stalk. CS wall thickness was $598 \pm 113.1 \mu \mathrm{m}$. CS lumen area was $12.03 \pm 2.703 \mathrm{~mm}^{2}$. We found that CCA thickness was $789 \pm 169.9 \mu \mathrm{m}$ and common carotid artery (CCA) lumen area was $15.98 \pm 3.405 \mathrm{~mm}^{2}$. External carotid artery (ECA) thickness was $488 \pm 107.8 \mu \mathrm{m}$ and ECA lumen area was $7.256 \pm 1.942 \mathrm{~mm}^{2}$. We found that CS thickness changed with age. Also, there was a positive correlation between CS thickness and CCA ( $r=0.742$; $\mathrm{p}=0.000)$ and between CS thickness and ECA ( $\mathrm{r}=0,688$; $\mathrm{p}=0.000$ ). In the conclusion, we think that the result of our survey will contribute to country's demography, to success on surgery. These data will also help the clinicians and contribute to the researchers who will study in this field. 


\section{PP-156}

\section{Variable origin of the vertebral arteries}

Dodevski $\mathrm{A}^{*}$, Menka Lazareska**, Lazarova $\mathrm{D}^{*}$, Julija Zhivadinovik J*, Cadikovska E*

Institute of Anatomy, Medical Faculty, Skopje, Republic of Macedonia*; Institute of Radiology, University Clinical Center, Skopje, Republic of Macedonia**

The vertebral artery arises from the first part of the subclavian artery on both sides. In some cases the vertebral artery may have anomalous origin, from a vessel other than the subclavian artery (the aortic arch, the thyrocervical trunk, the common carotid artery, the external carotid artery and brachiocephalic trunk). The anatomic and morphologic variations of the vertebral artery have significance not only for diagnostic examinations, but also for surgical and endovascular therapeutic considerations. This study was conducted to examine the variations in the origin of vertebral arteries and to discus their clinical implications while performing diagnostic and surgical procedures. Over a time span of 1 year, 50 patients (25 male and 25 females, age range from 25-79, mean age 55 years), underwent computed tomography angiography (CTA). The image quality was judged adequate for analysis, and 50 angiograms were reviewed with respect to the origin of the vertebral artery. The analysis obtained that in 48 patients (96\%) vertebral artery have origin from the subclavian artery. The results showed that two of the cases (4\%) have atypical arisen of the left vertebral artery from the aortic arch, as a branch between left common carotid and left subclavian artery. The described morphological variations have applications in different fields of Medicine. For the anatomists, the results obtained from this study present valuable teaching material for students and postgraduates. The applicative value for the radiologists is in diagnostic and intervention procedures such as CT, MRI, angiography and endovascular procedures. These data are valuable for vascular and neck surgeons during planning and accomplishing surgical procedures in order to prevent iatrogenic complications.

\section{PP-157}

Developmental anomalies of tracheobronchial tree: A multidetector computed tomography study

Ulusoy $M^{*}$, Kivrak AS**, Uysal II*, Karabulut AK*, Paksoy $\mathrm{Y}^{* *}$ Deparment of Anatomy, Selcuklu Medical Faculty, Selcuk University, Konya*; Deparment of Radiology, Selcuklu Medical Faculty, Selcuk University, Konya**

Objective: Tracheal bronchus and accessory cardiac bronchus are most frequent developmental anomalies. Tracheal bronchus is a lobar or segmental bronchus originating from trachea or main bronchus. Accessory cardiac bronch is an accessory bronchus originating from intermediate bronchus opposing the origin of right upper lobe bronchus or from the right main bronchus medial wall (0.09\%-0.5\%). The purpose of this study is to detect the incidence of these anomalies in Turkish population.

Methods: The axial, sagittal and coronal reformatted images of 4 slice computed tomography (CT) of 400 patients without any lung operation (0-79 year-old, 208 male and 192 female) were evaluated.

Results: The incidence of tracheobronchial tree anomalies in this study was higher than other previous studies (1\% versus $0.5 \%$ for tracheal bronchus; $0.5 \%$ versus $0.4 \%$ for cardiac bronch). Tracheal bronchus was detected in four case (1\%). Superior lobar bronchus was originating directly from trachea in one case (pig bronchus), and right apical segmental bronchus was originating directly from trachea (supernumerary tracheal bronchus). Accessory cardiac bronch was detected in two cases $(0.5 \%)$. In both cases accessory cardiac bronchus was originating at the level of origin of right superior lobar bronchus.

Conclusion: Tracheobronchial tree anomalies can be detected by MDCT. Knowledge about type and localization of anomalies is important for bronchoscopy.

\section{PP-158}

\section{Effects of the maternal obesity on the prenatal and postnatal development}

Malas MA*, $\underline{\text { Uzun } A^{* *}}$, Uguz $C^{*}$, Sener $\mathrm{EH}^{* * *}$

Department of Anatomy, Faculty of Medicine, Süleyman Demirel University, Isparta, Turkey*; Department of Anatomy, Faculty of Medicine, Eskişehir Osmangazi University, Eskişehir, Turkey**; Department of Nursing, School of Health, Mehmet Akif Ersoy University, Burdur, Turkey***

Objective: In this study we aimed to investigate the effects of maternal obesity before pregnancy on postnatal development of fetus.

Material and Methods: Seventeen female Wistar Albino rats weighing between 140 and 160 grams were included in the study. Experimental group was fed with high fat diet (lipid ratio $40 \%$ ) and control group was fed with control diet (lipid ratio $20 \%$ ) for a period of 15 weeks. The naso-anal length was measured to calculate body mass index. The weights of pregnant rats in the experimental and control groups were measured during the gestational period. At the end of the gestation, the gestation durations and pup numbers of all rats were determined. In the postnatal period the morphometrical growth 
parameters related to cranium, thorax, body and extremities were measured for each pup between newborn and adulthood.

Results: Maternal weight gain during gestation of rats in experimental group was greater than the control group. All measurements obtained from pubs were significant higher in experimental group when compared with the control group during postnatal period $(\mathrm{p}<0.05)$. Moreover, when data obtained between weeks 6 and 12 weeks were analyzed for males and female separately, morphometric measurements showed a significant difference between male to male and female to female in the experimental and control groups with larger measurements in the experimental group $(\mathrm{p}<0.05)$. And also there were significant differences between sex in each group $(\mathrm{p}<0.05)$.

Conclusion: In conclusion, maternal obesity during pregnancy adversely affects weight gaining and morphometric measurements of pubs during the postnatal period. The presented data in our study, considered to be a pioneer in the evaluation of the effects of maternal obesity on prenatal and postnatal periods of development and contribution of future studies.

\section{PP-159}

\section{Benefits of learning anatomy - Answers from undergraduate Romanian medical students}

\section{$\underline{\text { Chirculescu ARM* }}$, Lungu A**, Chirculescu $M^{* *}$,} Moxham BJ ${ }^{* * *}$

Department of Anatomy and Embryology, C. Davila University Bucharest, Romania and Department of Physiology, Anatomy and Genetics, University of Oxford*; Department of Anatomy and Embryology C.Davila University, Bucharest, Romania**; Cardiff School of Biosciences, Cardiff University, Wales, UK***

A matrix questionnaire was employed to evaluate how different teaching methods relate to different teaching aims/learning outcomes for an anatomy course for British, French, Turkish and Romanian undergraduate medical students (Moxham et al, $2005 ; 2006 ; 2009)$. As the previous study for Romanian medical students (2009) was performed on 257 students only, we extended it for 2009-11, to obtain a better statistic validity (558 respondents). We used the same questionnaire for 1st year students (before their first anatomy examinations) and 2nd year students (after their last examinations of the 4 anatomy exams), from the regular course in Romanian and the module for international students. Scores approaching 5 would suggest a good "fit" between a teaching method and a specific learning outcome, scores approaching 0 suggest no "fit". About $10 \%$ of answers were left blank or N/A. The students' age range was 18-42 years. The data show that, for learning outcomes relating to the acquisition of anatomical information, all teaching methods assessed scored this time between 1.97-4.23 (previously 3-4.9), showing a better evaluation of the issues. The highest scores were obtained from 1st year students, showing a sort of more enthusiastic view. The highest scores were associated with prosection and demonstration (3.51) and the lowest to models and CAL/e-learning (2.72-2.76) - as previously. In contrast to previous reported results, female students scored constantly lower than males. These data suggest that, for the Romanian students population studied, there are differences compared to British, French and Turkish students. Furthermore, of all teaching methods, using cadaveric material was considered most efficacious. Extending the study over a 5 years period made results more reasonable than those reported before. The data varied according to age, to the stage of study and also varies according to gender and nationality of the students.

\section{PP-160}

\section{MRI contribution in cerebral cavernous angiomas diagnosis}

Elena S, Stelian P, Daniela FC

Facultatea De Medicina, Disciplina De Anatomie Universitatea "Ovidius" Constanta, Romania

Purpose: Survey and highlighting the major role of magnetic resonance imaging in cavernous angiomas detection, evaluation and monitoring, with implications for treatment and prognosis.

Material and Method: We have evaluated retrospectively the results of the MRI exam for a period of one year, which included 7 patients, aged 3-70 years, hospitalized in the Neurology Clinic, for non-specific neurological symptoms or a clinical picture of a cerebral tumor substrate or a small intracerebral bleeding. The study was initially reviewed biological and with computed-tomography investigations, MRI being used as the final examination for completion of diagnostic algorithm.

Results: All seven cases were located over the tentorium, i.e. 3 in the frontal lobe, 2 in the parietal lobe, and 2 in temporal lobe. Four of them were in the gray-white substance junction and the other three in periventricular white matter, varying in size from 7 -- $30 \mathrm{~mm}$. In one case, we founded multiple lesions. MRI examination revealed in all cases, a heterogeneous structure of the lesions, with mixed signal hyperintensity T2, T2* GR and FLAIR, with areas of marked hyperintensity in T1weighted sequence (sub acute thrombosis in methaemoglobin / slow flow stage), with small central and peripheral ring hypointensity areas (haemosiderin / calcification). In all cases, the lesions were not complicated with sub acute hemorrhage, not led to mass effect on adjacent structures, were not accompanied by edema and were occult at MRA sequences.

Conclusions: MRI examination, with conventional sequences $\left(\mathrm{SE}, \mathrm{EG}^{*}\right)$, is an elective method in the diagnosis and characterization of arterio-venous malformations, and in monitoring complications. Most of them are silent, but some patients may 
show various nonspecific symptoms or neurological signs of a small intracerebral bleeding.

\section{PP-161}

\section{The evaluation of the hypoglossal canal types by multidetector computed tomography (MDCT)}

Yilmaz MT*, Cicekcibasi AE*, Tezer $M^{*}$, Kiresi D**, Buyukmumcu $M^{*}$

Department of Anatomy, Meram Medical Faculty, Selçuk University, Konya, Turkey*; Department of Radiology, Meram Medical Faculty, Selçuk University, Konya, Turkey**

Introduction and Purpose: The hypoglossal canal is the bony opening in occipital condylus which twelfth cranial nerves, innervating the lingual muscles, passes. The aim of this study was to determine the types of the hypoglossal canal according to gender and lateralization.

Tools and Method: This study was retrospectively composed on MDCT images of the 116 (female 51 - male 65) adult subjects. All MDCTA examinations were performed with a 64channel MDCTA scanner (Somatom Sensation 64, Siemens, Germany). The images were retrieved to the Leonardo workstation (Siemens Medical Solutions). The viewing hypoglossal canals were classified as follows: A classic single canal (type 1), one osseous spur located either in the internal or external orifice of the canal (type 2), two or more osseous spurs (type 3), complete osseous hypoglossal bridging of the internal or external portion of the canal (type 4), complete osseous bridging (double canal) extending along the entire canal (type 5).

Findings: In females, types 1, 2, 4, and 5 were detected in $82.35 \%, 1.96 \%, 1.96 \%$, and $13.75 \%$ of cases on the right side while $66.67 \%, 19.63 \%, 1.96 \%$, and $11.74 \%$ on the left side, respectively. In males, types $1,2,4$, and 5 were detected in $81.54 \%, 12.30 \%, 4.61 \%$, and $1.55 \%$ of cases on the right side while $83.08 \%, 15.30 \%, 0 \%$, and $1.55 \%$ on the left side, respectively. Type 3 was not observed in both genders. Besides, type 4 was not determined on the left side, in males.

Result and Interpretation: The knowledge of the hypoglossal canal morphology is important to avoid hypoglossal nerve injury at the posterior fossa approaches.

\section{PP-162}

\section{The sternalis muscle: a radiologic study}

Dogan NU*, Ozbek S**, Kivrak AS**, Fazliogullari Z*, Uysal II*, Karabulut AK*

Department of Anatomy, Selcuklu Medical Faculty, Selcuk University, Konya, Turkey*; Department of Radiology, Selcuklu Medical Faculty, Selcuk University, Konya, Turkey**
Introduction and Objective: The sternalis muscle is a supernaturary muscle located in the anterior thoracic region, parallel to the sternum. Muscle fibers arise from the sheath of the rectus abdominis muscle or adjacent structures and terminate upon the pectoralis fascia, upper sternum, costal cartilages, the sternum, or the medial heads of the sternocleidomastoid muscle. We studied the incidence and apperance of the sternalis muscle on multidetector computed tomography (MDCT) to permit the differentiation of the sternalis muscle from significant pathologic condition.

Methods: We resrospectively reviewed consecutive MDCT scans of the chest in 200 adult Anatolian patients at our hospital from September to December 2011. Images were analyzed, focused on distribution, size, shape, and location.

Result: The sternalis muscle was present in 12 (6\%) of 200 Anatolian patients. 6 patients showed bilateral sternalis muscles. Among 6 patiens with unilateral sternalis muscles, 4 patients showed right sternalis muscles and 2 patients showed left sternalis muscles. Right distribution was more common. Mean height, width, and depth were $50.5 \mathrm{~mm}, 4.2 \mathrm{~mm}, 13.6$ mm respectively.

Conclusion: Radiologists should be familiar with the image findings of sternalis muscle to avoid any confusing pathologic lesions.

\section{PP-163}

\section{Volumetric measuring method of the femoral head using MRI}

Florin SM, Daniela FC, Adriana R, Alexandru P, Dan G, Gratian D, Casiana S

Universitatea De Vest Vasile Goldis, Arad, Romania

Purpose: Finding a reliable method of volumetric measurement of the femoral head using MRI.

Methods: Our study tested the accuracy of a volumetric measuring method, of the femoral head, using Mathcad on computed magnetic resonance imaging. The test was conducted on 16 femoral heads from diffrent corpses in the Anatomy Department of the Medicine Faculty from Western University Vasile Goldis from Arad, Romania. The values were compared with direct anatomical measurements of the femoral head. Results: Volumetric measurement appeared to be very reliable. The mean absolute deviation between MRI and anatomical measurements was similar to that of two MRI sets.

Conclusions: Quantitative volumetric measurements appear to be the most reliable method to measure the true size of a three-dimension anatomical region. 


\section{PP-164}

Variants of basilar artery and communicating posterior cerebral artery: a report of seven cases

Arráez-Aybar LA*, Villar-Martin A**, Poyatos $C^{* * *}$, Rodríguez-Boto $\mathrm{G}^{* * * *}$, Conejo-Menor JL*, Martínez-Martínez A ${ }^{* * * * *}$, Arrazola-García J*****

Dpto Anatomía Y Embriología Humana II. Facultad De Medicina Ucm. Madrid. España*; Servicio Radiodiagnóstico Hospital "Virgen De Altagracia" Manzanares (ciudad real). España**; Servicio Radiodiagnóstico. Hospital Universitario "Dr. Peset" Valencia. España***; Servicio De Neurocirugía. Hospital Clínico "San Carlos" Madrid. España****; Servicio De Anatomía Patológica. Hospital Clínico "San Carlos" Madrid. España***** Servico Radiodiagnóstico. Hospital Clínico "San Carlos" Madrid. España******

Introduction: In embryos of 45 days $(21-24 \mathrm{~mm})$ cerebral arterial circle is completed. However, in the embryonic period, several developmental anomalies of the cerebral arteries can occur: persistent primitive arteries -otic, hypoglossal, trigeminal and proatlantal arteries, agenesis, hipoplasia, duplications and fenestrations. Fenestrations (segmental duplications) of the cerebral arteries are rare variants with a reported incidence as high as $11 \%$, resulting from incomplete fusion of primitive embryologic vessels. Anterior communicating artery, vertebrobasilar system and anterior cerebral artery are typical locations for fenestrations. They have been associated with aneurysms and other vascular anomalies.

Case report: We report seven case of basilar artery fenestration and found in a control Angio-NMR. Basilar artery (BA) fenestration is reported in $1-5 \%$ of autopsies and in $0.1-1.9 \%$ of angiographic series. It can occur anywhere along the course of the BA but is most frequent in the proximal basilar trunk, close to the vertebral arteries. Only one unilateral partial and other complete duplication of the posterior communicating artery were identified in in the reviewed literature. The knowledge of these anatomic variations is important to avoid the unnecessary surgery and to perform either neurological surgery or interventional radiology with safety.

\section{PP-165}

Morphological studies on the ductus deferens of wild rabbit in Libya

\section{Abughrien B, Saleh AM}

Faculty Vet Med., Alfateh University, Tripoli, Libya

The study on the morphology of the male reproductive system of wild rabbit are very sparse. The present study was undertaken to provide morphological features of ductus deferens in the Libyan wild rabbit. specimens of ductus deferens from five wild rabbit were immersion fixed in $4 \%$ neutral buffer formalde- hyde. 8 to $10 \mathrm{~m}$ thick paraffin sections stained with $\mathrm{H} \& \mathrm{E}$, alcian blue, PAS and Van Gisson stains and were observed under light microscope. Other specimens taken to exam by the scanning electron microscope. The tunica mucosa formed longitudinal folds along the deferent duct, from the epididimary region to the pelvic one, determining an irregular lumen. Lining epithelium was found to be poseudostratified columnar ciliated (stereocilia) in the proximal region and simple cuboidal and columnar in the abdominal part and ampulla of the ductus deferens. The histochemical study revealed the presence of polysaccharides in this last part. At the level of ampulla the mucous folded in various compartments appeared as angular nets. By the scanning electron microscope, the lining epithelium proximal segments of the deferent duct showed stereocilia, which decreased in length distally and replaced by microvilli in the ampullary zone. Tunica musculosa consisted of two laminae (less developed inner and well developed outer longitudinal). The tunica adventicia was consisted of dense conjunctive tissue with vessels and nerves was observed.

\section{PP-166}

\section{Study of effects of pine needle extract}

\section{Jafarpur S}

Department of Anatomy and Cell Biology; Faculty of Medicine Mashhad University of Medical Sciences, Iran

Introduction: Herb medication was common in ancient Iran and has continued yet. As chemical drugs, Some herbs have side effects too. Nevertheless only destructive effects of some herbs have known yet. Pine needle is a herb medication which is used as abortive agent in some communities. Is it abortive agent realy? Has it other destructive effects? These questions induced us to research.

Materials Methods: Sixteen female BALB/C mice were divided in control 1, control 2 and experimental groups. They expose to males mice for mating and pregnancy. Vaginal plaque was marked as zero day of gestation. Medication was begun from the first day of gestation and continued to end of pregnancy. Experimental mice received pine needle extract and control 1 mice received only normal saline through gavages. Control 2 group received nothing. Animals were cared and every abortion or death were recorded.

Results: We observed no abnormality in control groups, but all of mice were aborted in experimental group. Fifteen died at time of abortion. Histological evaluation of uterus and placenta af these mice, showed abnormal changes, such as blood congestion, inflammatory cells infiltration, atrophy and necrosis in placenta.

Conclusion: Other investigators had demonstrated mother death, abortion and histological abnormal changes by pine needle consumption. In this research, death, abortion and histo- 
logical abnormal changes were recorded too. By comparison of these two results we can conclude that pine needle extract may has destructive effects on mother and fetuses.

\section{PP-167}

\section{Densitometric analysis of the human ulna}

Henche SA, Martín AB, Rubio PS, Torres RR, Sánchez JC, Pellico LG

Department of Human Anatomy and Embriology, Faculty of Medicine, University of Alcalá, Spain

Introduction and Purpose: Studies on the spatial distribution of bone mineral density (BMD) in the whole bone, reflecting its morphological pattern, are scarce. This study set out to establish with dual energy X-ray absorptiometry (DXA) the internal design of the human ulna.

Tools and Method: A random sample of 41 ulnas from the collection of the Anatomy and Embryology Department of the University of Alcalá was studied with a DXA system using a Norland XR-26 densitometer. Two projections were performed, lateral and antero-posterior. In both projections five regions were selected: proximal (R1), proximal-intermediate (R2), intermediate (R3), distal-intermediate (R4) and distal (R5). All statistical calculations were performed using SPSS-15. The means and standard deviation (SD) for bone mineral density (BMD) and bone mineral content (BMC) were calculated. Paired data were analyzed using parametric Student t-Test to compare BMD and BMC in every region.

Results and Interpretation: Results show that the higher BMD is in the proximal-intermediate region (R2), which corresponds to the coronoid process, which is the part of the ulna that bears the higher force of traction. The higher BMC is found in the proximal region (R1). Lower BMD and BMC are found in the distal region (R5). The total BMD shows significant statistical differences ( $\mathrm{p} 4$ 0.001), which indicates the heterogeneous nature of bone mass in the studied bone. We conclude that bone densitometry, measured by the DXA technique, is useful for assessing trabecular architecture of the human skeleton. The human ulna presents a heterogeneous distribution of the BMD. This study confirm that the mechanical solicitations in the ulna are major in the proximal extremity that the distal extremity.

\section{PP-168}

\section{Ultrastructural changes of epididymal spermatozoa in alcohol consuming rats}

Talebi AR, Nayeri M

Research \& Clinical Center For Infertility, Shahid Sadoughi University of Medical Sciences, Yazd, Iran
Introduction: Ethanol is the most widely abused drug. It has been proven that alcohol can suppress reproductive function and sexual behavior in both laboratory animals and humans. Impotence, testicular atrophy, gynecomastia and loss of sexual interest are often associated with alcoholism in $50 \%$ of alcololic men. Ethanol produces a significant decrease in the percentage of motility, concentration and normal morphology of spermatozoa. To our knowledge, the impact of alcohol consumption on sperm ultrastructure is poorly understood. So, the aim of this study was the evaluation of epididymal sperm ultrastructure in chronic alcohol-consuming rats.

Materials and Methods: 16 male mature Wistar rats with same age 10 weeks were categorized into two different groups. Control group included 8 rats allowed free access to rat chow and water. Experimental group included 8 rats with free access to rat chow and $5 \%$ ethanol in the same volume (50 cc daily) as controls that received water. After 30 days, epididymal spermatozoa from two groups were aspirated for sperm electron microscopic study.

Results: No ultrastructural changes were observed in control group. In experimental animals, most of spermatozoa showed several alternations in their ultrastructures. Anomalies such as abnormal nuclear chromatin density, swollen area, rupture and lysis of plasmalemma, persistence of numerous cytoplasmic droplets, mitochondrial swelling and vaculization, absence of axonemal microtubules, complete degeneration of axoneme, deletion of one or more outer dense fibers and absence of tail plasmalemma were seen in majority of the alcohol-treated spermatozoa.

Conclusions: Spermatozoa from alcohol consuming rats show spectrum of anomalies in their head, middle and principle piece of tail. These may be one of the possible causes of subfertility or infertility due to alcohol consumption.

\section{PP-169}

Localizations of p97/VCP (valosin containing protein) and jab1/csn5 in the postnatal rat testis and epididymis

Cayli $\mathrm{S}^{*}$, Erdemir $\mathrm{F}^{* *}$, Tas $\mathrm{U}^{* * *}$, Ungor $\mathrm{B}^{* * *}$, Yener $\mathrm{T}^{* * * *}$, Kesici $\mathrm{H}^{*}$, Karaca $\mathrm{Z}^{*}$, Aslan $\mathrm{H}^{*}$

Department of Histology and Embryology, Medical Faculty, Gaziosmanpaşa University, Tokat, Turkey*; Department of Urology, Medical Faculty, Gaziosmanpaşa University, Tokat, Turkey**; Department of Anatomy, Medical Faculty, Gaziosmanpaşa University, Tokat, Turkey***; Experimental Animal Center, Medical Faculty, Gaziosmanpaşa University, Tokat, Turkey****

Objective: The ubiquitin proteasome system (UPS) is a key player in regulating the many cellular processes via proteasomal degradation of ubiquitinlated proteins. Although the presence of p97/VCP and Jab1/CSN5 in various tissues is well known, the expression of both proteins in the developing rat 
testis and epididymis has not been studied. The aim of the present study was to show the cellular localization of $\mathrm{p} 97 / \mathrm{VCP}$ and Jab1/CSN5 in the developing rat testis and epididymis.

Materials and Methods: Testicular and epididymal tissues from 5-, 15-, 30-, and 60-day-old rats were examined by immune-histochemistry and Western blotting techniques.

Results: In 5 days old rat testis, p97/VCP and Jab1/CSN5 were specifically expressed in gonocytes. p97/VCP and Jab1/CSN5 expressions significantly increased at day 15 and found in spermatogonia, Sertoli cells and spermatocytes. In 60 days old rat testis, p97/VCP was strongly expressed in Sertoli cells however, moderate to weak expression was observed in spermatogonia, spermatocytes, round and elongating spermatids. Jab1/CSN5 showed strong expression in spermatogonia and spermatocytes while relatively weak expression was observed in round and elongating spermatids in 30 and 60 days old rat testis. The expression of both proteins in epididymis was gradually increased from 5 to 60 day of age. After 2 weeks of age, expression of both proteins was mostly restricted to the basal and the principal cells of caput epididymis.

Conclusion: Our study suggests that p97/VCP and Jab1/CSN5 could be an important part of the UPS in the developing rat testis and epididymis and both proteins may be involved in the regulation of spermatogenesis and epididiymal epithelial functions.

\section{PP-170 \\ Localization of factors stimulating or inhibiting the development of lymph vessels in the bovine corpus luteum}

Sinowatz F*, Rodler D*, Schilffarth $\mathrm{S}^{* *}$, Schams D**, Meyer $\mathrm{H}^{* *}$

Department of Veterinary Sciences, Institute of Anatomy, Histology and Embryology, University of Munich, Germany*; Institute of Physiology, Weihenstephan, Technical University, Munich, Germany**

The existence of an extensive lymphatic system in the corpus luteum (CL) of primates has been shown just recently. The bovine ovary offers an excellent system for studying the mechanisms of lymphangiogenesis. The aim of this study was to study the localization of factors stimulating (VEGF-C, VEGFD, FLT-4 hypoxia inducible factor-1a (HIF-1a)) or inhibting (vasohibin 1, thrombospondin 1) the development of the lymphatic system during estrus cycle and pregnancy using immunohistochemical techniques. Additionally the mRNA expression and imunohistochemical localization of VEGF-R3 in bovine corpora lutea, which has been shown to be involved in lymphangiogenesis, has been studied. Samples of corpora lutea from the following stages of the estrus cycle day 1-2 3-4, 5-7, 8-12, 13-16, >18, and pregnancy: month 1-2, 3-4, 6-7, >8) were obtained. We could localize FLT4 but not VEGF-C and VEGF-D to granulosa lutein cells of the CL. We could clearly demonstrate factors that inhibit lymphangiogenesis (Vasohibin 1 and Thrombospondin 1). These factors mainly occurred in the endothelium of large lymphvessels in the connective tissue surrounding the corpus luteum and in connective tissue septa separating larger areas of the corpus luteum but not in the lymph capillaries within the CL. VEGF-R3 could be localized to large granulosa-lutein cells, but was negative in endothelial and stromal cells. The data of our study supports the idea that interactions of local pro- and antiangiogenic factors impacts the development of lymph vessels in the bovine CL.

\section{PP-171}

\section{Investigation of the effects of artificial food colorings exposed during prenatal period on the postnatal morphometric development}

Sener $\mathrm{EH}^{*}$, $\mathrm{Uguz}^{* *}$, Uzun $\mathrm{A}^{* * *}$, Ceyhan $\mathrm{BM}^{* * * *}$, Malas $\mathrm{MA}^{* *}$, Gultekin $\mathrm{F}^{* * * *}$

Department of Nursing, School of Health, Mehmet Akif Ersoy University Burdur, Turkey*; Department of Anatomy, Faculty of Medicine, Süleyman Demirel University, Isparta, Turkey**; Department of Anatomy, Faculty of Medicine, Osmangazi University, Eskişehir, Turkey***; Department of Biochemistry, Faculty of Medicine, Süleyman Demirel University, Isparta, Turkey****

Objective: In our study, we aimed to investigate the effects of intrauterin exposure to synthetic food colors on morphometric growth during neonatal-adult periods after delivery.

Materials and Methods: A total of 30 Wistar albino female rats weighing between 160 and 190 grams were included in the study. A mixture of nine food colors (E102, E110, E122, E123, $\mathrm{E} 124, \mathrm{E} 127, \mathrm{E} 129, \mathrm{E} 132, \mathrm{E} 133)$ was orally given daily to the study group with the amount of their ADI, and tap water was given to control group during period of four weeks from the preconception to birth. Regular feed diet and water ad libitum were given during the study. Weights of the pregnant rats were measured three times a week through the gestation. At the end of the gestation, the gestation durations and pup numbers of all rats were determined. Morphometric growth parameters related to cranium, thorax, body and extremities were measured for each pup at day 0 and 1st, $2 \mathrm{nd}, 3 \mathrm{rd}$, 4th, 5th, 6th, 10th and 12 th weeks through the neonatal-adult periods.

Results: It was observed that rats in the study group have gained less weight during their gestation, when compared with 
the controls. In measurement of the morphometric parameters of 119 pups from two groups (study 64, controls 55) it was found that study group has increased values generally $(\mathrm{p}<0.05)$. Whereas in parameters measured with regard to genders at 6 th week, it was determined that morphometric parameters of the female and male pups in study group were grater than the females and males in control group $(\mathrm{p}<0.05)$.

Conclusion: It was concluded that consumption of syntethic food colors before and during gestation affects maternal weight gaining and morphometric development of pups during newborn and lactation periods and adulthood.

\section{PP-172}

\section{Photographic ear soft tissue analysis of healthy Turkish young adults}

Ozdemir S*, Ocakoglu G**, Ercan I**

Department of Anatomy, Uludag University Faculty of Medicine, Bursa, Turkey*; Department of Biostatistics, Uludag University Faculty of Medicine, Bursa, Turkey**

The purpose of this study was to establish ear soft tissue norms for Turkish young adults.

Method: Anthropometric measurements of the ear were taken from 100 female and 83 male Turkish adults aged between 18 and 24 years. The soft tissue human ear profiles were digitally analyzed using linear measurements. All photographs were obtained in a natural head position (NHP) by using a 5.1megapixel digital camera. All distances were measured using the TPSDIG 2.04 program by the same investigator. Nine standard anthropometric measurements right and left ear on both sexes were obtained, including length of auricula, width of auricula, lobular length, lobular width, conchal length, conchal width, distance from tragus to helix, distance from tragus to antihelix, distance from preaurale to lobule anterior.

Results: A statistically significant difference was found between males and females in 3 of 9 measurements taken for right side $(\mathrm{p}<0.05)$. A statistically significant difference was found between males and females in 4 of 9 measurements taken $(p<0.05)$ for left side. When the left and right ear measurements of the male are compares, meaningful differences were seen for conchal and lobular width. $(\mathrm{p}<0.05)$. When side difference for female is considered, meaningful right-left difference was seen in four (width of auricula, preaurale-lobule anterior distance, lobular width, conchal length) of the nine lineer measurement $(\mathrm{p}<0.05)$. Results were compared with other ethnic groups.

Conclusion: The ear soft tissue measurements of Turkish young adults were analyzed by using $2 \mathrm{D}$ digital photogrammetry and average ear soft tissue data were obtained. Reference anthropometric data for ear are necessary for ethnic-related data banks. Besides, anthropometric measurements of the ear are necessary to analyze the morphologic differentiations, to plan the adequate timing of surgical treatment, and also to establish a standard for the industry manufacturing hearing instruments.

\section{PP-173}

\section{Tissue specific teratogenic effects of lithium carbonate and bupropion on differentiated and differentiating cardiomyocytes in vitro system}

\author{
Qureshi WMS, Parker T, Pratten MK \\ University of Nottingham, Nottingham, UK
}

Congenital malformations account for a high mortality rate in the new born. $1 \%$ of these anomalies are associated with the use of drugs and medications during gestation. Some antidepressants such as Lithium Carbonate and Bupropion used during pregnancy may increase the risk of congenital malformations, particularly increasing abortion rates and some cardiovascular malformations, with Ebstein's anomaly attributed to lithium therapy. Chick cardiomyocyte micromass and mouse embryonic stem cell (ESCs) differentiation into contracting cardiomyocytes were used to evaluate the possible teratogenic effects. For the Micromass system, cardiomyocytes from five day old chicks were cultured at high cell density to form beating foci, while for the Embryonic Stem Cell Test (EST) cells were differentiated into contracting cardiomyocytes using the hanging drop method. In both systems drugs were added and contractile activity was recorded, followed by the resazurin assay for cell viability and the kenacid blue protein assay. In the micromass, Bupropion did not significantly decrease contractile activity, which significantly declined with increased dose, along with cell viability, in the ESCs system. The kenacid blue assay showed Bupropion had cytotoxic effects on protein levels in both systems. Lithium carbonate did not alter any end point in the micromass system at all doses tested, while in the EST contractile activity ceased around $1500 \mu \mathrm{M}$, a dose close to its therapeutic serum levels. The Resazurin assay did not show any change in cell viability, while protein levels increased with increasing dose and reached significant levels at $2000 \mu \mathrm{M}$. To evaluate the possible effects of both drugs on gap junction proteins, Connexin43 and Phospho-Connexin43 expression was analysed using western blots but no significant variation was observed at any dose tested. Other possible mechanisms of teratogenicity are under investigation. Both drugs significantly affected the contractile activity in EST, while Bupropion was found to be more cytotoxic than Lithium Carbonate. 


\section{PP-174}

\section{Distribution and morphological structure of Pancreatic mast cells after administration of high dose sugammadex}

Kalkan $\mathrm{Y}^{*}$, Tumkaya $\mathrm{L}^{* *}$, Bostan $\mathrm{H}^{* *}$, Tomak $\mathrm{Y}^{* *}$, Altuner D $\mathrm{D}^{* * *}$, Yilmaz $\mathrm{A}^{* * * *}$, Basar $\mathrm{E}^{* *}$, Bedir $\mathrm{R}^{* * * * *}$

Department of Histology and Embryology, Rize University School of Medicine, Rize, Turkey*; Department of Anaesthesiology, Rize University School of Medicine, Rize, Turkey**; Department of Pharmacology, Rize University School of Medicine, Rize, Turkey***; Department of Biochemistry, Rize University School of Medicine, Rize, Turkey*** ; Department of Pathology, Rize University School of Medicine, Rize, Turkey*****

Sugammadex is a drug used for the elimination of the blockade created with recuronium. Our study examined that how it affects the density of mast cells in the pancreas and the effect of the drug complex on the distribution of mastocyte granules. Groups have been edited as the following; G1. Recuronium $1 \mathrm{mg}$ group, G2. Recuronium $1 \mathrm{mg}+16 \mathrm{mg}$ Sugammadex group, G3. Recuronium 1mg + 96mg Sugammadex group, G4. Sugammadex $16 \mathrm{mg}$ group G5. Sugammadex 96mg group, G6. The control group. In this study, we investigated the effect of both recuronium on the distribution of mast cells of the pancreatic connective tissue and the influence of, if any, sugammadex on this change. Mast cell density in the G1 group was much higher, had distinct nuclei and scattered granules (+++) than the rest of the groups. Although G4 and G5 were similar to controls, mast cell granule scatter was less intense (+). Mast cell numbers and granule intensities of G2 and G3 were less than those of $\mathrm{G} 1(++)$. Cell density distribution of $\mathrm{G} 4$ and $\mathrm{G} 5$ was similar to the control $(++)$. When comparing the singledose recuronium (G1) and sugammadex groups (G4 and G5) with the complex groups (G2 and G3), there was a significant reduction in the numbers of must cells of G2 and G3 (+). While both recuronium and sugammadex caused a sensitivity reaction, recuronium resulted in a more severe reaction than sugammadex. These results have shown that sugammadex rapidly returns neuromuscular blockade arising from rocuronium during anesthesia in animals. However, adverse reactions may sometimes occur, such as anaphylactic shock, but sugammadex prevents this. Our results show that sugammadex can be used for the treatment of allergic reactions caused by recuronium during induction of anesthesia in animals, which is supported by similar studies in humans.

\section{PP-175}

The effects of high-dose sugammadex on renal tissues of rat after rocuronium-induced neuromuscular blockade

Bostan $\mathrm{H}^{*}$, Kalkan $\mathrm{Y}^{* *}$, Tomak $\mathrm{Y}^{*}$, Tumkaya $\mathrm{L}^{* *}$, Altuner $\mathrm{D}^{* * *}$, Yilmaz $\mathrm{A}^{* * * *}$, Basar $\mathrm{E}^{*}$, Bedir $\mathrm{R}^{* * * *}$

Department of Anaesthesiology, Rize University School of Medicine, Rize, Turkey*;Department of Histology and Embryology, Rize University School of Medicine, Rize, Turkey**; Department of Pharmacology, Rize University School of Medicine, Rize, Turkey***; Department of Biochemistry, Rize University School of Medicine, Rize, Turkey***; Department of Pathology, Rize University School of Medicine, Rize, Turkey*****

Sugammadex is a cyclodextrin molecule designed to encapsulate rocuronium to facilitate reversal of neuromuscular blockade. We administered high doses of Sugammadex to rats paralysed with rocuronium to study its effects on renal biochemical markers and histological section. 180-200 day-old adult Sprague-Dawley 18 healthy male rats, weighing 140-170 g, born and raised at Veterinary Control and Research Institute, Erzurum, Turkey were used. The rats were anesthetized under ether anesthesia and rocuronium $1 \mathrm{mg} / \mathrm{kg}$. Study groups received either Rocuronium $1 \mathrm{mg} / \mathrm{kg} \mathrm{IV}+$ Sugammadex 16 $\mathrm{mg} / \mathrm{kg}$ IV (G1) or Rocuronium $1 \mathrm{mg} / \mathrm{kg}$ IV +Sugammadex 96 $\mathrm{mg} / \mathrm{kg}$ IV (G2). Control group received equal volumes of serum physiologic after ether anesthesia. Rats receiving Sugammadex were ventilated until resolution of spontaneous ventilation and followed for 72 hours. Blood samples were taken to measure urea, creatinine and electrolyte values and both kidneys were excised to prepare histopathological specimens. Mean elapsed times between the administration of drug and the resolution of spontaneous ventilation were 64.0 46.9 and 21.0 \pm 1.8 minutes in G1 and G2, respectively. In G2, an abundant inflammatory infiltrate consisting of mostly lymphocytes and eosinophils was observed in the renal interstitium. Mean urea level in G2 $(33.80 \pm 2.864 \mathrm{~min})$ was significantly lower than G1 (40.20 $3.271 \mathrm{~min})$. Mean potassium level was statistically significantly increased in both Group 1 and 2 compared to control group, and the difference between G1 and G2 was insignificant. Mean creatinine, sodium and chloride levels were not significantly different between groups. Increased doses of Sugammadex reduce time required to resolution of neuromuscular blockade, however also cause a dose-dependent inflammatory reaction in kidneys and its use may be detrimental to patients suffering from renal failure. 


\title{
Poster Presentations - B
}

\author{
(PP-176 - PP-358)
}

\author{
PP-176 \\ Morphometric analysis of C2 vertebra \\ Dektas NS, Kafa IM, Sendemir E \\ Department of Anatomy, School of Medicine, Uludag University, \\ Bursa, Turkey
}

C2 or second cervical vertebra of the cervical spine is named as axis, vertebra dentata or epistropheus. It has a prominent odontoid process called dens and has many characteristic peculiar features. C2 vertebra injuries are the most risky one of all spinal injuries and many surgical procedures are performed to decompress or to stabilize it. Our first aim was to analyze morphometric features of $\mathrm{C} 2$ vertebra extensively. We also aimed to estimate distinctive bony features from other measurements by help of correlation matrices and regression formulas. We used fifty six parameters to evaluate $\mathrm{C} 2$ features in total of seventy vertebrae of late Byzantine era which are preserved at the Department of Anatomy, Medical School, Uludag University. Vertebrae used in this study were excavated in 1984 from the archeological site of Nicea in Iznik, Turkey. All parameters measured digitally from photographs using Scion Image Software (ver. 4.0.3.2). Besides the various correlated parameters, area of the corpus vertebra, length between the transverse processes, antero-posterior length of axis, length between inferior articular facets and height of dens parameters were highly correlated with more than forty parameters $(\mathrm{p}<0.05)$. Multiple regression formulas were also derived to estimate for highly correlated parameters. Forensic anthropologists and bio-archeologists attention are focusing more on the estimation of measurable bony features from fragmented bones based on markers. We believe that our data are useful for applications on physical anthropology and forensic Medicine.

\section{PP-177}

\section{Morphometric characteristics and typology of the coronoid process of the mandible}

\section{Dagli Z, Kafa IM, Ari I}

Department of Anatomy, School of Medicine, Uludag University, Bursa, Turkey

The coronoid process of mandible is an eminence which is varies in shape and size. Its anterior border occasionally is convex and posterior border is concave. We aimed to investigate types of the coronoid process, the angle between coronoid process and condylar process and the area of the coronoid processes in total of ninety seven mandibles of two different time periods (sixty six, contemporary period; thirty one, Byzantine period) from Department of Anatomy, Medical School, Uludag University. We found that $60 \%$ and $64.2 \%$ of all coronoid processes were hook shaped, 20\% and 21,4\% were triangular shaped and $20 \%$ and $14.28 \%$ were round shaped at right and left sides respectively in contemporary group. $66.6 \%$ and $61.4 \%$ of all coronoid processes in Byzantine group were hook shaped, 17,9\% and $13.6 \%$ were triangular shaped and $15.4 \%$ and $25 \%$ were round shaped at right and left sides respectively. Bilaterality has been found $74.2 \%$ (65.2\% hook, $13 \%$ round, $21.8 \%$ triangular) in contemporary group and $21.2 \%$ (64.3\% hook, $14.3 \%$ round, $21.4 \%$ triangular) in Byzantine group. Mean area of the coronoid processes were $1.94 \mathrm{~cm}^{2}$ and $2.21 \mathrm{~cm}^{2}$ at right and left sides respectively in contemporary group and $2.00 \mathrm{~cm} 2$ and $2.22 \mathrm{~cm}^{2}$ on right and left sides respectively in Byzantine group. The means of the angle between coronoid process and condylar process were $99.14^{\circ}$ and $94,91^{\circ}$ for contemporary group and $101.03^{\circ}$ and $98.96^{\circ}$ for Byzantine group at right and left sides respectively. We found types of coronoid processes are significantly shows laterality for both groups $(\mathrm{p}<0.05)$. There is also a significant difference between areas of left and right sides of coronoid processes of contemporary group $(\mathrm{p}<0.05)$. Our results may valuable for anthropologists, forensic scientists and bioarcheologists on evolutionary and geometric morphometrics of mandible and its coronoid processes, and also for the reconstructive surgeries such as graft operations.

\section{PP-178}

Nasal bone length values in the anacephalic human fetuses. A human fetal cadaver study

Sener $\mathrm{EH}^{*}$, Esenlik E**, Uguz $\mathrm{C}^{* *}$, Malas MA***

Department of Nursing, School of Health, Mehmet Akif Ersoy Universtiy, Burdur, Turkey*; Department of Orthodontics, Faculty of Dentistry, Süleyman Demirel University, Isparta, Turkey**; Department of Anatomy, Faculty of Medicine, Süleyman Demirel University, Isparta, Turkey***

Objective: The aim of this study was to investigate nasal bone length values in the anencephalic human fetal cadavers.

Material and Methods: This study included 23 anencephalic fetuses aged between 20-40. Gestational weeks and 47 control fetuses without external anomalies having similar aged. Gestational age (GA) determined to crown-rump length (CRL), bi-parietal diameter (BPD), head circumference (HC), femur length (FL) and foot lenth (FtL) for normal fetuses and FL and FtL for anencephalic fetuses. The standardized lateral 
cephalometric films were taken from all fetal cadavers. Nasal bone length value was measured on the lateral cephalometric films by using Easy Dent 4 Viewer Software program.

Results: Means of nasal bone length and general parameters with respect to gestational age were calculated. Nasal bone length at different ages are increased with gestational age in both groups. Anencephalic fetuses had a larger nasal bone length than normal fetuses, however, there were not statistically differences between groups $(\mathrm{p}>0.05)$. There were significant correlation between nasal bone length and general parameters with gestaional age $(\mathrm{P}<0.0001)$.

Conclusion: Nasal bone can become useful for evaluation of the fetus in the prenatal period and also identifying fetal anomalies. In this study, we determined nasal bone length values of normal and anencephalic fetuses aged between 21-40 weeks of gestation. These findings may be important for the fetopatological examination and help to diagnose fetal anomaly and pathologies.

\section{PP-179}

\section{Determination of normal cephalometric values of adults Turkish society living in Anatolia}

Cinar $\mathrm{S}^{*}$, Ulger $\mathrm{H}^{*}$, Baysal A*, Ertas ET ${ }^{* * *}$, Nisari $\mathrm{M}^{*}$, Uysal T**

Department of Anatomy, Faculty of Medicine, Erciyes University, Kayseri, Turkey*; Department of Orthodontics, Faculty of Dentistry, Erciyes University, Kayseri, Turkey**; Department of Oral Diagnosis and Radiology, Faculty of Dentistry, Erciyes University, Kayseri, Turkey***

In this study, the facial values of Turkish adults were determined and the differences between male and female individuals were investigated. The measurements have been done on 76 participants aged between 18 and 29 from both sexes. Six measurements from bone and six measurements from soft tissue were taken on the lateral cephalometric films. Measurements were taken on soft tissue of the men and women in the facial height was $12.0( \pm 0.5) \mathrm{cm}$ and $13.2( \pm 0.6) \mathrm{cm}$, nose height was $5.7( \pm 0.4) \mathrm{cm}$ and $6.2( \pm 0.4) \mathrm{cm}$, upper facial height was 7.8 $( \pm 0.4) \mathrm{cm}$ and $8.6( \pm 0.4) \mathrm{cm}$, lower face height was $6.6( \pm 0.4) \mathrm{cm}$ and $7.3( \pm 0.7) \mathrm{cm}$, the upper lip height was $2.3( \pm 0.3) \mathrm{cm}$ and $2.6( \pm 0.3) \mathrm{cm}$, mandible height was $4.3( \pm 0.2) \mathrm{cm}$ and $4.7( \pm 0.4)$ $\mathrm{cm}$, respectively. Measurements were taken on hard tissue measurements of men and women in facial height was 12.3 $( \pm 0.6) \mathrm{cm}$ and $13.5( \pm 0.6) \mathrm{cm}$, nose height was $5.7( \pm 0.4) \mathrm{cm}$ and $6.0( \pm 0.3) \mathrm{cm}$, top face height was $8.6( \pm 0.5) \mathrm{cm}$ and $9.2( \pm 0.5)$ $\mathrm{cm}$, lower face height was $6.8( \pm 0.4) \mathrm{cm}$ and $7.7( \pm 0.5) \mathrm{cm}$; top alveolar height was $3.0( \pm 0.3) \mathrm{cm}$ and $3.3( \pm 0.3) \mathrm{cm}$, the lower alveolar height was $4.1( \pm 0.2) \mathrm{cm}$ and, $4.7( \pm 0.3) \mathrm{cm}$, respectively. Soft and hard tissue measurements values of the males are higher than the women $(\mathrm{p}<0.05)$. The results obtained from soft and hard tissue measurements show that there are differences between male and female individuals. We believe that to know the differences between soft and hard tissue measurements could be useful to evaluate the morphological changes on the face and facial skeleton.

\section{PP-180}

In vitro effect of BDNF and NGF on the axonal growth cones of cortical neurons from apolipoprotein E3 or apolipoproten E4 transfected newborn mice

Donthcev V, Bojilova A, Wladimir O

Department of Anatomy and Histology, Medical Faculty, Medical University of Sofia, Sofia, Bulgaria

The neurotrophins comprise a family of secreted proteins that promote the survival of distinct neuronal populations in the developing nervous system. They actively participate in the guidance of the axons toward their targets. In our study we investigate the role of two growth factors, the neurotrophins Brain-derived growth factor (BDNF) and Nerve growth factor (NGF) in the outgrowth development of cortical neurons taken from, transfected with human Apo E3 or Apo E4 gene newborn mice. The cells raised in conditioned media supplemented with B27, PSF and Glutamine. Both neurotrophins (NGF or BDNF) were added to some of the dishes. On the next day to some of the dishes were added additional amounts of correspondent neurotrophin for an hour. After 24 hours of growing all the dishes were fixed and viewed by light inverted microscope. The area of randomly selected axonal endings as well as latitude of axons was measured and scored. The morphology of the same neurons (lamelopodia and filopodia formation), depending whether they are NGF or BDNF responsive was described and compared. Our results indicated that the BDNF responsive neurons demonstrate higher growth activity compared with NGF responsive neurons regarding growth cones, but both groups are similar in their action over the axonal extension. We explain those findings with the involvement of some receptors like Nogo in the Neurotrophin /receptor interactions.

\section{PP-181}

\section{Biochemical and electron microscopic investigation of cholesterol distribution in different structures of rat auditory cortex, with aging}

\section{Dontchev V}

Department of Anatomy and Histology, Medical Faculty, Medical University of Sofia

The aim of the present work is to analyze the amount and distribution of the cholesterol in the membrane structures of rat brain, from animals of different ages. Of particular interest was the cholesterol distribution in neurons and especially the neu- 
rons which present sign of degeneration. Thin layer chromatography and electron microscopy with and without cholesterol specific markers were applied. With age the total cholesterol amount in the brain tissue does not change significantly, but decrease of the esterificated and increase of the nonesterificated cholesterol content were found. The electron microscopic study, with cholesterol specific marker, reveals high and low cholesterol loaded areas in the plasma membranes and some organelles. They are irregularly distributed in the cortex. The degree of this unequal distribution increases with senescence. In particular in the cells with sign of degeneration low cholesterol content was found in most of the organelles. The decrease of the cholesterol in these types of cells was probably accomplished by means of its transfer in multivesicullar endodomes, which could be founded inside of the cell cytoplasm. Those endosomes are the only organelles in the cells with degeneration, which are able to respond to the digitonin marker. That's mean that they are loaded with enough cholesterol to reach the required threshold for activation and visualization the membrane changes in result of digitonin - cholesterol interactions.

\section{PP-182}

\section{Analysis of attitudes \& behaviour of students who attend anatomy lectures by comparing with success rate}

$\underline{\mathrm{Ak} \mathrm{G}^{*}}$, Emirzeoglu $\mathrm{M}^{* *}$, Dikmetas $\mathrm{E}^{*}$, Tomak L** ${ }^{* *}$ Asik H*

Vocational High School of Health Services, Ondokuz Mayıs University, Samsun, Turkey*; Department of Anatomy, Medical Faculty, Ondokuz Mayıs University, Samsun, Turkey**; Department of Biostatistics, Medical Faculty, Ondokuz Mayıs University, Samsun, Turkey***

Introduction and Purpose: Anatomy is one of the most important department on health professionals' education in all over the world. Due to this, we try to analyze the attitudes and behavior of students who attend anatomy lectures at Ondokuz Mayis University Vocational High School of Health Services (OMU/SHMYO) by comparing with their success rate.

Tools and Method: We used following data as assessment tools: The Study Attitudes Development Scale (SADS) (Baltaş, 1999- Bahçeci 2006), Attitudes Scale For Anatomy Lectures (ASAL) (Bahçeci 2009) and mean of students term examination and final examination. At OMU/SHMYO there are six anatomy programs. The study groups consist of students from 2009-2010 and 2010-2011 educational years who attend anatomy lectures. All the students $(n=424)$ accepted for study without any obligation. 63\% (268/424) of them responded the questionnaires. Collected data studied by SPSS 13.0 statistical analyze program.

Findings: We didn't find any statistically important results when we compared six anatomy programs with SADS and ASAL data ( $>0.05)$. Grouping by the score of anatomy exams as: good -very good 107(25.0\%) students (score limit: 68-100), average
75 (35.7\%) students (score limit: 59-67), fail 86 (31,2\%) students (score limit: 29-58). When comparing SADS and ASAL questionnaire results with score based grouping, there wasn't any statistical importance between SADS and exam score groups ( $>0.05)$. There were statistically important difference between ASAL and exam score groups $(\mathrm{p}<0.05)$. ASAL scale results compared in between the three exam score groups and students who grouped as failed statistically different than other two $(\mathrm{p}<0.05)$.

Result: No statistically importance found on SADS scores and exam results of OMU/SHMYO students who attended anatomy lectures. We found difference between ASAL and anatomy exams score groups especially within four programs due to their numeric or oral nature.

\section{PP-183}

\section{Variations in origin of the intrarenal apical segment artery of the human kidney}

Jovevska S, Matveeva N, Zivadinovik J

Institute of Anatomy, Medical Faculty, Skopje, Republic of Macedonia

Based on the arterial distribution, the kidney is divided into five segments: apical, middle, lover and posterior. The apical segment artery is however, a vessel whose origin is the most diverse of all segmental arteries. Knowledge of the segmental distribution of the arteries is very important in segmental resection of the kidney. A total of 30 kidneys, obtained from subjects of both sexes and 35 renal angiographic pictures where analyzed. Dissectional, injectional and injection-corrosive methods were applied. In order of frequency, apical segment artery arises from the upper segment artery; from the junction of the anterior and posterior divisions; from the posterior division. The investigation has yielded a number of facts about the renal vascular pattern that have practical application in segmental or partial resection of the kidney.

\section{PP-184}

\section{Morphometry of auricle}

Tatlisumak E*, Yavuz MS**, Kutlu $\mathrm{N}^{* * *}$, Asirdizer $\mathrm{M}^{* *}$, Yoleri L $\mathrm{L}^{* * *}$, Aslan $\mathrm{A}^{* * * * *}$

Department of Anatomy, School of Medicine, Celal Bayar University, Manisa, Turkey*; Department of Forensic Medicine, School of Medicine, Celal Bayar University, Manisa, Turkey**; Department of Physiology, School of Medicine, Celal Bayar University, Manisa, Turkey***; Department of Plastic and Reconstructive Surgery , School of Medicine, Celal Bayar University, Manisa, Turkey****; Department of Otolaryngology, School of Medicine, Celal Bayar University, Manisa, Turkey*****

The auricles are the defining features of human face. The aim of this study was to determine various morphometric measure- 


\section{S108 Joint Meeting of Anatomical Societies, 19-22 May 2011, Bursa, Turkey}

ments of auricle, to investigate asymmetry and its relation with handedness in both sexes. The study group consisted of 200 male and 200 female university students with no history of trauma or congenital anomalies. The decision of handedness was done by applying Edinburgh Handedness Questionnaire to the subjects. Auricle projection (AP) was measured from the helix to the mastoid process at the level of the tragus with a digital caliper. Both ears of the subjects were photographed in a standardized manner with a digital photo camera. A cardboard containing a graph paper scale was placed to the side of the head surrounding the auricle. Auricle length (AL), auricle width (AW), earlobe length (EL), earlobe width (EW), distances from the tragus to helix (TH) and antihelix (TAH) and distance from the highest point of auricle to the bottom of the intertragic notch (TIN) were determined for each photographed ear by using the measuring scale. 183 male and 183 female (91.5\%) subjects were righthanded. 13 left-sided males (6.5\%) and 12 left-sided females $(6 \%)$ were present. 9 subjects had no hand preference. All the measurements of right and left auricles were higher in males than the same sides in females. All of the measurements of right auricle were higher than the measurements of left auricle except $\mathrm{AL}$ and TIN in both sexes. The measurements of the righthanded subjects presented higher values of right auricle except $\mathrm{AL}$ and TIN as the general population but in addition to them, EL of left auricle had a higher value in left-handed subjects. Knowledge concerning the anatomy of auricle is important in the diagnosis of congenital malformations, planning the plastic surgical procedures and designing the hearing devices.

\section{PP-185}

Enteric nervous system role in physiological activities of the fetal gastrointestinal tract

Sisu AM, Cebzan C, Petrescu C, Stana LG, Selaru M, Motoc A University of Medicine and Pharmacy "Victor Babes", Timisoara, Romania

Enteric nervous system (ENS) is part of the autonomic nervous system, having the myenteric plexus and the submucous plexus. The neurotransmitters of the ENS like acetylcholine and serotonin present different configurations in order to identify groups of neurons with certain functions (Costa and Brookes, 1999). Normal motility and secretory functions are depending on the anatomical integrity, chemical and functional of the plexuses network which forming ENS. Layers of the gastric wall in humans contain a rich cholinergic supply: mucosal layer has fibers with a strong reaction to acetylcholinesterase disposed both around gastric glands and in the muscularis submucosal layer. This presents the most number of cholinergic fibers gathered in fascicles, Auerbach plexus, with the most intense reaction at cell level. Our study was performed on 12 human foetuses with an average age of 6, 7 months. Specimens of 6 months showed a lack of submucosal nervous cells and led us to conclusion that these cells are still primitive, with different sizes and drawing prolongations for tending to form neuronal plexuses. After 21 weeks of intrauterine life the neuronal population will grow up to be mature, being integrated into command and control circuits.

\section{PP-186}

\section{Intrinsic regulatory elements unequal disposal in the gallbladder}

Sisu AM, Cebzan C, Petrescu C, Selaru M, Motoc A

University of Medicine and Pharmacy "Victor Babes", Timisoara, Romania

Argirofile cells migration from the extrinsic regulatory sources to control the visceral activity is supported by silver stain of the gallbladder and cystic duct. Present study was made on 10 human fetuses between 6 and 9 months gestational ages, using microscopic samples. Results showed (on gallbladder longitudinal section) the unbalanced presence in gallbladder wall a population made of argyrophil nervous cells, probably Dogiel I type, capable of colonising a gallbladder wall (mesenteric wall), meanwhile the antimesenteric wall is free of argyrophil cells. This could represent at 8 fetal months an intraparietal progressive colonisation with these type of cells or, in a finished colonisation, an unbalance of gallbladder wall intrinsic regulatory elements. At cystic canal level the density of argyrophil cells is bigger and their distribution in muscular and submucos layers is obviously. Cells from muscular layer show tends of grouping that can detect less in submucos argyrophil neurons. Argyrophil cells disposal in main bile duct at 8 gestational months, confounding with a neural fibres network very well represented in the wall, suggests a migratory primitive aspect, followed by main bile duct microganglia configuration. Localization of nervous plexuses in extrahepatic bile ducts are extremely important in medical practice related to gallstones and inflammation of the biliary segments. Abundant nervous fibers in bile duct explain the severity of pain in bile diseases with the bile duct peristalsis violence.

\section{PP-187}

\section{The morphology of the nutritional foramens of hip and long bones within the lower limb}

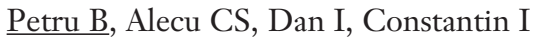

Department of Anatomy, Faculty of Medicine Constanta, Romania

Our study was performed on 21 hip bones, 44 femurs, 52 tibias and 46 fibulae, evaluating the nutritional foramens in terms of their location, number and size Within the hip bone is noted the large number of nutrient foramen, with size from 1-2 mm; more in number on the lateral face of the bone, located predominantly around the acetabulum. On the external iliac fossa 
there were 2-4 nutrient foramens. On the medial face of the hip bone is reported a small number of foramens (1-2 foramens, 1$1.5 \mathrm{~mm}$ diameter). Within the femur, the nutrient foramen was 1-2 $\mathrm{mm}$ in diameter. In 25 cases the nutrient foramen was single and in 19 cases was double. Most often they are located on the linea aspera and on the medial face of the diaphysis (closer to the linea aspera). When the nutritional foramen was double, one of them may be located on the lateral face of the diaphysis. The foraminal index was 36-39\% (single foramen) and 34.6$61.8 \%$ (double foramens). In the tibia, the nutrient foramen was located under the soleal line. In 5 cases we found 2 nutrient foramens. At tibial level we noticed the largest nutrient foramens, the diameter 1-2.5 $\mathrm{mm}$. The foraminal index was 28.7-34.3\%. Fibula showed a single nutrient foramen, (diameter $0.5-1.2 \mathrm{~mm}$ ), most often on the medial border of the diaphysis (19 cases), on the medial face (15 cases) and on the posterior diaphyseal face (12 cases). The shape was angular (foraminal index 37.8-51.3\%). We notice the major clinical importance of the nutrient foramen in bone fractures; a line of fracture that passes through the nutrient foramen may injure the nutrient artery, thus leading to a lack of fusion between that bony parts. Also, we notice a significant importance of the nutrient foramen within the bony grafts.

\section{PP-188}

\section{Biomathematical study of the cranial vault}

Motoc A, Niculescu MC, Jianu AM, Stana LG, Hogea GB, Moise M, Selaru M

Department of Anatomy and Embryology, "Victor Babes" University of Medicine and Pharmacy, Timisoara, Romania

The development of the bones of the cranial vault start from a few ossification centres, and thus ossification proceeds from the centre towards the periphery. In trying to establish the centre of the sphere corresponding to specific bones of the cranial vault, we have applied a geometrical approximation, using the biomathematical method. We have chosen the parietal as our model, as it is the only bone which is not in direct relation to the cranial base. Measurements have been carried out both on the isolated parietal and on the parietal as an integrating part of the cranial vault. In a central point of a part of a curved surface represented by the parietal bone, we can imagine a surface tangential plane and we can trace the normal of the plane, which also represents the normal of the surface. All the planes pass through the normal and intersect the internal plate, following an arch that is adjacent to a circle arch with a radius $\mathrm{R}$. We have determined the curvature of the parietal depending on several factors and using mathematical formulae. Following the results, the calculi show the development of the bones of the cranial vault is completed according to their degree of curvature, which increases with the development of cortical areas.

\section{PP-189}

Changes in bone mineral density, t-score and z-score of the hip and vertebral column with aging in osteoporosis

Niculescu MC*, Torok-Oance R**, Motoc A* ${ }^{*}$ Jianu AM*, Stana LG*, Folescu R*, Niculescu $\mathrm{V}^{*}$

Department of Anatomy and Embryology, "Victor Babes" University of Medicine and Pharmacy, Timisoara, Romania*; Department of Biology, Faculty of Chemistry, Biology and Geography, West University of Timisoara, Romania**

The bone resistance largely depends on the bone mineral density. Osteoporosis is a disease in which the bone resistance is affected, and establishing a diagnostic of certainty can be achieved by determining the bone mineral density. The present paper is based on the analysis of the results of the osteodensitometric investigation conducted on 361 patients who had been diagnosed with primary or secondary osteoporosis. The investigation took into account the hip bone and the vertebral column, and the following parameters have been analyzed: bone mineral content, bone mineral density, T-score and Zscore. We analyzed the evolution of the bone mineral density with increasing age, the existence of a connection between the bone mineral density determined at the vertebral column and at the hip, the connection between the $\mathrm{T}$ - and $\mathrm{Z}$-score and age at the population we took into account, as well as the existence and the type of discordance in diagnosis for the two points of determination. We found out that there is a decrease in the bone mineral density above the age of 40; concerning the $\mathrm{T}$ score, there is an obvious decrease tendency with ageing, both at the vertebral column and at the hip, while in the case of the $\mathrm{Z}$-score, the tendency is that of increasing with age. The most frequent discordance in diagnosis found in the population we analyzed is the minor one. The axial skeleton is preponderantly affected.

\section{PP-190}

An unusual variation of hyoid bone: bilateral absence of minor cornua and abnormal bone attachment to the corpus of the hyoid bone

\section{Kafa IM*, Gok E**, Fedakar R**}

Department of Anatomy, School of Medicine, Uludag University, Bursa, Turkey*; Department of Forensic Medicine, School of Medicine, Uludag University, Bursa, Turkey**

The hyoid bone is horseshoe shaped bone at the neck. It is a solitary bone that situated in the anterior midline of the neck just above the thyroid cartilage and it has attachments from nearby muscles but has no bony articulations. Hyoid bone consists of a body, two greater cornua and two lesser cornua and it 


\section{S110 Joint Meeting of Anatomical Societies, 19-22 May 2011, Bursa, Turkey}

is ossified from six centers: two for the body, and one for each cornu. During dissection of 16 years old male who is suspected case of hanging, we found an extra bone attached to the hyoid bone at left side of the corpus. Minor cornua were not found bilaterally and the abnormal bone was attached to the corpus at upper side and $1.59 \mathrm{~cm}$ left of the midline. It was approximately $3.79 \mathrm{~cm}$ long and $0.38-0.50 \mathrm{~cm}$ wide and head towards to the upper left of the hyoid bone. Antero posterior and transverse lengths of thyroid bone were $3.68 \mathrm{~cm} 4.96 \mathrm{~cm}$ respectively. Greater cornua lengths were 3.16 and 2.98 at right and left sides respectively. We believe that clinicians should be aware such abnormalities of hyoid bone, because both of its variations and abnormal relations within nearby structures have importance for surgical operations and radiological studies of the neck and it also have importance for forensic Medicine because of its susceptibility to fractures of hanging cases.

\section{PP-191}

\section{Morphological analysis of nasal bones of skulls from two different time periods}

$\underline{\text { Kafa IM }}{ }^{*}$, Coskun Ikiz I*, Akgun $Z^{* *}$

Department of Anatomy, School of Medicine, Uludag University, Bursa, Turkey*; High School of Health, Uludag University, Bursa, Turkey**

The nasal bones are two small bones which are located at the midline and upper part of the piriform aperture. These two bones articulate with each other at midline and also forming the bride of the nose. They are articulating with frontal bone superiorly, lateral cartilage of the nose at their inferior borders and septal cartilage at their vertical crest posteriorly. In our study, fifty human skulls (thirty skulls from contemporary period and twenty skulls from Byzantine period) from Department of Anatomy, Medical School, Uludag University were used for analysis of morphometric features of nasal bones and piriform apertures. Further, we also aimed to estimate total nasal bone area from other parameters. Parameters were height, width and area of the nasal bone, height, upper and lower widths of the piriform aperture, height of the piriform aperture (to the anterior nasal spine), and interior angles of piriform aperture and nasal bone. All parameters measured digitally from photographs using Scion Image Software (ver. 4.0.3.2). There were no significant differences between Byzantine period and contemporary period skulls. We therefore, include all skulls from both time periods for correlation analyses between all parameters. Area of the nasal bone and height of the nasal bone parameters were highly correlated with other parameters negatively or positively. Multiple regression formulas were also derived to estimate "nasal bone area" from other measurements used in the study except the "lower width of the piriform aperture" parameter which is show no significant correlation with "area of the nasal bone". We believe that, morphometric measure- ments and correlation analysis results of easily measurable parameters of the nasal bones may valuable for plastic surgeons and derived regression formulas may also for anthropologists and forensic scientists who are interested in the morphologic features of these bones from remnants or forensic cases.

\section{PP-192}

\section{Osseous orifice of the Eustachian tube}

\section{Ari I, Kafa IM}

Department of Anatomy, School of Medicine, Uludag University, Bursa, Turkey

The eustachian tube (tuba auditiva, auditory tube) is a very functional structure which plays important roles in drainage and aeration of middle ear and consists of a lumen, cartilaginous and osseos parts, surrounding muscles and soft tissue. It courses from tympanic cavity of the middle ear to the nasopharynx bilaterally. The proximal part of eustachian tube is made of bone (one third of total length) and distal part composed of cartilage (two third of total length). It is about $36 \mathrm{~mm}$ in length, and its direction is downward, forward, and medialward. They also form an angle of about 45 degrees with the sagittal plane. In our study, fifty human skulls (twenty eight skulls from contemporary period and twenty two skulls from Byzantine period) from Department of Anatomy, School of Medicine, Uludag University were used for analysis of morphometric features of the base of skull with selected parameters which are specially related with the osseous orifice of the eustachian tube. All parameters measured with a caliper. There were no significant differences between Byzantine period and contemporary period skulls. We therefore, include all skulls from both time periods for correlation analyses between all parameters. We found significant correlations $(\mathrm{p}<0.05)$ which are related to the osseous orifices with other parameters such as "distance between osseous orifices" and "distance between osseous orifice and medial pterygoid plate" measurements with "width of the palatum durum" and "distance between medial pterygoid plates" measurements. Obtained results in this study may have an importance for clinical approaches of the concerning area of the skull and understanding the structural relations of the eustachian tube with neighboring osseous landmarks.

\section{PP-193}

\section{Variant foramina at the base of the sphenoid bone}

\section{Ari I, Kafa IM}

Department of Anatomy, School of Medicine, Uludag University, Bursa, Turkey

Variants of the foramina of the middle cranial fossa and base of the skull have been studied by several authors extensively. 
Sphenoid bone is a butterfly or bat shaped and complex bone of the base of the skull and one of the seven bones that articulate to form the orbit. Absence, unilaterality, duplication, asymmetry or inequality of sizes of foramina of sphenoid bone has been also reported in literature. Foramen rotundum, ovale and spinosum are well-known foramina located at the cerebral surfaces of each great wing. Sometimes the foramen Vesalii may occasionally be seen medial to the foramen ovale. Pterygoalar foramen and canaliculus innominatus are other variations which are seen intermittently. Pterygoid processes of the sphenoid bone descend perpendicularly from sphenoid bone and each process consists of a two plates (medial and lateral). Divergence of the lateral pterygoid plate and the medial pterygoid plate form a $\mathrm{V}$-shaped fossa, the pterygoid fossa and a small depression, the scaphoid fossa. During the examination of the skulls from Department of Anatomy, School of Medicine, Uludag University we found unusual foramina at the base of the medial pterygoid plates at seven out of sixty skulls. These unusual foramina were observed at different size, number and locations at the base of the medial pterygoid plates. The probability of such foramina which may be associated with the sphenoidal sinuses should take into consideration when investigating base of the skull and during surgical approaches associated with the sphenoid bone.

\section{PP-194}

Determination of the infraorbital foramen using bone landmarks

Develi $\mathrm{S}^{*}$, Gokce $\mathrm{G}^{* *}$, Yazar F*, Ozan $\mathrm{H}^{*}$, Ceylan OM**

Department of Anatomy, Gulhane Military Faculty of Medicine, Ankara, Turkey*; Department of Ophthalmology, Gulhane Military Faculty of Medicine, Ankara, Turkey**

Purpose: The aim of this study was to determine the location of infraorbital foramen (IOF) and its relationships between some important cranial landmarks to provide better regional anesthesia before initiating surgery.

Methods: Twelve dry Turkish adult human skulls of unknown age and gender (24 sides) were studied. In each skull, measurements were made by using a digital caliper. Measurements were made from; a) superior wall of IOF to the inferior orbital rim (IOF-IOR), b) medial wall of IOF to the maxillary skeletal midline (IOF-MSM), c) medial wall of IOF to the lateral rim of the piriform aperture (IOF-PA), d) medial-inferior wall of IOF to the anterior nasal spine (IOF-ANS), e) superior wall of IOF to the zygomaticomaxillary suture at the level of the inferior orbital rim (IOF-ZMS). The longest diameter of IOF, vertical and horizontal diameter of orbital rims were also measured. Correlation between these measurements statistically were analyzed.
Results: According to our initial results no significant difference was found between the left and right sides in all measurements $(\mathrm{p}>0.05)$. There were significant correlations between the longest vertical diameter of the orbital rim and IOF-PA ( $\mathrm{p}=0.02 \mathrm{r}=0.632)$; IOF-MSM and IOF-ZMS ( $\mathrm{p}=0.01 \mathrm{r}=0.675)$; IOF-ANS and IOF-ZMS ( $\mathrm{p}=0.01 \mathrm{r}=0.673$ ); IOF-ANS and the longest diameter of IOF ( $\mathrm{p}=0.03 \mathrm{r}=0.619)$. The mean location of the IOF was about $6.71 \mathrm{~mm}$ inferior to IOR, about 23.15 $\mathrm{mm}$ from the midline, and about $29.6 \mathrm{~mm}$ above and lateral from the ANS.

Conclusion: Considering the positive correlation between IOF-ANS and the longest diameter of IOF, most of the IOFs that we studied are in the inferomedial rather than the vertical or horizontal direction on both sides. Knowledge of the anatomical characteristics of IOF is essential for local anesthesia procedures as well as preventing from anesthesia related complications in surgery and dentistry.

\section{PP-195}

Morphometry and morphology of the nasal bones and piriform apertures in the West Anatolian people

Ergur $\mathrm{I}^{*}$, Kiray $\mathrm{A}^{*}$, Ecevit $\mathrm{MC}^{* *}$, Zeybek $\mathrm{G}^{*}$, Cirpan $\mathrm{S}^{*}$, Dogan $\mathrm{E}^{* *}$

Department of Anatomy, Medical Faculty, Dokuz Eylul University, Izmir, Turkey*; Department of Otorhinolaryngology, Head and Neck Surgery, Faculty of Medicine, Dokuz Eylul University, Izmir, Turkey**

Aim: This study was made for to investigate the morphology and morphometry of nasal bones and piriform apertures in West Anatolian people.

Material and Method: Morphometric measurements were made from 168 dry skulls of unknown gender adults which belong to West Anatolian people found in Dokuz Eylul University Medical Faculty Anatomy laboratory. Digital photos were taken and morphometric analyses were made by using software (UTHSAA Image tool version 3.0 for Windows). Nasal bones and piriform apertures were classified according to their morphologies. Statistical analysis was made by using SPSS 15.0 program.

Results: Mean length of nasal bone was found $19.06 \mathrm{~mm}$ (min: $10.93 \mathrm{~mm}$, max: $35.39 \mathrm{~mm}$ ), width of nasofrontal suture was $12.46 \mathrm{~mm}$ (min: $3.57 \mathrm{~mm}$, max: $25.50 \mathrm{~mm}$ ), vertical length of piriform aperture was $34.26 \mathrm{~mm}$ (min: $18.39 \mathrm{~mm}$, max: 51.84 $\mathrm{mm})$. Totally eleven types of nasal bone were determined. Type 1 was seen $49.4 \%$ of the nasal bones.

Conclusion: This study provides morphologic knowledge of the noses in the West Anatolian population. This data's could be useful in antrophologic investigations. 
S112 Joint Meeting of Anatomical Societies, 19-22 May 2011, Bursa, Turkey

\section{PP-196}

Ultrastructural analysis of human bone marrow mesenchymal stem cells during in vitro osteogenesis and chondrogenesis

Mazzotti G, Teti G, Salvatore V, Grigolo B, Facchini A, Cavallo C, Falconi $M$

University of Bologna, Italy

The main purpose of this paper was to describe the morphology of mesenchymal stem cells (MSCs) differentiated in vitro towards osteogenic and chondrogenic lineages and to focus on the ultrastructural features associated with these processes. Human mononuclear cells (hMNC) were isolated, expanded and analysed for the expression of specific cell surface markers to demonstrate their stem cell characteristics. Moreover, hMNC were differentiated in vitro in an osteogenic sense and in a chondrogenic sense for 7, 14, 21 and 28 days. After treatment, they were processed using high resolution scanning electron microscopic analysis (FEISEM). Alizarin red and alcian blue staining was carried out to demonstrate the deposition of mineral salts and proteoglycans in the extracellular matrix. Undifferentiated MSCs showed a cell surface covered by filopodia and ondulopodia which allowed cells to migrate and to adhere to the substrate. During differentiation, the MSCs changed their shape from a round to a fibroblastic-like shape, showing numerous vesicles under the cell membrane. At the end of the differentiation, several filaments with a parallel orientation in the osteogenic samples as well as a network organisation in the chondrogenic samples were detected in the extracellular spaces. This study demonstrated that there are morphological features associated with the undifferentiated and differentiated states of the MSCs which could be utilised as new parameters for identifying and classifying these cells. Furthermore, they could be utilised to better understand the biological and physiological characteristics as well as the behaviour of the MSCs utilised in tissue repair and regenerative Medicine.

\section{PP-197}

A morphometric study of the jugular foramen in human adult skulls of South India

Hussain Saheb S, Mavishetter GF, Thomas ST, Prasanna LC, Muralidhar P

Department of Anatomy, JJM Medical College Davangere, Karnataka, India

It is well known that the jugular foramina are essential for venous drainage of the brain. The size of the foramenis related to the size of the sigmoid sinus and the presence or absence of prominent jugular bulb. An anatomical study was undertaken in order to investigate the size of jugular foramen in human adult skulls. A total of 125 human skulls were examined to determine the diameter, area and side predominance of the foramen. In $64.8 \%$ of cases the right foramina were larger than the left, in $24.8 \%$ of cases the left foramina were larger than the right and in $10.4 \%$ cases they were equal in size on both sides. The mean length of the foramen on the right and left were $23.62 \mathrm{~mm}$ and $22.86 \mathrm{~mm}$; the width measured $7.83 \mathrm{~mm}$ and $6.83 \mathrm{~mm}$ on the right and left respectively; the mean area on the right was $584.36 \mathrm{~cm}^{2}$ and on the left $493.30 \mathrm{~cm}^{2}$.

\section{PP-198}

\section{Femoral arterial system configuration}

\section{Soames R, Altalalwah W}

University of Dundee, UK

As it passes deep to the midpoint of the inguinal ligament the external iliac artery continues as the (common) femoral artery, which usually divides into superficial and deep femoral arteries with Scarpa's triangle: the medial and lateral circumflex femoral arteries can arise directly or indirectly from the common, superficial or deep femoral arteries. The superficial femoral artery becomes the popliteal artery after passing through the adductor canal, while the deep femoral artery gives perforating branches to the posterior aspect of the thigh. This study, undertaken on 74 lower limbs, focuses on the common femoral artery and its branches, which can be classified as being one of four types: nondivision, bifurcation, trifurcation and tetrafurcation. Non-division of the femoral artery at any level within the thigh was observed in 2 specimens; bifurcation into superficial and deep femoral arteries was observed in 41 specimens. Trifurcation was present in 19 specimens with either the medial (13) or lateral (6) circumflex femoral artery arising at the same level as the superficial and deep femoral vessels. Tetrafurcation was seen in 12 specimens with both circumflex arteries arising at the same level as the superficial and deep femoral arteries.

\section{PP-199}

\section{Variation of the origin of the obturator artery}

Soames R, Altalalwah W

University of Dundee, UK

The obturator artery usually arises as a branch of the anterior division of the internal iliac artery; however, this study of 48 specimens revealed that the origin can be highly variable. The obturator artery was a branch of the internal iliac arteries in all 48 specimens; however in only 30 specimens did it arise from directly (22) or indirectly (1 from the inferior gluteal artery, 3 from a gluteopundendal trunk, 1 from an obturatopundendal trunk and 2 from an umbilical artery) from the anterior divi- 
sion. In the remaining 16 specimens the obturator artery was a direct (1) or indirect ( 9 from the superior gluteal artery, 6 from a sciatic artery) branch from the posterior division. In 2 specimen the obturator artery arose from the bifurcation of the internal iliac artery.

\section{PP-200}

\section{The effects of simvastatin on ischemia-reperfusion injury of sciatic nerve in rats}

Pasbakhsh P, Gholami MR, Abolhasani F, Akbari M, Sobhani A, Mehrania K

Department of Anatomy, Medical School, Tehran University of Medical Science, Tehran, Iran

Severe ischemia to nerve results in fiber degeneration and Reperfusion results in oxidative injury to endothelial cells and augments fiber degeneration. Statins, 3 - hydroxy- 3 methyLgLutaryl coenzymeA (HMG-CoA) reductase inhibitors, the most widely used lipid lowering drugs, have been demonstrated to play a neuroprotective role. So we evaluated the effectiveness of simvastatin in protecting sciatic nerve from ischemia-reperfusion (I/R) injury using the model of experimental nerve ischemia. Sixty adult male Sprague-Dawley rats weighing 250-300 g were used. They were divided into ten groups ( $\mathrm{N}$ $=6$ per group). We used ischemia model in these groups. All ischemia groups were rendered ischemic for3 hours (h). Then followed by reperfusion durations of zero time(0hR), $3 \mathrm{~h}(3 \mathrm{hR})$, 7 days ( $7 \mathrm{dR}), 14$ days (14dR). Half of the groups had experimental simvastatin $\mathrm{IV}$ injection treatment via tail vein 1 hour before ischemia. The other half experienced only I/R as control groups. Behavioral data were obtained immediately before euthanasia. The score was based on coordination, racing reflex, toe spread and reaction to pinch. After euthanasia we took distal part of the sciatic nerve and made histological samples. Samples were observed to consider their fiber degeneration and edema changes. Functional changes were observed in I/R rats and also fiber degeneration and endoneurial edema were detected. In simvastatin treated I/R rats we had increase in functional recovery. The level of fiber degeneration and endoneurial edema were also decreased in these recent groups. In conclusion, preischemic administration of simvastatin exhibits neuroprotective properties in I/R nerve injury. (240 words)

\section{PP-201}

Presentation of cerebral blood vessels using injection corrosive method in 4-9 month old fetuses

Kulenovic A, Sarac-Hadzihalilovic A

Department for Anatomy, Faculty of Medicine, University of Sarajevo, Sarajevo, Bosnia and Herzegovina
We studied cerebral blood vessels in 25 fetuses of gestational age 16-36 weeks and in 10 cadavers of still-born babies by injection-corrosive method. In the early fetal life, arteries are thin with the straight flow, which is directly connected with the brain development. Progressive changes are observed in all the three cerebral arteries in 28-week old fetus, which straight flow becomes more and more tortuous. As in the 32nd week the brain develops faster and gyri and sulci are being formed, the arteries assume wavy flow and number of their rami increases. In a still-born baby, arteries are of rather bigger caliber; they branch abundantly; and due to their relatively broad cerebral sulci, it can be said that their flow is partly tortuous. Our results show evidently that position, flow and relation of cerebral arteries change concurrently with the brain development and appearance of cerebral gyri and sulci.

\section{PP-202}

The origin, in relation with the vertebral column and the morphometry of the superior mesenteric artery

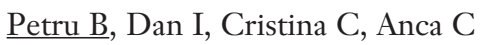

Department of Anatomy, Faculty of Medicine Constanta, Romania

The study was performed on 122 samples, using as methods the plastic injection followed by corrosion (28 cases), ultrasound examination (24 cases) simple (38 cases) and CT angiography (32 cases). We assessed the level of origin of the superior mesenteric artery related to the vertebral column and also its caliber, compared with the celiac trunk and the renal arteries. The origin of the superior mesenteric artery was evaluated on 52 samples; most often, the origin was placed on the upper third of the L1 vertebra (18 cases, $34.62 \%$ ), followed by the middle third of L1 (15 cases, $28.85 \%$ ), intervertebral disc T12L1 (10 cases, $19.23 \%$ ) and inferior third of the L1 (7 cases, $13.46 \%)$. In only 2 cases $(3.85 \%)$ the origin of the artery was located at the level of the inferior border of T12. The caliber of the superior mesenteric artery is highly variable, 5.7 to 10.2 $\mathrm{mm}$; most often is larger than the celiac trunk, followed by the samples with equal calibers and, rarely, the caliber was smaller than the caliber of the celiac trunk. Also, the caliber of the superior mesenteric artery is always larger than the caliber of the renal arteries. At the terminal level, the caliber was about 2 - $4 \mathrm{~mm}$, approximately equal with those of the final ileal artery or with the ileocolic artery. The morphology of the superior mesenteric artery and of its branches and, mostly, of its variations, shows a significant clinical importance in surgical practice, such as hemicolectomies, segmental colectomies, esophageal plasty with ileocolic ansa or isoperistaltic transverse colon. The echo-Doppler ultrasound and the angiography both shows a significant diagnostic relevance prior to the surgery, being able to notice the arterial stenosis and the modifications of flux within the arterial trunks. 
S114 Joint Meeting of Anatomical Societies, 19-22 May 2011, Bursa, Turkey

\section{PP-203}

Anatomic variation in the origin of aortic arch branches

Zhivadinovik I, Lazarova D, Dodevski A, Matveeva N

Institute of Anatomy, Medical Faculty, Skopje, Republic of Macedonia

The anatomic variations of the aortic arch and its branches are numerous and are often seen during autopsies, diagnostic or surgery procedures. The purpose of the present study was to describe variations of the aortic arch branches, using standard anatomical methods, in order to offer useful data to anatomists, radiologists, vascular, neck and thorax surgeons. The examination was made on 110 unselected human hearts without pathoanatomical changes, obtained after autopsy of newborns, fixed in $10 \%$ formaldehyde. The hearts were taken with the aortic arch, proximal part of thoracic aorta and ligamentum arteriosum. Standard anatomical methods, inspection and dissection were used. The analysis of the 110 heart specimens showed that 102 of them had the usual pattern of the aortic arch and its branches, with the usual topographic relations. Eight of the cases had irregular number of branches. Four of them had aortic arch with two branches, with left common carotid artery originating from brachiocephalic trunk. Three of the specimens had aortic arch with four branches, with separate origin of the left vertebral artery between left common carotid and left subclavian artery). One of the cases had aortic arch with five branches, with separate origin of the left vertebral artery as the fourth branch between left common carotid and left subclavian artery, and a fifth branch originating distal to left subclavian artery, probably branch of costocervical trunk. Despite the fact that the variations of the aortic arch branches are usually asymptomatic, they may cause dyspnea, dysphagia, intermittent claudication, misinterpretation of radiological examinations and complications during neck and thorax surgery. Furthermore, these variations may be accompanied by other congenital abnormalities.

\section{PP-204}

Third coronary artery: a case report

Dogan NU*, Uysal II** Dogan $\mathrm{KH}^{* *}$, Demirci $\mathrm{S}^{* *}$

Department of Anatomy, Selcuk University, Selcuklu Medical Faculty, Konya, Turkey*; Department of Forensic Medicine, Selcuk University, Meram Medical Faculty, Konya, Turkey**

The right coronary artery (RCA) begins at the right aortic sinus and goes along the coronary sulcus. It supplies the right atrium, right ventricle, atrioventricular septum and a part of the left ventricle. Along its course, it produces a conus branch, a right marginal branch and a posterior interventricular branch. Generally, there is only one orifice in the aortic sinus from which the RCA originates; however, more than one orifice is sometimes observed. It has been reported that the conus branch originates from this accessory orifice; in this case, the conus branch is called the third coronary artery. A third coronary artery orifice belonging to conus branch on the near the right aortic orifice was observed in a 62-year-old man at autopsy. The left anterior descending coronary artery and the right coronary artery arose normally from their respective aortic sinuses, and all coronary arteries were widely patent. The knowledge of the variations of coronary arteries is important when evaluating the coronary vessels for cardiac catheterization as well as during surgical procedures.

\section{PP-205}

\section{A case of coeliacomesenteric trunk}

Yilmaz MT, Tezer M, Cicekcibasi AE, Aydin AD, Salbacak A Department of Anatomy, Meram Medical Faculty, Selcuk University, Konya, Turkey.

Introduction and Purpose: The coeliac trunk and superior mesenteric artey are the anterior visseral branches of abdominal aorta. The anatomical variations of these arteries are due to development of the anterior splanchnic arteries. A case of coeliacomesenteric trunk was defined in this study.

Tools and Method: A coeliacomesenteric trunk was observed in a 70-year-old male cadaver during the routine dissection. The measurements were performed using a digital caliper ( $\mathrm{mm}$ ).

Findings: This trunk which the diameter was $13.98 \mathrm{~mm}$ originated from the anterolateral wall of the abdominal aorta after aortic hiatus $76.89 \mathrm{~mm}$. After $13.42 \mathrm{~mm}$ course, the trunk was divided to coeliac truncus which the diameter was $7.09 \mathrm{~mm}$ and superior mesenteric artery which the diameter was $5.25 \mathrm{~mm}$. Then, the coeliac truncus trifucated as splenic $(6.85 \mathrm{~mm}$ diameter), common hepatic $(5.31 \mathrm{~mm}$ diameter) and left gastric arteries (3.28 $\mathrm{mm}$ diameter). The root diameter of the superior mesenteric artery was also $5.25 \mathrm{~mm}$ and superior pancreaticoduodenal artery which the diameter was $3.20 \mathrm{~mm}$ was arised from it as first branch.

Result and Interpretation: Knowledge of variations of the coeliac trunk is important for both abdominal surgical approaches and radiological assessments.

\section{PP-206}

Evaluation of anatomical features of the coronary sinus $\underline{\text { Kafa IM }^{*}}$, Cetin S**, Durak D**, Coskun Ikiz I*

Department of Anatomy, School of Medicine, Uludag University, Bursa, Turkey*; Department of Forensic Medicine, School of Medicine, Uludag University, Bursa, Turkey**

The coronary sinus is located in the right atrium and posterior part of the coronary sulcus at the base of the heart. Between the 
orifice of the inferior vena cava vein and the right atrioventricular orifice, coronary sinus opens into the right atrium. It has a semicircular valve named as valve of the coronary sinus or Thebesian valve and this valve prevent the regurgitation of blood into the sinus during systole. The atrioventricular node also located near the orifice of the coronary sinus. The function of the coronary sinus is to collect blood which is coming from cardiac veins (small, middle, great and oblique cardiac veins and also from the left marginal vein and the left posterior ventricular vein) as a regulator and drains blood into the right atrium. We investigated total of twenty coronary sinuses from hearts of twenty adult (fifteen male and five female) cases aged between 20 and 60 . We found that mean area of the coronary sinus area was $15.53 \pm 3.61 \mathrm{~cm}^{2}$, mean perimeter of the coronary sinus was $16.08 \pm 2.54 \mathrm{~cm}$, mean ellipse major and minor axises were $5.07 \pm 1.04 \mathrm{~cm}$ and $3.33 \pm 0.53 \mathrm{~cm}$ respectively. The thickness for the left and right ventricles and the septum were $1.59 \pm 0.37 \mathrm{~cm}, 0.55 \pm 0.21 \mathrm{~cm}$ and $1.52 \pm 0.37 \mathrm{~cm}$, respectively. Coronary sinus, which is collect the blood by the cardiac veins that roughly track the coronary arteries, has very important roles at the blood supply of the heart. Increasing and reliable knowledge of accurate morphometric features of important structures of heart like coronary sinus provides more anatomical insights to the surgeons on various major cardiac surgeries and angioplasties.

\section{PP-207}

\section{Computed tomography of the heart: correlation between lumen and length of the left ventricle}

Escalona C, Bellmunt AP, Blasi M, Lorente M

Faculty of Medicine and Health Sciences, Universitat Internacional de Catalunya

Purpose: The purpose of this study is to describe the anatomy of the left ventricle of the heart by Computed Tomography (CT) in patients without heart disease in systole (S) and diastole (D). In this paper we specifically describe the correlation between the length and the lumen of the left ventricle, as well as the correlation between the variations they suffer between both phases of cardiac cycle. Spiral, multi-slice, 16 detectors system was used; an iodinated contrast solution was injected intravenously in coordination with a scanner. 69 patients finished the study. The study was approved by bioethics comitees of Universitat Interacional de Catalunya (UIC) and Centro Internacional de Medicina Avanzada (CIMA). Data analysis was performed on the basis of relevant scientific studies.

Results: The length has a linear relationship with the lumen on three levels; in basal and mid heights it was found in both phases, while in apical height this correlation occurred only in the phase of diastole (Values of Pearson/Spearman correlation coefficient: Basal: S:0.485 - D:0.549, Mid: S:0.262 -D:0.264;
Apical: S:0.193 - D:0.242). The relationship between variations of the lumen and the length was also calculated with Pearson/Spearman correlation coefficient, resulting to maintain a statistically significant linear relationship, in measurements made in three heights (Basal: 0.505; Mid: 0.289; Apical: 0.456).

Disussion: The length of the left ventricle is almost $10 \%$ less in systole than in diastole, while the thickness of the apex is $150 \%$ higher in systole. It follows that reducing the length of the ventricular cavity itself is very important and means a great contribution to the outflow of blood from the left ventricle, as though at some point (including the total length measurement of the left ventricle, which is the apex) is a growth of $150 \%$, overall length decreased by $10 \%$.

\section{PP-208}

\section{Histomorphometric study of the intracranial internal carotid artery in man}

Bharihoke $V^{*}$, Rustagi $\mathrm{S}^{* *}$

University College of Medical Sciences and Guru Teg Bahadur Hospital, Delhi, India*; Maharishi Markandeshwar Institute of Medical Sciences and Research, Mullana, Ambala, Haryana, India**

Cerebral arteries are unique muscular arteries in their histological structure in having thin walls and a prominent internal elastic lamina. The intracranial internal carotid arteries are one of the earliest vessels involved in cerebrovascular accidents. (Moossy 1966) The histological structure of the intracranial internal carotid arteries was studied in thirty healthy adults 2040 years of age who had succumbed to road accidents. The intracavernous part of the artery was dissected out and processed for paraffin sectioning. Seven micron thick cross sections were stained with Haematoxylin and Eosin, Masson's Trichrome and Verhoeff's stains. The outer diameter was found to be more on the left side $(4.32 \mathrm{~mm} \pm 0.44)$ as compared to the right side $(4.29 \mathrm{~mm} \pm 0.43)$. The mean thickness of the tunica intima was found to be more on the right side $(26.27 \mathrm{~mm}$ $\pm 12.71)$ as compared to the left $\operatorname{side}(25.23 \mathrm{~mm} \pm 9.59)$. The subendothelial tissue in the tunica intima was not uniform in thickness. It showed focal fibroelastic masses in three cases with migration of smooth muscle cells in the tunica intima from the tunica media. Splittings of the internal elastic lamina were seen bilaterally in six cases, in three cases only on the right side and in three case only on the left side. These cases are similar to the preatherosclerotic changes described by Simionescu and Simionescu (1977). These findings suggest that ageing changes begin in the vessels of apparently healthy individuals as early as third decade of life. This is an alarming sign indicating the need for lifestyle changes. 


\section{PP-209}

\section{C-kit positive interstitial cells and networks of the human heart}

$\underline{\text { Rusu MC }}$, Pop F* , Hostiuc $\mathrm{S}^{* *}$, Curca GC ${ }^{*}$, Jianu AM***

Carol Davila University of Medicine and Pharmacy Bucharest, Romania*; Mina Minovici National Institute of Legal Medicine, Bucharest, Romania**; Victor Babes University of Medicine and Pharmacy Timişoara, Romania***

Objectives: The cardiac telocytes (CTCs) are a distinctive class of interstitial Cajal-like cells (ICLCs) present within the cardiac structure. We hypothesized that c-kit positive cardiac ICLCs/telocytes could present morphological and topographical patterns that could separate them into distinctive classes and so we aimed to evaluate that hypothesis by studying cardiac tissues immunostained with c-kit antibodies.

Materials and Methods: Autopsy samples of cardiac tissues were obtained from thirteen young human cadavers, negative for cardiac pathology. Immunohistochemistry on formalinfixed paraffin-embedded tissues was performed using monoclonal antibodies for CD117/c-kit.

Results: Within the subepicardium we found c-kit+ CTCs: (a) within the walls of the subepicardial coronary arteries, at the media-adventitia border and (b) embedded in subepicardial fat. c-kit+ CTCs were also present within the deep subendocardium. Well-designed intramyocardial c-kit+ positive networks were present in walls of all four cardiac chambers. The CTCs of these networks presented were small-sized, bipolar (in the plane of section) and, seemingly, serially linked. A different type of c-kit+ CTCs, larger, was identified within myocardium - multipolar CTCs (in the plane of section), and located in larger myocardial interstitia.

Conclusions: c-kit+ CTCs of two classes build up a veritable cardiac interstitial system and may be analogous to the gastrointestinal interstitial Cajal cells (ICCs) that mediate neurotransmission or act as pacemakers. c-kit appears as a reliable marker for CTCs and could be used accurately for light microscopic diagnostics, for evaluating the cardiac ICLCs/telocytes involvement in various cardiac pathological states, especially in rhythm dysfunctions, prior to any long-lasting TEM examination. Acknowledgements: UEFICSU Grant 2642/2008, The Young Researchers Grant 8846/05.10.2009, "Victor Babeş" University of Medicine and Pharmacy, Timişoara, Romania and the SOP HRD, financed from the ESF and by the Romanian Government under the contract number POSDRU/ 89/1.5/S/64109.

\section{PP-210}

Anatomical and histological structure of vomenasal organ VNO (Jacobson organ) in Camelus domesticus Var.dromedaris persica

$\underline{\text { Karimi H }}$

University of Tabriz, Iran
The vomenasal organ VNO is tubular structure that lied in roof of nasal cavity. The important role of this organ is olfaction of sexual odor. Therefore, believed VNO is accessory olfactory system. Anatomical and histological structure of $\mathrm{VNO}$ was described in many different animals. In this research, position, anatomical structure and histological properties of VNO of Camelus Domesticus Var.dromedaris persica was studied.14 healthy male head was collected from Shahre Rey industrial slaughterhouse. 7 head was used for anatomical studies and 7 head was used for histological studies. The length of VNO (distance between incisive papillae and 2th premolar teeth), length and wideness of dental pad and number and wideness of palatine crests was studied. For anatomical studies, the mandible of heads was removed and maxilla and nasal cavity was sectioned longitudinal and transversely. For histological studies, the mandible was removed and initially $0.5 \mathrm{~cm}$ of $\mathrm{VNO}$ was sectioned. Then nasal cavity was cute in many segments with $2 \mathrm{~cm}$ distance. Obtained segments were brought for routine histological studies. The obtained results were disappeared which VNO of Camelus Domesticus Var.dromedaris persica have 3.85 \pm 0.31 $\mathrm{cm}$ diameter in initial part and $1.57 \pm 0.18 \mathrm{~cm}$ diameter in end part. The length of VNO was measured $15 / 61 \pm 0.59 \mathrm{~cm}$. Also, histological studies were disappeared which VNO was enclosed by J shape hyaline cartilage. The covering epithelium was respiratory epithelium in lateral wall and olfactory epithelium in medial wall of VNO. Lamina propria and tunica submucosa was vascular fibroelastic connective tissue with seromucose gland that serouse seratory unites were abundant. This research results were showed which the duct of VNO (not similar another animals opened) into nasal cavity. Observation respiratory epithelium and olfactory epithelium particularly was emphasis of important role of this organ in received sexual odor.

\section{PP-211}

\section{Effect of nilvadipine on the penicillin-induced epileptic seizures and hippocampal neuronal loss}

Onur $\mathrm{S}^{*}$, $\underline{\operatorname{Akdogan} \mathrm{I}^{*}}$, Genc O** , Hatip-Al-Khatib I***, Erken $\mathrm{H}^{* *}$, Yonguc $\mathrm{N}^{*}$, Ozdemir B*, Adiguzel E*

Department of Anatomy, Faculty of Medicine, Pamukkale University, Denizli, Turkey*; Department of Physiology, Faculty of Medicine, Pamukkale University, Denizli, Turkey**; Department of Pharmacology, Faculty of Medicine, Pamukkale University, Denizli, Turkey***

Introduction and Purpose: Epilepsy is a common neurological disorder due to uncontrolled, abnormal and intensive electrical discharges of a group of neurons in central nervous system. It results in neuronal deaths in various regions of brain especially in hippocampus. It is believed that calcium influx is one of the main mechanisms that stimulate cell death. The aim of this study is to research the effects of nilvadipine on penicillin-induced epilepsy epileptic seizures and number of hippocampal neurons in rat. 
Tools and Method: We used 4 month-old, 25 female adult Spraque-Dawley rats in our study. They were divided into five groups each of five rats: Sham (S), Control-saline (C), Epilepsy, Epilepsy+Nilva1 and Epilepsy+Nilva2. Epilepsy was induced by intracortical (i.c.) $1500 \mathrm{IU} / 2 \mu \mathrm{l}$ penicillin. Epilepsy+Nilva1 group had firstly received nilvadipine $(3.2 \mathrm{mg} / \mathrm{kg}$, i.p.) followed 30 min later by penicillin. In Epilepsy+Nilva2 group nilvadipine $(3.2 \mathrm{mg} / \mathrm{kg}$, i.p.) was administered for 7 days, and penicillin was administered $2 \mathrm{~h}$ after the last dose of nilvadipine. EEG (number and amplitude of spike wave) was recorded for all groups before and after operations. The rats were decapitated at the end of the experiments. Hippocampal neuron numbers were counted by the optical fractionator method (a stereological method) in transverse brain sections.

Findings: The results showed that in Epilepsy+Nilva1 and Epilepsy+Nilva2 groups the spike numbers and spike amplitudes where less than Epilepsy group $(\mathrm{P}<0.05)$, whereas sum of the neurons of hippocampal CA1-CA2-CA3 regions were higher than Epilepsy group ( $\mathrm{p}<0.05)$, but less than $\mathrm{C}$ group $(\mathrm{P}<0.05)$.

Results and Interpretation: According to the present results, nilvadipine reduces spike numbers and amplitudes in epileptic seizures and reduces neuron loss due to experimental penicillin-epileptic seizures in rat hippocampus.

\section{PP-212}

The effect of hyperbaric oxygen on nerve healing at acute thoracic spinal cord injury

Dagci $T^{* *}$, Erturk $M^{*}$, Dayan $K^{* * *}$, Sengul G*

School of Medicine Department of Anatomy, Ege University, Izmir*; School of Medicine Department of Physiology and Center for Brain Research, Ege University, Izmir**; Department of Orthopaedic and Trauma Surgery, Izmir Bozyaka Training and Research Hospital Department of Orthopaedic and Trauma Surgery, Izmir ***

Objective: In this study, the effect of HBO (hyperbaric oxygen) therapy on healing in experimental spinal cord injury was addressed via the comparison of HBO's effectiveness with preoperative, postoperative or preoperative and postoperative administration.

Material and Method: 48 male Sprague-Dawley rats (200-250 gr.) were divided into eight groups. The spinal cord injury (SCI) was applied with an aneurysm clip placed at the T9-11 level. Biochemical results of spinal cord samples and functional healing of rats were compared.

Results: When the laminectomy group was compared with the SCI group, the significant increase in SOD, GPx levels $(\mathrm{p}<0.05)$ and significant decrease in NOS and NO levels $(\mathrm{p}<0.05)$ indicated the adequacy of the trauma. When SCI and PreopSCI were compared, a significant increase $(\mathrm{p}<0.05)$ was detected in NOS and NO levels, while the comparison of SCI and PostopSCI revealed a significant decrease in SOD levels $(\mathrm{p}<0.05)$ and a significant increase in NOS and NO levels $(\mathrm{p}<0.05)$. The comparison of PreopOIH and PostopSCI indicated a significant decrease in SOD levels $(\mathrm{p}<0.05)$, while the same significant decrease in SOD levels was observed in the comparison of PreopSCI and PrepostSCI $(\mathrm{p}<0.05)$. In terms of functional healing, which was evaluated on the basis of $\mathrm{BBB}$ scoring, significant improvement was detected $(\mathrm{p}<0.05)$ in PostopSCI and PrepostSCI, when compared to the SCI group.

Conclusion: HBO therapy was found to be beneficial for thoracic spinal nerve damage in terms of biochemical parameters and functional healing. While HBO therapy is already beneficial in preoperative period, its benefits increase by orders of magnitude in postoperative period.

\section{PP-213}

\section{Volumetric changes and cranial anomalies in MRI of patients with chiari malformation type 1}

Alkoc $\mathrm{OA}^{*}$, Songur $\mathrm{A}^{* *}$, Eser $\mathrm{O}^{* * *}$, Toktas $\mathrm{M}^{* *}$, Gonul $\mathrm{Y}^{* * * *}$, Esi $\mathrm{E}^{* * * * *}$, Haktanir $\mathrm{A}^{* * * * * *}$

Department of Anatomy, School of Medicine, Duzce University, Duzce, Turkey*; Department of Anatomy, School of Medicine, Afyon Kocatepe University, Afyonkarahisar, Turkey**; Department of Neurosurgery, School of Medicine, Afyon Kocatepe University, Afyonkarahisar, Turkey***; Department of Anatomy, School of Medicine, Namık Kemal University, Tekirdag, Turkey****; Department of Radiology, Afyonkarahisar State Hospital, Afyonkarahisar, Turkey*****; Department of Radiology, School of Medicine, Afyon Kocatepe University, Afyonkarahisar, Turkey*****

Objective: The aim of our study was to measure the total, supratentorial (ST) and infratentorial(IT) intracranial volumes; examine the anomalies; measure foramen magnum diameter, posterior cranial fossa (PCF) volume and platybasia using magnetic resonance (MR) images of patients with Chiari Malformation (CM) Type1. We aimed at clarifying whether the volume difference between the compartments or congenital anomalies are more significant regarding etiology of CM Type1.

Material and Method: 33 patients with CM Type1, aged between 20-65 years, without intracranial lesions and 33 control subjects were included in our study. In sagittal MR images, tonsillar herniation length and concurrent anomalies were evaluated; ST, IT and total intracranial volumes were measured using Cavalieri Method; several cranial distances and angles used for evaluation of platybasia and PCF development were measured. Results obtained from control and CM Type1 group were analyzed statistically. Comparisons and relations between groups were assessed. 
Results: Tonsillar herniation was $9.091 \pm 3.392 \mathrm{~mm}$ in CM Type1 group. Tonsillar herniaton was mild in $66.7 \%$, moderate in $24.2 \%$ and severe in $9.1 \%$ of the patients. Tonsillar herniation and concurrent syringomyelia, concavity and defect of clivus, herniation of bulbus and fourth ventricle, basilar invagination and craniovertebral junction anomaly rates were found to be $30.3 \%, 27 \%, 18.2 \%, 3 \%$ and $3 \%$, respectively. Absence of cisterna magna was encountered in $87.9 \%$. ST, IT, total intracranial volumes were significantly decreased in patient group. Distance measurements used for evaluation of platybasia including distance between Chamberlain line and tip of dens axis, Klaus index, clivus length, distance between internal occipital protuberance and opisthion were significantly decreased in patient group. Welcher basal angle and Boogard angle were found to be increased, tentorial slope was found to be decreased.

Conclusion: Especially IT intracranial volume decrease is prominant. Regarding the findings, we think that congenital anomalies are more important in CM Type 1.

\section{PP-214}

\section{Histomorphometric changes in substantia nigra due to} lead toxicity in albino rat

Verma S*, Bharihoke $V^{* *}$, Ram Prakash $\mathrm{R}^{* *}$

Pondicherry Institute of Medical Sciences, Delhi, India*; University College of Medical Sciences, Delhi, India**

The widespread presence of lead on the earth has been a concern to the health workers. Many effects of lead toxicity have been shown histopathologically while other conditions still need to be confirmed at the microscopic level. Chronic exposure to lead leads to impairment of dopaminergic transmission at the straitum and hypothalamic levels in the rat (Memo et.al. 1981). This study was carried out in order to see if lead actually affects the dopamine producing cells in the Susbstantia nigra of rat or not. 45 adult albino rats were divided into three groups of 15 animals each consisting of a normal, control and experimental groups. The experimental group was given a daily dose $0.05 \mathrm{mg} / \mathrm{gm}$ body weight lead acetate in water, made acidic with acetic acid at pH 5.5 for 30 days. The animals were sacrificed on the 31 st day by perfusion with $10 \%$ formal saline. Serial paraffin sections of the midbrain were stained with $\mathrm{H} \& \mathrm{E}$, Glees silver and Cresyl violet stains. The substantia nigra was studied under the light microscope. Patchy degenartion of neurons of the substantia nigra and crus cerebri was seen in the experimental animals. These changes explain the manifestation of Parkinson type syndrome associated with chronic lead toxicity.

\section{PP-215}

The effect of dimensionality on growth and differentiation of neural progenitors from different regions of fetal rat brain in vitro

Luu $\mathrm{H}^{*}$, Searle $\mathrm{K}^{* *}$, Liu $\mathrm{Y}^{*}$, Parker T**

Institute of Neurobiology, Environment and Genes Related to Diseases Key Laboratory of Education Ministry, Xi'an Jiaotong University College of Medicine, Xi'an, p. R. China*; Institute of Neuroscience, Department of Human anatomy and cell biology, School of Biomedical Sciences, University of Nottingham, Nottingham, UK**

Three dimension cultures are currently used in a broad range of cell biology studies. However, the effect of dimensionality on bio-behaviour of neuroprogenitors remains an unmet need. Cells were isolated from cerebral cortex, cerebella and brainstem of fetal rat brain, then cultured in serum free DMEM/F12 medium and DMEM with $10 \%$ FBS. The character of neuroprogenitor cells was identified by morphological and immunocytochemical criteria. The growth and differentiation of neuroprogenitors in 3 dimensional spheroids was compared with that in 2 dimensional monolayer cultures. Results demonstrated that cell proliferation increased by up to $17 \%$ in spheroid diameter over 10 days. More neuroprogenitors undertook neuronal differentiation in spheroids than that in 2 dimensional monolayer cultures. In comparison with the undissociated brain tissue, the neuron/astrocyte ratio, as shown by NF: GFAP immunoreactivity, in spheroids from cerebral cortex and cerebella is similar to that found in adult rat tissue. However, neuron/astrocyte ration in spheroids from brainstem is more close to fetal brain tissue. Our results suggested that three-dimensional spheroid culture system mimics the in vivo cytoarchitecture to a greater extent that more closely reflects adult tissue cellular composition. The niche in spheroids is more favorable for neuronal precusors' survival and differentiation while the cue in artificial 2 dimensional monolayer cultures is favoring glial cells survival. The differences between brainstem and other two brain regions could be due to the specific spatio-temporal cues that are essential for neuronal development.

\section{PP-216}

Comparison of three methods for the estimation of the pineal gland volume using magnetic resonance imaging: a stereological study

$\underline{\text { Acer } \mathrm{N}^{*}}$, Ilica AT ${ }^{* *}$, Turgut AT ${ }^{* * *}$, Ozcelik O*, Turgut $\mathrm{M}^{\star * * *}$ Department of Anatomy, School of Medicine, Erciyes University, Kayseri, Turkey*; Department of Radiology, Gulhane Military Medical School, Ankara, Turkey**; Department of Radiology, Ankara Training and Research Hospital, Ankara, Turkey***; Department of Neurosurgery, School of Medicine, Adnan Menderes University, Aydin, Turkey**** 
The pineal gland has many physiological activities, such as circadian rhythm, but it is a very important neuroendocrine organ. Radiologically, it is usually difficult to distinguish small pineal tumors via magnetic resonance imaging (MRI), because it is small organ. Although many studies have estimated pineal gland volume by the mathematical approach the elliptic formula, there is no stereological study so far. The objective of the current study was to determine pineal gland volume using stereological methods and standard image analysis method named region of interest (ROI) on MRI. In this study, pineal gland volumes were estimated in a total of 62 subjects (36 females, 26 males,) who were free of any pineal lesions or tumors. The mean \pm SD pineal gland volumes for the point counting, planimetry and ROI groups were 99.55 \pm 51.34 , $102.69 \pm 40.39$, and $104.33 \pm 40.45$, respectively. No significant difference was found among methods for the pineal gland volume $(\mathrm{p}>0.05)$. The mean CE for pituitary gland volume estimates derived from the point counting technique was $5.8 \pm 2.2 \%$. From these results, it can be concluded that each techniques are unbiased, efficient and reliable methods and are ideally suitable for in vivo examination of MRI data for pineal gland volume estimation.

\section{PP-217}

Effects of grape seed extract on brain weight and CA3-2 pyramidal neuron number of hippocampus in the hypoxic-ischemic neonatal rats: preliminary results

Adiguzel E* $E^{*}$ Yonguc GN**, Yilmaz $\mathrm{Y}^{* *}$

Department of Anatomy, School of Medicine, Pamukkale University, Denizli, Turkey*; Department of Food Engineering, Faculty of Engineering, Pamukkale University, Denizli, Turkey**

Objectives: Grape seed extract have antioxidant activity by reason of its fenolic contents. The aim of this study is to investigate the effect of subcutaneously administered grape seed extract on brain weight and CA3-2 pyramidal neuron number in hypoxic-ischemic encephalopathy model of neonatal rats.

Materials and Method: Thirty Wistar rat pups were included in this study. Animals divided in four groups: 1- hypoxicischemic pups treated with grape seed extract 2- wild pups treated with grape seed extract, 3 - wild pups for normal control, 4- Experimental hypoxic-ischemic pups for injured control. A modification of Levine preparation was used to develop neonatal hypoxic-ischemic encephalopathy in the 1st and 4th Groups on the 12th day after delivery. Grape seed extract injection was performed in 1 st and 2 nd groups during three days after operation (50 mg per $\mathrm{kg} /$ day). Animals were perfused with paraformaldehyde solution and decapitated on the day 60th after operation. The brains were removed, weighted, frozen and cut with a thickness of $30 \mu \mathrm{m}$ in a cryostat. Sections were collected by systematic random sampling and stained with cresyl violet. Total pyramidal neuron number of the right hippocampuses CA2 and CA3 subfields were estimated using the optical fractionator method.

Results: Means of the brains' weights (g) were $1.98+/-0.06$, $2.21+/-0.04,2.40+/-0.06,2.19+/-0.04$ consecutively in the groups $1-4$ ( $\mathrm{p}$ values between the groups $1 \mathrm{vs} 2=0.006$, $1 \mathrm{vs} 3=0.000$, 1vs4=0.037). Although the mean of the total pyramidal neuron number in right hippocampal CA3-2 subfield was the highest in the 3 rd group, and the lowest in the 4th group, there was not statistically significant differences among the groups $(\mathrm{p}>0.05)$.

Conclusion: Results showed that the grape seed extract injection worsened the brain weight loss in the rats, suffered from hypoxic-ischemic encephalopathy. This study was supported by TUBİTAK (SBAG-3994. 108S157) and Pamukkale University research grant (2008TPF005)

\section{PP-218}

\section{Evaluation of intra-rater variation for estimation of volume of cerebral structures using the Cavalieri principle on magnetic resonance imaging}

\section{Elfaki A, Sahin B}

Department of Anatomy, Faculty of Medicine, Ondokuz Mayıs
University, Samsun, Turkey

Introduction and Purpose: Measurement of brain volume is regarded as an objective marker of neurodegenerative diseases. The purpose of this study is to evaluate intra-rater variation for the estimation of volume of cerebral structures using the Cavalieri principle on magnetic resonance (MR) images to determine its reproducibility.

Tools and Method: The MR images of 30 cases were analyzed using the same standard protocols of the Cavalieri principle in two sessions with one month intervals. The structural MR images were analyzed using the ImageJ software by the same observer. The threshold process was used for the cut surface area assessments. The volume of hemispheres, total brain, gray and white matters were estimated by means of the multiplication of cut surface are by the section interval. The same sections were used in both sessions. The results of two sessions were compared using Wilcoxon Signed Rank test. Findings and Result: The mean total brain, right and left hemispheres volumes of first session were $1089.53,544.82$ and $544.71 \mathrm{~cm}^{3}$, respectively. The mean total brain, right and left hemispheres volumes of second session were 1086.62, 543.99 and 542.63 $\mathrm{cm}^{3}$, respectively. There was no statistically significant difference between the data $(\mathrm{p}>0.05)$. The mean total gray and white matters volumes were $553.55,535.98$ and $549.32,537.31 \mathrm{~cm}^{3}$ 
for the first and second sessions, respectively. There was no statistically significant difference for the gray and white matters results $(p>0.05)$. Interpretation Our results showed that the reproducibility of the obtained data is good. The volume of cerebral structures could be estimated using the Cavalieri principle on MR images for comparative studies. We are planning to evaluate inter-rater difference in advance. This study was supported by the: - Third World Organization for Women in Science (TWOWS)

\section{PP-219}

\section{Effects of housing conditions on the hippocampal volumes of prenatally stressed rats}

Soztutar E, Topal F, Yucel F, Ulupinar E

Department of Anatomy, Faculty of Medicine, Eskişehir Osmangazi Universıty, Eskisehir, Turkey

In rodents, while majority of the neurons of the CA1-3 areas are produced during the last days of gestation; approximately $85 \%$ of the dentate's granule cells are produced postnatally. As both a target of glucocorticoids and an active participant of the HPA axis, the hippocampus is a primary target for the effects of early life stress exposure. In this study, we aimed to compare the effects of diverse housing conditions on the hippocampal volumes of prenatally stressed rats by using stereological analysis methods. Wistar rats were exposed to restraint stress during their last week of gestation, for 3 hours. After weaning, male offspring from different litters were grouped according to housing conditions (Enriched=EC, Standard=SC, Isolated = IC). In EC and SC, 12 and 4 animals were housed as a group, respectively; but in IC, animals were housed individually. After seven weeks, six animals from each group were perfused with $4 \%$ paraformaldehyde. The right hemispheres of each animal were cut into serial sections using vibratome and stained with Golgi-Cox method. A point counting grid was applied on the sampled sections in a systematic-random manner and the Cavalieri principle was used to estimate the volumes of related regions. The highest volume of dentate gyrus $\left(0.59 \pm 0,05 \mathrm{~mm}^{3}\right)$ and CA1-3 regions of the hippocampus $\left(1.41 \pm 0.03 \mathrm{~mm}^{3}\right)$ were found in pups housed in IC; followed by EC $\left(0.53 \pm 0.03 \mathrm{~mm}^{3}\right.$ and $1.37 \pm 0.06 \mathrm{~mm} 3)$ and SC $\left(0.50 \pm 0.03 \mathrm{~mm}^{3}\right.$ and $1.30 \pm 0.06$ $\mathrm{mm}^{3}$ ). However, these volumetric differences among the groups, as well as their ratios to cortical or cerebral volumes did not reach to a significance level when compared with one way ANOVA test. These results suggest that early adulthood hippocampal volumes might be influenced by extrinsic signals in addition to the individual genetic program. However, in order to understand the exact contribution of volumetric changes to hippocampal functions, further investigation needs at cellular and molecular level.
PP-220

Histopathological effects of the aqueous leaf extract of sage officinalis on the frontal cortex, liver, kidney and testes of adult male Sprague Dawley rats

Adekomi DA, Tijani AA, Adeniyi TD, Caxton-Martinsg ED

University of Ilorin, Ilorin, Kwara State, Nigeria

Sage has been used in the 'Old and New world' as a spice and a medicine. Salvia officinalis is also more commonly used medicinally, horticulturally, and commercially. Unlike other species of Salvia genus, the histopathological effects of the plant as a marker of tissue toxicity are not well documented. In recent years, the different potentials of this plant such as hypoglycemic, anti-nociceptive and anti-inflammatory, anti-oxidant, anti-ischemia, anticonvulsant, antiulcer effects, antibacterial activities and anti-mutagenic effects were evaluated. These effects introduce this plant for more toxicological evaluations as a herbal remedy. Ten male Sprague Dawley rats of about four-five months old were used. The animals were randomly assigned into two groups identified as groups A and B. Group A ( $n=5)$ served as the control group while group B $(n=5)$ was the treatment. $300 \mathrm{mg} / \mathrm{kg}$ body weight of the aqueous leaf extract of Salvia officinalis was administered once daily for 14 consecutive days orally through a sterilized flexible plastic orogastric tube, while the control rats received equal volume of phosphate buffered saline (PBS). The animals were sacrificed twenty-four hours after the last administration. The frontal cortices were excised, fixed in 10\% formol calcium for eighteen hours and were processed for routine light microscopic study using Hematoxylin and Eosin (H\&E) method. The histological findings revealed that the extract treated sections showed histological derangements ranging from degeneration, distortion, vacuolations and evidence of necrotic bodies while the sections from the animals in group A conformed to normal histological profile. These findings suggest a deleterious and toxic effects of aqueous leaf extract of Salvia officinalis on the frontal cortex of male Sprague Dawley rats.

\section{PP-221}

\section{Gender dependent influences of diverse rearing conditions on cerebral volumetric parameters of prenatally stressed rats}

Topal F, Soztutar E, Yucel F, Ulupinar E

Department of Anatomy, Faculty of Medicine, Eskisehir Osmangazi University, Eskisehir, Turkey

In animal experiments, prenatal stress exposure causes negative behavioral and cognitive outcomes by influencing the offspring's brain morphology. The present study was conducted to investigate whether opposing rearing conditions influence 
the cerebral growth parameters of prenatally stressed rats in a gender specific manner. Wistar rats were exposed to stress paradigm between 14-21th gestational days by keeping the dam immobile in wire-mesh cylinders, for 3 hours duration. After weaning, male and female offspring were assigned to enriched (EC) or isolated conditions (IC), and reared in special cages equipped with turning wheels, climbing ladders, platforms and stimulant objects or individually in metal cages, respectively. Seven weeks later, six offspring from each group and gender were sacrificed and their brains were sectioned on a vibratome. Golgi-Cox stained sections were used for volumetric measurements of the brains with the Cavalieri estimation method. While the volumes of cerebral cortex $(\mathrm{p}<0.001)$ and the right cerebral hemispheres $(\mathrm{p}<0.01)$ were significantly higher in males reared in $\mathrm{EC}$; hippocampal volumes including dentate gyrus and CA1-3 regions were found similar in both genders. Thus, the ratio of hippocampal volumes to cortical or total hemispheric volumes were significantly higher in females than those of males $(\mathrm{p}<0.01)$. In animals reared in IC, only cerebral hemisphere volume was higher in males $(\mathrm{p}<0.05)$. Since hippocampal and cortical volumes were similar in both gender, the ratio of hippocampus to cortex $(\mathrm{p}<0.05)$ and hemisphere $(\mathrm{p}<0.01)$ were again significantly higher in females. On the other hand, the ratio of female cerebral cortex to whole hemisphere was not significantly different than those of males, in both groups. Induction of physical and cognitive activities by rearing the animals in EC especially alters the hippocampal volumetric parameters in favor of females. Social isolation condition seems less remarkably affected the prenatally stressed male offspring.

\section{PP-222}

\section{Cerebellar neurotrophin levels of prenatally stressed rats reared in different environments}

$\underline{\text { Ulupinar } \mathrm{E}^{*}}$, Ay $\mathrm{H}^{*}$, Erol K**

Department of Anatomy, Faculty of Medicine, Eskisehir Osmangazi University, Eskisehir*; Department of Pharmacology, Faculty of Medicine, Eskisehir Osmangazi University, Eskisehir, Turkey**

Cerebellum, due to the earliest beginning of differentiation; but the latest end of maturation, is one of the most affected regions of the brain from adverse conditions. Exposure to prenatal stress decreases the synaptic plasticity capacity of cerebellar neurons by causing long-lasting structural alterations. The aim of present study was to investigate whether the environmental rearing conditions affect cerebellar neurotrophin levels of prenatally stressed Wistar rats. Prenatal stress paradigm was applied on 14-21st gestational days, for 3 hours. Weaned female and male offspring from different litters were reared in standard (=SC), enriched (=EC) or isolated conditions (=IC) for
6 weeks, and then sacrificed by decapitation ( $n=6$ for each group and gender). Neurotrophin concentrations in the left paravermal cerebellar hemispheres were measured by ELISA. NT-3 levels in the cerebellar hemispheres of different groups were not significantly different in both genders. Nerve growth factor (NGF) levels were not different in males; but it was found significantly lower in females reared in IC than those reared either in SC $(\mathrm{p}=0.008)$ or $\mathrm{EC}(\mathrm{p}=0.017)$. Brain derived neurotrophic factor (BDNF) levels, on the other hand, were not different in females; but significantly lower in males reared in EC than those reared in SC $(\mathrm{p}<0.001)$ and $\mathrm{EC}(\mathrm{p}=0.013)$. Glia derived neurotrophic factor (GDNF) levels, too, were significantly different in offspring reared in EC and IC. However, these differences were caused by the increases of GDNF levels in females; but decreases in males reared in EC. These results showed that cerebellar neurotrophin levels of pups reared in different environmental conditions exhibit gender dependent differences. It is thought that in females, reduction of NGF levels in social isolation; but in males, reduction of BDNF and GDNF levels in enriched environmental conditions negatively affects the adaptation capacity of neurons to environmental alterations. Supported by ESOGU-Scientific Research Projects Commission (\#200911016)

\section{PP-223}

\section{Anatomy of the facial nerve paralysis}

Gulbar S*, Kasirga UB*, Sonmez FT**, Tunali S*

Department of Anatomy, Faculty of Medicine, Hacettepe University, Ankara, Turkey*; Department of Emergency Medicine Diskapi Yildirim Beyazit, Training and Education Hospital Altindag, Ankara, Turkey**

Objective: In this study we aimed to provide new illustrations on the anatomy of the facial nerve and facial paralysis, for a better understanding of the facial nerve paralysis which is a common case in emergency medical services.

Materials and Methods: All available anatomy textbooks and atlases, as well as current literature were reviewed carefully. We further referred the computerized tomography images of the patients admitted to Yildirim Beyazit Education and Research Hospital Emergency Service. Watercolor and waterbased acrylic drawings were scanned to get digital versions. Finally, using Adobe Photoshop CS5 software, the illustrations were edited to better display fine details.

Results: We draw eight illustrations showing the nuclei of the facial nerve, course of the nerve, the structures that are innervated by specific branches, and the probable paralyses in facial nerve lesions.

Conclusion: We hope this new illustrations would help to understanding the anatomy and paralysis of the facial nerve, and contribute to the available bibliography on this subject. 


\section{PP-224}

Hot potatoes as a useful tool for student's self-revision when learning neuroanatomy

Ordonez FJ*, Rosety-Rodriguez $\mathrm{M}^{* *}$, Diaz-Ordonez $\mathrm{A}^{* *}$, Garcia-Gomez N**, Rosety I*, Fornieles G**, Garcia-Gomez $\mathrm{S}^{* * * *}$, Rosety $\mathrm{MA}^{* *}$, Camacho-Molina A***

Human Anatomy Department, University of Cadiz, Cadiz, Spain*; School of Sports Medicine. University of Cadiz, Cadiz, Spain**; Hospital Sas Juan Ramon Jimenez, Huelva, Spain***; Human Anatomy Department, University Complutense, Madrid, Spain****

Teaching and learning with ICT helps to support a range of learning styles which may not be catered for by traditional book methods. Further, they may improve both understanding and motivation by providing an alternative learning experience for revision or self-assessment. The Hot Potatoes suite is a set of Computer- Assisted Language Learning tools that lets teachers easily create interactive web-based exercises. The program is easy to use with Moodle and it is also free of charge for those working for non-profit educational institutions. The present study was designed to ascertain student's opinion regarding the use of Hot Potatoes as a revision tool in the optative subject "Fundamentals of Neuroanatomy". To get this goal 96 students, aged $20.4 \pm 1.6$ years-old, from the Faculty of Education (Cadiz University) volunteered for this cross-sectional study. These students are well trained in ITC and related fields. The Hot Potatoes software suite included six different modules to make interactive exercises (multiple-choice; short-answer; jumbled-sentence; crossword; matching/ordering; gap-fill) that can be used in and out of the classroom. Since all modules are similar in design and share basic features, learning one of the Hot Potatoes modules makes it easy to learn the other five. It should be also pointed out this study has been approved by an Institutional Ethic Committee. All participants reported that this ITC had increased their interest in the subject. Further $92 \%$ considered that it had improved their learning task. The most striking features were it allowed them to know the level of their own knowledge and to self-correct errors and mistakes during their learning process. It may be concluded Hot Potatoes suite was well-accepted by our students as a revision tool for Fundamentals of Neuroanatomy. Further studies on this topic are highly required.

\section{PP-225}

Morphological analysis of Purkinje cells in rats housed differrent environments after prenatal stress exposure

Ay $\mathrm{H}^{*}$, Erol K**, Ulupinar $\mathrm{E}^{*}$

Department of Anatomy, Faculty of Medicine, Eskisehir Osmangazi University, Eskisehir*; Department of Pharmacology, Faculty of Medicine, Eskisehir Osmangazi University, Eskisehir, Turkey**
The cerebellum is a part of the nervous system involved in not only motor movements, but also cognitive functions and emotional responses. Previous studies have shown that environmental conditions might cause neuroanatomical and behavioral changes in rodents. This study was conducted on prenatally stressed rats, to investigate the effects of diverse environmental conditions on morphological characteristics of the cerebellar Purkinje cells. Wistar rats were exposed to three-hour immobilization stress period between gestational days 14-21. After weaning, male offspring were divided into three groups and housed in standard (=SC), enriched (=EC) or isolated (=IC) conditions from postnatal day 21 to 65 . Animals in EC were physically and cognitively stimulated with various objects; whereas animals in IC were separated socially. At the end of housing period, all rats were behaviorally evaluated with rota-rod test, to assess their motor coordination abilities. Performances of animals were measured by latencies to falling off the rotating cylinder, during 3 subsequent days. Mean latencies were significantly $(\mathrm{p}<0,001)$ higher in SC than those of IC and EC. Interestingly, these animals were not able to improve their motor coordination abilities over time. After behavioral tests, animals were sacrificed and vibratome sections of paravermal cerebellar hemispheres were stained with Golgi-Cox procedure. Detailed morphometric analysis of Purkinje cells were conducted from the camera lucida drawings of neurons. The length of primary trunk, number of primary branches and total densities of dendritic branches were measured by using Scion Image Analysis program. In animals reared in EC, the length of primary trunk was found significantly $(p=0,001)$ shorter than those of SC. Dendritic complexity parameters, on the other hand, were found similar among groups. These results imply an impaired adaptation capacity in the prenatally stressed males to diverse environmental conditions at morphological and behavioral level.*Supported by ESOGU-Scientific Research Projects Commission (\#200911016).

\section{PP-226}

\section{Assessment of maturity and morphology of human oocytes after vitrification and IVM}

Khalili MA, Mohsenzadeh M, Jahromi V, Nazari S

Research \& Clinical Center For Infertility, Shahid Sadoughi University of Medical Sciences, Yazd, Iran

Background: In assisted reproduction technology (ART), 85\% of retrieved oocytes are mature (MII stage); while, the rests are at the GV and MI stages. These immature oocytes should be first matured in vitro (IVM), so they may be used in subsequent ART program. The aim was to evaluate the maturity and morphology of human oocytes after vitrification and IVM

Materials and Methods: The first group of immature oocytes ( $\mathrm{n}=103$ ) underwent frest IVM (fIVM) and second group $(\mathrm{n}=102)$ were vitrified first and stored in liquid nitrogen tank. After thawing, the samples underwent IVM (vIVM). Oocyte 
maturation was confirmed with presence of the first polar body under dissecting microscope. From morphology point of view, three oocyte characteristics were assessed: ooplasm coloration, PVS normality and shape. In both fIVM and vIVM groups, oocyte morphology and maturity were assessed.

Results: $27 \%$ of oocytes were immature (9.5\% MI and $17.5 \%$ GV) and $73 \%$ were mature (MII). The rates of maturation in fIVM $(61.2 \%)$ group was significantly higher than vIVM group (33.3\%). Although the difference was insignificant $(\mathrm{P}=0.197)$, the percentage of maturation of immature oocytes in women under age of 30 was higher in both groups of fIVM and vIVM. Also, data showed that the rate of maturation after IVM was higher in patients with male factor infertility.

Conclusion: Vitrification is a suitable technique for preservation of immature oocytes in stimulated ART cycles. It should be noted that immature oocytes at GV stage stand the $-198 \mathrm{C}$ better than MI oocytes. In ART, we recommend to conduct IVM technology for fresh MI, and vitrify GVs for future use in IVM.

\section{PP-227}

\section{Denticulate ligament: anatomical properties, functional and clinical significance}

Ceylan $\mathrm{D}^{* * *}$, Abdullaev $\mathrm{T}^{* *}$, Tatarli $\mathrm{N}^{* * *}$, Seker $\mathrm{A}^{* *}$, Yildiz $\mathrm{SD}^{*}$, Konya D**, Kilic T**, Cavdar S*

Department of Anatomy, School of Medicine, Marmara University, Istanbul, Turkey*; Department of Neurosurgery, School of Medicine, Marmara University, Istanbul, Turkey** ; Institute of Neurological Sciences, Marmara University, Istanbul, Turkey***

Objective: The stabilization of the spinal cord within the vertebral canal is attibuted to be the major function of the denticulate ligaments. The vertebral column at different levels consists of variable motion capacity. The amount of stabilization nesseccery for the different levels of the vertebral column can vary. Therefore, "does regional difference in the motion capacity of the vertebral column reflect to structure of the denticulate ligament". Further, detailed anatomical description of the was aimed in the present study.

Method: 5 formalin-fixed adult were used in the present study. The denticulate ligaments were exposed via posterior approach and detailed anatomy were documented.

Result: Denticulate ligament is a narrow fibrous sheath that extends between the craniovertebral junction to the level of T12. The results of the present observations are as follows: 1) the number of ligaments varied between 18 - 20 in the adult cadaver. 2) the triangular denticulate ligament showed regional differeces; the first denticulate ligament was the largest, at cervical levels they were in the form of small and at thoracic levels they consisted of larger triangles. In lumbar vertebral levels no denticu- late ligaments were observed in the adult cadaver. 3) The apical attachments of the denticulate ligaments to the dura at cervical levels were via fibrous bands (2-4 mm), however at thoracic levels direct attachments of the apices were observed, at lower thoracic levels attachments were via fibrous bands (21-26 mm), 4) The ligament attachments were always superomedial to the below intervertebral foramen. This distance was below $10 \mathrm{~mm}$ at cervical and upper thoracic, $10-15 \mathrm{~mm}$ at lower thoracic levels and reached 15-20 $\mathrm{mm}$ at T11 and T12 levels.

Conclusion: Accurate knowledge of the ligament can be important for the clinical procedures in the spinal cord and craniovertebral junction.

\section{PP-228}

\section{The effect of mandibular anthropometry to tongue movements}

Yilmaz A

Department of Anatomy, Faculty of Medicine, Trakya University, Edirne, Turkey

Aim: The tongue is responsible for many different functions in human body. The tongue provides a path for food to travel through the digestive system, it has taste buds so that we can enjoy the flavor of that food, and the tongue is largely responsible for speech, among other things. The purpose of this study is to investigate the relation of mandibular anathropometry with a new, alternative method suggested to be used in estimation of tongue movements.

Methods: 114 volunteers who were studying at our university participated in this study. Interincisial distance, lower face height, mandible length and bigoinal distance were measured by using vernier caliper. Horizontal and vertical lines used for determined of tongue movements. Horizontal lines passing through the mid points of the upper lip and mandible, and vertical lines passing through the right and left infraorbitale were constituted on each subject. And subjects were asked to protruding tongue maximally and downward, protruding tongue maximally and upward, and protruding tongue maximally and laterally in sitting position with the neck and head in neutral position. The scores corresponding with the movements of tongue were compared with the mandibular anthropometry.

Results: Interincisial distances $59.18 \pm 6.60 \mathrm{~mm}$, lower face height 71.25 \pm 7.69, mandible length $95.36 \pm 8.51 \mathrm{~mm}$ and bigoinal distance $115,87 \pm 8,84 \mathrm{~mm}$ respectively. Both protruding tongue maximally and downward and protruding tongue maximally and upward were significantly different between interincisial distance, mandible length and bigonial distance ( $\mathrm{p}<0.001, \mathrm{p}<0.001$ and $\mathrm{p}<0.001$, each all respectively).

Conclusion: The estimate of tongue movements are important in the diagnostic and treatment of the musculoskeletal and 
neurological diseases. This new method can be combined with the mandibular anthropometry.

\section{PP-229}

\section{Temporomandibular joint mobility and tongue movements}

Taskinalp O, Yilmaz A, Cigali BS, Onal T

Department of Anatomy, Faculty of Medicine, Trakya University, Edirne, Turkey

\begin{abstract}
Aim: The aim of our study was to determine the range of motion (ROM) of the Temporomandibular joint (TMJ) in male adults by using digital inclinometer and its correlation with the tongue movements.
\end{abstract}

Methods: 90 male volunteers were enrolled in this study. Maximum mouth opening, right- left excursion and protraction degress were determined with electronic digital inclinometer. Horizontal lines passing through the mid points of the upper lip and mandible, and vertical lines passing through the right and left infraorbitale were constituted on each subject for determine tongue movements. And subjects were asked to protruding tongue maximally and downward, protruding tongue maximally and upward, and protruding tongue maximally and laterally in sitting position with the neck and head in neutral position. The scores corresponding with the movements of tongue were compared with the ROM of the TMJ.

Results: The average values for our subject were found as follows: maximum mouth opening $28.57 \pm 5.48$; right excursion $4.52 \pm ; 1.45$ left excursion $4.44 \pm 1.31$ and protraction $12.08 \pm 2.82$, respectively. Protruding tongue maximally and downward and protruding tongue maximally and upward were significantly different between maximum mouth opening and protraction $(\mathrm{p}<0.001$ and $\mathrm{p}<0.001$, each all respectively).

Conclusion: The estimate of ROM values for the TMJ are important in the diagnostic and treatment of the musculoskeletal and neurological diseases affecting joint movement. We conclude that tongue movements may be a practical method for evaluation of the derangements in this joint.

\section{PP-230}

The pathological anatomy of the ventrolateral foramen magnum meningiomas

Yilmazlar S, Isik S, Kocaeli H, Kaplan T

Department of Neurosurgery, School of Medicine, Uludag University, Bursa, Turkey

Introduction: Craniovertebral junction is a transition region and the neural and vascular anatomy is critical due to the asso- ciation with neurovascular structures. Spinal cord, vertebrobasilar artery, the posterior inferior cerebellar artery (PICA), anterior spinal artery and lower cranial nerves (IX, X, $\mathrm{XI}, \mathrm{XII})$ are the most important anatomical structures of the region. Meningiomas arising from this region pose surgical difficulties due to the anatomical location and pathological relationship with neurovascular structures.

Case Presentations: We reviewed the recent records of 3 patients with craniovertebral junction meningiomas that were operated on in our clinic. MRI examinations of the patients showed extraaxial lesions in the ventrolateral part of the craniovertebral junction in all cases. All three patients were operated on at the sitting position. Accessory nerve(XI), vertebral artery, PICA, C1 roots and lower cranial nerves were specially noted in terms of their relation with the tumor.

Results: The course of accessory nerve may be variable which is usually found stretched over the meningiomas during the posterolateral exposure. In the first 2 patients, XI were found to course dorsally above the lesion whereas in the third case, it was surrounded by the tumor. If the nerves and vertebral artery cannot be mobilized, the radical excision cannot be achieved. PICA was found in front of the tumor in the first case. In the second case, PICA was located dorsal to the tumor. In the third case, PICA was not seen due to its high origin from the vertebral artery.

Conclusions: The course of the spinal accessory nerve and PICA may be variable in ventrolaterally located foramen magnum meningiomas. When viewed from posterolateral approach they cross the operative field from above to below and from lateral to medial. If the nerve and artery cannot be mobilized, the exposure of the tumor's origin will also be difficult.

\section{PP-231}

\section{Anatomic features of left atrium proper and openings of pulmonary veins in relation to clinical evaluations}

Ikiz ZAA, Ucerler H, Ozgur T

Department of Anatomy, Faculty of Medicine, Ege University, Izmir, Turkey

Objective: Left atrium(LA) and left atrial pulmonary venous anatomy have clinical importance for atrial fibrillation and cardiac tumor surgery. A detailed anatomic knowledge of these structures may faciliate clinical evaluations. The aim of this study was to assess LA size and to demonstrate other anatomic features of LA with pulmonary vein openings in normal population.

Materials and Methods: Fiftysix heart specimens obtained from formalin fixed adult cadavers were examined for present study in Ege University Faculty of Medicine Anatomy Department. None of the specimens showed any evidence of pathology or previous surgery. 
Results: The roof of LA was determined as convex(14.3\%), concave $(10.7 \%)$ or flat $(75 \%)$ in specimens. A roof pouch was present in $8.9 \%$ of specimens. The diameter of LA roof, the anteroposterior LA diameter and the LA diameter between septum and lateral wall were recorded. Thin areas on the atrial wall were observed in front of a light source around left atrial appendage orifice. The left lateral prominent ridge was seen in $91.1 \%$ of specimens. Septal band was found in $7.1 \%$ of specimens. The number of pulmonary vein openings into LA was observed. Different patterns of pulmonary vein openings were described such as common ostium and additional vein.

Conclusions: It is expected that the data from present study will be a guide to the clinicians during the surgical approaches or radiologic examinations of LA proper and pulmonary vein openings into LA.

\section{PP-232}

\section{Poland syndrome associated with opposite side retractile testicle}

Ozer $\mathrm{CM}^{*}$, Akca ASD**, Akca F***, Yildiz $\mathrm{S}^{* * * *}$

Department of Anatomy, Faculty of Medicine, Karaelmas University, Zonguldak, Turkey*; Department of Family Medicine, Faculty of Medicine, Karaelmas University, Zonguldak, Turkey**; Gokcebey State Hospital, Zonguldak, Turkey***; Department of Anatomy, Gulhane Military Medical Academy, Ankara, Turkey****

Poland syndrome is a rare congenital anomaly of unknown cause characterized by unilateral, partial or complete chest wall hypoplasia and ipsilateral hand abnormalities as especially on the right side. Many abnormalities have been described in association with this syndrome as dextrocardia, dextroposition, renal malformation and tumours. We report the case of a 37 years old man with absence of left pectoralis major muscle, hypoplasia of the ribs II and III so depending on this lung herniation on the anterior wall of the chest and reduced apex lung volume but with right retractile testicle. We recommend genitourinary system investigations to be performed on patients with Poland syndrome.

\section{PP-233}

Thiel's embalmed cadavers, an ultrasound phantom to describe the transversus abdominis plane (tap) blockade

Sáez JH*, Ferrer MS**, Sánchez Del Campo F* Ferrer MLS ${ }^{* *}$, Ortega ST*, Agulló JA*, Garcia VA**, Calpena FT**

Anesthesia Department, San Juan University Clinic Hospital*; Histology and Anatomy Department, Miguel Hernández University (Alicante), Spain**

Background: Ultrasound-guided transversus abdominis plane (TAP) blockade has been described, as a suitable analgesia after abdominal wall surgery. To realize a successful posterior TAP block, we have to inject local anesthetic in the TAP, in the lateral abdominal wall between the costal margin and the iliac crest. This block is useful for surgery below the umbilicus.

Objetive: The purpose of this report is to describe the anatomy of the lateral abdominal wall and the technique of the TAP block (single shot and continuous block). Thiel's embalmed cadavers will help to the anesthetists to know the sonographic anatomy and to learn technical skills to realize this block.

Conclusions: Thiel's embalmed cadavers - which color, consistency and transparency of the tissue are very well preserved- can be use as a phantom to familiarize with sonoanatomy of the lateral abdominal wall, in addition to learning the technique, needle placement and reliable method to detect the injectate spread of local anesthetic. Bibliography: Hebbard P, Barrington MJ, Vasey C. Ultrasound-Guided Continuous Oblique Subcostal Transversus Abdominis Plane Blockade. Regional Anesthesia and Pain Medicine. 2010; 35 (5): 436-441. Xu D, Abbas S, Chan VW. Ultrasound phantom for hands-on practice. Regional Anesthesia and Pain Medicine. 2005 Nov-Dec;30(6):593-4. Walter Thiel. Die Konservierung ganzer Leichen in naturliche Farben. Annals of Anatomy. 1992; 174: 185-195.

\section{PP-234}

Human left atrial appendage anatomy and overview to its clinical significance

\section{Ucerler H, Ikiz ZAA, Ozgur T}

Department of Anatomy, Faculty of Medicine, Ege University, Izmir, Turkey

Objective: The left atrial appendage (LAA) is an important anatomic region since it is a source of thromboemboli in patients with atrial fibrillation. Although this anatomic and clinical relation it has received little attention until recent years. A detailed anatomic knowledge of LAA and neighbouring structures may faciliate surgical apporaches to LAA.

Materials and Methods: From the archive of Ege University Faculty of Medicine Anatomy Department, 56 hearts of adult formalin fixed cadavers were examined for this study. The sections showed no evidence of pathology or trauma.

Results: The morphological apperance of the LAA was classsified into two types: slender like a crooked finger (in 73.2\%) and stump-like(in 26.8\%). The lobe number of LAA was two in $64.3 \%$ specimens and three in $35.7 \%$. The LAA orifice was oval-shaped in $37.5 \%$ and round-shaped in $62.5 \%$. The bridge on the orifice was determined in one specimen. The longest diameter of LAA orifice was $16.5 \pm 4 \mathrm{~mm}$. The presence of accessory atrial appendage was observed in one specimen. The distances between the orifices of left superior pulmonary veins, 


\section{S126 Joint Meeting of Anatomical Societies, 19-22 May 2011, Bursa, Turkey}

circumflex artery, mitral valve and left atrial appendage were also measured in this study. The distance between LAA orifice and oval fossa was $27.5 \pm 5.5 \mathrm{~mm}$.

Conclusion: We highlighted the anatomic features of LAA and LAA orifice. LAA os diameters had a wide range in our study. LAA diameters, shape and the relation between neighbouring structure in relation to atrial fibrillation and surgical approaches were discussed.

\section{PP-235}

\section{Revision of anatomical knowledge in medical practitioners: frequency and periodicity}

Arráez-Aybar LA*, Sanchez-Montesinos I**, Mirapeix RM*****, Mompeo-Corredera B***, Sañudo-Tejero JR****

Anatomy and Embryology Department 2, Medical School, Complutense University of Madrid, Spain*; Anatomy and Embryology Unit, Medical School, University of Granada, Spain**; Morphology Department, Health Sciences School, las Palmas University, Gran Canaria, Spain ${ }^{* *}$; Anatomy and Embryology Department 1, Medical School, Complutense University of Madrid, Spain****; Anatomy and Embryology Unit, Medical School, Autonomous University of

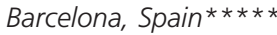

Aim: The aim of this study has been to evaluate the frequency and periodicity in revision of anatomical knowledge in different medical speciality professionals.

Materials and Methods: A total of 1250 questionnaires were distributed among 38 different medical speciality professionals. Answers were analyzed taking into account speciality (medical, surgery and others), professional status (training physician or staff member) and professional experience.

Results: The most frequently reviewed anatomical topics were the musculoskeletal system, head and neck anatomy, and nervous system, which were updated monthly by $19.8 \%, 15.7 \%$ and $11.2 \%$ respectively. The least frequently revised topic was embryology of which the necessity of updating was higher than a year in $67.5 \%$ of the cases. Our results showed that TP review anatomical topics more often than the medical specialists. There were statistically significant differences between TP and $\mathrm{SM}$ in cases of: nervous system $(\mathrm{p}=0.0082)$, cardiovascular system $(\mathrm{p}=0.0005)$, respiratory system $(\mathrm{p}=0.0048)$, digestive system $(\mathrm{p}=0.0029)$ and urogenital system $(\mathrm{p}<0.0001)$. The requirements varied according to different specialities. The group "others specialities" was the group that reported to revise anatomical knowledge more frequently, compared to "medical" and "surgical" group, in all systems. The "others" group showed statistically significant differences in relation to the great majority of systems: cardiovascular system $(\mathrm{p}<.0001)$, embryology $(\mathrm{p}<0.0001)$, head and neck $(\mathrm{p}<.0001)$, musculoskeletal system $(\mathrm{p}<0.0001)$, nervous system $(\mathrm{p}<0.0001)$ and respiratory system $(\mathrm{p}<0.0001)$.

\section{PP-236}

\section{Relations and morphology of the hamstrings muscle complex. Pilot study for a safer practice}

Bellmunt AP, Lorente M, Escalona C, Blasi M, Miguel M

Department of Basic Sciences, Faculty of Medicine and Health sciences. Universitat Internacional de Catalunya

Background: Hamstring muscle complex (HMC) injuries are often an issue of concern to clinical practice and medical specialities. Injuries in the HMC have one of the highest recurrence rates (1) and they can be observed in the majority of sports. The osseous avulsion of ischial tuberosity (as a consequence of a HMC contraction is increasing, but many surgeons do not operate because this anatomical complex and its relations are not well known. Also, in the neurology field, in order to avoid the risk of injury of the sciatic nerve during nerve and motor point blocking in the treatment of lower limb spasticity, there is a need of an accurate description of the HMC and its nerve and vascular structures.

Objective: The aims of this study are to detail the morphology and morphometry of the HMC, in order to know the relationship between vascular and muscular structures encountered during a surgery of a complete avulsion or to determine, safely, the never block injection point. Methods: Six fresh thighs were dissected. The morphology and the morphometry of all parts of the HMC were described. The relationship between their origin site to the surrounding neurologic and muscular structures were measured. The principal branches of the sciatic nerve to the hamstring muscles were located.

Results and Discussion: The macroscopic characteristics of the HMC help to develop models of relationships between structure and function, and propose injury and prevention models). The sciatic nerve, the posterior cutaneous nerve and the gluteal nerve and artery, may be at risk during surgical procedures to treat osseous avulsion of the ischial tuberosity. The description of the principal branches of the sciatic nerve might help when considering the right approach to nerve or motor point blocking.

\section{PP-237}

\section{Morphometric measurements of cerebral sulci in autopsy cases}

Gonul $\mathrm{Y}^{*}$, Songur A**, Uzun I***, Uygur R*, Alkoc OA****, Caglar $\mathrm{V}^{*}$, Kucuker $\mathrm{H}^{* * * *}$

Department of Anatomy, School of Medicine, Namık Kemal University, Tekirdag, Turkey*; Department of Anatomy, Faculty of Medicine, Afyon Kocatepe University, Afyonkarahisar**; Council of Forensic Medicine, Istanbul***; Department of Forencis Medicine, Faculty of Medicine, Sakarya University, Sakarya, Turkey****; Department of Anatomy, School of Medicine, Duzce University, Duzce, Turkey***** 
Cerebral sulci are anatomical structures that limit the gyri and separate them from other gyri making them more apparent. Also, they are known as the main microanatomic delimiting landmarks and surgical corridors in neurosurgery. In our study, making morphometric measurements of several sulci on the lateral aspects of the cerebrum in regard to main sulci and related reference key points was aimed. A total of 100 cerebral hemispheres from the 50 forensic autopsy cadavers were examined. The lengths of several sulci on the lateral aspect of the hemispheres were measured. Also, the measurements of the distances between each sulcus and the nearby sulci and reference key points were carried out. It was observed that the lengths of the anterior, ascending and posterior branches of lat-

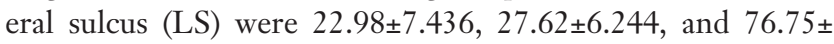
$10.10 \mathrm{~mm}$ respectively, whereas the length of external occipital fissure was $30.97 \pm 5.323 \mathrm{~mm}$. The distances between superior and inferior Rolandic (IR) points of central sulcus (CS), CS and LS, and IR and anterior Sylvian (AS) points were found $94.51 \pm 7.424,5.170 \pm 3.995$ and $29.59 \pm 5.093 \mathrm{~mm}$, respectively. The distances between the posterior extremity of the superior frontal sulcus and precentral sulcus (preCS), CS and interhemispheric fissure (IHF) were 2.500 $\pm 5.746,16.46 \pm 8.812$, and $24.44 \pm 5.686 \mathrm{~mm}$ respectively. In addition, the distances between the posterior extremity of the inferior frontal sulcus and preCS, CS, LS and AS point were measured as $3.880 \pm 4.985,17.13 \pm 6.405,33.35 \pm 5.665$ and $39.44 \pm 6.830 \mathrm{~mm}$ respectively. Also, while the measurements of the distances between the anterior extremity of intraparietal sulcus and postcentral sulcus, CS, and IHF were 4.400 $\pm 7.339,18.78 \pm 8.587$ and $32.03 \pm 7.428 \mathrm{~mm}$; the distance between posterior extremity of superior temporal sulcus and LS was observed $30.89 \pm 6.644 \mathrm{~mm}$. Altogether, some of our measurements were found to be compatible with the ones in the literature, while others were incompatible. Thus, we think that it may be important to consider racial and country-specific variations in both neurosurgery and anatomy education.

\section{PP-238}

\section{Situs inversus totalis: a case report}

Dogan NU*, Uysal II*, Dogan $\mathrm{KH}^{* *}$, Demirci $\mathrm{S}^{* *}$

Department of Anatomy, Selcuklu Medical Faculty, Selcuk University, Konya, Turkey*; Department of Forensic Medicine, Meram Medical Faculty, Selcuk University, Konya, Turkey**

Situs inversus totalis is a condition in which there is complete transposition (right to left reversal) of the thoracic and abdominal organs. The heart is not in its usual position in the left chest but is on the right. Specifically related to the heart, this is referred to as dextrocardia (literally, right-hearted). The stomach, which is normally in the left upper abdomen, is on the right. In patients with situs inversus totalis, all of the chest and abdominal organs are reversed and appear in mirror image when examined or visualized by tests such as x-ray filming. Incidences of situs inversus totalis range from 1 in 10,000 to 1 in 50,000. Situs inversus totalis was observed in a 39-year-old man at autopsy. In the thorax there was dextrocardia, right lung had two lobes, left lung had three lobes, in the abdomen liver was on the left, stomach and spleen were on the right, appendix was on the left. Situs inversus totalis may cause wrong interpretations during radiologic imaging and surgical procedures. In addition, the diagnosis of acute apendicitis may delay if the appendix is on the left.

\section{PP-239}

\section{Dextrocardia and situs inversus totalis in a Turkish subject: a case report}

$\underline{\text { Yilmaz } \mathrm{S}^{* *}}$, Demirtas $\mathrm{A}^{*}$, Akinsal E*, Sagiroglu A** Acer $\mathrm{N}^{* *}$, Aycan $\mathrm{K}^{* *}$

Urology Department, School Of Medicine, Erciyes University, Kayseri, Turkey*; Anatomy Department, School of Medicine, Erciyes University, Kayseri, Turkey**

This report describes an adult male patient with dextrocardia and situs inversus totalis in a Turkish subject. The photographic illustrations revealed transposition of some of the visceral organs such as the spleen was located right and the liver and gall bladder on the left. The heart was flattened and flipped to the right. Many people with situs inversus totalis are unaware of their unusual anatomy until they seek medical attention for an unrelated condition. So, early detection may lead to a successful surgical management and consequently offer a safer chance of survival. This report showed that dextrocardia and situs inversus can be seen amongst Turkish subjects.

\section{PP-240}

\section{Arterial supply of the thyroid gland and the relationship of the recurrent laryngeal nerve to the inferior thyroid artery in the human fetal cadavers}

\section{Ozguner G, Sulak O}

Department of Anatomy, Faculty of Medicine, Suleyman Demirel University, Isparta, Turkey

Objective: The present study aimed to determine arterial supply of the thyroid gland and the relationship between the inferior thyroid artery and recurrent laryngeal nerve using anatomic dissection method during the fetal period. 
Material and Methods: The study was performed on 200 human fetuses (100 males and 100 females) aged between 9-40 weeks of gestation. The fetuses were divided into four groups according to fetal age; 1st trimester, 2nd trimester, 3rd trimester and full term. After dissection of anterior cervical region, thyroid gland, superior and inferior thyroid artery and recurrent laryngeal nerve were exposed. The origins of the superior and inferior thyroid arteries were determined and lengths and diameters of the superior and inferior thyroid arteries were measured. The relationship between the inferior thyroid artery and recurrent laryngeal nerve also determined.

Findings: The origins of the superior thyroid arteries were determined as external carotid artery, common carotid artery, and thyrolingual trunk while the origins of the inferior thyroid arteries were determined as subclavian artery and common carotid artery. The diameters and lengths of the superior and inferior thyroid arteries were increasing with gestational age. No significant differences were observed between sexes for any of the parameters $(p>0.05)$. The relationship of the recurrent laryngeal nerve to the inferior thyroid artery was determined as nerve pass behind the artery, in front of the artery, between two branches of the artery and parallel to the artery.

Conclusion: Knowledge of variations in the origin of the superior and inferior thyroid arteries and the relationship of the recurrent laryngeal nerve to the inferior thyroid artery is of great importance in surgery and radiological examinations.

\section{PP-241}

The anatomical importance of lateral cutaneous nerve of thigh for meralgia paresthetica

Celik S, Govsa F, Bilge O, Pinar Y

Anatomy Department, Medical Faculty, Ege University, Izmir, Turkey

Objective: Terminal branching point (TBP) and passing types of lateral cutaneous nerve (LCNT) through the thigh near the anterior superior iliac spine (ASIS) are important to understand the pathogenesis and measure sensory conduction velocity for diagnosis and treatment of Meralgia Paresthetica (MP).

Material and Method: 38 thighs were dissected from 19 male cadavers. Terminal branching pattern, TBP and passing types to the thigh of nerve were examined. ASIS, inguinal ligament (IL), lateral and medial border of sartorius muscle (S) were used landmarks. TBP of nerves were described at five regions: Region I: In the pelvis, Region II: At the level of or under the IL, Region III: In the thigh, between IL and medial border of S, Region IV: In the thigh, between medial and lateral borders of S (on the muscle) and Region V: In the thigh, more lateral to the lateral border of $\mathrm{S}$.
Results: 30 bifurcations (\% 78.9) and 8 trifurcations (\%21.1) were observed. The symmetry were present at 17 cadavers (\%89.5), asymmetry were present at 2 cadavers (\%10.5). TBP were determined on Region I in 3 (\% 7.9), Region II in 1 (\%2.6), Region III in 4 (\% 10.5), Region IV in 23 (\%60.5) and Region V in 7 (\%18.4) of all cases. Passing types trough the thigh of LCNT were classified according to Ghent typing: Type A at 29 cases (\%89.5), Type B at 9 cases (10.5). Type C and D were not observed. The most observed type was Type A1 with 27 cases (84.2).

\section{PP-242}

\section{Sphenoidal concha: an endoscopic landmark for determining the location of sphenoidal sinus ostium}

Ecevit $\mathrm{MC}^{*}$, Zeybek $\mathrm{G}^{* *}$, Kiray $\mathrm{A}^{* *}$, Cirpan $\mathrm{S}^{* *}$, Ergur $\mathrm{I}^{* *}$, Dogan $E^{*}$, Semih $\mathrm{S}^{*}$

Department of Otorhinolaryngology Head and Neck Surgery, Faculty of Medicine, Dokuz Eylul University, Izmir, Turkey*; Department of Anatomy, Faculty of Medicine, Dokuz Eylul University, Izmir, Turkey**

Objective: This study is designed to determine whether sphenoidal concha can be used as a landmark to localize the sphenoidal sinus ostium.

Materials and Methods: One hundred and fifty two skulls were evaluated for measurements. Fifteen sides excluded due to lacking anatomical sites and 289 sides included into study. The endoscopic pictures were taken with Karl Storz Tele Pac and $30^{\circ}$ Endoscope. Each endoscopic picture included ostium of the sphenoidal sinus, sphenovomerine suture (SVS) and the articulation of sphenoidal process of palatine bone between body of the sphenoid. The measurements were taken software program (Image Tool for Windows version 3.00) on each picture. Horizontal and vertical lines crossing SVS and sphenoidal process of palatine bone were marked on pictures. The distance between the medial border of ostium and SVS, the angle between them, the distance between inferior border of ostium and horizontal line and the distance between the medial border of ostium and vertical line were determined.

Results: The distance between medial border of ostium and the vertical line was $0.73 \mathrm{~mm} \pm 0.90(0.00-3.93)$. The distance between SVS and medial border of ostium was $3.15 \mathrm{~mm} \pm 1.35$ (0.21-8.60). The distance between horizontal line and inferior border of sphenoid ostium was $5.99 \mathrm{~mm} \pm 2.38$ (1.25-14.99). The angle between SVS and medial border of ostium was $24.14^{\circ} \pm 9.73(3.65-54.91)$.

Conclusion: Sphenoidal conchae is a reliable landmark to identify ostium of the sphenoidal sinus in conjunction with SVS and articulation of sphenoidal process of the palatine bone. The validation of this data is planned by conduction of a study for the measurements during endoscopic sinus surgery. 
PP-243

New drawings for graduate education functions of related anatomical structures in the human voice formation

Baylan H, Yonguc GN, Akyer SP, Adiguzel E

Anatomy Department, Faculty of Medicine, Pamukkale University, Denizli, Turkey

Objective: A human can differentiate tones while talking which show significant differences among people as fingerprints. Differentiating is due to our ability to change the thickness and the tension of plica vocalis. The length and the width of larynx and pharynx are changed as the mouth and related structures change their anatomy. So, human voice can be used as a musical instrument. The functional anatomy of this tool is one of difficult tasks in anatomy. The purpose of this study is to make the basic mechanism of voice formation well understood and to understand why the human voices we hear when communicating are different with new anatomical demonstrations.

Material and Method: Related books and articles have been researched. The drawings that show the movements of laryngeal muscles, vocal cords during respiration, abdominal and thoracic structures for air flow to resonate vocal cords was performed. The glottic region is drawn anatomically in different speech styles. The changes of laryngeal positions while singing with squealing and making a chest note are drawn. Also the functions of suprahyoid and infrahyoid muscles that affect larynx are drawn with their innervations together. The locations of the tongue, palate, tooth and the structures in the mouth for some letters to spell were drawn. All the drawings were performed with pencil and photos of them were taken. Then they were transferred to a PC to be processed with the softwares called Paint. NET, MS Paint, Photobie and Microsoft Office Picture Manager.

Results: Visuality is important in education of anatomy. Different shapes and locations of the anatomical structures must be supported with visual materials especially in functional anatomy. The figures revealed in this study will either help to learn or facilitate to teach the anatomy of voice production. It will also enrich the figures existing in the literature.

\section{PP-244}

\section{A giant tumor of the kidney: radiological case report}

Yonguc GN*, Sahinli $\mathrm{S}^{* *}$, Adiguzel $\mathrm{E}^{*}$, Ozdemir $\mathrm{MB}^{*}$, Akyer SP*

Department of Anatomy, Faculty of Medicine, Pamukkale University, Denizli, Turkey*; Department of Urology, Denizli State Hospital, Denizli, Turkey**

Objective: An abdominal mass is a localized swelling or enlargement in the human abdomen and usually detected on routine physical examination. Most abdominal masses develop slowly therefore most of the patients with abdominal mass have no symptoms until the mass is getting very large and pressurizes on neighboring structures which causes tenderness, pain and functional disturbances. Kidney cancer can sometimes cause a mass in the abdomen.

Material and Method: A 74-year-old man presented with a 3weeks history of abdominal pain and gastrointestinal symptoms. Physical examination was normal except for a large, solid mass in the right upper quadrant extending to right lower quadrant. The patient underwent computed tomography (CT) exam.

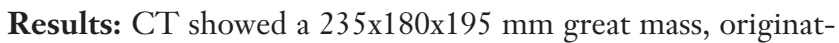
ing from the right kidney; filling right half of the abdominal cavity. The right lobe of the liver, gallbladder and loops of the small intestine were dislocated to the left and pressured to anterior abdominal wall. The mass was compressing the ascending colon and also it was compressing the inferior vena cava. The lower pole of the mass was located in right iliac fossa. The patient was treated surgically, right radical nephrectomy was performed. Histopathological examination revealed papillary renal cell carcinoma.

Conclusion: Most abdominal masses are discovered incidentally during routine physical examinations so clinicians should be careful. During physical examination, the clinician must identify the location of the mass, as well as characterize its location (usually specified in terms of quadrants). Also clinicians should be aware of how great masses can dislocate the anatomical structures while performing manipulations.

\section{PP-245}

\section{A short circuit resistance training improved body composition in adult men with metabolic syndrome}

Rosety I*, Diaz-Ordonez A**, Rosety MA**, Fornieles G**, Camacho-Molina A*** Garcia-Gomez $\mathrm{N}^{* *}$, Rosety-Rodriguez $\mathrm{M}^{* *}$, Garcia-Gomez $\mathrm{S}^{* * * *}$, Ordonez FJ*

Human Anatomy Department, University of Cadiz, Cadiz, Spain*; School of Sports Medicine, University of Cadiz, Cadiz, Spain**; Hospital Sas Juan Ramon Jimenez, Huelva, Spain***; Human Anatomy Department, University complutense, Madrid, Spain****

Contemporary prevalence of metabolic syndrome in western societies is clearly increasing. Fortunately regular exercise may play an important role in the management of these patients. Although exercise programs have traditionally emphasized aerobic training, research increasingly suggests that resistance training when appropriately prescribed and supervised has favorable effects in our health. For the reasons already mentioned the present study was designed to determine the influence of a resistance training program on body composition in men with metabolic syndrome. To get this goal 120 adult men 
with metabolic syndrome according to the criteria reported by the National Cholesterol Education Program Adult Treatment Panel III (NCEP ATP-III) volunteered for this study. Onehundred were randomly included in experimental group to perform a 12-week circuit resistance training, 3 days/week, consisting of warm up (10 min), main part (it will incluye several series of resistance exercises with 10-12 repetitions performed at 45$60 \%$ of 1 maximal repetition) and cool-down (10 min). Control group included 20 age, sex and BMI-matched women with metabolic syndrome that did not perform any training program. Written informed consent was obtained from all participants. Fat Mass (FM) and Fat Free Mass (FFM) were assessed by dual energy X-ray absorptiometry (DXA) twice: firstly 72-h before starting the program (pre-test) and finally $72-\mathrm{h}$ after its ending (post-test). Further it should be emphasized our protocol was approved by an Institutional Ethic Committee. After being exercised, fat free mass was increased significantly $(47.1 \pm 3.4 \mathrm{~kg}$ vs.49.2 $\pm 3.2 \mathrm{~kg} ; \mathrm{p}=0.016)$. whereas fat mass was significantly decreased $(40.6 \pm 3.5 \mathrm{~kg}$ vs. $38.7 \pm 2.9 \mathrm{~kg} ; \mathrm{p}=0.022)$. Conversely, no changes in body composition were reported in controls. A 12-week circuit resistance training improved body composition by increasing muscle mass percentage and decreasing fat mass percentage in men with metabolic syndrome. Further studies on this topic are required.

\section{PP-246}

The comparison of the rom values of hip joint between obese and normal weight womens

Cetkin $M^{*}$, Cikmaz S**

Department of Anatomy, Instute of Health Science, Adana Cukurova University, Adana, Turkey*; Department of Anatomy, Faculty of Medicine, Trakya University, Edirne, Turkey**

Objective: It's important to evaluate the human motion and functions in explaining many pathological case and developing new treatment methods. Therefore various tests and evaluation methods have been developed. With this idea, we aimed at comparing the Range of motion (Rom) values of hip joint in obese and normal weight women.

Material and Method: 20 obese women in the average age of 30.6 , and 20 normal weight women in the average age of 25.7 took part in our study. By their body mass index values, we determined if the participants were obese or not. We determined the individuals as obese who have $930 \mathrm{~kg} / \mathrm{m}^{2}$ body mass index and as normal who have $18.5-24.9 \mathrm{~kg} / \mathrm{m}^{2}$ body mass index. We measured the Rom values of hip joint by using Cybex electronic digital inclinometer. We measured abduction, adduction, flexion, extension, internal and external rotation movements which are the six main movements of hip joint.
Results: We found the average and standard deviation of Rom values of abduction, adduction, flexion, extension, internal and external rotation in obese and normal weight women respectively: $52.3^{\circ} \pm 5.9-56.7^{\circ} \pm 3.8 ; 20.6^{\circ} \pm 4.2-28.7^{\circ} \pm 2.7 ; 84.8^{\circ} \pm 11.7$ $-102.3^{\circ} \pm 5.5 ; 25.9^{\circ} \pm 5-29^{\circ} \pm 2.8 ; 37.1^{\circ} \pm 4.8-38.4^{\circ} \pm 4.5 ; 45.4^{\circ} \pm 4.6$ $-46.9^{\circ} \pm 4.5$.

Conclusion: In conclusion, increased body mass index in women limits the Rom values of abduction, adduction, flexion and extension.

\section{PP-247}

\section{MR findings in symptomatic individuals-distribution} characteristics

Matveeva N, ZivaDinovik J, Jovevska S

Institute of Anatomy, Medical Faculty, Skopje, Republic of Macedonia

The term degenerative disc changes covers various morphological alterations of the intervertebral disks. Modern imaging methods provide valuable information for the the type and the degree of the morphostructural changes of the degenerated discs. The presence of degenerative changes of the intervertebral discs doesn't indicate the presence of clinical symtoms caused by these changes, although low back pain can also be a symptom of degenerative disc disease. The imaging data of 150 patients with low back pain and radiculopathy were retrospectively reviewed.The aims of this study were to describe the prevalence of degenerative disc changes in symptomatic individuals and their association with the age and gender. The development of the various morphostructural alterations of the degenerated discs were analized, as well as their segmental anatomic distribution in the lumbar spine. The analysis of their anatomic distribution characterize degenerative disc disease as a cascade degenerative process.

\section{PP-248}

\section{Waist circumference was positively correlated to oxidized low density lipoprotein in women with metabolic syndrome}

Rosety $M^{*}$, Diaz-Ordonez A**, Rosety MA**, Rosety I*, Ordonez FJ*, Fornieles G*, Camacho-Molina A*, Garcia-Gomez $N^{* *}$, Rosety-Rodriguez $M^{* *}$

Human Anatomy Department, University of Cadiz, Cadiz, Spain*; School of Sports Medicine, University of Cadiz, Cadiz, Spain**; Hospital Sas Juan Ramon Jimenez, Huelva, Spain***

Although there has been significant debate regarding the definition and diagnostic criteria of metabolic syndrome, the 
emerging role of oxidative stress in its pathogenesis is widely accepted. In fact, high levels of oxidized LDL were associated with increased risk of future myocardial infarction, even after adjustment for LDL-cholesterol and other established cardiovascular risk factors. Accordingly, several originals have reported oxidative stress may be considered a potential therapeutic target. This study was designed to identify potential association between plasmatic levels of oxidized low density lipoprotein and waist circumference in women with metabolic syndrome. Sixty young women $\left(39.1 \pm 3.5\right.$ years; $\left.28.7 \pm 1.9 \mathrm{~kg} / \mathrm{m}^{2}\right)$ with metabolic syndrome according to the criteria reported by the National Cholesterol Education Program Adult Treatment Panel III volunteered for this study. Plasma carbonyl content was determined by mean of slightly modified Levine method and read in a plate-reading spectrophotometer obtaining the maximum absorbance between 360-390 nm. Waist circumference was assessed by a long-experienced anthropometrist. This protocol was approved by an Institution Ethic Committee. We found a significant and positive association between waist circumference and low density lipoprotein oxidation $(r=0.51$; $\mathrm{p}<0.01$ ). It was concluded waist circumference was significantly associated to plasmatic levels of oxidized low density lipoprotein in adult women with metabolic syndrome. Further longterm well-conducted studies are required in order to highlight potential clinical benefits of this finding in these patients.

\section{PP-249}

\section{Traumatic hematoma of left alar cartilage and its anatomical localization}

Kurkcuoglu A*, Yilmaz $\mathrm{H}^{* *}$, Pelin $\mathrm{C}^{*}$, Zagyapan $\mathrm{R}^{*}$

Department of Anatomy, Faculty of Medicine, Baskent University, Baglıca, Ankara, Turkey*; Depatrmant of Otorhinolaryngology, Yenimahalle State Hospital, Ankara, Turkey**

Introduction: The greater alar cartilage is a thin, flexible plate and bent upon itself in such a manner as to form the medial wall and lateral wall of the naris of its own side. The portions of the alar cartilages can be excised or augmented, depending on the modification desired. Symmetry between the domes can be achieved with suture or excisional in surgical approaches. Grafts for alar collapse and internal nasal valve obstruction can also be placed. Whereas haematomas of the nasal septum, upper lateral nasal cartilage, pinna and larynx are all well recognized causes of cartilaginous necrosis, haematomas of the alar cartilage have not been previously described. Case presentation A 22-year-old women came to the department of ear nose and throat with a swelling on the left side of her nose due to a blunt trauma, and complaining of congestion feeling and difficulty in breathing. During rhinoscopic examination severe pain, and left nasal passage blockage due to a purple blue swelling was reported. No associated septal or nasal bone deformity was observed. The patient had been hospitalized with a diagnose of alar cartilage hematoma due to trauma. Later under sterile operation room conditions hematoma at the left subpericondreal region had been drained by an incision from two millimeters above to standard left supracartilagenous incision up to the alar cartilage. The incision plase was left open and a Merocel compress was placed in to left nasal vestibule. Antibiotics and anti-inflammatory agents were given to the patient in order to prevent a potential infection. Merocel compress was removed in three days. Cure was obtained without any esthetic deformation and relapse.

Conclusion: The diagnosis like that of a septal haematoma, is dependent on the awareness of its existence by the clinician and the knowledge that, if left untreated, it may result in an unacceptable cosmetic deformity. Nasal obstruction may also ensue, as a very small change in the width of the nostril will markedly affect air flow.

\section{PP-250}

\section{Expression of mesenchymal stem cells markers from human adipose tissue around the vas deferens}

Akbulut $\mathrm{H}^{*}$, Cuce $\mathrm{G}^{* *}$, Aktan $\mathrm{TM}^{* *}$, Duman $\mathrm{S}^{* *}$

Department of Urology, Hospital of Medical Faculty, University of Bezmi Alem, Istanbul, Turkey*; Deparment of Histology and Embryology, Faculty of Meram Medicine, University of Selcuk, Konya, Turkey**

Introduction and Purpose: Mesenchymal stem cells (MSCs) have unique properties: they exhibit transdifferantiation. Adipose tissue is recognized as an important source of postnatal mesenchymal stem cells for regenerative Medicine applications. A cell must express at least CD105 (Endoglin), CD73 and CD90 to be accepted as a MSC. In lipoaspirate adiposse tissue, these markers expressed. In our study we investigated CD105, CD73 and CD90 expression in adipose tissue around the vas deferens.

Tools and Method: Adipose tissue around the vas deferens were obtained from 5 patients from the Urology deparment of Bezm-i Alem Medical Faculty Parafin sections were prepared from adipose tissue after routine histologic process. HE and Masson Tricrome staining and CD105, CD73, CD90 immunostaining were performed to parafin sections. Findings: The strongest expression belonged to CD105, expression of CD90 was moderate and expression of CD73 was weak compared to CD105 and CD90 in all patients.

Result and Interpretation: Stromal cells derived from adipose tissue around the vas deferens has smilar properties like liposuctioned adipose tissue according to expression of mesenchymal stem cells markers. It was reported that CD105 might be a useful cell surface marker to determine the differentiation status of MSCs. 
S132 Joint Meeting of Anatomical Societies, 19-22 May 2011, Bursa, Turkey

\section{PP-251}

Interstitial cells of the human trigeminal ganglion a TEM study

Rusu MC $\mathrm{C}^{*}$, Mirancea $\mathrm{N}^{* *}$, Pop $\mathrm{F}^{*}$, Curca GC*, Hostiuc S*, Jianu $\mathrm{AM}^{* * * *}$

"Carol Davila" University of Medicine and Pharmacy Bucharest, Romania*; Institute of Biology, Romanian Academy, Bucharest**; "Mina Minovici" National Institute of Legal Medicine, Bucharest, Romania*** "Victor Babes" University of Medicine and Pharmacy Timisoara, Romania****

Objectives: Interstitial Cajal like cells (ICLCs) and interstitial Cajal cells (ICCs) are present in various locations and act either as pacemakers, or mediate the neural input. We aimed to evaluate whether or not interstitial cells (ICs) are present within the trigeminal ganglion (TG) and to check whether or not these cells, if present, have convincing ultrastructural features to allow considering them ICLCs.

Materials and Methods: Human trigeminal ganglia were collected at the autopsy from 15 adult donors negative for neurodegenerative disorders, and were processed for transmission electron microscope (TEM) evaluation.

Results: ICs were identified within the TG neighboring microvessels, intraganglionic bundles of nerve fibers, and envelopes built up by the satellite glial cells (SGCs) around trigeminal neurons. Long processes appeared as a striking feature of the ICs we analyzed, and were positive for the presence of intermediate filaments. Dilations along these processes contained mainly mitochondria and endoplasmic reticulum. These ICs were caveolae-rich and positive for coated pits, vesicles, and microvesicular bodies. Contacts and stromal synapses were evidenced between processes of such ICs.

Conclusions: The ICs of the trigeminal ganglion have the complete set ("gold standard") of ultrastructural criteria that allow them to be considered as ICLCs. Moreover, some of these trigeminal ICLCs fully correspond to the standards recently stated for telocytes. Acknowledgements: (1) Research Grant UEFISCSU, 317/2007. (2) This study was also supported by the SOP HRD, financed from the ESF and by the Romanian Government under the contract number POSDRU/89/1.5/S/64109.

\section{PP-252}

Expression of HSP70 and iNOS in toluene inhalated and melatonin treated liver of rats

Tas $\mathrm{U}^{*}$, Ogeturk $\mathrm{M}^{* *}$, Sapmaz $\mathrm{HI}^{* * *}$, Kuloglu $\mathrm{T}^{* * * *}$, Kocaman $N^{* * * *}$, Ungor B*, Sarsilmaz $M^{* * * *}$

Department of Anatomy, Faculty of Medicine, Gaziosmanpasa University, Tokat, Turkey*; Department of Anatomy, Faculty of Medicine, Firat University, Elazig, Turkey**; Department of Anatomy, Faculty of Medicine, Inonu University, Malatya, Turkey***; Department of Anatomy, Faculty of Medicine, Firat University, Elazig, Turkey****; Department of Anatomy, Faculty of Medicine, Sifa University, Izmir, Turkey*****
Toluene is a clear, colorless, volatile hydrocarbon that is metabolized in liver, caused free oxygen radicals and can mediate cellular damage. Heat shock protein 70 (HSP 70) induction is often associated with a cellular response to a harmful environment or to adverse life conditions. iNOS, however, is readily upregulated in the liver under a number of conditions, including endotoxemia, ischemia-reperfusion, infection, and liver regeneration. Melatonin which is a pineal gland hormone is a very potent antioxidant. It can make the cellular membrane more durable against oxidative attacks and protect nuclear DNA from oxidative damage. This study aimed to investigate the protective effects of melatonin against toluene-induced hapatotoxicity in the liver of rats using by HSP 70 and iNOS immune positivity. 21 adult male Wistar-albino rats (200-220 g) were used in this study. They were randomly divided three experimental groups. Animals in group I were used as control. The rats in group II were exposed toluene (3000 ppm/1 hour/day) for 4 weeks, while the rats in group III treated with melatonin $(10 \mathrm{mg} / \mathrm{kg} / \mathrm{day}$, ip) plus toluene inhalation. At the end of 30-days experimental period, all rats were killed by decapitation. Then the liver tissues of rats were removed. For light microscopic examination, tissue specimens were embedded in paraffin blocks following routine histological procedures. Section obtained from paraffin blocks were used for immune detection of HSP 70 and iNOS. Increased HSP 70 and iNOS immune reactivity were determined in toluene inhalated group and melatonin treatment decreased this immune positivity.

\section{PP-253}

\section{Application of markers of proliferative activity and apoptosis in the assessment of the dynamics of the character and degree of morphological changes of the stomach in rats caused by toluene intoxication}

\section{Fedchenko SN, Klimotchkina YM, Galuzina IO}

State Institution, Luhansk State Medical University, Lugansk, Ukraine

The object of the study is to determine toxic inhalation effect of toluene on cellular renovation and apoptosis of epitheliocytes of the stomach in rats. Assessment of morphological changes with apoptosis. The method of fluorescence microscopy with the use of color Hoechst 33342 binding with DNA is considered to be very suitable for the purpose. In the result of changes in permeability of the plasma membrane apoptotic cells accumulate color Hoechst 33342 much more actively than the intact ones do that. To reveal cells with condensated or fragmented nucleus fluorescent nuclear color Hoechst 33342 was used. Analysis of proliferative activity of cells was based on immunofluorescent detection of nuclear antigen of dividing cells. As primary antibodies were used antibodies to antigen Ki67 (Sigma) by which the preparations were 
marked and then were being incubated for 30 minutes at 40C. Morphological assessment was made with fluorescence microscope MC 300 (Micros Austria) of 400-divisible magnification. The studies conducted showed that intoxication caused by toluene resulted in characteristic changes in proliferative activity and viability of epitheliocytes of the MMS. The findings obtained on the 1st and 7th day of the study showed that the processes of the cellular renovation demonstrated reliable lowering of proliferative ability and increase in the percentage of death of the cells in the form of apoptosis when compared with control group. Lowering of proliferative activity and increase in the number of apoptosis are typical processes only for the animals with erosion of the MMS. Revealed disorders in the cellular renovation promote accelerated displacement of the cells out of the generative zone and not undergoing full differentiation they appear in the places of usual localization of maturated, of special activity epitheliocytes. And it may result in weakening of the functional ability of the cells.

\section{PP-254}

Ultrastructural features histaminproducing (ECL) cells of the rat stomach after the using of zoledronic acid and its combination with hydrocortisone

Fedchenko SN, Kondaurova AY

State Institution "Luhansk State Medical University", Ukraine

The purpose of this study is to examine the influence of zoledronic acid (TSP) and its combination with hydrocortisone on different periods of using on the structure of histaminproducing (ECL) cells of the gastric mucosa (GM). The experiment was conducted on 125 white male rats that had wight 180-200 g. Animals of the first group were intraperitoneally injected with the drug "Zometa. The drug was used 1 time in 30 days at a dose of $0.362 \mathrm{mg} / \mathrm{kg}$ of body weight. The second group of animals received hydrocortisone acetate. Animals of the third experimental group received a combination of zoledronic acid with hydrocortisone in the same way. Digital images of electron slides were recorded on a CD - disks, then they were processed using the program «Morpholog». Morphometric data were exported to the program Exel for further statistical processing and storage, reliable considered probable error of less than $5 \%(\mathrm{p}<0,05)$. Vector of ratio of heterochromatin and euhromatin of Ecl-cell nuclei on 90 day of experiment was 0,88 $\pm 0,06$ and pointed to the dominance of the area of heterochromatin over the area of euchromatin indicating the decrease in synthetic processes. The thickness of the marginal chromatin increased on $41 \%$. Most of the ESL-cells were degranulated. This indicates on the decrease the total area of secretory granules in almost 2 times, and these dataes combined with a decrease of the area of cytoplasm occupied by the Golgi complex by 32\% ( $<<0.05)$. Established that endocrine cells respond to the application of TSP nonspecific changes in the organelles of general purpose, in this case along with the usual forms of secretion - molecular extrusion and exocytosis in endocrine cells release secretory granules is carried out by its lysis in numerous vacuoles (degranulation).

\section{PP-255}

Terotologic effect of adriamycin on septal development of early fetal heart

Ulger $\mathrm{H}^{*}$, Ozdamar $\mathrm{S}^{* *}$

Department of Anatomy, Faculty of Medicine, Erciyes University, Kayseri, Turkey*

Esophageal atresia is often associated with cardiovascular and other malformations. The present study tests the heart development may be abnormal in the rat model of esophageal atresia. Time-mated pregnant rats received $2 \mathrm{mg} / \mathrm{kg}$ Adriamycin intraperitoneally on days 6-9 of gestation. The heart malformations were investigated under the microscope in early term fetuses. Adriamycin caused resorption of most embryos and only seven embryos obtained from Adriamycin receved dams. Control fetuses had no heart malformations, whereas five of the seven fetuses exposed to Adriamycin had interatrial and interventricular septal defects besides esophageal atresia.

\section{PP-256}

Investigation of the effects of chemotherapeutic agents applied before pregnancy on rat fetus development and morphological structure: quercetin's possible protective role

Dogan $Z^{*}$, Erdemli E**, Kose $E^{*}$, Ekincioglu $Z^{*}$, Ekinci $\mathrm{N}^{*}$, Turkoz $\mathrm{Y}^{* *}$

Department of Anatomy, Medicine Faculty, Universtiy of Inonu, Malatya, Turkey*; Department of Biochemistry, Medicine Faculty, University of Inonu, Malatya, Turkey**

Objective: It was investigated the toxic effects of some drugs used in chemotherapy on the embryonic development and the protective role of quercetin against these toxic effects.

Methods: Totally 53 female Wistar albino rats were used in this study. 5 rats were used as control group and the remaining 48 rats were taken as experimental group. Rats divided into 5 groups: quercetin $(n=5)$, cyclophoshamide $(n=12)$, doxorubicin $(n=12)$, cyclophoshamide + quercetin $(n=10)$, and doxorubicin + quercetin $(n=9)$ rats. The doses of the chemotherapeutic drugs applied to rats were equivalent to human doses. Cyclophoshamide and doxorubicin were applied as a single dose, once every 3 weeks for 10 weeks. In 2 days after chemotherapy, all rats were mated and pregnancy was 


\section{S134 Joint Meeting of Anatomical Societies, 19-22 May 2011, Bursa, Turkey}

obtained. On the 20th day of gestation, the embryos were taken by cesarean section. At the end of the study, the growth parameters such as the number of abnormal embryos, embryo weight, and crown-rump length between the groups were evaluated by morphological methods.

Results: When the data from the experimental group compared with the control group, it has been observed that the chemotherapy reversed developmental parameters and caused general morphological abnormalities. It was observed that quercetin a protective agent against chemotherapeutic drugs, significantly decreased the negative effects of cyclophoshamide on the number of embryos and embryo weight, but can not affect the negative effects of doxorubicin.

Conclusion: Chemotherapy received before pregnancy caused the developmental toxicity on rat embryos during pregnancy. It was observed that quercetin given as a protective during chemotherapy could not effect the development of embryonic malformations from doxorubucin, but significantly inhibited the malformative effect of cyclophoshamide on the embryos. As a result, we recommended that quercetin could be used as an alternative preventative treatment against malformations in embryos after chemotherapy with cyclophoshamide.

\section{PP-257}

\section{Brachyury expression and allantois development in preimplantation pig and rabbit embryos}

Hassan W, Viebahn C

Department of Anatomy and Embryology, University of Göttingen, Göttingen, Germany

The allantoic core domain (ACD) is considered a possible stem cell niche in the proximal core of the murine allantois, the posterior extraembryonic structure contributing to the formation of the umbilical cord and placenta. Defined by dense mesoderm and the expression of Brachyury, a T-box transcription factor with a major role in embryonic vascularization and mesoderm establishment, the ACD has been proven to be fundamental for allantoic elongation and vascularization in the mouse. To find an ACD equivalent for human embryonic development the allantois and the existence and importance of the ACD for the embryonic-maternal connection needs to be analysed in other mammalian model organisms as well. The present study, therefore, examined the posterior part of preimplantation pig and rabbit embryos using high-resolution light microscopy and in-situ-hypridization for Brachyury expression. The rabbit allantois emerges at embryonic day (ED) 8-9 as a round structure containing loose vascularized mesoderm connected to embryonic mesoderm beyond the cloacal membrane. Into this allantoic mesoderm hindgut-endoderm stretches posteriorly forming several small diverticula. In the pig the allantois emerges at ED 11-12 sickle-shaped and con- taining, posterior to cloacal membrane, hindgut-endoderm which branches into two large lateral diverticula covered by thin vascularized mesoderm. Brachyury in rabbit and pig is expressed in notochord and primitive streak-mesoderm anterior to cloacal membrane (as in the mouse) but, remarkably, not in the allantois, where the expected ACD region is occupied by endodermal diverticula instead. ACD appears thus not to be defined in preimplantation pig and rabbit embryos. However, once implantation starts at more advanced stages and the umbilical cord and placenta will have to be established, Brachyury expression may still be expected to begin in the allantois defining an ACD equivalent as a possible stem cell niche important for building the embryonic-maternal connection in mammals including man.

\section{PP-258}

Light microscopic investigation of quercetin's effects on kidney injury induced by doxorubicin in rats

\author{
Yagmurca $M^{*}$, Yasar $Z^{* *}$, Bas $\mathrm{O}^{* * *}$ \\ Department of Histology and Embryology, Faculty of Medicine, Fatih \\ University, Ankara, Turkey*; Department of Histology and \\ Embryology, Faculty of Medicine, Afyon Kocatepe University, \\ Afyonkarahisar, Turkey**; Department of Anatomy, Faculty of \\ Medicine, Rize University, Rize, Turkey***
}

Objective: The anthracycline antitumor drug doxorubicine causes severe nephrotoxicity in a variety of experimental animals and may be nephrotoxic to humans. This effect may be the consequence of oxidative stress. The aim of the present study was to determine by light microscopy the doxorubicin-induced structural/ histological changes and protective effects of co-administration of quercetin in doxorubisin induced kidney injury.

Methods and Materials: Forty adult, male Wistar albino rats were used by dividing them into four groups: I, control $(n=10)$; II, doxorubicin ( $\mathrm{n}=10)$; III, doxorubicin + quercetin, $(\mathrm{n}=10) ; \mathrm{IV}$, quercetin only $(n=10)$. In the doxorubicin group; the animals were treated with single dose of $20 \mathrm{mg} / \mathrm{kg} / \mathrm{i} . \mathrm{p}$ doxorubicin. After administration of the drugs, the rats were decapitated and their kidneys were removed and fixed in 10\% neutral-buffered formalin. Tissue samples were stained with H\&E (HematoxylinEosin) and histologically examined by light microscope.

Results: In the doxorubicin-administered rat kidneys (or one kidney?), renal tubular dilation, tubular vacuolar changes, glomerular vacuolization, a decrease in space of bowman, bowman capsule thickening and interstitial infiltration were found under light microscope.

Conclusion: This study shows that quercetine may decrease kidney damage caused by doxorubicin. This study was supported by the Scientific Researches Fund of Kocatepe University (Project number:08.TIP.01). 
PP-259

Hassall's corpuscles as possible parameter of grades in accidental thymic involution in human

Lestarevic S, Andjelković $Z^{*}$, Miloseavic $M^{* *}$, Rancic $G^{* * *}$, Mandic $\mathrm{P}^{* * * *}$, Savic $\mathrm{S}^{*}$

Institut of Histology and Embriology, University of Pristina, Serbia*; Institut of Forensic Medicine, University of Pristina, Serbia**; Institut of Histology and Embriology, University of Nis, Serbia*** Institut of Anatomy, University of Pristina, Serbia****

Introduction: Accidental thymic involution represents nonspecific response on either pathogen or physiological stimuli. Numeric and morphological changes of Hassall's corpuscles (HC) are one of parameters of its progression. Aim: Histologic analysis $\mathrm{HC}$ in different stages of accidental involution in purpose to establish additional critheria of its grade.

Material and Methods: 34 thymuses of newborn have been obtained during necropsies. Applying standard histological techiques, specimen were fixed and stained with hematoxylineozin and Gomori Silver Methenamine technique. Pursuant histological properties, specimen has been divided in five groups. Control group consisted examples with classic histologic structure of thymus. Thymuses with altered cytoarchictectonical properties were, folowing stages of involution, classified in the next four groups.

Results: Control groupe characterized small and small numbered HC. In first stage of accidental involution, number and morphology were not marcedly changed, while in second stage $\mathrm{HC}$ were present in larger number with equilibrium of cellular and degenerative forms. Progredient enargement of HT was marked in III stage. In IV stage of accidental involution, numbered and degenerated $\mathrm{HC}$ occupie large space in central area of lobuluses. We observed one specimen with unusual form $\mathrm{HC}$, rich in lymphocytes.

Conclusion: Progression of accidental involution is followed with changed morfology, number and size of $\mathrm{HC}$, that could be important determinant of its grade.

\section{PP-260}

Evaluation of TGF beta1 expression and comparison the thickness of different aort layers in experimental diabetes

Cuce $\mathrm{G}^{*}$, Alkan $\mathrm{S}^{*}$, Esen $\mathrm{HH}^{* *}$

Department of Histology and Embryology, Faculty of Meram Medicine, University of Selcuk, Konya, Turkey*; Department of Pathology, Faculty of Meram Medicine, University of Selcuk, Konya, Turkey**

Introduction and Purpose: It was aimed to investigate the effects of experimental diabetes on TGF $\beta 1$ expression and tunica intima and media thickness in abdominal and thoracic aorta.

Tools and Method: Fourteen three months old female rats were divided into two groups, non-diabetic and streptozotocin (STZ) induced diabetic group. At the end of the 60. day without any other application, the abdominal and thoracic arteries were removed. Four study groups toracic control (TC), thoracic diabetic (TD), abdominal control (AC) and abdominal diabetic (AD) were formed. Routine histologic process were performed to aortic tissues. Hematoxylin-Eosin and Verhoeff's Van Gieson elastic staining and TGF $\beta 1$ immunohistochemistry staining were performed. Abdominal and thoracic intima and media thickness of aortas were measured with the oculometer. Findings: In the evaluation of intima and media thickness measurements, there were no significant statistical differences between $\mathrm{AD}$ and $\mathrm{AC}$ groups and TD and TC groups. TGF $\beta 1$ expression increased significantly in TD group compared to TC, AD and AC did not differ significantly.

Result and Interpretation: Abdominal aorta is predisposed to the development of atherosclerosis and we found negative TGF $\beta 1$ expression in the abdominal aorta, it can be interpreted for preparing an appropriate basis for the development of atherosclerosis, it could be developed with overtime. In the toracic aorta, it can be interpreted that TGF $\beta 1$ coordinates the cellular events such as tissue repair. 60 days duration of diabetes is not enough time for the pathological changes could lead to thickening in aortic intima-media layers.

\section{PP-261}

\section{Corneal Endothelial cell area values of healthy subjects}

Sanchis-Gimeno $\mathrm{J}^{*}$, Sanchez-Ferrer $\mathrm{MP}^{* *}$, Herrera $\mathrm{M}^{* * *}$, Pierre-Calzado O*, Garcia-Sandoval B*, Sanchez Del Campo $\mathrm{F}^{* *}$, Martinez-Soriano $\mathrm{F}^{*}$

University of Valencia, Spain*; University Miguel Hernandez of Elche, Spain**; University of Alicante, Spain***

Introduction: Specular microscopy makes it possible to study corneal endothelial cell area in vivo. A specular microscope is an optical system which relies on the reflected light from an incident beam projected onto the corneal surface which shows the cellular shape and configuration at a magnification determined by the optics of the system. In the light of the above we analyzed the corneal endothelial cell area values of healthy subjects by means of non-contact specular microscopy.

Methods: We carried out a prospective study on 419 eyes of 419 healthy subjects: volunteers with manifest sphere and manifest cylinder of \pm 1 diopters and of an age that ranged from 20 to 79 years $($ mean $\pm S D, 49.52 \pm 15.81)$. The work was performed in accordance with the World Medical Association's Declaration of Helsinki and written informed consent was 
obtained from all patients. We recorded the mean of three consecutive measurements of the corneal endothelial cell area using the Topcon SP-2000P non-contact specular microscope (Topcon Corp., Tokyo, Japan).

Results: The mean corneal endothelial cell area was $341 \pm 27$ microns 2 for the subjects $(n=42)$ that ranged from 20 to 29 years old, $343 \pm 32$ microns 2 for the subjects $(n=87)$ that ranged from 30 to 39 years old, $368 \pm 45$ microns 2 for the subjects $(\mathrm{n}=97)$ that ranged from 40 to 49 years old, $375 \pm 19$ microns 2 for the subjects $(n=59)$ that ranged from 50 to 59 years old, $382 \pm 30$ microns 2 for the subjects $(n=92)$ that ranged from 60 to 69 years old and $392 \pm 57$ microns 2 for the subjects $(n=41)$ that ranged from 70 to 79 years old. Lower corneal endothelial cell area values were found in young subjects than in older subjects ( $\mathrm{p}=0.002$; ANOVA test).

Conclusion: In healthy subjects there is an increase in mean corneal endothelial cell area values with age.

\section{PP-262}

Effects of the antiepileptic drug topiramate and co-administration of Nigella sativa on the hippocampus and cerebellum of adult male albino rat: histological immunohistochemical and morphometric studies

Abdel-Hamid K, Mostafa N, Fares S, Shawky O

Histology Department and Neurology and Psychiatry Department, Faculty of Medicine, Assiut University, Asssiut, Egypt

Introduction: Topiramate, is a recently-introduced antiepileptic drug, used for partial onset, primary generalized tonic- clonic, myoclonic seizure and in migraine prevention. Some data in the available literatures point to the presence of some adverse effects of this drug on the brain tissue upon prenatal exposure. Recently, the antioxidant effect of Nigella sativa upon most of body tissues have been widely established. Also, its role as an antiepileptogenic herbaceous plant was investigated in some experimental animal models. Aim of the work: Studying the histological effects of Topiramate which is neurostabilizing drug on the rat hippocampus and cerebellum and evaluating the role of co-administration of Nigella sativa upon these effects.

Materials and Methods: This study was conveyed on 30 adult male rats divided into three groups. Group I: control group. Group II: received $20 \mathrm{mg} / \mathrm{kg} /$ day of topiramate orally for two months. Group III: received $20 \mathrm{mg} / \mathrm{kg} /$ day of topiramate in addition to $100 \mathrm{mg} / \mathrm{kg} /$ dayof Nigella sativa suspension for two months.

Results: Many of the neuronal perikarya in the hippocampus and cerebellum of topiramate treated animals appeared shrunken with an irregular outline and deeply-stained cytoplasm and nuclei. Both of brain areas showed an increase in the immunohistochemical staining for GFAP which was statistically significant. All of these changes were minimized on co-administration of Nigella sativa.
Conclusion: Topiramate induced obvious degenerative changes in neurons to which glial cells reacted. Co-administration of Nigella Sativa is of value in minimizing the induced changes.

\section{PP-263}

\section{Histopathologic examination of the effects of long term-low dose cyclosporin-a therapy: does prednisolone prevent its side effects?}

Gulhan Aktas $\mathrm{R}^{*}$, Altaner $\mathrm{S}^{* *}$, Atasever $\mathrm{A}^{*}$

Department of Histology \& Embryology, Faculty of Medicine, Koc University, Istanbul, Turkey*; Department of Pathology, School of Medicine, Trakya University, Edirne, Turkey**

Introduction: Cyclosporin-A(CsA) is a very potent immunosuppressive agent which is widely used in transplantations and autoimmune diseases. It has been shown that CsA might cause histopathological findings of acute myocaridal infarction after high dose short term treatments. Knowledge about the effects of this drug for long term and low dose treatment after transplantations is limited. The microscopic effects of combined therapy with Prednisolone for long term has not been published. The aims of this experimental study were (i) to examine the effects of CsA on heart at morphologic level and to verify the tolerability of long-term, low-dose treatment with this drug; (ii) to analyze if Cremophor-EL (the vehicle in the intravenous form of $\mathrm{CsA}$ ) is responsible from any additional changes; (iii) to investigate if combined therapy with Prednisolone has different effects on myocardial tissue.

Material and Methods: 24 female Sprague Dawley rats were divided into four experimental groups: I.Group was control, II. Group had oral form of CsA (4 mg/ $\mathrm{kg} /$ day) for 10 weeks. III. Group had i.v. form of CsA ( $4 \mathrm{mg} / \mathrm{kg} /$ day) for 10 weeks. This form contains Cremophor-EL, which is different from oral form. III. Group had i.v. form of CsA ( $4 \mathrm{mg} / \mathrm{kg} /$ day) and prednisolone $(1 \mathrm{mg} / \mathrm{kg} /$ day) for 10 weeks. Specimens were embedded in paraffin and stained with Haematoxylene\&Eosin after sectioning. Histological changes were graded according to the previous studies.

Results: I. Group: Myocardial tissue demonstrated normal histologic features. II. Group: Myocardial desorganisation and inflammatory cell infiltration were clear. III. Group: Infiltration of polimorphonuclear leucocytes and structural desorganisation were primary findings. IV. Group: Similar light microscopic findings were seen in this group. Additionaly, focuses of hemorrhage were observed.

Conclusion: Long term/low dose CsA causes microscopic changes which are similar to the differences seen after short term/high dose therapy. Neither Cremophore EL nor steroids improves the findings or cause additional harmful effect at light microscope level. 
PP-264

Evaluation of the association between TGF-B1 expression and diabetes mellitus in coronary bypass grafts

Dursunoglu D

Department of Histology-Embryology, Selcuklu Medicine Faculty, Selcuk University, Konya, Turkey

Background and Aim: Patients with diabetes have an increased risk for atherosclerosis and its complications and diabetes is an independent risk factor for the development of neointimal hyperplasia, and subsequent vein graft failure, following coronary artery bypass grafting (CABG). However, the mechanism by which diabetes influences the vascular response remains unknown. It is thought that transforming growth factor- $\beta 1$ (TGF- $\beta 1$ ), is involved in the pathogenesis of intimal hyperplasia and arteriosclerosis through the induction of extracellular matrix proteins. This study aims to evaluate the association between TGF- $\beta 1$ expression and diabetes in Saphenous vein (SV), Internal mammarian artery (IMA) and Radial artery (RA) grafts. Material and Methods: In this study, 44 vessels (19 SV, 18 IMA and 7 RA) were collected from 34 patients during CABG. TGF- $\beta 1$ antibodies were used for immunohistochemical analysis and TGF- $\beta 1$ expressions were scored. The association between diabetes and TGF- $\beta 1$ expression was evaluated by the vessel groups.

Results: The expression of TGF- $\beta 1$ in SV grafts was significantly higher than in RA and IMA grafts. There was not any difference between IMA and RA grafts in terms of the expression of TGF- $\beta 1$. There was a strong association between diabetes and TGF- $\beta 1$ expressions in all vessel groups. The expression of TGF- $\beta 1$ in vessels with diabetes was significantly higher when compared to the vessels without diabetes.

Conclusion: TGF- $\$ 1$ expression is incrased in SV, IMA and RA grafts of diabetic patients and increased TGF- $\beta 1$ expression might be among various mechanisms that lead to graft failure that develops after CABG in diabetic patients. Due to their lower TGF- $\beta 1$ expression, IMA and RA grafts might be preffered for $C A B G$ in diabetic patients.

\section{PP-265}

\section{Expression of angiotensin converting enzyme 2 in} human ischemic myocardium

Balnyte I, Bunevicene V, Pangonyte D, Utkiene L, Peciulyte L, Valanciute A, Palubinskiene J, Kazlauskaite D, Ziuraitiene R

Medical Academy, Lithuanian University of Health Sciencies, Kaunas, Lithuania

Introduction: The most important role of angiotensin converting enzyme 2 (ACE-2) in metabolism of angiotensins is the production of angiotensin (1-7), which counteracts the effects of angiotensin II - i.e., causes vasodilatation and prevents fibrosis. The level of its expression in disease depends upon the origin of myocardial damage. Aim of the study was to evaluate the expression of ACE-2 in left ventricular myocardium in the presence of chronic myocardial ischemia.

Object and Methods: Heart specimens with persistent ischemia ( $n=43$, pre-infarction ischemic heart disease (IHD) group) and post-infarction scar ( $\mathrm{n}=32$, post-infarction IHD group) from dissected males who had died suddenly (within 6 h) during the first or repeated acute attack of IHD (without congestive heart failure) and heart explants ( $n=12$, end-stage ischemic heart failure group) were studied. Heart specimens $(n=29)$ selected at autopsy from individuals who died from accidents were used as controls. Slides of myocardium were incubated with rabbit polyclonal antibody against the ACE-2 (1:1000, ab15348, Abcam). Dako Real Envision visualisation system was employed to stain the samples. Expression of ACE2 in cardiomyocytes and vascular smooth muscle cells, endothelial cells and macrophages was assessed according to the 3-point system: 1 - weak, 2 - moderate, 3 - strong.

Results: ACE-2 expression in cardiomyocytes of preinfaction IHD and postinfarction IHD groups did not differ from the controls and was increased only in the postinfarction period when there was also total heart failure, i.e. in the end-stage ischemic heart failure group $(\mathrm{p}<0.05)$. ACE-2 expression in vascular endothelium, smooth muscle cells and macrophages of preinfarction IHD group did not differ from controls; but the number of ACE-2 expressing cells in the postinfarction IHD and in the end-stage ischemic heart failure groups was greater, as compared with preinfarcion IHD group and controls $(\mathrm{p}<0.05)$.

Conclusion: Expression of ACE-2 is increasing in progression of ischemia-induced myocardial dysfunction.

\section{PP-266}

The effects of different fixatives on morphology and immunohistochemical staining of skin tissue

Aktan $\mathrm{TM}^{*}$, Cuce $\mathrm{G}^{*}$, Tosun $\mathrm{Z}^{* *}$, Duman $\mathrm{S}^{*}$

Department of Histology Embryology Selcuk University, Faculty of Meram Medical, Konya, Turkey*; Department of Plastic Surgery, Faculty of Selcuk Medical, Selcuk University, Konya, Turkey**

Introduction and Purpose: We aimed to investigate the effect of Carnoy and \%10'luk formaldehyde fixatives on immunohistochemical staining properties and morphological structure of skin tissue.

Tools and Method: The fresh skins obtained from Department of Plastic Surgery, Faculty of Meram Medicine. The skins were fixed two days in Carnoy and \% 10 formalde- 
hyde solutions. At the end of two days, the same routine histological process applied to skins tissues. While immunohistochemical staining and other stainings, the same methods were applied to 5 micron thick paraffin sections from skin tissue. Hematoxylin-Eosin, Masson's Trichrome stains and F8, Actin, Ki67 immunohistochemical stains were applied to all sections prepared with Carnoy and \%10 formaldehyde. Findings:There was not a morphological difference between Carnoy and Formaldeyde fixed tissue sectiones according to HematoxylinEosin and Masson Trichrome staining. However, the immunohistochemical stained preparations were fixed with $10 \%$ formalin corroded and integrity was lost in the retrieval stage carried out with microwave. Wear more occurred in the reticular layer of dermis and no change was observed in tubular structures.

Result and Interpretation: Skin tissue is fixed by Carnoy can be said to be more successful in the retrieval stage carried out with microwave according to skin tissue fixed in $10 \%$ formalin.

\section{PP-267}

Russian biomechanical and morphological terminology of supporting-motor apparatus in the first half of the XIX-th century

Kapustin RF, Shilo EI

Belgorod State Agricultural Academy, Belgorod, Russia

The problem of terminological standards in the biomechanics and morphology is actual as application of the standard nomenclature allows to improve possibilities of the morphological information search and to provide integration of various information systems, but it requires the account of some specific features in the applied context. The historicism principle lies in the research methodology base. Comparative-historical studying of the basic Russian biomechanical and morphological terms and concepts of first half of XIX-th century was carried out (P.A. Zagorskiy «Abridged anatomy or manual to studying the organization of human body», 1801; E.O. Muhin «Anatomy Course», 1818; A.M. Filomafitskiy «Physiology published for a management of the listeners», 1836; I.P. Zhemchuzhin «Anatomy and physiology of man and animals», 1846). The logic-substantial and comparative analysis of biomechanical terminology allows to allocate some directions: the terms describing features of the form and a structure of bones and muscles: «ensisternum» - apex s. processus ensiformis [P.A. Zagorskiy, 1801]; «capping or a cartilage» [E.O. Muhin, 1818], definition of working muscles topography: «muscle, round and turning hand with palm looking downwards»-M. pronator teres [P.A. Zagorskiy, 1801]; the terms describing statics (poses of animals and man): «spinal column» [P.A. Zagorskiy, 1801], "stroma" [I.P. Zhemchuzhin, 1846]; The terms describing bones biomechanics (bones as levers): "handle" - manubrium [P.A. Zagorskiy, 1801]; the terms describing muscles biomechanics (muscles as «moving force»): «mobile point» - (cauda-tail) [I.P. Zhemchuzhin, 1846]; the terms describing biomechanics of the tendinous apparatus: «articular circumference»- ambitus s. circumferentia articularis [P.A. Zagorskiy, 1801] and so on. On the basis of the carried-out researches it has been shown that the account of not only morphological (anatomic) terminology itself, but also of its biomechanical aspect is necessary at transition from descriptive anatomy to physiologic and topographic anatomy in particular, and interdisciplinary use as a whole.

\section{PP-268}

\section{Evaluation of anatomoradiological landmarks in trigeminal neuralgia using cone beam computed tomography: prelimenary results}

Bilecenoglu B*, Aksoy $\mathrm{S}^{* *}$, Orhan $\mathrm{K}^{* *}$, Gorur DI ${ }^{* * *}$, Sakul U****, Kose $\mathrm{K}^{* * * * *}$

Department of Anatomy, Faculty of Medicine, Ufuk University,Ankara, Turkey*; Department of Oral, Teeth and Jaw Radiology, Faculty of Dentistry, near East University, Mersin, Turkey**; Department of Oral and Maxillofacial Surgery, Faculty of Dentistry, Ankara University, Ankara, Turkey***; Department of Anatomy, Faculty of Dentistry, Ankara University, Ankara, Turkey****; Department of Biostatistics, Faculty of Medicine, Ankara University, Ankara, Turkey*****

Purpose: Foramens which trigeminal nerve passing through, are of great surgical and diagnostic importance in procedures like percutaneous trigeminal rhizotomy for trigeminal neuralgia, transfacial fine needle aspiration technique in perineural spread of tumour and electroencephalographic analysis for seizure. This study presents the anatomic variations in dimensions, appearance and number of foramen ovale (FO), rotundum (FR) and fissura orbitalis superior (FOS). Materials and methods CBCT images (Netwom 3G, QR Verona) of 18 trigeminal neuralgia (V2 and V3) and 40 control patients retrospectively evaluated using a surface rendering program (Maxilim, Medicim). The distance among FOS, FR, FO and also the distance among all foramen to anterior, medius and posterior clinioids were measured. The cliniond calcifications were also noted in the study.

Results: It was found that trigeminal neuralgia occurs along with the increased age. It was also revealed that the trigeminal neuralgia patients have more distance between FOS, FR and FO than normal patients. Moreover, the distance between FOS -posterior cliniod and FR-posterior clinoic is higher than normal patients.

Conclusions and Discussion: It is concluded that Anatomical variations in size and shape of FOS, FR and FO is important esp. development of trigeminal neuralgia Our data suggest that the determination of some landmarks allows customization to individual patient anatomy and may help the surgeon for treatment. Besides CBCT can be a powerful tool for detecting skull base structures with less ionizing radiation. 
PP-269

Estimation of volume of spleen on computed tomography images using the cavalieri principle: a gold standard study

Emirzeoglu $M^{*}$, Sahin B*, Selcuk MB**, Uzun A*, Edizer $M^{*}$

Department of Anatomy, Faculty of Medicine, Ondokuz Mayıs University, Samsun, Turkey; ${ }^{\star}$ Department of Radiology, Faculty of Medicine, Ondokuz Mayıs University, Samsun, Turkey**

Introduction and Purpose: Size changes of spleen are often monitored using the computed tomography (CT) scans. However, we have not found a study evaluating the estimation of spleen using stereological methods. In the present study, we aimed to evaluate the accuracy of the estimation of volume of cadaveric spleen in CT imaging.

Tools and Method: Five spleens were obtained from the cadavers. The spleens were scanned with a CT machine. The section series with thicknesses of $0.5 \mathrm{~mm}$ were obtained. The cut surface areas of the spleens were obtained using ImageJ software. The spleen volumes were estimated using the Cavalieri principle of stereological methods. The actual spleen volumes were obtained by Archimedean principle.

Findings: The actual spleen volumes were 190, 146, 314, 178 and $165 \mathrm{~cm} 3$ respectively. The estimated spleen volumes from the CT images were 167.5, 126.6, 288.7, 156.6 and 142.5 respectively. The deviation percentages were $-13.5 \%,-15.4 \%$, $-8.8 \%,-13.7 \%$ and $-15.8 \%$, respectively. The difference from the actual volumes was statistically significant.

Results and Interpretations: Our results showed that the estimated spleen volume was changing negatively on thin section. Some researchers indicate that, while the volume was decreasing in thin sections, it was increasing in thick sections. We are planning to go further with thicker section to get more precise results. The usage of constant section thickness may standardize the deviations from the actual volumes depending on the section thickness.

Resources: This study was supported by the Project Management Office (PYO.TIP.1901.09.002) of the Ondokuz Mayıs University.

\section{PP-270}

Examination of the anatomy of the secretion ducts of parotid gland in Akkaraman sheep by corrosion method

Bagci G, Aycan K

Department of Anatomy, Faculty of Medicine, Erciyes University, Kayseri, Turkey

In literature, we could not find any study examining the structure of the secretory ducts of parotid gland in Akkaraman sheep with corrosion method. In this study, parotid glands of Akkaraman sheep were studied with corrosion method so that the parotid gland duct system could be put forth for consideration the heads of the ten Akkaraman sheep that were taken from the slaughterhouse were washed and cleared. Parotid gland and parotid duct dissection was performed. Parotid gland and duct parotid were dissected. It was found that the average weight of the parotid gland was $25.3 \mathrm{~g}$, the height $5.87 \pm 1.04 \mathrm{~cm}$, the tickness $0.1-1.30 \mathrm{~cm}$, the length of ductus parotideus $10.92 \pm 1.027$ $\mathrm{cm}$ and the volume of the parotid gland $12.50 \pm 2.48 \mathrm{~cm}^{3}$ and that parotid duct was opened into the mouth between the upper third premolar and the first molar teeth. In order to examine the the secretion ducts of parotid gland by corrosion method, polyster was injected into the duct parotid. The secretion ducts of parotid gland exposed were examined macroscopically and microscopically. It was determined that parotid gland consisted of lobes and lobules. It was also found that the number of the lobes of the parotid gland in sheep ranged from 6 to 17 (the mean 12), that an average of 3-16 lobules (the mean 9) existed in a lobe and that each lobe and lobule had one interlobaris duct and one interlobular duct. The secretion ducts of parotid gland from the acinus to the mouth comprised of the duct in the following order; 1-acinus, 2-intralobular duct (intercalated duct+Striat duct), 3-interlobular duct, 4-intralobar duct, 5Interlobar duct, 6-Ductus parotideus. In this study, we examined the anotomy of the secretion ducts of the parotid gland in Akkaraman sheep and we hope that this study will shed light into the studies in the future.

\section{PP-271}

Effects of inhaled formaldehyde on humoral immunity: an experimental study

Sapmaz HI* Sarsilmaz $\mathrm{M}^{* *}$, Godekmerdan $\mathrm{A}^{* * *}$, Ogeturk $M^{* * * *}$, Tas $\mathrm{U}^{* * * * *}$, Kose $\mathrm{E}^{*}$

Department of Anatomy, Faculty of Medicine, Inonu University, Malatya, Turkey*; Department of Anatomy, Faculty of Medicine, Sifa University, Izmir, Turkey**; Department of Immunology, Faculty of Medicine, Firat University, Elazig, Turkey***; Department of Anatomy, Faculty of Medicine, Firat University, Elazig, Turkey ${ }^{* * *}$; Department of Anatomy, Faculty of Medicine, Gaziosmanpasa University, Tokat, Turkey*****

Objective: This study was carried out to determine the variations that occur in humoral immunity of rats that were exposed to subacute (4 weeks) and subchronic (13 weeks) formaldehyde (FA) inhalation.

Material and Methods: For this purpose, male SpragueDawley rats were exposed to 5 or 10 ppm FA inhalation for either 4 weeks or 13 weeks (total 4 groups; n=7/group). FA was inhaled for 5 days per week and 8 hours per day. Control groups were kept under normal laboratory clean air for 4 or 13 weeks ( 2 groups; $n=5 /$ group). At the end of the study period, blood 
samples were collected from rats and the serums of the rats were separated by centrifuge. ELISA was used to determine serum immunoglobulin A (IgA), immunoglobulin M ( $\operatorname{IgM})$, immunoglobulin $\mathrm{G}(\mathrm{IgG})$ and complement 3 (C3) levels.

Results: IgA, IgM and C3 levels in subacute groups were determined to be increased significantly whereas IgG levels were determined to be decreased significantly compared with control groups. IgM levels which exposed 10 ppm FA inhalation and IgA levels in both of subchronic groups were determined to be increased significantly compared with control groups. IgG and C3 levels in subchronic groups didn't show statistically significance compared with control groups.

Conclusion: At the end of this study, we determined that formaldehyde was capable to stimulate the immune system and cause an increase in IgA, IgM and C3 levels, but seriously suppressed the secondary immune response $(\mathrm{IgG})$.

\section{PP-272}

Estrogen effects on GH/IGF-1 system in immune organs of Yersinia-exposed rainbow trout (Oncorhynchus mykiss)

Wenger $M^{*}$, Shved $N^{* *}$, Akgul G* , Segner $H^{* * *}$, Eppler $E^{*}$

Neuro-Endocrine Immune Interactions Research Group, Institute Of Anatomy, University of Zurich, Zurich, Switzerland; Center for Evolutionary Medicine, Institute of Anatomy, University of Zurich, Zurich, Switzerland; Center for Fish and Wildlife Health, Institute of Animal Pathology, University of Bern, Bern, Switzerland

Estrogens are known to interact with the neuro-endocrine system, which is also involved in immunological responses in fish via the growth hormone/insulin-like growth factor (GH/IGF) -system of the brain-pituitary-liver axis. GH thereby stimulates the synthesis of hepatic IGF-1 which in turn exerts a negative feedback loop on pituitary GH secretion. Previous studies have shown that external factors such endocrine disruption, salinity, temperature, and nutritional state influence this system. Especially endocrine disruption via estrogen receptor (ER) ligands is thought to be one of the major stressors of the aquatic environment. However, the effect of estrogenic exposure on the GH/IGF system and the possible interactions with the immune system remains to be elucidated. The current project aims at investigating these interactions between the GH/IGFaxis and immune-related factors to discover a possible connection between the neuro-endocrine system and the immune system in fish. To this end, juvenile rainbow trout were first exposed to different doses of 17 beta-estradiol and then infected with the bacteria Yersinia ruckeri, the etiological agent of the red mouth disease. The mRNA expression levels of GH receptor 1 and 2, IGF-1, IGF-2, estrogen receptor beta 1 (ERbeta1) in immune system-related organs (head kidney, spleen, liver) were measured via qPCR and results were then compared to immune system responses assessed by using a $2 \mathrm{~K}$-cDNA microarray platform. Results will be analyzed and discussed.

\section{PP-273}

An anatomical study on the three-headed biceps brachii in human fetuses and clinical relevance

Kervancioglu P, Orhan M

Anatomy Department, Medical Faculty, Gaziantep University Gaziantep, Turkey

The biceps brachii is as one of the most variable muscle in the human body, in terms of number and morphology of its heads. The most frequent variation is the presence of a third head which has been reported by several authors in different population. Our aim was to find the occurrence of the supernumerary head of biceps brachii in Turkish fetuses. Out of the 24 upper limbs of the fetuses' two (8.33\%) arms were found to have a three-headed biceps brachii. The variations were present unilaterally in the right arm of one male fetus and one female fetus. In one of the cases, the third head of biceps brachii originated from the anteromedial aspect of the humerus just distal to the insertion of the coracobrachilais, medial to the brachialis and in the other one, the third head was a thin muscle bundle which raised the lateral side of the insertion of coracobrachilais and over the origin of brachialis. Both of them were extended distally and join the common tendon. The occasional presence of the three-headed biceps brachii in the fetuses observed in the present study was similar those of adults reported in the previous studies. In conclusion, these variations are not rare and are interesting not only to anatomists, but also to orthopedic surgeons, plastic surgeons, traumatologists, physiotherapists, doctors dealing with sports Medicine and radiologists.

\section{PP-274}

\section{Semg performance associated to isokinetic velocity of the wrist muscles}

Carrascosa-Sánchez I, Spottorno-Rubio P, Bosch-Martín A, Aguado-Henche S, Slocker de Arce A, Gómez-Pellico L

Department of Human Anatomy and Embriology, Faculty of Medicine, University of Alcalá, Alcalá de Henares, Madrid, Spain

Introduction and Purpose: Kinesiologic electro-myography (KEMG) is used to evaluate muscle activity patterns during exercise. The purpose of this preliminary study is to asses EMG activity behaviour of flexo-extension muscles of the wrist during isokinetic exercises at different velocities.

Method: We studied EMG activity of the following muscles: Extensor carpi radialis longus (ECRL), Extensor carpi ulnaris (ECU), Flexor carpi ulnaris (FCU) and Flexor digitorum superficialis (FDS). A total of 10 healthy subjects between 18 and 21 years of age. All subjects signed an informed consent. The procedure for surface electrodes placement referred from 
SENIAM (1). EMG and isokinetic muscle activity was recorded simultaneously at 60 and $180^{\circ}$ s with a range of movement of $80^{\circ}$. Each cycle was defined by means of video synchronization. SEMG recordings: The Raw EMG signal was recorded using a device (ME6000, Mega Electronic). Isokinetic recordings: A Biodex System 3 PRO was used. Raw EMG data were analyzed using a Fast Fourier Transform algorithm acting on 1024 data points. Averaged EMG (aEMG) and Mean Power Frequency (MPF) were calculated in all studied muscles. We used Statgraphics plus to analyze the data. Inferential statistic was performed using ANOVA and Fisher test.

Result and Interpretation: When comparing aEMG and MPF variables for each registered muscle at each different isokinetic velocity, significant differences were found only in some of the studied muscles. Therefore, when defining the tables of averages, we observed a different behaviour of ulnaris wrist muscles (ECU and FCU) in comparison with radialis muscles (ECRL and FDS). ECU and FCU show an activation pattern, intensity and frequency more homogeneous and constant at 60 and $180 \%$ s. Referente (1) Hermens H.J, Freriks B, Merletti R, Hägg G, Stegeman D, Blok J et al, editors (1999). SENIAM 8: European recommendations for surface electromyography. Roessingh Research and Development, Netherlands.

\section{PP-275}

\section{Trunk isokinetic assessment in elite female fencers}

Bosch-Martin A, Spottorno-Rubio P, Carrascosa-Sanchez J, Aguado-Henche S, Gomez-Pellico L, Gomez-Pellico L

Department of Human Anatomy and Embriology, Faculty of Medicine, University of Alcalá, Alcalá de Henares, Madrid, Spain

Introduction: The association between specific fencers training, and strength characteristics of trunk extension and flexion were studied in high level fencers of the Autonomous Community of Madrid.

Material and Methods: Eight elite female fencers, between 14 to 23 years old (mean 19.38) were tested for concentric trunk flexion and extension muscle strength. All the fencers have similar training characteristics and signed a consent form. None of them have had any history of surgeries or trunk pain at least one year before the test. Maximal trunk forces were measured in two positions (semi-standing and seated-compressed) using Biodex System 3 electromagnetic dynamometer (specific back attachment). Subjects warmed up for 20 minutes before putting on the back attachment (10 minutes with a cycle ergometer and 10 minutes stretching specific muscles). The strength was tested at four angular velocities: $60 \% \mathrm{~s}, 90 \% \mathrm{~s}, 120 \% \mathrm{~s}$ (5 repetitions) and $180 \%$ s (10 repetitions) in each position (rest period: $60 \mathrm{sec}-$ onds between series) Range of motion studied: $90^{\circ}$. Warm-up repetitions were performed before all tests repetitions. During testing standard encouragement was given.
Results: Peak torque and agonist-antagonist ratio were separately analyzed in each velocity. Inferential statistic was performed using t-Student $(\mathrm{p} \leq 0.05)$. SPSS statistical software (17.0) was used. Results evidenced no significant differences for peak torque between the 2 positions studied. In both positions and all velocities we found an imbalance between agonist and antagonist muscles in favour of trunk flexors with higher values, double the average of normal population.

Conclusions: It is necessary to study the relationship between muscle trunk flexors and extensors in high level athletes to conclude if our findings are due to the training or to the specific fencing gesture. To prevent possible wall abdominal injuries or low back pain, specific strength training has to be introduced in the basis fencing training program.

\section{PP-276}

\section{Shoulder isokinetic assessment in high-level swimmers}

Spottorno-Rubio P, Bosch-Martin A, Carrascosa-Sanchez J, Aguado-Henche S, Gomez-Pellico L

Department of Human Anatomy and Embriology, Faculty of Medicine, University of Alcalá, Alcalá de Henares, Madrid, Spain

Introduction: This study aims to evaluate the behaviour of external and internal rotation (ER and IR) shoulder muscles in high-level female swimmers of Madrid region.

Methods: 20 female high level swimmers, 12 to 17 years old (mean 13.80) with similar training characteristics were bilaterally tested for concentric shoulder ER / IR muscle strength. All the subjects signed a consent form. None of them have ever had any upper limb surgery or injuries at least one year before the test. Swimmers warmed up for 10 minutes pre-test $(5 \mathrm{~min}$ utes in cycle ergometer and 5 minutes specific exercises with upper limbs). Maximal bilaterally shoulder concentric ER and IR forces were measured using an electromagnetic dynamometer Biodex System 3 (shoulder attachment). The strength was tested at $60 \%$ s (5 repetitions) and $180 \%$ s (15 repetitions). 30 seconds rest period between series and 5 minutes rest period between dominant and non-dominant shoulder. Range of motion: $90^{\circ}\left(35^{\circ} \mathrm{ER}-55^{\circ} \mathrm{IR}\right)$.

Results: All the data were analysed using SPSS statistical software (version 17.0). Inferential statistic was performed using Wilcoxon test to verify significant differences among Peak Torque in each studied velocities. No significant differences ( $p>0.05$ ) were found between dominant and non dominant shoulder in both speeds. When studying ER/IR ratio we found a $60 \%$ one swimmer had balanced forces, 3 had a misbalance towards ER and the rest of the sample had a misbalance towards IR. At $180 \%$ s only one swimmer continued with a misbalance towards ER, and the rest of the sample had IR misbalance.

Conclusions: In swimmers under 18, the use of isokinetic tests to measure shoulder strength can be effective to improve train- 


\section{S142 Joint Meeting of Anatomical Societies, 19-22 May 2011, Bursa, Turkey}

ings' planning and control. Findings showed strength training has to be done individually, considering balance between ER and IR in dominant and non-dominant extremity.

\section{PP-277}

\section{Connected superior and middle trunks of brachial} plexus: an unique variation of brachial plexus

Yildiz $\mathrm{S}^{*}$, Comert A**, Ozan $\mathrm{H}^{*}$

Department of Anatomy, Gulhane Military Medical Academy, Ankara, Turkey*; Department of Anatomy, Faculty of Medicine, Ankara University, Ankara, Turkey**

During treatment of tumors of nerve sheaths such as schwannomas and neurofibromas neurosurgeons should be aware of variations and abberant formations of brachial plexus. Variations of the branches of brachial plexus are common but its variations in the level of the roots and trunks are rare. Variations regarding inferior trunk were founded more frequent in previous studies. A unilateral an unusual variant of the brachial plexus was found during routine dissection of a 75year-old male cadaver. It was observed that middle trunk was connected to superior trunk. Deep cervical artery originating from subclavian artery passed between C6 root and middle trunk before their connection. Similar variations in the brachial plexus were not observed on the contralateral side. In available literature only two similar bilateral cases were reported. The details of this variation and its clinical significance were discussed. Knowledge about these rare variations in the trunks is very useful in surgical practice and anesthesia.

\section{PP-278}

\section{A rare case of inferior alveolar nerve in infratemporal fossa}

Mostafavi FS, Dashti GR

Department of Anatomical Sciences and Molecular Biology, Faculty of Medicine, Isfahan Medical Sciences University, Isfahan, Iran

The inferior alveolar nerve and maxillary artery were studied in 30 cadaver through infratemporal dissection; in one of the cadaver, on left side of the face of 50 years old woman, an unusual variation in the origin of the inferior alveolar nerve and its relationship with the surrounding structures was observed. The inferior alveolar nerve originated from the mandibular nerve by two roots and the second part of the maxillary artery was incorporated between them. An embryologic origin of this variation and its clinical implications is discussed. Because the maxillary artery ran between the two roots of the inferior alve- olar nerve, and the nerve was fixed between the foramen ovale and mandibular foramen, tension and compression of the nerve from arterial pulsation could cause mandibular neuralgia.

PP-279

\section{A peerless abnormality in the course of the musculocutaneous nerve}

\author{
Bakirci S, Sapmaz HI, Oner Z, Ekinci N \\ Department of Anatomy, Inonu University School of Medicine, \\ Malatya, Turkey.
}

During routine dissection of a white male cadaver, age 65 years old, we found abnormality in the course of the musculocutaneous nerve on the left upper extremity. Also there was no abnormality at five terminal branches and other "preterminal" or "collateral" branches of brachial plexus. Musculocutaneous nerve was arised lateral cord of the brachial plexus. It was piercing coracobrachial muscle and passing obliquely between the brachial muscle and the biceps brachi muscle. It was innervating with two different branches biceps brachi muscle and with a single branch brachialis muscle. It was terminated two branches on the lower part of arm. Lateral branch piercing deep fascia at the lateral border of tendon of biceps brachi muscle and was continueted into the forearm as lateral cutaneous antebrachial nerv. Medial branch was lying underly bicipital aponeurosis and passes above brachial artery. It was continueted between radial artery and ulnar artery and attached median nerve in the cubital fossa. A more frequently can observed communicating from proksimal part of musculocutaneous nerve and median nerve in the arms, but a rare seeing course abnormality of musculocutaneous nerve in the cubital fossa. Medial branch of the musculocutaneous nerve can be affected compression under bicipital aponeurosis due to muscular hypertrophy and may be caused press semptoms on vessels in the cubital fossa due to strenching of its. Therefore we think, that variation would be important particularly for plastic and pediatric surgeons.

\section{PP-280}

\section{Intracardiac ganglia of the frog heart}

Batulevicius D*, Skripkiene $\mathrm{G}^{*}$, Batuleviciene $\mathrm{V}^{* *}$, Skripka $\mathrm{V}^{*}$ Institute of Anatomy, Medical Academy, Lithuanian University of Health Sciences, Kaunas, Lithuania*; Faculty of Health Care, Kaunas College, Kaunas, Lithuania**

The histochemistry for acetylcholinesterase was used to quantify the distribution of the intrinsic neurons in the hearts of 12 frogs Rana temporaria. Sixty-six intracardiac neurons from 12 frogs were injected ionophoretically by the intracellular mark- 
ers Lucifer Yellow and AlexaFluor 586 to determine the neuronal morphology and projections. Total neuronal number per frog heart varied from 1060 to 1637 and was 1374 +/- 56 (Mean +/- SE), on average. About $46 \%$ of the neurons were located in the interatrial septum, $25 \%$ in the atrioventricular orifice, $12 \%$ on the sinus venosus, and $9 \%$ on the superior caval veins. Only $2 \%$ of the neurons were located on the external surface of atria, while $6 \%$ on the ventricles. The extracardiac nerves entered the frog heart by the superior caval veins. The dorsal and ventral septal nerves extended to the atrioventricular orifice and branched into the ventricle. Among the intracellularly labeled neurons, $67 \%$ were unipolar, $9 \%$ bipolar, $9 \%$ multipolar and $4 \%$ pseudounipolar. The frog ventricle received the axons of the neurons situated along the large nerve trunks. In contrast, frog atria received axons of the neurons situated further apart from the large nerve trunks in the septum. The axon hillocks of most of the neurons contained numerous thin subsidiary processes projecting onto adjacent neuronal somata and cardiac muscle fibers within septum. Vast majority of the intracardiac neurons were classified as Golgi I type neurons with long prominent axons. A few Golgi II type neurons lacking the prominent axons, the presumptive interneurons, were also found. Results of the present study demonstrate the wide morphological diversity of the frog intracardiac neurons.

\section{PP-281}

Human anatomic variation: doubled foramen rotundum and duplicated maxillary nerve

Rusu MC

"Carol Davila" University of Medicine and Pharmacy Bucharest, Romania

Even though the doubled foramen rotundum (FR) can be identified once within the literature, there are no details of the contents at that level. We present here an anatomical case demonstrating the maxillary nerve (MN) duplication at the level of an unilateral doubled FR: the accessory nervous trunk of the MN left the trigeminal ganglion on the outer side of the MN main trunk and coursed beneath the main trunk of the MN canal, within the sphenoidal greater wing, to join infero-medially that main trunk at the entrance in the pterygopalatine fossa. Previously undocumented, the MN duplication may interfere with various surgical exposures interfering with the foramen rotundum and may explain atypical sensory syndromes and functional impairment during skull base trauma or anesthesia. Acknowledgements: (1) Research Grant UEFISCSU, 317/2007. (2) This study was also supported by the SOP HRD, financed from the ESF and by the Romanian Government under the contract number POSDRU/89/1.5/S/64109.

\section{PP-282}

Trigeminal ganglionic neuronal death in a case positive for the botulinum neurotoxin $B$

Rusu $\mathrm{MC}^{*}$, Pop F* ${ }^{*}$, Hostiuc $\mathrm{S}^{* *}$, Curca GC**

"Carol Davila" University of Medicine and Pharmacy Bucharest, Romania*; "Mina Minovici" National Institute of Legal Medicine, Bucharest, Romania**

Botulinum neurotoxin B (BoNT-B) is a well known lethal agent in humans. In the last years botulinum neurotoxins have been used as therapeutic agents in pain management; their intimate effects on the peripheral nervous ganglia were however not thoroughly described. We present here a rare case of a BoNT-B serologically positive human adult in which trigeminal ganglia were found immunopositive for caspases 3 and 9 . Thus the intrinsic apoptotic pathway was proposed as the cell death mechanism involving primary trigeminal neurons. Scavengers such as macrophages and resident satellite glial cells, with a CD68 immunopositivity were also identified, presumably being involved in the process of eliminating apoptotic remnants. Taking into account that the down-regulation of metalloproteinases leads to inactivation of neuronal caspase 3, exogenous metalloproteinases, such as BoNT, may lead to apoptosis, as we evidenced in the trigeminal ganglion. If this causal relation, neurotoxin-to-apoptosis is valid, similar apoptotic processes can be presumed to occur in various ganglia, sensory and autonomic, involved in vital functions of the body. In conclusion, further morphological and experimental studies are needed to extensively evaluate the apoptotic effect of BoNT within the peripheral nervous ganglia in botulinum infections and BoNT treatments. Acknowledgements: (1) Research Grant UEFISCSU, 317/2007. (2) This study was also supported by the SOP HRD, financed from the ESF and by the Romanian Government under the contract number POSDRU/89/1.5/S/64109.

\section{PP-283}

Histological and morphometric characteristics of donor nerve grafts in the lower limb

Altun U*, Ucar Y*, Sarikcioglu L*, Gokhan G**, Gurer EI ${ }^{* *}$

Department of Anatomy, Faculty of Medicine, Akdeniz University, Antalya, Turkey*; Department of Pathology, Faculty of Medicine, Akdeniz University, Antalya, Turkey**

Purpose: Primary tensionless repair is the desired treatment for traumatically transected peripheral nerves. In the setting of segmental nerve loss resulting from avulsion, crushed and debrided margins, or contraction and scarring in delayed repair, interposition conduits are required to obtain a tension free coaptation. In the present study, we aimed to study histo- 
logic and morphometric characteristics (axon number, number of fascicles, total area of the connective tissue, cross-sectional area) of the donor nerve grafts in the lower limb.

Methods: Donor nerves of the lower limb (N. suralis, N. saphenous, N. peronealis superficialis) were included to the present study. After routine histological tissue processing, cutting, and staining methods cross sections of the graft samples were analyzed by image analysis system. Results: Comparison of the data revealed that each nerve had specific characteristics.

Conclusions: We suggest that histological and morphometric characteristics of the donor nerves should be remembered during selection of the appropriate donor nerve for reconstruction of recipient nerve.

\section{PP-284}

Size and localization of the sciatic nerve during the fetal period

Sulak O, Sakalli B, Ozguner G, Kastamoni Y

Department of Anatomy, Faculty of Medicine, Suleyman Demirel University, Isparta, Turkey

Objective: The aim of this study was to determine the morphometric development, localization and the variation of the sciatic nerve during the fetal period.

Material and Methods: 400 fetal sciatic nerve obtained from 200 human fetuses (103 males and 97 females) aged between 940 weeks were used in this study. Fetuses were divided into four groups between gestational weeks; 1st trimester, 2nd trimester, 3rd trimester and full term of gestation. At first, gluteus maximus muscle was dissected. The distances between sciatic nerve and greater trochanter, ischial tuberosity and distances between bifurcation point of the sciatic nerve and knee width were measured. The relation of sciatic nerve and piriform muscle was determined. The sciatic nerve lengths, and width (at the origin and at the bifurcation level) were measured.

Findings: Mean values and standard deviations of all parameters according to trimesters were calculated. It's found that all parameters were increased with age during the fetal period. No significant differences were observed between sexes for any of the parameters $(p>0.05)$. There was significant correlation between gestational age and all parameters $(\mathrm{p}<0.001)$. When the sciatic nerve was evaluated according to the piriformis muscle; the sciatic nerve exits below the piriformis muscle in $98.5 \%$ of the cases (in right and left side). When the remaining of the cases were evaluated; 3 (1.5\%) in right side and $3(1.5 \%)$ in left side were anomalies.

Conclusion: The present study has revealed that the morphometrical measurement of the sciatic nerve during the fetal period. We hope that present results can be considered as providing some useful findings for future studies.

\section{PP-285}

\section{Flexion furrows of the feet in Macedonian population}

Cadikovska E, Zafirova B, Trpkovska B

Institute of Anatomy, Medical Faculty, Skopje, Republic of Macedonia

The aim of the study is to establish flexion furrows of the feet in Macedonian population and attempt to detect regularities in their occurrence. Material and method: 400 examinees were included in the study, 200 males and 200 females, ages from 16-20 years. Sole prints have been taken using Cummins and Midlo's method, pattern types and flexion furrows were determined. Furrows running in various directions in relation to the long axis of the foot (longitudinal, transverse, oblique) were observed and they are classified as F1 and F2. Results: Transverse furrows from $\mathrm{F} 1$ and oblique furrows from $\mathrm{F} 2$ predominated. In $45 \%$ of females and almost $60 \%$ of males furrows were entirely absent on the feet.The arangment of the furrows was not related to sex. The arrangement of the furrows on the feet of the same individual was not symmetric. The number of the furrows on the right feet was higher. Conclusiones: Flexion furrows on the feet are characterized by marked individual variation, pertaining to their course, frequency and mutual arrangement, although in dermatoglyphic descriptions they are usually omitted.

\section{PP-286}

\section{The effect of glenoid version angle on rotator cuff pathology}

Dogan $\mathrm{M}^{*}$, Cay $\mathrm{N}^{* *}$, Tosun $\mathrm{O}^{* * *}$, Karaoglanoglu $\mathrm{M}^{* * *}$, Bozkurt $M^{* * * *}$

Department of Orthopedics, Yıldırım Beyazıt University, Faculty of Medicine, Ankara, Turkey*; Department of Radiology, Yuksek Ihtisas Education and Research Hospital, Ankara, Turkey**; Department of Radiology, Ataturk Education and Research Hospital, Ankara, Turkey***; Department of Orthopedics, Ataturk Education and Research Hospital, Ankara, Turkey****

Objective: The relationship between glenoid version angle and rotator cuff pathology has been described. However, the effect of glenoid version angle itself on rotator cuff pathology is still unknown. The aim of this study was to investigate whether there is an impact of glenoid version angle on rotator cuff pathology.

Materials and Methods: Shoulder MRI examinations of any cause performed in the study centers between August 2008 and August 2009 were evaluated retrospectively. Shoulder MRI exams having rotator cuff pathology such as trauma, degeneration, and acromion type 2-3-4 reported in previous studies were excluded from the study. Sixty two shoulder exams with rotator cuff pathology having type 1 acromion morphology and 60 shoulder exams without rotator cuff pathology were includ- 
ed in the study. Glenoid version angle was calculated in axial images. Rotator cuff was evaluated in fat-suppressed T2weighted and proton density-weighted images.

Results: The mean values for glenoid version angle were 2.41 and 0.61 in the control and the study groups, respectively. No statistically significant difference was found between 2 groups ( $>0.05$ ). In addition, $26.6 \%$ and $33.8 \%$ of the glenoids were retroverted and, $73.4 \%$ and $66.2 \%$ were anteverted in the control and the study groups, respectively (all p $>0.05$ ).

Conclusion: This study demonstrated no significant relationship between glenoid version angle and rotator cuff pathology. Therefore, the pathologies that can be related to cuff itself should be investigated if the pathology can not be explained by an extrinsic cause in subjects with rotator cuff pathology.

\section{PP-287}

The effect of morphometric relationship between the glenoid fossa and the humeral head on rotator cuff pathology

Cay $\mathrm{N}^{*}$, Tosun $\mathrm{O}^{* *}$, Dogan $\mathrm{M}^{* * *}$, Karaoglanoglu $\mathrm{M}^{* *}$, Bozkurt $M^{* * * *}$

Department of Radiology, Yuksek Ihtisas Education and Research Hospital, Ankara, Turkey*; Department of Radiology, Ataturk Education and Research Hospital, Ankara, Turkey**; Department of Orthopedics, Yildirim Beyazit University, Faculty of Medicine, Ankara, Turkey***; Department of Orthopedics, Ataturk Education and Research Hospital, Ankara, Turkey****

Objective: The aim was to investigate whether there is an impact of morphometrical association between the glenoid fossa and the humeral head on rotator cuff pathology.

Design: This was a non-randomized, retrospective and observational study. Setting: Shoulder MRI examinations of any cause performed in study centers between August 2008 and August 2009 were evaluated.

Patients/Participants: Shoulder MRI exams having rotator cuff pathology such as trauma, degeneration, and acromion type 2-3-4 were not included. Sixty two shoulder exams with type 1 acromion morphology and 60 shoulder exams without rotator cuff pathology (control group) were included. Intervention: Glenoid antero-posterior distance and the humeral head diameter in axial images, the humeral head and the glenoid articular surface diameters in coronal images, and their ratios were measured and calculated in both groups. In addition, the subacromial distance was measured in sagittal images. Rotator cuff was evaluated in fat-suppressed T2weighted and proton density-weighted images. Main outcome measurements: Subacromial distances were $8.94 \pm 1.43 \mathrm{~mm}$ and $10.96 \pm 1.62 \mathrm{~mm}$ in rotator cuff pathology and control groups, respectively $(\mathrm{p}<0.001)$.
Results: The humeral head diameter, the glenoid articular surface diameter, the glenoid antero-posterior distance, and their ratios were not statistically different in 2 groups (all p>0.05).

Conclusion: This study demonstrated no association between the humeral head and the glenoid articular surface which can result in rotator cuff pathology. The glenohumeral joint is compatible morphometrically. Therefore, the pathologies that can be related to cuff itself should be investigated if the pathology can not be explained by an extrinsic cause.

\section{PP-288}

The demonstration of the number, course and the location of nutrient artery canal of the femur by multidetector computed tomography

Imre N*, Battal B*, Acikel CH*, Comert A**, Yazar F*

Department of Anatomy, Gulhane Military Faculty of Medicine, Ankara, Turkey*; Department of Anatomy, Faculty of Medicine, Ankara University, Ankara, Turkey**

The aim of this study is to suggest the number, course, width and location of nutrient artery canal in femur using the multidetector computed tomography (MDCT). These canals have been assessed on the multiplanar-reformatted (MPR) and Volume Rendered (VR) images produced on the basis of axial sections. While the number of median nutrient artery is 2 , this has demonstrated the varied distribution between one and ten. It was detected that there was an inversion correlation between the nutrient canal number and the canal diameter. While the large majority of the canals have demonstrated upward course (95\%), it has been observed that a few canals have shown the direction to the transverse $(3 \%)$ or downward $(2 \%)$ course. And the locations of outer ostiums of the artery entrance holes according to linea aspera (foramina) were established in this study.

\section{PP-289}

\section{Are two methods better than one for teaching cardiac anatomy?}

Finn G, Sawdon M, Griksaitis M

Durham University, UK

Introduction: Many methods are available to teach cardiac anatomy, but little evidence supports the use of any one method over another. This study sought to determine whether there was an additive effect on students' knowledge by combining two modalities (ultrasound and prosections) to teach the same content.

Methods: First year medical students $(n=108)$ were divided into 2 equal groups. Initially all students sat a pre-test to estab- 
lish whether groups were randomised. Students were then taught cardiac anatomy using either ultrasound or prosections (session A) and subsequently sat a 10 question MCQ based on both ultrasound and cadveric images. Students then crossedover and received teaching using the other modality (session B) before completing the test again.

Results: The pre-intervention test scores showed no statistical significant difference in base line knowledge between the two study groups $(\mathrm{p}=0.948)$. After teaching session A there was no statistically significant difference between the scores of those taught using ultrasound versus those taught using prosections $(p=0.105)$. After teaching session $B$, there was still no significant difference between the two groups' scores $(\mathrm{p}=0.664)$. Considering the cohort as a whole, the increase in test scores after teaching using both modalities was not statistically significant $(\mathrm{p}=0.407)$ compared to after teaching session A alone.

Conclusions: Although students' knowledge did not significantly increase after teaching via two different modalities, we advocate the use of both methods in the undergraduate curriculum. Prosections serve to demonstrate gross anatomy, however imaging is a common form by which anatomy is encountered in clinical practice and therefore could be included to add interest and clinical relevance to gross anatomy teaching. Student feedback was positive with regard to the use of ultrasound as an adjunct to traditional didactic prosectionbased anatomy teaching.

\section{PP-290}

Renal length measured by ultrasound in healthy adult Turkish population: A preliminary study

Guven $\mathrm{G}^{*}$, Akçar Degirmenci N**, Urfali FE**, Ozden $\mathrm{H}^{*}$

Department of Anatomy, Faculty of Medicine, Eskişehir Osmangazi University, Eskişehir, Turkey*; Department of Radiolog, Faculty of Medicine, Eskişehir Osmangazi University, Eskişehir, Turkey**

Introduction and Purpose: Renal length measurements via ultrasound is an important parameter in clinical evaluation of kidney diseases and healthy donors (1). These measurements and their correlations in adult Nigerian population displayed different results when compared to literature (2).The aim of this study was to determine the normal limits of renal length in healthy adult Turkish population.

Tools and Method: Renal length of 145 healthy Turkish adults were measured by ultrasound. Means were analyzed for sex differences and their correlations with age, body weight, height and body mass index.

Findings: Mean age was $52 \pm 17$ years old, body mass index was $26.6 \pm 5.7 \mathrm{~kg} / \mathrm{m}^{2}$. Means renal lengths for left and right kidneys were $114.3 \pm 5.9 \mathrm{~mm}$ and $113.4 \pm 6.1 \mathrm{~mm}$, respectively. There wasn't any difference between sexes. Renal length correlated positively with weight and height.
Result and Interpretation: There are few studies measuring renal length in healthy Turkish population. Doğan et al examined a group of normal Turkish children and found some differences when compared to other populations (1). Renal length in our study was higher than those of Nigerian people. Our findings contribute to normal limits of renal length for Turkish adult population. Body weight and height are significant factors for renal lenght.

\section{PP-291}

\section{Common bile duct diameter in healthy adult Turkish population: measurement by ultrasonography}

Guven $\mathrm{G}^{*}$, Akcar Degirmenci N**, Urfali FE**, Ozden $\mathrm{H}^{*}$

Department of Anatomy, Faculty of Medicine, Eskişehir Osmangazi University, Eskişehir, Turkey*; Department of Radiology, Faculty of Medicine, Eskişehir Osmangazi University, Eskişehir, Turkey**

Introduction and Purpose: The measurement of common bile duct diameter is important in the biliary system to distinguish obstructive from non obstructive causes of jaundice (1). However, there are few studies assessing biliary system in healty adult Turkish population (2). The aim of this study was to determine range for normal common bile duct diameter in Turkish population.

Tools and Method: Common bile duct diameter, gall bladder sizes of 150 healty Turkish adults were measured by ultrasonography. Means were analysed sex differences and their correlations with age, body weight, height and body mass index.

Findings: Mean age was $53 \pm 19$ years old and body mass index was $26.1 \pm 5.7 \mathrm{~kg} / \mathrm{m}^{2}$. Mean common bile duct diameter was $3.6 \pm 0.9 \mathrm{~mm}$ and had correlation positively with weight, body mass index and gall bladder sizes. The subjects having gall bladder stone had larger duct. There wasnt' any difference between sexes.

Result and Interpretation: Admassie showed that common bile duct diameter increased with age and weight in Ethiopians (1). Our findings contribute to the standards for normal common bile duct diameter in Turkish population. Body mass index is a significant factor for common bile duct diameter.

\section{PP-292}

Radio-anatomical examination of sex determination between the distance of transverse foramens at first cervical vertebra

Sertel S*, $\underline{\text { Arifoglu } Y^{*}}$, Gurel S**, Buber A**

Department of Anatomy, Faculty of Medicine, Abant Izzet Baysal University, Bolu, Turkey*; Department of Radiology, Faculty of Medicine, Abant Izzet Baysal University, Bolu, Turkey** 
Determination of sex using skeletal bones is important for sciences like anatomy, forensic Medicine, archeology, anthropology and pathology. The aim of this study is to contribute to this issue by using a different method to determine whether first cervical vertebra, which is one of the best known bones in columna vertebralis, can be used in sex determination or not. A total of 284 cases were examined in this study in which cases were 200 neck CT examinations (100 women and 100 men) and 84 cases were dry atlas bones. First of all, 6 parameters were used while measuring the distance between both medial and lateral edge of the transverse foramen (FTM, FTL) which belongs to adult men's and women's CT images. Then by taking the averages of them, 2 more other parameters (FTMORT - FTLORT) were obtained. According to this, FTMORT is $4.92 \mathrm{~cm}( \pm 0.34)$ for men and $4.52 \mathrm{~cm}( \pm 0.28)$ for women, while FTLORT is $6.24 \mathrm{~cm}( \pm 0.38)$ for men and $5.70 \mathrm{~cm}( \pm 0.29)$ for women. By examining these parameters using ROC analysis, it was found that, the cut-off value of FTMORT is $4.64 \mathrm{~cm}$ and the cut-off value of FTLORT is $5.92 \mathrm{~cm}$. The accuracy of sex determination in CT images by FTMORT cut-off was 76\% while it was $80 \%$ for FTLORT. When these data were applied to 84 dry atlas bones whose sex were unknown, it has been anticipated that 49 of them were women, 22 were men and also it was estimated that 11 were children which were below women values. Two bones were excluded from the examination since the parameters could not be measured absolutely. In conclusion, it was assessed that, with the cut-off values found by measuring the distance between both medial and lateral edge of the transverse foramen, sex determination can be made in atlas bones with a $78.8 \%$ accuracy in the unknown genders.

\section{PP-293}

The stereological investigation of brain ventricular volumes of children with hydrocephalus on preoperative and postoperative tomographic images

Ozbag $\mathrm{D}^{*}$, Yuksel $\mathrm{ZK}^{* *}$, Senoglu $\mathrm{M}^{* *}$, Gumusalan $\mathrm{Y}^{*}$, Altun $\mathrm{I}^{* *}$, Tugtag $\mathrm{B}^{*}$

Departments of Anatomy Faculty of Medicine, Kahramanmaraş Sütçü Imam University, Kahramanmaraş, Turkey*; Departments of Neurosurgery, Faculty of Medicine, Kahramanmaraş Sütçü Imam University, Kahramanmaraş, Turkey**

Hydrocephalus is the gathering of neurologic and neuropsycologic symptoms due to the increase of cerebrospinal fluid that courses with complete or partial enlargement of cerebral ventricles. Most common way of treatment of this disorder is the application of ventriculoperitoneal shunt technic. The objective of this study is the stereological investigation of brain ventricular volumes of children with hydrocephalus on preoperative and postoperative tomographic images. Our study was conducted on computerized tomographic (CT) images of 12 hydrocephalic children ( 7 girls and 5 boys) the diagnosis, treatment and follow up of whom were performed between 2004 and 2010 in the department of neurosurgery. Primarily, the patients who were diagnosed as hydrocephalus were evaluated from the point of etiological reasons, clinical data and causes of complication. Then the changes on ventricular volumes were measured by Cavaliery stereologic volume evaluation method after and before the application of ventriculoperitoneal shunt technic. We found no difference between female and male patients, but statistically significant difference was obtained on preoperative and postoperative CT images of the same individuals between the values of left and right ventricular volumes $(\mathrm{p}<0.05)$. Postoperative right lateral ventricular volume was measured as $123.9 \mathrm{~cm}^{3}$ while it was $155.5 \mathrm{~cm}^{3}$ preoperatively as $\mathrm{x}$. Postoperative values of left lateral ventricular volume was 158.1 while it was measured as $211.8 \mathrm{~cm}^{3}$ preoperatively. Evaluating the application of ventriculoperitoneal shunt technic as the most commonly used method in the treatment of hidrocephalus with stereologic method can provide a substantial contribution to the numerical evaluation of clinical findings and easy determination of the rate of shrinkage of ventricular volume following the application of shunt technic.

\section{PP-294}

Development of corpus callosum, cerebrum, cerebellum and lateral ventricles by using stereological method on magnetic resonance images in children

Ozbag D*, Dilber $C^{* *}$, Gumusalan $\mathrm{Y}^{*}$, Tugtag B*,

Departments of Anatomy, Faculty of Medicine, Kahramanmaraş Sütçü Imam University, Kahramanmaraş, Turkey*; Departments of Pediadric Neurology, Faculty of Medicine, Kahramanmaraş Sütçü Imam University, Kahramanmaraş, Turkey**

The aim of this study is to investigate the development of corpus callosum, cerebrum, cerebellum and lateral ventricles by using stereological methods on Magnetic Resonance Images of children between $0-15$ years old This study was conducted on brain MR images of 150 healthy subjects $(75$ male and 75 female) who applied to the department of pediatric neurology between the years of 2008-2010 and were found to have no illnesses. An irregular increase was detected in the projection area of corpus callosum, and volumes of cerebrum, cerebellum and ventricles related to age. When the increases related to age were evaluated, projection area length values of corpus callosum and volumetric values of cerebrum, cerebellum and ventricles of male subjects were seen to be generally higher than females. Mean volumetric values in males were found as 989,7 $\mathrm{cm} 3$ for cerebrum, $88.06 \mathrm{~cm}^{3}$ for cerebellum, $5.5 \mathrm{~cm}^{3}$ for right lateral ventricle, $6.1 \mathrm{~cm}^{3}$ for left lateral and $2.5 \mathrm{~cm}^{3}$ for the third 
ventricle. Mean value for projection area length of corpus callosum was found as $14.25 \%$ in male. On the other hand, mean volumetric values in females were found as $969.7 \mathrm{~cm}^{3}$ for cerebrum, $89.02 \mathrm{~cm}^{3}$ for cerebellum, $5.3 \mathrm{~cm}^{3}$ for right lateral, 5.8 $\mathrm{cm} 3$ for left lateraland $2.3 \mathrm{~cm}^{3}$ for the third ventricle. Also the mean value for projection area length of corpus callosum was calculated as $12.7 \%$ in female. It may be provided very precious knowledges to experts of topic about evaluation of deseases related to this structures that neearly truly measurment of dimension with stereological methods upon normally magnetic resonans imaging of corpus callosum, cerebrum, cerebellum and ventriculs in $0-15$ yers old and more clear knowing of vertically growth border related to ages.

\section{PP-295}

The morphometric analysis of the coeliac trunk and its branches with multidetector computed tomography angiography (MDCTA)

Yilmaz MT* , Seker $M^{*}$, Paksoy $\mathrm{Y}^{* *}$, Cicekcibasi $\mathrm{AE}^{*}$,

Salbacak A*

Department of Anatomy, Meram Medical Faculty, Selçuk University, Konya, Turkey*; Department of Radiology, Selçuklu Medical Faculty, Selçuk University, Konya, Turkey**

Introduction and Purpose: The hepatic, splenic and left gastric arteries are considered as the "main classic branches" of the coeliac trunk. It was aimed to evaluate the efficiency of multidetector computed tomography angiography (MDCTA) reformations in defining the origin, localization, and variations of the coeliac trunk and its branches. Tools and method: The coeliac trunk structures of 150 (78 male, 72 female ) patients which were examined MDCTA images of the abdominal aorta because of various symptoms were retrospectively reviewed. All MDCTA examinations were performed with a 64-channel MDCTA scanner (Somatom Sensation 64, Siemens, Germany). The images were retrieved to the Leonardoworkstation (Siemens Medical Solutions).

Findings: The variational structure of the coeliac trunk was observed in $12 \%$ of cases while the normal structure was determined in $88 \%$. The splenomesenteric trunk, the hepatomesenteric trunk, the splenogastric trunk, and the splenogastric trunk were detected in $0.6 \%, 1.3 \%, 1.3 \%$, and $1.3 \%$ of cases, respectively. The gastroduodenal artery originated from the coeliac trunk as fourth branch in $3.3 \%$ whereas the right hepatic artery originated from in $2.6 \%$. In $1.3 \%$, we found the absent coeliac trunk and three main branches arised from abdominal aorta.

Result and Interpretation: MDCTA is a safe and noninvasive test and provides a excellent radiological method of preoperative vascular evaluation.

\section{PP-296}

The inferior mesenteric artery originating from the superior mesenteric artery with multidetector computed tomography angiography (MDCTA)

Yilmaz MT*, Seker M*, Cicekcibasi AE*, Paksoy Y**, Buyukmumcu $M^{*}$

Department of Anatomy, Meram Medical Faculty, Selçuk University, Konya, Turkey*; Department of Radiology, Selçuklu Medical Faculty, Selçuk University, Konya, Turkey**

Introduction and Purpose: The variations of the inferior mesenteric artery are extremely rare. In this study, it was reported a case of the inferior mesenteric artery which arised from superior mesenteric artery.

Tools and Method: The present case was retrospectively detected during reviewing on MDCTA images in Radiology Department.

Findings: The superior mesenteric artery which the stem diameter was $0.69 \mathrm{~mm}$ originated from anterolateral wall of the abdominal aorta $1.76 \mathrm{~cm}$ below of the coeliac trunk. In addition, the inferior mesenteric artery arised from $3.06 \mathrm{~cm}$ away from the superior mesenteric artery. The stem diameter was also $0.36 \mathrm{~mm}$. The distance between the superior mesenteric artery and aortic bifurcatio was $9.31 \mathrm{~cm}$.

Result and Interpretation: The knowledge of the developmental variations of the inferior mesenteric artery is very important in surgical procedures such as right hemicolectomy, resection of the transverse colon, left hemicolectomy, sigmoidectomy, and en bloc resection of the head of the pancreas

\section{PP-297}

The mandibular landmarks of the facial artery and vein by multidetector computed tomography angiography (MDCTA): an anatomical and radiological morphometric study

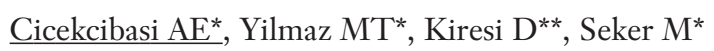

Department of Anatomy, Meram Medical Faculty, Selçuk University, Konya, Turkey*; Department of Radiology, Meram Medical Faculty, Selçuk University, Konya, Turkey**

Introduction and Purpose: The injuries of the facial vessels which commonly used for flap surgery should be avoided. The aim of this study to investigate the course of the facial vessels according to several mandibular landmarks in living individuals using multidetector computed tomography angiography (MDCTA) and to determine these data related to sex and side.

Tools and Method: In total, sixty faces from 30 specimens (15 males and 15 females) which had symptoms and signs of any 
vascular disease were evaluated for the facial vessels by MDCTA scan. The parameters about facial vessels were measured according to the reference points (mandibular angle, mental protuberence, mental foramen and facial midline). Findings: The distance from the point at which the facial artery first appears in the lower border of the mandible to the mandibular angle for right and left facial artery were observed as $3.53 \pm 0.66 \mathrm{~cm}$ and $3.31 \pm 0.73 \mathrm{~cm}$ in males, respectively. These distances were determined as $2.91 \pm 0.52 \mathrm{~cm}$ and $3.35 \pm 0.48 \mathrm{~cm}$ in females.

Result and Interpretation: MDCTA is a new, powerful, safe and noninvasive test to demonstrate the vasculature of the head. Bony structures and neighbouring vessel morphology can be evaluated by this technique in cases of trauma with suspected vessel injuries and when considering patient selection for flap surgery.

\section{PP-298}

The review of the clival canal by multidetector computed tomography (MDCT)

Cicekcibasi $\mathrm{AE}^{*}$, Yilmaz MT*${ }^{*}$, Tezer $M^{*}$, Kiresi D**, Salbacak A*

Department of Anatomy, Meram Medical Faculty, Selçuk University, Konya, Turkey*; Department of Radiology, Meram Medical Faculty, Selçuk University, Konya, Turkey**

Introduction and Purpose: The clival canal is an osseus canal occuring both in vertical and horizontal orientations. There are various hypotheses such as remnants of the notochord or the pathway for Rathke's pouch, first sclerotome, intercommunication among parts of the basilar venous plexus or between this plexus and the diploic space of the clivus as a cause of this canal. In this study, it was aimed to determine both the structure through this canal and its incidence.

Tools and Method: This study was retrospectively composed on MDCT images of the 116 (male 65 - female 51) adult subjects. All MDCTA examinations were performed with a 64 canal MDCTA scanner (Somatom Sensation 64, Siemens, Germany). The images were retrieved to the Leonardoworkstation (Siemens Medical Solutions).

Findings: In males, the double clival canal was detected in 1 (1.53\%) of cases, single clival canal in 4 (6.15\%), normal structure in $60(92.30 \%)$ while in females, single clival canal in 2 (3.92\%) of cases, normal structure in 49 (96.07\%). The double canal was not observed in females. Besides, there was no structure through this canal.

Result and Interpretation: We thought that the clival canal was negligible in cases of clival fracture or during neurosurgical operations on this region because of no structure via this canal.
PP-299

The morphometric analysis of the important bone structures on external skull base in living individuals with multidetector computed tomography (MDCT)

\author{
Gokce $\mathrm{C}^{*}, \underline{\text { Cicekcibasi AE }}{ }^{* *}$, Yilmaz MT** Kiresi D ${ }^{* * *}$
}

Ozel Onem of Special Education and Rehabilitation Center, Konya, Turkey*; Department of Anatomy, Meram Medical Faculty, Selcuk University, Konya, Turkey**; Department of Radiology, Meram Medical Faculty, Selcuk University, Konya, Turkey***

Introduction and Purpose: Multidetector computed tomography (MDCT) is the most advanced radiological imaging technique at 21. century. MDCT is new, powerful, reliable, and noninvasive technique which can be obtained the unique anatomical details. In this study, it was aimed to determine the reliable morphometric data of the important bone structures of the external skull base in living individuals which are necessary for many surgical approaches and to identify the differences of these data according to gender and lateralization.

Tools and Method: This retrospective study was created on MDCT images of the patients with acute nontraumatic subarachnoid hemorrhage or suspected cerebral aneurysm with symptoms such as headache, cranial neuropathy. It were examined images of the 100 adult individuals (50 males and 50 females) without cranial trauma, surgery or any bone deformity. The distance from the medial and lateral lamina of pterygoid processus to the midline (PP-ML, PP-LL), the sagittal - transvers diameters and area of foramen magnum (FMsgt, FMtrn, FMarea), the anterior and posterior intercondylar distances and the lenght of occipital condyles (AID, PID, OCn) were measured.

Findings: The significant differences were found between the right and left PP-ML, PP-LL, OCn according to the genders. In addition, the data of FMsgt, FMtrn, FMarea were significantly different $(\mathrm{P}<0.05)$. In males, all data were higher than females.

Result and Interpretation: We think that the results of this study can be contributed to diagnosis and treatment in radiology and surgical branches, gender determination in forensic Medicine, education in basic sciences.

\section{PP-300}

The morphometric analysis of the important bone structures on internal skull base in living individuals with multidetector computed tomography (MDCT)

Gokce $C^{*}$, Cicekcibasi AE ${ }^{* *}$, Yilmaz MT*** Kiresi D***

Ozel Onem of Special Education and Rehabilitation Center, Konya, Turkey*; Department of Anatomy, Meram Medical Faculty, Selcuk University, Konya, Turkey**; Department of Radiology, Meram Medical Faculty, Selcuk University, Konya, Turkey***

Introduction and Purpose: The parts of human skeleton have the different anthropometric data according to gender and 
race. The skull base is a very important region of the skeleton because of the implementation of the neurosurgical approaches and the entries of the vital structures (vessels - nerves). In this study, it was aimed to determine the reliable morphometric data of the important bone structures of the internal skull base with MDCT in living individuals.

Tools and Method: This study was retrospectively made on MDCT images. It was evaluated the images of the 100 (male 50 - female 50) adult subjects without cranial trauma or surgery and any deformity of the bone. The distance between the tuberculum sellae and foramen caecum (TS - FC), the distance between the tuberculum sellae and prechiasmatic sulcus (TS PCS), the distance between the tuberculum sellae and dorsum sellae (TS - DS), the distance between the dorsum sellae and the anterior margin of foramen magnum (DS - FM), the height and anteroposterior diameter of the hypophysial fossa (HFheight, HFanteroposterior) were measured.

Findings: It was observed that right and left data in all parameters were higher in males than females. It was usually determined higher values on the right side in both genders. In both genders, the some parameters were highly correlated $(\mathrm{P}<$ 0.001).

Result and Interpretation: We believed that the morphometric measurements were reliable and the error rate was minimal according to the measurements on dry bones and direct radyographic images. Because, the data were obtained from living individuals and computer environment with MDCT.

\section{PP-301}

The anatomic importance of multidetector computerize tomography angiography (MDCTA) in Leriche's syndrome

Yilmaz MT* ${ }^{*}$ Cicekcibasi $\mathrm{AE}^{*}$, Seker $\mathrm{M}^{*}$, Paksoy P**,

Department of Anatomy, Meram Medical Faculty, Selçuk University, Konya, Turkey*; Department of Radiology, Selçuklu Medical Faculty, Selçuk University, Konya, Turkey**

Introduction and Purpose: Leriche's syndrome which the main cause is an atherosclerosis is an aortoiliac occlusive disease, associated with ischemic symptoms such as intermittent claudication, atrophy of the lower extremities, impotence and weak-absent femoral pulses.

Tools and Method: A 57-year-old white man with Leriche syndrome presented. Multidetector computed tomography angiography (MDCTA) showed distal abdominal aorta occlusion and full right - left common iliac arteries occlusion.

Findings: There was an interesting anastomosis from the superior mesenteric artery to inferior mesenteric artery and extending through the left side of the abdomen. Both lower limbs were perfused by the anostomosis between internal mammary artery and inferior epigastric artery. Besides, the collaterals between the deep iliac circumflex artery and posterior intercostal arteries had bilaterally.

Result and Interpretation: When deciding for revascularization treatment of a patient with Leriche syndrome or about the graft choice for coroner revascularization, it is vital to detect the origin of collateral circulation to the lower limbs. MDCTA provides the best spatial resolution for almost all vascular territories.

\section{PP-302}

\section{The morphometric analysis of the inferior phrenic arteries with multidetector computed tomography angiography (MDCTA)}

Yilmaz MT*, Seker $M^{*}$, Paksoy $\mathrm{Y}^{* *}$, Cicekcibasi $\mathrm{AE}^{*}$, Buyukmumcu $M^{*}$

Department of Anatomy, Meram Medical Faculty, Selçuk University, Konya, Turkey*; Department of Radiology, Selçuklu Medical Faculty, Selçuk University, Konya, Turkey**

Introduction and Purpose: The inferior phrenic arteries usually arise from the abdominal aorta, less frequently from coeliac trunk, renal, hepatic or left gastric arteries. It is aimed to determine with multidetector computed tomography angiography (MDCTA) the origin, localization, and variations of the inferior phrenic arteries which especially can be a major arterial source for hepatocellular carcinoma. Tools and method: The inferior phrenic arteries of 75 (37 male, 38 female) patients which were examined MDCTA images of the abdominal aorta because of various symptoms were retrospectively reviewed and variations were recorded. All MDCTA examinations were performed with a 64-channel MDCTA scanner (Somatom Sensation 64, Siemens, Germany). The images were retrieved to the Leonardoworkstation (Siemens Medical Solutions). Findings: In both sexes, the average diameter of the inferior phrenic arteries was $0.13 \mathrm{~cm}$ (range $0.09-0.21 \mathrm{~cm}$ ). There was no significant difference between the sexes. The right and left inferior phrenic arteries which originated from only single root were observed in 11 (14.7\%) (9 male, 2 female). The single root which arised from the abdominal aorta, the coeliac trunk, and left gastric artery were seen 4 (5.3\%) of cases, $6(8 \%), 1$ $(1.3 \%)$, respectively. In 64 cases, the inferior phrenic arteries have separate origins and $44(58.6 \%)$ of them originated from outside of the abdominal aorta, 20 (26.7\%) of them originated from the abdominal aorta.

Result and Interpretation: The knowledge of the origins and variations of the inferior phrenic arteries is important for treatment of pathologies such as primary or metastatic hepatic tumors, cholangiocarcinoma. 
PP-303

\section{Ectopic kidney with varied vasculature: demonstrated by CT angiography}

Kara E*, Ozturk NC**, Kara A**, Ozgur A*, Yildiz A*, Ozturk $\mathrm{H}^{* *}$

Department of Radiology, Faculty of Medicine, Mersin University, Mersin, Turkey*; Department of Anatomy, Faculty of Medicine, Mersin University, Mersin, Turkey**

Objective: To report an ectopic kidney in a 20-year-old male patient.

Material and Method: Evaluated by CT angiography.

Results: The ectopic kidney was situated on the right side at the abdominopelvic junction, partly in the abdomen at the level of the intervertebral disc between L3-L4 superiorly and partly in the greater pelvis at the level of the promontorium and close to the inferior border of the sacroiliac joints. It was supplied by two arteries which were nearly in same calibre, and each of which branched from the common iliac arteries both close to the aortic bifurcation. There were two renal veins. The larger one which was emerging from the lateral part of the ectopic kidney was draining into the inferior vena cava. The smaller one which was the only hilar vessel of the ectopic kidney was draining into the left common iliac vein. The orthotopic left kidney was also supplied by two arteries from the abdominal aorta.

Conclusion: Ectopic kidneys pose a problem for any planned surgical intervention given their anomalous blood supply. Ectopic position and varied vasculature can predispose to iatrogenic trauma during interventional radiological and laparoscopic procedures, and emergency operations.

\section{PP-304}

\section{The effects of erdosteine on testis tissues of rats exposed to hypobaric hypoxia}

Guven Bagla $A^{*}$, Ickin $\mathrm{M}^{*}$, Uzun $\mathrm{O}^{* *}$, Balbay $\mathrm{O}^{* * *}$

Department of Histology and Embryology University of Çanakkale Onsekiz Mart, Faculty of Medicine, Çanakkale, Turkey* ; Department of Pharmacology, University of Abant Izzet Baysal, Faculty of Medicine, Bolu, Turkey**; Department of Pulmonary Medicine University of Düzce, Faculty of Medicine, Düzce, Turkey***

The purpose of present study was to examine the effects of hypobaric hypoxia on testis morphology and possible protective effects of erdosteine on this tissue. HIF-1 $\alpha$ and Caspase- 3 immunostainings were applied to detect response of testicular tissue to hypoxia. Adult male Wistar rats were placed in a hypobaric hypoxic chamber (hypoxic group, $n=8$ ). Rats in erdosteine group $(n=8)$ were exposed to the same conditions and treated orally with erdosteine at the same time, $20 \mathrm{mg} / \mathrm{kg}$ daily, from the first day of hypoxic exposure for 2 weeks. Normoxic group $(\mathrm{n}=7)$ was evaluated as control. Light microscopy: Testis tissues exposed to hypoxia reflected a decrease in height of spermatogenic epithelium in some seminiferous tubules. Vacuolization in spermatogenic epithelial cells, deterioration in basal membrane were observed. Increase in number of blood vessels, and edema was observed in interstitial area. The height of spermatogenic epithelium was normal in erdosteine group, vacuolization was diminished. HIF-1 $\alpha$ immunostaining: No immunostaining was observed in normoxic group. No immunostaining was observed in germ cells of spermatogenic epithelium in any groups. Number of immunostained Sertoli cells and Leydig cells in hypoxic group was more than those in erdosteine group. Caspase-3 immunostaining; In all groups, immunostaining was observed in germ cells of spermatogenic epithelium located near to the lumen of some seminiferous tubules. We observed that number of seminiferous tubules having Caspase-3 immunostained germ cells was higher in hypoxia group, when compared with erdosteine group. Number of immunostained tubules was lowest in normoxic group. In normoxic group, immunostaining of Leydig cells was observed in cytoplasm, while it was observed in both cytoplasm and nucleus in hypoxia and erdosteine groups. Considering hypoxic and erdosteine groups, we observed erdosteine reduced number of Caspase-3 immunostained seminiferous tubules. Consequently, we suggest that erdosteine protects testis tissue from hypoxic injury by reducing apoptotic cell death.

\section{PP-305}

\section{Beneficial effects of melatonin and $\mathrm{Bq}-123$ on the rat testis damage caused by cigarette smoke}

$\underline{\text { Ungor B* }^{*}}$ Kesici $\mathrm{H}^{* *}$, Karaca $\mathrm{ZI}^{* *}$, Cayli $\mathrm{S}^{* *}$, Ozyurt B*, Tas $\mathrm{U}^{*}$, Cihan $\mathrm{OF}^{* * *}$, Aslan $\mathrm{H}^{* *}$

Department of Anatomy, Gaziosmanpasa University School of Medicine, Tokat, Turkey*; Department of Histology and Embryology, Gaziosmanpasa University School of Medicine, Tokat, Turkey**; High School of Health, Gaziantep University, Gaziantep, Turkey***

Objective: Several studies have demonstrated that cigarette smoking has detrimental effects on testicular function. However, it is unknown whether melatonin or BQ-123 have beneficial effects on the rat testis damage caused by cigarette smoke. The aim of the present study was to investigate the beneficial effects of melatonin or BQ-123 on the testicular damage caused by cigarette smoke.

Materials and Methods: In this study, fifteen Wistar rats, 3-4 months old and weighing 250-300 gr, were exposured to cigarette smoke for 4 weeks. After exposing to cigarette smoking, the first group (n:5) was administered to melatonin (25 $\mathrm{mg} / \mathrm{kg} /$ day, ip) five days in a week for four weeks and the sec- 
ond group (n:5) was administered to BQ-123 (1 mg/kg, iv) once in a week for four weeks. The third group (n: 5) was exposed to only cigarette smoke for four weeks. Control rats (n: 5) were exposed to room air. At the end of four weeks all the rats were sacrificed by using ketamine $(30 \mathrm{mg} / \mathrm{kg}$, ip) and xylazine $(5 \mathrm{mg} / \mathrm{kg}$, ip) anesthesia before removal of the testis. Testes were fixed in $10 \%$ formalin for $12 \mathrm{~h}$ immediately upon collection, dehydrated and embedded in paraffin for histologic and stereologic investigations. $30 \mu \mathrm{m}$ sections were sampled by systematically randomized manner and stained with hematoxylin-eosin. By using stereology workstation total number of spermatogonia and spermatocytes were estimated.

Results: All the control testes demonstrated complete spermatogenesis. There was a significant decrease in the germ cells of rats exposed to cigarette smoking for 4 weeks. After applying of melatonin or BQ-123, total number of spermatogonia and spermatocytes in the testes were significantly increased.

Conclusion: Based on these findings, melatonin and BQ123 are able to minimize the degenerative effects cigarette smoke by increasing the germ cells number.

\section{PP-306}

\section{An anatomo-radiological study of the pattern of branching of the left portal vein}

Macchi V, Porzionato A, Stecco C, Morra A, Picardi E, Parenti A, De Caro R

Department of Human Anatomy and Physiology, University of Padua, Padua, Italy

Branching anomalies of the left portal vein can be managed safely with new surgical techniques but they can represent contraindications for living donor transplantation. The aim of the present study was to investigate the pattern of ramification of the left portal vein (PV) in a casistics of 200 patients who underwent abdominal CT examinations. Two observers evaluated both thin axial sections and $3 \mathrm{D}$ reconstructions. Classic morphology of the left liver was found in $35 \%$, a small left lobe in $23.5 \%$, elongated left lobe in $19.6 \%$, globose left lobe in $8 \%$. The portal vein presented a trifurcation in $10 \%$ of cases: left branch, right paramedian branch and right lateral branch in $4 \%$; right lateral branch, right paramedian branch and left branch in 2\%; branch for VI e V, branch for VII e VIII and left branch in $4 \%$. In proximity to the angle between the left PV and the left paramedian vein (PMV) the branches for segment II origin with a mean number $1.1 \pm 0.3$. From the cul de sac, lateral pedicles origin, destined to segments III and IV with a mean number of $3.1 \pm 1.2$ and $5.1 \pm 0.5$ respectively. CT-angiography can be extremely useful as pre-operatory examination to visualize the liver vascular pattern.

\section{PP-307}

The effect of r-FSH and u-FSH administration on endometriotic implants in experimental endometriosis

Oner $\mathrm{Z}^{*}$, Ekinci $\mathrm{N}^{*}$, Celik $\mathrm{O}^{* *}$, Ozercan $\mathrm{MR}^{* * *}$, Kose $\mathrm{E}^{*}$, Sapmaz HI*

Department of Anatomy, Faculty of Medicine, Inonu University, Malatya, Turkey*; Faculty of Medicine, Inonu University, Malatya, Turkey**; Department of Pathology, Faculty of Medicine, Firat University, Elazig, Turkey***

Introduction: The objective of this study is to investigate immunohistochemically the effects of r-FSH, u-FSH and aromatase inhibitors on the development of experimental endometriotic implants in rats.

Material and Methods: Endometriosis was induced in 46 female animals surgically by using the method described by Vernon and Wilson. Four weeks after this procedure, relaparotomy was performed. The vesicles at the sutures region were observed and the rats were graded according to average vesicle diameter (D) as: Grade 1 (for cases in which the implant had disappeared or, if it was visible, never became a cyst), Grade 2 (D < $2 \mathrm{~mm}$ ), Grade $3(2 \mathrm{~mm}<\mathrm{D}<4.5 \mathrm{~mm})$ or Grade $4(\mathrm{D}>4.5 \mathrm{~mm})$. Rats were randomly divided into four groups. The groups were labelled as the control (n:10), the r-FSH (n:12), the u-FSH (n:12) and the aromatase inhibitor (n:12). At the end of four days drug administration, the endometrial implants were excised and processed for immunohistochemical studies.

Results: It was observed that $93.5 \%$ of the implanted rats developed vesicles in the suture zone. The rats that somehow did not develop any vesicle were assigned as Grade 1 (6.5\%). In $24 \%$ of the subjects were Grade 2, in 56,5\% were Grade 3 and in $13 \%$ were Grade 4 . After the treatment with $\mathrm{r}-\mathrm{FSH}$ we did not find any Grades 4 vesicles whereas the percentage of grades 3 vesicles increased significantly. After the treatment with u$\mathrm{FSH}$ and aromatase inhibitor the percentage of Grade 3 and 4 vesicles increased, whereas the percentage of Grade 2 vesicles decreased. The post-treatment implant volumes were increased in the control group. Glycodelin expression levels were similar in each groups. Conclusions: In a rat endometriosis model, r$\mathrm{FSH}$ and u-FSH administration have showed significant increase implant number and size.

\section{PP-308}

\section{Macroscopic evaluation indexes of placentas from premature births with fetuses presenting developmental abnormalities}

Daniela FC, Adriana R, Florin SM, Casiana S

Universitatea De Vest Vasile Goldis, Arad, Romania

The purpose was to establish the importance of the macroscopic placental abnormalities in prematurity and developmental disorders. There are statistical proofs that there is a serious increase 
in the number of pregnancies with fetuses that present developemental abnormalities in Europe in general and particulary in Romania. Also there is a prominent increase in premature births during the last five years (2006-2010). All these facts are in favor for an accurate and detailed research on the fetus - placental unit. Methods. Our study focuses on the macroscopically study of the placentas that were obtained from premature births with developemental abnormalities new borns. The evaluated parameters weare: placental shape, surface weight, size, and the ration between placental weight/ fetal weight. The hereby study was conducted on 311 placentas from premature births all of that were associated with developmental fetal problems. Results and discutionsAfter studying such a large number of placentas and finding highly variable results assessed parameters that we considered must specify, in our opinion is mandatory to introduce a new pregnancy predictor parameter such as the placental surface which in fact represents the contact surface between the placenta and the uterine wall occupied by it, which is not necessary similar to the exchange uterine - placental surface. Conclusions. We considered necessary to introduce an observation on placental form that contain all relevant and mandatary data.

\section{PP-309}

\section{An "in vitro"study on lung cells exposed to fluoroedenite fibres. Biological role of retinoblastoma proteins}

Musumeci G, Cardile V, Carnazza ML, Martinez G, Loreto C Department of Bio-Medical Sciences, University of Catania, Italy

Introduction: In this study we addressed the expression of $\mathrm{Rb}$ and $\mathrm{pRb}$ protein in human lung epithelial A549 and MeT-5A cell cultures treated with three concentrations of fluoro-edenite fibres to gain insights into the biomolecular mechanisms underlying the interaction between these environmental particulates/fibres and human respiratory epithelium. Several asbestos-like mineral fibres, including fluoro-edenite, may cause lung cancer and/or other lung diseases. However, biological and molecular mechanisms linked to cancer development after mineral fibres exposure were not fully investigated.

Methods: In the present study, mesothelial (MeT-5A) and human bronchoalveolar alveolar epithelial (A549) cell lines were incubated with rising concentrations of fluoro-edenite to evaluate the expression of retinoblastoma $(\mathrm{Rb})$ protein, which has been demonstrated to play an important role in cell cycle control and tumour progression.

Results: Intriguingly, these results show that $\mathrm{Rb}$ expression was unchanged, while the level of the phosphorylated protein increased significantly in a dose-dependent manner, suggesting an involvement of this regulator protein in the pathogenesis of the lung diseases induced by mineral fibres.

Conclusion: In conclusion, fluoro-edenite regulates the expression of $\mathrm{pRb}$ to trigger a network of signals strictly connected with cell proliferation and neoplastic cell transformation.

\section{PP-310}

\section{The relationship between lateral wall of nasal cavity and septal deviation}

Sevinc $\mathrm{O}^{*}$, Barut $\mathrm{C}^{* *}$, Kacar D***, Is $\mathrm{M}^{* * * *}$

Department of Anatomy, School of Medicine, Çanakkale Onsekiz Mart University, Çanakkale, Turkey*; Department of Anatomy, School of Medicine, Zonguldak Karaelmas University, Zonguldak, Turkey**; Department of Anatomy, School of Medicine, Zonguldak Karaelmas University., Zonguldak, Turkey***; Department of Neurosurgery, Dr. Lütfi Kırdar Kartal Education and Research Hospital, Istanbul, Turkey****

Sinonasal variations are suggested to have a role in sinusitis etiology. Concha bullosa (CB) and septal deviation (SD) are amongst these variations. The aim of this study was to evaluate the sinonasal variations in a certain population sample. Septal deviation is displacement of the nasal septum towards the nasal passage. Diffuse pneumatization of the middle nasal concha (MNC) is called concha bullosa. The pneumatization of the concha was classified based on the location as lamellar concha bullosa (LCB), bulbous concha bullosa (BCB) and extensive concha bullosa (ECB). Coronal paranasal sinus CT images of 115 individuals (65 women, 50 men) were reviewed retropectively and presence of $\mathrm{CB}$, the effect of hypertophy of $\mathrm{MNC}$ and inferior nasal concha (INC) on nasal septum was evaluated. Of the 76 individuals CB was present unilaterally or bilaterally. A total of $125 \mathrm{CB}$ (27 unilateral and 49 bilateral) was evaluated and the incidence of each type was determined. In case one side of the MNC and INC was greater than the opposite side it was considered as dominant concha. 86 of the 115 individuals participated in the study had SD. Among those individuals 21 of them was not effected from the size of the MNC and INC. 34 cases had dominant MNC, 20 cases had dominant INC and 11 cases had both dominant MNC and dominant INC and all of those cases had SD towards the opposite side. The results of this study may not be generalized to the whole population but may bring insight into the knowledge about such variations.

\section{PP-311}

\section{Topical decongestants and glucocorticosteroids:} interaction in nasal mucosa of animal model

\author{
Akpinar $\mathrm{M}^{*}$, Yigit $\mathrm{O}^{*}$, Akakin $\mathrm{D}^{*}$, Sarioz $\mathrm{O}^{* * *}$, Ozkan $\mathrm{N}^{* * * *}$, \\ Yildiz SD***, Azizli E*, Sehirli US*** \\ Second Clinic of Otolaryngology Head and Neck Surgery, Istanbul \\ Trainning and Research Hospital, Istanbul, Turkey*; Department of \\ Histology and Embryology, Marmara University School of Medicine, \\ Istanbul, Turkey**; Department of Anatomy, Marmara University School \\ of Medicine, Istanbul, Turkey***; School of Health Sciences, Marmara \\ University, Istanbul, Turkey****
}

Objective: The aim of this study is to investigate the consequences and safety of combined nasal glucocorticosteroids and 
xylometazoline $\mathrm{HCl}$ usage and the efficacy of combined use in minimizing the oxymetazoline $\mathrm{HCl}$ dependent rebound congestion and tachyphylaxis.

Methods: Twenty New Zealand male rabbits were grouped as control (6), xylometazoline $\mathrm{HCl}$ (8) and xylometazoline $\mathrm{HCl}$ fluticasone furoate (6). The group I constituted the controls with no treatment. Group II and group III were administered two puffs of xylometazoline $\mathrm{HCl} 0.5 \mathrm{mg} / \mathrm{ml}$ twice daily (tid) and two puffs of xylometazoline $\mathrm{HCl} 0.5 \mathrm{mg} / \mathrm{ml}$ tid plus one puff of $27.5 \mu \mathrm{g}$ of fluticasone furoate nasal spray tid respectively. At the end of the three weeks all rabbits were sacrified. The nasal cavities were dissected and mucosa of nasal septum were excised. The parafin sections $(5 \mu \mathrm{m})$ of the nasal mucosa were stained with Hematoxylin-Eosin. The presence of inflamatory cell infiltration, edema, congestion, degeneration of epithelium and nerve endings in the mucosa were investigated under light microscope semiquantitatively. In slides revealing inflammatory cell infiltration, the cells were counted in three areas under the 400X magnification. Total cell numbers were taken as criterion for statistical analysis. The study was approved by Marmara University Experimental Animal Research Committee.

Results: Statistically significant difference was detected between group II and III in terms of edema, congestion and inflammatory cell infiltration $(\mathrm{p}=0.0015, \mathrm{p}=0.0307, \mathrm{p}=0.0056$, $\mathrm{p}=0.0089$ ). The degree of epithelial and nerve ending degeneration was more prominent in group II $(2.19,1.54)$ when compared to group I $(1.67,1.10)$, the difference between two groups was not significant ( $\mathrm{p}=0.148, \mathrm{p}=0.244)$.

Conclusion: The combined simultaneous administration of oxymetazoline $\mathrm{HCl}$ with fluticasone furoate decreased the long-term oxymetazoline $\mathrm{HCl}$ dependent rebound congestion, edema and inflammatory cell infiltration in rabbit model.

\section{PP-312}

\section{Developmental changes affecting lectin binding in the vomeronasal organ of domestic pigs}

Shin $\mathrm{T}^{*}$, Park $\mathrm{J}^{*}$, Taniguchi $\mathrm{K}^{* *}$

Jeju National University, Jeju, South Korea*; Kitasato University, Tokyo, Japan**

This study investigated the developmental changes of glycoconjugate patterns in the porcine vomeronasal organs (VNOs) and associated glands (Jacobson's glands) from prenatal (9 weeks of gestation) and postnatal (2 days after birth) to the sexually mature stage (6 months old). The VNO of pigs (Sus scrofa) was examined using the following: Dolichos biflorus agglutinin (DBA), Bandeiraea simplicifolia agglutinin isolectin B4 (BSI-B4), Triticum vulgaris agglutinin (WGA), Ulex europaeus agglutinin I (UEA-I), and soybean agglutinin (SBA). At the fetal stage, all lectins examined were detected mainly in the free border of the vomeronasal epithelium, but few (WGA and UEA-I) and or absent in the VNO cell bodies. At the post- natal and sexually mature stages, the reactivity of some lectins, including WGA, UEA-I, DBA and SBA, were shown to increase in the VNO sensory epithelium as well as the free border. The increased reactivity of lectins as development progressed was also observed in Jacobson's gland acini. These findings suggest that binding sites of lectins, including those of WGA, UEA-I, DBA, and SBA, increase during development from fetal to postnatal growth, possibly contributing to the increased ability of chemoreception in the pig.

\section{PP-313}

\section{Macular choroidal osteoma associated with subretinal neovascularization}

Gokce $\mathrm{G}^{*}$, Durukan $\mathrm{AH}^{*}$, Ceylan $\mathrm{OM}^{*}$, Uysal Y*, Develi S**, Ozan $\mathrm{H}^{* *}$

Department of Ophthalmology, Gulhane Military Faculty of Medicine, Ankara, Turkey*; Department of Anatomy; Gulhane Military Faculty of Medicine, Ankara, Turkey**

Aim: The aim of this study to report the choroidal osteoma located in macular region.

Method: A 30-year-old male was referred to our hospital with acut decreased visual acuity in his left eye. Ocular findings, fundus fluorescein angiography (FFA), optical coherence tomography (OCT) results and radiological images were described.

Findings: His best-corrected visual acuity was 20/20 in the right eye (OD) and counting fingers in the left (OS). Fundus examination of OS showed subretinal mass with depigmented area associated subfoveal choroidal neovascular membrane (CNVM) with haemorrhagic pigment epitelyum detachment (PED) at the fovea. OCT showed high reflectivity and elevation of retina at the tumor area. Fluorescein angiography revealed early hyperfluorescence with late staining and Ultrasound examination demonstrated intense reflectivity from the tumor and acoustic shadowing behind the calcified plaque. Computerized tomography (CT) scan revealed a focal area of calcification involving the submacular choroid. Multifocal electroretinography (mfERG) demonstrated a central depression in the area overlying the osteoma. The acut decrease in OS visual acuity was presumed to be a result of the haemorrhage from CNVM touching upon the fovea. On follow-up at 2 months the haemorrhage had resolved and the VA of OS reached to $4 / 10$ without any treatment.

Conclusion: The development of CNVM is the leading cause of decreased vision in choroidal osteoma patients. Early detection is essential to preserve VA. Frequent fluorescein angiography may be most helpful diagnostic test in order to detect new occult CNVM during follow-up. Osteoma of the choroid also have to be differentiated from other fundus tumours, especially from malignant melanoma of the choroid. A complete ocular examination is essential for the differantial diagnosis from malignant melanoma. 


\section{PP-314}

Morphometric analysis of optic nerve head using spectral domain optical coherence tomography

\section{Gokce $\mathrm{G}^{*}$, Develi $\mathrm{S}^{* *}$, Ceylan $\mathrm{OM}^{*}$, Ozan $\mathrm{H}^{* *}$}

Department of Ophthalmology, Gulhane Military Faculty of Medicine, Ankara, Turkey*; Department of Anatomy, Gulhane Military Faculty of Medicine, Ankara, Turkey**

Purpose: The aim of this study to evaluate the optic nerve head $(\mathrm{ONH})$ measurements, obtained by a spectral domain optical coherence tomography (SD-OCT).

Methods: One hundred eyes of 50 healthy subjects with no ocular disease were analyzed. Optic disc measurements including vertical and horizontal disc diameter and the distance between medial edge of $\mathrm{ONH}$ and center point of macula (umbo) obtained by SD-OCT (Spectralis ${ }^{\circledR}$; Heidelberg Engineering, Germany). Kolmogorov-Smirnov test was performed to evaluate the normal distribution of the variables. Differences between the measurement of both sides were analyzed by Student's t test. The Pearson test was also used for correlation analysis.

Results: No significant difference was found between the left and right sides in all measurements $(\mathrm{p}>0.05)$. The mean vertical diameter of $\mathrm{ONH}$ was $1665.88 \pm 183 \mu$. The mean horizontal diameter of $\mathrm{ONH}$ was $1529.32 \pm 160 \mu$. The mean distance between $\mathrm{ONH}$ and umbo was $3607.30 \pm 289 \mu$. This distance was 2.18 times longer than the vertical diameter and 2.37 times longer than the horizontal diameter of ONH. There was significant correlation between vertical diameter of $\mathrm{ONH}$ and the distance of $\mathrm{ONH}$ to umbo $(\mathrm{p}<0.01, \mathrm{r}=0.395)$. No significant correlation was observed between horizontal diameter of $\mathrm{ONH}$ and the distance of $\mathrm{ONH}$ to umbo ( $\mathrm{p}=0.07, \mathrm{r}=0.25)$.

Conclusion: The size of the optic disc may be related to various disease such as glaucoma, high refractive errors, optic nerve diseases and anomalies. Measurements of $\mathrm{ONH}$ size in previous studies were based on fundus photography. However it is subjective and variable between observers. SD-OCT is an noninvasive method which provides accurate $\mathrm{ONH}$ measurements with good image quality in a short period of time.

\section{PP-315}

\section{Comparative morphogenesis of the lower} axio-appendicular junction

\section{Johannes S}

Integrative Morphology Group, Medical University of Vienna, Vienna, Austria

Introduction: While the question "How to make a limb" is extensively addressed in terms of pattern formation, morphogenesis, and genetic background, the ontophyletic scenario of attaching the limb to the axial skeleton via girdles has been paid much less attention.
Methods and Materials: In a comparative study we have investigated pelvis morphogenesis and axio-appendicular linkage in species from different vertebrate classes. Series of developmental stages from frog tadpoles(1), chick embryos and mouse embryos(2) were serially sectioned, and 3D-reconstructed(3). The 3D-models were aligned to 4D-visualizations of developmental dynamics and further analyzed.

Results: Our data reveal, that in all three species - at the morphological level - the pelvic element originates from a single anlage in close relation to the femur. Shape and orientation, however, differ already at initial stages and are followed by divergent subsequent morphogenesis. The timing and patterns of ossification are significantly different. In frog tadpoles a connective tissue strand serves a guiding function for attachment to the sacral vertebra1.

Interpretation: These results demonstrate substantial differences in the establishment of the axio-appendicular linkage among vertebrates and prompt for clarification of their genetic and epigenetic background.

\section{PP-316}

\section{Functional significance of sacrum morphotype}

Yeryomin A, Luzin V

State Institution, Luhansk State Medical University, Lugansk, Ukraine

The purpose of our work - to study the effect of the sacrum type on functions of the lumbosacral joint.

Materials and Methods: Construction of lumbosacral segments models were conducted using algorithms solid parametric modeling. Mathematical analysis of 3D models was made by Gauss's finite element method for the static analysis of the compression with the tentative findings of biomechanical research on native preparations. Three $3 \mathrm{D}$ models of the segment were created before it: with homobasal, hyperbasal and hypobasal sacrum. All models were tested for static compression: the power applied to the upper surface L5 disc perpendicular to its was equal to $1.331 \mathrm{~N}$, auricular surface of sacrum is fixed.

Results: In tests of 3D model segment homobasall sacrum set: the stress on the front surface of L5 vertebra made - 25.67 MPa, on the pelvic surface of sacrum - 38.66 MPa, on the front surface of the intervertebral disc L5/S1 - $124 \mathrm{MPa}$. Thus, the stress on the drive was less than a vertebra in $50.3 \%$ and less than $62,4 \%$ on the sacrum. For the other two models, the distribution of stresses in the vertebrae and intervertebral discs was different. So, for the hyperbasal segment model of sacrum maximum stresses were recorded on the anterior surface, where the zone in which the stresses exceed the level of $20 \mathrm{MPa}$. Compression testing of $3 \mathrm{D}$ model with the hypobasal sacrum set: the maximum stress is recorded on the lower edge of the vertebra, and the concentration of stress does not reach the level of $20 \mathrm{MPa}$.

Conclusions: The classical form of the sacrum is the best to transfer compression loads at the lumbosacral junction. Extreme 
forms of individual variability of the sacrum, by changing the geometry lead to a decrease in the functionality of the system.

\section{PP-317}

Effect of eicosapentaenoic acid on bone changes due to the methylprednisolone in rats

Nazem MN, Rezaian M, Shomali T, Asadi F

Faculty of Veterinarian Medicine, Tehran University

The present study was conducted to investigate the effect of eicosapentaenoic acid on glucocorticoid - induced bone changes in rats, and to compare them with those of alendronate. Thirty six male Wista rats, 2.5 months of age, were divided into six groups $(\mathrm{n}=6$ each $)$ and treated with $0.9 \%$ $\mathrm{NaCl}$ ( control ), methylprednisolone $7 \mathrm{mg} / \mathrm{kg}$, once a week subcutaneously + alendronate $20 \mathrm{ppb}$, twice a week subcutaneously and methylprednisolone +80 or 160 or $320 \mathrm{mg} / \mathrm{kg}$ eicosapentaenoic acid, per day orally, for 6 weeks. At the end of the experiment, serum and urinary parameters of bone metabolism determined and bone histomorfometric analyses performed on cancellous bone of femoral epiphysis and cortical bone of tibial diaphysis. There were no significant differences in serum and urinary parameters among groups. Decrease of epiphyseal and metaphyseal trabecular width, epiphyseal bone area/tissue area and increase of epiphyseal trabecular separation observed in the methylprednisolone group compared to control. Alendronate restored all of these parameters except metaphyseal trabecular width, which increased significantly by eicosapentaenoic acid at the dose of 80 and $160 \mathrm{mg} / \mathrm{kg}$. Effects of alendronate and $160 \mathrm{mg} / \mathrm{kg}$ eicosapentaenoic acid on bone area/tissuearea, alendronate and eicosapentaenoic acid at the dose of 80 and $160 \mathrm{mg} / \mathrm{kg}$ on trabecular separation and alendronate and eicosapentaenoic acid at dose of $160 \mathrm{mg} / \mathrm{kg}$ and $320 \mathrm{mg} / \mathrm{kg}$ on epiphyseal trabecular width were statistically similar. Methylprednisolone did not significantly change cortical bone parameters including cortical width and marrow area/cortical area. eicosapentaenoic acid, especially, at the dose of $160 \mathrm{mg} / \mathrm{kg}$ exerts beneficial effects on methylprednisolone induced bone changes in rats; these effects are similar or sometimes even better than alendronate.

\section{PP-318}

\section{Sulcus nervi dorsalis penis/clitoridis: an anatomic study}

\section{Tastekin Aksu F}

Department of Anatomy, Faculty of Medicine, Dokuz Eylul University, izmir, Turkey

The aim of this study was to investigate the rate of presence and the anatomical features of Sulcus Nervi Dorsalis
Penis/Clitoris (SNDP/C) which is located on pubis and which has clinical significance. This study was conducted on 90 dry hip bones (36 male, 23 female and 31 unsexed). We investigated the presence of SNDP/C on the bones and the length and width of symphisis pubis, the length of SNDP/C on the inferior pubic ramus, the width and the length of SNDP/C on the anterior pubis were measured. SPSS 15.0 program was performed for the analysis of the findings. The rate of SNDP/C on the female and male pubic bones was $43.5 \%$ and $36.2 \%$, respectively. Developed SNDP/C (1.5 $\mathrm{mm}$ in depth and over) were found to be $8.7 \%$ and $13.8 \%$ in the male and female pubic bones, respectively. The length of Symphisis pubis (SU) was $36.85 \pm 4.06 \mathrm{~mm}$ and $41.68 \pm 6.19 \mathrm{~mm}$; The width symphisis pubis (SG) was $13.98 \pm 2.43 \mathrm{~mm}$ and $16.47 \pm 2.50 \mathrm{~mm}$; the length of SNDP/C on the inferior pubic ramus was $11.40 \pm 5.92 \mathrm{~mm}$ and $8.60 \pm 3.09 \mathrm{~mm}$; the width of SNDP/C on the anterior pubic bone was found $5.22 \pm 1.42 \mathrm{~mm}$ and $5.30 \pm 1.12 \mathrm{~mm}$; the length of SNDP/C on the anterior pubic bone was found $11.94 \pm 3.96 \mathrm{~mm}$ and $13.43 \pm 4.02 \mathrm{~mm}$ in the female and male pubic bones, respectively. SU and SG values were significantly higher in men than in women. The ratio of SNDP/C in women is greater than in men. The compression of the last branch of pudendal nerve passing over SNDP/C causes Alcock Syndrome and that's why the importance of SNDP/C in urological surgery is increasing every day. In addition, it makes gender discrimination on the intact pelvis possible in forensic Medicine. For this reason, good knowledge of the anatomy of SNDP/C will shed light on future studies.

\section{PP-319}

\section{Evaluation of piriform aperture according to gender}

Barut $C^{*}$, Kaçar D*, Dogan $\mathrm{A}^{* *}$, Ceylan $\mathrm{T}^{* * *}$, Sevinc $\mathrm{O}^{* * * *}$, Ceri $\mathrm{NG}^{* * * * *}$, Basaloglu $\mathrm{HK}^{* * * * *}$

School of Medicine, Zonguldak Karaelmas University, Zonguldak, Turkey*; Faculty of Arts and Sciences, Ahi Evran University, Kahramanmaras, Turkey**; Faculty of Arts and Sciences, Zonguldak Karaelmas University, Zonguldak, Turkey***; Department of Anatomy, School of Medicine, Çanakkale Onsekiz Mart University, Çanakkale, Turkey****; Department of Anatomy, School of Medicine, Adnan Menderes University, Aydin, Turkey*****

The aim of this study was to evaluate the morphology the piriform aperture according to gender. Morphometric analysis were performed on the photographs of 47 (10 females, 37 males) skulls taken in standard positions with appropriate photographic techniques. Initially 4 linear measurements were performed using 7 landmarks and area of the piriform aperture was measured with classical morphometry. Mann-Whitney U test was used for statistical analysis of these measurements. There were no statistically significant differences between the 
linear measurements according to gender $(\mathrm{p}>0.05)$. The area of the piriform aperture of males were greater than females and the difference was statistically significant $(\mathrm{p}<0.05)$. Afterwards piriform aperture was evaluated using 9 landmarks with geometric morphometrics. There were no statistically significant differences between shape of piriform apertures of males and females depending on Manova and pairwise analysis ( $p>0.05)$. Centroid size data were analysed with one-way ANOVA, and the differences between genders were statistically significant $(p<0.05)$. Depending on this finding it can be suggested that piriform aperture of males were greater than those of females regardless of shape. This finding is in accordance with the area measurement obtained with classical morphometry. The findings of this study broaden our knowledge about the morphology of the piriform aperture and may provide contribution for future studies related with sex determination from this feature.

\section{PP-320}

\section{Evaluation of foramen magnum according to gender}

Barut $C^{*}$, Dogan $\mathrm{A}^{* *}$, Kacar D*, Celik B***, Sevinc O****, Ceri $\mathrm{NG}^{* * * * *}$, Basaloglu $\mathrm{HK}^{* * * * *}$

School of Medicine, Zonguldak Karaelmas University, Zonguldak, Turkey*; Faculty of Arts and Sciences, Ahi Evran University, Kahramanmaras, Turkey**; Faculty of Arts and Sciences, Zonguldak Karaelmas University, Zonguldak, Turkey***; Department of Anatomy, School of Medicine, Çanakkale Onsekiz Mart University, Çanakkale, Turkey****; Department of Anatomy, School of Medicine, Adnan Menderes University, Aydin, Turkey*****

The aim of this study was to evaluate the morphology the foramen magnum according to gender. Morphometric analysis were performed on the photographs of 51 (11 females, 40 males) skulls taken in standard positions with appropriate photographic techniques. Initially 3 linear measurements were performed using 6 landmarks and the area of the foramen magnum was measured with classical morphometry. Mann-Whitney U test was used for statistical analysis of these measurements. The linear measurement values and area of the foramen magnum of males were greater than females and the difference was statistically significant $(\mathrm{p}<0.05)$. Afterwards foramen magnum was evaluated using 10 landmarks with geometric morphometrics. There were no statistically significant differences between shape of foramen magnum of males and females depending on Manova and pairwise analysis $(\mathrm{p}>0.05)$. Centroid size data were analysed with one-way ANOVA, and the differences between genders were statistically significant $(\mathrm{p}<0.05)$. Depending on this finding it can be suggested that foramen magnum of males were greater than those of females regardless of shape. This finding is in accordance with the results of classical morphometry. The results of this study may bring insight into the morphological differences of the foramen magnum according to sex.

\section{PP-321}

\section{Expression and regulation of pax-9 and msx-1 in embryonic and post-natal dental stem cells}

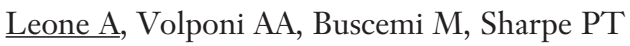

University of Palermo, Palermo, Italy; Kings College of London, London, UK

Introduction: Msxland Pax9, are genes expressed in mesenchymal cells during the odontogenesis that are required for tooth formation. Our investigation aimed to study the expression of these genes in two sources of dental mesenchymal cells. Stem Cells from Human Exfoliated Deciduous teeth (SHED) and Human Embryonic Dental Mesenchymal Cells (HTM) were used. Regulation of gene expression in these cells by FGF, Wnt, BMP4 and Shh signalling pathways together with the effects of tooth matrix proteins (EMD) was investigated.

Materials and Methods: Cells were grown in 6 well plates in 3 ml MSCGM medium (Lonza) containing Glutamax, 10\% FBS and gentamicin Sulfate. Cells were grown to confluence and when $80 \%$ confluent, they were treated for 24 hours with either $20 \mathrm{ng}$ per $\mathrm{ml}$ BIO (Gsk3 inhibitor), $1.5 \mu \mathrm{l}$ per $\mathrm{ml}$ of porcine dental matrix proteins (EMD), $0.4 \mu \mathrm{l}$ per $\mathrm{ml}$ of Fgf8 and $4 \mu \mathrm{l}$ per $\mathrm{ml}$ of DMSO (control). RNA was extracted and purified according the RNeasy-kit-protocol from Qiagen. RNA quantitation was done using a Bio Photometer (Eppendorf) in 2:100 dilutions of the samples with nuclease-free water. Quantitative analysis was performed using Rotor Gene Q-serier softhware. 10 $\mu$ l of mixture was made with $5 \mu \mathrm{l}$ of Sensimix- syber green from Quantace, $0.2 \mu \mathrm{l} \mathrm{F-primer,} 0.2 \mu \mathrm{l}$ R-primer and 4.6 $\mu \mathrm{l}$ of cDNA.

Results: Complex patterns of changes in the levels of gene expression were observed that were both cell and treatment specific. In particular BMP4 treatment resulted in large upregulation of Msx1 and Pax9 gene expression in both cell types. Differences in gene expression observed between HTM and SHED cells identify possible reasons why SHED cells respond differently to HTM in tissue recombinations directed towards producing biological replacement teeth.

Conclusions: Data obtained can help to understand and imitate the natural process of embryonic tooth development using adult dental cells.

\section{PP-322}

\section{Renal structures response to new implants with nanomodified surface}

Zhernakova NI*, Dolzhikov AA*, Kapustin $\mathrm{RF}^{* *}$, Sudakov $\mathrm{MV}^{*}$, Kovalenko IB*, Shkodkin $\mathrm{SV}^{*}$, Dmitriev VN*

Belgorod State University, Belgorod, Russia*; Belgorod State Agricultural Academy, Belgorod, Russia**

Searching for ways to improve the qualities of materials used for making stents, endo-prosthesis (implants) and medical sutures, 
we have undertaken an experiment with white laboratory rats and examined the renal structures response to metal implants of various composition without the surface modification and with the application of nano-coatings by original techniques. Five types of implants were studied: titanium, nickelid titanium, with carbon, carbon-nitrogen and carbon-argentum coatings. Using the morphologycal method we determined varios types of tissues reaction depending on the type of the implant. In all the cases, an encapsulation area was formed around the im-plant on the 14th day after the operation. The diameter of the implants bed is identical to their diameter and ranged from 200 to $220 \mathrm{mcm}$. The morphometric parameters of the forming encapsulants and their qualitative composition differed at the application of im-plants without surface modification and implants with nanocoatings. The thickness of the encapsulants around the implant is, to a certain degree, the integral index of the tis-sue response level to foreign material. According to this criterion the implants with carbon-argentum coating showed the best features. The thickness of the encapsulation varied from 21 to $35 \mathrm{mcm}$, its structure consists of 4-7 layers of thin collagenic fibers and fibroblastic elements, including singular lympho-histiocytic cells. Cells of the foreign body type were not found. In the series of tests with carbon and carbon-nitrogen coat-ings inflammatory changes were minimal, but the inner surface of the implant encapsu-lation was formed by a layer of epithelioid histiocytes; around the implants with carbon coating singular cells of foreign bodies were detected. The implants without the surface modification (titanium and nickelidtitanium) were also characterized by the full encapsu-lation but its thickness reached $80-100 \mathrm{mcm}$; around there was wide infiltrate of lympho-histiocytic elements in all the cases.

\section{PP-323}

\section{Personal formaldehyde exposure level in the gross anatomy dissecting room at College of Medicine King Saud University Riyadh}

\section{Vohra S}

Department of Anatomy, King Khalid University Hospital, King Saud University, Riyadh, Saudi Arabia

This study was conducted to correlate the personal formaldehyde (FA) exposure levels of instructors and students with the indoor FA concentrations in gross anatomy laboratory of college of Medicine at King Saud University. The personal FA exposure levels of instructors and students are higher than the indoor FA concentration in the gross anatomy laboratory.

Materials and Methods: The gross anatomy laboratory at college of Medicine, King Saud University Riyadh, was observed for indoor FA concentration and the personal exposure levels of ten instructors and the twenty medical students during the 4th 10th and 14th weeks of the dissection sessions. All air samples were collected by the diffusive sampling device and analyzed by using high performance liquid chromatography (HPLC).
Results: The personal exposure level of FA was higher than the indoor concentration, and the personal exposure levels of instructors were higher than that of the students. The concentration of FA was also higher in the center of the room than the corners and near the doors.

Conclusion: Both the indoor FA concentrations and the degree of personal FA exposure levels are higher near the dissecting table than at locations away from it during the gross anatomy laboratory sessions. Thus, the instructors and students are exposed to the higher concentration of FA than the dissection class witnesses.

\section{PP-324}

\section{Morphological considerations regarding the topography of segmental renal arteries}

Zahoi DE, Daescu E, Enache A, Sztika D,Taban S

University of Medicine and Pharmacy "Victor Babes", Timisoara, Romania

The renal artery presents great morphological variability. The number and distribution of the segmental branches, as well as how they approach the renal parenchyma are variable. The accessibility of the segmental arteries for their intraoperatory ligation is in close correlation with the place and branching of the renal artery. The study was performed on 60 unfixed kidneys, by dissection and microdissection of the renal artery and the segmental branches. The legislation in force was observed. In 52 cases, the renal artery trunk had pre-hilar branches, and in 9 cases it branched at the level of the hilum. The segmental arteries detached themselves either as individual branches of the renal artery, or as common (presegmental) trunks. The artery of the superior segment was the individual branch in 5 cases, the artery of the superior anterior segment in 8 cases, the artery of the inferior anterior segment in 6 cases, the artery of the inferior segment in 10 cases and the artery of the posterior segment in 23 cases. The artery of the superior segment was extrahilar only in $5 \%$ of cases; the artery of the superior anterior segment in $16.66 \%$ of cases; the artery of the inferior anterior segment $-23.33 \%$; the artery of the inferior segment $-26.66 \%$. The most frequently identified extrahilum artery was the artery of the posterior segment ( $40 \%$ of cases). Familiarity with the branching typology of the renal artery trunk and the location of the segmental arteries is extremely important in performing partial nephrectomies.

\section{PP-325}

\section{Morphological aspects regarding the origin of supernumerary renal arteries}

\section{Zahoi DE, Daescu E, Barsasteanu F, Ureche M, Pusztai A}

University of Medicine and Pharmacy "Victor Babes", Timisoara, Romania

The predominant morphological variations in renal arteries are numerical ones. This study was conducted on a sample of 50 
dissection pieces from the department's laboratory and 50 CT angiographies (performed using a Siemens Somatom Sensation 64 CT). The incidence of supernumerary renal arteries was $22 \%$, more frequent in males (58\%). Regarding their presence, most were encountered unilaterally $(88 \%)$, predominantly on the left side $(65 \%)$. The other $12 \%$ of cases presented bilateral multiple arteries - nine cases with two renal arteries and three cases with three arteries. In five of the cases, the origin of the supernumerary arteries was different than that of the main renal artery (four stemming from anterior side of the aorta and one from the left gonadal artery. In $76 \%$ of cases, the right supernumerary arteries had the same origin as the main artery (out of which $20 \%$ at the $\mathrm{L} \neg 1-\mathrm{L} 2$ intervertebral disc, one case at T12-L1 and three cases at L2-L4). On the left side, the origin of the main artery and the supernumerary arteries coincided in $73 \%$ of cases $(21 \%$ at the L1-L2 level, two cases at T12L1, three cases at L2-L4 and in one case stemming from the left gonadal artery). The distance between the origin of the main artery and that of the supernumerary arteries was found to vary between 0.1 and $5.7 \mathrm{~cm}$, with an average of $2.9 \mathrm{~cm}$. Awareness and knowledge of the aspects described are necessary in the surgical field, for interventions such as kidney transplants and vascular surgery.

\section{PP-326}

The effects of montelucast against cisplatin-induced acute renal damage

Beytur $\mathrm{A}^{*}$, Kose $\mathrm{E}^{* *}$, Sapmaz $\mathrm{HI}^{* *}$, Dogan $\mathrm{Z}^{* *}$, Cetin $\mathrm{A}^{* * *}$, Turkoz $\mathrm{Y}^{* * * *}$, Ekinci $\mathrm{N}^{* *}$

Department of Urology, Inönü University, Faculty of Medicine, Malatya, Turkey*; Department of Anatomy, Inönü University, Faculty of Medicine, Malatya, Turkey**; Department of Histology-Embriology, Inönü University, Faculty of Medicine, Malatya, Turkey***; Department of Biochemistry, Inönü University, Faculty of Medicine, Malatya, Turkey****

Objective: In the current study, the therapeutic and protective effects of montelucast against cisplatin-induced acute renal damage were investigated.

Material and Method: For this purpose 35 wistar albino female rats divided into 5 groups as follows: Group 1: Control; Group 2: Control+montelukast $(10 \mathrm{mg} / \mathrm{kg}$, daily for 10 days p.o.); Group 3: Cisplatin: (single dose $7 \mathrm{mg} / \mathrm{kg}$ i.p.); Group 4: Cisplatin (single dose $7 \mathrm{mg} / \mathrm{kg}$ i.p.) + montelukast $(10 \mathrm{mg} / \mathrm{kg}$, daily for 10 days p.o., after 3 days injected cisplatin); Group 5: Montelukast (10 mg/kg, daily for 10 days p.o.) + cisplatin (single dose $7 \mathrm{mg} / \mathrm{kg}$ i.p, after the last dose of montelukast). At the end of the experiment, all rats were sacrified and serum and the kidney tissues of rats collected. MDA and GSH-R levels of kidney tissues and serum BUN and Cr levels were determined. In addition, kidney tissues were examined histologically.
Results: The levels of MDA, BUN and $\mathrm{Cr}$ of group III increased when compared to control group $(\mathrm{p}<0.05)$. The MDA, BUN and Cr levels of group IV decreased when compared to group III $(\mathrm{p}<0.05)$. The GSH levels of group III decreased when compare to the control group. However the levels of GSH of group IV increased when compered to the only cisplatin injected group (Group III). In the histological findings, group III showed renal damage including marked swelling of epithelial cell, tubular dilatation, tubular desquamation and loss of brush border in kidney. Group IV showed only slight degeneration at tubular cells.

Conclusion: Montelukast treatment after cisplatin injection, reduced MDA levels and improve histological damage, in cisplatin-induced acute kidney damage.

\section{PP-327}

\section{Protective effects of erdosteine on cyclosporine A-induced nephrotoxicity}

Tutanc $M^{*}$, Arica $V^{*}$, Yilmaz $\mathrm{N}^{* *}$, Nacar $\mathrm{A}^{* * *}$, Zararsiz $\mathrm{I}^{* * * *}$, Fatmagul B*, Tutanc OD*****

Department of Pediatrics, Mustafa Kemal University, Hatay, Turkey*; Department of Biochemistry, Mustafa Kemal University, Hatay, Turkey**; Department of Histology and Embriology, Mustafa Kemal University, Hatay, Turkey***; Department of Anatomy, Mustafa Kemal University, Hatay, Turkey****; Department of Radiation Oncology, Antakya State Hospital, Hatay, Turkey*****

Aim: Cyclosporine A (CsA) is used for the management of malignant, autoimmune and inflammatory disorders. In CsA induced toxicity, oxidative stress has been implicated as a potential responsible mechanism. The use of antioxidants like erdostein reduces the adverse effects of CsA. Therefore we aimed to investigate the protective role of erdostein against CsA induced nephrotoxicity in terms of tissue oxidant/antioxidant parameters and light microscopy in rats.

Materials and Methods: Wistar albino rats were randomly separated into four groups. Group 1 rats were treated with sodium chloride as control, group 2 with CsA, group 3 with CsA plus erdostein, and group 4 with erdostein alone. Animals were sacrificed and blood samples were analyzed for blood urea nitrogen (BUN), serum creatinine (Cr), uric acid (UA), Total protein (TP), albumine (ALB) levels. Kidney sections were analyzed for malondialdehyde (MDA) and nitric oxide (NO) levels and superoxide dismutase (SOD), catalase (CAT), and glutathione peroxidase (GSH-Px) activities, as well as effected by histopathological changes.

Results: In the CsA group, MDA, GSH-Px, BUN, creatinine levels were increased. The total protein and albumin levels were decreased. These changes had been improved by erdosteine administration. Other biochemical parameters did not show any significant change. 
Conclusion: These results indicate that erdostein produces a protective mechanism against CsA-induced nephrotoxicity and suggest a role for oxidative stress in pathogenesis.

\section{PP-328}

Immunohistochemical studies on the lactating bovine
mammary gland

Alkafafy $\mathrm{M}^{*}$, Rashed $\mathrm{R}^{* *}$, Helal $\mathrm{A}^{* * *}$

Department of Cytology and Histology, Faculty of Veterinary Medicine, Minufiya University, Sadat City Branch, Egypt*; Department of Anatomy and Embryology, Faculty of Veterinary Medicine, Minufiya University, Sadat City Branch, Egypt**; Department of Anatomy and Embryology, Faculty of Veterinary Medicine, Zagazig University, Zagazig, Zagazig, Egypt $^{\star * *}$

Introduction: The mammary gland consists of an extensively branched ductal network enclosed within a distinctive basement membrane and embedded in a stromal compartment. Mammary epithelium is organized as luminal secretory and basal myoepithelial cells. The most important portion of the mammary gland development occurs postnatally, with distinct periods of intensive morphogenesis taking place during pregnancy and lactation.

Aim: The study aimed to evaluate the validity of immunohistochemistry (IHC) in the differential labeling of the diverse components of lactating mammary gland.

Materials and Methods: Paraffin-embedded sections of lactating bovine mammary glands were staind by conventional methods and immunohistochemical techniques using AvidinBiotin-Complex (ABC) Method. Primary antibodies against S100, alpha smooth muscle actin ( $\alpha$-SMA), cytokeratin-14 (Ck14), galactosyltransferase (GalTase), angiotensin converting enzyme (ACE) and vascular endothelial growth factor (VEGF) were applied on paraffin sections.

Results: Strong cytoplasmic and nuclear S100 immunoreactivity (IR) was mainly expressed by alveolar epithelium and to lesser variable extent by ductal epithelium. The Golgi zone of the epithelial cells expressed a strong GalTase-IR. Myoepithelial cells displayed a strong IR for $\alpha$-SMA and Ck14, but not for S100. Vascular endothelium showed a moderate (for VEGF) to strong IR (for ACE). Discussion and conclusion: The histochemical spatial distribution of different proteins in the diverse compartments of lactating mammary tissue displayed a variable IR. The luminal epithelial cells expressed binding sites only for S100 and GalTase, reflecting their synthetic and secretory activities. The myoepithelial cells showed a distinct IR for both of Ck14 and $\alpha$-SMA; highlighting their ectodermal origin and contractile function. In addition to the VEGF-binding sites expressed by the interstitial mast cells, the vascular endothelium displayed distinct binding sites for VEGF and ACE. This may underline their key role in regulation of the mammary gland angiogenesis, circulation and immunity

\section{PP-329}

The study of ovine digital veins by means of corrosion casting method

Ahmadmahmudi MZ

Department of Clinical Sciences, Faculty of Veterinary Medicine, University of Tehran, Tehran, Iran

Investigation on vessel structures is necessary to diagnose the mechanism of laminitis. The importance of vessel structure has been shown in pathogenesis of the equine and bovine laminitis. The published studies on ovine digital veins are confined. Ovine digital vessels were studied using corrosion casting method. The hind limbs of 15 lambs of 3 months ages were separated from tarsus joint. After the dissection of dorsal metatarsal artery, normal saline and resin metacrylat were injected into the vessels. In this species the common dorsal digital and abaxial proper digital veins drain all venous blood from the claw. The dorsal proper digital vein arises from the numerous smaller veins which are joined to form the deep and superficial venous networks of coronary margin and wall. This vein delivers blood to the common dorsal digital vein. The axial proper plantar vein drained into the abaxial proper plantar vein. Two main vessels which arise from venous network of wall, sole and plantar surface of the bulb, drain into the abaxial proper plantar vein. Unlikely registered information by nickel et al and Vermount at al the main responsibility of blood supply in hoof is on the common dorsal digital, abaxial and axial proper digital veins. Previous studies suggested that axial proper digital vein also drains blood from hoof.

\section{PP-330}

\section{Liver histopathology and enzyme indicators}

\section{Valdovska A*, Pilmane $M^{* *}$}

Faculty of Veterinary Medicine, University of Agriculture of Latvia, Jelgava, Latvia*; Institute of Anatomy and Antropology, Riga Stradins University, Riga, Latvia**

Introduction and Purpose: The dystrophy of mink liver is widely distributed in mink farms of Latvia. However, clinical diagnostics of the liver dystrophy is quite difficult. The hypothesis of this study was to clarify indicators of ALAT (alanine aminotransferase) and SAP (alkaline phosphatase) as criteria in early diagnostics of mink liver injury. The goal of this study was to determine the potential correlation between the expressed functional indicators and histopathology of the liver.

Tools and Methods: Blood samples were obtained from ten minks, seropositive against the Aleutian disease. Liver injury was assessed histologically. HGF as well as the basal membrane (BM) components were established in the mink liver by immunohistochemistry, but liver apoptosis was determined by TUNEL reaction. For assessment of cytokines, a semi-quantitative counting method was used. 
Results: The results showed apoptosis of the hepatocytes in the whole area of the liver parenchyma and calculated statistical coherence shows that the influence of inflammation cells on apoptosis is not a vital one. We find the high expression of the ALAT activity at the middle (++) apoptotic cell count whereas we did not find the expression of the ALAT activity at the little (+) or significant (+++). Fatty dystrophy was observed in all mink liver samples. We established correlation between middle $(++)$ steatosis and weak HGF expression that indicate to ability of mink liver regeneration. Blood serum SAP level was increased at all mink samples. From BM components only fragmentar appearance of collagen type IV was detected in bile ducts and blood vessels.

Conclusions: There is serious BM damage and weak regeneration of liver (due to still persisting appearance of HGF) in hepatitis affected minks without any correlation between the ALAT and SAP and the level of this damage. However, ALAT/SAP activity increases during the acute necrotic/steatotic liver injury.

\section{PP-331}

Immunohistochemical studies on the epididymal duct in the dromedary camel (Camelus dromedarius)

\author{
Alkafafy $M^{*}$, Rashed $R^{* *}$, Emara $S^{*}$, Nada $M^{*}$ \\ Department of Cytology and Histology, Faculty of Veterinary Medicine, \\ Menoufiya University, Sadat City Branch, Egypt*; Department of \\ Anatomy and Embryology, Faculty of Veterinary Medicine, Menoufiya \\ University, Sadat City Branch, Egypt**
}

Introduction: The present work was conducted to underscore the spatial distribution of some biologically active proteins within the epididymal duct in the dromedary camel.

Materials and Methods: Paraffin-embedded sections from different regions (caput, corpus and cauda) were stained conventionally and by immunohistochemistry (IHC) using AvidinBiotin-Complex (ABC) method. Primary antibodies against six proteins [angiotensin converting enzyme (ACE), S100, galactosyltransferase (GalTase), alpha smooth muscle actin $(\alpha-$ SMA), connexin-43 (Cx43) and vascular endothelial growth factor (VEGF)] were used in this study.

Results and Discussion: The camel epididymal epithelium consisted of five cell populations: principal, basal, apical, dark and halo cells. Cell populations other than principal (PCs) and basal (BCs) cells showed different regional distribution. The histochemical findings revealed that there were no binding sites for VEGF or $\mathrm{Cx} 43$. PCs displayed variable IR for ACE, S100 and GalTase throughout the whole length of the duct. Whereas the apical surfaces of most (in caput) and some (in corpus) PCs expressed intense ACE-IR, those in cauda manifested alternating negative and strong positive IR. Likewise, a moderate S100-IR was exhibited by cytoplasm and nuclei of all PCs in caput, few PCs in corpus and several PCs alternating with negative ones in cauda. Conversely, only some PCs showed weak to strong GalTase-IR in the different regions. BCs and halo cells failed to express IR for almost all proteins. Apical cells (ACs) displayed strong IR for ACE, S100 and GalTase with some regional differences. Dark cells (DCs) expressed weak to moderate IR for both ACE and S100 only in caput region; however, they failed to display such IR for other proteins. The peritubular and vascular smooth muscle cells reported a strong $\alpha$-SMA-IR in all regions.

Conclusion: IHC showed region-specific topographic distribution of different cell types indicating that the caput and cauda were metabolically more active than did the corpus.

\section{PP-332}

Spot examination for assessing practical neuroanatomy knowledge - Results from Bucharest Medical School

Chirculescu M, Lungu A, Chirculescu ARM

Department of Anatomy and Embryology, C. Davila University, Bucharest, Romania

Spot examination is a controversial method to evaluate students' anatomical knowledge. A common criticism is that it requires only simple recall of information/ identification of a pinned structure, thus having little worth in global evaluation of the knowledge level. But, it gives the best quantitation of correct/wrong answers for all students examined at a time. As compared to other chapters of anatomy (loco-motor, head and neck, thorax, abdomen, pelvis), neuroanatomy seems by far the most suitable for such an examination, because identification of a structure and its level within the CNS are well correlated to function and clinical applications. As we observed that some structures were more frequently identified by our students and some other not, we performed a longitudinal analysis of the answers given during 2000-2010 exams of neuroanatomy, to one of the 4 standard sets of 36 tagged elements to identify on Weigert-stained slides, slices of fixed brains, CT/MRI images. The frequency of good, wrong and no answers was registered. The corpus calosum (but not its parts - rostrum or splenium), insula, substantia nigra, lentiform nucleus were the most frequently identified, alveus, amygdala, geniculate bodies not; mammillary bodies and colliculi were mismatched, as well as parts of the internal capsule. Scores were usually better on slices than on slides. These results allow evaluate notions to be identified in easy, moderate and difficult. The relevance of the method can be improved by asking 3 types of questions: identification, functional correlation and clinical application, as suggested by Plater and Skidmore (2010). Anyhow, neuroanatomy is the most suitable for such examination, all these 3 categories being equally relevant. ARMC is a Senior Visiting Fellow of the Anatomical Society in DPAG - Anatomy, University of Oxford, UK 
S162 Joint Meeting of Anatomical Societies, 19-22 May 2011, Bursa, Turkey

\section{PP-333}

Anatomy and histology of vomeronasal organ in the snake, Eirenis collaris

Abbasi $M^{*}$, Gharzi A**, Usefi $\mathrm{P}^{*}$

Department of Basic Sciences, School of Veterinary Medicine, Lorestan University, Khorramabad, Lorestan, Iran*; Department of Biology, Faculty of Basic Sciences, Lorestan University, Khorramabad, Lorestan, Iran**

Vomeronasal organ (VNO) in snakes is engaged in chemical communications and hunting. This organ is located in the roof of the mouth, below the nasal cavity. In contrast to mammals, in snakes, the VNO opens only into oral cavity. Environmental stimuli are collected by the forked tongue and transported into to the VNO ducts where their sensory epithelium produced nervous impulses and send to neural centers in brain. In this survey 10 snakes of Eirenis collaris were collected from their natural habitats and transferred to the laboratory where they were killed and their head were cut and detached from the rest of the body. The heads were then processed for histological examinations. Based on our anatomical and histological investigations, it was found that: like other snakes, in Eirenis collaris, the VNO is located on the roof oral cavity and beneath the nasal cavity. This organ possesses two ducts that open into oral cavity. Sensory and non-sensory epithelium is found in this organ but secretary component is absent in this species.

\section{PP-334}

\section{Morphology and histology of ovary in the lacertid lizard, Acantodactylus boskianus}

Gharzi A*, Abbasi $M^{* *}$, Yari $\mathrm{A}^{* * *}$

Department of Biology, Faculty of Basic Sciences, Lorestan University, Khorramabad, Iran*; Department of Basic Science, School of Veterinary Medicine, Lorestan University, Khorramabad, Iran**; Department of Biology, Faculty of Basic Sciences, Lorestan University, Khorramabad, Iran***

The Bosc's fringe-toed lizard Acantodactylus boskianus (lacertidae) has been recently identified in west of Iran. There is not any record concerning the existence of this taxon in other regions of Iranian plateau. In this research we studied macroscopical and microscopical characteristics of the ovaries in females of this taxon. We found that in this species ovaries are spherical, small (in average $0.4 \mathrm{~g}$ ) and located under the gut and above the kidneys where they were covered and attached to posterior side of abdominal cavity by mesovarium. Under this tissue a thin layer of epithelium surround the gonads. A single germinal bed that contains numerous oogonia and developing primordial follicles are derived from the ovarian epithelium and is found next to its hilum. The ovarian stroma which is a loose connective tissue and rich in blood vessels contains follicles at different stages of developments. The follicular epithelium at first consists of a single layer of small cells but as the oocyte enlarge this layer become a bilayer granulose consisting inner large cells with bright cytoplasm and round nucleus, and outer small cells with dark cytoplasm and oval nucleus. At final stage this bilayer structure is turned into a polymorphic granulose including small, intermediate and large pyriform cell types are formed around the oocyte. Of these, the large flask shaped, pyriform cells, characterized by the narrow protoplasmic extension are placed between inner intermediate cells and outer small cells. Surrounding the ovarian follicle, including the granulosa layer, are the thecal layers, the theca interna and theca externa, composed of connective tissue, blood vessels, fibroblasts and secretory cells.

\section{PP-335}

\section{The Anatomy of vomeronasal organ (VNO) of East Azerbaijan Grey fox}

$\underline{\text { Karimi H}^{*}}$, Emami SJ**, Tahapour $\mathrm{K}^{* *}$

Department of Veterinary Anatomy, Faculty of Veterinary Medicine, University of Tabriz, Tabriz, Iran*; Student of Veterinary Medicine, Faculty of Veterinary Medicine, University of Tabriz, Tabriz, Iran**

The most mammals have an accessory olfactory system which it called Vomeronasal organ (VNO) or Jacobson organ. Its function is to receive feremons. VNO cause perforation interesting phenomena in equinata which it is called Flehman reaction. In trhis research histology structure of VNO of East Azerbaijan grey fox were studied. 5 head of East Azerbaijan grey fox that expired by automobile accident or villagers thruma were collected. The heads were frozen and transmitted to anatomy laboratory of faculty of veterinary Medicine of university of Tabriz. Firstly, the mandibles were removed, inlet of $\mathrm{VNO}$ was detected and venoject $0.2-0.25 \mathrm{~mm}$ was entered into orifice of $\mathrm{VNO}$. The latex was injected into venoject for detection of rout of $\mathrm{VNO}$ and a sagittal section was prepared from nasal cavity with injected VNO. The length and diameter of it was measured. This research results were showed which the $\mathrm{VNO}$ orifice has not a mucosal valve it's diameter was $0.2-0.25 \mathrm{~mm}$ in beginning area and it's length was $17.46 \pm 0.33 \mathrm{~mm}$. The distance between two VNO orifices was $4.83 \pm 0.28 \mathrm{~mm}$. It is beginning $3.16 \pm 0.28 \mathrm{~mm}$ back of incisive teeth and cranially to first palatine ridge. The distance, it terminated about first and second premolar teeth. The orifice of VNO in camel has a mucosal valve. The length of $\mathrm{VNO}$ is related to length of nasal cavity completely. Also the orifice of $\mathrm{VNO}$ is in nasal cavity but it was observed in roof of mouth in fox.

\section{PP-336}

The lumbar dorsal rami of the wild pig: The intermedial branch

Kurtul I, Ates S, Ozmen E

Department of Anatomy, Faculty of Veterinary Medicine, Mustafa Kemal University, Hatay, Turkey 
This study has visualized the intermedial branch of the dorsal rami of the spinal nerves, along with the lateral and medial branches, by the ventral approach in the lumbar region in four already dead adult wild pigs. Trunks of the cadavers fixed through embalming with $10 \%$ formalin were divided longitudinally into two components at the medial plane. Upon the dissection of the 32 dorsal rami at L1-4 levels, 24 were displayed to construct three discrete branches while the remaining 8 were observed to form two typical branches. The dorsal rami were remarkably short, giving their branches in a fan-like spreading fashion. The lateral branch sent 2-3 smaller sub branches to the iliocostalis lumborum muscle, then became the cutaneous nerve. The medial branch innervated the medial component of the intertransversal muscle, finally terminating within the multifidus muscle through sending several branches. The intermedial branch directed between the lateral and medial branches into the longissumus lumborum muscle, ramifying hereby and sending no cutaneous branches to the related skin area. Four out of eight dorsal rami dissected at L5 sent off the intermedial branch with roughly similar pattern and distribution to those seen at L1-4. The dorsal branches at L6 were relatively longer, advanced dorsally, giving a constant branch presumably the intermediate branch to the caudal edge of the longissimus lumborum muscle. It then continued as the cutaneous nerve, coursed upward through the subcutaneous fascia, terminating thereby. No such branch correspondence with the medial one was observed at this level.

\section{PP-337}

\section{Assembly of the avian inner perivitelline layer - An immunohistochemical and ultrastructural study in the quail (Coturnix Japonica)}

\author{
Rodler D*, Sasanami T**, Sinowatz $\mathrm{F}^{*}$ \\ Institute of Anatomy, Histology and Embryology, Department of Veterinary \\ Sciences, University of Munich, Munich, Germany*; Department of \\ Applied Biological Chemistry, Shizuoka University, Shizuoka, Japan**
}

The avian inner perivitelline layer (IPVL) is a homologous structure of the mammalian zona pellucida, which is deposited between the granulosa cells and the oocyte during folliculogenesis. Its glycoprotein meshwork forms a three-dimensional matrix and possesses important functions for fertilization: it contributes to the species-specific binding of avian spermatozoa to the oocyte and induces the acrosomal exocytosis reaction. Contrary to the mammalian zona pellucida, the IPVL does not prevent polyspermy, which is regarded as physiologic in birds. Previous studies have shown that in the Japanese quail (Coturnix japonica) at least five glycoproteins are constituents of the perivitelline membrane. In this study, we investigate the spatio-temporal pattern of the assembly of the IPVL during folliculogenesis using immunohistochemical and ultrastructural methods. Our data clearly show that the glycoproteins are incorporated into the perivitelline membrane at distinct time points of follicular development, supporting the hypothesis that ZP2 and ZP4 form a kind of prematrix into which ZP1, ZP3, ZPD are integrated at a later stage of development.

\section{PP-338}

\section{Clinically important variations of the brachiocephalic artery}

Biasutto SN, Aliendo M, Ceccon GAF, Bortolin PA, De La Rosa M, Repetto E

Institute of Normal Anatomy, Faculty of Medical Sciences, National University of Cordoba, Cordoba, Argentina

Characteristics of vascular division are an important risk factor for arterial occlusion and causes frequent difficulties during catheterization. Not as often as carotid artery bifurcation, but the brachiocephalic artery division may also be affected by obstructive disease; and arterial catheterization finds there the main procedure obstacle. The objective was to study the morphologic elements in the brachiocephalic artery that could represent a risk factor for vascular disease or cause difficulties during invasive procedures. We used 40 fetuses, with a crown-rump length of 64.4 to $175 \mathrm{~mm}$ (12 to 22 weeks of gestation), fixed by immersion in $4 \%$ aqueous solution of formaldehyde. Only $16 \%$ were female. After dissecting the great vessels, veins were resected (internal jugular, subclavian, brachiocephalic and superior cava veins). The brachiocephalic artery was carefully dissected. The segment including brachiocephalic, right common carotid and right subclavian arteries were cut off and opened. We measured the length, width and the angle between the subclavian and carotid arteries Brachiocephalic artery length varied from 1.9 to $6.72 \mathrm{~mm}$ (mean: $3.91 \mathrm{~mm}$ ) and the artery width was between 0.5 and $5.07 \mathrm{~mm}$ (mean: $1.3 \mathrm{~mm}$ ). There was not a direct relation between fetus age and the vessel measures. Bifurcation angle was also very different from one to another specimen, varying from $24^{\circ}$ to $99^{\circ}$ (mean: $63^{\circ}$ ). Opening the arteries, we found that $20 \%$ of the brachiocephalic ones had a partial septum dividing the fused carotid and subclavian arteries. Another 20\% showed a ridge at the level of the bifurcation. Bifurcation angle of those vessels with septum and ridge was wider than the average (mean: 76.9). This is a preliminary presentation, but it shows that some brachiocephalic arteries have embryological rests that increase the risk for vascular occlusion and catheterization difficulties, and those rests are associated with wider bifurcation angle.

\section{PP-339}

\section{Abdominal wall: study of arcuate line in fetuses}

Biasutto SN, Repetto E, Aliendo M, Altamirano BR, Diz MC Institute of Normal Anatomy, Faculty of Medical Sciences, National University of Cordoba, Cordoba, Argentina

Muscles of the abdominal wall are well developed in fetuses older than 10 weeks of gestation, instead there are further changes in the structures depending on them (e.g.: inguinal canal, etc.). This study objective is to provide information about changes and variations on the arcuate line during fetal growth. The study included 26 fetuses, between 15 and 22 
weeks of gestation. Female were $31 \%$ of the total fetuses kept by immersion in $4 \%$ aqueous solution of formaldehyde. After dissecting recti abdominis muscles, they were displaced to observe the arcuate lines behind them. We measured crownrump length, xiphoid-pubis length and arcuate-pubis length. Histological study was performed to confirm the location of the arcuate lines. Crown-rump length varied from 106.5 to 185 $\mathrm{mm}$. Xiphoid-pubis length (linea alba) was 25.99 to $58.9 \mathrm{~mm}$. Arcuate-pubis length was 6.4 to 16.5 on the right side and 0 to 16.5 on the left side. We couldn't establish any relation between these measures. The most surprising difference was between crown-rump length and linea alba length. The relation between arcuate pubis length and linea alba length varied from $1 / 3$ to $1 / 6.3$, except one left side where the rectus abdominis muscle was absent and the arcuate line too. This is a preliminary presentation of this study, but our current results demonstrate that the arcuate line location varies significantly from one to another fetus, instead we could not demonstrate any relation with other measured parameters, but it is clear that its presence depends on the rectus abdominis muscle.

\section{PP-340}

\section{Role of epithelial progenitors in gastric carcinogenesis}

Karam SM, El-Marzoqee F, Branicki F

Faculty of Medicine and Health Sciences, UAE University, Al-Ain, United Arab Emirates

Advances have been made in identifying the gastric epithelial stem cells and their differentiation pathways into cell lineages secreting mucus, acid, pepsinogen and various hormones. In addition, the development of gastric cancer in genetically engineered mouse models recapitulated some events that occur during development of chronic atrophic gastritis and gastric adenocarcinoma and revealed the involvement and amplification of epithelial stem cells. To examine whether these findings also occur in humans, we have recently examined gastric mucosal tissues from informed individuals undergoing endoscopy (due to upper gastrointestinal symptoms) and gastrectomy (for gastric adenocarcinoma). Tissues were processed for morphological, immunohistochemical and western blot analysis. Eighty nine biopsies were examined and categorized as: normal (33\%), mild superficial gastritis (34\%) and severe atrophic gastritis (33\%). About $5 \%$ of the latter exhibited evidence of intestinal metaplasia. Cancer tissues obtained from three patients were examined in three regions: safe resected margin, tumor edge and tumor center. Progressive changes in mucosal thickness, dysplasia and cellular transformation were observed, and when compared with alterations in biopsies, all appeared to represent a continuum of progression toward invasive adenocarcinoma. In addition, epithelial progenitor cells were amplified during the early stages of gastric carcinogenesis. This study would advance our knowledge of gastric stem cells and would help in the design of new modalities for early detection and/or prevention of gastric cancer. (Funded by Terry Fox Foundation for Cancer Research)

\section{PP-341}

The common peroneal nerve passing between two bellies of the piriformis: a case report and short review of the piriformis syndrome

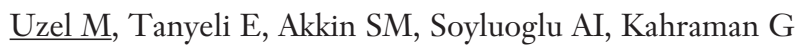

Department of Anatomy, Cerrahpasa Medical Faculty, Istanbul University, Istanbul, Turkey

The ischiadic nerve -or its components- passing between the bellies of the piriformis muscle can cause piriformis syndrome. There are many etiologies of the piriformis syndrome. It can be a result of trauma to the pelvis and buttock, leg length discrepancies, piriformis hypertrophy or myositis. The pain can be felt on the buttock region, and can radiate to the back of the thigh or lower back regions. The syndrome can be mistaken for a herniated disc, spinal stenosis, and trochanteric bursitis. There may be history of trauma, palpable mass in the gluteal region, gluteal atrophy, and pain. Although the diagnosis is mainly based on clinical findings, CT, electromyography, and MRI have been reported to have diagnostic value. Management of the syndrome includes physical therapy, ultrasound therapy, local injection therapy, and surgery. In this paper we present case of a common peroneal nerve passing through the two bellies of a double piriformis muscle on the right side of a 67-year-old male cadaver during our routine dissections. The piriformis muscle had two distinct bellies which did not unite each other any point. The common peroneal nerve passed between those two bellies and did not unite to the tibial nerve to form the ischiadic nerve. The tibial nerve passed through the infrapiriform foramen as usual. Our case corresponds to Beaton and Anson type B variation of ischiadic nerve and piriformis muscle which can cause the piriformis syndrome.

\section{PP-342}

The comparison of biomechanical properties of glass ionomer cement for repair of incudostapedial joint discontinuity of two different diameters: experimental study

Alkan $Z^{*}$, Server EA*, Yigit O*, Acioglu E*, Bekem A**, Unal $\mathrm{A}^{* *}$, Akkin $\mathrm{SM}^{* * *}$

IInd Clinic of Ear Nose and Throat, Istanbul Training and Research Hospital, Istanbul, Turkey*; Department of Metallurgy and Material Engineering, Faculty of Chemistry and Metallurgy, Yıldiz Technical University, Istanbul, Turkey**; Department of Anatomy, Cerrahpasa Medical Faculty, Istanbul University, Istanbul, Turkey***

The aim of this study was to investigate the biomechanical properties of glass ionomer cement which is used for repair of incudostapedial joint bridge of 2 different distances by compression test. A master block made from cold worked tool steel (2080) used to locate the incus and stapes with a 91 degree angle constant. Two experimental groups were composed according to the 
distances between adult's incus and stapes which were adjusted as 1.0 and $2.0 \mathrm{~mm}$. The mixture of bone cement was applied to the gap between incus and stapes and adhere them together. After the bone cement hardened, sample was taken from the master block. Diameters (D, mm) of the bone cement parts of the samples were measured. Compression tests were performed to the samples. During the test, maximum force (Fmax, N) at the time of fracture was determined. It was divided to cross sectional area (D2, mm2) to obtain strength ( $\mathrm{Rmax}, \mathrm{MPa}$ ) of the adhesion. The outputs of the compression tests, which were stated as compression force and compression strength, were compared. Finally, fracture surface images were taken by using scanning electron microscope (SEM) and investigated according to fracture formation. Group 1 and 2, each have 15 samples were evaluated separately and irrelevant values were excluded from the study. Thus, 6 samples for each group were not considered. It was seen on those samples that fracture occurred between the interface of bone cement and bone, not in bone cement. The average Fmax was 5.4217 N for Group 1, and 6.5311 N for Group 2. Their diameters were approximately $1.76 \mathrm{~mm}$ and $2.18 \mathrm{~mm}$ for Group 1 and 2, respectively. Rmax was calculated as 2.2433 for Group 1 , and 1.6711 for Group 2. No significant difference was found between two groups in terms of Fmax $(p=0.312)$, D value of Group 2 was significantly higher than Group 1 ( $\mathrm{p}=0.006)$. However, Rmax of Group 1 were significantly higher than Group 2 ( $\mathrm{p}=0.042)$. The fragility of bone cement used in $2 \mathrm{~mm}$ gap increased $25.5 \%$ compared to $1 \mathrm{~mm}$ gap. We speculate that bone cement use in the repair of short incudostapedial defects might be safer.

\section{PP-343}

Effects of mobile phone exposure to ventral cochlear nucleus in the adult female rat: a stereological and histopathological study

Tumkaya L*, Kalkan $\mathrm{Y}^{*}$, Bas $\mathrm{O}^{* *}$, Bostan $\mathrm{H}^{* * *}$

Department of Histology and Embryology, Rize University School of Medicine, Rize, Turkey*; Department of Anatomy, Rize University School of Medicine, Rize, Turkey**; Department of Anaesthesiology, Rize University School of Medicine, Rize, Turkey***

Background: Mobile phone usage is so widespread that its effects on the central nervous system have to be investigated. The mobile communications corporations are concerned about the effects of electromagnetic fields on human health. It is very likely that the most susceptible area to mobile phone irradiation is the cochlear nucleus. The aims of this study were to investigate the effect of exposure to mobile phone on the number of neuron cells and to assess histopathological changes in the ventral cochlear nucleus (VCN) of the adult female rats.

Materials and Methods: A total of 12 female Wistar albino rats were used. The study was performed in two groups of rats.
The first group was exposed to mobile phone $1 \mathrm{~h} /$ day for 45 days, while the control group remained unexposed. The mobile phone was left open the speaker facing the rat cage from approximately $50 \mathrm{~cm}$. After perfusion fixation, brainstems (six in each group) were processed with routine paraffin histology and cut exhaustively in the coronal plane with a nominal section thickness of $40 \mu \mathrm{m}$. The optical fractionator method was used for determining the total number of neurons. They were stained with cresyl fast violet, hematoxylin-eosin and photographed.

Results: The exposed group showed statistically significant loss of VCN neurons versus controls. A semi-quantitative analysis showed that the number of dark cells was higher in exposed rats than that of the controls.

Discussion: These results show that decrease mobile phone exposure is associated with neural degeneration, even at a distance of $0.5 \mathrm{M}$ away from the source.

\section{PP-344}

\section{Effects of mobile phone exposure to hippocampus in} the adult female rat: a stereological, histopathological and behavioural study

Tumkaya $\mathrm{L}^{*}$, Kalkan $\mathrm{Y}^{*}$, Bas $\mathrm{O}^{* *}$, Bostan $\mathrm{B}^{* * *}$

Department of Histology and Embryology, Rize University School of Medicine, Rize, Turkey*; Department of Anatomy, Rize University School of Medicine, Rize, Turkey**; Department of Anaesthesiology, Rize University School of Medicine, Rize, Turkey***

Background: Today, more than \% 80 of the population in the world uses mobile phones. The rapid development of mobile communications contributes to the general argument and concern about the effects of electromagnetic fields (EMFs) on human health. Studies reported that women use mobile phones more and longer than men. Therefore, women are more exposed to EMFs than men. The aims of the current study were to investigate the effect of exposure to mobile phone EMFs on the number of neuron cells, learning test and histopathological.

Materials and Methods: Fourteen female Wistar albino rats were used. The study was performed in two groups $(n=7$ in each group) of rats. The first group was exposed to mobile phone 1 h/day for 45 days, while the control group remained unexposed. Rats were tested by passive avoidance training test (PAT). After this behavioural test, the rats were sacrificed by perfusion, the brains were removed and, after the dehydration procedures, the samples were embedded in paraffin. Serial sections of 40 and $5 \mu \mathrm{m}$ thicknesses were obtained from paraffin blocks. They were stained with cresyl fast violet and hematoxylin-eosin. The optical fractionator method was used for determining the total number of neurons. 
Results: The results showed that mobile phone exposed rats were slower than controls in PAT, which was statistically significant. A semi-quantitative analysis of dark cell counts showed that the number of dark cells was higher in exposed rats than that of the controls. Exposed group showed statistically significant loss of hippocampal neurons versus controls.

Discussion: We propose that the decrease passive avoidance learning is associated with neural degeneration or a reduction in the number of synapses.

\section{PP-345}

Role of the cell surface protease seprase / fibroblast activation protein-alpha (FAP) in astroglial tumors

Hattermann $\mathrm{K}^{*}$, Held-Feindt $\mathrm{J}^{* *}$, Mentlein $\mathrm{R}^{*}$

Institute of Anatomy, University of Kiel, Kiel, Germany*; Department of Neurosurgery, University of Schleswig-Holstein Medical Center, Campus Kiel, Germany**

Purpose: The tendency to diffusely infiltrate the nervous system which prevents their complete surgical reception is a characteristic feature of glial tumors and is one of the main reasons for the low survival times of the patients. Since proteases play a decisive role in this malignant process, we investigated the expression and role of the cell surface protease seprase / fibroblast activation protein- $\alpha$ (FAP) in astroglial tumors.

Methods: FAP expression was analyzed by quantitative RTPCR, Western blot and immunohistochemistry. Cell invasion without / with silencing FAP by siRNA was assayed by Boyden chamber assays and by migration of fluorescent-labeled cells through brain slices. Brevican degradation was monitored by Western blots.

Results: FAP- $\alpha$ was elevated in whole glioblastoma tissues and in particular in most glioma cells in situ and in vitro. In glioma stem-like cells (gliomaspheres), FAP- $\alpha$ was detected at low levels; however, FAP- $\alpha$ was considerably induced upon differentiation with $10 \%$ fetal calf serum. To explore its functional role, FAP- $\alpha$ was silenced by siRNA transfection. In Boyden chamber assays, FAP- $\alpha$ silenced cells migrated similar as control cells through non-coated or Matrigel (basal lamina)-coated porous membranes, but significantly slower through membranes coated with gelatin or brevican, a major component of brain extracellular matrix. Furthermore, FAP- $\alpha$-silenced glioma cells migrated through murine brain slices much slower under the conditions tested than differentially fluorescentlabeled control cells.

Conclusion: FAP- $\alpha$ is highly expressed on the surface of glioma cells and contributes to diffuse glioma invasion through brain extracellular matrix components.
PP-346

Aortic depressor nerve ultrastructure and morphometry in chronic experimental diabetes

Oliveira FS*, Nessler RA**, Salgado $\mathrm{HC}^{*}$, Fazan VP*

School of Medicine of Ribeirão Preto - University of São Paulo, Sao Paulo, Brasil*; Central Microscopy Research Facility, The University of lowa, lowa City, lowa, USA**

Most of the reports about an altered baroreflex sensitivity attribute this alteration to the diabetic efferent neuropathy being the $\mathrm{ADN}$ (afferent arm of the baroreflex) less explored. The aim of this study was to evaluate morphological and morphometric alterations in the ADN of rats, due to chronic experimental diabetes. Wistar rats received a single intravenous injection of streptozotocin $(40 \mathrm{mg} / \mathrm{kg}) 12$ weeks $(\mathrm{N}=18)$ before the experiments. Control animals $(\mathrm{N}=15)$ received vehicle (citrate buffer). Under pentobarbital anesthesia the ADNs were isolated and had their spontaneous activity recorded. Afterwards, nerves were prepared for light and transmission electron microscopy studies as we reported previously. ADN of the chronic diabetic animals showed axonal atrophy for myelinated fibers, but alterations of the myelin sheath were more evident, such as myelin infolding and outfolding, presence of myelin bodies and very thin myelin sheath in relation to the axonal size, particularly for the small myelinated fibers. Unmyelinated fibers were more preserved in this experimental group as were most of the endoneural capillaries. Morphometry showed a larger number and density of unmyelinated fibers in diabetic animals compared to controls but fiber diameter was significantly smaller. These alterations were more evident in the distal segments of the nerves and were prevented by insulin treatment. No differences were observed in myelinated fibers number and their density as well as on the fascicular area. Our results provide morphological support to the previous reports of an altered function of the baroreflex in this model of autonomic diabetic neuropathy. Support: CNPq, FAPESP and FAEPA.

\section{PP-347}

Investigation of the effect of atropine sulphate on the fenthion-induced injury in the rat hippocampus by stereological and morphometric methods

Bas $\mathrm{O}^{*}$, Toktas $\mathrm{M}^{* *}$, Songur $\mathrm{A}^{* * *}$, Tumkaya $\mathrm{L}^{* * *}$, Yagmurca $M^{* * * * *}$

Department of Anatomy, Faculty of Medicine, Rize University, Rize, Turkey*; Department of Anatomy, Faculty of Medicine, Afyon Kocatepe University, Afyonkarahisar, Turkey**; Department of Anatomy, Faculty of Medicine, Afyon Kocatepe University, Afyonkarahisar, Turkey***; Department of Histology and Embryology, Faculty of Medicine, Rize University, Rize, Turkey****; Department of Histology and Embryology, Faculty of Medicine, Fatih University, Ankara, Turkey***** 
Objective: The aim of our study was to investigate the toxic effect of fenthion on pyramidal neurons in CA area of hippocampus using stereologic methods and light microscopy and to determine whether atropine has protective effects.

Methods: Eighteen male adult Wistar albino rats were divided into three groups. Control group received a single dose of 0.8 $\mathrm{g} / \mathrm{kg}$ subcutaneoussaline followed by $2 \mathrm{mg} / \mathrm{kg} / \mathrm{h}$ saline intraperitoneally for four hours. Fenthion group received single dose of $0.8 \mathrm{~g} / \mathrm{kg}$ subcutaneous fenthion followed by $2 \mathrm{mg} / \mathrm{kg} / \mathrm{h}$ saline intraperitoneally for four hours. Fenthion+atropine group received a single dose of $0.8 \mathrm{~g} / \mathrm{kg}$ subcutaneous fenthion followed by $2 \mathrm{mg} / \mathrm{kg} / \mathrm{h}$ atropine intraperitoneally for four hours. On the fifth day, animals were sacrificed and sequential sections of hippocampus were stained with Cresyl Fast Violet. Optical dissector/fractionator sampling method was used to estimate total neuronal number. Also, area, circumference and radius of neurons were measured.

Results: Pyramidal cell number was found to be $620803 \pm$ $30818,459291 \pm 47077$ and $551328 \pm 51710$ in control, fenthion and fenthion+atropine groups, respectively. These differences among the groups were statistically significant $(\mathrm{p}<0.05)$. Compared to fenthion group, a statistically significant decrease was observed in area, circumference and radius of neurons in fenthion+atropine group. There wereno statistically significant differences between in fenthion+atropine and control groups regarding these parameters. Swelling, pyknosis and karyolysis were observed in fenthion group. Only pyknosis was seen in fenthion+atropine group.

Conclusion: Fenthion significantly reduces total pyramidal neuronal number in hippocampus CA area, increases apoptosis, causes neuronal swelling and increase in area, circumference and radius of neurons. Toxic effects of fenthion can be reduced completely or partly by atropine.

\section{PP-348}

Identification of potential diagnostic and prognostic biomarkers in Sardinian MS patients

Sotgiu $\mathrm{MA}^{*}$, Tolu $\mathrm{P}^{*}$, Asara $\mathrm{Y}^{*}$, Farace $\mathrm{C}^{*}$, Sotgiu $\mathrm{S}^{* *}$, Bandiera $\mathrm{P}^{*}$, Montella A*, Madeddu R*

Department of Biomedical Science, University of Sassari, Sassari, Italy*; Department of Neurosciences, University of Sassari, Sassari, Italy**

Introduction: Multiple sclerosis (MS) is a chronic acquired inflammatory demyelinating disease of the central nervous system (CNS). MS lesions are characterized by inflammation, demyelination/ remyelination, axonal damage and gliosis. Injured axons release several molecules from their proximal extremity into the intercellular space. Although these molecules can be measured, at present there are no biological markers with proven reliability as measures of disease activity and progression in MS patients. The aim of this study was to see if are present informative levels of CNS-derived proteins in cerebrospinal fluid (CSF) of MS patients such that they can be considered biomarkers of disease activity. Our attention was directed to evaluate some proteins linked to axonal damage, such as neurofilament light protein (NFL), glial fibrillary acidic protein (GFAP) and $\beta$ - tubulin isophorms II and III.

Materials and Methods: For the study 51 individuals were enrolled. Of those, 29 were affected by MS (case group) and 18 by other neurological disorders than MS (control group). CSF samples, obtained by lumbar puncture, were cooled at $4^{\circ}$, centrifuged and were dot-blotted in triplicate on nitrocellulose membrane. Kruskal-Wallis test was performed to compare the differences in biomarkers among the different groups of MS and controls.

Results and Conclusions: We found that beta II-tubulin, NFL and GFAP are detectable in cerebral spinal fluid (CSF) of MS patients at levels significantly higher than controls. Identification of markers that could predict the development of MS in high-risk populations would allow for intervention strategies that may prevent evolution to definite disease.

\section{PP-349}

Presentation of quantitative morphometric study of cross sections of embryonic spinal cord using computer software

\section{$\underline{\text { Kaka G }}$}

Applied Neuroscience Research Center of Baqiyatallah Medical Sciences University, Tehran, Iran

Background: Morphometric study by computer visualization is an ideal tool for the evaluation of the embryonic development of spinal cord and other soft tissue injuries where multiple pathological features may be embedded within each other.

Methods: This study offers a histologic examination of the embryonic spinal cords quantitavely with the aid of computerassisted morphometry. For quantitative investigation of the spinal cord segments of 17-day mouse embryos were embedded in paraffin after fixation and dehydration; the paraffin blocks were serially sectioned from foramen magnum to caudal end of spinal cords at 5 microns. Total cross-sections were stained with hematoxylin and eosin methods.

Results: For white matter analysis, cross-sectional profiles were divided into six radial sectors: the dorsal funiculus (Sector 1), left and right dorsal lateral funiculi (Sectors 2 and 6), left and right ventral lateral funiculi (Sectors 3 and 5), and ventral funiculus (Sector 4). For gray matter analysis, cross-sectional profiles were also divided into six sectors: the anterior horns (Sectors 1 and 6), posterior horns (Sectors 3 and 4), intermediate area or horns 
(Sectors 2 and 5). For detection of special area of spinal cord epedymal canal and antero-posterior and transverse diameters of cross section of spinal cord are the landmarks.

Conclusion: The software can measure and calculate all region of spinal cord with high validity.

\section{PP-350}

The immunostaining of dystroglycan complex and the leakage of the blood-brain-barrier

Kalman M, Mahalek J, Sadeghian S, Farago E

Semme/weis University, Budapest, Hungary

Former studies revealed characteristic alterations of cerebral vessels following lesions: laminin which usually not detectable in the formaldehyde fixed, intact tissues (except some circumventricular organs) becomes detectable, whereas the immunoreactivity of the lamina basalis-receptor beta-dystroglycan, which delineates the vessels of intact brain, disappeares. These alterations are supposed to be indirect markers of gliovascular detachment which occurs following lesions and may have role in the impairment of blood-brain-barrier. The aims of the presents study are: a) to estimate the temporal and territorial correlations between the extravasate-penetrated area and the abovementioned cerebrovascular immunohistochemical alterations; b) to compare the stainings of circumventricular organs having 'leaky' blood-brain-barrier to that of the nonleaky intact vessels and to that of the lesioned areas with 'leaky' vessels. Cryostate lesions were performed in deep ketaminexylazine anaesthesia with a copper rod cooled surrounded with dry ice and contacted the brain surface covered only with leptomeninx. Extravasation was estimated with immunohistochemical detection of plasma-fibronectin and immunglobulins extravasated and by filling the animals with rhodamine dye at ten minutes to decapitation. Condition of vessels was estimated by the immunohistochemical staining of laminin, beta-dystroglycan as well as alpha-dystrobrevin and utriophin, also components of the gliovascular beta-dystroglycan complex and changing (disappearing and appearing, respectively) following lesions. The main results: a) the different markers mark similar areas of vessel leakage following lesion; laminin immunopositivity appears at that territory and outside, it can follow the course of the corpus callosum; beta-dystroglycan immunopositivity disappears from this area but it doesn't follow the corpus callosum; changes of vascular staining precede the leakage; b) leaky vessels occurring post-lesional and circumventricularorgan have different immunohisto-chemistries since in circumventricular organs extravasate doesn't cover the vessels (except in the case of rhodamine) and dystroglycan and dystrobrevin immunoreactivity doesn't lack.

\section{PP-351}

\section{Study of the effects of oral morphine on the fertilization of Balb/c mice}

Kaka G

Applied Neuroscience Research Center of Baqiyatallah Medical Sciences University, Tehran, Iran

Background: In addition to have side effects on adults and embryos, abused drugs by affective sex and hypophysial hormones can reduce germ cells and sexual drive resulting infertility in human. In the present study, the effects of addiction to morphine-induced infertility in Balb/c mice were investigated.

Methods: For this propose, a control $(n=6)$ and 21 experimental $(n=363)$ groups were used. Then oral morphine with initial doses $(0.01,0.05$ and $0.1 \mathrm{mg} / \mathrm{ml}$ of water) in a 3 weeks period was administered to the animals and then the animals were crossed to each other. On 17th day of pregnancy, the pregnant and non-pregnant rats were anesthetized by ether and the embryos were taken out surgically.

Results: Our results showed that: in all groups the birth chance were reduced according to the control group. The reduction was severing in the grope, which treated with 0.1 morphine.

Conclusion: It is concluded from our experiments that: morphine extremely reduced the birth chance in mice and induced in fertility in animals and this may be reliable for human.

\section{PP-352}

Determining the heavy metal levels of malleus and incus by optical emission spectroscopy (ICP-OES)

Ocal H*, Unur E*, Leblebici $Z^{* *}$, Susar $\mathrm{H}^{*}$

Department of Anatomy, Medical Faculty, Erciyes University, Kayseri, Turkey*; Department of Biology, Faculty of Science, Erciyes University, Kayseri, Turkey ${ }^{* * * *}$

There are three mobile auditory ossicles (malleus, incus, stapes) between the ear membrane in middle ear and inner ear. We used 10 malleus and 10 incus in our study. We obtained these bones from bone collection and cadaver those used for education of Erciyes University in Faculty of Medicine in Anatomy department. We determined the heavy metal content of the ossicles (ferrum, zinc, cadmium, copper, nickel, lead, manganese) by optical emission spectroscopy (ICP-OES). Heavy metal analysis processes have been carried out in the Ecology Laboratuary of Erciyes University in Science Faculty in Biology Department by the Liberty ICP-OES Sequential device called Varian. 0.5 grams of powdered samples were brought ossicles taken by adding $10 \mathrm{ml} \mathrm{65 \%} \mathrm{HNO3} \mathrm{CEM-}$ Marsh 5(CEM Corporation Mathews) was dissolved in 
microwave sample-solving device. We drew calibration charts for each element. We read each sample for three times. According to the heavy metal analysis processes, these heavy metal contents have been determined in malleus as below; ferrum 0.3060-0.2987 $\mu \mathrm{g}$ g-1, zinc 0.2503-0.1143 $\mu \mathrm{g}$ g-1, cadmium 0.0028-0.0007 $\mu \mathrm{g}$ g-1, lead 0.0483-0.0125 $\mu \mathrm{g}$ g-1, copper 0.0334-0.0092 $\mu \mathrm{g}$ g-1, manganese 0.0121-0.0214 $\mu \mathrm{g}$ g-1, nickel 0.0129-0.0045 $\mu \mathrm{g} g-1$. These heavy metal contents have been determined in incus as below; ferrum 0.1902-0.1216 $\mu \mathrm{g} \mathrm{g-1,}$ zinc 0.2572-0.1214 $\mu \mathrm{g}$ g-1, cadmium 0.0021-0.0006 $\mu \mathrm{g}$ g-1,

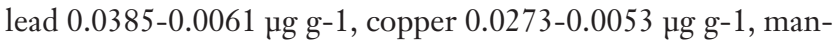
ganese 0.0043-0.0045 $\mu \mathrm{g}$ g-1, nickel 0.0129-0.0045 $\mu \mathrm{g} g-$ 1.Other research results are much higher at levels than ours. And this situation indicates that there are no heavy metal dirtiness in the malleus and incus that we made analysis.

\section{PP-353}

\section{Anatomy of the temporary teeth: proposition of a} teaching tool in 3 dimensions

\author{
Morice $\mathrm{A}^{*}$, Frapart $\mathrm{Y}^{*}$, Wolikow $\mathrm{M}^{* *}$, Tilotta $\mathrm{F}^{*}$ \\ Urdia, University Paris Descarte, Paris, France*; Dental School, \\ University Paris Descartes, Paris, France**
}

The aim of this study is to work out a teaching tool in 3 dimensions meant to teach the anatomy of temporary teeth and thus meet the needs for distance education of the odontology students at the University Paris Descartes.

Material and Method: The temporary human teeth of a dental arch have been scanned. Data capture sections have been made. The selection of regions of interest and then the choice of suitable densities have made it possible to make out the pulp duct as well as the basic anatomical pattern of each tooth.

Results: for each tooth, importing native sections into the software CTAn and CTvol has ensured the piecing together in 3 $\mathrm{D}$ of the 5 dental views of reference and the working out of videos. In a second time, improvements concerning the editing and visual layout (animations, captions) have been made.

Conclusion: The disposal of these videos on the digital learning platform is part of e-learning. Thanks to the $3 \mathrm{D}$ reconstruction which supplements the usual 2D representation of the anatomy pattern, the student is more likely to comprehend the complex detailed anatomy of human teeth.

\section{PP-354}

\section{Anatomical study of leg perforator shows pattern} complexity of inter-perforator anastomosis

Uhl JF, Ami O, Chahim M, Gillot C, Delmas V

Urdia EA4465, Laboratory of Anatomy, University Paris Descartes, Paris, France
Introduction: The study of Van Rij [JVS 1996] shows that incompetent perforator recurrence after surgery is far more common than previously recognized ( $76 \%$ at 4 years). It is due to either neovascularization or the development of perforator's incompetence rather than to poor surgery. This could be related to the anatomical complexity of the perforator pattern.

Material: About 500 limbs of 250 non-selected, non-embalmed cadaveric subjects of a high average age were used. Methods The anatomical technique of injection uses 5 steps: Catheterization of the medial marginal vein of the foot, directed towards the toes. Insertion of a tube in the femoral vein to perform washing and irrigation with soapy water. Then green neoprene latex was injected with the foot catheter (120 to 150 $\mathrm{ml}$ per limb). Dissection was started the next day. A colored segmentation of each vein was then made using direct painting.

Results: This study shows inter perforator anastomosis (IPA) in more than half of the cases. We can distinguish 2 types of IPA, vertical and horizontal: Vertical IPA is usually located in the upper half of the leg, joining the paratibial perforators. They include 2 to 5 perforator veins connected together by vertical arcades. Horizontal IPA is more frequently seen in the lower half of the leg connecting with posterior tibial and calf perforators.

Conclusion: The perforator veins of the leg usually make a complex network due to IPA. This could explain the high recurrence rate after their ligation. Better anatomical knowledge of the perforator vein pattern, but also better imaging, mapping and marking, as well as the use of intraoperative ultrasound provide scope for improvement. The surgeon therefore needs not only to treat each incompetent perforator by ligation below the aponeurosis, but also to disconnect the whole IPA network

\section{PP-355}

\section{Anatomical study of the veins of the lower limb:} 3D modelling by CT scan of the injected material

Uhl JF, Camard A, Chabert J, Munoz G, Oiseau C, Mesnier J, Heissat AS, Sygiel R, Delmas V

Urdia EA4465, Laboratory of Anatomy, University Paris Descartes, Paris, France

Objective: Build a database of anatomy of the lower limb's veins including segmented slices and a $3 \mathrm{D}$ interactive vectorial model for educationnal purpose.

Material and Methods: Injection of the venous system was made after washing with green neoprene latex. Diluted contrast iodin was added. A multislice CT (64 detectors Siemens) was performed resulting with 1000 slices of $1.2 \mathrm{~mm}$ thickness. A colored segmentation of the slices was done by using the Photoshop ${ }^{\circledR}$ CS2 software. Then a semi-automatic drawing of the boundaries was achieved with Winsurf ${ }^{\circledast}$ software (version 3.5 ) completed by a manual expertise for the nerves and musles. 
An interactive vectorial 3D model was obtained of all anatomical structures, excepting the arteries (not injected).

Results: 500 slices have been colored with labels. The 3D model includes about 50 anatomical structures with: skin, bones, muscles, nerves and veins (superficial, deep system and perforators). Finally, an anatomical dissection was performed with colored segmentation and several photographs, so we could find out the advantages and insufficiency of the 3D reconstruction and traditional anatomy by comparing them with each other.

Conclusion: The 3D modelling tools play an important role today in medical teaching and clinical training to show the complex multidimensional structure of the venous system. The virtual environment could be used as a nice educational tool, but also as preoperative designing, so it has an irreplaceable and an encouraging future in surgical education and simulation training.

\section{PP-356}

\section{3d modelling of leg veins from MRI slices: Interest of volume quantification to assess the effects of compression therapy}

Uhl JF, Ami O, Lapeyre M, Leonard CA, Touma Y, Choukroun R, Delmas V

Urdia EA4465, Laboratory of Anatomy, University Paris Descartes, Paris, France

Background: Direct mechanical compression of the veins seems to be the main mechanism of action of compression therapy in chronic venous disease. New imaging techniques allow a quantitative evaluation of the biophysical impact of compression on the $3 \mathrm{D}$ anatomy of the leg, particularly on the venous system.

Objective: To use 3D modeling and volume quantification in order to better understand the anatomical effects of compression therapy on the venous system.

Material and Methods: A total of 15 individuals were studied by T2 weighted MRI of the calf or thigh in different body positions (supine, prone, upright) before and after application of different stockings and bandages. In every case the interface pressure was measured by the use of Picopress ${ }^{\circledR}$ pressure transducer. Compression devices producing different pressures and stifness were assessed. 3D vectorial models were built with Winsurf $^{\circledast}$ software from cross sectional pictures by manual segmentation of all important anatomical structures (bone, muscles, skin, superficial and deep veins). A realistic interactive $3 \mathrm{D}$ vectorial model of the extremity was obtained for each leg showing the influence of compression on the leg's anatomy not only in a single cross-sectional slice but for the whole calf.

Results: Even low external pressure is able to induce deformations of the underlying muscle compartments. These shifts of tissue go along with changes of venous caliber and are some- times unrelated to the balance between intravenous pressure and external compression on the skin. Discrepant findings concerning the narrowing of superficial and deep veins are obtained depending on the body position.

Conclusions: 3D modeling and volume quantification renders clear graphic images of the anatomical structures of the leg demonstrating the effect of different kinds of compression on the veins.

\section{PP-357}

Real-scan: a revolutionary new tool for educational anatomy and research

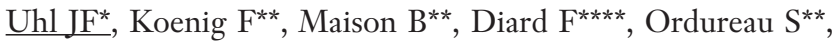
Lester $\mathrm{D}^{* * *}$, Roche $\mathrm{P}^{* * *}$, Ami $\mathrm{O}^{*}$, Delmas $\mathrm{V}^{*}$

Urdia EA4465, Laboratory of Anatomy, University Paris Descartes, Paris, France*; Useful Progress, University Paris Descartes, Paris France**; Real Scan Company, Seattle, Washington, USA***; Nvidia Company, Santa Calra, California, USA****

Purpose: To provide a powerful interactive $3 \mathrm{D}$ modeling webtool for educational anatomy and easy transmission of patient's data.

Methods: The system is based on a computing server powered by nVidia GPUs. The user upload patients DICOM by a secured connection, then he can watch and manipulate the volume rendering view inside his web browser in real-time. He is able to create movies, change LUT, navigate inside slices or export organs in triangle mesh. The web viewer is also $3 \mathrm{D}$ ready on nVidia 3DVision systems and Alioscopy screens. The website is provided by Real-scan, the web viewer software and server software is developed by the Useful Progress Company in partnership with Nvidia. The laboratory of anatomy of the Paris Descartes University (URDIA EA 4465), provides the anatomical and case reports database for teaching use.

Results: As a result, the students, doctors and patients can access and dial together with a weak tool. They don't need an expensive computer and software to use the system, only a high-speed internet connection is required. The main functions of the software are:

- Pre-configurated LUT (bones, skin, muscles)

- Full-HD interactive 3D volume rendering with stereo vision with and without glasses.

- Slices views and measures tools

- Movie generator

- Mesh exporter (to export organs in triangles for 3D softwares)

- 3D labelling system (for teaching) The anatomical database is still under construction and the softwares evolve to add new tools and functionalities. 
Conclusion: This new tool has a lot of advantages:

- Easily accessible by students for e-learning or MDs for their patients exams.

- Affordable: Today's institutions cannot afford \$200,000 visualization equipment. Here, only a high-speed internet access is needed.

- The software makes possible virtual dissections and virtual inspection for anatomy teaching

\section{PP-358}

Multiple variations of the renal and testicular vessels: a case report

Uysal II, Fazliogullari Z, Dogan NU, Ulusoy M, Karabulut AK Department of Anatomy, Selcuklu Faculty of Medicine, Selcuk University, Konya, Turkey

Multiple variations of the renal vessels, testicular veins, left testicular artery and renal pelvisses were found during routine dis- section in a 65 -year-old male cadaver. The right renal vein was formed by five large veins leaving the hilum of the kidney. There were two right testicular veins. One of the testicular veins terminated into fifth branch of the right renal vein while the other testicular vein terminated into the inferior vena cava. The left renal vein was formed from three branches. It received the left suprarenal, testicular and eleventh intercostal veins before terminating into the inferior vena cava. Right renal artery was divided into five branches before reaching the hilum of the kidney, while left renal artery was divided into six branches, one of which was upper polar artery. The left testicular artery originated from the abdominal aorta and ran upward and had a twist around the left renal vein. The artery was initially above the renal vein, but later travelled anterior and inferior to it. The rest of the course of the left testicular artery was normal. The calices were subsequently formed by branching form the renal pelvis. The caliceal branching for both renal pelvisses were in the dendritic (ramified) pattern. Such variations of the kidney might prove to be important for urologists, radiologists and surgeons. 